\title{
Abstracts from the 53rd European Society of Human Genetics (ESHG) Conference: e-Posters
}

(c) European Society of Human Genetics 2020. Modified from the conference website and published with permission 2020

Volume 28 I Supplement 1

Virtual Conference

June 6-9, 2020

Sponsorship: Publication of this supplement was sponsored by the European Society of Human Genetics. All content was reviewed and approved by the ESHG Scientific Programme Committee, which held full responsibility for the abstract selections.

Disclosure Information: In order to help readers form their own judgments of potential bias in published abstracts, authors are asked to declare any competing financial interests. Contributions of up to EUR 10 000.- (Ten thousand Euros, or equivalent value in kind) per year per company are considered "Modest". Contributions above EUR 10 000.- per year are considered "Significant".

Presenting authors are indicated with bold typeface in the contributor lists.

\section{e-Posters}

\section{E-P01 Reproductive Genetics/Prenatal Genetics}

\section{E-P01.07}

Epidemiological monitoring of congenital malformations in Yakutia from 2007 to 2018

\author{
A. I. Fedorov ${ }^{1}$, A. L. Sukhomyasova ${ }^{1,2}$, A. N. Sleptsov ${ }^{2,1}$, N. \\ R. Maksimoval
}

${ }^{1}$ Research Laboratory "Molecular Medicine and Human Genetics", Medical Institute, M. K. Ammosov NorthEastern Federal University, Yakutsk, Russian Federation, ${ }^{2}$ Medical Genetic Center, Republican Hospital №1 "National Medical Center", Yakutsk, Russian Federation

Introduction: Congenital malformations represent an urgent problem that affects the structure of infant and child mortality. In Yakutia, the prevalence of congenital malformations is recording in Register of Medical Genetic Center, Republican Hospital №1 - "National Medical Center". The purpose of epidemiological monitoring is to improve the prevention and treatment of congenital malformations.

Material and methods: The data from Medical Genetic Center Register was analyzing by the MedCalc 15.8 program.

Results: The incidence rate of congenital malformations over a 12-year period averaged 29.4 cases per 1000 newborns with a standard deviation of 2.9. It's high indicator In Russia (>20\%). At this period, the average annual number of births was 15725.7 (SD 1174.3). The high frequencies of malformations in 2012, 2017 and 2018 was observing (Table 1). The observed differences are statistically significantly $(\mathrm{p}<0.05)$.

Table 1. Congenital malformations prevalence

\begin{tabular}{ll}
\hline Years & Incidences with $95 \%$ CI \\
\hline 2007 & $0.0296(0.02694-0.03246)$ \\
2008 & $0.02734(0.02479-0.03008)$ \\
2009 & $0.02743(0.02492-0.03012)$ \\
2010 & $0.02893(0.02636-0.03168)$ \\
2011 & $0.02981(0.02723-0.03258)$ \\
2012 & $0.03336(0.03067-0.03622)$
\end{tabular}




\begin{tabular}{ll}
\hline Years & Incidences with 95\% CI \\
\hline 2013 & $0.0273(0.02485-0.02992)$ \\
2014 & $0.02704(0.02463-0.02963)$ \\
2015 & $0.02527(0.02289-0.02783)$ \\
2016 & $0.02977(0.0271-0.03263)$ \\
2017 & $0.03265(0.02973-0.03579)$ \\
2018 & $0.03464(0.03154-0.03797)$ \\
\hline
\end{tabular}

Conclusion: Thus, Yakutia is region with a high frequency of congenital malformations, and in recent years an increase the frequency of their registration. The prevalence of congenital malformations is vary at approximate intervals of 4-5 years and increase tendency may persist for several years because improved diagnostic methods.

Research is part of the project FSRG-2020-0014 "Arctic Genomics: epidemiology, heredity and pathology".

A.I. Fedorov: None. A.L. Sukhomyasova: None. A.N. Sleptsov: None. N.R. Maksimova: None.

\section{E-P01.09}

Analysis of the minor allele frequency of WNT7A (rs2639607), WNT4 (rs7521902), TWIST1 (rs4721745) and $H O X A 10$ gene (exon2) in women with presumably hereditary forms of endometriosis.

N. S. Osinovskaya ${ }^{1,2}$, N. Y. Shved ${ }^{l}$, O. V. Malysheva ${ }^{l}$, M. I. Yarmolinskaya $^{1}$, V. S. Baranov ${ }^{1}$

${ }^{1}$ Ott's Scientific Research Institute of Obstetrics, Gynecology and Reproductology, St. Petersburg, Russian Federation, ${ }^{2}$ North-Western State Medical University named after I.I. Mechnikov, Saint-Petersburg, Russian Federation

Introduction: According to GWAS, over 100 genes are associated with development of endometriosis (EM) common gynecologic disorder. The present work was devoted to the study of exon 2 polymorphism in $\mathrm{HOXA10}$ gene as well as the frequency of minor allelic variants of the WNT7A, WNT4, TWIST1 genes.

Materials and methods: DNA samples extracted from peripheral blood of 41 patients with laparoscopically proved endometriosis and 43 ones from the control cohort were used. Real-time PCR was applied to analyze allelic variants of the WNT7A, WNT4, TWIST1 genes. Sanger sequencing of the 2 nd exon of the HOXA10 gene was performed in 41 patients with proved endometriosis.

Results: The frequencies of the minor variants: rs12639607 (WNT7A), rs4721745 (TWIST1), rs7521902 (WNT4) corresponded its rate in European populations. In the HOXA10 gene, we identified two variants: rs34957925 and rs560654095. The frequency of the rs34957925 minor variant in the endometriosis group corresponded its rate in European populations. The frequency of the minor rs560654095 variant was 0.061 (G-0.00001, 1000 Genomes).

Conclusions: We did not found statistically significant differences in allele frequencies of EM patients and control groups for the polymorphic variants the WNT7A, TWIST1 and WNT4 genes. In endometriosis patients a high representation $(6 \%)$ of the rs560654095 minor allele was found. The HOXA10 gene product binds to the $\beta$-actin promoter and regulates its expression in the endometrium. Further studies are in progress to check this unusual finding. This work was financially supported by the Russian Science Foundation (No. 19-15-00108)

N.S. Osinovskaya: None. N.Y. Shved: None. O.V. Malysheva: None. M.I. Yarmolinskaya: None. V.S. Baranov: None.

\section{E-P01.12}

NFE2L2 and JUN transcription study in fetal and maternal tissues of women with pregnancy loss in the first trimester

\section{E. Mashkina, K. Kovalenko}

Southern Federal University, Rostov-on-Don, Russian Federation

Background: The cells constantly interact with many signaling molecules. This leads to dynamic changes in the spectrum of expressed genes. Nrf2 and JUN are transcription factors that are involved in maintaining intracellular redox homeostasis, which is necessary for cells proliferation and differentiation. These transcription factors activate the antioxidant defense of cells from oxidative stress. In current work, we investigated the expression of NFE2L2, JUN, $S O D 1$ genes in chorionic and decidual tissues after spontaneous abortion compared to normal first trimester pregnancies.

Materials and methods: Samples of chorionic tissue and decidua were taken after surgical termination of normally progressing pregnancies and spontaneous abortion in 5-9 week of gestation. RNA expression was analyzed using quantitative real-time PCR method. Data were analyzed using the 2- $\Delta \Delta \mathrm{CT}$ method.

Results: The transcription level of the NFE2L2, JUN, SOD1 genes is significantly higher in decidual tissue cells compared to chorion cells during normal pregnancy $(\mathrm{p}<0.0001)$. Thus, the tissue-specific transcription level of the NFE2L2, JUN, SOD1 genes has been revealed for physiological pregnancy. The correlation in the mRNA levels of the JUN, NFE2L2 and SOD1 genes was detected 
in decidua. In chorionic tissue during normal pregnancy, the level of mRNA of the $S O D 1$ gene is directly proportional to the mRNA of the NFE2L2 gene $(\mathrm{r}=0.81 \mathrm{p}=0.0002)$. With miscarriage the level of mRNA of the $J U N$ gene in the chorionic tissue is significantly higher compared to the control.

Conclusion: The results demonstrated that high JUN transcription level in chorionic tissue could be associated with miscarriage in first trimester of pregnancy.

E. Mashkina: None. K. Kovalenko: None.

\section{E-P01.14}

Prenatal diagnosis of Xq28 microdeletion including exon 10 of IKBKG gene in male fetus with increased nuchal translucency and hydrops and a family history of Incontinentia Pigmenti

\author{
S. Koka ${ }^{1}$, O. Lobel ${ }^{1}$, E. Zivi ${ }^{1}$, Z. Ben-Neriah ${ }^{1}$, A. Frumkin ${ }^{2}$, \\ O. Weiss ${ }^{l}$, S. Zeligson ${ }^{l}$, R. Segel ${ }^{l}$
}

${ }^{1}$ Shaare Zedek Medical Center, Jerusalem, Israel, ${ }^{2}$ Cytogenetics Lab, Department of Genetics, Hadassah Medical Center, Jerusalem, Israel

Introduction: Incontinentia Pigmenti (IP), is an X-linked dominant Male-lethal disorder, caused by devastating mutations in IKBKG gene. A recurrent exon 4_10 deletion in the IKBKG gene accounts for 60 to $80 \%$ of IP-causing mutations, but other point mutations along the gene as well as exon 10 (which include the zinc-finger domain) truncating mutation were described previously.

Materials and methods: Chromosomal Microarray Analysis (CMA) was performed in chorionic villi sample of aborted pregnancy of fetus with increased nuchal translucency and hydrops, and his 31 years old healthy mother.

Results: CMA of the fetus found a 34kbp deletion on Xq28 chromosome including at least exon 10 of IKBKG gene - arr[hg19]Xq28(153,792,620-153,826,362)x0. Preliminary results show that the healthy mother carries the deletion in a mosaic state. Family history includes another termination of pregnancy of a male fetus with increased nuchal translucency, two spontaneous early abortions, a girl with suspected clinical diagnosis of IP and one health boy. The recurrent exon 4_10 deletion in IKBKG was previously excluded in the symptomatic girl.

Conclusions: Here we describe a $34 \mathrm{kbp}$ deletion on Xq28 chromosome including exon 10 of IKBKG gene inherited from a healthy mother as a cause for increased nuchal translucency and hydrops in male fetus with family history suspicious for familial IP. The deletion segregation in the family is now in progress and we believe it will explain the girl phenotype as well as the obstetric history.
This report stresses the importance of molecular diagnosis in IP for genetic counseling of the entire family.

S. Koka: None. O. Lobel: None. E. Zivi: None. Z. BenNeriah: None. A. Frumkin: None. O. Weiss: None. S. Zeligson: None. R. Segel: None.

\section{E-P01.18}

Reciprocal $7 p$ deletion-7q duplication in a malformed fetus due to maternal inversion of chromosome 7 cytogenetic and microarray analysis

M. Hristova-Savova ${ }^{1}$, R. Bozhilova ${ }^{2}$, V. Peycheva ${ }^{2}$, E. Dimova ${ }^{3}, K$. Belemezova ${ }^{1}$, S. Yovinska ${ }^{1}$, M. Rizov $^{l}$, P. Chaveeva ${ }^{I}$, A. Shterev ${ }^{I}$, R. Kaneva ${ }^{2}$, I. Dimova ${ }^{l, 2}$

${ }^{1}$ SAGBAL "Dr Shterev", Sofia, Bulgaria, ${ }^{2}$ Molecular Medicine Center, Medical University Sofia, Sofia, Bulgaria, ${ }^{3}$ RWTH, Aachen, Germany

Pericentric inversions occur in human with a frequency of $1-2 \%$ of general population. These balanced chromosomal rearrangements do not cause any problem for the carrier, but during meiosis there is a risk of inversion loop formation in the offspring leading to de novo duplication, deletion or a combination of both [duplication q/deletion $\mathrm{p}$ (dup q/del p) or del q/dup p]. Significant numbers of recombinants are produced when the inverted segment size is $>50 \%$ of the total length of the affected chromosome. We report here a case of prenatal diagnostics of a fetus with multiple anomalies: severe growth retardation, enlarged third ventricle, agenesis of ductus venosus, and cyst of the blood cord. Cytogeneic analysis was performed after amniocentesis and pathologic duplication of chromosome $7 \mathrm{q}$ was revealed. To clarify the aberration we made array $\mathrm{CGH}$ analysis; the results showed combined aberrations: $3.96 \mathrm{Mbp}$ deletion of 7p (arr[hg19] 7p22.3p22.2(54,185-4,017,864)x1) and 47 Mbp duplication of 7q (7q31.1q36.3(111,668,521-159,118,566)x3). We tested the parents by cytogenetic analysis in a meantime. The mother was revealed to be a carrier of pericentric inversion in chromosome $7-46, X X, \operatorname{inv}(7)$ (p22;q31). Thus, we clarify the genetic diagnosis in the fetus - the recombinant chromosome 7 [rec(7)dup(7q)inv (7)(p22q31)mat] must have arisen through meiotic crossing-over between the inverted chromosome and the normal chromosome 7 in the maternal germline. Conclusion: Array $\mathrm{CGH}$ is the most powerful technique in determining the exact size and boundaries of the unbalanced segments in case of rearranged chromosomes and, in combination with parental cytogenetic analysis, for providing the adequate genetic counselling.

M. Hristova-Savova: None. R. Bozhilova: None. V. Peycheva: None. E. Dimova: None. K. Belemezova: None. 
S. Yovinska: None. M. Rizov: None. P. Chaveeva: None. A. Shterev: None. R. Kaneva: None. I. Dimova: None.

\section{E-P01.19}

The impact of maternal inherited thrombophilia on intra uterine growth restriction

\section{K. Kartvelishvili ${ }^{1,2}$, N. Pirtskhelani ${ }^{2,3}$, N. Kochiashvili ${ }^{2}$, L. Makhaldiani ${ }^{4}$}

${ }^{1}$ Tbilisi State Medical University, Tbilisi, Georgia, ${ }^{2}$ National Forensics Bureau/Forensic Biology (DNA) Department, Tbilisi, Georgia, ${ }^{3}$ Tbilisi Open University, Tbilisi, Georgia, ${ }^{4}$ K. Eristavi National Center of Experimental and Clinical Surgery, Tbilisi, Georgia

Introduction: Intrauterine growth restriction (IUGR) is defined as estimated fetal weight below gestational age. Inherited thrombophilia is a blood coagulation disorder, which predisposes to thrombosis. Since the placenta is rich in blood vessels, it is reasonable to infer that clot formation within the placental vasculature may result in placental insufficiency, intrauterine growth restriction and other complications.

Aim: The aim of this study was to determine the intensity of connection between inherited thrombophilia (Factor V Leiden, Prothrombin G20210A and MTHFR C677T gene mutations) and IUGR.

Materials and methods: 48 Georgian women with pregnancy complication (IUGR of unknown origin) and 100 controls (women with three or more uncomplicated pregnancies) were investigated for detection of inherited thrombophilia by PCR analyses.

Results: Relationships between IUGR and FVL (12.5\% in patients and $0 \%$ in control; $\chi 2(1, \mathrm{~N}=148)=13.028$, $\mathrm{p}=0.001)$ and Prothrombin $(8.33 \%$ in patients and $1 \%$ in control; $\left.\chi^{2}(1, \mathrm{~N}=148)=5.343, \mathrm{p}=0.038\right)$ mutations were significant. Relationship between IUGR and MTHFR mutation $(6.25 \%$ in patients and $1 \%$ in control; $\chi 2(1$, $\mathrm{N}=148)=3.399, \mathrm{p}=0.1)$ was weak. The combined double and triple mutations were seen in 4 cases, which was not seen in control group. It is important to note that 46 $(95.83 \%)$ patients had a history of other pregnancy complications and thrombosis, 19(39.53\%) patients had a history of stillbirth.

Conclusion: There are few studies concerning the association between IUGR and thrombophilia. Our study reconfirms impact of studied mutations (totally 23\%) on IUGR. Further research will help to clarify who should receive thromboprophylaxis to prevent adverse pregnancy outcomes in women with inherited thrombophilia.
K. Kartvelishvili: None. N. Pirtskhelani: None. N. Kochiashvili: None. L. Makhaldiani: None.

\section{E-P01.20}

Panels of genetic tests in infertile men with severe spermatogenic failure

\section{S. Yovinska ${ }^{l}$, M. Hristova-Savova ${ }^{l}, K$. Belemezova ${ }^{l}$, M. Rizov ${ }^{l}$, T. Milachich ${ }^{l}$, T. Timeva ${ }^{l}$, P. Andreeva ${ }^{l}$, D. Savoval, M. Yunakova ${ }^{l}$, A. Shterev ${ }^{l}$, I. Dimova ${ }^{2}$}

${ }^{1} S A G B A L$ "D Shterev", Sofia, Bulgaria, ${ }^{2} S A G B A L$ "D Shterev"; Department of Medical genetics, Medical University Sofia, Bulgaria, Sofia, Bulgaria

We offered in our clinics different kinds of genetic analyses with the aim to clarify diagnosis in men with severe spermatogenic failure. We have performed cytogenetic analysis in 770 men attending reproductive clinics (group I), 88 with confirmed diagnosis of oligoastenozoospermia (group II) and 43 with confirmed azoospermia (group III). Numerical sex chromosomal aberrations (incl. mosaicism) were found in $3.3 \%$ of group I, $3.4 \%$ of group II (mainly polysomy Y) and $9.3 \%$ of group III (mainly Klinefelter syndrome); female karyotype was diagnosed in $0.6 \%$ of men. Structural chromosomal aberrations (mainly translocations) were detected in $1.2 \%$ of group I, $3.4 \%$ of group II and none of group III. Chromosomal heteromorphism was revealed, respectively, in $13 \%, 13.6 \%$ and $11.5 \%$. DNA analysis for 10 pathogens (CT, MG, MH, UP, UU, TV, NG, TP, HS1 and 2) was performed in ejaculate of men with oligoastenozoospermia and azoospermia. The test was positive in $61.5 \%$ of azoospermic men and in $21 \%$ of oligoastenozoospermic $(\mathrm{p}<0.01)$ as double infection was revealed in $38 \%$ and $12 \%$, respectively $(\mathrm{p}<0.05)$. The statistically higher frequency was established for Ureaplasma parvum (54\% vs $15 \%)$ and Herpes simplex (23\% vs $3 \%$ ). Genotyping for 24 mutations and IVS 8 intron polymorphism in CFTR gene was performed in 52 men with severe male factor. Both delf508 mutation and IVS 85 T was found in $5.8 \%$ of the analyzed men. In conclusion, by applying all of the above mentioned analyses in 52 men with severe male factor, we were able to make diagnosis in $60 \%$ of them.

S. Yovinska: None. M. Hristova-Savova: None. K. Belemezova: None. M. Rizov: None. T. Milachich: None. T. Timeva: None. P. Andreeva: None. D. Savova: None. M. Yunakova: None. A. Shterev: None. I. Dimova: None.

\section{E-P01.21}

Recurrent congenital microcephaly: a case report 
M. N. Komnenić Radovanović ${ }^{1}$, I. Novaković ${ }^{2}$, G. $\check{C}_{\text {uturilo }}^{3,2}$, J. Ruml Stojanović ${ }^{3}$, B. Petrović ${ }^{1}$, O. KontićVučinić, ${ }^{1,2}$

${ }^{1}$ Clinic for gynecology and obstretrics, Clinical centre of Serbia, Belgrade, Serbia, ${ }^{2}$ Faculty of Medicine, University of Belgrade, Serbia, Belgrade, Serbia, ${ }^{3}$ University Children's Hospital, Belgrade, Serbia, Belgrade, Serbia

Introduction: We report the case of a couple with three lost pregnancies, two with primary congenital microcephaly (the first and the third pregnancy, both with male fetuses). The second pregnancy ended as a missed abortion. Karyotyping was perfomed to the couple and results were normal karyotypes. Couple negated the family burden, but X-linked and primary autosomal recessive microcephaly was suspected.

Materials and methods: After fetal ultrasonogram showing recurrent primary microcephaly in the third pregnancy, fetal blood sample obtained by cordocentesis. The sample was used for standard cytogenetic analysis, aCGH and clinical exome next generation sequencing.

Results: The fetal karyotyping showed a normal male karyotype $(46, \mathrm{XY})$, confirmed by aCGH analysis. Clinical exome testing and bioinformatic analysis of the genes known to be associated with microcephaly and similar pathological conditions detected pathogenic and likely pathogenic variant in the ASPM (abnormal spindle-like microcephaly-associated) gene. Pathogenic variant was ASPM c.7324C> T, known stop gained mutation with confirmed maternal origin. Likely pathogenic variant was newely described fremeshift deletion ASPM c.10148 delC, with confirmed paternal origin.

Conclusions: Comprehensive genetic testing of affected fetus showed compound heterozygous mutations in ASPM gene, designed as MCPH5 (microcephaly primary hereditary) locus. An autosomal recessive pattern of inheritance of the detected mutations is most likely involved in the formation of primary congenital microcephaly. Patients are genetically informed about the mode of inheritance of these mutations, possibility of prenatal testing, as well as of the possibility of in vitro fertilization for the purpose of preimplantation genetic diagnosis of detected mutations.

M.N. Komnenić Radovanović: None. I. Novaković: None. G. Čuturilo: None. J. Ruml Stojanović: None. B. Petrović: None. O. Kontić-Vučinić: None.

\section{E-P01.24}

PGT-A because of male 9qh chromosomal heteromorphism revealed only unbalanced embryos
M. Rizov ${ }^{l}$, K. Belemezova ${ }^{l}$, M. Hristova-Savova ${ }^{l}$, S. Yovinska ${ }^{1}$, T. Milachich ${ }^{2}$, G. Ganeva ${ }^{3}$, T. Timeva ${ }^{3,4}$, A. Shterev ${ }^{3}$ I. Dimoval

${ }^{1}$ Genetics laboratory, SAGBAL "Dr Shterev", Sofia, Bulgaria, Sofia, Bulgaria, ${ }^{2} I V F$ laboratory, SAGBAL "Dr Shterev", Sofia, Bulgaria, Sofia, Bulgaria, ${ }^{3} S A G B A L$ "Dr Shterev", Sofia, Bulgaria, Sofia, Bulgaria, "Angel Kanchev" University of Ruse, Ruse, Bulgaria

Structural chromosomal abnormalities are responsible for different reproductive issues such as infertility and recurrent pregnancy loss. Polymorphic variations are known to occur in the general population. They include varying sizes of heterochromatin blocks, satellite or repeat sequence regions and inversions. However, previous studies have reported that heterochromatin polymorphisms are more frequent in infertile men. This may be associated with infertility and unsuccessful pregnancy. Preimplantation genetic testing for aneuploidy (PGT-A) is a common method in the assisted reproduction techniques (ART) to determine the genetic status of human embryos. In the presented case we report an infertile couple with recurrent implantation failures (RIF). Karyotyping by Gbanding of cultured peripheral blood lymphocytes was performed on both patients. After the analysis, in the male partner was detected heterochromatin polymorphism, karyotype- 46, XY, 9qh+. After genetic counseling, PGD-A was used. For detection of chromosomal imbalances a laser-assisted biopsy was performed on blastocyst stage embryos followed by whole genome amplification (WGA) and next generation sequencing (NGS) was performed according to manufacturer's instructions. 6 embryos were biopsied and analyzed. None of the embryos was euploid. Various aberrations - from single to complex monosomies and trisomies, were detected. Due to the lack of an euploid embryo, transfer was not performed. This case report is a good example for the reproductive failures at carriers of chromosomal heterochromatin polymorphisms. NGS allows screening of all 24 chromosomes. It's effective, ensuring a high level of confidence in the results and subsequent selection of euploid embryos for transfer.

M. Rizov: None. K. Belemezova: None. M. HristovaSavova: None. S. Yovinska: None. T. Milachich: None. G. Ganeva: None. T. Timeva: None. A. Shterev: None. I. Dimova: None.

\section{E-P01.25}

Chromosomal centromeric polymorphisms and couple infertility 
P. Gkika ${ }^{l}$, D. Athanasiou ${ }^{l}$, P. Symeonidis ${ }^{2}$, F. Gkaitatzi $i^{2}, E$. Kontomanolis $^{2}$, N. Koutlaki ${ }^{2}$, T. S. Lialiaris ${ }^{l}$

${ }^{1}$ Demokritus University of Thrace, Medical School, Dept of Genetics, Alexandroupolis, Greece, ${ }^{2}$ Demokritus University of Thrace, Medical School, Dept of Obstetrics \& Gynecology, University Hospital of Alexandroupolis, Alexandroupolis, Greece

Introduction: A possible association between the appearance of pericentric chromosomal polymorphisms in cytogenetic examinations and idiopathic reproductive failure in northeastern Greek population was examined.

Material and methods: Lymphocyte cultures of peripheral blood from 150 individuals, mainly couples, were harvested and processed according to standard laboratory protocols. The samples were visualized using C-banding technique. At least 15 metaphases were analyzed visually per case, and 5 of them were karyotyped using MetaSystems Ikaros software and a Zeiss Axio Imager. Z.2 microscope.

Results: Chromosomal variations in centromere area of 1,9 and 16 chromosomes were identified in 115 of 150 individuals with failed reproductive clinical histories. After dividing the results by gender, a higher frequency of the chromosome 9 polymorphism was observed in women. The frequency of chromosomal polymorphisms in chromosome 1 and 16 were broadly similar for both genders. As for the couples, we observed that appearance of chromosomal centromeric polymorphisms in both partners was significantly higher, reaching a considerable number of $74 \%$.

Conclusions: Increasing evidence from our research has confirmed that infertility and subfertility are closely related to chromosomal polymorphisms as $80 \%$ of the patients were identified with a centromeric chromosomal variation. Nevertheless, their mechanism and reason of appearance is yet unclear and to explain the potential reasoning and the meaning of this phenomenon further studies will be needed.

P. Gkika: None. D. Athanasiou: None. P. Symeonidis: None. F. Gkaitatzi: None. E. Kontomanolis: None. N. Koutlaki: None. T.S. Lialiaris: None.

\section{E-P01.26}

Prenatal detection of a 3p-duplication / 6q-deletion by array-Analysis not visible in GTG-banding

U. Siebers-Renelt ${ }^{1}$, I. van Deest ${ }^{2}$, J. Steinhard ${ }^{2}$, C. MüllerHofstede $^{l}$, Y. Stratis ${ }^{l}$, A. Bohring ${ }^{l}$, A. Röpke ${ }^{l}$

${ }^{1}$ Institut für Humangenetik, University Hospital, Muenster, Germany, ${ }^{2}$ Zentrum für Pränatalmedizin, Muenster, Germany
We report on a non-consanguineous couple who presented in the $25^{\text {th }}$ week of their second pregnancy. The fetus showed mild cerebral ventriculomegaly, hypoplasia of the cerebellum and a peculiar posture of the fingers without further abnormalities. Family history revealed that his 1.5 year old sister postnatally showed a fixed posture of the fingers and a general developmental delay but no diagnostics had been done so far. In order to exclude trisomy 18, the couple decided for amniocentesis. Array-analysis showed a gain of $14.7 \mathrm{Mb}$ in the chromosomal region 3p25.1-p26.3 and a loss of $5.3 \mathrm{Mb}$ in $6 \mathrm{q} 27$ not visible in conventional karyotype (GTG-banding, 400 bands). The sister clinically exhibits a striking similarity with features already described in partial trisomy $3 p$ like hypertelorism, round face, short neck, down slanting palpebral fissures, psychomotor delay and behavioral problems. Chromosomal analysis and array-analysis were performed and revealed the same abnormalities as in the fetus. We therefore suspected a balanced translocation in one of the parents and indeed the mother carries a balanced reciprocal translocation $(46, \mathrm{XX}, \mathrm{t}(3 ; 6)(\mathrm{p} 25.1 ; \mathrm{q} 27))$. Despite the clinical variability, the parents felt reassured because they did not experience their daughter as a handicapped child. Therefore, they decided to continue pregnancy. Our case illustrates, that minor findings in prenatal ultrasound and detailed family history may be crucial in prenatal diagnosis. Moreover, it stresses the importance of array-analysis in the prenatal setting. We postulate that genetic counselling should be offered early in pregnancy to all families with retarded children.

U. Siebers-Renelt: None. I. van Deest: None. J. Steinhard: None. C. Müller-Hofstede: None. Y. Stratis: None. A. Bohring: None. A. Röpke: None.

\section{E-P01.29}

\section{Expression Profile of Complement Activation at Decidual Tissue in Patients with Recurrent Pregnancy Loss}

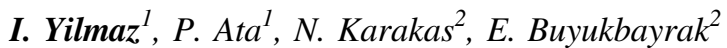

${ }^{1}$ Marmara University School of Medicine Medical Genetics, Istanbul, Turkey, ${ }^{2}$ Marmara University School of Medicine Dept of Obstetrics and Gynecology, Istanbul, Turkey

Recurrent pregnancy losses (RPL) are seen in approximately $1 \%$ of fertile women. RPL are defined as spontaneous and terminating at least two or more consecutive pregnancies, seen in $0.5-3 \%$ of all pregnancies. Damage in decidual tissue occuring with the effect of maternal antibodies, has a contribution of complement activation. Seventeen percent of women with at least 2 recurrent 
abortions and thirtyseven percent of those with more than 3 abortions were detected in idiopathic RPL patients having no autoimmune background. The complement system, is a first defense mechanism towards unwanted pathogens and host elements. Decay accelerating factor (DAF,CD55), MAC inhibitory protein (MAC-IP,CD59) and membrane co-factor protein (MCP,CD46) are major complement inhibitory proteins which are highly expressed in normal placenta. Herein, we had nine women with spontaneous abortion as our control group and seventeen women with RPL as our study group. TSH, T3, T4, and progesterone, also C3, C4 serum levels were determined. Since CD55, CD46 and CD59 of fetal trophoblast and maternal tissues are crucial to provide tissue homeostasis, we analyzed the expression of these genes using CFX real-time PCR setting. The statistical analysis according to average $\mathrm{CT}$ values were done through Qiagen GeneGlobe assay tool. As a preliminary finding of this research, CD55 expression level was reasonably elevated at decidual tissues of our cases compared to that of controls. Although, CD46 expression levels were different among two groups, the difference was not remarkable as CD55 expression ( $\mathrm{p}>0.05)$. To support pregnancy in RPL, regulation of complement activation might be crucial.

I. Yilmaz: None. P. Ata: None. N. Karakas: None. E. Buyukbayrak: None.

\section{E-P01.31}

Progesterone Receptivity and Expression of Progesterone Triggered Genes at the Decidual Tissue in Patients with Recurrent Pregnancy Loss.

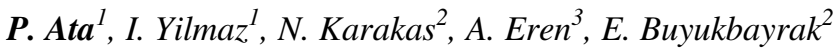

${ }^{1}$ Marmara University School of Medicine Medical Genetics, Istanbul, Turkey, ${ }^{2}$ Marmara University School of Medicine Dept of Obstetrics and Gynecology, Istanbul, Turkey, ${ }^{3}$ Koc University College of Sciences Molecular Biology and Genetics, Istanbul, Turkey

Recurrent pregnancy losses (RPL) are defined as spontaneous termination of at least two or more consecutive pregnancies. It accounts $0.5-3 \%$ of all pregnancies. Its etiology is still unknown in about half of the cases. Since progesterone stabilizes endometrial tissue, it affects decidual tissue during differentiation by changing the expressivity of different genes. Progesterone unresponsiveness due to decreased expression of its receptor and downstream genes, rather than hormone deficiency may effect the fate of pregnancy itself. Herein, nine women with spontaneous abortion as control groups, and seventeen women with RPL as our case group were analyzed; and TSH, T3,T4, progesterone, and $\mathrm{C} 3, \mathrm{C} 4$ levels were determined in the patients' sera. Patient group had higher progesterone level and lower TSH level compared to controls. We evaluated the expression level of Progesterone Receptor Gene (PGR), and downstream genes; Inositol 1,4,5-Trisphosphate Receptor Type 3 (ITPR3), Extracellular Signal-Regulated Kinase 1 (MAPK1) and Proline Rich Transmembrane Protein 2 (PRRT2) of the decidual tissue. The expression analysis using CFX real-time PCR platform were done through Qiagen GeneGlobe assay tool. Internal control gene was GAPDH for analysis. As a preliminary finding, PGR gene expression level was diminished in our case group compared to that of controls. Conversely, ITPR 3 expression was elevated and there was a slight increase in MAPK1 gene expression in patients compared to controls $(\mathrm{p}>0.05)$. As protective effect of progesterone on decidual tissue is strongly associated with expression of PGR and its downstream genes, possible future treatment targets could be identified for RPL.

P. Ata: None. I. Yilmaz: None. N. Karakas: None. A. Eren: None. E. Buyukbayrak: None.

\section{E-P01.32}

\section{Maternal pathogenic $K C N Q 1$ variant and predisposition} to recurrent fetal loss

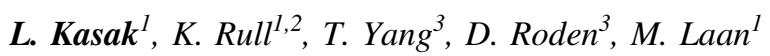

${ }^{1}$ Institute of Biomedicine and Translational Medicine, University of Tartu, Tartu, Estonia, ${ }^{2}$ Women's Clinic, Tartu University Hospital, Tartu, Estonia, ${ }^{3}$ Departments of Medicine, Pharmacology and Biomedical Informatics, Vanderbilt University Medical Center, Nashville, TN, United States

Introduction: Recurrent pregnancy loss (RPL) concerns up to $5 \%$ of women of childbearing age. Up to $25 \%$ of RPL cases remain idiopathic, suggesting a possible monogenic origin. The study aimed to identify the genetic cause of idiopathic late RPL in a family with multiple miscarriages without fetal malformations or chromosomal abnormalities.

Materials and methods: Exome sequencing was applied to three miscarried fetuses and the mother, who had experienced five live births, intersected by three early pregnancy losses, and six fetal deaths. Interestingly, 4/5 karyotyped fetal losses had a normal female karyotype, whereas all but one live-born children are male.

Results: The miscarried conceptuses do not share any rare single nucleotide variants not detected in live-born children. However, the mother carries a KCNQ1 p. Val173Asp substitution (rs199472695), previously reported in a patient with long QT syndrome (LQTS; PMID:16414944). KCNQ1 protein forms potassium 
channels and regulates the cardiac action potential duration. Consistent with a recent epidemiological study (PMID:31520628), mothers carrying pathogenic heterozygous variants in LQTS genes have an elevated risk of fetal death regardless of the carrier status of the fetus. Functional assay confirmed pathogenicity of the p.Val173Asp variant. The mutant channel significantly reduced total activating and deactivating currents, with dramatically positive shift of voltage dependence of activation by $\sim 10 \mathrm{mV}$.

Conclusions: Dysfunction of KCNQ1 contributes not only to the risk of sudden death from LQTS, but also as a maternal risk factor to RPL. The exact molecular mechanism behind this effect is still to be clarified.

Funding: Estonian Research Council grant IUT34-12

L. Kasak: None. K. Rull: None. T. Yang: None. D. Roden: None. M. Laan: None.

\section{E-P01.33}

Prenatal diagnosis of a complex skeletal dysplasia case deciphered by exome sequencing and postmortem pathological examination

G. Christopoulou ${ }^{l}$, A. Oikonomaki ${ }^{1}$, S. Samara ${ }^{l}$, A. Xefteris ${ }^{2}$, A. Konstantinidou ${ }^{3}$, P. Constantoulakis ${ }^{1}$

${ }^{1}$ Genotypos Science Labs MSA, Athens, Greece, ${ }^{2}$ Eurogenetica S.A., Thessaloniki, Greece, ${ }^{3}$ Department of Pathology, Unit of Perinatal Pathology, National \& Kapodistrian University of Athens (NKUA), Athens, Greece

Introduction: We present a prenatal and postmortem diagnosis of diastrophic dysplasia (DD) in a fetus conceived by a mother with hypochondroplasia.

Materials and methods: Prenatal testing at 13 weeks gestation was requested due to a maternal hypochondroplasia phenotype of unknown genetic background. Clinical exome sequencing was performed on DNA extracted from chorionic villi and maternal blood, using Sophia Genetics' Clinical Exome Solution v2. Following preparations according to the manufacturer's protocol, DNA libraries were sequenced on an Illumina NextSeq-500 genetic analyser. Data processing, variant calling and pre-classification were conducted by SOPHiA DDM $^{\circledR}$ bioinformatics pipelines.

Results: A FGFR3 pathogenic variant was identified in the mother, confirming her phenotype; this variant was not detected in the fetus. However, one maternally inherited pathogenic and one of unknown significance SLC26A2 variants were identified, related, according to the literature, to four distinct genetic skeletal dysplasias of various severity and prognosis. On $2^{\text {nd }}$ trimester ultrasound the fetus presented short long bones and, following clinical genetic counselling, the pregnancy was terminated.
Postmortem radiographical and histopathological findings were consistent with Diastrophic Dysplasia.

Discussion: Targeted FGFR3 mutation testing, based on the mother's phenotype, would have missed the Diastrophic Dysplasia diagnosis in the fetus, preventing informed decision for this pregnancy and proper genetic counselling for the family. Therefore, this case highlights the utility of exome sequencing for complex overlapping prenatal phenotypes such as skeletal dysplasia, and underscores the contribution of postmortem pathological examination to the phenotype - genotype correlation, which is essential for the correct interpretation of exome sequencing results.

G. Christopoulou: None. A. Oikonomaki: None. S. Samara: None. A. Xefteris: None. A. Konstantinidou: None. P. Constantoulakis: None.

\section{E-P01.34}

Homozygous hypomorphic SOX9 mutation in two 46, XY sisters without skeletal malformations

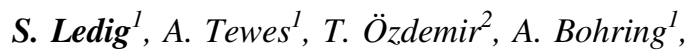 \\ P. Wieacker ${ }^{l}$
}

${ }^{1}$ Institute for Human Genetics, Münster, Germany, ${ }^{2}$ Department of Medical Genetics, Izmir, Turkey

Haploinsufficiency of the SOX9 gene in humans causes the skeletal malformation syndrome campomelic dysplasia (CD), which is a semilethal disorder characterised by congenital bowing of the long bones, cleft palate, absence of olfactory bulbs and tracts, heart and renal malformations, hypoplastic lungs, narrow thoracic cage, and delayed bone age. CD patients generally die from respiratory distress. Together with the bone disease $75 \%$ of CD-patients show testicular dysgenesis leading to sex reversal. Here, we describe two sisters with the suspicion of non-syndromic XY DSD (disorder of sexual development) and no signs of skeletal malformations. Causative mutations in SRY, NR5AI and $D H H$ have been excluded by Sanger-sequencing before whole-exome-sequencing was performed and surprisingly revealed in both sisters homozygosity for undescribed variant c. $1518 \mathrm{C}>\mathrm{G}$ p.(Leu506Val) in SOX9. The consanguine parents are heterozygous for c. $1518 \mathrm{C}>\mathrm{G}$ p.(Leu506Val) in SOX9. Functional characterization of SOX 9 c. $1518 \mathrm{C}>\mathrm{G}$ p. (Leu506Val) by luciferase assay showed no decrease of transactivating function on Col2a1 promotor in contrast to two SOX9 mutations (c.347C $>\mathrm{T}$ p.(Ala116Val) and c.358C $>\mathrm{T}$ p.(Arg120Cys)) known to be associated with CD, explaining the non-skeletal phenotype of our patients. Therefore, we suggest that SOX9 variant c. $1518 \mathrm{C}>\mathrm{G}$ p. (Leu506Val) is a hypomorphic mutation that causes $\mathrm{XY}$ DSD without raising any $S O X 9$ related skeletal phenotype. 
S. Ledig: None. A. Tewes: None. T. Özdemir: None. A. Bohring: None. P. Wieacker: None.

\section{E-P01.35}

Vascular endothelial growth factor serum level during pregnancy and $V E G F$ rs2010963 polymorphism among women with congenital heart defects in the fetus

\section{Y. Sharhorodska ${ }^{1,2,3}$, I. Haiboniuk ${ }^{l}$, A. Malahova ${ }^{l}$, U. Dorofeyeva ${ }^{3}$, O. Shkolnyk ${ }^{l}, H$. Makukh $^{l}$}

${ }^{1}$ Institute of Heredetary Pathology NAMS, Lviv, Ukraine, ${ }^{2}$ Danylo Halytsky Lviv National Medical University, Lviv, Ukraine, ${ }^{3}$ Medical Center Intersono Medicover Group, Lviv, Ukraine

The aim of the study to determine serum level of vascular endothelial growth factor (VEGF) during pregnancy as a possible diagnostic marker of congenital heart defects in offspring. Materials and methods. The serum concentration of VEGF by ELISA was measured among 24 pregnant women (term 20-30 weeks of gestation) with prenatally diagnosed congenital heart defects (CHDs) in fetus. For control group we studied the serum of 45 pregnant women without complicated genetic and obstetric history. For the analysis of rs2010963 VEGF the PCR - RFLP method was used. Results. Maternal VEGF 405GG genotype was significantly correlated with congenital heart defects of the fetus $(\mathrm{p}=0.017, \mathrm{OR}=$ 3.45 (95\% CI: 1.22 - 9.72)). In the CHD group, compared with controls, VEGF serum levels were significantly higher $(285.6 \pm 4.5$ vs. $268,0 \pm 5,2 \mathrm{pg} / \mathrm{mL}, \mathrm{p}=0.029)$. The VEGF serum level in pregnant women with genotype VEGF $405 G G$ was higher compare with another genotypes group 405GC and $405 C C$. Conclusions. Our study suggests that evaluation of VEGF serum level during pregnancy may provide prognostic data, and help identify patients who would have CHD of fetus. Mothers rs2010963 VEGF genotype may play a role in the susceptibility of conhenital heart defects in offspring.

Y. Sharhorodska: None. I. Haiboniuk: None. A. Malahova: None. U. Dorofeyeva: None. O. Shkolnyk: None. H. Makukh: None.

\section{E-P01.36}

Detection of complete delection of CRRPA gene in prenatal CGH-Array performer by eco anomalies. A case report.

\section{Perez Sanchez ${ }^{l}$, M. Bellido díaz ${ }^{l}$, T. De Haro Romero. ${ }^{l}$,} A. González Ramírez ${ }^{2}$

${ }^{1}$ Servicio Andaluz de Salud (SAS)., Granada, Spain, ${ }^{2}$ FIBAO., Granada, Spain
Introduction: Wlaker-Warburg syndrome (WWS) is clinically defined as congenital muscular dystrophy acompanied by a variety of brain and eye malformations. Its represents the most severe clinical phenotype in a spectrum of alpha-dystroglycan posttranslational proccesing abnormalities, wich share a defect in laminin bindin glycan synthesis. Homozygotics or compound heterozygotics mutations in several genes had been described as causative for WWS, but very low cases with complete homozygotics delections. Here we described two cases of prenatal diagnosis in a couple with complete CRPPA gene delection. In the first pregnancy, amniocentesis was done for genetics prenatal diagnosis, when by eco at 16 gestation week, was detected absence of cerebellar vermis, megacisterna magna with occipital bone defects and. At a second pregnancy chorionic villis sampling was performed at 12 gestation week.

Materials and methods: Karyotype was performed with the standar methods. Comparative genomic hybridation (CGH-Array) was performed with the Agilent platform, suplied by PerkinElmer.

Results: In the first fetus, the fetal Karyotype was normal $(46, \mathrm{XX})$, but the CGH-Array detected an homozygotic $354,58 \mathrm{~Kb}$ delecttion at $7 \mathrm{p} 21.2$. CGH-Array of both parents presented the same chromosomal microdelection in heterozygosis and was recomended genetic prenatal diagnosis for another pregnancies. In the next pregnancy, chorionic villis sample was analided with de same reults; normal karyotype $(46, \mathrm{XY})$ and homozygotic $354,58 \mathrm{~Kb}$ delection at $7 \mathrm{p} 21,2$. In both cases the gestation was finised.

Conclusions: The use of CGH-Array in prenatal diagnosis when ecografics anomalies are detected is the election method for detection of chromosomal microdelections and microduplications.

M. Perez Sanchez: None. M. Bellido díaz: None. T. De Haro Romero.: None. A. González Ramírez: None.

\section{E-P01.37}

Fetal mosaicism in dichorionic twin pregnancies: challenges for accurate diagnosis and results interpretation

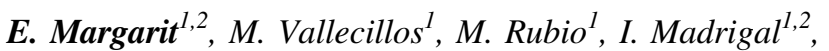 C. Badenas ${ }^{1,2}$, A. Soler ${ }^{1,2}$}

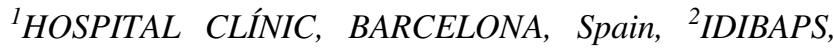
Barcelona, Spain

The risk of fetal structural abnormalities and chromosomal aneuploidy is increased in twin pregnancies compared with singleton gestations. In many cases, the chromosome 
constitution of twin fetuses is discordant, so an accurate prenatal diagnosis is needed. We report two dichorionic/diamniotic twin pregnancies presenting with ultrasound anomalies affecting only one of the fetuses (fetus B). A chorionic villus sample (CVS) was obtained separately for each one of the fetuses, allowing individual analyses to determine possible chromosomal abnormalities. In both cases, samples were first analyzed by QF-PCR to detect possible common aneuploidies. In three of the fetuses, an $\mathrm{X}$ chromosome aneuploidy in mosaic form was detected. Additional analyses by conventional cytogenetics, QF-PCR and/or array were performed first in CVS and later in amniotic fluid (AF) samples in order to interpretate the results, detecting possible confined placental mosaicism and allowing a definitive diagnosis for each fetus. Detailed results and possible embryological interpretations are presented.

\begin{tabular}{|c|c|c|}
\hline & Fetus A & Fetus B \\
\hline \multicolumn{3}{|l|}{ CASE 1} \\
\hline Ultrasound1 & $\begin{array}{l}\text { normal fetal } \\
\text { structure }\end{array}$ & $\begin{array}{l}\text { edema+increased nuchal fold } \\
+ \text { ductus agenesis }\end{array}$ \\
\hline $\begin{array}{l}\text { QF-PCR } \\
\text { (CVS) }\end{array}$ & $\begin{array}{l}\operatorname{mos} 90 \% \mathrm{X} / \\
10 \% \mathrm{XX}\end{array}$ & $\operatorname{mos} 10 \% \mathrm{X} / 90 \% \mathrm{XX}$ \\
\hline $\begin{array}{l}\text { CVS-short } \\
\text { culture }\end{array}$ & $45, X$ & - \\
\hline $\begin{array}{l}\text { CVS-long } \\
\text { culture }\end{array}$ & $\begin{array}{l}\operatorname{mos} 45, X[3] / 46 \\
X X[27]\end{array}$ & - \\
\hline Array(CVS) & - & $\operatorname{mos} 10 \% \mathrm{X} / 90 \% \mathrm{XX}$ \\
\hline QF-PCR(AF) & $\approx \mathrm{XX}$ & $\mathrm{X}$ \\
\hline AF-culture & $\begin{array}{l}\operatorname{mos} 45, X[2] / 46 \\
X X[78]\end{array}$ & $45, X[20]$ \\
\hline $\begin{array}{l}\text { Control } \\
\text { ultrasound }\end{array}$ & $\begin{array}{l}\text { normal fetal } \\
\text { structure }\end{array}$ & subcutaneous edema + ascitis \\
\hline \multicolumn{3}{|l|}{ CASE 2} \\
\hline Ultrasound 1 & $\begin{array}{l}\text { normal fetal } \\
\text { structure }\end{array}$ & $\begin{array}{l}\text { edema }+ \text { increased } \mathrm{NT}+\text { reverse } \\
\text { ductus venosus }\end{array}$ \\
\hline $\begin{array}{l}\text { QF-PCR } \\
\text { (CVS) }\end{array}$ & $\begin{array}{l}\operatorname{mos} \mathrm{X} / \\
\mathrm{XX} \text { or } \mathrm{XXX}\end{array}$ & $\mathrm{XX}$ \\
\hline $\begin{array}{l}\text { CVS-long } \\
\text { culture }\end{array}$ & $45, X$ & $46, \mathrm{XX},+11, \operatorname{der}(11 ; 22)(\mathrm{p} 10 ; \mathrm{q} 10)$ \\
\hline Array(CVS) & $\begin{array}{l}\operatorname{mos} \mathrm{X} / \\
\mathrm{XX} \text { or } \mathrm{XXX}\end{array}$ & dup11p \\
\hline QF-PCR(AF) & $\mathrm{XX}$ & - \\
\hline Array(AF) & XX & - \\
\hline $\begin{array}{l}\text { Control } \\
\text { ultrasound }\end{array}$ & $\begin{array}{l}\text { normal fetal } \\
\text { structure }\end{array}$ & no fetal activity \\
\hline
\end{tabular}

E. Margarit: None. M. Vallecillos: None. M. Rubio: None. I. Madrigal: None. C. Badenas: None. A. Soler: None.
E-P02 Sensory Disorders (Eye, Ear, Pain)

\section{E-P02.04 \\ Congenital bilateral iris coloboma in a patient with 16q23.1q23.2 deletion}

B. Aleksiūniene $\dot{e}^{l}$, B. Tumiene $\dot{e}^{l}$, R. Strupaitè-Šileikiene $\dot{e}^{2}$, A.
Utkus

${ }^{1}$ Department of Human and Medical Genetics of Institute of Biomedical Sciences, Faculty of Medicine, Vilnius University, Vilnius, Lithuania, ${ }^{2}$ Centre of Eye Diseases, Vilnius University Hospital Santaros Klinikos, Vilnius, Lithuania

Clinical and molecular characterization of rare chromosomal abnormalities helps to detect the candidate genes for congenital anomalies. Congenital ocular coloboma is a birth defect caused by abnormal development of the eye during embryogenesis. We report on a 33-year-old man with bilateral congenital coloboma of the iris at 6 o"clock. The patient has a high myopia and myopic astigmatism, BCVA of $\mathrm{BE}-0,7$. Choroidal nevi of both eyes were also noticed. He has no other specific complaints as photophobia or nictalopia, the color vision is full. There were no dysmorphic signs or developmental issues. Chromosomal microarray analysis using the HumanCytoSNP-12v2.1 BeadChip (Illumina Inc., San Diego, CA, USA) was performed and revealed a $2.96 \mathrm{Mb}$ in size deletion in the $16 \mathrm{q} 23.1 \mathrm{q} 23.2$ region, $\operatorname{arr}[\mathrm{GRCh} 37] 16 \mathrm{q} 23.1 \mathrm{q} 23.2$ $\left(78473430 \_81434676\right) \times 1$. The deleted region encompasses 12 protein-coding genes, including 5 disease-associated genes $W W O X, M A F, G C S H, B C O 1$ and GAN. Three genes WWOX, GCSH and GAN are associated with autosomal recessive disorders. $M A F$ is associated with Ayme-Gripp syndrome and various types of cataracts. $B C O 1$ encodes beta-carotene oxygenase 1 , which is a key enzyme in the production of vitamin A from carotenoids. Deficiency of this enzyme results in hypercarotenemia and secondary vitamin A deficiency. We speculate that haploinsufficiency of $\mathrm{BCOl}$ may be related to congenital bilateral iris coloboma in our patient.

B. Aleksiūnienè: None. B. Tumienè: None. R. StrupaitėŠileikienè: None. A. Utkus: None.

\section{E-P02.07}

First reported case of Doyne Honeycomb Retinal Dystrophy (Malattia Leventinese/Autosomal Dominant Drusen) in Scandinavia 
I. N. Sheyanth ${ }^{1,2,3}$, M. B. Petersen ${ }^{1,2,3}$, L. P. Kiruparajan ${ }^{4}$, S. K. Abildgaard ${ }^{4}$

${ }^{1}$ Research and Knowledge Center in Sensory Genetics, Aalborg, Denmark, ${ }^{2}$ Department of Clinical Medicine, Aalborg University, Aalborg, Denmark, ${ }^{3}$ Department of Clinical Genetics, Aalborg University Hospital, Aalborg, Denmark, ${ }^{4}$ Department of Ophthalmology, Aalborg University Hospital, Aalborg, Denmark

Introduction: Doyne Honeycomb Retinal Dystrophy (DHRD)/ Malattia Leventinese (ML) is an autosomal dominant, progressive eye disorder characterized by massive central retinal drusen often partly coalescent forming a characteristic honeycomb pattern. Debut of vision loss often occurs in mid-early adulthood and the degree varies. A single mutation in EFEMPlc.1033C >T p.(Arg345Trp) has been identified as the cause.

Materials and methods: We report a 57-year-old Scandinavian woman of non-consanguineous Danish Caucasian parents presenting with vision loss and metamorphopsia. Ophthalmological examination revealed massive hard drusen on both eyes in the macular region and nasal to the disc as well as macular hyperpigmentation. Optical coherence tomography on the left eye showed partly affected foveal configuration with slightly increased foveal thickness. The patient was clinically diagnosed with DHRD/ML and followed annually with stable conditions. Ten years later reduced visual acuity primarily on the left eye and increased atrophy with subretinal fluid were observed. Anti-vascular endothelial growth factor (antiVEGF) was administrated, but without effect. Family history revealed no other cases of reduced vision. Diagnostic next generation sequencing of seven genes (Amplexa FleckRet7 Panel) associated with flecked retina was performed at Amplexa Genetics using NovaSeq 6000. The mutation was verified by bidirectional Sanger sequencing.

Results: A known pathogenic mutation NM_001039348 (EFEMP1): c.1033C > T, p.(Arg345Trp) previously seen in relation to $\mathrm{DHRD} / \mathrm{ML}$ was identified in a heterozygote state in the index. No other causative variants were identified.

Conclusion: To our knowledge this case represents the first molecular genetically verified case of DHRD/ML in Scandinavia. Grants: The Obel Family Foundation

I.N. Sheyanth: B. Research Grant (principal investigator, collaborator or consultant and pending grants as well as grants already received); Significant; The Obel Family Foundation. M.B. Petersen: B. Research Grant (principal investigator, collaborator or consultant and pending grants as well as grants already received); Significant; The Obel Family Foundation. L.P. Kiruparajan: None. S.K. Abildgaard: None.

\section{E-P02.08}

LOXL1 and CACNA1A SNPs associated with exfoliation syndrome susceptibility in a sample of Northern Spanish population, revealed by high-resolution melting analysis.

L. Escudero-Arrarás ${ }^{1}$, A. Lara $^{l}$, M. Rodríguez-Hidalgo ${ }^{1}, T$. Alberdi $^{2}$, A. Basasoro ${ }^{2}$, I. Rodríguez-Agirretxe ${ }^{2,3,4}$, J. Mendicute ${ }^{2}$, J. Ruiz Ederra ${ }^{1,4}$

${ }^{I}$ Biodonostia Health Research Institute, San Sebastián, Spain, ${ }^{2}$ Department of Ophthalmology, Donostia University Hospital, San Sebastián, Spain, ${ }^{3}$ Instituto Clínico-Quirúrgico de Oftalmología, Bilbao, Spain, ${ }^{4}$ RETICS OFTARED, National Institute of Health Carlos III, Ministry of Economy and Competitiveness, Madrid, Spain

Introduction: Exfoliation syndrome (XFS) is a systemic disease associated with age and characterized by the formation and deposition of a whitish fibrillar substance in the anterior segment of the eye. XFS is a multifactorial disease with genetic and non-genetic factors associated. LOXL1 and CACNA1A are the main genes associated with an increased risk.

Materials and methods: High resolution melting (HRM) is a simple and high-throughput assay, which has been widely applied in sequence polymorphism identification. Our main goal was to test the suitability of HRM analysis as a genetic screening technique for XFS. We analyzed 26 XFS patients and 52 controls. We analyzed LOXL1 (rs1048661, rs3825942, rs2165241, rs16958477, rs12914489, rs11638944, rs7173049) and CACNA1A (rs4926244) genes. Detected variants were confirmed by Sanger sequencing.

Results: The allelic and genotypic frequencies of the SNPs are shown in the Table 1. Most XFS SNP were properly resolved by this technique, with the exception of rs1048661 and rs3825942. This is like due to the high percentage of $\mathrm{CG}(\sim 70 \%)$ surrounding these SNP.

Conclusion: Our method provides a genotyping strategy for identifying individuals with XFS-SNPs in a costeffective way for most variants analyzed.

LEA is supported by fellowships from Fundación Jesús de Gangoiti Barrera and from the Health Department of the Basque Government (2018111062 and MTVD19/BD/006). Supported by grants from the ISCIII and FEDER (PI18/ 00507) and BEGISARE. 
Table 1. Allelic and genotypic frequencies for the most prevalent SNPs in LOXL1 and CACNA1A.

\begin{tabular}{|c|c|c|c|c|c|c|c|c|c|c|c|}
\hline SNP & $\begin{array}{l}\text { XFS } \\
(\mathrm{n}=24)\end{array}$ & $\begin{array}{l}\text { Controls } \\
(\mathrm{n}=52)\end{array}$ & SNP & $\begin{array}{l}\mathrm{XFS} \\
(\mathrm{n}=24)\end{array}$ & $\begin{array}{l}\text { Controls } \\
(\mathrm{n}=52)\end{array}$ & SNP & $\begin{array}{l}\mathrm{XFS} \\
(\mathrm{n}=24)\end{array}$ & $\begin{array}{l}\text { Controls } \\
(\mathrm{n}=52)\end{array}$ & SNP & $\begin{array}{l}\text { XFS } \\
(\mathrm{n}=24)\end{array}$ & $\begin{array}{l}\text { Control } \\
(\mathrm{n}=52)\end{array}$ \\
\hline \multicolumn{3}{|c|}{$r s 2165241$} & \multicolumn{3}{|c|}{ rs1048661 } & \multicolumn{3}{|c|}{ rs 16958477} & \multicolumn{3}{|c|}{ rs11638944 } \\
\hline \multicolumn{3}{|c|}{ Allele } & \multicolumn{3}{|c|}{ Allele } & \multicolumn{3}{|c|}{ Allele } & \multicolumn{3}{|c|}{ Allele } \\
\hline C & 0.885 & 0.702 & G & 0.923 & 0.933 & A & 0.788 & 0.865 & C & 0.942 & 0.981 \\
\hline $\mathrm{T}$ & 0.115 & 0.298 & $\mathrm{~T}$ & 0.077 & 0.067 & C & 0.212 & 0.135 & G & 0.058 & 0.019 \\
\hline \multicolumn{3}{|c|}{ Genotype } & \multicolumn{3}{|c|}{ Genotype } & \multicolumn{3}{|c|}{ Genotype } & \multicolumn{3}{|c|}{ Genotype } \\
\hline $\mathrm{CC}$ & 0.808 & 0.673 & GG & 0.885 & 0.885 & $\mathrm{AA}$ & 0.731 & 0.577 & $\mathrm{CC}$ & 0.885 & 0.962 \\
\hline CT & 0.154 & 0.058 & GT & 0.077 & 0.096 & $\mathrm{AC}$ & 0.269 & 0.423 & CG & 0.115 & 0.038 \\
\hline TT & 0.038 & 0.269 & TT & 0.038 & 0.019 & $\mathrm{CC}$ & 0 & 0 & GG & 0 & 0 \\
\hline \multicolumn{3}{|c|}{$r s 4926244$} & \multicolumn{3}{|c|}{$r s 3825942$} & \multicolumn{3}{|c|}{$r s 12914489$} & \multicolumn{3}{|c|}{$r s 7173049$} \\
\hline \multicolumn{3}{|c|}{ Allele } & \multicolumn{3}{|c|}{ Allele } & \multicolumn{3}{|c|}{ Allele } & \multicolumn{3}{|c|}{ Allele } \\
\hline $\mathrm{T}$ & 0.971 & 0.981 & G & 1 & 0.981 & G & 0.808 & 0.827 & A & 0.981 & 0.942 \\
\hline C & 0.029 & 0.019 & A & 0 & 0.019 & A & 0.192 & 0.173 & G & 0.019 & 0.058 \\
\hline \multicolumn{3}{|c|}{ Genotype } & \multicolumn{3}{|c|}{ Genotype } & \multicolumn{3}{|c|}{ Genotype } & \multicolumn{3}{|c|}{ Genotype } \\
\hline TT & 0.942 & 0.962 & GG & 1 & 0.962 & GG & 0.615 & 0.654 & AA & 0.962 & 0.885 \\
\hline TC & 0.058 & 0.038 & GA & 0 & 0.038 & GA & 0.385 & 0.346 & AG & 0.038 & 0.115 \\
\hline $\mathrm{CC}$ & 0 & 0 & AA & 0 & 0 & AA & 0 & 0 & GG & 0 & 0 \\
\hline
\end{tabular}

L. Escudero-Arrarás: None. A. Lara: None. M. Rodríguez-Hidalgo: None. T. Alberdi: None. A. Basasoro: None. I. Rodríguez-Agirretxe: None. J. Mendicute: None. J. Ruiz Ederra: None.

\section{E-P02.11}

Overview of mutations causing early nonsyndromic hearing loss among Czech patients with excluded pathogenic mutations in GJB2 gene.

D. Šafka Brožková ${ }^{1}$, S. Poisson Markovál, J. Laštůvkováa D. Raškováa ${ }^{3}$ A. Uhrová Mészárosovál, P. Seeman ${ }^{1}$

${ }^{1}$ 2nd Faculty of Medicine, Charles University and University Hospital Motol, Prague, Czech Republic, ${ }^{2}$ Department of Medical Genetics, Masaryk Hospital in Usti nad Labem, Regional Corporation, Usti nad Labem, Czech Republic, ${ }^{3}$ Centre for Medical Genetics and Reproductive Medicine, Gennet,, Prague, Czech Republic

Nonsyndromic autosomal recessive hearing loss is a heterogeneous disease where over 80 genes has been reported to be associated with. We examined a group of patients with early/prelingual nonsyndromic hearing loss (NSHL) in which we excluded pathogenic mutations in GJB2 gene. We examined 421 unrelated patients for deletions of STRC gene with quantitative comparative fluorescent PCR (QCF PCR) and 201 unrelated patients with next generation sequencing by custom designed NSHL gene panel. We discovered the disease cause in 54 patients, from which 43 we diagnosed with NSHL panel examination $(21.4 \%)$. The most frequent type of nonsyndromic hearing loss is DFNB16, caused by biallelic mutations in the STRC gene and detected to be a cause of hearing loss in 23 patients, almost half of the clarified patients (43\%). The spectrum of STRC gene mutations was as follows; A) twelve patients were homozygotes for STRC gene deletion, B) eight were compound heterozygotes for STRC gene deletion and point inactivating mutation and $\mathrm{C}$ ) three were compound heterozygotes for point inactivating mutations. Other biallelic pathogenic mutations were detected in genes MYO15A (six patients), LOXHD1 (five patients), TMPRSS3 (five patients), CDH23 (four patients), OTOG (two patients). Other genes with biallelic pathogenic mutations were detected only once among patients. Supported by AZV 1631173A.

D. Šafka Brožková: None. S. Poisson Marková: None. J. Laštůvková: None. D. Rašková: None. A. Uhrová Mészárosová: None. P. Seeman: None.

\section{E-P02.12}

Possible digenic inheritance of mutations in OTOG and OTOGL as cause of prelingual mild hearing loss

\section{Sinnema ${ }^{1}$, V. Vernimmen ${ }^{1}, H$. Yntema ${ }^{2}$}

${ }^{1}$ Department of Clinical Genetics, MUMC, Maastricht, Netherlands, ${ }^{2}$ Department of Human Genetics, Radboud University Medical Centre, Nijmegen, Netherlands

Normal 021 false false false NL X-NONE X-NONE /* Style Definitions */ table.MsoNormalTable \{mso-stylename:Standaardtabel; mso-tstyle-rowband-size:0; msotstyle-colband-size:0; mso-style-noshow:yes; mso-stylepriority:99; mso-style-parent:""; mso-padding-alt:0cm $5.4 \mathrm{pt}$ $0 \mathrm{~cm}$ 5.4pt; mso-para-margin-top:0cm; mso-para-marginright:0cm; mso-para-margin-bottom:10.0pt; mso-para-margin-left:0cm; line-height:115\%; mso-pagination:widoworphan; font-size:11.0pt; font-family:"Calibri" "sans-serif”; mso-ascii-font-family:Calibri; mso-ascii-theme-font:minorlatin; mso-hansi-font-family:Calibri; mso-hansi-theme-font: minor-latin; mso-fareast-language:EN-US;\} Background: Autosomal recessive non-syndromic hearing loss (DFNB) is a genetically heterogeneous disorder. OTOG and $O T O G L$ were identified as autosomal recessive hearing loss genes. Patient: We describe a 20-year old girl with moderate prelingual hearing loss. Whole exome sequencing showed a pathogenic nonsense mutation in the OTOG gene $($ c. $2500 \mathrm{C}>\mathrm{T})$ and a partial gene deletion of the OTOGL gene (deletion exon 9-41). Additional Sanger sequencing of both genes did not show a second mutation in one of the genes. The father of the proband is the only family member with the same audiologic profile. Co-segregation between the hearing loss phenotype and the potential causative mutations was verified in all family members by Sanger sequencing. Father and daughter are the only family members with the combination of the OTOG and OTOGL mutation. Conclusions: We identified heterozygous mutations in OTOG 
and $O T O G L$ in a girl and her father with prelingual hearing loss. OTOG and OTOGL show striking similarities in terms of structure, expression as well as clinical phenotype. To the best of our knowledge, this is the first report of possible biallellic inheritance of mutations in $O T O G$ and OTOGL.

M. Sinnema: None. V. Vernimmen: None. H. Yntema: None.

\section{E-P02.13}

Whole exome sequencing in hearing loss Mexican family cases

M. Arenas-Sordo ${ }^{l}$, P. Linares Mendoza ${ }^{l}, J$. Doll $^{2}, P$. Bahena $^{2}$, M. Hofrichter ${ }^{2}$, B. Vona ${ }^{2}$, K. Peñuelas-Romero ${ }^{l}$, T. Haaf ${ }^{2}$

${ }^{1}$ Instituto Nacional de Rehablitacion, Mexico City, Mexico, ${ }^{2}$ Institute of Human Genetics, Julius Maximilians University, Würzburg, Germany

Introduction: Congenital hearing loss is one of the most frequent and genetically heterogeneous sensory diseases in humans. Its prevalence is $1-2$ per 1000 live newborns. Globally, the GJB2 gene is responsible for almost $20 \%$ of congenital hearing loss but in Mexico it is lower. Mutations in about 200 other genes have been associated. Current studies of new generation sequencing (NGS) has a diagnostic rate of about $40 \%$.

Material and method: Ten familial cases of bilateral sensorineural hearing loss, severe to profound, nonsyndromic, with complete audiological studies and tomographic image of the ear, were studied. Sanger sequencing of GJB2 was done and when no pathogenic variants were found in this gene, WES was performed.

Results: 1 patient presented pathogenic variants of GJB2 (c.139G $>\mathrm{T}$ and c.551G> C), the rest of the results are shown in the table. In 4 patients, the origin of the hearing loss could not be determined. The results found, for the most part, are those expected according to what is reported in other populations, especially variants in genes that cause Usher syndrome.

Conclusion: The aetiological diagnosis of hearing loss requires the clinical study of patients and their families and molecular studies, starting with the one with the highest prevalence to subsequently move to the different possibilities. It is not possible to date to know the etiology in $100 \%$ of cases.

\begin{tabular}{lllll}
\hline Gene & Variant & Protein & Zygosity & Related disease \\
\hline GPR98 & $\begin{array}{llll}\text { c.5167C }>\text { G/ } \\
\text { c.14939T }>\text { C }\end{array}$ & $\begin{array}{l}\text { p. } \\
\text { (Pro1723Ala)/ }\end{array}$ & $\begin{array}{l}\text { Compound } \\
\text { Heterozygosity }\end{array}$ & $\begin{array}{l}\text { Usher syndrome } \\
\text { type 2C }\end{array}$
\end{tabular}

\begin{tabular}{|c|c|c|c|c|}
\hline Gene & Variant & Protein & Zygosity & Related disease \\
\hline LRP5 & c. $442 \mathrm{C}>\mathrm{T}$ & $\begin{array}{l}\text { p. } \\
\text { (Val4980Ala) } \\
\text { p.(Gln148*) }\end{array}$ & Heterozygosity & $\begin{array}{l}\text { Osteopetrosis } \\
\text { Dominant } 1\end{array}$ \\
\hline$T N C$ & c. $5317 \mathrm{G}>\mathrm{A}$ & $\begin{array}{l}\text { p. } \\
\text { (Val1773Met) }\end{array}$ & Heterozygosity & DFNA 56 \\
\hline$H K 1$ & c. $60+2 \mathrm{~T}>\mathrm{A}$ & p.? & Homozygosity & $\begin{array}{l}\text { Neuropathy } \\
\text { hereditary } \\
\text { sensorimotor } \\
\text { (Russe Type) }\end{array}$ \\
\hline USHIG & c. $742 \mathrm{C}>\mathrm{T}$ & p. $\left(\mathrm{G} \ln 248^{*}\right)$ & Homozygosity & $\begin{array}{l}\text { Usher Syndrome } \\
\text { type } 1 \mathrm{G}\end{array}$ \\
\hline
\end{tabular}

M. Arenas-Sordo: None. P. Linares Mendoza: None. J. Doll: None. P. Bahena: None. M. Hofrichter: None. B. Vona: None. K. Peñuelas-Romero: None. T. Haaf: None.

E-P02.14

Mutational spectrum of the SLC26A4 gene and its contribution to the etiology of hearing loss in the indigenous peoples of Southern Siberia (Russia)

V. Y. Danilchenko ${ }^{l}$, M. V. Zytsar ${ }^{l}$, M. S. Bady-Khoo ${ }^{2}$, E. A. Maslova $^{1,3}$, A. A. Bondar, I. V. Morozov ${ }^{3,4}$, N. A. Barashkov $^{5,6}$, O. L. Posukh ${ }^{1,3}$

${ }^{1}$ Institute of Cytology and Genetics, Siberian Branch of Russian Academy of Sciences, Novosibirsk, Russian Federation, ${ }^{2}$ Perinatal Center of the Republic of Tyva, Kyzyl, Russian Federation, ${ }^{3}$ Novosibirsk State University, Novosibirsk, Russian Federation, ${ }^{4}$ Institute of Chemical Biology and Fundamental Medicine, Siberian Branch of the Russian Academy of Sciences, Novosibirsk, Russian Federation, ${ }^{5}$ Yakut Scientific Centre of Complex Medical Problems, Yakutsk, Russian Federation, ${ }^{6}$ M.K. Ammosov North-Eastern Federal University, Yakutsk, Russian Federation

Introduction: Mutations in the SLC26A4 gene (7q22-q31) lead to recessively inherited deafness (DFNB4) and Pendred syndrome. More than 500 pathogenic SLC26A4-variants are currently revealed in deaf patients in different populations worldwide. This study aimed to investigate the SLC26A4 pathogenic contribution in deafness with unknown etiology in patients belonging to indigenous peoples of Southern Siberia: Tuvinians (the Tyva Republic) and Altaians (the Altai Republic).

Materials and methods: The study included 232 patients (171 Tuvinians, 61 Altaians) with hearing loss of unknown etiology. Analysis of the SLC26A4 complete sequence including all 21 exons and flanking regions was performed by Sanger sequencing.

Results: High contribution of the SLC26A4 mutations in deafness was found in Tuvinian patients (28.2\%) in contrast 
to Altaian patients (4.3\%). Both known (c.170C >A, c.919$2 \mathrm{~A}>\mathrm{G}, \quad$ c. $2027 \mathrm{~T}>\mathrm{A}, \quad$ c. $2034+1 \mathrm{G}>\mathrm{A}, \quad$ c. $2168 \mathrm{~A}>\mathrm{G})$ and several novel pathogenic variants as well as a wide range of benign variants were found in the $S L C 26 A 4$ sequences of patients. High frequency of mutation c.919-2A $>\mathrm{G}$ in Tuvinians $(69.9 \%$ of all mutant alleles detected in patients and carrier frequency reaching to $5.1 \%$ in controls) is probably due to the founder effect.

Conclusion: This is the first study to address the SLC26A4 mutations contribution in deafness in Siberian populations. Contrast differences in the proportion of deafness caused by SLC26A4 mutations were revealed in Tuvinians and Altaians despite of their related ethnicity and close residence of these indigenous peoples of Southern Siberia. Obtained data significantly contribute to the SLC26A4 allelic diversity worldwide.

Study was supported by Projects \#0259-2019-0010-C-01, \#0324-2019-0041-C-01 and RFBR grants \#17-2906016_ofi-m, \#20-015-00328_a.

V.Y. Danilchenko: None. M.V. Zytsar: None. M.S. BadyKhoo: None. E.A. Maslova: None. A.A. Bondar: None. I.V. Morozov: None. N.A. Barashkov: None. O.L. Posukh: None.

\section{E-P02.16}

Copy number variation analysis in a group of previously unsolved patients with inherited retinal dystrophies: a comparative study

M. Rodríguez-Hidalgo ${ }^{l}$, M. Ezquerra-Inchausti ${ }^{l}$, A. Lara-López ${ }^{l}$, L. Escudero-Arrarás ${ }^{1}, M$. Galdós ${ }^{2}$, G. Garay-Aramburu ${ }^{3}$, C. Cruchaga ${ }^{4}$, C. Irigoyen ${ }^{1,5}$, J. RuizEderra $^{1,6}$

${ }^{1}$ IIS Biodonostia, San Sebastián, Spain, ${ }^{2}$ Cruces University Hospital, Bilbao, Spain, ${ }^{3}$ Araba University Hospital, Vitoria-Gasteiz, Spain, ${ }^{4}$ Washington University School of Medicine, St. Louis, MO, United States, ${ }^{5}$ Donostia University Hospital, San Sebastián, Spain, ${ }^{6}$ RETICS OFTARED, Madrid, Spain

Introduction: Inherited retinal dystrophies (IRD) are an heterogeneous group of diseases with $>250$ genes involved. Despite substantial progress in mass sequencing techniques, the genetic cause of $35-45 \%$ of the cases of IRD is still unknown. Since the participation of CNVs is involved in at least 1 in 13 patients, here we present the results of analyzing $\mathrm{CNV}$ in a cohort of Basque patients using 2 different approaches.

Materials and methods: 48 IRD patients and 2 positive controls were analyzed. We compared 2 hybridization arrays: Affymetrix CytoScan Xon arrays (ACX) and Agilent Custom arrays (ACA), with 123 IRD related genes.
Results: Affymetrix CytoScan Xon array: A mean of 56 $\mathrm{CNV}$ /patient, both positive control and 10 potentially disease-causing CNVs were detected. Agilent Custom array: A mean of $8 \mathrm{CNVs} /$ patient, only one positive control and 6 potentially disease-causing CNVs were found.

Conclusions: ACA are easier to analyze, since their results derive from only 123 IRD genes. Moreover, they include information from both exonic and intronic regions. In contrast, ACX delivers information of CNV throughout the whole genome, with most probes enriched on exonic regions. Therefore information from exons is more robust in ACX arrays. As a general conclusion, Affymetrix CytoScan Xon arrays have more sensitivity but less specificity than our customized Agilent CGH array.

MRH is supported by a fellowship from Gobierno Vasco, Spain (Pre-2019-1-0325). Work supported by grants from the ISCIII and fondos FEDER (PI17/01413), from Departamento de Salud of Gobierno Vasco (2018111062 and MTVD19/BD/006) and from BEGISARE.

M. Rodríguez-Hidalgo: None. M. Ezquerra-Inchausti: None. A. Lara-López: None. L. Escudero-Arrarás: None. M. Galdós: None. G. Garay-Aramburu: None. C. Cruchaga: None. C. Irigoyen: None. J. Ruiz-Ederra: None.

\section{E-P02.17}

How prepare a corneal epithelium for cone-related transcriptomic profile assessment in keratoconus?

\section{K. Jaskiewicz ${ }^{1}$, M. Maleszka-Kurpiel ${ }^{2,3}$, M. Rydzanicz ${ }^{4}$, R. Ploski ${ }^{4}$, M. Gajecka ${ }^{1,5}$}

${ }^{1}$ Institute of Human Genetics, Poznan, Poland, ${ }^{2}$ Optegra Eye Health Care Clinic, Poznan, Poland, ${ }^{3}$ Chair of Ophthalmology and Optometry, Poznan University of Medical Sciences, Poznan, Poland, ${ }^{4}$ Department of Medical Genetics, Medical University of Warsaw, Warsaw, Poland, ${ }^{5}$ Department of Genetics and Pharmaceutical Microbiology, Poznan University of Medical Sciences, Poznan, Poland

Introduction: Keratoconus is a multifactorial eye ectasia affecting 1 per 2000 individuals worldwide, characterized by pathological cone formation decentered from pupil axis. Because all corneal layers are involved in disease progression we aimed to establish a protocol for transcriptomic profile based on three distinct corneal epithelium regions.

Materials and methods: The 10 corneal epithelia before the corneal cross-linking procedure were stampted to maintain correct orientation for cutting on microscope slides with light microscope's control. As non-keratoconus control 5 myopia patients undergoing refractive error correction were included. From each corneal epithelium the central, middle, and periphery corneal region samples 
were proceeded based on epithelial thickness mapping and corneal tomography maps. The RNA samples were extracted using Norgen Biotek kits (Single Cell RNA Purification Kit, RNA/DNA/Protein Purification Plus Micro Kit), and RNA quality/quantity were compered (RNA 6000 Pico Kit, Agilent).

Results: Study design included evaluation of unaffected curvature of cornea and different physiological/functional states of epithelial cells from keratoconus cone and noncone corneal regions. After protocol establishment we obtained high quality RNA for RNA-seq libraries preparation.

Conclusions: The corneal material proceeded in accordance with the presented protocol will allow for detailed keratoconus epithelium study involving the phenotypical and transcriptional assessments of keratoconus progression.

Support: The National Science Centre grant no. 2018/31/ B/NZ5/03280. Co-financed by the European Social Fund within the framework of the Operational Programme Knowledge Education Development, a non-competition project 'International scholarship exchange of doctoral students and academic staff', implemented within the framework of the Action specified in the application No. POWR.03.03.03.00-00-00-PN13/18.

K. Jaskiewicz: None. M. Maleszka-Kurpiel: None. M. Rydzanicz: None. R. Ploski: None. M. Gajecka: None.

\section{E-P02.18}

Beta tubulin gene mutation in association with congenital Leber herediter amaurosis syndrome

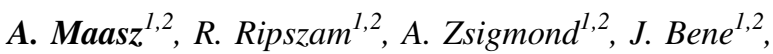 A. Gyenesei ${ }^{2}$, B. Melegh ${ }^{1,2}$, K. Hadzsiev ${ }^{1,2}$}

${ }^{1}$ University of Pecs, Department of Medical Genetics, Pecs, Hungary, ${ }^{2}$ Szentagothai Research Centre, Pecs, Hungary

Introduction: Beta-tubulin 4B isotype is one of the subunits of microtubules, which is responsible for the maintenance of microtubules stability. The beta-tubulin $4 \mathrm{~B}$ protein is encoded by TUBB $4 B$ gene located on chromosome 9. Genetic defects of microtubules have been associated with several disorders like tubulinopathies, which show huge heterogeneity. In $T U B B 4 B$ gene, only a few mutations have been identified to have implications in disorders, so far.

Patients and methods: The authors report a Hungarian family with undiagnosed rare disorder. The family members suffer from also ophthalmologic and otologic impairments. The authors analyzed the proband and her affected children and the unaffected mother of the proband using whole genome sequencing (WGS). The authors found that all of the affected family members carry the c.1171C $>$ T missense mutation in $T U B B 4 B$ gene, which causes Arg to Cys change at 391 amino acid position. The mutation was absent in the sample of the unaffected family member. The clinical evaluation of the findings reveals that the mutation fulfills the criteria of pathogenic mutation and the clinical symptoms of the affected family members matches to Leber congenital amaurosis syndrome, which has been previously correlated to this $T U B B 4 B$ mutation.

Conclusion: Our unique findings with three affected family members carrying the TUBB4B mutation highlights the importance of next generation sequencing in the discovery of new pathogenic variants and in diagnosis of rare disorders; additionally, it will be important knowledge in gene therapy even in ophthalmology and otology. This work was supported by the grant NSRP K119540 and GINOP-2.3.2-15-2016-00039.

A. Maasz: None. R. Ripszam: None. A. Zsigmond: None. J. Bene: None. A. Gyenesei: None. B. Melegh: None. K. Hadzsiev: None.

\section{E-P02.20}

Mutational analysis of the most frequent genes in Romanian patients with congenital non-syndromic hearing loss

I. Resmerital ${ }^{1}$ S. Cozma ${ }^{2}$, M. Panzaru ${ }^{l}$, E. Gorduza ${ }^{l}$, R. Popescul ${ }^{1}$ L. Radulescu ${ }^{2}$, L. Butnariu ${ }^{l}$, C. Rusu ${ }^{l}$

${ }^{1}$ University of Medicine and Pharmacy "Grigore T. Popa" Iasi - Medical Genetics Department, Iasi, Romania, ${ }^{2}$ University of Medicine and Pharmacy "Grigore T. Popa" Iasi ENT Department, Iasi, Romania

Introduction: Hearing loss is the most common congenital sensory impairment worldwide, with a genetic etiology in more than $60 \%$ of the cases. It is estimated that more than 70 loci may contribute to non-syndromic deafness, the most commonly involved genes being GJB2, GJB3, GJA1, MYO6, SLC26A4, POU3F4. The knowledge on the prevalence of mutations in a certain population allows the development of adjusted screening programs.

Materials and methods: We included in this study 144 unrelated Romanians with non-syndromic congenital severe to profound hearing loss (NSHL). All of the individuals were screened by Multiplex ligation-dependent probe amplification (MLPA) for mutations in GJB2, GJB3, GJB6, WFS1 and POU3F4 genes, and Sanger sequencing for variants in $G J B 2$ gene.

Results: We found 54 homozygotes or compound heterozygotes individuals (38\%) and 14 carriers of different mutations on $G J B 2$ gene. In the study group we found a total of 10 different mutations in GJB2 gene. The $35 \mathrm{delG}$ was the most common form of mutation that was present in 
23 out of $54(43 \%)$ mutated alleles. The next frequent types of mutation were WT313del14, IVS1 and c.71G>A. We also found 2 patients with mutations in WFS1 gene.

Conclusions: The etiology of deafness is an additional argument, besides audiological tests, for early treatment (best choice being cochlear implant) and an essential element for the correct management of the individuals. Moreover, identification of the genetic basis of hearing loss in a child facilitates correct genetic counselling with adequate molecular testing of the parents and subsequent prenatal diagnosis.

I. Resmerita: None. S. Cozma: None. M. Panzaru: None. E. Gorduza: None. R. Popescu: None. L. Radulescu: None. L. Butnariu: None. C. Rusu: None.

\section{E-P02.24}

Next-generation sequencing for germline mutation analysis in patients with retinoblastoma

D. R. Pencheva, K. Kamenarova, K. Mihova, V. Petkova, I. Dimova, M. Nacheva, V. Mitev, R. Kaneva

Genome Diagnostics Laboratory, Molecular Medicine Center, Department of Medical Chemistry and Biochemistry, Medical Faculty, Medical University of Sofia, Sofia, Bulgaria

Introduction: Retinoblastoma is the most common pediatric ocular tumor affecting the retina, initiated mainly by mutations in the tumor suppressor gene RB1. Both hereditary and sporadic forms of the disease are known.

Materials and methods: In the current study we included two Bulgarian patients diagnosed with retinoblastoma. DNA was isolated from blood. Next-generation sequencing was performed on MiSeq/Illumina platform with TruSight Cancer panel including 94 cancer related genes.

Results:We found two heterozygous probably pathogenic variants both in RB1, encoding RB transcription corepressor 1: c.2606C>T (p.Pro869Leu; rs775273085) in exon 25 and c. $1960+1 \mathrm{G}>\mathrm{C}$ in intron 19 of the RB1 gene.

Conclusions:The missense variant c.2606C $>\mathrm{T}$ (p. Pro869Leu) in exon 25 is of low frequency in the population (one heterozygote $\mathrm{C} / \mathrm{T}$ is detected in gnomAD, 1/251337). This mutation was also detected in a heterozygous condition in the mother of the patient, who was diagnosed with renal angiomyolipoma with focal hemorrhages and micro necrosis. The other variant c. $1960+1 \mathrm{G}>\mathrm{C}$ found in intron 19 is new for data bases. Single-point replacements of $G>A$ and $\mathrm{G}>\mathrm{T}$ in the same position have been shown to be pathogenic in patients with retinoblastoma. According to the program used to evaluate the effect of intron variants, replacement of c. $1960+1 \mathrm{G}>\mathrm{C}$ would affect mRNA splicing (ADA score 0.99). All of the six commonly used programs to assess the pathogenicity of genetic alterations identify the variant as potentially pathogenic.

Funding: NSP "Young Scientists and Postdoctoral Fellows"

D.R. Pencheva: None. K. Kamenarova: None. K. Mihova: None. V. Petkova: None. I. Dimova: None. M. Nacheva: None. V. Mitev: None. R. Kaneva: None.

\section{E-P02.27}

The utility of whole-exome sequencing in establishing specific diagnosis in three cases with syndromic cataracts

\section{P. Liskova, L. Dudakova, J. Moravikova, M. Magner, T. Honzik}

First Faculty of Medicine, Prague, Czech Republic

Introduction: Congenital and juvenile cataracts can be associated with a range of systemic signs. Herein we aim to report results of molecular genetic investigation in six probands with syndromic cataracts without a definitive clinical diagnosis.

Materials and methods: Whole exome sequencing (WES) was performed in all index cases. Variants with minor allele frequency less than 0.005 as per gnomAD v.2 were filtered and those located in genes listed in database Cat-Map were given a priority for further evaluation. Conventional sequencing was used to confirm the presence of the variants and for segregation analysis within the families. Variant-classification was based on the American College of Medical Genetics and Genomics guidelines.

Results: One proband carried a heterozygous variant c.3491del in COL2A1 predicted to interfere with pre-mRNA splicing. Subsequent re-evaluation of his clinical data confirmed the diagnosis of Stickler syndrome, which was also present in the mother. One proband had a de novo arising heterozygous variant c.512T $>\mathrm{G}$; p.(Leu171Trp) in MYH9 allowing to make a diagnosis of MYH9-related disease. The third case had a known heterozygous change c.284G>C; p.(Gly95Ala) in RIT1 causing Noonan syndrome. His mother was subsequently found to be also mildly affected. No possibly pathogenic variant was identified in three other probands.

Conclusions: WES allowed for providing definitive diagnosis in three probands with syndromic cataracts. Our study shows that there is a need for raising awareness among healthcare professionals regarding variable association of many syndromes with congenital or juvenile cataracts. Supported by AZV 17-30500A.

P. Liskova: None. L. Dudakova: None. J. Moravikova: None. M. Magner: None. T. Honzik: None. 


\section{E-P02.29}

Clinical exome sequencing reveals Usher syndrome pathogenic $P C D H 15$ and $A D G R V 1$ variants in two pedigrees with hearing and vision loss

\section{K. Kamenarova ${ }^{1}$, S. Cherninkova ${ }^{2}, K$. Mihova $^{l}$,} F. Shakola ${ }^{l}$, V. Mitev $^{l}$, R. Kaneva ${ }^{l}$

${ }^{1}$ Molecular Medicine Center, Medical University - Sofia, Sofia, Bulgaria, ${ }^{2}$ Department of Neurology, University Hospital Alexandrovska, Medical University - Sofia, Bulgaria, Sofia, Bulgaria

Introduction: Usher syndrome (USH) is a rare disorder causing retinitis pigmentosa (RP), and sensorineural hearing loss. Due to the phenotypic and genetic heterogeneity of this disease, the best method to screen the causative mutations is by high-throughput sequencing. By next-generation sequencing of clinical exome including known USH-causative genes, we aimed to identify the disease-causing mutation in two USH-pedigrees.

Materials and methods: Using Illumina ${ }^{\circledR}$ platform, we identified two pathogenic variants in two USH genes (PCDH15 and ADGRVI) segregating with vision and hearing loss in two unrelated Bulgarian families.

Results: In a USH-family, an affected woman was homozygous for a splice-site mutation in $P C D H 15$ (c.3983+1G>T,p.?), predicted to cause splice error - a known mutation for USH type-1F. Ophthalmological examination of the patient confirmed typical RP and prompted a corrected USH type diagnosis. Patient's unaffected parents and brother were heterozygous for the mutation. In an unrelated clinical case, two siblings with RP were homozygous for a novel nonsense mutation in ADGRVI (c.5709G>A,p.Trp1903*), predicted to create a very premature stop codon and shortened protein. Clinical follow-up subsequently confirmed pre-existing hearing loss since early childhood in the two patients, resulting in a reassigned diagnosis of USH. Asymptomatic parents and brother were heterozygous carriers of the mutation.

Conclusions: We have recruited two pedigrees with USH and found homozygous mutations in $\mathrm{PCDH} 15$ and ADGRV1. The proteins encoded by these genes are thought to interact with one another to form a network in the sensory cells of the retina and inner ear. Grant references: D01285/17.12.2019/D-230/17.12.2019/NSF/MES and КП-06H33/12/ NSF/MES.

K. Kamenarova: None. S. Cherninkova: None. K. Mihova: None. F. Shakola: None. V. Mitev: None. R. Kaneva: None.

\section{E-P02.30}

Usher syndrome type 3 in Russian population: 6 first reported cases.

M. E. Ivanova ${ }^{1,2}$, E. R. Lozier ${ }^{3}$, V. S. Kaimonov ${ }^{2}$, I. V. Mironova $^{2}$, E. A. Pomerantseva ${ }^{2}, F$. A. Konovalov ${ }^{3}$, A. A. Isaev $^{2}$

${ }^{1}$ Oftalmic, Moscow, Russian Federation, ${ }^{2}$ Genetico, Moscow, Russian Federation, ${ }^{3}$ Clinical bioinformatics laboratory, Moscow, Russian Federation

Introduction: Usher syndrome is one of the most frequent reasons of combined blindness and deafness. There are three types of Usher syndrome, type 3 is mild and can be clinically evaluated quite accurately. There is no known effective treatment of Usher syndrome type 3.

Materials and methods: 120 patients with isolated and syndromic retinitis pigmentosa participated in the study under NCT03901391 clinical trial protocol. Out of 120 cases six were CLRN1 confirmed. Complete ophthalmologic, ENT, and vestibular medical tests were done for clinical characterization. WES, Sanger sequencing were provided for genetic analysis and diagnosis confirmation.

Results: 6 patients ( 4 male, 2 female) aged $30.2 \pm 6.9$ yrs were diagnosed with CLRN1 associated retinopathy which showed classical retinitis pigmentosa bone spicules in the peripheral retina combined with hearing loss starting from early childhood. Four patients were having mild myopia and two were having emmetropia. Four of these patients were unrelated to each other and two were sibs (brother and sister). All of the patients had c.567T $>\mathrm{G}$ p. Tyr 189* mutation in homozygous state, which probably can denote the founder effect on the territory of Russia.

Conclusions: There were no previouslydescribed and published Usher syndrome type 3 clinical cases in Russia confirmed with CLRN1 gene mutations. There might be attractive perspectives for the treatment strategies for such group of patients not only because CLRN1 gene has only 6 exonsand mRNA is relatively short $(2,39 \mathrm{~kb})$, but also because mutation is homogenous and includes only c.567T $>$ G p.Tyr189* mutation in homozygous state. Funding: Charity fund Con-nection.

M.E. Ivanova: None. E.R. Lozier: None. V.S. Kaimonov: None. I.V. Mironova: None. E.A. Pomerantseva: None. F. A. Konovalov: None. A.A. Isaev: None.

\section{E-P03 Internal Organs \& Endocrinology (Lung, Kidney, Liver, Gastrointestinal)}

\section{E-P03.01}

Hyperaldosteronism in a patient with ATP1A1 deficiency 
S. Gisladottir, H. Gisladottir, J. J. Jonsson, H. T. Bjornsson

\section{University Hospital of Iceland, Reykjavik, Iceland}

We report a patient with episodes of hypertensive crisis and lactic acidosis from infancy. These episodes were found to be related to hyperaldosteronism and ill treatable with conventional blood pressure medication. At four months old, she was diagnosed with pulmonary hypertension and refractory seizures. She also has developmental delay compounded by multiple brain infarcts. She is treated with alpha-agonists and ACE-inhibitors and responds well. She is also receiving anticonvulsants and PDE inhibitors. WGS was performed for her in 2017 which identified a heterozygous missense variant, p.Asp897Asn, in the ATP1A1 gene (in the extracellular domain) that encodes for the Alpha-1 isoform of the $\mathrm{Na}(+) / \mathrm{K}(+)$-ATPase, which helps establish electrochemical gradient over plasma membranes. In 2018 similar variants were described in the ATPIAl gene in three children (all in the intramembranous domain). Their phenotype has been described as a new disease called hypomagnesemia, seizures and mental retardation 2 (MIM\#618314) but none of these patients have hyperaldosteronism. Our patient has demonstrated mild hypomagnesemia as well as seizures and developmental delay. Interestingly, somatic ATPIAl disease causing variants, have been found in aldosterone-producing adenomas. Additionally in 2018, variants in the gene were found to be a novel cause of Charcot-Marie-Tooth syndrome (all in the intracellular domain), so this gene can lead to multiple phenotypes, which could differ depending on the location of the variants in the domains of the protein. We propose that her episodes of hypertensive crisis and hyperaldosteronism may be an expansion of the phenotype or a novel additional phenotype related to this gene.

S. Gisladottir: None. H. Gisladottir: None. J.J. Jonsson: None. H.T. Bjornsson: None.

\section{E-P03.04}

Novel variants in the $A V P$-NPII gene associated with neurohypophyseal diabetes insipidus
A. Garcia $^{l}$, L. Madariaga ${ }^{l, 2,3}$, G. Pérez de Nanclares ${ }^{l}$, A. Vela ${ }^{1,3,2}$, I. Rica ${ }^{1,2}$, S. Gaztambide ${ }^{1,2,3}$, R. Martinez $^{l}$, I. Martinez de LaPiscina ${ }^{l}$, I. Urrutial ${ }^{l}$, A. Aguayo ${ }^{l}$, O. Velasco ${ }^{4}$, L. Castaño $o^{1,2,3}$

${ }^{1}$ IIS Biocruces Bizkaia, CIBERDEM, CIBERER, Barakaldo, Spain, ${ }^{2}$ Hospital Universitario Cruces, Barakaldo, Spain, ${ }^{3}$ UPV/EHU, Leioa, Spain, ${ }^{4}$ IIS Biocruces Bizkaia, Barakaldo, Spain
Introduction: Familial neurohypophyseal diabetes insipidus is a rare disease producedby a deficiency in the secretion of antidiuretic hormone and is caused by mutations inthe AVP-NPII gene.

Materials and methods: Clinical, biochemical and genetic characterization of a group ofpatients clinically diagnosed with Familial neurohypophyseal diabetes insipidus. TheAVP-NPII gene was screened for mutations by polymerase chain reaction followed bydirect Sanger sequencing in 15 different unrelated families from Spain.

Results: The 15 probands presented with polyuria and polydipsia as the most importantsymptom at the time of diagnosis. In these patients, the disease was diagnosed at amedian of 6 years of age (range: $0.75-48$ years). We observed 11 presumed pathogenicvariants in 15 families: 8 missense, 1 nonsense and 2 small deletions of 3 nucleotides. Four of the AVP-NPII variants were novel (p.(Tyr21Cys), p. (Gly45Ser), p.(Cys75Tyr),p.(Gly88Cys)).

Conclusions: We found autosomal dominant FNDI in the 15 probands studied due tolikely pathogenic variants in the $A V P$-NPII gene confirming the clinical diagnosis. Fourof the $A V P-N P I I$ variants were novel.

A. Garcia: None. L. Madariaga: None. G. Pérez de Nanclares: None. A. Vela: None. I. Rica: None. S. Gaztambide: None. R. Martinez: None. I. Martinez de LaPiscina: None. I. Urrutia: None. A. Aguayo: None. O. Velasco: None. L. Castaño: None.

\section{E-P03.05}

The intriguing role of bitter taste receptor TAS2R38 in primary ciliary dyskinesia "PCD"

\section{A. Robino ${ }^{1}$, G. Girotto $^{2}$, U. Ambrosetti ${ }^{3}$, G. Piatti ${ }^{4}$ \\ ${ }^{1}$ IRCCS-Burlo Garofolo Children Hospital, Trieste, Italy, ${ }^{2}$ IRCCS-Burlo Garofolo Children Hospital-University of Trieste, Trieste, Italy, ${ }^{3}$ IRCCS Ca' Granda, Audiology Unit- University of Milan, Milano, Italy, ${ }^{4}$ IRCCS Ca' Granda, Bronchopneumology Unit-University of Milan, Milano, Italy}

PCD is a rare genetic disease due to malfunctioning and/or altered ultrastructure of motile cilia leading to recurrent respiratory infections of upper and lower airways. Chronic rhinosinusitis and bronchiectasis are very common in PCD patients. Recently, it has been shown the presence of taste receptors in the respiratory tract and the possible involvement of bitter taste receptor TAS2R38 gene, in susceptibility to respiratory infections and rhinosinusitis. The aim of this study is to evaluate the frequency of TAS $2 R 38$ polymorphisms in PCD patients and their possible correlations with clinical outcomes of the disease. Genetic and 
phenotypic data of 35 PCD patients were collected. Clinical evaluation includes: number of respiratory infections per year and pathogens; Lund McKay score; number of lung lobes involved; modified Bhalla score. As regards genetic data, 3 polymorphisms (rs1726866,rs713598, rs10246939) within TAS2R38 gene were analyzed and patients classified as PAV/PAV, PAV/AVI and AVI/AVI. A significant difference in the distribution of TAS2R 38 haplotype between patients with and without neonatal respiratory distress emerged ( $\mathrm{p}$-value $=0.01$ ). In particular, a lower percentage of PAV/PAV individuals showed a high number of pulmonary exacerbations $(\geq 2 /$ year $)$ (pvalue $=0.04)$ compared to those with AVI/AVI and AVI/ PAV haplotypes. Moreover, no patients homozygous for PAV/PAV haplotype presented with any chronic colonization by Pseudomonas aeruginosa thus supporting the possible role of $T A S 2 R 38$ in susceptibility to respiratory infections. In conclusion, here we report, for the first time, a possible association of TAS2R38 polymorphisms with PCD phenotype. A larger collection of patients is in progress and updated results will be presented and discussed.

A. Robino: None. G. Girotto: None. U. Ambrosetti: None. G. Piatti: None.

\section{E-P03.06}

Clinics and genetics in CF patients with large deletions

\section{N. Yaneva ${ }^{1}$, M. Libik ${ }^{2}$, G. Petrova ${ }^{3}$, I. Bradinova ${ }^{1}$, M.} Nikolova $^{4}$, M. Macek ${ }^{2}$, A. Savov ${ }^{1}$

${ }^{I}$ National Genetic Laboratory, University Hospital of Obstetrics and Gynecology "Maichin Dom", Sofia, Bulgaria, ${ }^{2}$ Department of Biology and Medical Genetics, Charles University-2nd Faculty of Medicine and Motol University Hospital, Prague, Czech Republic, ${ }^{3}$ Pediatric cilinic, UMHAT "Alexandrovska", Sofia; Pediatric department, Medical University of Sofia, Sofia, Bulgaria, ${ }^{4}$ Pediatric clinic, UMHAT"Sveta Marina", Varna; Medical Univcersity Varna, Varna, Bulgaria

Introduction: Cystic fibrosis (CF) is the most common lifeshortening condition with autosomal recessive inheritance. It is associated with at least one mutation on each allele on CF transmembrane conductance regulator (CFTR). After screening for point mutations and small indel, using Sanger sequencing and NGS, in some patients the second disease causing mutation remains unidentified.

Materials and methods: We performed MLPA analysis for detection of large deletions and duplications in CFTR gene, using P091 MLPA MRCHolland kit in a group of 44 $\mathrm{CF}$ patients with only one mutation.

Results: The MLPA screening detected large deletions in eight patients (18\%): deletion of exons 4-11 in one patient with R1158X mutation, deletion of exons 18-20 in three patients with F508del mutation and in one with R334W, deletion of exons 2 and 3 in two patients with F508del, two deletions of exons 2 and 13 in one patient. The last finding is novel and not described in databases. The clinical presentation of $\mathrm{CF}$ in all of them was with early onset (in the first year of life), meconium ileus, failure to thrive, pancreatic insufficiency, chronic obstructive lung disease and abnormal sweat test levels.

Conclusion: We established that overall frequency of the large deletions in our patients is about $1 \%$ from all mutations that we found in CFTR gene. It must be kept in mind that $>99 \%$ of all known point mutations have allele frequencies that are below $0.1 \%$. Therefore we believe that it is suitable to use MLPA of CFTR gene for routine molecular diagnostics of CF.

N. Yaneva: None. M. Libik: None. G. Petrova: None. I. Bradinova: None. M. Nikolova: None. M. Macek: None. A. Savov: None.

\section{E-P03.07}

Differential molecular diagnosis of Cystic fibrosis and Shwachman-Diamond syndrom

M. Kazaryan ${ }^{1,2}$, T. Adyan ${ }^{2,1}$, A. Polyakov ${ }^{2}$

${ }^{I}$ RNRMU, Moscow, Russian Federation, ${ }^{2}$ Research Centre for Medical Genetics, Moscow, Russian Federation

Cystic fibrosis (CF) and Shwachman-Diamond syndrome (SDS) are autosomal recessive disorders, associated with mutations in CFTR and SBDS genes respectively. CF and SDS have general symptom - exocrine pancreatic insufficiency. This study aims to describe the possibility of misdiagnosis in case of conditions with common symptoms, such as CF and SDS. The first cohort of 94 patients aged from 0 to 40 years with primary SDS diagnosis were investigated for the presence of 30 CFTR gene mutations that are most frequent in Russian Federation. The second cohort of 361 patients with primary CF diagnosis was analyzed for the presence of 2 common $S B D S$ gene mutations (this sample was divided into 2 groups: 246 probands aged from 0 to 5 years and 115 probands aged from 6 to 10 years). MLPA-method was used to determine point mutations; deletion/insertion mutations were tested by multiplex AFLP-method and automated sequencing as well. We identified one heterozygous carrier of F508del mutation in the CFTR gene in SDS sample (sequencing of the entire coding region of this gene and MLPA-analysis did not reveal a second pathogenic variant in this patient). In a group of 0-5 years $\mathrm{CF}$ patients 4 probands were found to have both mutations in the SBDS gene and one proband have 1 heterozygous mutation. In a group of 6-10 years $\mathrm{CF}$ 
patients only 1 proband had both mutations. Our results demonstrate that patients with suspected $\mathrm{CF}$ and undetected mutations in the CFTR gene are necessary to differential diagnosis with a SDS.

M. Kazaryan: None. T. Adyan: None. A. Polyakov: None.

\section{E-P03.08}

Thyroid peroxidase (TPO) mutations in Macedonian patients with congenital hypothyroidism

N. Zdraveska ${ }^{1}$, V. Anastasovska ${ }^{1}$, N. Schoenmakers ${ }^{2}$, A. Nicholas ${ }^{2}$, M. Kocoval

${ }^{1}$ University Children's Hospital Skopje, Skopje, Macedonia, The Former Yugoslav Republic of, ${ }^{2}$ University of Cambridge Metabolic Research Laboratories, Wellcome TrustMedical Research Council Institute of Metabolic Science, Addenbrooke's Hospital, Cambridge, United Kingdom

Introduction: Congenital hypothyroidism $(\mathrm{CH})$ due to thyroid dyshormonogenesis is a heterogenic disorder caused by impairment in any stage of the thyroid hormone biosynthesis pathway. Mutations in seven genes may be associated with thyroid dyshormonogenesis: SLC5A5 (NIS), SCL26A4 (PDS), TG, TPO, DUOX2, DUOXA2, and IYD (DHEAL1). Defects in thyroid peroxidase (TPO) gene are reported as the most frequent cause of dyshormonogenesis with permanent $\mathrm{CH}$ characterized with permanent total iodide organification defect.

Methods: A comprehensive, phenotype-driven, approach was used to identify underlying mutations in Macedonian cohort of $\mathrm{CH}$ patients, by sequentially screening known dyshormonogenesis-associated genes. Genomic DNA was extracted from peripheral blood leukocytes, and Sanger sequencing was used to screen for TPO gene mutations in all coding exons and exon/intron boundaries amplified by PCR specific primers.

Results: Analysis of the TPO gene revealed variants in 3 cases including 2 siblings who each harboured a heterozygous frameshift variant, p.A397Pfs*77, inherited from a euthyroid father. An unrelated case was compound heterozygous for a maternally inherited missense mutation, $p$. $\mathrm{R} 438 \mathrm{H}$, and a paternally inherited frameshift variant, $\mathrm{p}$. E17Dfs*77. All 3 cases exhibited severe $\mathrm{CH}$ at diagnosis, with significantly elevated serum TSH values (mean: $103 \mathrm{mU} / \mathrm{L}$, range $>75-159 \mathrm{mU} / \mathrm{L}$ ) and low fT4 values (mean $<4 \mathrm{ug} / \mathrm{dl})$. Goitre was diagnosed on the neonatal thyroid ultrasound and during foetal ultrasound examination in the third case.

Conclusion: Finding TPO gene mutations in a neonate with $\mathrm{CH}$ indicates that the subject will require life-long treatment with thyroid hormone and that future pregnancies should be carefully monitored for the presence of prenatal goiter.

N. Zdraveska: None. V. Anastasovska: None. N. Schoenmakers: None. A. Nicholas: None. M. Kocova: None.

\section{E-P03.10}

Molecular investigation of Cystic Fibrosis: CFTR mutation spectrum among Greek population

M. Argyraki, M. Chatziapostolou, S. Vittas

DNA - Diagnosis N Analysis Laboratory, Thessaloniki, Greece

Introduction: Cystic Fibrosis (CF) represents the second most common congenital disorder among Greek population. Approximately $4-5 \%$ of Greek population represents asymptomatic $\mathrm{CF}$ mutation carriers. This in conjunction with the limited knowledge about this disease necessitates the implementation of carrier screening programs.

Materials and methods: The study population consisted of 485 Greek individuals screened for CF mutations. We used a targeted NGS to sequence all CFTR gene coding regions, intron/exon splice sites and UTRs, combined with MLPA analysis.

Results: Molecular analysis showed that 96 individuals $(19.8 \%)$ represent CF mutation carriers, consisting of 24 individuals $(4.75 \%)$ carrying typical CF-causing mutations, 38 individuals $(7.52 \%)$ carrying CFTR-RD (CFTR-related disorders) mutations and 38 individuals (7.52\%) carrying variants of uncertain significance (VOUS). Seven cases of complex alleles that carry two mutations were also observed, a factor that can complicate CFTR diagnosis and clinical evaluation. The most common typical mutation identified in 15 carriers was $\Delta F 508$, followed by $621+1 \mathrm{G}>\mathrm{T}$ mutation occurring in 3 carriers. Likewise, R75Q mutation represents the most common CFTR-RD mutation identified (8 carriers), followed by L997F and R1162L (found in 6 and 4 carriers respectively). Lastly, c. $2620-15 \mathrm{C}>\mathrm{G}$ mutation was identified in 11 carriers, representing the most common VOUS mutation in the population studied.

Discussion: Aim of the current study was to estimate the CFTR mutation spectrum and the prevalence of $\mathrm{CF}$ mutation carriers among Greek population. Accurate knowledge of $\mathrm{CF}$ mutation spectrum provides information for $\mathrm{CF}$ prevention programs and may prove particularly important for providing more sufficient genetic counselling to the Greek population.

M. Argyraki: None. M. Chatziapostolou: None. S. Vittas: None. 


\section{E-P03.13}

Correlation of $10 \mathrm{SNP}$ polymorphisms in modifier genes (TNF, GST, HFE) with phenotype of Slovak cystic fibrosis (CF) patients

\section{E. Tóthová Tarová ${ }^{1}$, A. Soltysova ${ }^{2}$, A. Ficek ${ }^{3}, P$. Celec $^{4,5,3}$, H. Kayserova ${ }^{6}$, L. Kádasi ${ }^{3,2}$}

${ }^{1}$ Department of Biology, Faculty of Education, J. Selye University, Komárno, Slovakia, ${ }^{2}$ Institute of Molecular Physiology and Genetics, Slovak Academy of Sciences, Bratislava, Slovakia, ${ }^{3}$ Department of Molecular Biology, Comenius University, Bratislava, Slovakia, ${ }^{4}$ Institute of Pathological Physiology, Faculty of Medicine, Comenius University, Bratislava, Slovakia, ${ }^{5}$ Institute of Molecular Biomedicine, Faculty of Medicine, Comenius University, Bratislava, Slovakia, ${ }^{6}$ Center of Cystic Fibrosis, Department of Pediatric Pneumology, University Hospital, Bratislava, Slovakia

Cystic fibrosis (CF) is still the most serious autosomal recessive disease in the white population despite improved treatment of CF patients. More than 2000 identified mutations have been previously described in the CFTR gene. Even in CF patients with identical CFTR genotypes, there are a large number of serious lung diseases and other symptoms. Environment and polymorphisms in other genes play a particularly important role in the phenotypic modification of disease. The main aim of our study was the correlation analysis of 10 SNP polymorphisms/variants in selected modifier genes (TNF $\alpha$, GSTM, GSTP, GSTT and HFE) and subsequent correlation analysis with clinical symptoms of patients, mainly spirometry measurements of lung function. To identify these polymorphisms, we used methods such as PCR and direct sequencing. Our results show a significant association of the $\mathrm{TNF} \alpha$ gene polymorphism (rs1800629) with cardiomyopathy, another polymorphism ( $\mathrm{rs} 361525)$ with hypoalbuminemia and distal obstructive syndrome, and two other polymorphisms (rs1800630, rs1799724) with bacterial infections of Pseudomonas aeruginosa. The GSTT1 gene show significant association with the nasal polyps, GSTP1 gene (rs1695) with a worse pulmonary function by vital capacity of lung $\left(\mathrm{FEV}_{1}\right)$, also HFE gene polymorphism (rs1799945) with worse pulmonary function $\left(\mathrm{FEV}_{1}\right)$. Our results can be used as a prognostic marker in the treatment of $\mathrm{CF}$ patients.

E. Tóthová Tarová: None. A. Soltysova: None. A. Ficek: None. P. Celec: None. H. Kayserova: None. L. Kádasi: None.

\section{E-P03.14}

The c.7G $>A$ variant in $E H H A D H$ associated with Fanconi renotubular syndrome is also responsible for hepatic failure

P. Rollier ${ }^{1,2}$, M. Barth ${ }^{1}$, V. Milon ${ }^{1}$, N. Gueguen ${ }^{1}$, C. Bris ${ }^{1}$, L. Damaj ${ }^{2}$, A. Ryckewaert ${ }^{3}$, S. Odent ${ }^{2}$, V. Procacciol, A. Ziegler ${ }^{1}$, D. Bonneau ${ }^{1}$

${ }^{1}$ Department of Biochemistry and Genetics, Angers University Hospital, Angers, France, ${ }^{2}$ Department of Clinical Genetics, Rennes University Hospital, Rennes, France, ${ }^{3}$ Department of Pediatrics, Rennes University Hospital, Rennes, France

Introduction: $E H H A D H$ was first associated to a human disease by Klootwijk et al. in 2014 who reported that the pathogenic variant c.7G>A;p.E3K was responsible for an isolated Fanconi renotubular syndrome. This variant causes an addressing defect of this peroxisomal protein leading to its abnormal localization to mitochondria. We report a second case harbouring the same mutation in $E H H A D H$ as that previously reported who was affected with a broader phenotype including recurrent hypoglycemia and hepatic cytolysis associated to Fanconi syndrome.

Materials and methods: Recruitment of the patient, exome sequencing and functional analyses were carried out at the Angers University Hospital.

Results: At 4 months of age, the individual presented with hypoglycemia, ketonuria, lactic acidosis and hepatic cytolysis. Subsequently, he presented recurrent episodes of vomiting and/or diarrhea with hypoglycemia after long fasting requiring glucose infusions. Additional features included severe Fanconi tubulopathy with glucosuria, tubular proteinuria, hypouricemia, and nephrocalcinosis. Mitochondrial respiratory chain analyses performed on hepatocytes showed a decreased activity of complex I and IV, including a $70 \%$ decrease in complex IV/citrate synthase ratio which was confirmed by the decrease of the same complexes using Western blot analyses. Exome analysis evidenced the missense mutation c.7G $>$ A;p.E3K in $E H H A D H$. This mutation was inherited from the father who was asymptomatic at age 54 .

Conclusions: We expand the phenotypic spectrum of the c.7G $>$ A mutation in EHHADH with the description of hepatic failure, including cytolysis and hypoglycemic episodes, associated with Fanconi tubulopathy. This disease is an autosomal dominant disorder with a great variability in expression.

P. Rollier: None. M. Barth: None. V. Milon: None. N. Gueguen: None. C. Bris: None. L. Damaj: None. 
A. Ryckewaert: None. S. Odent: None. V. Procaccio: None. A. Ziegler: None. D. Bonneau: None.

\section{E-P03.15}

Renal phenotype expansion in known syndromes using whole exome sequencing

\section{Schüle ${ }^{l}$, K. Häffner ${ }^{1,2}$, J. Kracht ${ }^{l}$, G. Ruzaike ${ }^{l}$, \\ E. Lausch ${ }^{l}$, D. Matheisl ${ }^{1}$, R. Hentschel ${ }^{l}$, M. Pohl ${ }^{1}$, M. Schmidts ${ }^{l}$}

${ }^{1}$ Clinic for General Pediatrics, Adolescent Medicine and Neonatology, University of Freiburg, Freiburg im Breisgau, Germany, ${ }^{2}$ Clinic for Internal Medicine IV Nephrology and General Medicine, University of Freiburg, Freiburg, Germany

Background: Patients with rare syndromes often have mutations in genes that are not covered by organ specific NGS Panels. Whole exome sequencing (WES) helps finding the underlying genetic cause of end-stage renal disease (ESRD) especially in patients with extrarenal features.

Methods and results: We report two cases with ESRD in patients with extrarenal organ involvement. A 14 year old boy with a history of perinatal asphyxia presented with a first seizure and ESRD. In addition he showed a psychomotor developmental retardation, muscular hypotonia and facial dysmorphism. The second patient was a newborn with congenital kidney failure, cleft palate, microcephaly, hypothyroidism and also facial dysmorphism. WES revealed a heterozygous variant in the KANSL1 gene (c.1284delA:p.V428fs) in the 14 year old patient not detected in the mother (father unavailable) and a heterozygous de novo variant in the KMT2D gene (c.C8434T:p. Q2812X) in the newborn. Both variants have not been previously described in literature.

Discussion: KANSL1 and KMT2D mutations cause Koolen-de Vries and Kabuki Syndrome. While clinical features observed in our patients fit well with those conditions, renal disease is extraordinarily severe in our cases: no ESRD has been previously reported for Koolen-de Vries Syndrome and likewise, congenital ESRD has not been described in the literature for Kabuki syndrome so far. Our findings extend the kidney phenotype in these syndromes and show the utility of WES to unravel the cause of ESRD in children that would be missed by gene panel based NGS.

I. Schüle: None. K. Häffner: B. Research Grant (principal investigator, collaborator or consultant and pending grants as well as grants already received); Significant; Greenovation Biotech Gmbh. J. Kracht: None. G. Ruzaike: None.
E. Lausch: None. D. Matheisl: None. R. Hentschel: None. M. Pohl: None. M. Schmidts: None.

\section{E-P03.18}

Juvenile nephronophthisis due to NPHP1 homozygous deletion revealed by whole exome sequencing

\section{G. Christopoulou ${ }^{l}$, A. Oikonomaki ${ }^{l}$, S. Samara ${ }^{l}$, S. Bournazos $^{1}$, C. Melexopoulou ${ }^{2}$, J. Boletis ${ }^{2}, \boldsymbol{P}$. Constantoulakis $^{1}$}

${ }^{1}$ Genotypos Science Labs MSA, Athens, Greece, ${ }^{2}$ Department of Nephrology and Renal Transplantation, Laiko Hospital, Athens, Greece

Introduction: Renal failure is a major cause of morbidity and mortality worldwide and revealing a potential genetic background is essential for the patients' management and families' counselling.

Materials and methods: A 17-year-old female with tubulointerstitial nephritis was referred for whole exome sequencing (WES). WES was performed on DNA extracted from peripheral blood, using the Twist Human Exome Core-v2 kit (Twist Bioscience). Following preparations according to the manufacturer's protocol, libraries were sequenced on an Illumina NextSeq-500 genetic analyser. Data processing, variant calling and pre-classification were conducted by SOPHiA DDM $^{\otimes}$ bioinformatics pipelines. Multiplex Ligation-dependent Probe Amplification (MLPA) was performed (P387-C1, Coffalyser net, MRC-HOLLAND) to further confirm results.

Results: No pathogenic/likely pathogenic SNVs/Indels were detected, related to the phenotype. Gene coverage analysis revealed a homozygous NPHPl whole gene deletion, subsequently confirmed by MLPA. Deletions including at least NPHPI are associated with Juveline nephronophthisis, which is in concordance with the phenotype. Parental DNA MLPA analyses revealed NPHP1 heterozygous deletions in both, whereas the proband's sister was completely normal thus, substantiating inheritance and further guiding the selection of the appropriate family member for possible kidney transplantation.

Discussion: Optimization in WES protocols allows greater confidence in calling copy number variations. By applying this approach, definite diagnosis of the nephropathy was achieved, otherwise challenging with traditional methods. Whole exome sequencing permits a large number of genes investigated, for different types of variants, establishing diagnosis and leading to accurate and timely decisions for the patient's and families' healthcare. 
G. Christopoulou: None. A. Oikonomaki: None. S. Samara: None. S. Bournazos: None. C. Melexopoulou: None. J. Boletis: None. P. Constantoulakis: None.

\section{E-P03.19}

Polymorphisms of calcium metabolism genes associated with kidney stone disease in Russian population

M. M. Litvinova ${ }^{1,2}$, T. V. Filippova ${ }^{l}$, D. V. Svetlichnaya ${ }^{l}, S$. Z. Nuralieval, A. D. Matsvay ${ }^{3}$, K. Khafizov ${ }^{3}$

${ }^{1}$ I.M. Sechenov First Moscow State Medical University (Sechenov University), Moscow, Russian Federation, ${ }^{2}$ The Loginov Moscow Clinical Scientific Center of Moscow Health Department, Moscow, Russian Federation, ${ }^{3}$ “Center of Strategic Planning” of the Ministry of Health of the Russian Federation, Moscow, Russian Federation

Introduction: Kidney stone disease (KSD) is an urgent problem of modern medicine due to high prevalence and recurrence rate of the pathology. The particular scientific interest is currently focused on the genetic mechanisms of urolithiasis. The aim of this study was to investigate an association between polymorphic loci of the calcium metabolism genes and the risk of KSD development in Russian population.

Materials and methods: Based on the analysis of scientific literature and genomic databases, the list of genes and polymorphisms associated with KSD in different countries was formed. The information was obtained from case-control studies and GWAS. Finally, we formed a genetic panel which included SNPs of VDR, CASR, CALCR, SLC34A1, CLDN14, TRPV5, TRPV6, KLOTHO, ORAI1, ALPL, RGS14, OPN, MGP, PLAU, AQP1, DGKH genes. The study group consisted of 77 patients with KSD. The control group included 76 persons without urolithiasis. Genetic analysis was carried out by next generation sequencing using the Ion S5 platform. We also studied the presence of familial cases of KSD in the observed group.

Results: Among patients with KSD 54\% had family history of urolithiasis, $44 \%$ had recurrent course of the disease. 9 SNPs in 4 genes showed statistically significant association with KSD: rs219781, rs219780, rs219779, rs219778, rs219777, rs1544410, rs731236, rs11746443, rs6486795. The odds ratio ranged from 1.2 to 2.5 depending on polymorphism.

Conclusions: Targeted genetic panel for detecting the genetic predisposition to urolithiasis was designed and validated. Polymorphic loci of genes CLDN14, VDR, $S L C 34 A 1$, and ORAII are associated with KSD in Russian population.
M.M. Litvinova: None. T.V. Filippova: None. D.V. Svetlichnaya: None. S.Z. Nuralieva: None. A.D. Matsvay: None. K. Khafizov: None.

\section{E-P03.20}

A rare presentation of $\mathrm{NPHP3}$ nephronophthisis

L. Ramos $^{1,2}$, S. Fernandes ${ }^{1}$, C. S. Rosas ${ }^{1}$,

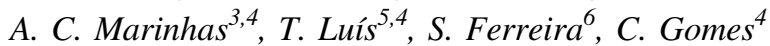

${ }^{1}$ Medical Genetics Unit, Hospital Pediátrico, Centro Hospitalar e Universitário de Coimbra, Coimbra, Portugal, ${ }^{2}$ Faculty of Health Sciences, Universidade da Beira Interior, Covilhã, Portugal, ${ }^{3}$ Pediatric Unit, Hospital Pediátrico, Centro Hospitalar e Universitário de Coimbra, Coimbra, Portugal, ${ }^{4}$ Pediatric Nephrology Unit, Hospital Pediátrico, Centro Hospitalar e Universitário de Coimbra, Coimbra, Portugal, ${ }^{5}$ Pediatric Unit, Centro Hospitalar do Baixo Vouga, Aveiro, Portugal, ${ }^{6}$ Pediatric Hepatology Unit, Hospital Pediátrico, Centro Hospitalar e Universitário de Coimbra, Coimbra, Portugal

Introduction: Nephronophthisis (NPHP) is an autosomal recessive cystic kidney disease that usually progresses to end-stage renal disease (ESRD). It is caused by mutations in genes that encode proteins involved in the function of primary cilia, basal bodies and centrosomes, resulting in renal and extrarenal manifestations. Prenatal history of oligohydramnios and kidney cysts can be present. Infantile type of NPHP develops ESRD in the first three years of age and is the most severe form. It is usually caused by mutations in NPHP2 and NPHP3 genes.

Objective: We intend to demonstrate how difficult the genotype/phenotype correlation in NPHP is, and reinforce the use of gene panel or exome in this disease.

Materials and methods: Report of clinical manifestations, laboratory examination and imaging data in family related foetus and child with different features of NPHP. A NGS panel for cholestasis was performed.

Results: Previously healthy child presents pruritus and laboratory examination with renal/liver dysfunction at the age of 21 months. Ultrasound showed normal-sized kidneys with cysts and absent hepatobiliar, pancreatic or cardiac changes. The first pregnancy of the parents was complicated due to nephromegaly with microcysts and anamnios - the diagnosis of NPHP was evoked. The NGS panel identified a homozygous NPHP3 variant, c.3601G >T p.(Glu1201*), and molecular testing to her consanguineous parents is being performed.

Conclusion: NPHP is characterized by a significant genetic and clinical heterogeneity. Gene panel or exome is the most appropriate approach when NPHP is suspected. 
The etiologic diagnosis is important for management and genetic counselling.

L. Ramos: None. S. Fernandes: None. C.S. Rosas: None. A.C. Marinhas: None. T. Luís: None. S. Ferreira: None. C. Gomes: None.

\section{E-P03.21}

A novel pathological variant in the $P H O X 2 B$ gene associated with a respiratory/ Hirschsprung phenotype in a three-generation family

\section{Borg ${ }^{1,2}$, M. Pace Bardon ${ }^{3}$, N. Pace P $^{4,5}$}

${ }^{1}$ Department of Pathology, University of Malta, Msida, Malta, ${ }^{2}$ Medical Genetics Unit, Department of Pathology, Mater Dei Hospital, Msida, Malta, ${ }^{3}$ Department of Medicine, Mater Dei Hospital, Msida, Malta, ${ }^{4}$ Department of Anatomy, University of Malta, Msida, Malta, ${ }^{5}$ Centre for Molecular Medicine and Biobanking, University of Malta, Msida, Malta

Introduction: Mutations in the $P H O X 2 B$ gene cause congenital central hypoventilation syndrome (CCHS), a rare autonomic nervous system dysfunction disorder characterised by a decreased ventilatory response to hypercapnia. Affected subjects require lifelong ventilatory support, and develop hypoventilation during the non-REM phase of sleep where autonomic control of breathing predominates. CCHS may be isolated or associated with disorders characterised by defective migration/differentiation of neural crest derivatives, including aganglionic megacolon or milder gastrointestinal phenotypes, such as constipation. Most cases of CCHS are de-novo, caused by heterozygosity for polyalanine repeat expansion mutations (PARMs) in exon 3. The remaining $10 \%$ of cases are due to heterozygous non-PARM missense, nonsense or frameshift mutations.

Methodology: We describe a three-generation MalteseCaucasian family with a variable CCHS/Hirschsprung phenotype, characterised by chronic constipation, three siblings with Hirschsprung disease necessitating surgery and chronic hypoxia requiring non-invasive ventilation.

Results: Bidirectional sequencing of $P H O X 2 B$ revealed a novel heterozygous c. $241+2$ delT splice variant that segregates with the CCHS/Hirschsprung phenotype in the family. The deletion lies in the donor splice site of exon 1 and has not been reported in literature or genomic databases. Bioinformatic analysis predicts that the mutation will result in skipping of exon 1 and the formation of a non-functional protein product. The mutation is pathogenic according to ACMG, PVS1, PM2 and PP1 criteria.

Conclusion: This report expands the mutational spectrum of $P H O X 2 B$. It is significant as no splice-site mutations have been previously reported for $P H O X 2 B$, and highlights the variability in clinical expression and disease severity of non-PARM mutations.

I. Borg: None. M. Pace Bardon: None. N. Pace: None.

\section{E-P03.22}

GeNepher: building a data- and biobank for (suspected) hereditary renal disease

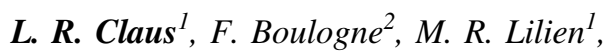
M. B. Rookmaaker ${ }^{l}$, A. van der Zwaag ${ }^{1}$, T. Q. Nguyen ${ }^{l}$, M. C. Verhaar ${ }^{1}$, N. V. A. M. Knoers ${ }^{2}$, P. Deelen ${ }^{1,2}$, L. Franke ${ }^{2}$, M. H. de Borst ${ }^{2}$, A. M. van Eerde ${ }^{l}$

${ }^{1}$ University Medical Center Utrecht, Utrecht, Netherlands, ${ }^{2}$ University Medical Center Groningen, Groningen, Netherlands

Introduction: A substantial part of patients with renal disease has an undiscovered monogenic cause. The timely awareness of a potential genetic etiology in kidney disease patients has profound implications for diagnosis and subsequent care pathways, for family members (e.g. in presymptomatic testing and family donation) and for options regarding establishing a family.

Methods: We will set-up the GeNepher Data- and Biobank. Dutch patients with known and suspected hereditary renal disease are eligible for inclusion. Informed consent will cover use of available medical data, use of available DNA/sequencing data and in selected cases consent for additional blood sampling, skin biopsy or urine collection.

The GeNepher Data- and Biobank will be used to:

1. Answer questions regarding etiology. In unsolved cases we will perform add-on analyses to identify new candidate genes for renal disease. In order to optimally identify new candidates, and to gain new insights, we are developing a kidney specific gene network using kidney derived RNA-sequencing data. Ultimately we will perform functional studies to validate plausible candidates. In GeNepher there is an infrastructure for return of relevant findings.

2. Study modifiers of progressive renal disease like NPHP1-nephronophtisis.

3. Contact patients for opportunities in treatment development or when diagnose specific trials are started or treatment is approved.

Results: Inclusions will start in Q2 of 2020.

Conclusions: The GeNepher Data- and Biobank will improve diagnosis of renal disease by identifying genetic causes, provide insight into pathophysiology and contribute to development of new therapies. 
Grant references: Dutch Kidney Foundation, Kolff Postdoc Senior Grant

L.R. Claus: None. F. Boulogne: None. M.R. Lilien: None. M.B. Rookmaaker: None. A. van der Zwaag: None. T.Q. Nguyen: None. M.C. Verhaar: None. N.V.A.M. Knoers: None. P. Deelen: None. L. Franke: None. M.H. de Borst: None. A.M. van Eerde: None.

\section{E-P03.23}

Founder effect of SLC2A9 mutation p.(T125M) in Spanish families with hereditary renal hipouricemia type 2

E. Córdoba-Lanús ${ }^{1,2}$, A. Perdomo-Ramírez ${ }^{1}$, G. Mura-Escorche ${ }^{1,3}$,S. Carinelli ${ }^{1}$, E. Ramos-Trujillo ${ }^{1}$, F. Claverie-Martín ${ }^{1}$

${ }^{1}$ Unidad de Investigación, Hospital Universitario Nuestra Señora de Candelaria, Santa Cruz de Tenerife, Spain, ${ }^{2}$ IUETSPC, Universidad de La Laguna, Santa Cruz de Tenerife, Spain, ${ }^{3}$ Universidad de La Laguna, Santa Cruz de Tenerife, Spain

Introduction: Renal hypouricemia (RHUC) is a rare inherited disorder characterized by impaired urate reabsorption in the proximal tubule. Some patients may present severe complications such as exercise-induced acute renal failure and nephrolithiasis. RHUC is caused by inactivating mutations in the SLC22A12 (RHUC type 1) or SLC2A9 (RHUC type 2) genes, which encode urate transporters URAT1 and GLUT9, respectively. Recently, we found that mutation c.374C $>\mathrm{T}$, p.(T125M) is recurrent in Spanish families with RHUC2. Therefore, we hypothesized that this might be due to a founder effect.

Methods: Thirteen new patients with RHUC were analyzed by sequencing the SLC22A12 and SLC2A9 coding exons. To determine whether patients with the $\mathrm{p}$. (T125M) mutation had a common p.(T125M)-linked haplotype a SNPs-haplotype analysis was performed. A total of nine patients from apparently unrelated families were included in this analysis. Six informative SNPs were analyzed by PCR amplification from genomic DNA and direct sequencing.

Results: Seven patients carried SLC22A12 mutation p. (T467M), and six presented the recurrent SLC2A9 mutation p.(T125M). Alleles carrying the p.(T125M) mutation shared a common SNP haplotype. Every patient harboring this mutation in homozygous state had a common haplotype (0.7 frequency), G-A-C-T-G-T-C-T (mutant allele in bold). While the haplotype G-G-C-C-G-T-C was the most frequent between controls (0.42).

Conclusions: Mutation p.(T125M) is the most common variant in Spanish patients with RHUC2. Haplotype analysis confirmed that p.(T125M) is a founder mutation. This study was supported grant PI17/00153 co-financed by the ISCIIISubdirección General de Evaluación y Fomento de la Investigación and the ERDF "Another way to build Europe".

E. Córdoba-Lanús: None. A. Perdomo-Ramírez: None. G. Mura-Escorche: None. S. Carinelli: None. E. RamosTrujillo: None. F. Claverie-Martín: None.

\section{E-P03.25}

Molecular study of Wilson disease in a Spanish cohort

M. Antolin ${ }^{1}$, I. Paramanov ${ }^{l}$, P. Fernandez ${ }^{l}$, L. Iranzo ${ }^{l}$, D. Martinez $^{1}$, E. García-Arumíl,2, E. Tizzano ${ }^{1}$

${ }^{I}$ Department of Clinical and Molecular Genetics and Medicine Genetics Group, VHIR, University Hospital Vall d'Hebron, Barcelona, Spain, ${ }^{2}$ CIBERER, Centro de Investigación Biomédica en Red de Enfermedades Raras, ISCIII, Madrid, Spain

Introduction: Wilson disease (WD) is a disorder caused by biallelic pathogenic variants in $A T P 7 B$ gene, resulting in abnormal copper metabolism and deposition, leading to a combination of hepatic, neurologic, and psychiatric features. Diagnosis is confirmed by molecular analysis of $A T P 7 B$ gene.

Materials and methods: 34 patients with WD were studied using two long range PCR (exon 2-9; exon 10-21 $A T P 7 B$ gene). Fragmentation, indexing, library preparation (NEBNext Ultra DNA library prep kit) and sequencing in a MiSeq platform (Illumina) were performed. Exon 1 was analysed by Sanger sequencing.

Results: Genetic diagnosis was achieved in 18 WD patients whereas only one pathogenic variant was found in 5. Familiar studies have allowed the identification of 2 WD patients before clinical manifestations. $86 \%$ of the variants were described as pathogenic whereas $14 \%$ were not described previously. A prevalent $A T P 7 B$ allelic variant, p.(Met645Arg), was found in 5 patients $(28 \%)$, in trans with other allelic variants. The splicing mutation c.1708-1G>A was found in 3 patients $(17 \%)$ whereas the c.1708-1G>C was found in one $(6 \%)$, all in homozygous state. The most prevalent pathogenic variant in Europe p.(His1069Gln) was found in heterozygosis in 2 patients, representing only the $4,4 \%$ of pathogenic alleles in our cohort.

Conclusions: The most common allelic variant among our WD patients was p.(Met645Arg) followed by the splicing variant c.1708-1G>A. Familiar genetic study of WD by NGS allows the molecular diagnosis of asymptomatic patients and prevent disease.

M. Antolin: None. I. Paramanov: None. P. Fernandez: None. L. Iranzo: None. D. Martinez: None. E. GarcíaArumí: None. E. Tizzano: None. 
E-P04 Skeletal, Connetive Tissue, Ectodermal and Skin Disorders

\section{E-P04.02}

The first missense mutation in CSTA gene causes Acral Peeling Skin Syndrome in a Turkish family, Review of the literature

\section{A. C. Ceylan ${ }^{1}$, S. Emre Em $^{2}$ G. G. Ceylan ${ }^{1}$}

${ }^{1}$ Yildirim Beyazit University Medical School Department of Medical Genetics, Ankara, Turkey, ${ }^{2}$ Yildirim Beyazit University Medical School Department of Dermatology, Ankara, Turkey

Introduction: Acral Peeling Skin Syndrome (APSS) is a rare, autosomal recessive genodermatosis. The disease is characterized by superficial peeling of the cornified epidermal layer. Skin fragility often affects hands and feet. It is most commonly caused by mutations in the TGM5 (*603805). CSTA $(* 184600)$ rarely causes APSS. We intend to invest if there is a new mutation in genes related with APSS.

Materials and methods: Two siblings were presented us because of chronic and superficial itchy skin lesions. After the dermatological examinations, clinical exome solutionwas performed for the molecular diagnosis of one of the patients. Sanger sequencing was performed for the other patient to find if there is the same mutation.

Results: A novel homozygote missense mutation was found in CSTA gene in both of the siblings. This variant fulfills PM1, PM2, PP3 and PP4, and should therefore be regarded as a likely pathogenic according to the joint consensus recommendation of the ACMG and AMP.

Conclusion: Peeling skin syndromes appears to be heterogeneous clinically and genetically. Inhere, we report sixth family with a novel homozygote missense CSTA mutation and clinical findings. It is shown that different type of CSTA mutations can also cause APSS unlike previously reported cases, and will contribute to better illumination of the phenotype regarding with the literature review.

A.C. Ceylan: None. S. Emre: None. G.G. Ceylan: None.

\section{E-P04.03}

Molecular genetic investigation of ACE inhibitor induced angioedema

C. Mathey ${ }^{1}$, A. F. Forstner ${ }^{1,2}$, C. Maj ${ }^{3}$, O. Borisov ${ }^{3}$, E. R. Rassmussen ${ }^{4}$, A. Bygum ${ }^{5,6}$, C. von Buchwald ${ }^{4}$, M. Wadelius ${ }^{7}$, P. Hallberg ${ }^{7}$, M. Karawajczyk ${ }^{8}$, N. Eriksson ${ }^{7,9}$, B. Wedi ${ }^{10}$, D. Wieczorek ${ }^{10}$, P. M. Amann ${ }^{11}$, H. Löffler ${ }^{11}$, L. Koch ${ }^{12}$, C. Schöffl ${ }^{12}$, N. Evertz ${ }^{13}$, J. Greve ${ }^{14}$,
G. Wurpts ${ }^{15}$, S. Moebus ${ }^{16}$, P. M. Krawitz ${ }^{3}$, J. Stingl ${ }^{17}$, B. Sachs* ${ }^{15,18}$, M. M. Nöthen ${ }^{* 1}$, * equally shared

${ }^{I}$ Institute of Human Genetics, University of Bonn, School of Medicine \& University Hospital Bonn, Bonn, Germany, ${ }^{2}$ Center for Human Genetics, University of Marburg, Marburg, Germany, ${ }^{3}$ Institute for Genomic Statistics and Bioinformatics, University Hospital Bonn, Bonn, Germany, ${ }^{4}$ Department of Otorhinolaryngology - Head and Neck Surgery and Audiology, University Hospital of Copenhagen, Rigshospitalet F-2071, Copenhagen, Denmark, ${ }^{5}$ Department of Clinical Genetics, Odense University Hospital, Odense, Denmark, ${ }^{6}$ Research Unit of DermatoVenerology, Clinical Institute, University of Southern Denmark, Odense, Denmark, ${ }^{7}$ Department of Medical Sciences, Clinical Pharmacology and Science for Life Laboratory, Uppsala University, Uppsala, Sweden, ${ }^{8}$ Department of Medical Sciences, Clinical Chemistry, Uppsala University, Uppsala, Sweden, ${ }^{9}$ Uppsala Clinical Research Center, Uppsala, Sweden, ${ }^{10}$ Department of Dermatology and Allergy, Comprehensive Allergy Center, Hannover Medical School, Hannover, Germany, ${ }^{11}$ Department of Dermatology, SLK Hospital Heilbronn, Heilbronn, Germany, ${ }^{12}$ Department of Dermatology and Venerology, Medical University of Graz, Graz, Austria, ${ }^{13}$ Clinic and Polyclinic for Dermatology and Allergology, University Hospital Bonn, Bonn, Germany, ${ }^{14}$ Department of Otorhinolaryngology, Head and Neck Surgery, Ulm University Medical Center, Ulm, Germany, ${ }^{15}$ Department of Dermatology and Allergology, Aachen Comprehensive Allergy Center, University Hospital of RWTH Aachen, Aachen, Germany, ${ }^{16}$ Centre of Urban Epidemiology, Institute of Medical Informatics, Biometry, and Epidemiology, University of Essen, Essen, Germany, ${ }^{17}$ Institute for Clinical Pharmacology, University Hospital of RWTH Aachen, Aachen, Germany, ${ }^{18}$ Federal Institute for Drugs and Medical Devices, Research Division, Bonn, Germany

Angioedema are known adverse drug reactions of ACE inhibitors (ACEi) that can become a potentially life-threatening event. Results of previous research suggest that susceptibility to ACEi induced angioedema (ACEi AE) is dependent on a genetic predisposition and non-genetic risk factors. However, as the biological mechanisms contributing to these angioedema are still largely unknown, further research is needed. The aim of the present study was to investigate common and rare genetic variation in ACEi AE patients. In a targeted sequencing approach, we investigated the occurrence rate of known pathogenic mutations associated with monogenic forms of angioedema in 69 German and 68 Danish patients with ACEi AE. All samples were sequenced on an Illumina NextSeq platform using single molecule molecular inversion probes. In 
addition, all samples were genotyped on the Illumina Infinium Global Screening Array to identify common genetic variants contributing to angioedema risk under ACEi. Analysis of the sequencing data revealed no known pathogenic mutations in genes associated with the monogenic forms of angioedema. However, we identified 19 other rare (MAF $\leq 0.05)$, non-synonymous variants for which a contribution to ACEi AE risk cannot be excluded. Quality control and imputation of the array data is currently ongoing. Furthermore, we are currently applying the above investigation strategies to an independent Swedish sample $(n=77)$. Follow-up of the variants identified by targeted sequencing in larger patient samples as well as subsequent biostatistics/-informatics analyses of the GWAS data will help to gain further insights into the underlying molecular mechanisms of ACEi AE.

C. Mathey: None. A.F. Forstner: None. C. Maj: None. O. Borisov: None. E.R. Rassmussen: None. A. Bygum: None. C. von Buchwald: None. M. Wadelius: None. P. Hallberg: None. M. Karawajczyk: None. N. Eriksson: None. B. Wedi: None. D. Wieczorek: None. P.M. Amann: None. H. Löffler: None. L. Koch: None. C. Schöffl: None. N. Evertz: None. J. Greve: None. G. Wurpts: None. S. Moebus: None. P.M. Krawitz: None. J. Stingl: None. B. Sachs*: None. M.M. Nöthen*: None.

\section{E-P04.04}

ACTN3 rs1815739 polymorphism is associated with anterior cruciate ligament (ACL) rupture in physically active people from Italian, Japanese, Polish and Russian cohorts

V. Pushkarev ${ }^{1,2,3}, N_{\text {Kikuchi }}^{4}$, H. Kumagai ${ }^{5}$, N. Kamiya ${ }^{6}$, E. Miyamoto-Mikami ${ }^{7}$, N. Fuku ${ }^{7}$ C. Calo ${ }^{8}$, M. Massidda ${ }^{8}$, D. Dyatlov ${ }^{9}$, E. Bykov ${ }^{10}$, P. Cieszczyk ${ }^{11}$

${ }^{1}$ Medical Genetic Centre "Progen", Moscow, Russian Federation, ${ }^{2}$ Moscow Center of Advanced Sport Technologies, Moscow, Russian Federation, ${ }^{3}$ Genetic laboratory, Ural State University of Physical Culture, Chelyabinsk, Russian Federation, ${ }^{4}$ Department of Training Science, Nippon Sport Science University, Tokyo, Japan, ${ }^{5}$ Graduate School of Health and Sports Science, Juntendo University, Chiba, Japan and Reseach fellow of Japanese Society for the Promotion of Science, Tokyo, Japan, ${ }^{6}$ Faculty of Budo and Sport Studies, Tenri University, Nara, Japan, ${ }^{7}$ Graduate School of Health and Sports Science, Juntendo University, Chiba, Japan, ${ }^{8}$ Department of Environmental and Life Science, University of Cagliary, Calgary, Italy, ${ }^{9}$ Department of the Theory of Physical Culture and Biomechanics, Ural State University of Physical Culture,
Chelyabinsk, Russian Federation, ${ }^{10}$ Department of sports medicine and physical rehabilitation, Ural state university of physical culture, Chelyabinsk, Russian Federation, ${ }^{11}$ Department of Physical Education, Gdansk University of Physical Education and Sport, Gdansk, Poland

ACL rupture is one of severe multifactorial injuries. The ACTN3 rs1815739 polymorphism affect muscle structure and metabolism and it has been associated with muscle injuries. Resistance to fatigue and muscle stiffness are considered risk factors for ACL injury. The aim of our study was to determine the influence of ACTN3 rs1815739 on ACL rupture in Italian, Japanese, Polish and Russian population samples. A total of 1706 subjects ( $\mathrm{n}=596$ ACL rupture patients (cases); $\mathrm{n}=1113$ controls) from Italy ( $\mathrm{n}=52$ cases; $\mathrm{n}=81$ controls), Japan $(n=74$ cases $; n=395$ controls), Poland $(n=237$ cases; $n=230$ controls) and Russia ( $n=233$ cases; $n=407$ controls) participated in the study. DNA samples were genotyped by TaqMan. $\mathrm{R}$ packages were used for statistical analysis. The genotype distributions of all control groups were in HWE. Since the Italian, Polish and Russian samples had similar genotype distributions (all $\mathrm{p}>0.05$ ) they were pooled together (European cases and controls).

Genotypes distribution (\% and counts) in European and Japanese case and controls groups

\begin{tabular}{llll}
\hline & $\mathrm{RR}$ & $\mathrm{RX}$ & $\mathrm{XX}$ \\
\hline European cases $(\mathrm{n}=522)$ & $0,370(193)$ & $0,500(261)$ & $0,130(68)$ \\
European controls $(\mathrm{n}=718)$ & $0,372(267)$ & $0,450(323)$ & $0,178(128)$ \\
Japanese cases $(\mathrm{n}=74)$ & $0,270(20)$ & $0,527(39)$ & $0,203(15)$ \\
Japanese controls $(\mathrm{n}=395)$ & $0,220(87)$ & $0,463(183)$ & $0,316(125)$ \\
\hline & & &
\end{tabular}

Significant differences in ACTN3 rs1815739 genotype frequencies were found between cases and controls in the European sample (Fisher's Exact Test, $\mathrm{p}=0,048$ ). The XX genotype was underrepresented in the cases (odds ratio $(\mathrm{OR})=0.69 ; 95 \%$ confidence interval $(\mathrm{CI}), 0.50-0.95$, $\mathrm{P}=0.021)$ in comparison with controls. The same trend has been showed between cases and controls in the Japanese population $(\mathrm{OR}=0.55$; 95\% CI, 0.30-1.006, $\mathrm{P}=0.05)$. The XX ACTN3 rs1815739 seems to protect against ACL rupture in three European and Japanese cohorts.

V. Pushkarev: None. N. Kikuchi: None. H. Kumagai: None. N. Kamiya: None. E. Miyamoto-Mikami: None. N. Fuku: None. C. Calò: None. M. Massidda: None. D. Dyatlov: None. E. Bykov: None. P. Cieszczyk: None.

\section{E-P04.05}

Congenital contractural arachnodactyly syndrome in Polish family with c.4216T/c variant in FBN2 gene. 
M. A. Malarska, H. Moczulska, K. Antosik, P. Jakiel, P. M. Pachniak, M. Borowiec

Department of Clinical Genetics, Lodz, Poland

The congenital contractural arachnodactyly (CCA, Beals syndrome, OMIM: 121050) is an autosomal dominant condition including contractures, crumpled ears, arachnodactyly, dolichostenomelia, scoliosis and pectus deformities. The congenital contractural arachnodactyly has overlapping features with the Marfan syndrome. Both syndromes result from mutations in genes from the fibrillin family, $F B N 1$ and $F B N 2$ respectively. We identified the c. $4216 \mathrm{~T}>\mathrm{C}$ variant in the $F B N 2$ gene in a Polish family with the congenital contractural arachnodactyly. The variation in phenotypic expression was high in the family. In some subjects there was only an arachnodactyly, but in others there were many features like severe kyphoscoliosis with dolichostenomelia, arachnodactyly, camptodactyly, talipes equinovarus and ureteropelvic junction obstruction. We found for the first time a hydronephrosis in the congenital contractural arachnodactyly syndrome. There were no crupled ear in the analysed family. The c.4216T $>C$ variant had been previously found in a Chinese family with modest family variation in phenotypic expression. Our observation confirms the relationship between c.4216T $>C$ variant in the $F B N 2$ gene and the congenital contractural arachnodactyly.

M.A. Malarska: None. H. Moczulska: None. K. Antosik: None. P. Jakiel: None. P.M. Pachniak: None. M. Borowiec: None.

\section{E-P04.06}

ISSAID and EMQN best practice guidelines for gene panel based diagnosis of autoinflammatory diseases

Y. Shinar ${ }^{1}$, I. Ceccherini ${ }^{2}$, D. Rowczenio ${ }^{3}$, I. Aksentijevich $^{4}$, J. Arostegui ${ }^{5,6}$, E. Ben-Chétrit ${ }^{7}$, G. Boursierr, ${ }^{8}$. M. Gattorno ${ }^{9}$, H. Hayrapetyan ${ }^{10}$, H. Ida ${ }^{11}$, N. Kanazawa ${ }^{12}$, H. J. Lachmann $^{3}$, A. Mensa-Vilaro ${ }^{5}$, R. Nishikomori ${ }^{11}, C$. Oberkanins $^{13}$, L. Obici ${ }^{14}$, O. Ohara ${ }^{15}$, S. Ozen ${ }^{16}$, T. Sarkisian $^{17}$, K. Sheils ${ }^{18}$, N. Wolstenholme ${ }^{18}$, E. ZonneveldHuijssoon $^{19}$, I. Touitou ${ }^{20}$, M. E. van Gijn ${ }^{19}$

${ }^{1}$ Sheba Medical Center, Ramat Gan, Israel, ${ }^{2}$ IRCCS Istituto Giannina Gaslini, Genova, Italy, ${ }^{3}$ UCL Medical School, London, United Kingdom, ${ }^{4}$ National Human Genome Research Institute, Bethesda, MD, United States, ${ }^{5}$ Hospital Clínic, Barcelona, Spain, ${ }^{6}$ Institut d'Investigacions Biomèdiques August Pi i Sunyer (IDIBAPS), Barcelona, Spain, ${ }^{7}$ Hadassah-Hebrew University Medical Center, Jerusalem, Israel, ${ }^{8}$ CHU Montpellier, Univ Montpellier, Montpellier, France, ${ }^{9}$ IRCCS G. Gaslini, Genova, Italy, ${ }^{10}$ Center of Medical Genetics and Primary Health Care, Yerevan State
Medical University, Yerevan, Armenia, ${ }^{11}$ Kurume University School of Medicine, Kurume, Japan, ${ }^{12}$ Wakayama Medical University, Wakayama, Japan, ${ }^{13}$ ViennaLab Diagnostics, Vienna, Austria, ${ }^{14}$ Fondazione IRCCS Policlinico San Matteo, Pavia, Italy, ${ }^{15}$ Kazusa DNA Research Institute, Kisarazu, Japan, ${ }^{16}$ Hacettepe University, Ankara, Turkey, ${ }^{17}$ Center of Medical Genetics and Primary health Care, Yerevan State Medical University, Yerevan, Armenia, ${ }^{18}$ European Molecular Quality Network (EMQN), Manchester Centre for Genomic Medicine, St Mary's Hospital, Manchester, United Kingdom, ${ }^{19}$ University of Groningen, University Medical Center Groningen, Groningen, Netherlands, ${ }^{20}$ INSERM, Montpellier, France

Introduction: A growing number of monogenic autoinflammatory diseases (AIDs) has been identified since 1997 . Their clinical diagnosis remains a challenge in face of overlapping inflammatory symptoms. The previous guidelines for genetic testing of AIDs were limited to Sanger based diagnosis of four prototypic recurrent fevers $(M E F V$, $M V K, T N F R S F 1 A$, and NLRP3 genes). Revised guidelines employing next generation sequencing (NGS) are needed to improve diagnosis and precision of AIDs treatment.

Methods: Based on a survey regarding gene scope and available methodology in AIDs laboratories (Rowczenio et al, 2019), and on annotation of AIDs gene variants by experts' consensus (van Gijn et al, 2018; Infevers data base), a draft of recommendations was written by European Molecular Genetics Quality Network members. The recommendations were discussed by a panel of experts of the International Society for Systemic AIDs (ISSAID) during a consensus meeting.

Results: Four additional disease genes are recommended for the core screen of auto-inflammatory diseases $(A D A 2$, NOD2, PSTPIP1, and TNFAIP3). The initial screen, either a single gene or panel screen is oriented by the clinical autoinflammatory suspicion. In overt cases complementary methods to identify large deletions or insertions (i.e. copy number variation) or search for somatic mosaicism are recommended. A genotype table based on the 5-category variant pathogenicity classification with distinction between rare/novel or common variants of unknown significance, and consideration of dose effect, provides the clinical significance of prototypic genotypes per gene and disease.

Conclusions: These guidelines will improve the practice of AIDs diagnosis provided by the medical community. ERare-3 grant 9003037603.

Y. Shinar: None. I. Ceccherini: None. D. Rowczenio: None. I. Aksentijevich: None. J. Arostegui: None. E. BenChétrit: None. G. Boursier: None. M. Gattorno: None. H. Hayrapetyan: None. H. Ida: None. N. Kanazawa: None. H.J. Lachmann: None. A. Mensa-Vilaro: None. R. Nishikomori: None. C. Oberkanins: None. L. Obici: None. O. Ohara: None. S. Ozen: None. T. Sarkisian: None. 
K. Sheils: None. N. Wolstenholme: None. E. ZonneveldHuijssoon: None. I. Touitou: None. M.E. van Gijn: None.

E-P04.08 TGDS pathogenic variants cause Catel-Manzke syndrome without Manzke dysostosis

F. Boschann ${ }^{1}$, K. Stuurman ${ }^{2}$, C. de Bruin ${ }^{3}$, M. van Slegtenhorst ${ }^{2}, H$. van Duyvenvoorde ${ }^{4}$, S. Kant ${ }^{2,4}$, N. Ehmke ${ }^{l}$

${ }^{1}$ Charité-Universitaetsmedizin Berlin, Berlin, Germany, ${ }^{2}$ Department of Clinical Genetics, Erasmus MC, University Medical Center Rotterdam, Rotterdam, Netherlands, ${ }^{3}$ Department of Pediatric Endocrinology, Leiden Uni, Rotterdam, Netherlands, ${ }^{4}$ Department of Clinical Genetics, Leiden University Medical Center, Leiden, Netherlands

Introduction: Catel-Manzke syndrome, also known as micrognathia-digital-syndrome, is characterized by the combination of the two cardinal features Pierre-Robin sequence (with or without cleft palate) and bilateral hyperphalangism. Hyperphalangism is caused by an accessory ossification center in the second metacarpo-phalangeal joint leading to radial deviation and ulnar clinodactyly of the index fingers. Individuals without one of the two major hallmarks or with additional hand malformations have been described as atypical or Catel-Manzke-like syndrome. Biallelic pathogenic variants in $T G D S$ have been described in nine individuals including one fetus with Catel-Manzke syndrome.

Material and methods: We performed trio exome sequencing in two individuals who presented with disproportionate short stature, micrognathia as well as mild bilateral radial deviation and ulnar clinodactyly of the index fingers, but without radiological signs of hyperphalangism.

Results: We found known pathogenic variants in TGDS in both individuals. The two individuals described here are the first with confirmed TGDS pathogenic variants who present with almost normal hand radiographs and therefore do not show one of the clinical hallmarks of Catel-Manzke syndrome. In addition, disproportionate short stature has not been described as a feature of Catel-Manzke syndrome.

Conclusion: Our findings extend the phenotypic spectrum of Catel-Manzke syndrome. TGDS-associated Catel-Manzke syndrome should be considered in individuals with mild ulnar clinodactyly and radial deviation of the index fingers even without radiological evidence of hyperphalangism.

F. Boschann: None. K. Stuurman: None. C. de Bruin: None. M. van Slegtenhorst: None. H. van Duyvenvoorde: None. S. Kant: None. N. Ehmke: None.

\section{E-P04.09}

A novel pathogenic variant in the CLDN10 gene associated with HELIX syndrome
M. C. Martínez-Romero ${ }^{1,2,3}$, M. Barreda-Sánchez ${ }^{1,3}$, M. E. Pérez-Tomás ${ }^{l}$, M. J. Ballesta-Martínez ${ }^{1,2,3}$, V. LópezGonzález $^{1,4,2}$, M. J. Sánchez-Soler ${ }^{1,2,3}$, A. T. SerranoAntón ${ }^{1,2,4}$, L. Rodríguez-Peña ${ }^{l}, M$. E. Hernández ${ }^{l}, T$. Martínez-Menchón ${ }^{1,4}$, P. Sánchez-Pedreño ${ }^{1,4}$, G. Glover ${ }^{1,2}$, P. Carbonell $^{1,2}$, V. Cabello-Chaves ${ }^{5}$, Grupo de Investigación Español de Displasias Ectodérmicas, E. Guillén-Navarro ${ }^{1,2,4}$

${ }^{I}$ Sección de Genética Molecular (Centro de Bioquímica y Genética Clínica), Sección de Genética Médica (Servicio de Pediatría), Servicio de Medicina Interna y Servicio de Dermatología. Hospital Clínico Universitario Virgen de la Arrixaca. Instituto Murcia, Murcia, Spain, ${ }^{2}$ CIBERER, Centro de Investigación Biomédica en Red de Enfermedades Raras, Instituto de Salud Carlos III, Madrid, Spain, ${ }^{3}$ Cátedra de Genética Médica. UCAM- Universidad Católica de Murcia, Murcia, Spain, ${ }^{4}$ Departamentos de Pediatría y Dermatología. Facultad de Medicina. UMUUniversidad de Murcia, Murcia, Spain, ${ }^{5}$ Servicio de Nefrología. Hospital Universitario Virgen del Rocío, Sevilla, Spain

Introduction: HELIX syndrome (OMIM\#617671) is an autosomal recessive genetic disorder, described in 2017, characterized by hypohidrosis, electrolyte imbalance, lacrimal glands dysfunction, ichthyosis, and xerostomia; and associated with pathogenic variants in $C L D N 10$ gene that encodes a member of claudins proteins (integral membrane components of tight junction). So far, only 4 families have been reported.

Materials and methods: Subjects. Two Spanish adult brothers (41/38 years old) born to consanguineous parents presenting with a long history of hypohidrosis, dry skin, alacrimia and xerostomia, diagnosed as ectodermal dysplasia (ED). Complementary exams showed renal loss of $\mathrm{NaCl}$ with secondary hyperaldosteronism, hypercalcemia, hypermagnesemia and hypokalemia. Genetic analysis. CLDN10 gene was designed with Primer3 software for all coding exons. Sanger sequencing was performed using BigDye terminator (Life-Technologies), analysed in ABI3130 Genetic Analyser (Applied-Biosystems) and aligned with human reference genome GRCh37/hg19; the identified variant was segregated in progenitors.

Results: The sequence analysis revealed a frameshift genetic variant in CLDN10 (NM_006984.4): c.322_329del8/p.Gly108fs, in exon 2, in homozygosity in both affected brothers and in heterozygosity in parents. This variant, not previously described, was found in a highly conserved region by in silico prediction software, being categorized as pathogenic.

Conclusions: 1. A new CLDN10A pathogenic variant associated to HELIX syndrome in the first Spanish affected 
family is described, supporting the implication of this gene in this rare disorder.2. Clinical manifestations such as lacrimal dysfunction, xerostomia and electrolyte imbalance in suspected ED without specific molecular diagnosis are the clue for HELIX syndrome.

This project has been funded by ISCIII-Madrid-SpainFEDER (PI/14/01259 and PI/17/00796) and AADE.

M.C. Martínez-Romero: None. M. Barreda-Sánchez: None. M.E. Pérez-Tomás: None. M.J. Ballesta-Martínez: None. V. López-González: None. M.J. Sánchez-Soler: None. A.T. Serrano-Antón: None. L. Rodríguez-Peña: None. M.E. Hernández: None. T. Martínez-Menchón: None. P. Sánchez-Pedreño: None. G. Glover: None. P. Carbonell: None. V. Cabello-Chaves: None. E. GuillénNavarro: None.

\section{E-P04.10}

Identification a novel mutation of LTBP4 gene in a patient with congenital cutis laxa: a case report

M. Mazaheri ${ }^{1,2},{\text { M. } \text { yavari }^{3}, \text { R. Mirjalili }}^{2}$, A. Razavi $^{3}$

${ }^{1}$ Shahid Sadoughi University of Medical Sciences, Yazd, Iran, Islamic Republic of, ${ }^{2}$ Mother and Newborn Health Research Center Shahid Sadoughi University of Medical Sciences, Yazd, IRAN., Yazd, Iran, Islamic Republic of, ${ }^{3}$ Dr. Mazaheri's Medical Genetics lab Yazd, IRAN., Yazd, Iran, Islamic Republic of

Normal 0 false false false EN-US X-NONE FA /* Style Definitions */ table.MsoNormalTable \{mso-style-name:"Table Normal"; mso-tstyle-rowband-size:0; mso-tstyle-colband-size:0; mso-style-noshow:yes; mso-style-priority:99; mso-style-parent:"'; mso-padding-alt:0cm 5.4pt 0cm 5.4pt; mso-para-margin:0cm; mso-para-margin-bottom:.0001pt; mso-pagination:widow-orphan; font-size:10.0pt; fontfamily:"Times New Roman","serif"; \} Cutis laxa is a rare disorder characterized by degenerative changes in elastic fibres. Congenital cutis laxa is more common than acquired cutis laxa. The recessively inherited form is severe and more frequent. Cutis laxa affects all races and both the sexes equally. LTBP4, an extracellular protein that is closely related to fibrillins, consists of epidermal growth factor-like domains interspersed with four 8-cys domains (including a hybrid domain). The third 8-cys domain covalently binds the small latent TGF $\beta 1$ complex. A proline -rich hinge region provides $\cdots$ exibility to the protein. The $\mathrm{N}$-terminus of LTBP4 binds to the microibrillar structures. A 4-monthold full term normally delivered neonate was referred with complaints of respiratory distress from his birth. Physical examination revealed laxity of the skin and heart problem and swelling around the eyes. Length, weight, and head circumference percentiles at birth were $55 \mathrm{~cm}, 4100 \mathrm{gr}$ and
$36 \mathrm{~m}$ respectively. whole- exome sequencing test was done for the patient. The results showed a novel homozygous mutation (c.533-1G>A) in exon 6 of the LTBP4 gene. After this observation we perform the Sanger sequencing for his parents. As a result, his parents have a heterozygous mutation in the same gene and same location. This finding can be useful in genetic counseling of cutis laxa in this family.

M. Mazaheri: None. M. yavari: None. R. Mirjalili: None. A. Razavi: None.

\section{E-P04.11}

Analysis of xenobiotics biotransformation and DNA repair genes as factors of etiology and pathogenesisin children with congenital spine deformities

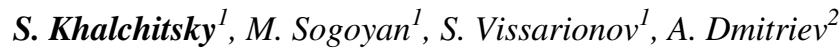

${ }^{1}$ The Turner Scientific Research Institute for Children's Orthopedics, Saint-Petersburg, Russian Federation, ${ }^{2}$ Institute of Experimental Medicine, Saint-Petersburg, Russian Federation

Introduction: Spine congenital curvatures formed as a result of anomalies in the development of vertebral bodies leads to severe and rigid deformities of the spinal column in the adolescent age and are often accompanied by irreversible neurologic disorders. There for genetic markers study of the congenital malformations is an important and urgent task.

Materials and methods: The study of 200 children with congenital spine deformities (CSD) and 96 healthy children without pathology of the spine was carried out. Molecular diagnostics was carried out by analysis of multiple polymorphic regions in the genes of detoxification and DNA repair. Genotyping of the gene polymorphism was performed using polymerase chain reaction.

Results: The polymorphisms of the genes CYP1A2, GSTM1, GSTT1, GSTP1, NAT2, XRCC1, XRCC3 and their frequency distribution among patients with CSD and control group were investigated. Significant differences in the distribution of genotypes compared with the control group were found in the polymorphic regions of the genes CYP1A2, GSTM1, GSTT1, NAT2, XRCC3. However, in the GSTP1 and XRCCl genes, no significant differences between the CSD patients and the control group was observed.

Conclusions: All patients with congenital spine deformities had significant changes in some candidate genes alleles. It was found that the number of minor alleles in the genes under investigation correlate with the severity of congenital deformity and the variability of vertebral anomalies. It was determined that in children with 
combined defects in the spine development, there is a greater number of mutations compared to children with isolated vertebral anomalies.

S. Khalchitsky: None. M. Sogoyan: None. S. Vissarionov: None. A. Dmitriev: None.

\section{E-P04.12}

Another family with compound heterozygosity for variants in $\mathrm{MYH3}$

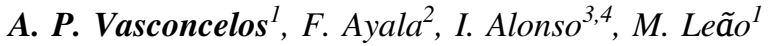

${ }^{1}$ Department of Medical Genetics, Centro Hospitalar Universitário São João, Porto, Portugal, ${ }^{2}$ Department of Medical Genetics, Universidade Estadual de Campinas, Campinas, Brazil, ${ }^{3} I B M C$ - Institute for Molecular and Cell Biology, i3S Instituto de Investigação e Inovação em Saúde, Universidade do Porto, Porto, Portugal, ${ }^{4}$ Genetyca-ICM, Instituto de Estudos Celulares e Moleculares, Porto, Portugal

Contractures, pterygia, and variable skeletal fusions syndrome 1B (CPSKF1B) (OMIM \#618469) is an autosomal recessive disorder, characterized by contractures of proximal and distal joints, multiple pterygia involving the neck, elbows, fingers, and/or knees, and variable vertebral, carpal and tarsal fusions, firstly described by Cameron-Christie et al. All those patients, displaying remarkable phenotypic variability, were compound heterozygous for variants in the MYH3 gene (Cameron-Christie et al., 2018). We describe an 11 years-old male patient, born from non-consanguineous parents, with no relevant family history, whose phenotype was consistent with CPSKF1B, including arthrogryposis of the hips, knees and elbows, multiple pterygia, severe scoliosis and vertebral fusions at cervical, dorsal, lumbar and lumbosacral levels. At the age of 9 there was no radiological evidence of carpal synostosis. Additionally, he has triangular shaped face, low-set ears, bilateral down slanted palpebral fissures, ptosis, and micrognatia. Genetic analysis identified three heterozygous variants in MYH3 [NM_002470.3: c.5072del,p.(Ala1691Valfs*52) in exon 35; c.749G $>$ A,p.(Arg250Gln) and c.787G $>$ T,p.(Asp263Tyr) in the same allele in exon 9]. Genetic testing of his parents identified the first of these variants in the mother and the others in the father, suggesting an autosomal recessive pattern of inheritance. Our patient presents several dysmorphic features not previously reported by Cameron-Christie et al. References: CAMERON-CHRISTIE, S. R., WELLS, C. F., SIMON, M., WESSELS, M., TANG, C. Z., WEI, W., TAKEI, R., AARTS-TESSELAAR, C., SANDARADURA, S. \& SILLENCE, D. O. 2018. Recessive Spondylocarpotarsal synostosis syndrome due to compound heterozygosity for variants in MYH3. The American Journal of Human Genetics, 102, 1115-1125.

A.P. Vasconcelos: None. F. Ayala: None. I. Alonso: None. M. Leão: None.

\section{E-P04.13}

Case report: cellular interference highlights an unusual $\mathrm{X}$-linked mode of inheritance and seemingly genetic paradox in craniofrontonasal dysplasia

\section{J. Ronez, S. Morlot, B. Schlegelberger, H. Wallaschek}

Department of Human Genetics, Hannover Medical School, Hannover, Germany

Introduction: craniofrontonasal dysplasia is a rare form of frontonasal dysplasia caused by mutations in the EFNBI gene on the $\mathrm{X}$ chromosome. EFNB1 encodes for ephrin-B1, a transmembrane ligand of the $\mathrm{EphB}$ receptor with crucial roles in cell migration and pattern formation during skeletal development. The phenotype is more pronounced in females, who characteristically present with frontonasal dysplasia and coronar craniosynostosis, while the manifestation in males is usually milder, often only characterized by hypertelorism.

Case report: We, here, present an 11-week-old girl who presented with dysmorphic facies. Leading characteristics were plagiocephaly, hypertelorism, a cleft palate, slanted eyes, dysplastic ears and a broad nasal bridge. Molecular genetic analyses of the girl revealed a pathogenic de novo nonsense variant in the EFNBI gene, which triggers nonsense mediated decay.

Background: Females with a heterozygous mutation in the EFNB1 gene usually display a more severe phenotype than hemizygous males. The embryonal $\mathrm{X}$-inactivation leads to a heterogenic population of cells either expressing the mutated or the wildtype allele, disrupting the correct formation of boundaries within the frontal bone precursors during embryogenesis. Hemizygous males display a homogenous cell population, since no functional ephrin-B1 can be produced at all, in which case other ephrin-B family members are likely to compensate by assuming ephrin-B1 function.

Conclusion: Due to cellular interference, craniofrontonasal dysplasia does not follow a classic Mendelian mode of inheritance. Our case report is a good example for this mechanism and highlights the need to be aware of such unusual modes of inheritance in genetic counselling.

J. Ronez: None. S. Morlot: None. B. Schlegelberger: None. H. Wallaschek: None.

\section{E-P04.15}

Heterozygous 7q32.3-q35 deletion causes syndromic craniosynostosis 
E. Bukowska-Olech ${ }^{1}$, D. Simon ${ }^{2}$, D. Larysz ${ }^{3}$,

A. Jamsheer ${ }^{1,2}$

${ }^{I}$ Department of Medical Genetics, Poznan University of Medical Sciences, Poznan, Poland, ${ }^{2}$ Centers for Medical Genetics GENESIS, Poznan, Poland, ${ }^{3}$ Department of Radiotherapy, The Maria Skłodowska Curie Memorial Cancer Centre and Institute of Oncology, Gliwice Branch, Gliwice, Poland, Gliwice, Poland

Background: The most commonly recognisable syndromes with craniosynostosis (CS) are regarded as monogenic disorders and result from different pathogenic variants affecting genes, that encode fibroblast growth factor receptors (FGFRs) such as FGFR1, FGFR2, and FGFR3. However, a couple of studies have also pointed to copy number variations (CNVs) as molecular causes of syndromic CS.

Materials and methods: The female patient with psychomotor delay, intellectual disability and multiple CS and her healthy parents were subjected to standard karyotyping analysis. Next, we have implemented array comparative genomic hybridisation (array CGH) for the proband (SurePrint G3 Human CGH Microarray 4×180; Agilent Technologies), followed by microarray-based genes expression profiling (one-color SurePrint G3 Unrestricted GE 8x60K microarray; Agilent Technologies).

Results: Karyotyping analysis revealed a heterozygous deletion on chromosome 7 (der7). Furthermore, with the use of array CGH, we have shown, that the aberration was 12.782 $\mathrm{Mb}$ in size and mapped to locus 7q32.3-q35 (HG19 chr7:131521826-144304164). The CNV encompassed 143 genes including the $B R A F$, that encodes B-Raf. B-raf belongs to one of the molecules involved in RAS/MAPK pathway.

Conclusion: Our finding supports the contribution of CNVs to syndromic CS development. We also pointed to the existing interaction between RAS and FGFs signaling cascades and conclude that dysregulation of those intracellular pathways may give rise to CS. Finally, we strongly suggest screening of RAS-associated genes in patients with Noonan-like syndrome and skull abnormalities. This work was supported by the grant from the Polish National Science Centre, Poland UMO-2016/ 23/N/NZ5/02577 to Ewelina Bukowska-Olech.

E. Bukowska-Olech: None. D. Simon: None. D. Larysz: None. A. Jamsheer: None.

\section{E-P04.16}

\section{Genetic risk factors for hip dysplasia}

K. K. Jacobsen ${ }^{\text {I, L. B. Laborie }}{ }^{2,3}$, H. Kristensen ${ }^{4,5}$, A.

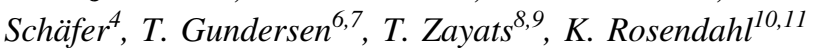

${ }^{I}$ Department of Orthopedic Surgery, Helse Førde, Forde, Norway, ${ }^{2}$ Department of Radiology, Helse Bergen, Bergen,
Norway, ${ }^{3}$ Helse Vest, Bergen, Norway, ${ }^{4}$ Department of Pediatrics, Helse F $\phi$ rde, F $\phi r d e$, Norway, ${ }^{5}$ Department of Clinical Science, Universtity of Bergen, Bergen, Norway, ${ }^{6}$ Department of Orthopedic Surgery, Helse Bergen, Bergen, Norway, ${ }^{7}$ Department of Clinical Medicine, University of Bergen, Bergen, Norway, ${ }^{8}$ Massachusetts General Hospital, Boston, MA, United States, ${ }^{9}$ Stanley Center for Psychiatric Research, Harvard/MIT, Boston, MA, United States, ${ }^{10}$ Department of Radiology, University Hospital of North Norway, Troms $\phi$, Norway, ${ }^{11}$ Department of Clinical Medicine, University of Troms $\phi$, Troms $\phi$, Norway

Introduction: Developmental dysplasia of the hip (DDH) is a congenital condition affecting 2-3\% of all infants (1). DDH increases the risk of osteoarthritis and is the cause of $30 \%$ of all total hip arthroplasties in adults $<40$ years of age (2). We aim to explore the genetic background of DDH in order to improve diagnosis and personaliz treatment.

Materials and method: We conducted a structured PubMed-search and added selected studies from our archives. A total of 66 papers were included for detailed review. In addition, we are conducting a heritability analysis in the Norwegian Twin Registry using Falconer's method.

Results: The results of the heritability analyses will be presented at the conference. Similar studies have found a heritability of $\sim 80 \%$. The literature review revealed that the majority of published papers on the genetics of DDH were mostly underpowered for detection of any association. One large genome wide association study has been published, establishing GDF5 as a plausible risk factor. This study found a single nucleotide polymorphism-based heritability of $55 \%$, however GDF5 only explains $\sim 1.5 \%$ (3).

Conclusion: DDH is known to be congenital and heritable, with family occurrence of DDH already included as a risk factor in most screening programs. Despite this, high quality genetic research is scarce, and no genetic risk factors have been soundly established, prompting the need for more research.

(1) Rosendahl et al, 1996, Pediatric Radiology (2) Engesæter et al, 2011, Acta Orthopaedica (3) Hatzikotoulas et al, 2018, Communications Biology

Grants: Helse Vest (F-12550-D11544)

K.K. Jacobsen: None. L.B. Laborie: None. H. Kristensen: None. A. Schäfer: None. T. Gundersen: None. T. Zayats: None. K. Rosendahl: None.

\section{E-P04.17}

Ectodermal Dysplasia Type 4: Phenotype-Genotype Correlation 


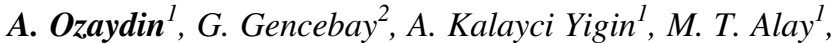
M. Seven ${ }^{1}$

${ }^{1}$ Department of Medical Genetics, Cerrahpasa Medical Faculty, Istanbul University-Cerrahpasa, Istanbul, Turkey, ${ }^{2}$ Department of Skin and Venereal Diseases, Cerrahpasa Medical Faculty, Istanbul University-Cerrahpasa, Istanbul, Turkey

Introduction: Ectodermal dysplasia is a rare hereditary disease that is characterized by elliptical nodules located on the hair shaft. Fragile hair fibers are found in the temporal and occipital region, resulting in alopecia. Patients are usually normal at birth, but the hair becomes fragile in the first few months of life. In severe cases, eyebrows, eyelashes, and other body hairs may also be affected. The disease can be isolated to the scalp, and may be accompanied by tooth and nail disorders. Ectodermal dysplasia is generally caused by mutations in KRT81, KRT83, KRT85 and KRT86 genes.

Clinical Findings and Method: In the physical examination of mother and her three children; localized alopecia, brittle hair-body hair and nail dystrophy were detected and ectodermal dysplasia was considered. DNA was isolated from peripheral blood samples of mother and her children. Due to the pre-diagnosis of ectodermal dysplasia clinical exom analysis was performed in the $K R T$ gene family.

Result: As a result of the analysis, the change of c. G565A (p.D189N) in exon 2 of KRT85 gene in mother and her children was detected as heterozygous and confirmed by Sanger sequencing. This change was evaluated according to the American College of Medical Genetics criteria and compared with genetic databases.

Conclusion: As a result of the evaluation, it is considered as a variant of unknown clinical significance (VUS) and as a pathological variant in silico databases.

A. Ozaydin: None. G. Gencebay: None. A. Kalayci Yigin: None. M.T. Alay: None. M. Seven: None.

\section{E-P04.18}

\section{A case of Classical and concomitant Periodontal Ehlers- Danlos syndrome}

\section{F. Stock ${ }^{l}$, M. R. Hanisch ${ }^{2}$, S. Lechner ${ }^{3}$, S. Biskup ${ }^{3}$, A. Bohring ${ }^{1}$}

${ }^{1}$ Institut für Humangenetik, Universitätsklinikum Münster, Münster, Germany, ${ }^{2}$ Klinik für Mund-, Kiefer- und Gesichtschirurgie, Universitätsklinikum Münster, Münster, Germany, ${ }^{3}$ Praxis für Humangenetik Tübingen, Tübingen, Germany
Ehlers-Danlos syndrome (EDS) is a generic term for a clinically and genetically heterogeneous group of connective tissue disorders. According to the clinical picture and underlying genetic mutation 13 subtypes are distinguished. We saw our proband as a five year old girl with severe periodontitis and premature loss of several teeth. Moreover she displayed a short stature, joint hypermobility, chronic pain, recurrent luxation of knee caps, flat feet, a soft and hyperelastic skin with multiple subcutaneous papules, easy bruising, and pretibial hyperpigmentation. Due to those signs and symptoms EDS was suspected and genetic testing brought about. NGS analysis of 44 connective tissue-related genes revealed a pathogenic frameshift mutation in COL5A1, confirming the diagnosis Classical EDS. This diagnosis was consistent with the proband's joint and skin related phenotype, yet did not explain her severe dental phenotype. As the dental status was highly suggestive of periodontal EDS, analysis of the underlying genes $C 1 R$ and $C 1 S$ was performed. The detection of a pathogenic missense mutation in $C 1 R$ confirmed the additional diagnosis periodontal EDS. Analysis of the parents showed the COL5AI mutation being maternally inherited whereas the $C 1 R$ mutation occured de novo. Our patient's mother experienced many signs of Classical EDS already in her childhood, yet this diagnosis was never suspected in her before it was confirmed in her daughter. To our knowledge, this is the first reported case of Classical and Periodontal EhlersDanlos Syndrome in one single person.

F. Stock: None. M.R. Hanisch: None. S. Lechner: None. S. Biskup: None. A. Bohring: None.

\section{E-P04.19 \\ Role of TANGO1 gene in hypermobile type of Ehlers- Danlos syndrome}

\section{A. Junkiert-Czarnecka, M. Pilarska-Deltow, A. Bak, M. Heise, O. Haus}

Department of Clinical Genetics Nicolaus Copernicus University, Bydgoszcz, Poland

Introduction: Hypermobile Ehlers-Danlos syndrome (hEDS) is a non-inflammatory, autosomal connective tissue disorder. It is perhaps the most common hereditary connective tissue disorder with a prevalence estimate at about 1/5000. hEDS unlike other types of EDS, has no known genetic etiology. One of disfunctions in hEDS patients' cells is impaired transport of extracellular matrix proteins from cells to intercellular space. Protein taking part in this process is TANGO1 encoded by TANGO1 gene. The aim of study is a sequencing analysis of TANGO1 gene and also the evaluation of the potential role of detected variants in 
these gene on their role in the etiology of the hypermobile type of Ehlers-Danlos syndrome.

Methods: The study was carried out in a group of 100 patients with hypermobile type of Ehlers-Danlos syndrome. Control group consisted of 100 persons from general population. Sequencing analysis of gene TANGO1 was performed using Sanger sequencing technique. Results Among 100 hEDS patients in two c.1099A $>$ T (p. Thr367Ser) variant and in next three patients c.2637C $>\mathrm{A}$ (p.Asp879Glu) variant were detected. All patients were not mutation carriers of other connective tissue protein coding genes (previous study). According to ACMG guideline variants were evaluated as VUS.

Conclusion: This study is the next small step in the recognition and understanding of the complex picture of hEDS. Analysis of genes encoding of ECM protein carrier may be a new approach to evaluate genetic background of hypermobile type of Ehlers-Danlos syndrome. This investigation was financed by grant of National Science Centre Poland (2018/29/N/NZ5/00345).

A. Junkiert-Czarnecka: None. M. Pilarska-Deltow: None. A. Bak: None. M. Heise: None. O. Haus: None.

\section{E-P04.20}

Skeletal dysplasia in the era of genomic testing: first experience of a single genetic outpatient clinic from Serbia

\section{Mijovic ${ }^{l}$, J. Ruml Stojanovic ${ }^{l}$, A. Miletic $^{l}$, B. Bosankic ${ }^{l}$, H. Janeski ${ }^{1}$, B. Peterlin', A. Maver ${ }^{2}$, G. Cuturilo ${ }^{1,3}$}

${ }^{1}$ University Children's Hospital, Department of Clinical Genetics, Belgrade, Serbia, ${ }^{2}$ Clinical Institute of Medical Genetics, University Medical Centre Ljubljana, Ljubljana, Slovenia, ${ }^{3}$ Faculty of Medicine, University of Belgrade, Belgrade, Serbia

Introduction: Skeletal dysplasia are very heterogeneous group of genetic disorders, for which in most cases genomic testing (NGS technology) is the first-choice method in genetic testing. We want to present our diagnostic rate, distribution of frequencies between subgroups from classification of skeletal dysplasia and we try to give an answer about impact of diagnosis.

Materials and methods: We present our results for pediatric patients with different skeletal dysplasia who were first assessed in genetic outpatient clinic at Department of Clinical Genetics, University Children's Hospital in Belgrade and referred for diagnostic whole exome sequencing (WES), from 2015 to 2019. WES was performed for all 51 patients.

Results: Causative gene variants were identified in 38 patients $(74.5 \%, \mathrm{n}=38 / 51)$. We have diagnosed patient(s) with Otopalatodigital Spectrum Disorder, Dyggve-MelchiorClausen syndrome, Immuno-osseus dysplasia Schimke type, Acromesomelic dysplasia, Spondyloepimetaphyseal displasia with joint laxity type 2, Osteogenesis imperfecta, Osteopetrosis autosomal recessive type 1, Hypochondroplasia, Mucolypidosis type IV, Mucopolisaccharidosis type IIIA, Craniofrontonasal dysplasia, Oculo-dento-digital dysplasia, Crouzon syndrome, Craniosynostosis type 3, Short sature - brachydactyly - intellectual developmental disabilities - seizures syndrome, 3MC syndrome, Trichorhinophalangeal syndrome and other rare skeletal disorders. In seven cases we performed prenatal diagnosis.

Conclusion: To what extent early genomic diagnosis prevents additional unnecessary diagnostics and the opportunity for anticipatory guidance-related management in patients with skeletal dysplasia? We tried to answer this question based on the first experiences in our single genetic outpatient clinic. This study represent the pilot study with an aim to establish the multidisciplinary skeletal dysplasia Center.

M. Mijovic: None. J. Ruml Stojanovic: None. A. Miletic: None. B. Bosankic: None. H. Janeski: None. B. Peterlin: None. A. Maver: None. G. Cuturilo: None.

\section{E-P04.21}

Identification of rare mutation in ext2 gene among patients with multiple hereditary exostoses

\section{A. Yakovleva, A. Danilova, M. Savvina, D. Petukhova, A. Sukhomyasova, N. Maksimova}

North-Eastern Federal University, Yakutsk, Russian Federation

Multiple hereditary exostoses (MHE) is an autosomal dominant hereditary disorder and the prevalence in various populations ranges is estimated from 1-2 per 100 thousand people or 1 in 7000 orthopedic patients. According to data obtained from the "register of hereditary and congenital pathology of the republic of Sakha (Yakutia)" 80 patients and their relatives from different ethnic groups were registered. Genetic analysis was conducted for 65 patients with MHE and their relatives from 30 unrelated families. Next-generation sequencing (NGS) and Sanger sequencing methods were used. The EXT2 gene from a patient with MHE was sequenced using NGS method. The analysis revealed nonsense mutation c.751>T (p.Gln251*) in exon 5 of EXT2 gene in heterozygous condition and it also were confirmed in the rest 79 patients and their relatives by the Sanger's sequencing method. This mutation was found and confirmed in 16 patients from four families with MHE. Thus, for the first time in the Republic of Sakha (Yakutia), a molecular genetic study of the EXT2 gene was performed in patients with MHE and their relatives. A rare 
nonsense mutation c.751C> T (NM_001178083) was detected in exon 5 of the EXT2 gene, which leads to the replacement of the coding codon with a stop codon at the 251 position of the protein (p.Gln251*, NP_001171554). In the future, the diagnostic of this mutation can be included in the program of genetic testing for medical and genetic counseling. The research is supported by the project FSRG-2020-0014 "Genomic of Arctic: epidemiology, hereditary and pathology"

A. Yakovleva: None. A. Danilova: None. M. Savvina: None. D. Petukhova: None. A. Sukhomyasova: None. N. Maksimova: None.

\section{E-P04.22}

Mosaicism in the PHEX gene. Is it more common than we thought?

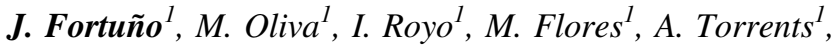
F. Martínez ${ }^{2}$, N. Pérez ${ }^{2}$, C. Camprubíl

${ }^{1}$ Reference Laboratory, L'Hospitalet de Llobregat, Spain, ${ }^{2}$ Hospital Universitario Nuestra Señora de Candelaria, Santa Cruz de Tenerife, Spain

Introduction: Familial X-linked hypophosphatemic rickets (XLHR) is defined as a group of disorders characterized by rickets with bone deformities, short stature, dental anomalies, hypophosphatemia and increased activity of serum alkaline phosphatases. According to the bibliography, pathogenic variants in the $P H E X$ gene are the most common cause of XLHR and only a few cases of mosaicism have been described worldwide. We present two unrelated Canary Islands (Spain) family cases referred with XLHR clinical suspicion in whom mosaic variants have been identified.

Materials and methods: Genomic DNA was isolated from EDTA blood using a standard protocol. Next Generation Sequencing (NGS) was used to analyse $P H E X$ gene in the probands. Sanger sequencing was used to perform the study in the mother's proband.

Results: In one family, a nonsense pathogenic variant ( $p$. Gln394Ter) was found in mosaicism in the proband. In the other family, the proband was hemizygous for a missense variant of uncertain significance (VUS) (p.Ala267Glu). The family analysis reveals that his unaffected mother was mosaic.

Conclusions: To our knowledge, the identified cases are the first families with mosaic variants in $P H E X$ gene reported in Spain. In the genetic counselling context, the "recurrence" of mosaicism in PHEX gene, emphasize to consider unaffected males as possible mosaic carriers. The advantage of performing $P H E X$ gene analysis by NGS is noteworthy: it allows detection of mosaic variants with high sensibility and reliability, as well as it enables discarding aneuploidy, for example a possible Klinefelter karyotype.

J. Fortuño: None. M. Oliva: None. I. Royo: None. M. Flores: None. A. Torrents: None. F. Martínez: None. N. Pérez: None. C. Camprubí: None.

\section{E-P04.23}

Agenesis of corpus callosum - a new phenotypic aspect in fibrodysplasia ossificans progressiva?

N. Meier, N. Knoll, S. Kleiner, M. Ritthaler, E. Wohlleber, J. Kohlhase

SYNLAB MVZ Humangenetik Freiburg GmbH, Freiburg, Germany

Introduction: Mutations in ACVR1 cause fibrodysplasia ossificans progressiva (FOP). The classical clinical feature of FOP is progressive heterotopic ossification, but affected individuals often display first toe and thumb malformations. We report on a one year old patient with motor development delay, corpus callosum agenesis, bilateral hand deformities, hypospadias and bilateral agenesis of the first toes. SmithLemli-Opitz syndrome had been suspected as the cause for limb malformations and hypospadias, and the child was referred to genetics in order to rule out or to confirm this diagnosis.

Material and Methods: normal results for 7-dehydrocholesterol levels made Smith-Lemli-Opitz syndrome unlikely, and we suspected a submicroscopic chromosomal anomaly or an unknown syndrome as the underlying cause. We performed karyotyping and $\mathrm{CGH}$, which revealed normal results, followed by whole exome sequencing (WES).

Results: WES detected a heterozygous de novo variant in ACVR1: c. $1067 \mathrm{G}>\mathrm{A}, \mathrm{p} . \mathrm{G} 356 \mathrm{D}$. A literature search showed that patients with absent first toes and mild brain abnormalities were previously reported in FOP, but agenesis of the corpus callosum had not yet been observed. Furthermore, hypospadias was reported once in an atypical case of FOP.

Conclusions: The combination of first toe anomalies, especially agenesis of first toes, in combination with hypospadias and brain malformations may be the clinical presentation of FOP before the characteristic heterotopic ossifications appear. Agenesis of corpus callosum may be part of the phenotypic spectrum of FOP. The diagnosis of FOP prior to the first heterotopic ossification can lead to an improved clinical management to prevent early heterotopic ossifications in the child.

N. Meier: None. N. Knoll: None. S. Kleiner: None. M. Ritthaler: None. E. Wohlleber: None. J. Kohlhase: None. 


\section{E-P04.24}

GDF5 clinical and molecular spectrum: expanding current knowledge on genotype-phenotype correlations

D. Guadagnolo ${ }^{l}$, M. Genovesi $i^{l}$, E. Marchionni ${ }^{l}$, S. Bernardo ${ }^{l}$, A. Giovannetti ${ }^{2}$, N. Panzironi ${ }^{1}$, A. Traversa ${ }^{2}$, F. Di Palma ${ }^{l}$, G. Mastromoro ${ }^{1}$, P. Palumbo ${ }^{3}$, M. Carella ${ }^{3}$, T. Mazza ${ }^{4}$, V. Caputo ${ }^{1}$, A. Pizzuti ${ }^{l}$

${ }^{I}$ Department of Experimental Medicine, Sapienza University of Rome, Italy, Rome, Italy, ${ }^{2}$ Laboratory of Clinical Genomics, Fondazione IRCCS Casa Sollievo della Sofferenza, San Giovanni Rotondo, Italy, ${ }^{3}$ Laboratory of Medical Genetics, Fondazione IRCCS Casa Sollievo della Sofferenza, Rome, Italy, ${ }^{4}$ Laboratory of Bioinformatics, Fondazione IRCCS Casa Sollievo della Sofferenza, San Giovanni Rotondo, Italy

Introduction: Brachydactyly is a bone development abnormality presenting with variable phenotype and different transmission patterns. Mutations in GDF5 (growth/ differentiation factor 5, MIM: 601146) gene account for a significant amount of cases. A 3-generation family showing isolated brachydactyly was studied. The proband and his grandfather showed features of $\mathrm{C}$ and A1 brachydactyly types, his mother showed only subtle signs.

Materials and methods: Whole Exome Sequencing (WES) was performed on the two affected individuals. An in-depth analysis of GDF5 genotype-phenotype correlations was performed through literature reviewing and retrieving information from several databases to elucidate GDF5 related molecular pathogenic mechanisms.

Results: WES analysis disclosed a known pathogenic variant in GDF5 (NM_000557.4:c.157dupC), segregating with the phenotype. The frameshift variant was previously associated with Brachydactyly type $\mathrm{C}$ in heterozygosis and the severe Grebe chondrodysplasia in homozygosis. We extended and updated the spectrum of GDF5 genotypephenotype correlations. In total, fifty pathogenic mutations were associated with nine clinical phenotypes and autosomal dominant and recessive transmissions even for the same phenotype. Presentations range from severe defects in limb morphogenesis to conditions with redundant ossification. We propose that this clinical gradient can be linked to a continuum of GDF5-activity variation, with variable loss of GDF5-activity underlying bone development defects, and gain-of-function mutations causing disorders with excessive bone formation.

Conclusions: Our in-depth analysis on GDF5 pathogenicity mechanisms furtherly supports that mutation and zygosity backgrounds resulting in the same level of GDF5 activity may lead to similar phenotypes. This information can aid in interpreting new variants and promote appropriate genetic counselling.

D. Guadagnolo: None. M. Genovesi: None. E. Marchionni: None. S. Bernardo: None. A. Giovannetti: None. N. Panzironi: None. A. Traversa: None. F. Di Palma: None. G. Mastromoro: None. P. Palumbo: None. M. Carella: None. T. Mazza: None. V. Caputo: None. A. Pizzuti: None.

\section{E-P04.25}

Identification and characterization of a $G L M N$ splice site variant in a three-generation-family with glomuvenous malformations

\author{
D. Skowronek ${ }^{1}$, M. Hebebrand ${ }^{2}$, R. Erber ${ }^{3}$, A. Agaimy ${ }^{3}$,
} C. Zweier ${ }^{2}$, U. Felbor ${ }^{1}$, M. Rath ${ }^{1}$

${ }^{1}$ Department of Human Genetics, University Medicine Greifswald, and Interfaculty Institute of Genetics and Functional Genomics, University of Greifswald, Greifswald, Germany, ${ }^{2}$ Institute of Human Genetics, FriedrichAlexander-Universität Erlangen-Nürnberg, Erlangen, Germany, ${ }^{3}$ Institute of Pathology, University Hospital Erlangen, Comprehensive Cancer Center Erlangen-EMN, Friedrich-Alexander University Erlangen-Nürnberg (FAU), Erlangen, Germany

Introduction: Glomuvenous malformations (GVMs) are painful vascular lesions with distended venous lumina that are surrounded by multiple layers of smooth-muscle-like glomus cells. Familial GVMs (OMIM: 138000) are inherited in an autosomal dominant manner and associated with loss-of-function germline variants in the GLMN gene.

Material and Methods: We here describe the natural history of GVM disease in a three-generation family and report on the identification and characterization of a GLMN splice site mutation by NGS gene panel sequencing and transcript analysis.

Results: At first clinical examination, the 16-year-old male index patient presented multiple, sharply defined bluish papules on various locations. His 35-year-old mother had approximately ten papules clustering on her back and his 7-year-old maternal half-sister presented with two bluish papules on her neck. The index patient's maternal aunt and grandfather were also reported to have one or two bluish spots each. Using NGS gene panel sequencing, we identified the heterozygous splice site variant c.1299 $+1 \mathrm{G}>\mathrm{A}$ in the GLMN gene of the index case which had not been reported in the literature before. Heterozygosity for the substitution at the donor splice site of exon 14 was also shown for the index patient's mother and maternal halfsister. A $G L M N$ transcript analysis finally verified that this variant causes out-of-frame skipping of exon 14. Following 
the latest ACMG guidelines for variant interpretation, it was classified as pathogenic for autosomal dominant GVM.

Conclusion: The three-generation-family reported here is a testimony for the variable expressivity of GVMs, best explained by Knudson's two-hit model.

D. Skowronek: None. M. Hebebrand: None. R. Erber: None. A. Agaimy: None. C. Zweier: None. U. Felbor: None. M. Rath: None.

\section{E-P04.26}

Identification of novel candidate genes for the hypermobile type of Ehlers-Danlos Syndrome

\section{T. I. Kadurina ${ }^{1}$, L. R. Lonishin ${ }^{2}$, E. A. Serebryakova ${ }^{1,3}$,} L. N. Abbakumova ${ }^{4}$, A. S. Glotov ${ }^{3}$

${ }^{1}$ North-Western State Medical University named after I.I. Mechnikov, St. Petersburg, Russian Federation, ${ }^{2}$ Peter the Great St. Petersburg Polytechnic University, St. Petersburg, Russian Federation, ${ }^{3}$ D.O. Ott Research Institute of Obstetrics, Gynecology and Reproductology, St. Petersburg, Russian Federation, ${ }^{4}$ Saint-Petersburg State Pediatric Medical University, Russia, St. Petersburg, Russian Federation

Introduction: There has been valuable progress in the use of different molecular diagnostic technologies to clarify the classification of the hypermobile type of EhlersDanlos Syndrome (hEDS). Diagnosis of hEDS is only based on clinical criteria, due to the absence of an identified gene and a protein, thus the main objective was to identify possible molecular genetic causes of hEDS development.

Materials and methods: For research, genomic DNA samples of unrelated patients with hEDS were used (9 females and 23 males). In all cases, the diagnosis was established by international recommendations for 2017 EDS. Whole-exome sequencing was performed on a HiSeq2500. Assessment of the pathogenicity of the identified variants was based on the recommendations of ACMG and Sherloc on the interpretation of data obtained by the next-generation sequencing method.

Results: As a result of the pathogenicity assessment, 7 pathogenic and 5 probably pathogenic variants were identified in the encoding regions of the ABCA4, ABCC9, CBS, CCDC8, FIG4, GJB2, HGD, KRT14, LMNA, MEFV, PROM1, RYR1, SLC26A2 and USH2A genes in the heterozygous state. Also, 15 genes were selected with variants of unknown clinical significance based on data on protein expression and high specificity of physical interactions among themselves that may be involved in the development of hEDS.
Conclusions: Considering the results of the study, we cannot exclude that the genes we identified may have a pathogenic effect, forming the hEDS phenotype. The research was partly supported by project 558-2019-0012 (№AAAA-A19-119021290033-1) of FSBSI «D.O. Ott Research Institute of Obstetrics, Gynaecology and Reproductology».

T.I. Kadurina: None. L.R. Lonishin: None. E.A. Serebryakova: None. L.N. Abbakumova: None. A.S. Glotov: None.

\section{E-P04.27}

Genotype-phenotype correlations in hereditary multiple exostoses

I. Akalin ${ }^{1}$, K. Kurosawa ${ }^{2}$, B. Yeter ${ }^{3}$, E. Akbas ${ }^{4}$, G. Nishimura ${ }^{5}$,W. Wuyts ${ }^{6}$, N. H. Elcioglu ${ }^{3}$

${ }^{1}$ Istanbul Medeniyet University, Faculty of Medicine, Department of Medical Genetics, Istanbul, Turkey, ${ }^{2}$ Kanagawa Children's Medical Center, Department of Medical Genetics, Yokohoma, Japan, ${ }^{3}$ Marmara University, Faculty of Medicine, Department of Pediatric Genetics, Istanbul, Turkey, ${ }^{4}$ Istanbul Medeniyet University, Faculty of Medicine, Istanbul, Turkey, ${ }^{5}$ Center for Intractable Diseases, Saitama Medical University Hospital, Saitama, Japan, ${ }^{6}$ Department of Respiratory Diseases, University Hospitals Leuven, Leuven, Belgium

Hereditary Multiple Exostoses (HME) is a complex hereditary musculoskeletal disorder diagnosed at either pediatric or adult age. HME is characterized by progressive exophytic osseous projections that form next to the growth plates of many skeletal elements, including long bones, ribs and vertebrae. In addition to pain and functional problems particular forehand deformities are main complaints of the patients in the clinics that the treatment is restricted to surgical excision of the lesions. The main complication is development of osteochondromas. Mutations in EXT1 or EXT2 genes that encode glycosyltransferases are responsible for the disease and represented locus heterogeneity. Nonetheless, genotype-phenotype interventions are still important we presented $20 \mathrm{HME}$ cases among 9 families and discussed genotype-phenotype correlations. This project has been founded by The Scientific And Technological Research Council Of Turkey (TUBITAK; Project \#217S675)

I. Akalin: None. K. Kurosawa: None. B. Yeter: None. E. Akbas: None. G. Nishimura: None. W. Wuyts: None. N.H. Elcioglu: None.

\section{E-P04.28}

Recurrent bone pain - more than tumors and inflammation 
M. M. Khelifa ${ }^{l}$, S. V. Hardenberg ${ }^{l}, B$. Auber $^{l}, B$. Schlegelberger ${ }^{1}$, S. Mukodzi ${ }^{2}$, J. Wiens ${ }^{2}$, A. K. Bergmann ${ }^{1}$

${ }^{I}$ Medizinische Hochschule Hannover, Hannover, Germany, ${ }^{2}$ Klinikum Wolfsburg, Wolfsburg, Germany

Hyperostosis-hyperphosphatemia syndrome (HHS) and hyperphosphatemic familial tumoral calcinosis (HFTC) are the clinical manifestations of a rare autosomal recessive disease caused by the inactivation of the fibroblast growth factor 23 (FGF23), Polypeptide N-Acetylgalactosaminyltransferase 3 (GALNT3) or Klotho genes, which in turn leads to an elevated blood phosphate level resulting mainly from an excessive proximal renal tubular phosphate reabsorption. We report the case of a 5-year-old female of healthy non-consanguineous parents of German and Italian descent. She initially presented with recurrent lower leg pain. Bone biopsy was inconclusive, as well as infectious work-up. Imaging identified a diaphyseal sclerosis and hyperostosis of the ribs. Hyperphosphatemia was observed. Thus, panel diagnostics of the genes $F G F 23, G A L N T 3$ and $K L$ was performed. Two heterozygous variants in GALNT3 (NM_004482.3) were identified, one previously undescribed missense variant c. $782 \mathrm{G}>\mathrm{A}$ p.(Arg261Gln), classified as a variant of uncertain significance. Protein-modeling of this variant suggested the disruption of GALNT3 activity, leading to an increased inactivation of the phosphaturic hormone FGF23 causing HHS/HFTC. The second variant c.803dupC p.(Thr269Asnfs*3) was previously described in a HHS/HFTC patient and classified as likely pathogenic (ACMG guidelines). In HHS/HFTC, no genotype-phenotype correlation was described so far, and suggested treatment regimes are heterogeneous. Thus, in addition to the detailed case, we present an overview of genotypephenotype correlation and a collection of follow-up descriptions of disease courses on patients reported before 2015. In conclusion, we aim to raise awareness of HHS/ HFTC as a syndrome presenting primary with bone pain and radiographic changes.

M.M. Khelifa: None. S.V. Hardenberg: None. B. Auber: None. B. Schlegelberger: None. S. Mukodzi: None. J. Wiens: None. A.K. Bergmann: None.

\section{EN-P04.29}

Clinical and genealogical characteristics of hereditary forms of ichthyosis in the Republic of Sakha (Yakutia)

\section{M. Sofronova, A. L. Sukhomyasova, N. R. Maksimova}

North-Eastern Federal University, Yakutsk, Russian Federation
Introduction: Hereditary ichthyosis is common name for many dry and scaly skin disorders ranging in frequency from common to very rare. Clinical genealogical analysis of hereditary forms of ichthyosis (from 1989 to 2018) are conducted in the Republic of Sakha (Yakutia), Russia.

Materials and methods: Data was collected from the genetic history of patients of the "Republican Genetic Register of Hereditary and Congenital Pathology of the Republic of Sakha (Yakutia)" of the Medical Genetics Center of the Republican hospital №1 "National center of medicine".

Results: The number of patients with ichthyosis is 126 patients (104 families) according to the genetic register of Yakutia according to date of 2018. The frequency of ichthyosis was about $1 / 7675$, prevalence is 13,0 to 100000 . Gender: 94 men (74.6\%), 32 women (25.4\%). Ethnicity: Yakut - 109 (86.5\%), Russian - 17 (13.5\%). In four families affected patients of both genders and in ten cases only males. All patients had dryness, peeling of the skin. In some cases, more severe symptoms were noted in the form of pityriasis peeling, follicular keratosis. The manifestation varied from birth to adolescence.

Conclusions: It is difficult to establish the type of inheritance and further medical and genetic counseling because of genetic heterogeneity and the similarity of the clinical features of ichthyosis. Thus, there is a strong need in a highly specific diagnostic method and in-depth research study for precise diagnosis, treatment and prevention of these diseases. Project: FSRG-2020-0014 "Genomics of Arctics: epidemiology, hereditary and pathology"

V.M. Sofronova: None. A.L. Sukhomyasova: None. N.R. Maksimova: None.

\section{E-P04.31}

\section{Jeune Syndrome with a novel DYNC2H1 mutation}

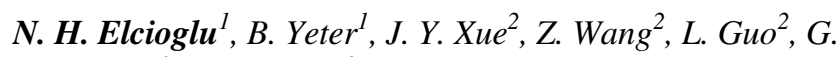
Nishimura $^{3}$, S. Ikegawa ${ }^{2}$

${ }^{1}$ Department of Pediatric Genetics, Marmara University Medical School, Istanbul, Turkey, ${ }^{2}$ Laboratory of Bone and Joint Diseases, Center for Integrative Medical Sciences, RIKEN, Tokyo, Japan, ${ }^{3}$ Center for Intractable Diseases, Saitama Medical University Hospital, Saitama, Japan

Jeune Asphyxiating Thoracic Dystrophy(OMIM\%208500, ATD, Short-rib thoracic dysplasia with or without polydactyly) is an autosomal recessive skeletal dysplasia characterized by short stature and pulmonary hypoplasia secondary to the small thoracic cage. Polydactyly, glomerular sclerosis, biliary dysgenesis, hirshprung disease, retinal dysplasia and cardiac defects are the other findings. Here, we will a male patient who was diagnosed as Jeune 
syndrome at the age of 3 month-old. He was born to a 28 years old G3P3L3 mother by ceserean section with a birth weight of $2685 \mathrm{~g}$ at 36 weeks of gestation. His birth length was $44.5 \mathrm{~cm}$, head circumference was $35 \mathrm{~cm}$. He was hospitalized in a newborn intensive care unit for 3 months due to respiratory distress. Except that the parents were far blood related there was no particular familial history. In his last follow-up at the age of 4.5 years, his measurements were weight: $14 \mathrm{~kg}(-1.9$ sds), height: $97 \mathrm{~cm}(-2.4$ sds), HC:50 $\mathrm{cm}(10-25 p)$. The patient had a narrow thorax and pectus carinatum. On the radiographs, horizontal and short ribs, hypoplastic iliac wings and horizontal acetabulum were detected. Mild sleep apnea was defined in polysomnography. A novel homozygous missense mutation c. G6439T in the DYNC2H1 gene was detected on exome sequencing for the etiologicall cause. Jeune syndrome is a genetically heterogeneous disease nearly forty genes have been identified and defined as ciliopathy. There may be slight to moderate short stature although respiratory difficulties eventuate in all survivors. Moreover, laboratory evaluation of urine/blood, abdominal US, spirometry and ophtalmological exam should be recommended for renal, hepatic and eye involvement

N.H. Elcioglu: None. B. Yeter: None. J.Y. Xue: None. Z. Wang: None. L. Guo: None. G. Nishimura: None. S. Ikegawa: None.

\section{E-P04.33}

Severe osteoporosis in Larsen syndrome: genetic and bone morphological investigations - a case report

\section{T. M. Juul ${ }^{1,2}$, C. Ejersted $^{3}$, L. Folkestad ${ }^{2,3}$, K. Brusgaard ${ }^{1,2}$, S. Hansen ${ }^{4,5}$, C. M. Andreasen ${ }^{6}$, L. K. Thomsen ${ }^{6}$, J. S. Thomsen $^{7}$, T. L. Andersen ${ }^{2,7,8}$, A. L. Frederiksen ${ }^{1,2,9}$}

${ }^{1}$ Dept. of Clinical Genetics, Odense University Hospital, Odense, Denmark, ${ }^{2}$ Dept. of Clinical Research, Faculty of Health, University of Southern Denmark, Odense, Denmark, ${ }^{3}$ Dept. of Endocrinology M, Odense University Hospital, Odense, Denmark, ${ }^{4}$ Dept. of Medicine, Hospital of South West, Esbjerg, Denmark, ${ }^{5}$ Dept. of Regional Health Research, University of Southern Denmark, Odense, Denmark, ${ }^{6}$ Clinical Cell Biology, Dept. of Pathology, Odense University Hospital, Odense, Denmark, ${ }^{7}$ Dept. of Biomedicine, Aarhus University, Aarhus, Denmark, ${ }^{8}$ Dept. of Forensic Medicine, Aarhus University Hospital, Aarhus, Denmark, ${ }^{9}$ Dept. of Clinical Genetics, Aalborg University Hospital, Aalborg, Denmark

Introduction: Larsen syndrome is a genetic condition characterized by facial dysmorphology and skeletal deformities. The syndrome has a dominant inheritance and is caused by heterozygous pathogenic variants in the Filamin
B encoding gene $(F L N B)$. FLNB is an essential cytoskeletal protein which is thought to play a key role in bone morphogenesis. However, the skeletal phenotype of Larsen syndrome has not yet been characterized in details.

Materials and methods: A case-study. Two Caucasian women (mother and daughter; age 63 and 33 years) with Larsen syndrome carrying a novel $F L N B$ c.688G $>$ T, p. (Val230Phe) variant were included. A bone morphologic evaluation was performed including DXA-scanning and high-resolution peripheral quantitative tomography (HRpQCT scan) evaluating their bone microstructure. Furthermore, an iliac crest bone biopsy from the mother was evaluated by $\mu \mathrm{CT}$.

Results: Both women showed signs of severe osteoporosis. The HR-pQCT scan showed a low estimated bone strength and volume density as well as decreased trabecular number compared to data from a population-based group of Danish women. The $\mu \mathrm{CT}$ analysis of the bone biopsy showed similar trabecular changes. The porosity of the cortical bone was found to be normal or decreased in both the HR-pQCT and the $\mu \mathrm{CT}$ analysis.

Conclusions: Our findings include an early onset of severe osteoporosis, which is likely due to structural changes in the trabecular bone compartment. On the other hand, the cortical bone compartment shows no osteoporotic characteristics. This highlights that the investigated FLNB variant may have a very different effect on the trabecular and cortical compartments.

T.M. Juul: None. C. Ejersted: None. L. Folkestad: None. K. Brusgaard: None. S. Hansen: None. C.M. Andreasen: None. L.K. Thomsen: None. J.S. Thomsen: None. T.L. Andersen: None. A.L. Frederiksen: None.

\section{E-P04.35}

Maffucci syndrome-like manifestations deciphered as a compound heterozygote of Gronblad-Strandberg syndrome by exome sequencing.

A. Ikonomidis $^{1}$, E. Oikonomidou ${ }^{2}$, A. Malamidou ${ }^{2}$, A. Arampatzi $i^{2}, N$. Peroulis ${ }^{2}, K$. Krapis $^{3}$, T. Konstantoula ${ }^{3}$, P. Katsiafiloudis ${ }^{3}$, A. Papadogoulas ${ }^{3}$, M. Hatzimichail ${ }^{3}$,

T. Zafeiridis ${ }^{3}$, E. Neou ${ }^{3}$, D. Papadopoulos ${ }^{3}$,

P. Papamichalis $^{3}$, S. Karagiannis ${ }^{3}$, A. Komnos ${ }^{3}$, K. Karakousis ${ }^{3}$, A. Xefteris $^{2}$

${ }^{1}$ Eurogenetica Thessalias, Larissa, Greece, ${ }^{2}$ Eurogenetica, Thessaloniki, Greece, ${ }^{3}$ Koutlimpanio General Hospital of Larissa, Larissa, Greece

Introduction: Exome sequencing is a state of the art technique that may clarify the genotype of complex overlapping phenotypes. We present a case of a female with a typical clinical phenotype of Maffucci syndrome which was 
finally deciphered as a compound heterozygote of Gronblad-Strandberg syndrome.

Materials and methods: A 20-year-old female suspected of Maffucci syndrome presented multiple cartilage deformities as well as hemangiomas and lymphangiomas disseminated along her body. Clinical exome sequencing was performed on the DNA extracted from patient's blood, using Sophia Genetics' Clinical Exome Solution v2. Following preparations according to the manufacturer's protocol, DNA libraries were sequenced on an Illumina NextSeq-500 genetic analyser. Data processing, variant calling and pre-classification were conducted by SOPHiA $\mathrm{DDM}^{\circledR}$ bioinformatics pipelines.

Results: The patient carried wild-type isocitrate dehydrogenase 1 (IDH1) and isocitrate dehydrogenase 2 (IDH2) alleles related to Maffucci syndrome. Instead, she was found to be a compound heterozygote for ATP-bindingcassette-subfamily-C-member-6 gene (ABCC6) related to pseudoxanthoma elasticum or Gronblad-Strandberg syndrome. In particular, she carried the pathogenic c. $1652 \mathrm{~T}>\mathrm{C}$ (Phe551Ser) mutation and the c.2836C $>$ A (Leu946Ile) mutation which is of debatable pathogenicity.

Conclusions: Exome sequencing revealed a compound heterozygote with Gronblad-Strandberg syndrome initially suspected to present a case of Maffucci syndrome. Pseudoxanthoma elasticum leads to mineralization of skin, eye, cardiovascular and gastrointestinal systems and commonly present with papules in the skin and/or with angioid streaks of the retina. Thus, in a great extent, the clinical manifestations are in discordance with the underlying genotype which suggests a novel phenotypic manifestation of Gronblad-Strandberg syndrome.

A. Ikonomidis: None. E. Oikonomidou: None. A. Malamidou: None. A. Arampatzi: None. N. Peroulis: None. K. Krapis: None. T. Konstantoula: None. P. Katsiafiloudis: None. A. Papadogoulas: None. M. Hatzimichail: None. T. Zafeiridis: None. E. Neou: None. D. Papadopoulos: None. P. Papamichalis: None. S. Karagiannis: None. A. Komnos: None. K. Karakousis: None. A. Xefteris: None.

\section{E-P04.36}

\section{A case report of somatic mosaicism for a FBN1 nonsense} variant

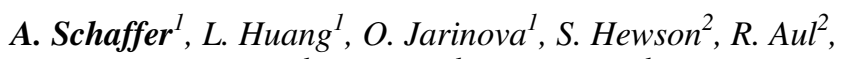
J. McGowan-Jordan ${ }^{l}$, A. Smith ${ }^{l}$, L. Bronicki ${ }^{l}$

${ }^{1}$ Department of Genetics, Children's Hospital of Eastern Ontario, Ottawa, ON, Canada, ${ }^{2}$ Department of Genetics, Hospital for Sick Children, Toronto, ON, Canada

Marfan syndrome (MS) is an autosomal dominant disorder caused by mutations in the fibrillin-1 gene (FBN1). It is a connective tissue disorder characterized by a combination of cardiovascular, musculoskeletal, ophthalmic and pulmonary manifestations. The wide clinical variability of this disorder can make its recognition difficult. Diagnosis is usually established based on the defined clinical criteria of the Ghent-2 nosology. Approximately 1800 pathogenic or likely pathogenic variants have been identified in FBN1, the majority of which are specific to a single family, and $25 \%$ occurring de novo. Somatic and/or germline mosaicism have been reported in rare cases.

Here we describe an asymptomatic 50-year-old female who presented for genetic counselling after her two daughters, from different partners, were both diagnosed with MS. A known pathogenic variant, c.1693C $>$ T, p. Arg565* in FBN1 (NM_000138.4), was confirmed in both affected half-siblings. However, targeted Sanger sequencing for this variant was negative in the mother. Repeat testing via Next-Generation-Sequencing (NGS) of FBN1 was offered to the patient.

NGS analysis of FBN1 revealed the presence of the variant in $9 \%$ of the sequencing reads. This is well below the allele frequency expected for a heterozygous germline variant $(\sim 50 \%)$. Based on the family history, this variant was reported at a mosaic level of $9 \%$.

These results are consistent with somatic and germline mosaicism in this patient. The recurrence risk for any future offspring is now known to be up to $50 \%$. Additionally, she has been referred to Cardiology and Genetics to assess for any clinical manifestations of MS in herself.

A. Schaffer: None. L. Huang: None. O. Jarinova: None. S. Hewson: None. R. Aul: None. J. McGowan-Jordan: None. A. Smith: None. L. Bronicki: None.

\section{E-P04.37}

Next Generation Sequencing in Marfan syndrome: rare genetic variants improve genotype phenotype correlation

N. Marinakis, C. Sofocleous, E. Tsoutsou, D. Veltra, M. Sviggou, J. Traeger-Synodinos, E. Fryssira

National \& Kapodistrian University of Athens, Athens, Greece

Introduction: Marfan syndrome (MFS) is an autosomal dominant disorder of connective tissue with a prevalence of 1 per 5000 individuals. MFS is characterized by early aortic thoracic dilatations/aneurysms, defects of the skeletal and ocular systems, tall stature, arachnodactyly, pectus excavatum, joint hypermobility and ectopia lentis. Mutations in the Fibrillin-1 gene (FBN1) are the most common cause of MFS. Nevertheless, mutations in other genes (e.g. TGFBRI, FLNA, COL5A1, SLC2A10, ADAMTSL4, CBS, SMAD3, 
TGFB2, MED12, FBN2, PLOD1, BMPR2 etc.) have also been associated with MFS. New technologies, such as Next Generation Sequencing, contribute to the identification of known and novel mutations in these genes.

Materials and methods: Clinical information and peripheral blood samples were collected from 25 patients with Marfan or Marfan-like phenotype and heart defects. DNA extraction and Whole Exome Sequencing (WES), analysing approximately 19.000 genes dispersed throughout the genome-were applied.

Results: WES analysis revealed positive results in 17 patients, accomplishing a diagnostic yield of $68 \%$. Causative alterations included 14 known and novel pathogenic variants in the FBN1 gene, 1 in the $F B N 2$ gene, 1 in the $B M P R 2$ gene and 1 in the $S L C 2 A 10$ gene.

Conclusion: WES can support definitive diagnosis in patients with Marfan or Marfan-like phenotypes, contributing to precise prognosis, systematic monitoring, and when appropriate, identification of other family members. NGS supports marked improvement in diagnostic-yield for numerous inherited disorders including Marfan Syndrome, especially in the case of non $F B N 1$ variants. The study was carried out as part of the Erasmus+ project:N-GeneS

N. Marinakis: None. C. Sofocleous: None. E. Tsoutsou: None. D. Veltra: None. M. Sviggou: None. J. TraegerSynodinos: None. E. Fryssira: None.

\section{E-P04.39}

\section{A novel pathogenic variant in the $C H R N G$ gene linked to} Escobar variant of multiple pterygium syndrome

H. Kathom ${ }^{1}$, D. Avdjieva-Tzavella ${ }^{l}$, T. Delchev ${ }^{1}$, S. Atemin ${ }^{2,3}$, T. Todorov ${ }^{3}$, A. Maver ${ }^{4}$, B. Peterlin ${ }^{4}$, V. Mitev $^{2}$, A. Todorova ${ }^{2,3}$

${ }^{1}$ University paediatric hospital "prof. Ivan Mitev", Sofia, Bulgaria, ${ }^{2}$ Department of Medical Chemistry and Biochemistry, Medical University Sofia, Sofia, Bulgaria, ${ }^{3}$ Genetic Medico-Diagnostic Laboratory "Genica", Sofia, Bulgaria, ${ }^{4}$ Clinical Institute of Medical Genetics, UMC Ljubljana, Ljubljana, Slovenia

Introduction: Multiple pterygium syndromes (MPS) comprise a group of multiple congenital anomaly disorders characterized by webbing (pterygia) of the neck, elbows, and/or knees and joint contractures (arthrogryposis) $)^{\mathrm{a}}$. The multiple pterygium syndromes are phenotypically and genetically heterogeneous but are traditionally divided into prenatally lethal and nonlethal (Escobar) types. In this report we present a case of Escobar type MPS with novel pathogenic variant in $C H R N G$.

Materials and methods: We present a 25 months old girl from fifth pregnancy with complications. She was born small for gestational age with dysmorphic facial features, skeletal abnormalities, arthrogryposis, and hypoplastic labia majora, without typical webbing.

Results: WES analysis was conducted after negative findings in karyotype, MLPA screening for microdeletions, subtelomeric deletions and duplications, urinary GAGs, NGS for congenital arthrogryposes. Two pathogenic variants were identified in the CHRNG gene, c.241C $>\mathrm{T}$ previously reported in homozygous state in patient with a similar clinical picture, and c.1143G $>$ A which has not been previously described.

Conclusions: Polimalformative syndromes including skeletal defects, and joint contractures, even without the typical webbing, should be tested for Escobar variants of MPS, because standard arthrogryposis gene panels can miss the diagnosis.

H. Kathom: None. D. Avdjieva-Tzavella: None. T. Delchev: None. S. Atemin: None. T. Todorov: None. A. Maver: None. B. Peterlin: None. V. Mitev: None. A. Todorova: None.

\section{E-P04.40}

Compound heterozygous variant in $N B A S$ as a case of bone fragility by the type of osteogenesis imperfecta

\section{A. Petukhova ${ }^{1}$, E. Gurinova ${ }^{2}$, A. Sukhomyasova ${ }^{1,2}$, N. Maksimova ${ }^{1}$}

${ }^{1}$ Research Laboratory "Molecular Medicine and Human Genetics", Medical Institute, M. K. Ammosov NorthEastern Federal University, Yakutsk, Russian Federation, ${ }^{2}$ Medical Genetic Center, Republican Hospital №1 «National Medical Center», Yakutsk, Russian Federation

Introduction: Biallelic mutations in the $N B A S$ gene have been reported to cause three different clinical signs: short stature with optic nerve atrophy and Pelger-Huët anomaly (SOPH) syndrome, infantile liver failure syndrome 2 (ILFS2) and a combined severe phenotype including both SOPH and ILFS2 features.

Material and Methods: Here, we describe a case of a 6year-old Yakut girl who presented with clinical signs of SOPH syndrome, acute liver failure (ALF) and bone fragility by the type of osteogenesis imperfecta (OI). Targeted panel sequencing for 494 genes of connective tissue diseases was performed on our patient. To predict the protein-damaging effects of the suspected pathogenic variations in NBAS, three different software programs were used: Polyphen-2, SIFT, MutationTaster.

Results: The molecular genetic study revealed the known heterozygous variant c.5741G $>\mathrm{A}$ (p.Arg1914His) in 45 exon of NBAS. Also, in 23 exon of NBAS was identified a previously unreported missense heterozygous variant 
c.2535G >T (p.Trp845Cys). Mutation affect evolutionarily conserved amino acid residues and predicted to be highly damaging.

Conclusion: Timely health care of patients with such a set of clinical spectrum of SOPH syndrome, ALF and bone fragility can contribute to establishment coordinated multispecialty management of the patient focusing on the health problems issues through childhood.

Grant: The study is supported by the Ministry Education and Science of Russian Federation (Project No. FSRG2020-0014 "Genomics of Arctic: epidemiology, hereditary and pathology").

D.A. Petukhova: None. E. Gurinova: None. A. Sukhomyasova: None. N. Maksimova: None.

\section{E-P04.43}

Nonsyndromic orofacial clefts - Identifying putative causative genes by $\mathrm{CNV}$ analysis of whole exome sequencing data

S. Hölzel ${ }^{l}$, N. Ishorst ${ }^{l}$, C. Greve ${ }^{l}, F$. Degenhardt $^{l}, D$. Drichel $^{2}$, C. Maj ${ }^{3}$, M. Nothnagel' ${ }^{2}$ J. Y. Hehir-Kwa ${ }^{4}$, J. A.

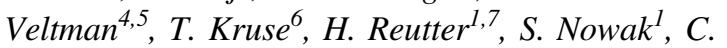
Carels $^{8,9}$, I. van Rooij ${ }^{10}$, K. U. Ludwig ${ }^{1}$, E. Mangold ${ }^{1}$

${ }^{1}$ Institute of Human Genetics, University of Bonn, School of Medicine and University Hospital Bonn, Bonn, Germany, ${ }^{2}$ Cologne Center for Genomics, University of Cologne, Cologne, Germany, ${ }^{3}$ Institute for Genomic Statistics and Bioinformatics, University of Bonn, School of Medicine and University Hospital Bonn, Bonn, Germany, ${ }^{4}$ Department of Human Genetics, Donders Institute, Radboud University Medical Center, Nijmegen, Netherlands, ${ }^{5}$ Institute of Genetic Medicine, Newcastle University, Newcastle-uponTyne, United Kingdom, ${ }^{6}$ Department of Orthodontics, University of Cologne, Cologne, Germany, ${ }^{7}$ Department of Neonatology, Children's Hospital, University of Bonn, Bonn, Germany, ${ }^{8}$ Department of Human Genetics, $K U$ Leuven, Leuven, Belgium, ${ }^{9}$ Orthodontics, University Hospitals KU Leuven, Leuven, Belgium, ${ }^{10}$ Department for Health Evidence, Radboud Institute for Health Sciences, Radboud university medical center, Nijmegen, Netherlands

Nonsyndromic cleft lip with/without palate (nsCL/P) and cleft palate only (nsCPO) are frequent congenital malformations with high heritability estimates. Common risk loci explain only some of their heritability. Rare, highly penetrant copy number variations (CNVs) encompassing possible clefting susceptibility genes might partially explain the missing heritability. Aim of this study was to identify such genes by detecting de novo CNVs from whole-exome sequencing (WES) trio data. CNVs were called from WES data of $50 \mathrm{nsCL} / \mathrm{P}$ and $43 \mathrm{nsCPO}$ Central European patient/ parent trios using XHMM, CoNIFER and EXCAVATOR2. Defining XHMM as primary algorithm we established a filtering pipeline considering sample wide frequency, de novo status, calling intersection (CoNIFER and/or EXCAVATOR2), annotation to RefSeq-genes, overlap with regions of segmental duplications and population frequency. CNVs were validated using qPCR. Three interesting de novo $\mathrm{CNVs}$ were identified: A $86 \mathrm{~kb}$ deletion on $\mathrm{Chr} 8$ in an nsCL/P patient, a $16 \mathrm{Mb}$ deletion on $\mathrm{Chr} 1$ in an $\mathrm{nsCPO}$ patient, and an $877 \mathrm{~kb}$ duplication on $\mathrm{Chr} 2$ in an $\mathrm{nsCPO}$ patient. Interesting candidate genes at these loci are $L H X 8$, $D L X 1$ and $D L X 2$, all suggested to contribute to craniofacial development: Targeted mutations of $L h x 8$ in mice lead to an isolated cleft palate. In embryonic mice $D l x 1$ and $D l x 2$ are expressed in both the maxillary and the mandibular component of the first pharyngeal arches, and Dlx $1 / 2^{-/-}$mutant mice exhibit a cleft palate. Our results indicate that rare highly penetrant CNVs can contribute nsCL/P and nsCPO risk. To achieve more evidence for our findings, replication studies in larger patient/control cohorts are essential.

S. Hölzel: None. N. Ishorst: None. C. Greve: None. F. Degenhardt: None. D. Drichel: None. C. Maj: None. M. Nothnagel: None. J.Y. Hehir-Kwa: None. J.A. Veltman: None. T. Kruse: None. H. Reutter: None. S. Nowak: None. C. Carels: None. I. van Rooij: None. K.U. Ludwig: None. E. Mangold: None.

\section{E-P04.44}

Glis3 expression in working model murine osteoarthritis

\section{E. Rothzerg}

\section{University of Western Australia, Perth, Australia}

Osteoarthritis (OA) is characterised by cartilage degradation, hypertrophy and inflammation. Chondrocytes are the only cells normally found in cartilage, producing and maintaining the cartilaginous matrix. Chondrocyte hypertrophy is an essential process required for endochondral ossification in mammals and it is also plays a role in the development of OA. Furthermore, genetic factors have been found to be strong determinants of OA and are likely to operate via chondrocyte function. I previously identified some novel candidate murine genes with a potential role in OA pathophysiology. However, one of the candidate genes, Glis3, has showed a potential by expressed in hypertrophic chondrocytes in OA knee joints obtained from the collaborative cross mouse model, using immunohistochemistry. In conclusion, this study has implicated several molecular mechanism in the pathogenesis of cartilage degeneration, thus improving our understanding of $\mathrm{OA}$ and offering potential molecular targets for novel therapeutic approaches. 
E. Rothzerg: None.

\section{E-P04.47}

A novel homozygous SLURP1 variant in a boy with Mal de Meleda born to non-consanguineous parents

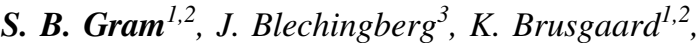 A. Bygum ${ }^{4,2}$, L. B. Ousager ${ }^{1,2}$}

${ }^{I}$ Department of Clinical Genetics, Odense University Hospital, Odense, Denmark, ${ }^{2}$ Department of Clinical Research, University of Southern Denmark, Odense, Denmark, ${ }^{3}$ Department of Clinical Genetics, Aarhus University Hospital, Aarhus, Denmark, ${ }^{4}$ Department of Dermatology and Allergy Centre, Odense University Hospital, Odense, Denmark

Introduction: Mal de Meleda (MDM) is an autosomal recessive disorder characterized by transgredient hyperkeratosis and erythema of palms and soles with well-demarcated borders. There might also be symptoms as hyperhidrosis, macerations and malodour due to secondary bacterial infections. Associated perioral erythema, brachydactyly, scaly plaques on elbows and knees as well as nail dystrophy has also been described. The disease presents early in childhood and has a progressive cause. MDM is associated with variants in SLURPI. The HGMD database includes 23 different variants in $S L U R P 1$ related to MDM or palmoplantar keratoderma.

Material \& methods: A 4-year-old boy presented with an approximately two-year history of erythema, scaling and diffuse, yellowish, transgredient keratoderma of palms and soles. He had associated interdigital macerations, dystrophia of several nails and recurrent fungal and bacterial infections. He was born as the second child of non-consanguineous parents and there was no family history of similar skin disease. The parents were from families living in different regions of Denmark. Genetic testing was performed using a next-generation sequencing-based gene panel targeting 28 genes related to palmoplantar keratoderma.

Results: A homozygous nonsense variant was identified in SLURP1 (c.259del, p.(Ala87Profs*4)). The variant, predicted to result in a premature stop codon, has not previously been reported. A more distal frameshift variant has, however, been reported as disease-causing supporting interpretation of the variant as likely pathogenic. The parents were heterozygous for the variant.

Conclusion: We identified a novel homozygous variant in SLURP1 in a Caucasian boy with MDM, who was born to non-consanguineous parents.

S.B. Gram: None. J. Blechingberg: None. K. Brusgaard: None. A. Bygum: None. L.B. Ousager: None.

\section{E-P04.49}

Molecular genetic diagnostic in skeletal disorders - a Western Romanian delineation

\section{E. Jurca-Simina ${ }^{1,2}$, A. Chirita-Emandi ${ }^{1,2}$,}

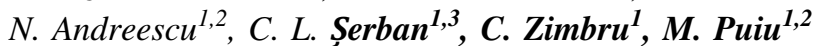

${ }^{1}$ Center of Genomic Medicine in Victor Babes University of Medicine and Pharmacy, Timisoara, Romania, ${ }^{2}$ Department of Medical Genetics, Louis Turcanu Emergency Hospital for Children, Timișoara, Romania, ${ }^{3}$ Functional Sciences Department, University of Medicine and Pharmacy Victor Babes, Timișoara, Romania

Introduction: Genetic skeletal disorders(GSD) represent a heterogeneous and large group, widening the more the sequencing technology thrives. We aim to contribute by describing the regional Romanian cohort of patients with GSD and their molecular features.

Methods: The clinical workup was performed for each affected individual. NGS was performed using the Illumina TruSight One (4813 genes) and TruSight Cardio (174 genes) Sequencing Panels on MiSeq Illumina platform, as by amplicon-based approach, but also PCR and Sanger were used.

Results and discussions: 110 patients with GSD phenotypic component addressed the Genetics department (July 2015-December 2019). 54.55\% had confirmed diagnosis, while $30.9 \%$ were tested negative for the suspected disorder and $14.55 \%$ were not tested. $10 \%$ were found with variants of uncertain significance, needing further testing. According to the Mortier's et al. 2019 revision of nosology and classification of GSD, we have found the following categories of disorders: $25 \%$ of patients with overgrowth syndromes with skeletal involvement (Marfan, Loeys-Dietz, Congenital contractural arachnodactyly), $10 \%$ with lysosomal storage diseases with skeletal involvement (Mucopolysaccharidosis), 5\% with Limb hypoplasia-reduction defects group (Fanconi anemia and Cornelia de Lange), 4\% with Osteogenesis imperfecta and decreased bone density, $2 \%$ with Brachydactylies with extraskeletal manifestations (Coffin Siris) and unique patients in other several disorders like Spondyloperipheral dysplasia, Atelosteogenesis type 3, Chondroectodermal dysplasia, Asphyxiating thoracic dysplasia, etc.

Conclusion: Skeletal disorders are rare entities needing proficient clinical and imagistic workup to reach diagnosis. We provide our Center's diagnostic yields in GSD together with particularities and discussions on our cohort of patients.

I.E. Jurca- Simina: D. Speakers Bureau/Honoraria (speakers bureau, symposia, and expert witness); Modest; Sanofi. Other; Modest; Sanofi, Takeda. A. Chirita-Emandi: Other; 
Modest; Sanofi, Takeda. N. Andreescu: Other; Modest; Sanofi, Takeda. C.L. Șerban: None. C. Zimbru: None. M. Puiu: Other; Modest; Sanofi, Takeda.

\section{E-P04.50}

A case report of a patient diagnosed with two skeletal dysplasias: osteogenesis imperfecta and pseudoachondroplasia

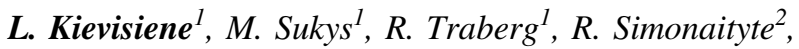 V. Asmoniene ${ }^{l}$}

${ }^{I}$ Department of Genetics and Molecular Medicine, Lithuanian University of Health Sciences, Kaunas, Lithuania, ${ }^{2}$ Department of Pediatric Surgery, Lithuanian University of Health Sciences, Kaunas, Lithuania

We present a case report of a 7 years old boy, the only child born to a man with osteogenesis imperfecta (OI) and a woman suspected to have pseudoachondroplasia, later on confirmed to have COMP gene pathogenic heterozygous variant (c.1393G>C, p.Gly465Arg). Since birth, patient had seven spontaneous fractures, predominantly in the lower limbs. At the age of seven the patient exhibited disproportionately short-limb short stature with height measured $96 \mathrm{~cm} ;<3$ rd percentile. The patient had brachydactyly and ligamentous laxity with deformity of the lower limbs. $\mathrm{He}$ also had characteristic radiological changes of pseudoachondroplasia: short tubular bones with widened and deformed metaphyses and vertebral anomalies. Dual energy $\mathrm{x}$-ray absorptiometry detected osteoporosis. Next generation sequencing was performed for the patient, OI genes and COMP gene known familial variant were analysed. It confirmed the same pathogenic variant of COMP gene and in COL1A2 gene two heterozygous variants were found (c.2317C $>$ T, p.Pro773Ser and c.2329_2331delCGT, p. Arg777del). The former was considered to be variant of uncertain significance because in silico predictors assessed it as benign, while the latter was assigned to be likely pathogenic because computational predictors assessed it as pathogenic. None of these variants were described in population databases. Both of $C O L 1 A 2$ variants were found in father's COL1A2 gene, meaning that they locate in the same allele. It remains unclear if these variants are diseasecausing since neither of fathers' family members were affected with OI. Therefore, further sequencing of father's first-degree family members will be performed to confirm if these variants are truly de novo.

L. Kievisiene: None. M. Sukys: None. R. Traberg: None. R. Simonaityte: None. V. Asmoniene: None.

\section{E-P04.51}

Further clinical and molecular delineation of spondylocostal dysostosis type 3

S. L. Schuhmann ${ }^{1}$, H. Koller
, H. Sticht

${ }^{1}$ Institute of Human Genetics, Friedrich-Alexander-Universität Erlangen-Nürnberg FAU, Erlangen, Germany, ${ }^{2}$ Klinikum rechts der Isar, Technische Universität München, Munich, Germany, ${ }^{3}$ Institute of Biochemistry, FriedrichAlexander-Universität Erlangen-Nürnberg FAU, Erlangen, Germany

Spondylocostal dysostosis (SCDO) is a heterogeneous group of rare spine disorders defined by multiple vertebral segmentation defects and rib anomalies. Patients present with shortened trunk in proportion to height and significant scoliosis. Seven subtypes of SCDO have been identified based on deleterious variants in one affected gene each (DLL3, MESP2, LFNG, HES7, TBX6, RIPPLY2, DMRT2), of mostly autosomal recessive inheritance. Most subtypes include only few cases, like SCDO3 $(L F N G)$ where only two affected individuals are reported to date with a total of three variants.

In a large study on genetic causes of skeletal dysplasia we recognized a 17 -year-old individual from healthy consanguineous parents with severe vertebral segmentation defects, rib and further non skeletal anomalies including solitary pelvic kidney, uterine dysgenesis, absence epilepsy and inner ear deafness. Performing WES we identified a homozygous missense variant c.59C $>\mathrm{T}$, p.(Thr20Ile) in $L F N G$, neither previously described in $\mathrm{SCDO} 3$ patients, nor listed in genetic databases. Both parents were heterozygous. The variant is predicted to destabilize a hydrogen bond leading to an impairment of protein function, likewise previously described variants inactivating the protein with altered Notch signaling.

While the two previously reported cases had isolated skeletal anomalies the here reported individual presented with various additional symptoms. As no other variants were identified neither in the exome nor in CMA we hypothesize that the phenotypic spectrum of this syndrome can be much broader, perhaps depending on the localization and nature of the affected variant, although we cannot exclude a blended phenotype due to an undetected additional variant.

S.L. Schuhmann: None. H. Koller: None. H. Sticht: None. C. Kraus: None. M. Krumbiegel: None. S. Uebe: None. A. Ekici: None. A. Reis: None. C.T. Thiel: None. 


\section{E-P04.52}

First reported case of Steel syndrome in the European population: a novel homozygous mutation in COL27A1

E. Kritioti ${ }^{1,2,3}$, A. Theodosiou ${ }^{l}, N$. Nicolaou ${ }^{2}$, A. Alexandrou $^{l}$, I. Papaevripidou ${ }^{l}$, E. Efstathiou ${ }^{4}, V$. Christophidou-Anastasiadou ${ }^{5}$, C. Sismani ${ }^{1,3}$,

G. A. Tanteles $^{2}$

${ }^{1}$ Cytogenetics and Genomics Department, The Cyprus Institute of Neurology and Genetics, Nicosia, Cyprus, ${ }^{2}$ Clinical Genetics Department, The Cyprus Institute of Neurology and Genetics, Nicosia, Cyprus, ${ }^{3}$ The Cyprus School of Molecular Medicine, The Cyprus Institute of Neurology and Genetics, Nicosia, Cyprus, ${ }^{4}$ Pediatric Department, Larnaca General Hospital, Larnaca, Cyprus, ${ }^{5}$ Clinical Genetics Department, Archbishop Makarios III Medical Centre, Nicosia, Cyprus

Steel syndrome is an autosomal recessive disorder that primarily affects the skeletal system causing a variety of manifestations. Sixteen individuals with Steel syndrome, mainly Puerto Ricans (11/16), were previously reported to carry bi-allelic mutations in the COL27Al gene. Here, we present the first patient with Steel syndrome in Europe and the sixth non-Puerto Rican carrying a novel homozygous mutation in COL27A1. The patient is a 4-year-old boy born to non-consanguineous healthy parents, with dysmorphic facial features, absent hip ossification centres, external rotation of both feet, relatively short stature, mild skin syndactyly, short mid phalanges and bilateral sensorineural hearing loss. Whole exome sequencing (WES) revealed a novel homozygous missense variant [chr9.hg:19: g.116982108G $>$ A; c. $2409 \mathrm{G}>\mathrm{A} ; \quad$ p.(Gly802Glu)] in COL27Al (NM_032888.4). The homozygous mutation was confirmed by Sanger sequencing in the proband and carrier status was confirmed in both parents and his unaffected sibling. According to online and in-house minor allele frequency (MAF) databases, this is the first COL27Al mutation reported in the European population. Additional screening of healthy Greek-Cypriot individuals was thus performed, which did not reveal any additional carriers in the population for the variant in question.

E. Kritioti: None. A. Theodosiou: None. N. Nicolaou: None. A. Alexandrou: None. I. Papaevripidou: None. E. Efstathiou: None. V. Christophidou-Anastasiadou: None. C. Sismani: None. G.A. Tanteles: None.

\section{E-P04.53}

Platyspondylic dysplasia, Torrance type resembling achondrogenesis type $1 \mathrm{~A}$
M. van den Born ${ }^{l}$, M. Joosten ${ }^{l}$, P. Schut ${ }^{l}, J$. Cornette $^{l}$, A. van der Hout ${ }^{2}$, G. Mortier ${ }^{3}$

${ }^{1}$ Erasmus MC, Rotterdam, Netherlands, ${ }^{2}$ University Medical Centre Groningen, Groningen, Netherlands, ${ }^{3}$ University of Antwerp, Antwerp, Belgium

To diagnose a specific lethal skeletal dysplasia purely based on ultrasound data can be quite challenging in a prenatal setting. However, molecular testing of fetal DNA in combination with radiographic evaluation and autopsy postdelivery usually allows the clinician to come up with a specific diagnosis. We report a case of platyspondylic lethal skeletal dysplasia, Torrance type (PLSD-T) that was initially diagnosed as achondrogenesis type 1A. Advanced ultrasound at 20 weeks of gestation showed, in addition to generalized edema, shortened and curved long bones with a thoracic-AC ratio of 0.68 . At 29 weeks of gestation a male neonate was born who died directly after birth. Achondrogenesis type $1 \mathrm{~A}$ was initially suggested based on the ultrasound abnormalities and clinical examination postnatally. Radiographs showed skeletal abnormalities reminiscent of achondrogenesis type 1A: severe micromelia with flared and cupped metaphyses, relatively large head, short ilia and delayed vertebrae ossification. All genes (TRIP11, SLC26A2 and COL2A1) causing the different forms of achondrogenesis (IA;IB;II) were sequenced. In TRIP11, only heterozygosity for a class 3 variant was identified and no variant could be identified on the other allele. However, a class 4 variant p.(lle1 389dup), in COL2Al was identified in the portion coding for the C-propeptide of type II collagen. This finding led to the diagnosis of PLSD-Torrance type. This case demonstrates the phenotypical overlap between achondrogenesis type 1A and Torrance dysplasia.

M. van den Born: None. M. Joosten: None. P. Schut: None. J. Cornette: None. A. van der Hout: None. G. Mortier: None.

\section{E-P04.55}

Novel variant in the EZH2 gene causing Weaver syndrome

\section{E. M. Vaiteniene ${ }^{l}$, L. Cimbalistiene $e^{l, 2}$, G. Zukauskaite ${ }^{l}$,} A. Utkus ${ }^{l}$

${ }^{I}$ Department of Human and Medical Genetics, Institute of Biomedical Sciences, Faculty of Medicine, Vilnius University, Vilnius, Lithuania, ${ }^{2}$ Centre for Medical Genetics, Vilnius University Hospital Santaros Klinikos, Vilnius, Lithuania 
Background: Overgrowth syndromes frequently share overlapping manifestations and are difficult to diagnose based on clinical features. Accurate diagnosis is important for prognosis and correct tumour surveillance program and can be achieved with next-generation sequencing.

Results: A 4-year-old girl presenting with excessive growth of prenatal onset, advanced bone age, macrocephaly, development delay, intellectual disability, generalized seizures and peculiar face was referred to our clinic. She was a third child in a healthy non-consanguineous family, born prematurely at 36 weeks gestation with birth weight $3100 \mathrm{~g}$ and height $58 \mathrm{~cm}$. Her karyotype revealed interstitial inversion 46,XX, inv $(X)(\mathrm{p} 22 ; \mathrm{q} 28)$ which was inherited from her healthy mother. No pathogenic variants were observed in the NSDl gene. At the age of 22 years, her height is $195 \mathrm{~cm}$, weight $140 \mathrm{~kg}$; limited shoulder, elbow and wrist movement, kyphosis, hypertrophic soft tissue around knees were observed besides previously mentioned features. Her IQ score is 40. Next-generation sequencing for known overgrowth conditions revealed a novel missense heterozygous EZH2 mutation c.373G>A (NM_004456.4:c.[373G>A]; [373=], NP_004447.2:p.(Glu125Lys) within exon 5. Based on 11 in silico tools of pathogenicity prediction detected variant was characterized as pathogenic. In addition to the typical findings of Weaver syndrome, our patient has polythelia, atrial septal defect and lumbar spinal stenosis.

Conclusion: An increasing number of diagnoses with next-generation sequencing helps to gather more phenotypic information, improve management and surveillance of overgrowth syndromes. This example also highlights the importance of periodic case reviews for patients not diagnosed on initial testing as more information becomes available in the medical literature.

E.M. Vaiteniene: None. L. Cimbalistiene: None. G. Zukauskaite: None. A. Utkus: None.

\section{E-P04.56}

Novel pathogenic variant in $E B P$ gene is associated to $\mathrm{X}$-linked dominant chondrodysplasia punctata in a female fetus.

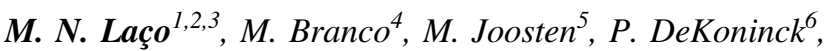 E. Galhano ${ }^{4}$, F. Ramos ${ }^{1,4}$}

${ }^{1}$ Medical Genetics Unit, Hospital Pediátrico, Centro Hospitalar e Universitário de Coimbra, Coimbra, Portugal, ${ }^{2}$ Faculty of Medicine, University of Coimbra, Coimbra, Portugal, ${ }^{3}$ Faculty of Health Sciences, University of Beira Interior, Covilhã, Portugal, ${ }^{4}$ Prenatal Diagnosis Center, Maternidade Bissaya Barreto, Centro Hospitalar e Universitário de Coimbra, Coimbra, Portugal, ${ }^{5}$ Department of Clinical Genetics, Erasmus MC, University Medical Center Rotterdam, Rotterdam, Netherlands, ${ }^{6}$ Department of
Obstetrics and Gynaecology, Erasmus MC, University Medical Center Rotterdam, Rotterdam, Netherlands

Introduction: X-linked dominant chondrodysplasia punctata (CDPX2) is an inherited condition present almost exclusively in females, characterized by skeletal dysplasia, skin defects and congenital cataracts and caused byheterozygous pathogenic variants in the EBP gene. Prenatal ultrasound diagnosis has been reported in some cases after detection of asymmetric shortening of the long bones with cartilage stippling, representing foci of calcification, usually in the second trimester of pregnancy.

Materials and methods: A female fetus was diagnosed with asymmetric bilateral short long bones and stippling of the proximal epiphyses of the humerus by prenatal ultrasound examination at 21 weeks of gestation. Absence of nasal bones was also observed previously at 13 weeks. Amniocentesis was performed for molecular analysis through array $\mathrm{CGH}$ and a 531 genes NGS panel for skeletal dysplasia. The pregnancy was terminated at 23weeks of gestation. Fetal gross examination, postnatal X-rays and steroid chromatography in amniotic liquid were performed to assist in the diagnosis.

Results: The NGS skeletal dysplasia panel identified a de novo heterozygous missense variant c.388G $>A$ p. (Gly130Arg), in the EBP gene. This variant was not reported in the literature nor in gnomAD or other databases and in silico analysis predicts it as likely pathogenic.

Conclusions: To our knowledge this is a novel EBP pathogenic variant, albeit missense variants changing the same Gly130 have been previously associated to CDPX2. CDPX2 should be a diagnosis to bear in mind when short fetal long bones and stippling are present in second trimester of pregnancy.

M.N. Laço: None. M. Branco: None. M. Joosten: None. P. DeKoninck: None. E. Galhano: None. F. Ramos: None.

\section{E-P05 Cardiovascular Disorders}

\section{E-P05.01}

A new missense variant in $A B C A 1$ gene, p.Ala2058Gly, in a myocardial infarction patient.

\section{R. Pan-Lizcano ${ }^{1}$, L. Nunez ${ }^{1}$, P. Pinon ${ }^{2}$, X. Flores ${ }^{2}$, G. Aldama ${ }^{2}$, R. Calvino ${ }^{2}$, N. Blanco-Menendez ${ }^{1}$, N. Vazquez-Gonzalez, J. M. Vazquez-Rodriguez, ${ }^{3}$, M. Hermida-Prieto ${ }^{1}$}

${ }^{1}$ Cardiology Research Group. Instituto de Investigación Biomédica de A Coruña (INIBIC)-CHUAC-UDC, A Coruña, Spain, ${ }^{2}$ Department of Cardiology, Complexo Hospitalario Universitario A Coruna (CHUAC)-INIBIC, A Coruña, Spain, A Coruña, Spain, ${ }^{3}$ Department of Cardiology, Complexo Hospitalario Universitario de A Coruña 
(CHUAC)-CIBERCV, Instituto de Investigación Biomédica de A Coruña (INIBIC), Universidad de A Coruña (UDC), A Coruña, Spain

Background: Acute myocardial infarction (AMI) is a complex multifactorial and polygenic disorder that leads the causes of death in Western societies. However, the understanding of the genetic contribution to ischemic heart diseases is very limited. It has been suggested that variants in genes related to pathways involved in atherothrombosis could increase the risk of development cardiac events.

Methods: Genetic variants in 24 genes which encoded proteins related to pathways involved in atherothrombosis were analyzed by next generation sequencing in 24 patients with AMI. Mutation found was confirmed by Sanger sequencing. Visualization of the mutation using pdb viewer and in silico prediction of the effects produced by the mutation (Mutation Tester, SNAP2, SIFT2, Polyphen, PhDSNP) were performed.

Results: In one patient, p.Ala2058Gly was identified in $A B C A 1$ gene that encodes the ATP-binding cassette transporter 1 , which is crucial in the initial step of highdensity lipoprotein (HDL) formation and in reverse cholesterol transport. All the in silico tools used suggested a pathological role of this variant.

Conclusion: Our results are in concordance with previously reports that associated different variants in $A B C A 1$ gene with an increased risk of coronary artery disease and ischemic heart disease. Thus, mutations in $A B C A 1$ gene could have a role in the development of AMI. Funding Acknowledgements: This work was supported by a grant from Instituto de Salud Carlos III (PI18/01737) and a non-conditional grant from Abbott Vascular.

R. Pan-Lizcano: None. L. Nunez: None. P. Pinon: None. X. Flores: None. G. Aldama: None. R. Calvino: None. N. Blanco-Menendez: None. N. Vazquez-Gonzalez: None. J. M. Vazquez-Rodriguez: None. M. Hermida-Prieto: None.

\section{E-P05.02}

\section{VUS or non VUS in ACTA2?}

E. Micaglio ${ }^{1}$, S. Benedetti ${ }^{2}$, S. Marelli ${ }^{3}$, A. G. Pini ${ }^{3}$,

R. Cotugno ${ }^{l}$, M. M. Monasky ${ }^{l}$, G. Ciconte ${ }^{l}$, P. Carrera $^{2}, C$. Pappone $^{l}$

${ }^{1}$ Arrhythmology and Clinical Electrophysiology, IRCCS Policlinico San Donato, San Donato Milanese, Italy, ${ }^{2}$ Laboratory of Clinical Molecular Biology and Cytogenetics, IRCCS Ospedale San Raffaele, Milan, Italy, ${ }^{3}$ Cardiovascular Genetics, IRCCS Policlinico San Donato, San Donato Milanese, Italy
Many ACTA2 missense variants are associated with aortic dissection but their role is still controversial. We describe a 53 year old male proband with a history of sudden death (maternal grandmother) and thoracic aortic aneurysm in the father. The proband bicycled regularly and experienced aborted cardiac arrest at the age of 52 during a normal daylight activity. ECG showed transient ischemic patterns with increased troponin-T values. A CT scan demonstrated a type A aortic dissection (AD) not involving coronary arteries, surgically corrected without complications. During the follow up, occasional seizures were observed, successfully treated with Levetiracetam. Ajmaline challenge test resulted negative, no arterial tortuosity neither signs of intracranial vessel involvement have been demonstrated by Angio MRI. The proband was studied with NGS including panels for Brugada syndrome, LQTS and AD genes. Only the c.1004C $>$ T (p.Pro335Leu) heterozygous missense variant in the ACTA2 gene was found. Proband's only daughter is asymptomatic, negative at clinical evaluation and does not carry the paternal variant. No other relatives are available to date. The c.1004C $>$ T (p.Pro335Leu) in the ACTA2 gene is predicted to be "pathogenic" by several bioinformatics tools, but to date its clinical significance is still uncertain. This case suggests the importance to consider clinical history in VUS evaluation for specific conditions. Further studies are required to clarify the role of ACTA2 missense variants in type $\mathrm{A}$ aortic dissections.

E. Micaglio: None. S. Benedetti: None. S. Marelli: None. A.G. Pini: None. R. Cotugno: None. M.M. Monasky: None. G. Ciconte: None. P. Carrera: None. C. Pappone: None.

\section{E-P05.03}

Molecular diagnosis of primary hypoalphalipoproteinemia disease by Next Generation Sequencing

\section{E. Sevilla, C. Rodríguez Jiménez, J. Sanguino, A. Carazo, V. Fernández-Montaño, I. Rueda-Arenas, F. Arrieta, S. Rodríguez-Nóvoa}

\section{INGEMM, Madrid, Spain}

Introduction: Hypoalphalipoproteinemia primary 2 (\# 618463), is generally an autosomal recessive disorder associated with extremely low levels of apoA-I and HDL-C in serum and, in most of the patients, premature atherosclerotic cardiovascular disease, xanthomas and corneal opacities ${ }^{1}$. Currently, 30 families have been described. This disease is caused by pathogenic variants in APOA1 gene (MIM 107680) that code for the Apolipoprotein A-I (apoAI), the major structural component of HDL. Herein we 
report a case of a male with suspicious of hypoalphalipoproteinemia.

Materials and methods: A 58-year-old woman with xanthomas, corneal clouding and familial history of isquemic cardiopathy, was remitted to our service for genetic testing. The patient had extremely low levels of apoA-I $(<16.0 \mathrm{mg} / \mathrm{dL})$ and HDL-C $(6 \mathrm{mg} / \mathrm{dL})$ in serum. Genes involved in cholesterol metabolism were analyzed by Next Generation Sequence (NGS) using a customized panel of 411 genes related with metabolic diseases.

Results: The frameshift mutation APOA1_NM_ 000039.2:c.85dupC;p.(Gln29Profs*29) was found in homozygosis in the patient resulting a truncated apoA-I. In silico analysis showed damaging and conservation for this variant. Sanger sequencing confirmed the variant found in APOA1.

Conclusions: This variant c.85dupC;p.(Gln29Profs*29) has been previously described as a cause of ApoA1 deficiency ${ }^{2}$. ApoA-I deficiency is generally associated with markedly increased risk of atherosclerotic cardiovascular disease. However, in this study we identified a homozygous frameshift mutation of the APOAl gene as the molecular basis of apoA-I deficiency in a 58-year-old woman without isquemic cardiopathy. A familiar study would be required for a better understanding of this rare disease-causing mutation.

E. Sevilla: None. C. Rodríguez Jiménez: None. J. Sanguino: None. A. Carazo: None. V. FernándezMontaño: None. I. Rueda-Arenas: None. F. Arrieta: None. S. Rodríguez-Nóvoa: None.

\section{E-P05.04}

\section{Study functional in $A P O B$ in HepG2 from patient with} Familial Hypercholesterolemia.

C. Rodriguez Jimenez ${ }^{1}$, D. Gómez-Coronado ${ }^{2}$, J. Sanguino ${ }^{1}$, E. Sevilla ${ }^{1}$, A. Carazo ${ }^{1}$, N. Gallego-Onís ${ }^{l}$, V. Gómez del Pozol , F. Arrietal, S. Rodríguez-Nóvoa ${ }^{l}$

\section{${ }^{1}$ INGEMM, Madrid, Spain, ${ }^{2}$ Hospital Ramón y Cajal, Madrid, Spain}

Introduction: Familial defective apoB100 (FCHL2, \# 144010) is a genetic disorder of LDL metabolism characterized by hypercholesterolemia and premature atherosclerosis. FCHL is caused by heterozygous mutation in the $A P O B$ gene. APOB is the ligand for the LDLreceptor and there are some essential residues that are decisive for the APOB-LDL receptor affinity ${ }^{1}$.

Materials and methods: A 56-year-old man with raised levels of LDL-c in serum, with family history and premature coronary artery disease. The DNA sample from patient was analysed by NGS using a customized panel
METABOL-v2 of 411 genes. LDL isolated from patient and the control were incubated with HepG2 cells. Uptake and binding were measured by flow cytometry in order to determine the impact of $A P O B$ variant.

Results: A variant of unknown significance (VUS) was found in $A P O B \_$exon26_NM_000384.2:c.11401T $>A ; \mathrm{p}$. (Ser3801Thr) in silico study showed damaging and conservation. However the LDL isolated of patient had the same impact in uptake and binding in LDLR of HepG2 cells that the LDL isolated of control.

Conclusions: This variant had conflict in the interpretation of results in ClinVar has been classified as benign and as VUS. It is well known that exon 26 is critical in folding of APOB protein also, in silico analysis reflected impact in the variant. The functional characterization of variant in $A P O B$ has allowed us to classify it as benign avoiding the "uncertain significance".

\section{References}

1- Miserez, A.R. and Keller, U. (1995) Differences in the phenotypic characteristics of subjects with familial defective apolipoprotein B-100 and familial-hypercholesterolemia. Arterioscler. Thromb. Vasc. Biol,

C. Rodriguez Jimenez: None. D. Gómez-Coronado: None. J. Sanguino: None. E. Sevilla: None. A. Carazo: None. N. Gallego-Onís: None. V. Gómez del Pozo: None. F. Arrieta: None. S. Rodríguez-Nóvoa: None.

\section{E-P05.05}

Phenotype-genotype correlation in Russian patients with arrhythmogenic right ventricular cardiomyopathy

\section{A. Shestak ${ }^{1}$, A. Bukaeval, O. Blagova ${ }^{2}$, Y. Lutokhina ${ }^{2}$, M. Polyakl', S. Dzemeshkevich ${ }^{1}$, E. Zaklyazminskayal}

${ }^{1}$ Petrovsky National Research Center of Surgery, Moscow, Russian Federation, ${ }^{2}$ The Department of Cardiology of the V.N. Vinogradov Faculty therapeutic clinic, I.M. Sechenov First Moscow State Medical University, Moscow, Russian Federation

Arrhythmogenic right ventricular cardiomyopathy (ARVC) is an inherited disease leading to fibro-fatty replacement of right ventricle and sudden cardiac death. Identification of mutation(s) in causative genes is one of the major diagnostic criteria of ARVC. Methods: Clinical, instrumental examination, medical and genetic counseling were performed for 70 ARVC probands (mean age 41,9 y. o.). Screening of 16 genes was performed by highthroughput semiconductor sequencing combined with Sanger sequencing. Potential role of genetic variants was evaluated according to ACMG criteria. Mutational spectrum of desmosomal and non-desmosomal genes in ARVC probands was studied in comparison with control group of 
46 DCM probands. Results: Definite, borderline or possible ARVC diagnosis based on the Task Force Criteria of 2010 was established for 70 probands. Some patients in our cohort had signs of dilated/restrictive cardiomyopathy $(\mathrm{N}=17)$, LVNC $(\mathrm{N}=10)$, BrS patterns $(\mathrm{N}=4)$. Likely pathogenic genetic variants and VUCSes were identified in $46 \%$ of ARVC probands in desmosomal (PKP2, DSG2, $D S P, D S C 2, J U P)$ and non-desmosomal (LMNA, SCN5A, MYH7, MYBPC3, TTN, FLNC, LDB3) genes. Mutational spectrum of genes in ARVC probands with a predominance of variants in desmosomal genes (64\%) was different from one in the DCM control group. Genetic variants in non-desmosomal genes prevailed in patients with ARVC overlapping phenotypes. Conclusion: Likely pathogenic genetic variants and VUCSes were identified in $46 \%$ of ARVC probands. Identification of mutations in desmosomal and non-desmosomal genes in addition to the deep phenotyping results allowed us to study the genetic heterogeneity of ARVC. This work was supported by Russian Science Foundation grant №16-1510421.

A. Shestak: None. A. Bukaeva: None. O. Blagova: None. Y. Lutokhina: None. M. Polyak: None. S. Dzemeshkevich: None. E. Zaklyazminskaya: None.

\section{E-P05.06}

Testing the association between ACE ID and MTHFR C667T gene polymorphisms and arterial hypertension in the Caucasian population

C. Barbarosie ${ }^{1}$, R. Nica ${ }^{2}$, O. Aparcioaie ${ }^{1}$, A. Ducal, P. Apostol ${ }^{3}$, O. Alexiu-Tomal, I. Radu ${ }^{l}$, R. Sionel, M. Toma ${ }^{2}$, D. Cimponeriu ${ }^{1}$, S. Nica ${ }^{4}$

${ }^{1}$ Faculty of Biology, University of Bucharest, Bucharest, Romania, ${ }^{2}$ Central Military University Emergency Hospital "Dr. Carol Davila", Bucharest, Romania, ${ }^{3}$ Genetic Expert Center, Sante Clinic, Bucharest, Romania, Bucharest, Romania, ${ }^{4}$ Bucharest Emergency University Hospital, Bucharest, Romania

Introduction: Genetic polymorphisms of various genes play a significant role in the predisposition for arterial hypertension $(\mathrm{AH})$. The relation between several genes and $\mathrm{AH}$ have been investigated in different populations but results were inconsistent and inconclusive. The aim of this study was to evaluate whether polymorphisms in two candidate genes are associated with $\mathrm{AH}$ in Romania's adult Caucasian population.

Materials and methods: We analysed data from 1660 unrelated Romanian Caucasian subjects (835 with $\mathrm{AH}$ and 825 normotensive). The average age of the hypertensive patients and controls were $54.66 \pm 4.30$ and $47.73 \pm 4.05$, respectively. The ACE ID and MTHFR C677T polymorphisms were genotyped using PCR based methods. Statistical tests were conducted with StatsDirect. The significance of the differences in observed frequencies of polymorphisms was assessed by chi-squared test $(p<0.05)$. The distribution of the genotypes in all lots respects Hardy Weinberg equilibrium condition.

Results: Significant associations were detected between $\mathrm{HA}$ and the ACE DD $(\mathrm{OR}=1.44, \mathrm{p}=0.0007)$ and MTHFR TT $(\mathrm{OR}=1.46, \mathrm{p}=0.0009)$. The ACE $\mathrm{D}$ allele was associated with earlier onset of $\mathrm{AH}$ in patients with $\mathrm{AH}$ $(\mathrm{p}=0.00005)$. ACE DD $(\mathrm{OR}=1.88, \mathrm{p}=0.0006)$, ACE $\mathrm{D}$ $(\mathrm{OR}=1.92, \mathrm{p}<0.0001)$, and MTHFR TT $(\mathrm{OR}=2.28$, $\mathrm{p}<0.0001)$ were significantly associated with $\mathrm{AH}$ among subjects whose parents also have AH. Smoking subjects with MTHFR TT genotype $(\mathrm{OR}=1.68, \mathrm{p}=0.0004)$ and those heterozygous $(\mathrm{OR}=1.46, \mathrm{p}=0.0002)$ or homozygous $(\mathrm{OR}=1.62, \mathrm{p}=0.0005)$ for ACE $\mathrm{D}$ presented higher risk for $\mathrm{AH}$. ACE DD and alcohol consumption predispose to $\mathrm{AH}$ $(\mathrm{OR}=5.6, \mathrm{p}=0.0004)$.

Conclusions: This study suggests that ACE and MTHFR genes are associated with $\mathrm{AH}$ in the studied population.

C. Barbarosie: None. R. Nica: None. O. Aparcioaie: None. A. Duca: None. P. Apostol: None. O. Alexiu-Toma: None. I. Radu: None. R. Sionel: None. M. Toma: None. D. Cimponeriu: None. S. Nica: None.

\section{E-P05.08}

A novel EMD mutation in patient with dilated cardiomyopathy

L. Piherova ${ }^{l}$, P. Melenovska ${ }^{l}$, V. Baresova ${ }^{l}, J$. Sovova $^{l}$, D. Musalkova $^{l}$, J. Sikora ${ }^{l}$, H. Hartmannova ${ }^{l}$, K. Hodanova ${ }^{l}$, T. Schimerova ${ }^{2}$, M. Kubanek ${ }^{2}$, S. Kmoch ${ }^{1}$

${ }^{1}$ RURD, Prague 2, Czech Republic, ${ }^{2}$ IKEM, Prague 4, Czech Republic

Emery-Dreifus muscular dystrophy is an inherited degenerative myopathy. One of the earliest manifestation of this disorders are muscle contractures causing joint deformities. Almost all patients with Emery-Dreifuss muscular dystrophy develop abnormalities in heart rhythm, such as atrial fibrillation, bradycardia, syncope, heart failure or an increased risk of sudden death. Among Emery-Dreifuss Xlinked myopathy belongs degenerative myopathy caused by mutation in EMD gene. Emerin is a member of the nuclear lamina-associated protein family and with other proteins takes part in membrane anchorage to the cytoskeleton. Emerin is a serine-rich protein and composed of three domains: nucleoplasmic domain, transmembrane domain and short lumenal domain. More than 100 mutations are known in $E M D$ gene but only one in lumenal domain. We 
present a patient diagnosed with Emery-Dreifuss muscular dystrophy and dilated cardiomyopathy caused by new EMD gene mutation at the luminal domain and its biological consequences. LM201509 NCLG, AZV 15-27682A, SVV260367, NV19-08-00122

L. Piherova: None. P. Melenovska: None. V. Baresova: None. J. Sovova: None. D. Musalkova: None. J. Sikora: None. H. Hartmannova: None. K. Hodanova: None. T. Schimerova: None. M. Kubanek: None. S. Kmoch: None.

\section{E-P05.10}

The influence of the circadian genes CLOCK and ARNTL on myocardial infarction

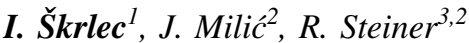 \\ ${ }^{1}$ Hystology, Genetics, Cellular and Molecular Biology Laboratory, Faculty of Dental Medicine and Health, Josip Juraj Strossmayer University of Osijek, Osijek, Croatia, ${ }^{2}$ Faculty of Medicine Osijek, Josip Juraj Strossmayer University of Osijek, Osijek, Croatia, ${ }^{3}$ Clinical Department of Cardiovascular Diseases and Intensive Care, Clinic for Internal Medicine, University Hospital Osijek, Osijek, Croatia}

Introduction: The circadian rhythm regulates various physiological mechanisms, and its disruption can promote many disorders. Many cardiovascular events show morning circadian preferences, such as myocardial infarction (MI), dissection of aortic aneurysms, and stroke. Disturbance of endogenous circadian rhythms enhances the chance of MI, showing that circadian clock genes could have a crucial function in the onset of the disease. It was hypothesized that the polymorphisms of one nucleotide (SNP) in two circadian rhythm genes (CLOCK and ARNTL) could be associated with MI.

Materials and methods: This case-control study was performed on 1057 participants. The sample included 431 patients (243 males, 188 females) of Croatian origin with non-fatal acute myocardial infarction and 626 participants in the control group without MI. Three SNPs were analyzed in CLOCK (rs13124436, rs6811520, and rs11932595), and three in the ARNTL gene (rs12363415, rs4757144, and rs3789327).

Results: Statistically significant differences, estimated by the chi-square test, were found in the distribution of alleles and genotypes between MI and no-MI groups of the CLOCK (rs6811520 and rs13124436) and ARNTL (rs3789327 and rs12363415) genes. Some of the tested SNPs were associated with cardiovascular risk factors in MI patients.

Conclusion: According to the results of the present study, the polymorphisms in the CLOCK and ARNTL genes could be related to MI. Polymorphisms in those clock genes might be an additional risk factor for myocardial infarction. Grant no. FDMZ-2019-IP8

I. Škrlec: None. J. Milić: None. R. Steiner: None.

\section{E-P05.12}

Genetic components of congenital heart defects in children

V. Galagan ${ }^{1,2}$, A. Dorokhina ${ }^{2,1}$, M. Tsyhankova ${ }^{l}$, S. Kulbalaieval, Y. Dudierina ${ }^{2}$, V. Samilyk ${ }^{2}$

${ }^{I}$ National children's specialized hospital «OKHMATDYT» of Ministry of Health of Ukraine, The Center of Medical Genetics, Kyiv, Ukraine, ${ }^{2}$ O.O. Bogomolets' National Medical University, Kyiv, Ukraine

Introduction: Among congenital malformations in children the greatest number is taken by defects of the cardiovascular system. According to Ukrainian and foreign researchers, congenital heart defects (CHD) occur at an average frequency of 59-60 per 10000 newborns. The purpose of this study was to determine the frequency of CHD in newborns and genetic components of this pathology in Kyiv.

Materials and methods: The following methods were used in this work: clinical-genealogical, cytogenetic including cytomolecular (FISH), biochemical (TMS) and conventional clinical and instrumental methods.

Results: The frequency of isolated CHD in newborns was determined based on medical-genetic counseling (MGC) practice at the Specialized Center of Medical Genetics in Kyiv. In 2018 the frequency was 90 per 10000 live births, in 2019- 93 per 10000. All cases were confirmed by cardiologist-ultrasound-diagnostics at specialized cardiac centers and maternity hospitals. During the study period (2018-2019) the weighted percentage of an isolated CHD was 53\% among all congenital malformations in live births in Kyiv. Analysis of genetic components including syndromes of various forms of the CHD (2018-2019), first discovered during MGC, showed that the causes of the CHD were due to chromosomal pathology- $52 \%$, isolated forms- $24 \%$, genetic pathology- $13 \%$, undifferentiated multiple malformations- $11 \%$.

Conclusions: Every child with a validated form of the CHD should pass the MGC and screening at a specialized genetic institution to eliminate syndromal forms, calculate various genetic risks for the family and prevent them. The mean frequency of isolated CHD in newborns is 92 per 10000 in Kyiv.

V. Galagan: None. A. Dorokhina: None. M. Tsyhankova: None. S. Kulbalaieva: None. Y. Dudierina: None. V. Samilyk: None. 


\section{E-P05.15}

Genomic markers in the determination of long-term risk in patients with stable angina undergoing coronary bypass surgery

\section{A. Ponasenko, M. Sinitsky, A. Tsepokina, M. Asanov, O. Barbarash}

Research Institute for Complex Issues of Cardiovascular Diseases, Kemerovo, Russian Federation

Introduction: Cardiovascular mortality in Russian Federation is $57 \%$. It is known that genetic polymorphism play the key role in cardiovascular diseases development. The aim of this research was to determine the most important polymorphic variants of genes involved in five pathogenetic ways of coronary artery atherosclerosis among people from large industrial region.

Materials and methods: 78 polymorphic variants of 41 genes involved in inflammation, lipid metabolism, endothelial function, blood coagulation and innate immune response were analyzed in 260 coronary artery disease patients with stable angina and 300 healthy donors (control) resident in Kemerovo Region using PCR method.

Results: It was discovered that coronary artery disease patients from Kemerovo Region are characterized by the difference in genotype frequencies compared to overall European population. Genes of inflammation and endothelial function (NOS3 rs1799983, EDN rs5370 and CRP rs3093077) more contribute to coronary artery disease in resident of large industrial region. Polymorphism in genes of innate immune response (TLRI rs5743551, ILIF9 rs17659543) and coagulation factor II (rs1799963) can play protective role. $A P O B$, TLRI and TREMI associated with increased mortality from cardiovascular diseases; and SELPLG - with high risk of acute cerebrovascular accident development. Genes of lipid and calcium metabolism have no significant contribution in coronary atherosclerosis. This study was supported by basic research project of Research Institute for Complex Issues of Cardiovascular Diseases №0546-2015-0012.

A. Ponasenko: None. M. Sinitsky: None. A. Tsepokina: None. M. Asanov: None. O. Barbarash: None.

\section{E-P05.16}

Functional gene analysis indicates shared heritability of coronary heart disease and Alzheimer's disease

M. B. Ganev ${ }^{l}$, D. Serbezov ${ }^{I}$, L. Balabanski, ${ }^{1,2}, R$. Vazharova $^{2,3}$, S. Karachanak-Yankova ${ }^{1,4}$, O. Antonova ${ }^{l}, D$. Nikolova $^{l}$, M. Mihaylova ${ }^{l}$, R. Staneva ${ }^{l}$, V. Spasova ${ }^{l}$, V. Damyanova $^{l}$, D. Nesheva ${ }^{l}$, Z. Hammoudeh ${ }^{l}, S$.
Hadjidekova $^{1}$, S. Mehrabian ${ }^{5}$, M. Petrova ${ }^{5}$, L. Traykov ${ }^{5}$, D. Toncheva ${ }^{1,2}$

${ }^{I}$ Department of Medical Genetics, Medical University of Sofia, Sofia, Bulgaria, ${ }^{2}$ Gynecology and assisted reproduction hospital "Malinov", Sofia, Bulgaria, ${ }^{3}$ Department of Biology, Medical genetics and Microbiology, Faculty of Medicine, Sofia University "St. Kliment Ohridski", Sofia, Bulgaria, ${ }^{4}$ Department of Genetics, Faculty of Biology, Sofia University "St. Kliment Ohridski", Sofia, Bulgaria, ${ }^{5}$ Depatment of Neurology, UH "Alexandrovska”, Medical University of Sofia, Sofia, Bulgaria

Introduction: Clinical and epidemiological studies have demonstrated a link between Alzheimer's disease (AD) and high levels of blood lipids such as cholesterol, a common feature of coronary heart disease (CHD), yet the biological mechanisms remain unknown.

Materials \& Methods: Whole-exome sequencing was performed on two DNA pools set up of Alzheimer patients $(\mathrm{n}=70)$ and healthy individuals $(\mathrm{n}=100)$, respectively. After reads quality filtering, 143,493 genetic variants detected in both pools were annotated. A list of variants associated with CHD was compiled using the publicly available ENSEMBL database $(n=1479)$. We surveyed the pool data for presence of CHD associated variants and selected those present in both pools.

Results: Of the 92 CHD associated variants detected in our pool data, 24 variants in 22 genes were estimated to have significantly higher frequency in $\mathrm{AD}$ patients compared to the healthy individuals $(\mathrm{FDR}<0.05)$. Functional analysis was performed on the 22 genes using ToppGene platform. Results indicate lipid metabolism and lipoprotein transport associated pathways to be most significantly overrepresented in $\mathrm{AD}$ patients compared to controls.

Conclusion: The analysis of CHD associated variants in AD patients and healthy controls suggests shared heritability in genes involved in overrepresented lipid pathways.

Acknowledgment:

KP-06-N33/5 from 13.12.2019 - National Science Fund of Bulgaria.

DN 03/7 from 18.12.2016 - National Science Fund of Bulgaria.

M.B. Ganev: None. D. Serbezov: None. L. Balabanski: None. R. Vazharova: None. S. Karachanak-Yankova: None. O. Antonova: None. D. Nikolova: None. M. Mihaylova: None. R. Staneva: None. V. Spasova: None. V. Damyanova: None. D. Nesheva: None. Z. Hammoudeh: None. S. Hadjidekova: None. S. Mehrabian: None. M. Petrova: None. L. Traykov: None. D. Toncheva: None. 


\section{E-P05.18}

A DNA repair pathway polymorphism in angiographically proven coronary artery patients of southern Iran

\section{Bijanzadeh, S. Seyedian, F. Ahmadi, S. Hosseini}

Ahvaz Jundishapur University of Medical Sciences, Ahvaz, Iran, Ahvaz, Iran, Islamic Republic of

Introduction: Coronary artery disease (CAD) is among the most common causes of death and is based on the interaction between environmental risk factors and predisposing genes. X-ray repair cross-complementing group 1 (XRCC1), a DNA repair protein involved in single-strand breaks (SSBs) and base excision repair (BER) pathways, was reported to be responsible for the efficient repair of single strand breaks and damaged bases in DNA. Arg399Gln (rs25487) is one of the common XRCC1 polymorphisms that might be associated with increased risk for CAD.

Materials and methods: A case-control study was performed to investigate the relation between this polymorphism and the CAD development. 290 patients and 216 controls were screened for the mentioned SNP by Restriction Fragment Length Polymorphisms (RFLP) method.

Results: The frequency of the GA genotype of XRCC1 Arg399Gln was significantly higher in CAD patients than in controls ( $\mathrm{p}=0.002$, OR: $1.21,95 \% \mathrm{CI}(1.06-1.37))$ and also dominant mode (AA + GA) genotype of XRCC1 Arg399Gln had a 1.851-fold increased risk for CAD $(\mathrm{p}=0.005)$.

Conclusion: Our findings demonstrated that Arg399Gln polymorphism of XRCC1 has a significant relationship and a probable predisposing role in CAD. Our results support the role of DNA damages and the malfunctions of DNA repair system in CAD patients. Introducing of this candidate gene along with other related genetic variants can help the development of early diagnosis and effective interventional strategies that are safe, necessary and individualized for CAD patients. This study (project no. CVRC-9714) was granted by research affairs of Ahvaz Jundishapur University of medical sciences.

M. Bijanzadeh: None. S. Seyedian: None. F. Ahmadi: None. S. Hosseini: None.

\section{E-P05.20 The FLNC gene in myopathies and cardiomyopathies}

E. Vanhoutte ${ }^{l}$, J. Verdonschot ${ }^{l}$, G. Claes ${ }^{l}$, A. Heldermanvan den Enden ${ }^{l}$, J. Hoeijmakers ${ }^{l}$, D. Hellebrekers ${ }^{l}$, A. de Haan $^{1}$, I. Christiaans ${ }^{2,3}$, R. Lekanne-Deprez ${ }^{2}$, E. van Craenenbroeck $^{4}$, H. Boen ${ }^{4}$, B. Loeys ${ }^{4}$, Y. Hoedemakers ${ }^{3,5}$, C. Marcelis ${ }^{5}$, M. Kempers ${ }^{5}$, E. Brusse , J. van Waning $^{6}$, A.
Baas $^{7}$, D. Dooijes ${ }^{8}$, F. Asselbergs ${ }^{7}$, D. Barge-Schaapveld ${ }^{9}$, P. Koopman ${ }^{10}$, A. van den Wijngaard ${ }^{1}$, S. Heymans ${ }^{1,11,12}$, I. Krapels $^{1}$, H. Brunner ${ }^{1,5}$

${ }^{I}$ Maastricht University Medical Center, Maastricht, Netherlands, ${ }^{2}$ Amsterdam University Medical Center, Amsterdam, Netherlands, ${ }^{3}$ University Medical Centre Groningen, Groningen, Netherlands, ${ }^{4}$ Antwerp University Hospital, Antwerp, Belgium, ${ }^{5}$ Radboud University Medical Centre, Nijmegen, Netherlands, ${ }^{6}$ Erasmus MC University Medical Centre, Rotterdam, Netherlands, ${ }^{7}$ University Medical Center Utrecht, Utrecht, Netherlands, ${ }^{8}$ University Medical Center Utrecht, Maastricht, Netherlands, ${ }^{9}$ Leiden University Medical Center, Leiden, Netherlands, ${ }^{10}$ Heart Center Hasselt, Hasselt, Belgium, ${ }^{11} K U$ Leuven, Leuven, Belgium, ${ }^{12}$ The Netherlands Heart Institute, Nl-HI, Utrecht, Netherlands

Filamin C (FLNC) variants are associated with cardiac and muscular phenotypes. Originally, FLNC variants were described in myofibrillar myopathy (MFM) patients. Later, high-throughput screening in cardiomyopathy cohorts determined a prominent role for FLNC in isolated hypertrophic and dilated cardiomyopathies (HCM and DCM). FLNC variants are now among the more prevalent causes of genetic DCM. FLNC-associated DCM is associated with a malignant clinical course and a high risk of sudden cardiac death. The clinical spectrum of FLNC suggests different pathomechanisms related to variant types and their location in the gene. Proper functioning of FLNC is crucial for structural integrity and cell signaling of the sarcomere. The secondary protein structure of FLNC is critical to ensure this function. Truncating variants with subsequent haploinsufficiency are associated with DCM and cardiac arrhythmias. Interference with the dimerization and folding of the protein leads to aggregate formation detrimental for muscle function, as found in HCM and MFM. Variants associated with HCM are predominantly missense variants which cluster in the ROD2 domain. This domain is important for binding to the sarcomere and ensure proper cell signaling. We here review FLNC genotype-phenotype correlations based on available evidence.

E. Vanhoutte: None. J. Verdonschot: None. G. Claes: None. A. Helderman-van den Enden: None. J. Hoeijmakers: None. D. Hellebrekers: None. A. de Haan: None. I. Christiaans: None. R. Lekanne-Deprez: None. E. van Craenenbroeck: None. H. Boen: None. B. Loeys: None. Y. Hoedemakers: None. C. Marcelis: None. M. Kempers: None. E. Brusse: None. J. van Waning: None. A. Baas: None. D. Dooijes: None. F. Asselbergs: None. D. BargeSchaapveld: None. P. Koopman: None. A. van den Wijngaard: None. S. Heymans: None. I. Krapels: None. H. Brunner: None. 


\section{E-P05.21}

From genomics data to multiscale simulation - portable pipeline for extracting cardiomyophaty specific parameters

N. Ilic Raicevic, L. Lenhardt Ackovic, V. Kovacevic, S. Stojanovic, J. Randjelovic, M. Mattioni, A. S. Yavuz, M. Lazarevic, T. Borovski

Seven Bridges Inc, Boston, MA, United States

Introduction: Hypertrophic and dilated cardiomyopathy are the most common inherited cardiovascular diseases which lead to increased mortality and morbidity if not diagnosed in early stages. Design and development of new drugs and therapies which would prevent initiation and progression of familial cardiomyopathies is achievable today due to improved simulation methods and genomic data availability. The goal of this study was to establish a portable pipeline for annotating variants associated with familial cardiomyopathy and to determine induced changes at protein level. The outputs combined with the clinical data could then be used for simulation of structure and function of the heart.

Materials and methods: Analyzing steps encompass variant calling, variant annotation with best fitting tool using apposite databases, and final refinement of the variants. The workflow consists of BWA-GATK best practices, Ensembl Variant Effect Predictor and bix2mus (custom-made tool). In this study, 100 samples of individuals with diagnosed cardiomyopathy are processed giving a statistically powerful dataset. The workflow was written in the CWL.

Results: The composed workflow produced a subset of annotated variants that are cardiomyopathy specific. Selected annotated variants were further enriched with custom parameters which can be used as inputs for the MUSICO heart function simulation.

Conclusions: The developed workflow for discovery and annotation of variants associated with analyzed forms of inherited cardiomyopathy is operating system-independent, runnable on cloud or locally, ensuring portability, reproducibility and reliability. This workflow provides an innovative way to infer simulation parameters starting from genomics data.

This research was funded by the EU project SILICOFCM.

N. Ilic Raicevic: None. L. Lenhardt Ackovic: None. V. Kovacevic: None. S. Stojanovic: None. J. Randjelovic: None. M. Mattioni: None. A.S. Yavuz: None. M. Lazarevic: None. T. Borovski: None.

\section{E-P05.23}

A newly detected variant in the $M Y B P C 3$ gene in a patient with hypertrophic cardiomyopathy

S. Josifovska ${ }^{l}$, R. Vazharova ${ }^{2,3}$, L. Balabanski ${ }^{2}$, M. Malinov ${ }^{2}$, S. Panov ${ }^{1}$, M. Ganev ${ }^{4}$, D. Toncheva ${ }^{4}$

${ }^{1}$ Faculty of Natural Sciences and Mathematics, SS Cyril and Methodius University, Skopje, Macedonia, The Former Yugoslav Republic of, ${ }^{2}$ Genome Centre, GARH Malinov, Sofia, Bulgaria, ${ }^{3}$ Department of Biology, Medical Genetics and Microbiology, Faculty of Medicine, Sofia University St. Kliment Ohridski, Sofia, Bulgaria, ${ }^{4}$ Department of Medical Genetics, Medical University of Sofia, Sofia, Bulgaria

Introduction: Hypertrophic cardiomyopathy $(\mathrm{HCM})$ is a kind of cardiomyopathy, a cardiac dysfunction due to diseases of the myocardium (WHO,1995). HMC causes partial enlargement of the myocardium.

Materials and methods: We report on middle aged patient with familial history and developed HCM. Exome sequencing was performed on the patient's extracted DNA, followed by a targeted analysis of genes from the "Cardio" gene panel. Results were validated by Sanger sequencing.

Results: The analysis resulted in detection of a heterozygous, probably pathogenic variant in the $M Y B P C 3$ gene (NM_000256.3: c.2965delG; NP_000247.2:p.Glu989Serfs*3). This variant has not been previously reported in individuals with HCM. The MYBPC3 encodes for the Cardiac myosin-binding protein $\mathrm{C}$. The MYBPC3 has a structural role as it is located in striated muscle sarcomere A-bands and binds myosin heavy chain in thick filaments and titin in elastic filaments causing muscle contraction. Pathogenic variants in the MYBPC3 gene account for approximately $15 \%$ of cases of familial HCM. The deletion that we detected leads to a frameshift resulting in an amino acid substitution of glutamic acid to serine at codon 989 . Furthermore, due to the new reading frame, a stop codon can be detected 3 codons downstream, likely affecting the protein function because of a possible truncated protein form. The inheritance is autosomal dominant. No other known pathological or probably pathological variants associated to HCM were found.

Conclusions: The genetic causes underlining HMC are complex and identifying novel variants may contribute to better distinction of this dysfunction and assessment of the risk of familial predisposition.

S. Josifovska: None. R. Vazharova: None. L. Balabanski: None. M. Malinov: None. S. Panov: None. M. Ganev: None. D. Toncheva: None. 


\section{E-P05.25}

\section{Genetic structure of hypertrophic cardiomyopathy in Russia}

\author{
E. V. Filatova ${ }^{l}$, N. S. Krylova ${ }^{2}$, I. N. Vlasov ${ }^{l}$, \\ M. S. Maslova ${ }^{2}$, N. Poteshkina ${ }^{2}$, P. A. Slominsky ${ }^{\text {, }}$ \\ M. I. Shadrina ${ }^{l}$
}

${ }^{1}$ Institute of Molecular Genetics RAS, Moscow, Russian Federation, ${ }^{2}$ Pirogov Russian National Research Medical University, Moscow, Russian Federation

Introduction: Hypertrophic cardiomyopathy (HCM) is the most common heritable cardiovascular disease, classically defined as the presence of idiopathic left ventricular hypertrophy. HCM is predominantly a single gene disorder with an autosomal dominant pattern of inheritance. However, pathogenic alleles are not identified in at least $25 \%$ of patients with HCM. Moreover, the whole spectrum of genes and mutations that contribute to the development of this disorder in Russian population has not been fully described. Therefore, the aim of our study was to identify genetic variants associated with the HCM in patients from Russia.

Materials and methods: The study cohort included 99 unrelated patients with HCM. We performed targeted exome sequencing and a subsequent bioinformatic analysis using the various algorithms for prediction of the potential pathogenicity of variants, as well as the ClinVar database.

Results: The frequency of pathogenic and potentially pathogenetically significant variants in the main HCMrelated genes was $18 \%$ in our patients with this disorder. We also identified 22 potentially pathogenetically significant variants, including novel ones, in other HCM genes and 52 potentially pathogenetic variants in other 35 genes with known associations to 17 different inherited cardiac conditions.

Conclusion: Thereby the prevalence of individual pathogenic variants in HCM-related genes in Russian population is very low. Therefore, it is necessary to continue the search for new genes and genetic variants associated with the development of HCM. This work was supported by the Russian Foundation for Basic Research (grants no. 19-015-00343, 18-015-00322) and the Russian Science Foundation (grants no. 20-15-00293).

E.V. Filatova: None. N.S. Krylova: None. I.N. Vlasov: None. M.S. Maslova: None. N. Poteshkina: None. P.A. Slominsky: None. M.I. Shadrina: None.

\section{E-P05.27}

Variants in KCNJ11 and SCN10A associated to ventricular fibrillation during acute myocardial infarction.
L. Nuñez ${ }^{1}$, R. Pan-Lizcano ${ }^{1}$, P. Piñon ${ }^{2}$, X. Flores ${ }^{2}$, G. Aldama ${ }^{2}, R$. Calviño ${ }^{2}$, N. Vázquez-González ${ }^{3}$, J. M. Vazquez-Rodriguez ${ }^{3}$, M. Hermida-Prieto $^{I}$

${ }^{1}$ Cardiology Research Group. Instituto de Investigación Biomédica de A Coruña (INIBIC)-CHUAC-UDC, A Coruna, Spain, ${ }^{2}$ Department of Cardiology, Complexo Hospitalario Universitario A Coruna (CHUAC)-INIBIC, A Coruna, Spain, ${ }^{3}$ Department of Cardiology, Complexo Hospitalario Universitario de A Coruña (CHUAC)CIBERCV, Instituto de Investigación Biomédica de A Coruña (INIBIC), Universidad de A Coruña (UDC), A Coruna, Spain

Background:Sudden death (SD) due to ventricular fibrillation (VF) during acute myocardial infarction (AMI) is one of the leading causes of death in Western societies. It has been suggested that the risk of SD due to VF in AMI is the result of the interaction between genetic factors and environmental factors. Moreover, it has been proposed that variants in genes related to cardiac excitability, mainly SCN5A, could play an important role in VF during AMI.

Methods:Genetic variants in 36 genes encoded proteins related to pathways involved in atherothrombosis were analyzed by next generation sequencing in 12 patients with FV during AMI. Mutations found were confirmed by Sanger sequencing. Visualization of the mutation using pdb and in silico prediction of the effects produced by the mutation (Mutation Tester, SNAP2, SIFT2, Polyphen, PhDSNP) were performed.

Results:In one patient, p.Trp83Ser was identified in KCNJ11 gene that encodes a forming KATP channel subunit, whereas in another patient p.Thr738Pro in SCN10A, encoding the forming subunit of the sodium channel, were identified. In both variants, more than four in silico tools suggested deleterious function of the protein.

Conclusion:Mutations in gene encoded forming channels subunits, as KCNJ11 and SCN1OA, could have a role in the development of FV during AMI. Thus, it is essential to use an ambitious strategy, including all genes related to cardiac excitability, to clarify the pathophysiological basis of ventricular fibrillation in acute myocardial infarction.

Funding Acknowledgements:This work was supported by a grant from Instituto de Salud Carlos III(PI18/01737) and a non-conditional-grant from Abbott Vascular.

L. Nuñez: None. R. Pan-Lizcano: None. P. Piñon: None. X. Flores: None. G. Aldama: None. R. Calviño: None. N. Vázquez-González: None. J.M. Vazquez-Rodriguez: None. M. Hermida-Prieto: None. 


\section{E-P05.28}

\section{A new case of Fish-eye disease in Spain.}

J. Sanguino, C. Rodríguez Jiménez, E. Sevilla, A. Carazo, V. Gómez del Pozo, N. Gallego-Onís, F. Arrieta,

S. Rodríguez-Nóvoa

\section{INGEMM, Madrid, Spain}

Introduction: Fish-eye disease (FED; MIM\#136120) is a rare disease with inheritance pattern autosomal recessive caused by Lecithin:Cholesterol Acyltransferase Deficiency 1. This disorder is characterized by raised levels of total cholesterol, triglycerides and decreased levels of HDL-c, apoA, and apoB in serum. We report a new case of suspicion of Fish-eye disease send to our laboratory to confirm the diagnosis genetic.

Patient and method: A 42-years-old male, with corneal opacity, very level low of HDL-c in serum, splenomegaly, without anemia or kidney disease, and raised levels of triglycerides, liver enzymes and homocysteine in serum, was remitted to our laboratory. The patient's DNA sample was analyzed by NGS using a customized panel MTB-v2 of 411 genes. The variant found was confirmed by Sanger sequencing Results We found an homozygous missense variant in $L C A T$, NM_000229.2: c.491G >A p.(Arg164His). In silico analysis showed damaging and conservation for this variant.

Conclusion: In this work, we report a new case of Fish -eye disease. The variant found in LCAT c.491G>A p. (Arg164His) had been previously reported in three siblings with typical triad: corneal opacity, anemia and kidney dysfunction and the functional study showed lack of activity in $\mathrm{LCAT}^{2}$. However, our patient exhibited a mild phenotype despite of display of same variant suggesting the existence of others factors that decreased the clinical manifestations.

J. Sanguino: None. C. Rodríguez Jiménez: None. E. Sevilla: None. A. Carazo: None. V. Gómez del Pozo: None. N. Gallego-Onís: None. F. Arrieta: None. S. RodríguezNóvoa: None.

\section{E-P05.29}

\section{Genetics of familial left venticular noncompaction cardiomyopathy in Russia}

A. Meshkov ${ }^{l}$, O. Kulikova ${ }^{l}$, R. Myasnikov ${ }^{l}$, A. Kiseleva $^{l}$, M. Divashuk ${ }^{1,2}$, A. Zharikova ${ }^{l}$, O. Skirko ${ }^{l}$, I. Efimova ${ }^{l}$, M. Pokrovskaya $^{l}$, E. Mershina ${ }^{3}$, V. Sinitsyn ${ }^{3}$, E. Basargina ${ }^{4}, N$. Sdvigova ${ }^{4}$, S. Boytsov ${ }^{5}$, O. Drapkina ${ }^{1}$

${ }^{I}$ National Medical Research Center for Preventive Medicine, Moscow, Russian Federation, ${ }^{2} R S A U-M A A$ named after $K$. A. Timiryazev, Moscow, Russian Federation, ${ }^{3}$ Lomonosov Moscow State University, Moscow, Russian Federation,
${ }^{4}$ National Medical Research Center for Children's Health, Moscow, Russian Federation, ${ }^{5}$ National Medical Research Center of Cardiology, Moscow, Russian Federation

Introduction: Left venticular noncompaction cardiomyopathy (LVNC) is a genetically determined cardiomyopathy, characterized by the presence of increased trabecularity and pronounced intertrabecular spaces extending into the left ventricle chamber. This study was aimed at finding new key links in LVNC development.

Materials and methods: The study included 26 families of LVNC patients. For all individuals was performed exome sequencing on Illumina Nextseq 550.

Results: Clinical and instrumental examination of 102 people was done: $41(40.2 \%)$ were healthy, in $61(59.8 \%)$ patients the diagnosis of LVNC was confirmed, $15(14 \%)$ were children. According to the examination results, the following clinical phenotypes of LVNC can be distinguished: LVNC dilatation phenotype, LVNC hypertrophic phenotype, LVNC and a combination of hypertrophic with dilatational phenotype, isolated LVNC, a combination of LVNC with congenital heart defects. The pathogenicity was evaluated for the identified nucleotide variants in 66 genes associated with the development of LVNC and in 122 genes associated with the development of other cardiomyopathies. Pathogenic (PV) or probably pathogenic (PPV) variants were found in 16 families: 11 variants in LVNC genes (5 PPV in MYH7, $2 \mathrm{P}$ variants in $T T N$, one PV and one PPV in MYBPC3, one PPV in TPMI and one PPV in SCN5A) and 5 variants in genes associated with other cardiomyopathies (2 PPV in DES, 1 $\mathrm{PPV}$ in $R B M 20,1 \mathrm{PV}$ in $V C L, 1 \mathrm{PV}$ in $D S G 2$ ).

Conclusions: The study of LVNC development on 26 families revealed mutations in $M Y H 7, T T N, M Y B P C 3$, TPM1, DES, RBM20, VCL and DSG2 genes.

A. Meshkov: None. O. Kulikova: None. R. Myasnikov: None. A. Kiseleva: None. M. Divashuk: None. A. Zharikova: None. O. Skirko: None. I. Efimova: None. M. Pokrovskaya: None. E. Mershina: None. V. Sinitsyn: None. E. Basargina: None. N. Sdvigova: None. S. Boytsov: None. O. Drapkina: None.

\section{E-P05.30}

Expression of miRNAs as a marker for cardiovascular risk in patients with type 2 diabetes mellitus

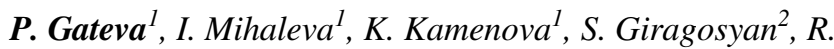 Dodova $^{2}$, R. Kaneva ${ }^{2}$, I. Dimova ${ }^{3}$}

${ }^{I}$ Department of Pharmacology and Toxicology, Medical University - Sofia, Sofia, Bulgaria, ${ }^{2}$ Laboratory of Genomic Diagnostics, Molecular Medicine Center, Medical University - Sofia, Sofia, Bulgaria, ${ }^{3}$ Department of Medical Genetics, Medical University - Sofia, Sofia, Bulgaria 
The use of circulating miRNAs as biomarkers is a potential additional option in the diagnosis and prognosis of diseases, complementary to established protein-based biomarkers. In our study, we isolated miRNAs from plasma of diabetic patients with and without cardio-vascular disease (CVD). The analysis included 6 patients with type 2 diabetes mellitus without CVD (control group) and 9 patients with type 2 diabetes mellitus and CVD (target group). Following reverse transcription and subsequent real-time PCR analysis of an equal amount of baseline miRNAs, the following miRNAs were successfully analyzed: miR-16-5p, miR-155-3p, miR155-5p,miR-210-3p, miR-221-3p miR-424-5p. The comparative analysis of expression showed a statistically significant increase in the expression of all these miRNAs in the target group compared to the control - see table bellow.

\begin{tabular}{lc}
\hline miRNA & Relative expression (RQ) \\
\hline miR-16-5p & 2.5 \\
miR-155-3p & 2.2 \\
miR-155-5p & 7.5 \\
miR-210-3p & 5.1 \\
miR-221-3p & 6.2 \\
miR-424-5p & 10.5 \\
\hline
\end{tabular}

The highest level of increase was established for miR155-5p (its target is ICAM-1 - with role in inflammation and in the regulation of vascular permeability, affected in atherosclerosis) and miR-424-5p (its target is HIF1A - the master transcriptional regulator of the adaptive response to hypoxia). In conclusion, CVD risk assessment, supported by emerging circulating biomarkers, such as miRNAs, is important for stratifying high-risk individuals, optimizing treatment strategies, and enhancing our understanding of basic biology. Acknowledgment: This study was supported by Grant NoD-66/2019, Medical University Sofia.

P. Gateva: None. I. Mihaleva: None. K. Kamenova: None. S. Giragosyan: None. R. Dodova: None. R. Kaneva: None. I. Dimova: None.

\section{E-P05.31}

In vitro induced DNA alkylation in endotheliocytes leads to genotoxic stress and secretion of proatherosclerotic cytokines

\section{Sinitsky, A. Kutikhin, D. Shishkova, A. Tsepokina, M. Asanov, A. Ponasenko}

Research Institute for Complex Issues of Cardiovascular Diseases, Kemerovo, Russian Federation

Introduction: Atherosclerosis is a leader in morbidity and mortality among all cardiovascular pathologies. There are evidences that somatic mutations can play a role in endothelial dysfunction, and atherosclerotic plaques are characterized by increased level of DNA adducts. The aim of this research was to study the level of genotoxic stress markers; secretion and gene expression of proatherosclerotic cytokines IL- 6 and IL- 8 by endothelial cells exposed to alkylating mutagen mitomycin $\mathrm{C}$ (MMC).

Materials and methods: Primary human coronary(HCAEC) and internal thoracic artery endothelial cells (HITAEC) were used in this research. Cytokinesis-block micronucleus assay was performed to score genotoxic stress markers in exposed and control cell cultures. Concentration of IL- 6 and IL- 8 in culture medium and mRNA level of the corresponding genes were measured immediately after 6 hours of cell incubation with mutagen and 24 hours after elimination of MMC from cell cultures, using ELISA and RT-qPCR.

Results: Exposed cells were characterized by increased level of all studied cytogenetic markers compared to control. After 6 hours of exposure, a significant decrease in the secretion and gene expression of IL-8, and decrease in the mRNA level of the $I L-6$ gene in the exposed cultures was discovered. At the second time point $(6+24 \mathrm{~h})$, on the contrary, the concentration and mRNA level of IL-8, as well as expression of the IL6 gene sharply increased in cultures exposed to MMC compared to control, and the HCAEC were more sensitive to mutagenic effects than HITAEC. This study was supported by basic research project №05462015-0012.

M. Sinitsky: None. A. Kutikhin: None. D. Shishkova: None. A. Tsepokina: None. M. Asanov: None. A. Ponasenko: None.

\section{E-P05.36}

Association of gene polymorphisms MTHFR in Kazakhstani patients with implanted left ventricular assist devices

\section{Zhalbinova ${ }^{1,2}$, S. Rakhimova ${ }^{2}$, M. Bekbosynova ${ }^{3}$, S. Andosova ${ }^{3}$, B. Abdirova ${ }^{3}$, A. Akilzhanova ${ }^{2,1}$}

${ }^{1}$ L.N. Gumilyov Eurasian National University, Nur-Sultan, Kazakhstan, ${ }^{2}$ National Laboratory Astana, Center for Life Sciences, Nur-Sultan, Kazakhstan, ${ }^{3}$ JSC "National research cardiac surgery center", National medical holding, NurSultan, Kazakhstan

Introduction: Chronic heart failure $(\mathrm{CHF})$ is one of the main health problems in Kazakhstan, which requires heart transplantation (HT) to extend the patient's life. The popular treatment of $\mathrm{CHF}$ is the implantation of left ventricular assist device (LVAD) which extends patient's life before HT. However, LVAD causes risk of thrombosis and 
bleeding in cardiac patients. Patients are normally prescribed with an antithrombotic therapy to prevent risks of thromboembolism. The purpose of this study is to evaluate patients for genetic variants responsible for risk of thrombosis: MTHFR $(* 1)$ and MTHFR $(* 2)$.

Materials and methods: Venous blood samples were recruited from patients $(n=100)$ with implanted LVADs (HW, HM2, HM3) at the National Research Center for Cardiac Surgery. Warfarin was prescribed according to clinical protocol $(\min =0.9 \mathrm{mg} ; \max =7.2 \mathrm{mg}$; Std.=1.15). Genotyping was done for MTHFR $(* 1)$ rs1801133 and MTHFR (*2) rs 1801131 by real-time polymerase chain reaction with TaqMan probes.

Results: Genotyping results were analyzed on IBM SPSS Statistics 23 for $\mathrm{n}=98$ patients ( $>18$ years old). Results presented that most of the patients have wild type of genotype which proves absence to the risk of thrombosis: MTHFR $(* 1)(\mathrm{G} / \mathrm{G}=47 \% ; \mathrm{G} / \mathrm{A}=41 \% ; \mathrm{A} / \mathrm{A}=10 \%, \mathrm{p}=$ 0.563); MTHFR (*2) (T/T =54\%; $\mathrm{T} / \mathrm{G}=38 \% ; \mathrm{G} / \mathrm{G}=6 \%, \mathrm{p}$ $=0.288)$. Results are not statistically significant.

Conclusions: Research advices to study other SNPs which will allow us to identify the reasons of thrombosis and bleeding in LVAD patients and also to study more about coagulation factors, aspirin resistance, warfarin metabolism. Study needs to add more samples to be able to compare results with other samples.

M. Zhalbinova: None. S. Rakhimova: None. M. Bekbosynova: None. S. Andosova: None. B. Abdirova: None. A. Akilzhanova: None.

\section{E-P06 Metabolic and Mitochondrial Disorders}

\section{E-P06.01}

The case of atypical form of 3-methylglutaconic aciduria

\section{O. Vernigor, O. Grechanina, M. Kanyuka}

Interregional Specialized Medical Genetic Center - Center of Rare (Orphan) Diseases, Kharkiv National Medical University, Kharkiv, Ukraine

Methylglutonic aciduria (MGA) is clinically and genetically heterogeneous disease. Genes responsible for development known diseases: OPA3, AUH, TAZ, DNAJC19. One of the diagnostic methods of MGA is an analysis of the spectrum of organic acids in urine by gas chromatography-mass spectrometry, as there are biomarkers of the disease - 3methylglutaric and 3-methylglutaconic acid increase is characteristic. Materials and methods: in our center there are 3 children with an atypical form of 3-methylglutaconic aciduria, a description of one clinical case. The child is 3 years old, applied with poor weight gain, a slight speech development delay, poor physical exercise tolerance. At an early age - regurgitation, lack of body weight. The diagnosis has not been established. Examination has revealed diffuse hypotension, asthenia, phenotypically signs of connective tissue dysplasia, Laboratory studies: increased transaminases, increased lactate level, hepatosplenomegaly. According to clinical criteria - mitochondrial Nijmegen disease. Diagnostic assessment (Wolf NI, Smeitink JA. Mitochondrial disorders) in a child confirmed mitochondrial pathology. As the boy grew, there was an increase of clinical symptoms: increased weakness, fatigue, muscle hypotension, hypomimia. GC-MS in dynamics: titer 3metylglutaric and 3- metylglutaconic increase, MRS- confirmed mitochondrial neurocyte activity. Diagnosed with atypical forms of 3-methylglutaconic aciduria (3-methylglutaconic deficiency - CoA-hydratases) Prescription: mitochondrial therapy during rehabilitation. It led to temporary positive dynamics. Conclusions: timely diagnosis of a rare disease based on biomarkers can improve the condition of the patient due to pathogenetic therapy.

O. Vernigor: None. O. Grechanina: None. M. Kanyuka: None.

\section{E-P06.03}

X-linked ALG13-CDG resulting from c.320A $>G$ variant - can transferrin profile change and correlate with the clinical phenotype?

\section{A. Jezela-Stanek ${ }^{1}$, A. Bogdańska ${ }^{2}$, P. Pokora ${ }^{3}$,} J. Paprocka ${ }^{4}$, A. Tylki-Szymańska ${ }^{3}$

${ }^{I}$ National Institute of Tuberculosis and Lung Disease, Department of Genetics and Clinical Immunology, Warsaw, Poland, ${ }^{2}$ The Children's Memorial Health Institute, Department of Biochemistry, Radioimmunology and Experimental Medicine, Warsaw, Poland, ${ }^{3}$ Children's Memorial Health Institute, Department of Pediatrics, Nutrition and Metabolic Disorders, Warsaw, Poland, ${ }^{4}$ Medical University of Silesia, Department of Pediatric Neurology, School of Medicine in Katowice, Katowice, Poland

Introduction: Congenital Disorders of Glycosylation (CDG) are multisystemic metabolic disorders, in general, caused by defects of $\mathrm{N}$-linked, O-linked oligosaccharides pathways. Their clinical presentation and aetiology are highly heterogeneous, which may cause a diagnostic dilemma. So far, $A L G 13$ c. $320 \mathrm{~A}>\mathrm{G}$ variant was reported in ten females and one male. Contrary to other known ALG13$\mathrm{CDG}$, in probands harbouring this mutation normal glycosylation and mass spectrometry pattern were observed (two and one individuals, respectively). There is, however, one 
another report, giving evidence of slightly reduced glycosylation with the absence of one glycan in MS. Since these laboratory data are not conclusive, and the number of reported patients is still very limited, we like to present novel individuals, pointing out their transferrin IEF results.

Materials: Three female probands with de novo ALG13 c. $320 \mathrm{~A}>\mathrm{G}$ variant $(\mathrm{P} 3$, with the most severe psychomotor impairment, also heterozygous for $c .3433 \mathrm{C}>\mathrm{T}$ in the CACNAIA gene).

Methods: Transferrin isoforms examined by isoelectric focusing (IEF) of iron saturated serum in agarose gel in a $\mathrm{pH}$ gradient 5-7 with immunofixation, demonstrated as the percentage of each isoform fraction in a densitometric scan.

Results: Transferrin isoforms showed normal profiles in P1, while in P2 - slight elevation of asialo, monosialo, disialo fraction; P3 - first analysis: normal; second (in another sample): slight elevation of only disialo fraction.

Conclusions: Obtained IEF data delineate the characteristics of ALG13-CDG caused by c.320A $>\mathrm{G}$ variant, providing evidence of variability or specificity of glycosylation defect. These may be significant for genetic results verification but also clinical assessment and correlation.

A. Jezela-Stanek: None. A. Bogdańska: None. P. Pokora: None. J. Paprocka: None. A. Tylki-Szymańska: None.

\section{E-P06.04}

Cerebrotendinous xanthomatosis with novel compound heterozygous mutation of CYP27A1gene

\section{H. N. Cesur Baltacı ${ }^{1}$, B. Sağlam Ada ${ }^{2}$, N. Yürür Kutlay ${ }^{1}, A$. Tükün ${ }^{2}$, S. Tıraş Teber ${ }^{3}, T$. Coşkun ${ }^{4}$}

${ }^{1}$ Medical Genetics Department, Ankara University, Ankara, Turkey, ${ }^{2}$ Department of Medical Genetics, Düzen Laboratories Group, Ankara, Turkey, ${ }^{3}$ Department of Pediatric Neurology, Ankara University School of Medicine, Ankara, Turkey, ${ }^{4}$ Departments of Pediatric Metabolism, Hacettepe University Faculty of Medicine, Ankara, Turkey

Introduction: Cerebrotendinous xanthomatosis (CTX) is a rare lipid storage disease characterized by the accumulation of cholesterol and cholestanol in various tissues. It is inherited in an autosomal recessive manner caused by the mutations of CYP27Al gene localized on 2q35. This gene encodes sterol 27-hydroxylase. Here we present a 8-yearold male with attention deficit and hyperactivity disorder. $\mathrm{He}$ was born to non-consanguineous parents. He was consulted to us for $C Y P 27 A 1$ gene analysis by the information of a homozygous deletion in exon 6 of CYP27Al gene in his mother. Additionally, his 7-dehydrocholesterol and plasma cholestanol levels increased consistently with CTX. His father had epilepsy together with limited intellectual capacity.
Material and Method: Genomic DNAs were extracted from the patient and his father, followed by CYP27Al gene sequencing. Furthermore, in order to demonstrate the deletion, multiplex ligation-dependent probe amplificationof the CYP27Al gene was performed in the proband.

Results: We found a novel heterozygous c.508_509ins16 (p.E170Vfs*16) mutation in CYP27A1 gene in the proband's father. Consequently, a compound heterozygous mutation was detected in the proband. The effect of this novel point mutation on CYP27Alfunction was predicted to be damaging by in silico analysis. After genetic counseling, his grandmother who was his legal guardian, consented to kenodeoxycholic acid (CDCA) treatment. Cholestanol decreased to normal levels and some symptoms improved at the end of two months treatment.

Conclusion: Genetic testing for presymptomatic family members of affected patients should be suggested especially to offer an opportunity to prevent or decrease the disease manifestations with CDCA therapy.

H.N. Cesur Baltacı: None. B. Sağlam Ada: None. N. Yürür Kutlay: None. A. Tükün: None. S. Tıraş Teber: None. T. Coşkun: None.

\section{E-P06.05}

A novel $C T C F$ variant in a patient with congenital anomalies

N. Okamoto ${ }^{l}, F . M i y a^{2,3,4}, T$. Tsunoda $a^{2,3,4}, K . K_{\text {Kosaki }}{ }^{5}$

${ }^{1}$ Department of Medical Genetics, Osaka Women's and Children's Hospital, Osaka, Japan, ${ }^{2}$ Department of Medical Science Mathematics, Medical Research Institute, Tokyo Medical and Dental University, Tokyo, Japan, ${ }^{3}$ Laboratory for Medical Science Mathematics, RIKEN Center for Integrative Medical Sciences, Yokohama, Japan, ${ }^{4}$ Medical Science Mathematics, Department of Biological Sciences, Graduate School of Science, The University of Tokyo, Tokyo, Japan, ${ }^{5}$ Center for Medical Genetics, Keio University School of Medicine, Tokyo, Japan

Introduction: The DNA-binding protein CCCTC-binding factor (CTCF) and the cohesin complex play important roles in chromatin architecture in vertebrates. Pathogenic variants in CTCF were reported in patients with neurodevelopmental disorders with distinctive facial features. We report a patient with a novel variant in $C T C F$ with intellectual disability (ID) and multiple anomalies.

Clinical Report: The 6-year-old male was the first child of healthy and non-consanguineous Japanese parents. He showed congenital cardiac anomalies, duodenal atresia, and atresia ani. His developmental milestones were also retarded. He also showed characteristic facial features. 
Conventional cytogenetic studies and microarray analysis gave normal results.

Method: With the approval of our institutional ethics committee, DNA samples from the patient and her parents were analyzed by WES.

Results: The patient was heterozygous for $C T C F$ frameshift variant (NM_006565:exon3:c.723delT:p.N241fs). The variant was de novo.

Discussion: Cornelia de Lange syndrome, a classical cohesinopathy, may show multiple malformations including CHD and gastrointestinal malformations. CTCF may regulate organogenesis. Mutations in $C T C F$ may also cause a multiple anomaly syndrome with ID.

N. Okamoto: None. F. Miya: None. T. Tsunoda: None. K. Kosaki: None.

\section{E-P06.07}

Two forms of a disease in the same family: compound heterozygosity in the father and heterozygosity in his sister both with corneal cystinosis, and heterozygous daughter and his other sister both having risks for nephropathic cystinosis

\section{B. Celtikci ${ }^{1}$, B. Kazanci ${ }^{2}$}

${ }^{1}$ Hacettepe University, Ankara, Turkey, ${ }^{2}$ Ulucanlar Eye Hospital, Ministry of Health, Ankara, Turkey

Cystinosis is a rare and autosomal recessive inherited disorder resulting from the accumulation of intracellular cystine, due to the mutations of the CTNS gene encoding the cystinosin protein that carries the lysosomal amino acid cystine. Its incidence is high in Turkey, because of the frequent consanguineous marriage and manifesting heterozygotes. The most common clinical diagnosis is kidney involvement. Corneal form occurs later. Some cases are diagnosed with photophobia, halo and glare style visual findings, depending on the density of corneal crystals. Spindle- and fusiform-shaped corneal crystals were detected in our patient and his sister. Sequencing of CTNS gene was performed on a MiSeq (Illumina). VCF files were analyzed by Variant studio (Illumina) and Geneticist Assistant (SoftGenetics) Software. Sequence variant classification was done according to the ACMG Standards and Guidelines. Detected variants were confirmed by Sanger sequencing. Our patient with corneal cystinosis has the compound heterozygosity of c.589G A (p.G197R)(p. Gly197Arg) /c.1015G’A (p.G339R)(p.Gly339Arg) mutations, correlated with cystinosis and his daughter is a heterozygote for c.1015G'A (p.G339R)(p.Gly339Arg) mutation, correlated specifically with nephropathic cystinosis. As expected, his mother is a heterozygote for
c.589GA (p.G197R)(p.Gly197Arg) mutation and his father is a heterozygote for c.1015G'A (p.G339R)(p.Gly339Arg) mutation. One of his asymptomatic sister is a heterozygote for c. 1015G ${ }^{>}$A (p.G339R)(p.Gly339Arg) mutation. Another sister with corneal cystinosis is a heterozygote for c.589G A (p.G197R)(p.Gly197Arg) mutation. Overall, we can trace the (potential) phenotype of these patients using their mutation. This study shows us the genetic heterogenity of cystinosis in Turkish population and helps us to understand the biochemical-genotype-phenotype relationship in this disease.

B. Celtikci: None. B. Kazanci: None.

\section{E-P06.08}

A unique combination of two lysosomal diseases in a child revealed as a result of a screening

O. Buhaiova, O. Grechanina, Y. Hrechanina, L. Molodan, N. Pokaziy, A. Bulavina, D. Shkolnikova

Interregional Specialized Medical Genetic Center - Center of Rare (Orphan) Diseases, Kharkiv National Medical University, Kharkiv, Ukraine

Introduction: Lysosomal storage disorders are characterized by comorbidity, polymorphism and genetic heterogeneity, which makes their timely diagnosis difficult.

Materials and methods: To identify patients with atypical forms of MPS, Pompe, Gaucher, Fabry disease among residents of Kharkov region in 2018. In Kharkiv region, selective screening for accumulation diseases has begun.

Results: determination of excretion of total glycosaminoglycans (GAGs) in daily urine (2733patients); determination of the level of fractional blood GAGs-(683patients), biochemical study of the level of enzymes and molecular genetic diagnostics (712patients). This revealed: Combination of Fabry disease and Pompe disease in 1 patient. In a child with diffuse muscular hypotension, retarded physical and statokinetic development, hepatomegaly, decreased activity of the lysosomal enzyme acid $\alpha$-glucosidase, decreased Lyso-Gb3, the GLA gene (heterozygous variant c.31G> C p. (Gly11Arg)) was detected. GLA - encodes a homodimeric glycoprotein. A mutation in the gene affects the synthesis, processing and stability of the enzyme that causes Fabry disease, which results from the inability to catabolize alpha - D-galactosyl glycolipid moieties. GAA The gene produces an acid alpha glycosidase enzyme. The body uses GAA to break down the glycogen used to produce energy.

Conclusions: The introduction of a selective screening system for diseases of accumulation made it 
possible to diagnose early and to start adequate pathogenetic personalized therapy in a timely manner for this group of patients. For the first time, a combination of Fabry and Pompe diseases was detected in 1 patient.

O. Buhaiova: None. O. Grechanina: None. Y. Hrechanina: None. L. Molodan: None. N. Pokaziy: None. A. Bulavina: None. D. Shkolnikova: None.

\section{E-P06.10}

\section{Spectrum of mutations in Russian patients with hypercholesterolemia}

\section{O. N. Ivanova, T. D. Krylova, N. A. Semenova, P. A.} Vasiluev, N. V. Milovanova, E. Z. E.Y. Zakharova

Federal State Budgetary Scientific Institution "Research Centre for Medical Genetics", Moscow, Russian Federation

-Introduction: We presented a genetic spectrum of hypercholesterolemia in a cohort of 72 unrelated patients from Moscow region. -Materials. The patients with high level of total cholesterol $(>6,7 \mathrm{mmol} / \mathrm{l})$ and/ or low-density lipoprotein cholesterol $(>4,9 \mathrm{mmol} / \mathrm{l})$ were screened on targeted NGS panel on IonS5 (Thermofisher Scientific) for the following genes: LDLR(NM_000527), APOB(NM_000384), PCSK9(NM_174936), ABCG5 (NM_022436), ABCG8(NM_022437), APOA2 (NM_001643), LIPA(NM_000235), LPL(NM_000237). Informed consents have been obtained from all patients or their legal representatives to conduct the study. The average age of the patients was $12,04+/-1,27$ years old. The nomenclature of variants and the assessment of their pathogenicity has been carried out in accordance with HGVS, ACMG recommendations. Variant validation has been performed by Sanger sequencing.

-Results: We found 44 variants among 72 of cases (61\%). Known pathogenic mutations have been detected in 27 patients, 21 of them were in LDLR gene, and six in the genes APOB, LIPA, ABCG8. 17 patients harbor new variants with varying degrees of pathogenicity (see table below).

-Conclusions: Pathogenic LDLR variants have been detected in $47 \%(34 / 72)$ of unrelated patients with hypercholesterolemia, the same results were shown in the literature. The most common variant in this gene is the known pathogenic mutation c.1775G $>$ A(p.G592E) (6.9\%) and the previously unpublished splicing variant c.940 +1_c.940+4delGTGA $(5,5 \%)$. The study has confirmed the genetic heterogeneity of hypercholesterolemia in the Russian cohort.

\begin{tabular}{|c|c|c|c|c|}
\hline & pathogenic & likely pathogenic & $\begin{array}{l}\text { probably } \\
\text { pathogenic }\end{array}$ & $\begin{array}{l}\text { variant of uncertain } \\
\text { significance }\end{array}$ \\
\hline LDLR & $\begin{array}{l}\text { c.316_328delCCCAAGAC } \\
\text { GTGCT: p.(K107Rfs*95); }\end{array}$ & $\begin{array}{l}\text { c.325T>G: p. } \\
(\mathrm{C} 109 \mathrm{G})\end{array}$ & c. $2389+5 \mathrm{G}>\mathrm{C}$ & c.616A>C: p.(S206R); \\
\hline LDLR & c. $940+1 \_c .940+4$ delGTGA; & $\begin{array}{l}\text { c. } 401 \mathrm{G}>\mathrm{C}: \mathrm{p} . \\
\text { (C134S) }\end{array}$ & & $\begin{array}{l}\text { c.663_683dupCTGCAAGGAC } \\
\text { AAATCTGACGA; }\end{array}$ \\
\hline LDLR & c. $1186+1 \mathrm{G}>\mathrm{T}$ & & & c.798T>A: p. (Asp266Glu); \\
\hline LDLR & $\begin{array}{l}\text { c. } 1684 \text { 1691delTGGCCCAA: } \\
\text { p.(P563̈Hs*14) }\end{array}$ & & & c. $1187-7 \mathrm{C}>\mathrm{G}$ \\
\hline LPL & & & & c.472T>C: p. $(\mathrm{Y} 158 \mathrm{H})$ \\
\hline APOA2 & & & & $\begin{array}{l}\text { c.122_123insACCGT: } p . \\
\text { (V41=fs*2); }\end{array}$ \\
\hline APOA2 & & & & c. $53 \mathrm{G}>\mathrm{A}: \mathrm{p} .(\mathrm{G} 18 \mathrm{E})$ \\
\hline
\end{tabular}

O.N. Ivanova: None. T.D. Krylova: None. N.A. Semenova: None. P.A. Vasiluev: None. N.V. Milovanova: None. E.Z. E.Y. Zakharova: None.

\section{E-P06.11}

Pathogenic variant p.E191K in ALPL is common in mild forms of hypophosphatasia in Northwest Russia

M. Fedyakov ${ }^{l}$, Y. Eismont ${ }^{l}$, T. Ivaschenko ${ }^{2}$, I. Sosnina ${ }^{3}, Y$. Snegova ${ }^{3}$, S. Scherbak ${ }^{l}$, Y. Barbitoff, A. Shikov ${ }^{1}$, O. Glotov ${ }^{1}$

${ }^{I}$ City Hospital 40, Saint-Petersburg, Russian Federation, ${ }^{2}$ D.O. Ott Research Institute of Obstetrics, Gynecology and Reproductology, Saint-Petersburg, Russian Federation, ${ }^{3}$ Consultative and diagnostic center for children, SaintPetersburg, Russian Federation, ${ }^{4}$ Bioinformatics institute, Saint-Petersburg, Russian Federation

Introduction: Hypophosphatasia (HPP) is a rare heritable metabolic disorder characterized by defective mineralization of bone and/or teeth in the presence of reduced activity of unfractionated serum alkaline phosphatase (ALP). The overall prevalence of severe HPP is range from 1/100 000 to $1 / 300$ 000. Mild forms of HPP are more frequent than severe forms - expected prevalence can reach 1/6000 in Western populations. Russian prevalence of mild and severe HPP is still unknown. Genetic analysis provides determining of diagnosis in cases with suspected HPP.

Materials and methods: We analyzed genomic DNA samples from 217 unrelated individuals with suspected HPP (inclusion criteria: low and/or recurrent low levels of ALP, low growth, recurrent fractures and others). Primers' system for Sanger sequencing was designed and validated for coding 2-12 exons of ALPL gene. Exome data of 353 of unrelated individuals (in-house control group) was used for genetic variant estimation of prevalence in Northwest Russia.

Results: Detection rate was $11 \%-21$ in heterozygous and 3 in compound-heterozygous. Mild HPP was founded in $87,5 \%$ positive cases (21/24). 5 novel mutations (3 missense, 2 frameshift) were detected. Most frequent pathogenic variant in ALPL gene was p.E191K in exon 6. 
The prevalence of this mutation was: $2.8 \%$ in suspected HPP group (12/434), 0.28\% (2/706 chromosome) in home controls, $0.25 \%$ in gnomAD.

Conclusions: Mild forms of HPP predominate in Northwest Russian patients with suspected HPP. Mutation p. E191K in exon 6 is 20 times common in patients with low levels of ALP compare general population.

M. Fedyakov: None. Y. Eismont: None. T. Ivaschenko: None. I. Sosnina: None. Y. Snegova: None. S. Scherbak: None. Y. Barbitoff: None. A. Shikov: None. O. Glotov: None.

\section{E-P06.14}

High genetic heterogeneity of lysosomal storage diseases in Ukraine

N. Olkhovych ${ }^{1,2}$, N. Pichkur ${ }^{1,2}$, N. Trofimova ${ }^{1,2}$, S. Kormoz ${ }^{2}$, I. Gregul ${ }^{2}$, Y. Gyvitsa ${ }^{2}$, N. Samonenko ${ }^{2,3}$, Y. Doronina ${ }^{2}, M$. Tcygankova ${ }^{2}, O$. Radzihovskaya ${ }^{2}$, N. Gorovenko ${ }^{1,3}$

${ }^{1}$ Institute of Genetic and Regenerative Medicine, Kyiv, Ukraine, ${ }^{2}$ National Children Hospital Ohmatdyt, Kyiv, Ukraine, ${ }^{3}$ National Medical Academy of Postdiploma Education named P.L.Shupika, Kyiv, Ukraine

Introduction: Lysosomal storage diseases (LSD) are inherited metabolic diseases that are caused by genetic alterations in lysosomal enzymes genes. Most of the human disease is characterized by marked genetic heterogeneity, far greater than previously appreciated. This degree of allelic, locus, and phenotypic heterogeneity has important implications for gene discovery and has important implications for development of molecular treatments for individual patients.

Aim: Analysis of genetic heterogeneity of the most common lysosomal storage diseases in patients from Ukraine.

Materials and methods: The frequencies and spectrum of causes mutations in patients with Gaucher $(n=62)$, Niemann-Peak A/B $(n=16)$, metachromatic leukodystrophy (MLD) $(\mathrm{n}=28)$, mucopolysaccharidosis type I (MPS I) $(\mathrm{n}=17)$, mucopolysaccharidosis type IIIA (MPS III A) $(n=21)$, GM1-gangliosidosis $(n=32)$ were analyzed. Mutation analysis was performed using classical PCR analysis and Sanger sequencing. Comparative analysis of the common mutations in different populations was performed according to the published data.

Results: Our research has shown that the frequencies of common mutations in patients with Niemann-Peak A/B, MLD and GM1-gangliosidosis in Ukraine is significantly different from other European. Frequencies of common mutations in patients with Gaucher, MPS I and MPS IIIA is close to that in the nearest neighbors - Poland, Hungary and Slovakia, but are lower than European average, and the genetic heterogeneity of these diseases in Ukraine is pronounced.

Conclusions: Existence of high genetic heterogeneity of LSD in Ukraine creates significant problems during medical genetic counseling for families and requires the development of specific diagnostic algorithm that regulates the stages of diagnostic search using DNA analysis.

N. Olkhovych: None. N. Pichkur: None. N. Trofimova: None. S. Kormoz: None. I. Gregul: None. Y. Gyvitsa: None. N. Samonenko: None. Y. Doronina: None. M. Tcygankova: None. O. Radzihovskaya: None. N. Gorovenko: None.

\section{E-P06.15}

A novel de novo variant in MT-ATP6 in a patient with Mitochondrial myopathy, Lactic Acidosis and Sideroblastic Anemia (MLASA) plus: expanding the clinical phenotype

J. Dupont ${ }^{1}$, P. Janeiro ${ }^{2}$, M. J. Palaré ${ }^{3}$, S. Quintas $^{4}$, A. B. Sousal

${ }^{1}$ Serviço de Genética Médica, Departamento de Pediatria, Hospital de Santa Maria, Centro Hospitalar e Universitário Lisboa Norte, Centro Académico de Medicina de Lisboa, Lisbon, Portugal, ${ }^{2}$ Centro de Referência de doenças hereditárias do metabolismo, Departamento de Pediatria, Hospital de Santa Maria, Centro Hospitalar e Universitário Lisboa Norte, Centro Académico de Medicina de Lisboa, Lisbon, Portugal, ${ }^{3}$ Unidade de Hematologia Pediátrica, Departamento de Pediatria, Hospital de Santa Maria, Centro Hospitalar e Universitário Lisboa Norte, Centro Académico de Medicina de Lisboa, Lisbon, Portugal, ${ }^{4}$ Unidade de Neurologia Pediátrica, Departamento de Pediatria, Hospital de Santa Maria, Centro Hospitalar e Universitário Lisboa Norte, Centro Académico de Medicina de Lisboa, Lisbon, Portugal

Introduction: MLASA is a mitochondrial disorder characterized by myopathy, lactic acidosis, and sideroblastic anemia, associated with biallelic mutations in PUS1 and YARS2 genes. In 2014, Burrage et al. reported an MLASAlike phenotype (OMIM 500011) in a patient harboring a novel heteroplasmic point mutation in mitochondrial ATP synthase 6 (MT-ATP6). Pathogenic variants in MT-ATP6 are a cause of NARP (neurogenic muscle weakness, ataxia, and retinitis pigmentosa) and Leigh syndrome.

Case description: The proband is an 8-year-old girl with an early infantile presentation of failure to thrive, sideroblastic anemia, axial hypotonia, nystagmus and mild developmental delay. Slow-growing, sparse and brittle 
striking hair was noticed since infancy. She evolved with developmental stagnation and severe ID. Recently, clinical deterioration occurred during acute illness. On physical examination she has tip-toe ataxic gait, dystonia, bradykinesia, rigidity, and muscle weakness with atrophy. Ophthalmologic assessment showed salt-and-pepper retinopathy. Persistent hyperlacticaemia and respiratory chain complex $\mathrm{V}$ deficiency in muscle were documented. WES and $\mathrm{CNV}$ analysis were negative. Mitochondrial genome sequencing in blood identified a novel de novo homoplasmic variant in MT-ATP6 gene (NC_012920.1: m.9014A $>$ G), classified as uncertain according to ACMG guidelines, but considered likely pathogenic applying the ClinGen SVI Bayesian classification framework. Functional studies are ongoing.

Conclusions: MLASA plus is a distinctive phenotype associated with MT-ATP6 uncommon variants. MTATP6-related disease is best defined as a mitochondrial disease spectrum disorder, as demonstrated by the presence of NARP and Leigh features in our patient. Hair abnormalities are distinctive and might lead the clinician to suspect the diagnosis before multi-organ involvement develops.

J. Dupont: None. P. Janeiro: None. M.J. Palaré: None. S. Quintas: None. A.B. Sousa: None.

\section{E-P06.16}

Management and outcomes in pregnant women with GCK-MODY and HNF1A-MODY from Poland

\section{Szopa, K. Zawadzka, K. Cyganek, P. Witek, M. T. Malecki}

\section{JUMC, Krakow, Poland}

Introduction: Mutations in the glucokinase $(G C K)$ and hepatocyte nuclear factorla (HNF1A) gene are the most common causes of maturity-onset diabetes of the young (MODY). The management of both MODY types during pregnancy is challenging and differs from other forms of diabetes. The aim was to assess clinical management and pregnancy outcomes among women with GCK-MODY and HNF1A-MODY.

Material and Methods: We included 13 GCK and 14 HNF1A-MODY patients.

Results: There was no significant difference in age of pregnancy between GCK-and HNF1A-MODY women; those with the HNF1A gene mutation were diagnosed with MODY earlier (28vs.30years). HNF1A-MODY patients preconception body weight was higher $(67.28 \mathrm{~kg})$ compared to GCK-MODY $(62.71 \mathrm{~kg})$, but the body weight gain during pregnancy was significantly higher among GCK-MODY patients $(18.5 \mathrm{vs} .12 .78 \mathrm{~kg}, \mathrm{p}<0.005)$. Treatment modification during pregnancy was more common among GCK-MODY patients: most switched from only diet to insulin. Total insulin per day in the $1^{\text {st }}$ trimester was significantly higher in GCK-MODY (34.57vs. 30.5units, $\mathrm{p}<0.005$ ) patients and the difference between percentage of basal insulin was also significantly higher $(34.99 \mathrm{vs} .20 .87 \%, \mathrm{p}<0.005)$, the latter of which remained significant until the end of pregnancy. There were no macrosomic or low birth weight neonates.

Conclusions: GCK-MODY pregnant women received a higher daily dose of insulin, especially in percentage of basal insulin, compared to HNF1A-MODY patients. This could be due to attempts to achieve target fasting glucose levels during pregnancy established for gestational diabetes despite different specificities of GCK-MODY. The higher dose of given insulin could be responsible for the greater weight gain among GCK-MODY patients.

M. Szopa: None. K. Zawadzka: None. K. Cyganek: None. P. Witek: None. M.T. Malecki: None.

\section{E-P06.18}

Mucopolysaccharidosis IIIA - a clinical case

\section{E. S. SHELBY, M. Axente, A. I. Grigoriu, L. Pădure;}

CNC RNC Dr. Nicolae Robanescu, Bucharest, Romania.

Introduction: We present the case of a 16 year old boy admitted in our hospital in May 2019 with no significant birth or family history, normal mental and motor development until one year and six months and progressive onset of symptoms consisting in macrocrania, hepatosplenomegaly, cognitive and motor retardation, hetero-aggressive behaviour, insomnia, incontinence, hirsutism, coarse facial features, muscle atrophies and scoliosis. Testing for several storage diseases was normal. MRI showed cortical atrophy, right maxillary mucocele, corpus callosum hypoplasia, occipital cyst. He had optic atrophy and aortic insufficiency. A diagnosis of mucopolysaccharidosis was suspicioned.

Objectives: The goal was to establish a diagnosis for optimal medical management and for establishing the recurrence risk in order to offer genetic counselling to the family.

Method: Further investigations were performed. CT showed skull hyperostosis, thickening of left clavicle and vertebrae (dysostosis multiplex) and femoral head necrosis. EEG was hypovoltated with epileptiform discharge. Genetic testing (panel for mucopolysaccharidoses) was performed.

Results: Patient was homozygous for SGSH c.1297C >T, p.(Arg433Trp) mutation, classified as pathogenic. He was diagnosed with Sanfilippo syndrome (MPZ type IIIA).

Conclusions: As treatment for several types of mucopolysaccharidoses exists, diagnosis must be made as soon as possible. To our knowledge, cortical atrophy and corpus 
callosum hypoplasia were described in other Sanfilippo subtypes but - except for two Israeli Arab sisters from a consanguineous family, in no other cases of Sanfilippo type A syndrome. This is the third case with these features, indicating they might also be associated with Sanfilippo type A syndrome.

E.S. Shelby: None. M. Axente: None. A.I. Grigoriu: None. L. Pădure: None.

\section{E-P06.20}

A novel homozygous pathogenic PEX26 gene's variant in patients with Zellweger syndrome from Dagestan

N. Semenova ${ }^{l}$, M. Kurkina ${ }^{l}$, E. Dadaly ${ }^{l}$, D. Guseva ${ }^{l}$, T. Strokiva ${ }^{2}$, N. Taran ${ }^{2}$

${ }^{1}$ Reseach Center for Medical Genetics, Moscow, Russian Federation, ${ }^{2}$ Federal Research Centre of Nutrition and Biotechnology, Moscow, Russian Federation

Introduction: Peroxisome biogenesis disorders (PBD) are a heterogeneous group of autosomal recessive disorders that affect multiple organ systems. Approximately $80 \%$ of PBD patients are classified in the Zellweger syndrome spectrum, which are caused generally by mutations in the PEX1, PEX6, PEX10, PEX12, or PEX26 genes.

Materials and methods: We present two unrelated families of Dagestan origin with three 1-year-old male members with cholestasis hepatopathy and development delay. Parents of one family were consanguineous. All patients were born at term, had appropriate birth weights for gestational age. From 2 months of age they have had poor feeding, development delay. Physical examination showed jaundiced, hepatosplenomegaly and coagulopathy. At 1year of age, they couldn't sit and stand on their own. Two sibs (monozygous twins) had very low cholesterol level and normal GGT. The concentrations of $\mathrm{C} 26$ and phytanic acid in plasma were increased and made up respectively $13.2 \mathrm{mM} / \mathrm{ml}$ (normal $0.22-2.2 \mathrm{nN} / \mathrm{ml}$ ) and $59.42 \mathrm{mg} / \mathrm{ml}$ (normal $0-3.11 \mathrm{mg} / \mathrm{dl}$ ); ratio of C26/C22 was 0.149 (normal 0.009-0.018). Another patient had normal phytanic acid concentration in plasma $(0.01 \mathrm{mg} / \mathrm{ml})$ but high level of C26 and C26/C22 (15.4 and 0.312 respectively). All children had normal bile acid concentration in urine.

Results: NGS was used to analyze 52 genes responsible for hereditary diseases with cholestasis. A nucleotide variant in PEX26 gene was identified: c.347T>A (p. Leu116Gln) in homozygous state. This variant was confirmed with Sanger sequencing. Parents are healthy and heterozygous for mutant allele.

Conclusions: Our clinical cases describe the phenotype associated with the c.347T $>$ A (p.Leu116Gln) variant in PEX26 gen of Dagestan origin
N. Semenova: None. M. Kurkina: None. E. Dadaly: None. D. Guseva: None. T. Strokiva: None. N. Taran: None.

\section{E-P06.21}

BH4-deficient hyperphenylalaninemia in Russia

P. Gundorova ${ }^{l}$, I. A. Kuznetsova ${ }^{l}$, A. A. Stepanova ${ }^{l}$, A. V. Polyakov ${ }^{1}$, Y. S. Itkis ${ }^{2}$

${ }^{I}$ Federal State Budgetary Institution "Research Centre for Medical Genetics", Moscow, Russian Federation, ${ }^{2}$ Research Centre for Medical Genetics, Moscow, Russian Federation

Introduction: Tetrahydrobiopterin-deficient hyperphenylalaninemia (BH4-HPA) is a group of heterogeneous diseases, caused by impaired synthesis or regeneration of tetrahydrobiopterin (BH4), a cofactor of the phenylalanine, tyrosine and tryptophan hydroxylases. Six different genes are known as responsible for BH4 metabolism. The proportion of BH4-HPA and the mutation spectrum of $\mathrm{BH} 4$ synthesis and regeneration genes is unknown in Russia.

Materials and methods: During the 20 years, more than 3,100 patients with PKU and HPA were diagnosed in the DNA-diagnostic laboratory of "RCMG". Among them, 30 patients with BH4-HPA were identified. Sanger sequencing of PTS and QDPR genes and custom panel NGS sequencing of all HPA genes and DNAJC12 were performed.

Results: In 25 patients "HPA-BH4A" diagnosis was confirmed, 18 different PTS variants were found on 50 alleles. The most common variant were p.Thr106Met $(32,0 \%)$, p.Asn72Lys (20,0\%), p.Arg9His $(6,0 \%)$. Seven novel variant were found, including the whole PTS gene deletion. In 4 patients "HPA-BH4C" diagnosis was confirmed, 5 different $Q D P R$ variants were found on 8 alleles. Three of them were not previously reported. Three patients were homozygous and one compound-heterozygous. In addition, one patient with DOPA-responsive dystonia and homozygous variant c.524C $>$ A (p. Ala175Asp) in SPR gene was identified.

Conclusions: The proportion of BH4-HPA in Russia is about $1 \%$ of all HPA. PTS gene mutations lead to the BH4HPA in $86 \%$ of cases, QDPR mutations in $14 \%$. The detection of BH4-HPA patients should be performed via differential diagnostic methods. Nowadays the reported cohort is involved in setting up a pterin method into a practice.

P. Gundorova: None. I.A. Kuznetsova: None. A.A. Stepanova: None. A.V. Polyakov: None. Y.S. Itkis: None. 


\section{E-P06.26}

Screening for genetic variants associated with cardiovascular diseases in patients with type 2 diabetes mellitus

\section{Mihaleva ${ }^{1}$, M. Mincheva ${ }^{2,3}$, M. Hristova-Savova ${ }^{3}$, K. Belemezova ${ }^{3}$, M. Rizov ${ }^{3}$, S. Yovinska ${ }^{3}$, R. Dimova ${ }^{4}$, T. Tankova ${ }^{4}$, P. Andreeva-Gateva ${ }^{1}$, I. Dimova ${ }^{2,3}$}

${ }^{1}$ Department of Pharmacology and Toxicology, Medical University Sofia, Sofia, Bulgaria, ${ }^{2}$ Laboratory of Genomic diagnostics, Molecular Medicine Center, Medical University Sofia, Sofia, Bulgaria, ${ }^{3}$ Genetic Laboratory, SAGBAL "Dr Shterev”, Sofia, Bulgaria, ${ }^{4}$ Department of Endocrinology, Medical University Sofia, Sofia, Bulgaria

A strong association between type 2 diabetes mellitus (DM) and cardiovascular diseases (CVD) exists. CVD are the leading cause of morbidity and mortality among patients with diabetes. Our objective was to study the allelic and genotypic frequencies of genetic variants that have shown a strong association with CVD in diabetic patients with and without cardiovascular complications and to assess the additional contribution of genetic variation in determining the risk for such complications. We have used CVD StripAssay kit (Vienna Lab) based on polymerase chain reaction (PCR) and reverse hybridization. The following mutations were studied: FV G1691A (Leiden), FV H1299R (R2), Prothrombin G20210A, Factor XIII V34L, $\beta$-Fibrinogen -455 G-A, PAI-1 4G/5G, GPIIIa L33P (HPA1), MTHFR C677T, MTHFR A1298C, ACE I/D, Apo B R3500Q, Apo E2/E3/E4. 36 diabetic patients divided in 2 groups were analyzed: 1) 20 diabetic patients with CVD and 2) 16 diabetic patients without CVD. Results: We found higher than population frequency for the following alleles/ genotypes - 5.5\% for FV Leiden allele, 9.7\% for FVR2 allele, $38.9 \%$ for $\beta$-Fibrinogen genotype $-455 \mathrm{G} / \mathrm{A}, 58.9 \%$ for PAI-1 4G allele, 36.1\% for ACE D/D genotype. Statistically higher frequency was established for $\beta$-Fibrinogen $-455 \mathrm{G}-\mathrm{A}$ in the patients with CVD compared to non-CVD (55\% vs. $18.7 \%$ ). Conclusion: We detected high frequency of $\beta$-Fibrinogen - $455 \mathrm{G} / \mathrm{A}$ genotype in diabetic patients, especially in these with CVD. Based on its pro-inflammatory role and its connection to possible thrombotic events, patients would benefit from anti-inflammatory treatment. Acknowledgment: This study was supported by Grant NoD66/2019, Medical University Sofia.

I. Mihaleva: None. M. Mincheva: None. M. HristovaSavova: None. K. Belemezova: None. M. Rizov: None. S. Yovinska: None. R. Dimova: None. T. Tankova: None. P. Andreeva-Gateva: None. I. Dimova: None.

\section{E-P06.28}

Variant nonketotic hyperglycinemia due to novel compound heterozygous LIAS mutations: a case with a branchpoint mutation

L. Pölsler ${ }^{1}$, B. Mühlegger ${ }^{1}$, S. Gang ${ }^{2}$, P. Traunfellner ${ }^{1}$, A. Amberger ${ }^{1}$, K. Wimmer ${ }^{1}$, S. Rudnik' ${ }^{1}$, J. Zschocke ${ }^{l}$

${ }^{1}$ Institute of Human Genetics; Medical University Innsbruck, Innsbruck, Austria, ${ }^{2}$ Department of Pediatrics and Adolescent Medicine; Academic Teaching Hospital LKH Feldkirch, Feldkirch, Austria

Nonketotic hyperglycinemia (NKH) results from defects in the glycine cleavage system and is characterized by neonatal seizures, psychomotor delay, and elevated glycine levels. It is caused by bi-allelic mutations affecting the subunits of the mitochondrial glycine cleavage system (including $G L D C$ ). The term "variant $\mathrm{NKH}$ " refers to the deficiency of one of the glycine cleavage cofactors, such as lipoate. This organosulphur compound is also part of the oxoacid dehydrogenase complexes such as PDHC and requires lipoyl synthase (LIAS gene) for its biosynthesis.

$\mathrm{NKH}$ was suspected in a boy who presented with neonatal intractable seizures from day 2 and died at 6 weeks of age. Blood and CSF glycine concentrations were elevated, lactate was normal.

Massive parallel sequencing showed heterozygosity for the known GLDC mutation c.2316-1G>A and the nonsense mutation c.985A $>$ T, p.(Lys329*) in LIAS. Additionally, a novel intronic variant c.551-24A $>\mathrm{T}$ affecting a predicted branch point in LIAS intron 5 was found. Analysis of mRNA transcripts in lymphocytes of the heterozygous LIAS c.551-24A $>\mathrm{T}$ carrier father showed retention of the 82-bp intron. Presumably due to nonsense-mediated mRNA decay, this splice effect, leading to the premature stop codon p.(Asp184Glyfs1*), was seen only in a small proportion of the father's transcripts. A minigene experiment confirmed this effect in the majority of the minigene transcripts.

This case report represents the 6th patient with variant $\mathrm{NKH}$ due to bi-allelic LIAS mutations. It illustrates the difficulties encountered by identification of single heterozygous coding or splice donor/acceptor variants in more than one candidate gene for a recessive condition.

L. Pölsler: None. B. Mühlegger: None. S. Gang: None. P. Traunfellner: None. A. Amberger: None. K. Wimmer: None. S. Rudnik: None. J. Zschocke: None.

\section{E-P07 Immunology and Hematopoietic System}




\section{E-P07.1}

Novel Indel variants in the $C A L R$ gene in mieloproliferative neoplasm patients

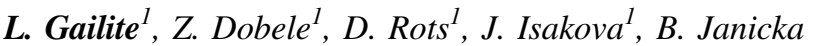
Kupra $^{2}$, K. Bernate ${ }^{3}$, L. Belajeva ${ }^{2}$, L. Zarina ${ }^{2}$, S. Lejniece ${ }^{l}$

${ }^{1}$ Riga Stradins University, Riga, Latvia, ${ }^{2}$ Riga East Clinical University Hospital, Riga, Latvia, ${ }^{3}$ Pauls Stradins University Hospital, Riga, Latvia

Objectives: Mieloproliferative neoplasm (MPN) subtypes are diagnosed based on clinical and genetical data. JAK2 gene variants serves as diagnostic criteria for polycythemia vera (PV), essential thrombocythemia (ET) and primary myelofibrosis (PMF), while CALR gene variants - for ET and PMF. The aim of the study was to summarize molecular data and utility of $C A L R$ gene indel testing in a clinical MPN cohort.

Material and methods: In the study 354 patients with MPN were included. All patients were tested for JAK2 p. V617F variant by qPCR and CALR gene indels by fluorescent-fragment length analysis (PCR-FFLA). Sanger sequencing was performed to confirm and identify previously detected variants.

Results: $J A K 2$ gene variant p.V617F was identified in 208 (58.8\%) patients. Indels in CALR gene were identified in $38(10.7 \%)$ patients: 52bp deletion c.1099_1150del52 in 20 cases and 5bp insertion c.1154_1155insTTGTC in 12 cases. The rest six patients were identified to have rare variants: c.1103_1148del (2 patients), c.1111_1141del and novel variants - c.1107_1140del and complex indel c.1131_1154delinsTTGTC (2 patients), that could be identified only with Sanger sequencing not by PCR-FFLA. $C A L R$ variant identification in two patients with previously diagnosed PV resulted in re-analysis of the clinical data and change of diagnosis to ET. For one patient JAK2 and CALR variant co-occurrence was identified.

Conclusions: $C A L R$ testing should be performed for all MPN cases. CALR testing can help to distinguish PV from other MPN forms. Validation with Sanger sequencing is necessary to confirm and identify rare $C A L R$ variants.

L. Gailite: None. Z. Dobele: None. D. Rots: None. J. Isakova: None. B. Janicka Kupra: None. K. Bernate: None. L. Belajeva: None. L. Zarina: None. S. Lejniece: None.

\section{E-P07.3}

Young adult with co-occurrence of common variable immunodeficiency and Loeys-Dietz syndrome: near the end of the diagnostic odyssey?
D. F. Osorio ${ }^{l}$, M. L. Agudelo ${ }^{1}$, O. I. Beltrán ${ }^{1,2}$

${ }^{1}$ Facultad de Medicina, Universidad Militar Nueva Granada, Bogotá DC, Colombia, ${ }^{2}$ Unidad de Genética. Organización Colsanitas SA, Bogotá DC, Colombia

Introduction: Patients with rare diseases often go undiagnosed for years while suffering from acute and/or chronic symptoms and signs that resulting in numerous health service use, multiple specialist involvement, and poor-quality life. The case report here describes a female adult with familial articular hyperlaxity due to Loeys-Dietz Syndrome found on exome sequencing and primary immunodeficiency confirmed by hypogammaglobulinemia

Methods: a retrospective analysis of family history, detailed clinical history since infancy and adulthood, physical evaluation by geneticist for contrasting phenotype combined with immunological tests and trio exome sequencing

Results: a 27-year-old woman of non-consanguineous parents; mother and aunts with symptoms of chronic myalgia, joint hyperlaxity, frequent sprains. Sister presented multiple dislocations that have required surgical interventions. Father shows a history of asthma, various atopies and depression. Since childhood, she presents mild hypotonia, myalgia, recurrent ankle and rotula sprains and chronic fatigue without an identified cause. During high school has suffered asthma, myopia, astigmatism, multiple foods, and medication allergies, inflammatory bowel disease, gastritis, overweight, depression and difficulties in learning. Face with downslanting palpebral fissures, cutaneous and joint hyperlaxity and skin striae. Normal echocardiography. Functional test revealed low serum $\operatorname{IgG}(5.76 \mathrm{~g} / \mathrm{L})$ and $\operatorname{IgA}(0.16 \mathrm{~g} / \mathrm{L})$, normal postvaccinal antibodies production, and a trio-based exome sequencing reporting a probably pathogenic variant (c.980G $>$ A, p. Arg327Gln) in TGFB2 gene.

Conclusion: Usually, cases with medical complexity can be explained by an extremely rare disease. However, another hypothesis diagnostic maybe that constellation of symptoms is due to the co-occurrence of two or more rare disease in an individual

D.F. Osorio: None. M.L. Agudelo: None. O.I. Beltrán: None.

\section{E-P08 Intellectual Disability}

\section{E-P08.02}

Further clinical and molecular delineation of Alazami syndrome associated with variants in $L A R P 7$ 
E. Gumuslu ${ }^{l}$, K. Karaer ${ }^{2}$, E. Gumus ${ }^{3}$, A. Ekici ${ }^{l}$, C. Kraus ${ }^{l}$, A. Reis ${ }^{l}$

${ }^{1}$ Institute of Human Genetics, Friedrich-Alexander-Universität Erlangen-Nürnberg, Erlangen, Germany, ${ }^{2}$ Gaziantep Genetik Hastaliklar Tani Merkezi, Gaziantep, Turkey, ${ }^{3}$ Sitki Kocman University School of Medicine, Medical Genetics Department, Mugla, Turkey

Autosomal recessive neurodevelopmental disorders are extremely heterogeneous and for many only few affected individuals have been described hampering delineation of the full spectrum of clinical presentation. In a large study of Turkish consanguineous families we identified a family with 3 affected siblings (two males 11 and 8 y.o. and one 5 y.o. female) all presenting with intellectual disability, severe verbal deficiency (2-3 words), short stature $(<3 p)$, delayed walking, seizures and dysmorphic features including malar hypoplasia, broad nose, short philtrum, wide mouth and full lips. After excluding aneuploidies exome sequencing revealed a novel frameshift insertion c.475_478dup, p. (Lys160Ilefs*2) in the LARP7 gene. LARP7 is a negative transcriptional regulator of polymerase II, acting by means of the 7SK RNP system.

Frameshifting variants in $L A R P 7$ have been associated with Alazami Syndrome (OMIM 615071) characterized by ID, severe growth restriction and characteristic facial dysmorphisms. To date, 15 patients were reported. By comparing this family with the previously reported cases, we strengthen the key features of AS: ID, growth restriction, and highlight the facial features including malar hypoplasia, broad nose, full lips and teeth abnormalities. Interestingly, no cases with seizures were described, so far. As we failed to identify in exome sequencing any variant in seizure associated genes and all three siblings are concordant we wondered if this symptom is part of the clinical spectrum of AS. Although we cannot exclude an undetected cause independent of $L A R P 7$, we propose that seizures are a so far undescribed symptom associated with Alazami Syndrome.

E. Gumuslu: None. K. Karaer: None. E. Gumus: None. A. Ekici: None. C. Kraus: None. A. Reis: None.

\section{E-P08.03}

Genomic and clinical characterisation of microduplications in a patient with developmental delay

D. Nestoroska, V. Anastasovska, E. Sukarova-Angelovska, M. Pesevska, G. Ilieva

Genetic Laboratory, University Pediatric Clinic, Skopje, Macedonia, The Former Yugoslav Republic of

Introduction: The implementation of array Comparative
Genomic Hybridization (aCGH) allows efficient genetic diagnosis of pathological conditions before their full clinical manifestation. Application of novel "genotype-first" diagnosis is of great importance to the patient's health and medical outcome due to higher rates of chromosomal abnormalities detection compared to conventional karyotyping and multiple ligation-dependent probe amplification (MLPA) analysis.

Materials and methods: The aCGH technique was used to determine the genetic background of developmental abnormalities in a 8-year-old female Macedonian patient. The blood-derived DNA sample was analyzed using the Affymetrix $^{\circledR}$ CytoScanTM 750K Array (Applied Biosystems) that includes $550 \mathrm{k}$ non-polymorphic and $200 \mathrm{k}$ SNP markers. The data were interpreted by using Chromosome Analysis Suite (ChAS) Software (v4.0).

Results: The patient showed the following clinical conditions: motor delay, deafness, lack of concentration and several dysmorhological features (blepharophymosis, telecanthus, narrow palpebral fissures, wide nose, low set ears, small mouth and arched eyebrows). Noteworthy, the girl was operated from atrial septal defect and was previously diagnosed with Ohdo Sy. Karyotyping results revealed a normal female 46, XX karyotype, while the MLPA analysis for microdeletions was negative. The aCGH analysis detected a pathological microduplication occurring at the 10q26.3 cytoregion ( $787 \mathrm{~kb}, 26$ genes included), as well as mosaic presence of 3 copies of the Xq26.3q28 cytoregion $(15,864 \mathrm{~kb}, 115$ genes included).

Conclusion: The present chromosomal abnormalities detected by aCGH analysis could be responsible for the developmental delay of the patient, considering their pathological expression according to ClinVar database. Further studies are needed to confirm these preliminary findings.

D. Nestoroska: None. V. Anastasovska: None. E. Sukarova-Angelovska: None. M. Pesevska: None. G. Ilieva: None.

\section{E-P08.05}

BRAT1-related neurodevelopmental disorders: two unrelated patients - one previously undescribed mutation

\section{B. Eichhorn $^{l}$, S. Weidensee ${ }^{2}$, H. Reichenbach ${ }^{3}$, M. Klaus ${ }^{l}$}

${ }^{l}$ MVZ Mitteldeutscher Praxisverbund Humangenetik GmbH, Dresden, Germany, ${ }^{2}$ MVZ Mitteldeutscher Praxisverbund Humangenetik GmbH, Erfurt, Germany, ${ }^{3} M V Z$ Mitteldeutscher Praxisverbund Humangenetik GmbH, Leipzig, Germany

BRAT1 gene mutations cause lethal neonatal rigidity and multifocal seizure syndrome (RMFSL) or 
neurodevelopmental disorder with cerebellar atrophy with or without seizures (NEDCAS), inherited in an autosomalrecessive manner. RMFSL is characterized by lack of developmental progress, neonatal onset hypertonia, microcephaly, intractable seizures and death before the age of two years, whereas NEDCAS results in milder clinical course. Here, we report a $2 \%$-year-old girl with severe global developmental delay (absent speech, no sitting), short stature, microcephaly, mild facial dysmorphism, brain ventricle enlargement and stereotypic hand movements. The second female patient showed also profound developmental disability (no head control), lack of eye contact with ocular motility disturbance, microcephaly, and mild facial dysmorphism. Epileptic seizures occurred at the second day of life. She died at 17 months. A panel diagnostic via sequencing by synthesis was conducted, including candidate genes contributing to neurodevelopmental disorders. Patient 1 and 2 each carried a well-described mutations causing either RMFSL or milder phenotype (c.638dupA/p. (Val214Glyfs*189)) and involved in a RMFSL (c.2125_2128delTTTG/p.(Phe709Thrfs*17)), respectively. Additional, both girls harbour the identical previously undescribed variant c.2164_2165delinsGA/p.(Leu722Asp) resulting in a missense mutation. Generally, amino acid substitutions in BRAT1 gene in homozygous or compound heterozygous state have been described to be causative for neurodevelopmental disorders. Segregation analysis in our families confirmed compound heterozygosity. There is a strong evidence for the disease causing effect of the unknown mutation in the BRAT1 gene, due to the evidence in two unrelated cases. Although a genotype-phenotype correlation is not noticeable for this variant.

B. Eichhorn: None. S. Weidensee: None. H. Reichenbach: None. M. Klaus: None.

\section{E-P08.06}

Phenotypic presentation of two additional individuals with heterozygous variants in BRSK2

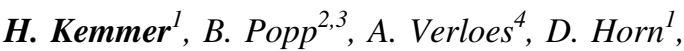 M. Holtgrewe ${ }^{5}$, C. Zweier ${ }^{3}$, N. Ehmke ${ }^{I}$}

${ }^{1}$ Institute of Medical Genetics and Human Genetics, Charité-Universitaetsmedizin Berlin, Berlin, Germany, ${ }^{2}$ Institute of Human Genetics, University of Leipzig Hospitals and Clinics, Leipzig, Germany, ${ }^{3}$ Institute of Human Genetics, Friedrich-Alexander-Universität Erlangen-Nürnberg, Erlangen, Germany, ${ }^{4}$ Department of Genetics, APHP-Robert DEBRE University Hospital, Denis Diderot School of Medicine, Paris University, Paris, France, ${ }^{5}$ Core Unit Bioinformatics - CUBI, Berlin Institute of Health, Berlin, Germany
Introduction: Loss-of-function variants in BRSK2 have recently been described as causative for a neurodevelopmental phenotype in 12 individuals with speech delay and variable additional features including intellectual disability, motor delay, behavioral issues and autism. Here we provide a detailed clinical description of two further individuals with variants in BRSK2.

Materials and methods: We performed phenotype analysis and exome sequencing on two individuals with intellectual disability and developmental delay of unknown cause.

Results: We identified two previously undescribed heterozygous variants in BRSK2: the frameshift variant c.1398del; p.(Ser467Alafs*42) and the de novo missense variant c.209T $>$ C; p.(Leu70Pro). Both individuals presented with developmental delay/mild intellectual disability with pronounced speech delay. One individual had unilateral renal agenesis and inguinal hernia, which have not been described in individuals with BRSK2 variants before.

Conclusion: Our data provide further insight into the BRSK2-associated phenotype. So far, no genotype-phenotype correlation can be determined.

H. Kemmer: None. B. Popp: None. A. Verloes: None. D. Horn: None. M. Holtgrewe: None. C. Zweier: None. N. Ehmke: None.

\section{E-P08.08}

New insights into the phenotype of $C O L A A 3 B P$-related intellectual disability: an emerging Golgipathy

\section{J. R. Alves ${ }^{1}$, J. Dupont ${ }^{1}$, J. P. Monteiro ${ }^{2}$, A. B. Sousa ${ }^{l}$}

${ }^{1}$ Serviço de Genética Médica, Hospital de Santa Maria, Centro Hospitalar e Universitário Lisboa Norte, Lisboa, Portugal, ${ }^{2}$ Serviço de Pediatria, Hospital Garcia de Orta, Almada, Portugal

Introduction: $C O L A A 3 B P$ encodes a protein implicated in intracellular ceramide transport to the Golgi complex. De novo missense variants in this gene have been linked to developmental disorders (DD) in a large-scale study of patients with severe undiagnosed DD (Fitzgerald et al., 2015). Only five patients have been reported to date and clinical data remains very scarce. We report a new patient with $C O L A A 3 B P$-related intellectual disability (ID) aiming to contribute to the recognition of this condition.

Materials and methods: Clinical data was collected from the patient's medical records and compared with previous reports from scientific literature and databases.

Results: A 5 year-old girl with unremarkable family history, pregnancy and delivery, presented with postnatal microcephaly, axial hypotonia, and severe global 
developmental delay. Short stature and facial dysmorphisms were noted and, at 24 months, she developed generalized seizures. Currently, she does not walk unaided, says only one word, and has poor visual contact and marked stereotypic behavior with bruxism. Whole exome sequencing identified a de novo pathogenic variant in COLAA3BP (S260L).

Conclusions: This missense variant was previously found in 4 out of 5 known patients, suggesting a mutational hotspot in this gene. Former functional evidence indicates it results in intracellular imbalances in ceramide, pivotal to the synthesis of sphingolipids. Our case has significant overlap with clinical data from previous patients. This report adds evidence to an emerging consistent phenotype associated with $C O L A A 3 B P$ mutations, characterized by severe ID, postnatal microcephaly, epilepsy, autistic behavior including striking bruxism, and unspecific dysmorphic features.

J.R. Alves: None. J. Dupont: None. J.P. Monteiro: None. A.B. Sousa: None.

\section{E-P08.09}

\section{Paediatric patient with deletion on chromosome 10q11.22 diagnosed by aCGH}

\author{
M. Pesevska, V. Anastasovska, E. Sukarova-Angelovska, D. \\ Nestoroska, G. Ilieva
}

University Pediatric Clinic, Skopje, Macedonia, The Former Yugoslav Republic of

Introduction: The microchip-based diagnostic practices become widely introduced in postnatal diagnosis of individuals with developmental delay and dysmorphism. Array Comparative Genomic Hybridization (aCGH) technology to whole human genome screening revealed an unexpectedly large number of deletions and duplications.

Materials and methods: aCGH analysis was perform in pediatric patient with dysmorphia and motor delay using the CytoScan_750k Array platform (Affymetrix) which comprises $550 \mathrm{k}$ non-polymorphic and $200 \mathrm{k}$ SNP markers by the Chromosome Analysis Suite (ChAS) Software (v4.0).

Results: Our patient was born with small birth weight with idiopathic thrombocytopenia during the neonatal period. At the age of 4 years, she has short stature and following sings of dysmorphia - sparse hair, baldness in infancy, slow growing hair, wide fontanel, dry skin, no sweating, small teeth, deep set eyes, wide nasal tip, smooth philtrum, up slanted palpebraes, protruded tongue in infancy, prominent forehead and some missing teeth-upper incisors. Hydronephrosis on the left kidney, reflux vesicaurinaria, atrial septal defect, dilatated pulmonary artery and CNS plexus hypertrophy were noticed by ultrasonography. Also, subclinical hypothyreosis was present. She has mild motor delay, started to walk at 20 months, first words spoken at 2.5 years with poor vocabulary at the moment. Good social skills were present. Karyotype was normal. aCGH analysis showed 1 pathogenic copy of the segment on the chromosome 10q11.22 (437 kb) including GPRIN2(611240) gene associated with brain development, according to aDGV and ClinVar databases.

Conclusions: The application of microarray will expand the spectrum in diagnosing cytogenomic abnormalities by including complex structural variants.

M. Pesevska: None. V. Anastasovska: None. E. Sukarova-Angelovska: None. D. Nestoroska: None. G. Ilieva: None.

\section{E-P08.10}

Deletion 7q31.2q31.31 segregating in a family with speech and language deficiencies

M. Rieger ${ }^{l}$, M. Krumbiegel ${ }^{l}$, M. Reuter ${ }^{\text {, }}$, A. Schützenberger ${ }^{2}$, A. Reis ${ }^{1}$, C. Zweier ${ }^{1}$

${ }^{1}$ Institute of Human Genetics, Friedrich-Alexander-Universität Erlangen-Nürnberg FAU, Erlangen, Germany, ${ }^{2}$ Division of Phoniatrics and Pediatric Audiology, Department of Otorhinolaryngology, Head and Neck Surgery, FriedrichAlexander-Universität Erlangen-Nürnberg FAU, Erlangen, Germany

Deletions of chromosome 7q31 have been described in individuals with variable neurodevelopmental disorders and speech impairment. These deletions usually contain FOXP2, in which loss-of-function mutations represent a major cause for specific language and speech phenotypes. Recently, a single patient with developmental delay as well as speech and language impairment carrying a $3.2 \mathrm{Mb}$ deletion in 7q31.2q31.31 excluding FOXP2 was reported. By chromosomal microarray analysis we now identified a similar microdeletion in three family members presenting with variable speech, language and neurodevelopmental phenotypes. The index patient presented with normal motor milestones but delayed speech development, reduced comprehension and an incomplete sentence structure at age 5 years. At age 10 years he attended a school for children with special needs and presented with microcephaly, attention deficits and slurred speech. His sister was referred at age 3 years with normal first milestones but a delay in speech development. Linguistic testing revealed impairment of all language and speech levels with limited vocabulary and slurred and imprecise pronunciation. The mother had attended a school focusing on speech and language deficiencies and now presented with slurred speech. Segregation analysis in the healthy, maternal grandparents did not detect the microdeletion, thus indicating de novo occurrence in the mother. The deletion contains 17 genes and is located 
in close proximity to the 3' end of FOXP2, but not including it. Though positional effects on FOXP2 expression cannot be excluded, our findings support a new locus on $7 \mathrm{q} 31.2 \mathrm{q} 31.31$ associated with mild neurodevelopmental and more prominent speech and language phenotypes.

M. Rieger: None. M. Krumbiegel: None. M. Reuter: None. A. Schützenberger: None. A. Reis: None. C. Zweier: None.

\section{E-P08.11}

Clinical significance of $2 q 37.1$ duplication in patients with intellectual disability and facial dysmorphism

Z. Capkova ${ }^{1,2}$, P. Capkova ${ }^{1,2}$, J. Srovnal ${ }^{1,3}$, K. Staffova ${ }^{1,3}$, K. Adamova ${ }^{1,2}$, J. Pazderova ${ }^{l}$, M. Hajduch ${ }^{3}$, M. Prochazka ${ }^{1,2}$

${ }^{1}$ Department of Medical Genetics, University Hospital Olomouc, Olomouc, Czech Republic, ${ }^{2}$ Department of Medical Genetics, Faculty of Medicine and Dentistry, Palacký University Olomouc, Olomouc, Czech Republic, ${ }^{3}$ Institute of Molecular and Translational Medicine, Faculty of Medicine and Dentistry, Palacký University Olomouc, Olomouc, Czech Republic

Background: 2q37 duplications including only $A L P P$, $A L P P L 2, D I S 3 L 2$ have been already described in patients with ID and facial dysmorphia. However, these duplications were concluded as benign variations based on inheritance from health parents.

Aim: We report two unrelated patients with ASD, ID and facial dysmorphic features.

Methods: Karyotyping, exclusion of CGG triplet repeats expansion in FMRI and chromosomal microarray (CMA) were performed in both patients after informed consent collecting from their parents.

Results: Chromosomal aberration and Fragile- $X$ syndrome were excluded in both. 2q37 duplication including $A L P P, A L P P L 2, D I S 3 L 2$ was revealed by CMA. In patient 1 the duplication was inherited from father. Inheritance was unknown in patient 2 . No other clinically relevant CNVs were observed.

Clinical and genetics features of both patients

\begin{tabular}{|c|c|c|}
\hline Feature & Patient 1 & Patient 2 \\
\hline Height & $<3^{\text {th }}$ percentile & $40^{\text {th }}$ percentile \\
\hline Head circumference & $<3^{\text {th }}$ percentile & $34^{\text {th }}$ percentile \\
\hline Weight & $<3^{\text {th }}$ percentile & $23^{\text {th }}$ percentile \\
\hline $\begin{array}{l}\text { Body mass } \\
\text { index (BMI) }\end{array}$ & 15.02 & 15.50 \\
\hline Add clinical features & microcephaly & none \\
\hline
\end{tabular}

\begin{tabular}{lll}
\hline \multicolumn{2}{l}{ Clinical and genetics features of both patients } \\
\hline Feature & Patient 1 & Patient 2 \\
\hline Coordinate (GRCh37) & $233195538-$ & $233195538-$ \\
& 233318065 & 233311912 \\
Length & $123 \mathrm{~kb}$ & $116 \mathrm{~kb}$ \\
Overlapped & $A L P P, A L P P L 2$, & ALPP, ALPPL2, \\
coding genes & DIS3L2 & DIS3L2 \\
Inheritance & father & unknown
\end{tabular}

Discussion and conclusion: Duplication of $2 \mathrm{q} 37$ including DIS $3 L 2$ is assumed as benign (DECIPHER, ISCA, ClinVar). We described two unrelated patients with ASD, ID and facial dysmorphia who carried the duplication of 2q37 including DIS3L2. We suggest that this CNV might cause of abnormal phenotype with variable penetrance. Of course, we cannot exclude a nucleotide level changes which could explain a variable penetrance of $2 \mathrm{q} 37$ duplication. Acknowledgment: Supported by MH_CZ_DRO_FNOL_00098892, IGA_LF_2020_007

Z. Capkova: B. Research Grant (principal investigator, collaborator or consultant and pending grants as well as grants already received); Modest; University Hospital Olomouc, Palacký University Olomouc. P. Capkova: B. Research Grant (principal investigator, collaborator or consultant and pending grants as well as grants already received); Modest; University Hospital Olomouc, Palacký University Olomouc. J. Srovnal: B. Research Grant (principal investigator, collaborator or consultant and pending grants as well as grants already received); Modest; University Hospital Olomouc, Palacký University Olomouc. K. Staffova: B. Research Grant (principal investigator, collaborator or consultant and pending grants as well as grants already received); Modest; University Hospital Olomouc, Palacký University Olomouc. K. Adamova: B. Research Grant (principal investigator, collaborator or consultant and pending grants as well as grants already received); Modest; University Hospital Olomouc, Palacký University Olomouc. J. Pazderova: None. M. Hajduch: None. M. Prochazka: None.

\section{E-P08.14}

Early neurodevelopmental milestones acquisition in fragile $\mathrm{X}$ syndrome, a caregivers online report

\author{
C. Azevedo Soares ${ }^{1,2}$, D. Gonzaga ${ }^{3}$, I. Vaz Matos ${ }^{3}$, \\ N. Tkachenko ${ }^{I}$, A. Fortuna ${ }^{1,2}$, G. Soares ${ }^{l}$, A. Prior $^{3}$ \\ ${ }^{1}$ Centro de Genética Médica Jacinto Magalhães, Porto, \\ Portugal, ${ }^{2}$ Unit for Multidisciplinary Research in Biomedi- \\ cine, Instituto de Ciências Biomédicas Abel Salazar/ \\ Universidade do Porto, Porto, Portugal, ${ }^{3}$ Unidade de
}


Neurodesenvolvimento, Serviço de Pediatria, Centro Materno-Infantil do Norte, Centro Hospitalar Universitário do Porto, Porto, Portugal

Introduction: Fragile X syndrome (FXS) is a genetic disorder affecting neurodevelopment caused by FMRI dysfunction. The identification of developmental delay patterns could accelerate the diagnostic of FXS in a population of children with development delay.

Methods: We designed an online survey in which FXS patients' caregivers indicated patients characteristics. We used the Haizea-Llevant development scale as a reference of normalcy. Time of acquisition was expressed as months old (mo) and for each milestone timing it was calculated the median (M) and P25 and P75 (P25-P75).

Results: Fifteen cases were considered for analysis: three females and 12 males. The median age for postnatal diagnosis was 48 mo: 36 mo for males and 72 mo for females. Male FXS patients showed a delayed acquisition of early milestones such as cervical control $(\mathrm{M}=6 \mathrm{mo}, 2-12$ mo) with normal median time of acquisition of the first word $(M=13 \mathrm{mo}, 11.75-21 \mathrm{mo})$, and delayed acquisition of autonomous walking $(\mathrm{M}=22 \mathrm{mo}, 16.5-24 \mathrm{mo})$. Given the small number of female FXS in our study, it was not possible to conclude if females had a characteristic pattern of developmental delay.

Conclusions: FXS patients present a biphasic delayed development in the first two years of life with an important delay on the acquisition of gross motor skills. Nevertheless, the clinical presentation was highly variable to allow the discrimination these patients with FXS by their pattern of developmental delay. Notwithstanding, this work bring the attention to the early gross motor skills impairment in FXS, that can be improved with targeted interventions.

C. Azevedo Soares: None. D. Gonzaga: None. I. Vaz Matos: None. N. Tkachenko: None. A. Fortuna: None. G. Soares: None. A. Prior: None.

\section{E-P08.16}

A case of autosomal recessive spinocerebellar ataxia type 18 with a novel mutation in GRID2

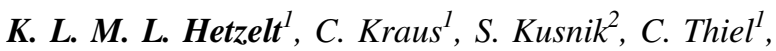 \\ A. B. Ekici ${ }^{l}$, A. Reis ${ }^{l}$, C. Zweier ${ }^{l}$
}

${ }^{1}$ Institute of Human Genetics, Friedrich-Alexander-Universität Erlangen-Nürnberg FAU, Erlangen, Germany, ${ }^{2}$ Department of Pediatrics, University Hospital for Children and Adolescents, Friedrich-Alexander-Universität Erlangen-Nürnberg FAU, Erlangen, Germany

Autosomal-recessive spinocerebellar ataxia type 18 is a rare genetic neurologic disorder. It is caused by homozygous or compound-heterozygous mutations in the GRID2 gene, encoding an ionotropic glutamate receptor. So far, seven affected individuals from three unrelated families have been reported. The disorder is characterized by delayed psychomotor development, intellectual disability, severely impaired gait due to progressive cerebellar ataxia, ocular movement abnormalities, and progressive cerebellar atrophy in brain imaging. By trio exome sequencing, we now identified a novel homozygous variant $(c .568 \mathrm{C}>\mathrm{T}$; $\mathrm{p}$. Gln190*) in GRID2 in a four year old female patient from a consanguineous Iraqi family. Both parents were confirmed to be heterozygous carriers. The patient was born after uneventful pregnancy and showed early-onset severe delayed psychomotor development. At the age of 4 years, she does not sit, walk or speak and presents with severe muscular hypotonia. Further clinical characteristics are a distinct truncal and appendicular progressive ataxia and binocular vertical nystagmus with a high frequent horizontal component, central hearing loss and incomplete loss of sight. She does not fix objects or interacts with people. She is dystrophic with a weight under the third percentile of the central European standard. Eating hair and gnashing of teeth are striking behaviors. In addition, cerebral magnetic resonance imaging (MRI) showed cerebellar hypoplasia. Our findings further delineate the mutational and clinical spectrum of GRID2 associated spinocerebellar ataxia type 18 .

K.L.M.L. Hetzelt: None. C. Kraus: None. S. Kusnik: None. C. Thiel: None. A.B. Ekici: None. A. Reis: None. C. Zweier: None.

\section{E-P08.17}

A novel patient helps to define the phenotype, developmental trajectory and clustering of de novo missense mutations in the HECW2 syndrome

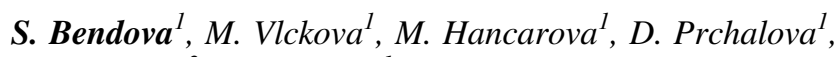 V. Stranecky ${ }^{2}$, Z. Sedlacek ${ }^{1}$}

${ }^{1}$ Department of Biology and Medical Genetics, Charles University 2nd Faculty of Medicine and University Hospital Motol, Prague, Czech Republic, Prague, Czech Republic, ${ }^{2}$ Department of Pediatrics and Adolescent Medicine, Diagnostic and Research Unit for Rare Diseases, Charles University 1st Faculty of Medicine and General University Hospital, Prague, Czech Republic, Prague, Czech Republic

De novo variants in HECW2, which encodes the HECT, C2 and WW domain containing E3 ubiquitin protein ligase 2, were initially identified in large exome sequencing (ES) studies. A series of seven patients clearly associated HECW2 with a neurodevelopmental disorder with hypotonia, seizures and absent language (OMIM \#617268) in 2017. Since then only two novel case reports have been published. We present 
the genotype, phenotype and developmental trajectory of a new patient, and review all previous cases. The 8-year-old boy has hypotonia, microcephaly, failure to thrive, severe intellectual disability, absent speech, and seizures. His facial features include prominent upper lip and strabismus. Trio ES identified de novo variant Chr2(GRCh37):g.197106886C>T, NM_001348768.1(HECW2):c.3572G>A, p.(Arg1191Gln). The variant has been reported in three previous patients, and is considered causal for the HECW2 syndrome. The phenotype of the patient fits the previous cases; however, he also has a ventricular septum defect, and he neither has abnormal brain MRI findings nor requires gastrostomy tube feeding. Long-term follow-up shows regression of motor skills around 12 months, abnormal EEG findings since 14 months (prompting anti-epileptic therapy), and self-injurious behaviour and sleep disturbances since 45 months of age. Our analysis (including variants from large studies and databases) indicates that deleterious missense HECW2 variants cluster in exons encoding the HECT domain and its adjacent region (exons 20-29, with residues Arg1191, Arg1330 and Glu1445 being the hotspots), and possibly also the WW2 domain (exons 12-13). Concurrently, these regions are deserts for missense variation in normal individuals. Supported by $17-$ 29423A, LM2018132 and 00064203.

S. Bendova: None. M. Vlckova: None. M. Hancarova: None. D. Prchalova: None. V. Stranecky: None. Z. Sedlacek: None.

\section{E-P08.18}

\section{A novel PGAP3 mutation in two siblings with hyperpho-} sphatasia with mental retardation syndrome

\section{F. Hazan ${ }^{1}, S$. Gürsoy ${ }^{2}, P . K_{\text {Karaoğlu }}^{3}, \ddot{U} . Y_{\imath l m a z}{ }^{3}$}

${ }^{1}$ Department of Medical Genetics, Dr. Behcet Uz Children's Hospital, Izmir, Turkey, ${ }^{2}$ Department of Pediatric Genetics, Dr. Behcet Uz Children's Hospital, Izmir, Turkey, ${ }^{3}$ Department of Pediatric Neurology, Dr. Behcet Uz Children's Hospital, Izmir, Turkey

Introduction: Hyperphosphatasia with mental retardation syndrome (HPMRS) OMIM \# 239300), is an autosomal recessive disorder characterized facial dysmorphism, intellectual disability, seizures, cardiac abnormalities, developmental delay and elevated alkaline phosphatase. Six subgroups of HPMRS were idefined in which pathogenic mutations affect genes involved in either synthesis (PIGV, PIGW, PIGO, PIGY) or remodeling (PGAP2, PGAP3) of the anchor proteins.

Material and methods: A two year-old girl patient was referred to our genetic clinic for intellectual disability and hypotonia. There was a first-degree consanguinity between the parents. This patient had microcephaly, facial dysmorphism including upslanting palpebral fissure, bulbous nose, cleft lip and palate, pectus excavatum, intellectual disability, developmental delay, hypotonia and increased alkaline phosphatase level. One out of three sisters of index patient had similar clinical features. The other family members were healthy. Whole exome sequencing (WES) was performed to the index patient. Sanger seguencing analysis was used for family segregation analysis.

Results: A novel homozygous mutation (c.715_717del TGG; p.Trp239del) of PGAP3 gene was detected with WES analysis in the index patient (NM_033419.5). Family segregation analysis was performed and bioinformatic tools were used for the evaluation of this novel variant. The same homozygous mutation was noted by sanger seguencing analysis in her younger sister, who had similar clinical features. Both parents and the other healthy sisters were heterozygous for this mutation.

Conclusions: We report e novel homozygous mutation of PGAP3 gene. HPMRS should be kept in mind in patients with developmental delay, hypotonia, cleft palate and mild to moderate serum ALP elevation.

F. Hazan: None. S. Gürsoy: None. P. Karaoğlu: None. Ü. Yilmaz: None.

\section{E-P08.19}

A 3-year old girl with Hypotonia, Ataxia, And DelayedDevelopment Syndrom (HADDS)

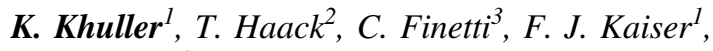 A. Kuechler ${ }^{1}$}

${ }^{1}$ Institut für Humangenetik, Universitätsklinikum Essen, Universität Duisburg-Essen, Essen, Germany, ${ }^{2}$ Institut für Medizinische Genetik und Angewandte Genomik, Tübingen, Germany, ${ }^{3}$ SPZ, Elisabeth-Krankenhaus, Essen, Germany

Hypotonia, ataxia, and delayed development syndrome (HADDS, OMIM \#617330) is a neurodevelopmental syndrome characterized by congenital hypotonia, delayed psychomotor development, variable intellectual disability with speech delay, variable dysmorphic facial features, and ataxia, often associated with cerebellar hypoplasia. In 2017, heterozygous variants in $E B F 3$ are described for the first time by three parallel publications in 2017 as causative for HADDS (Chao et al., 2017, Harms et al., 2017, Sleven et al., 2017). At present, 23 patients with different types of mutations including missense, frameshift or nonsense mutations as well as variants affecting conserved splice elements are reported. All reported cases showed a heterogenous phenotype. We report on a case of a 3-year old girl with a class 5-mutation in EBF3, detected by Next- 
Generation-Sequencing. She presented with delayed development, microcephaly, ataxia, reflux, and feeding difficulties. She also showed a thermoregulatory dysfunction, which was not observed in other affected individuals so far. We present detailed clinical data of our patient in the context of the current literature thus expanding the phenotypic spectrum of the HAAD syndrome.

K. Khuller: None. T. Haack: None. C. Finetti: None. F.J. Kaiser: None. A. Kuechler: None.

\section{E-P08.20}

TMEM222 is a new cause of autosomal recessive intellectual developmental disorder

\section{O. Levchenko, N. Demina, A. Lavrov}

Research centre for medical genetics, Moscow, Russian Federation

Introduction: The prevalence of autosomal recessive intellectual developmental disorder (ARIDD) in affected children of an outbred population is estimated about $10 \%$. There are 72 types of ARIDD described in OMIM. TMEM222 was once described as a candidate gene for ARIDD with moderate intellectual disability, speech delay, mild hypotonia, aggressiveness and body shivering.

Materials and methods: Whole exome sequencing (WES) was performed using IlluminaTruSeq ${ }^{\circledR}$ ExomeKit and IDT $\mathrm{xGen}^{\circledast}$ Exome Research Panel on Illumina NextSeq 500.

Results: We describe the Caucasian 31-year-old men born from consanguineous parents. Birth weight was 4800 $\mathrm{g}$, condition severe. He had infantile spasms when woke up during first 2-8 months. He walks since 3 years with ataxia, speaks few words, understands speech and uses computer. Phenotype: hypotonia, microcephaly, hypothelorism, big nose, short philtrum, big mouth, full lips, gingival hyperplasia, small chin, broad and short distal phalanges, narrow feet with valgus deformity, broad big toe. His brother 11 years old has similar phenotype but with prominent incisors and seizures since birth. He cannot walk and speak. His birth weight was $4700 \mathrm{~g}$. Their healthy sister had birth weight $3500 \mathrm{~g}$. WES revealed homozygous splice variant c.539+2T>C NM_032125 in TMEM222. Sanger sequencing confirmed this variant as heterozygous in parents and sister, and homozygous in affected brother.

Conclusions: Given the similarity of the phenotype with the previously described, the correspondence of segregation to the autosomal recessive type of inheritance, we suppose this variant to be causative. We describe new ARIDD with developmental delay, hypotonia, seizures, high birth weight and dimorphisms.
O. Levchenko: None. N. Demina: None. A. Lavrov: None.

\section{E-P08.23}

Patient with the Tatton-Brown-Rahman syndrome \&lt de novo constitutive DNMT3A variant

\section{Jaszczuk ${ }^{1,2}$, I. Winkler ${ }^{3}$, A. Sobczyńska-Tomaszewska ${ }^{4}$, M. Lejman ${ }^{5}$, A. Pietrzyk ${ }^{4}$, M. Kacprzak ${ }^{4}$}

${ }^{1}$ University Children's Hospital, Department of Pediatric Hematology, Oncology and Transplantology, Lublin, Poland, ${ }^{2}$ Department of Cancer Genetics with Cytogenetic Laboratory, Medical University, Lublin, Poland, ${ }^{3}$ St'Johns Center Oncology, Lublin, Poland, ${ }^{4}$ MedGen Medical Centre, Warsaw, Poland, ${ }^{5}$ Genetic Diagnostics, Department of Pediatric Hematology, Oncology and Transplantology, Medical University, Lublin, Poland

Introduction: Tatton-Brown-Rahman syndrome (TBRS; OMIM 615879) is an overgrowth and intellectual disability syndrome, first described in 2014. Additional clinical features include dysmorphic features, obesity, musculoskeletal disorders (hypotonia, hypermobility, kyphoscoliosis) and behavioural problems (autistic spectrum disorders) and afebrile seizures. The underlying cause of the syndrome is constitutive variants in the DNMT3 gene. We present an 11old boy with recognized Tatton-Brown-Rahman syndrome and confirmed variant in the DNMT3 gene.

Materials and methods: During molecular diagnostic procedure analysis of FMRI gene for FRAXA syndrome and panel of 264 genes correlated with autism and mental retardation (NGS) were performed.

Results: For FMRI gene expansion of CGG repeats was not identified. NGS analysis allowed identification of known heterozygotic variant p.Pro904Leu (c.2711C $>$ T) in the DNMT3A. This variant is reported in dbSNP database (rs149095705) and ClinVar Database as likely pathogenic. Molecular analysis of p.Pro904Leu was also performed for proband's mother and sister were mutation was not identified. Unfortunately the father's material was not available for genetic analysis. There is high probability of de novo character of identified variant.

Conclusions: In the case of patients with intellectual disability and obesity, Tatton-Brown-Rahman syndrome should be considered in the differential diagnosis. Studies using the NGS technique facilitate the diagnosis of patients with intellectual disabilities.

I. Jaszczuk: None. I. Winkler: None. A. SobczyńskaTomaszewska: None. M. Lejman: None. A. Pietrzyk: None. M. Kacprzak: None. 


\section{E-P08.24}

Biallelic NALCN variant detected by homozygosity mapping and whole exome sequencing in a consanguineous family from Turkey

\section{S. Susgun ${ }^{1,2,3}$, E. Yucesan ${ }^{1}$, O. Kocaturk ${ }^{4}$, M. Calik $^{5}$, U. Ozbek ${ }^{2,6}$, S. Ugur Iseri ${ }^{2}$}

${ }^{1}$ Department of Medical Biology, Faculty of Medicine, Bezmialem Vakif University, Istanbul, Turkey, ${ }^{2}$ Department of Genetics, Aziz Sancar Institute of Experimental Medicine, Istanbul University, Istanbul, Turkey, ${ }^{3}$ Graduate School of Health Sciences, Istanbul University, Istanbul, Turkey, ${ }^{4}$ Department of Neurology, Faculty of Medicine, Harran University, Sanliurfa, Turkey, ${ }^{5}$ Department of Pediatric Neurology, Faculty of Medicine, Harran University, Sanliurfa, Turkey, ${ }^{6}$ Department of Medical Genetics, School of Medicine, Mehmet Ali Aydinlar Acibadem University, Istanbul, Turkey

Neurodevelopmental disorders (NDD) are a large and heterogeneous group of diseases including intellectual disability, developmental delay and autism spectrum disorders. Several ultra rare NDD associated variants in numerous genes specific almost to single families have been identified by unbiased next generation sequencing technologies. To determine the genetic component of NDD in a consanguineous family from Turkey, we have performed SNPbased homozygosity mapping in the family along with whole exome sequencing (WES) in the affected sib pair.

The SNP genotyping data generated on two different array chips was combined with PLINK to detect homozygous stretches common to the affected sib pair. The WES data was filtered for rare alleles with predicted pathogenic impact on protein function in the homozygous loci. This collective effort has led us to identify a loss of function variant in NALCN in the homozygous state. Sanger sequencing has confirmed segregation of this variant with the NDD phenotype in the pedigree. NALCN encodes a member of sodium leak channel complex and is associated with a recessive OMIM phenotype; Hypotonia, infantile, with psychomotor retardation and characteristic facies 1 (MIM:615419)

Our study presents the combined analysis of linkage and exome analysis as a powerful and precise tool to diagnose a clinically rare and recessively inherited condition on molecular level. We are planning to analyze possible functional effects for the variant using in vitro studies to enlighten the molecular pathogenesis.

This work has been supported by the grants of TUBITAK (113S331) and TUBA GEBIP 2019 program and Bezmialem Vakif University Scientific Research Support Unit (Project no:12.2019/9)
S. Susgun: None. E. Yucesan: None. O. Kocaturk: None. M. Calik: None. U. Ozbek: None. S. Ugur Iseri: None.

\section{E-P08.27}

Laryngomalacia, recurrent overgrowth of pharyngeal lymphoid tissue, mild developmental delay and macrocephaly in a girl with a de novo heterozygous mutation in $Z B T B 7 A$

C. von der Lippe ${ }^{l}$, K. Tveten ${ }^{l}, \emptyset$. L. Holla ${ }^{l}$, Ø. L. Busk ${ }^{l}$, M. G. Haug $^{2}$, W. Thorstensen ${ }^{3,4}$, A. K. Aasebøst $l^{3}$, T. Prescott ${ }^{l}$

${ }^{I}$ Dept of Medical Genetics, Telemark Hospital Trust, Skien, Norway, ${ }^{2}$ Dept of Medical Genetics, St. Olavs University Hospital, Trondheim, Norway, ${ }^{3}$ Dept of Otolaryngology, Head and Neck Surgery, St. Olavs University Hospital, Trondheim, Norway, ${ }^{4}$ Dept of Neuromedicine and Movement Science, Norwegian University of Science and Technology (NTNU), Trondheim, Norway

Case: This 12-year-old girl had a congenital umbilical hernia and laryngomalacia, as well as frequent upper airway infections, obstructive sleep apnea and serious otitis media from infancy. Strikingly, recurrent pharyngeal lymphoid hyperplasia has necessitated 17 surgical procedures; starting at age 15 months old. Her thymus is described as large for her age. Generalized lymphadenopathy / hepatosplenomegaly are absent. She has mild motor and language delay. Current measurements are height $25 \%$, weight $4 \mathrm{~kg}>97,5 \%$, head circumference $97,5 \%$. WES-trio detected a de novo nonsense variant in ZBTB7A, NM_015898.3:c.832G>T p.(Glu278*). The variant is not present in gnomAD, and the gene is LoFintolerant ( $\mathrm{pLI}=0.96 ; \mathrm{O} / \mathrm{e}=0: 07$ ) and is predicted to result in NMD. No other SNVs of interest were detected, and a 180k array $\mathrm{CGH}$ analysis was unremarkable.

Discussion: ZBTB7A (OMIM \#605878) encodes the leukemia/lymphoma related factor, LRF $Z B T B 7 A$ is a candidate gene for some phenotypic features reported in individuals with microdeletions of chromosome 19p13.3; notably macrocephaly, obesity, sleep apnea, umbilical hernia, learning difficulties and developmental delay $(1,2)$ - all features present in the girl we describe. Ohishi et al. (3) recently reported a boy with macrocephaly, intellectual disability and sleep apnea who had a de novo likely pathogenic heterozygous missense variant in ZBTB7A.

Conclusion: We believe that the de novo ZBTB7A variant in our patient is the cause of her distinct phenotype -laryngomalacia, recurrent overgrowth of pharyngeal lymphoid tissue, macrocephaly and delayed motor and language development. 
C. von der Lippe: None. K. Tveten: None. Ø.L. Holla: None. Ø.L. Busk: None. M.G. Haug: None. W. Thorstensen: None. A.K. Aasebøstøl: None. T. Prescott: None.

\section{E-P08.31}

Study of diagnostic yield of genetic studies in neuronal migration disorders

N. Ortiz Cabrera, M. Vilar Egea, B. Fernández Garoz, V. Cantarín Extremera, A. Duat Rodríguez, L. González Gutierrez Solana, M. Jimémez Legido, V. Soto Insuga, M. Ruiz Falcó, J. García Peñas

\section{Hospital Infantil Universitario Niño Jesús, Madrid, Spain}

Neuronal migration is the key factor in lamination of cerebral cortex. At 12th day stage of development neuronal progenitors begin migration. Excitatory neurons are born in the dorsal ventricular zone and migrate superficially to the cortical plate. These neurons migrate along the vertical fibers of the radial glia. The layers of the cortex form in an "inside-out" sequence with later born neurons migrating past their predecessors in the cortical plate so that they are more superficial in their final position than earlier born neurons(1).

The neuronal migration disorders (NMD) include: lissencephaly, periventricular heterotopia, polymicrogyria (PMG) and cortical dysplasias(2). NMD present with: epilepsy, developmental delay (DD), intellectual disability (ID) and other malformation could be associated. Although the majority of this disorders have a genetic base, some environmental causes such as intrauterine infections could cause them(3). Today more than 100 genes have been related to NMD (3) including genes related to tubulinopathies (2).

We did an observational study of NMD patients seen at the Clinical Genetic clinic from june 2018 until december 2019. We analyzed the results of NGS based genetic studies.

Results: 29 patients were studied, the global diagnostic yield was $28 \%$. We were able to detect large deletions using NGS. We found pathogenic variants in ARFGEF2, ARX, DEPDC5, DYNC1H1, NEDD4L, PAFAH1B and TCF20. Pathogenic variants in DEPDC5 were the most frequent pathogenic finding $29 \%$ of positive results.

Conclusions: One third of patients with NMD will benefit from a genetic diagnosis. DEPDC5 is a frequently involved in this disorders.

N. Ortiz Cabrera: None. M. Vilar Egea: None. B. Fernández Garoz: None. V. Cantarín Extremera: None. A. Duat Rodríguez: None. L. González Gutierrez Solana: None. M. Jimémez Legido: None. V. Soto Insuga: None. M. Ruiz Falcó: None. J. García Peñas: None.

\section{E-P08.32}

The clinical utility of targeted NGS in neurodevelopmental disorders: a case of a girl with pontocerebellar hypoplasia caused by TSEN54 gene pathogenic variants

\section{Wayhelova' ${ }^{1,2}$, V. Vallova ${ }^{1,2}$, E. Hladilkova $^{2}$, H. Filkova ${ }^{2}$, J. Oppelt ${ }^{3}$, J. Soukalova ${ }^{2}$, P. Kuglik ${ }^{1,2}$}

${ }^{1}$ Department of Experimental Biology, Faculty of Science, Masaryk University, Brno, Czech Republic, ${ }^{2}$ Department of Medical Genetics, University Hospital Brno, Brno, Czech Republic, ${ }^{3}$ CEITEC-Central European Institute of Technology, Masaryk University, Brno, Czech Republic

Next-generation sequencing (NGS) techniques represent a widely used, powerful tool for the identification of the genetic causes of neurodevelopmental disorders and congenital abnormalities. We present a case of a girl $(* 2016)$ from the $3^{\text {rd }}$ gravidity of healthy non-consanguineous couple (mother $* 1984$, father $* 1978$ ). She was diagnosed a global developmental delay, microbrachycephaly, severe dysplasia of brainstem and muscle hypertonia. She has a normal female karyotype $46, \mathrm{XX}$ and normal array-CGH profile $\operatorname{arr}(1-22, \mathrm{X}) \times 2$. She was investigated by targeted NGS by gene panel ClearSeq ${ }^{\mathrm{XT}}$ Inherited Disease (Agilent Technologies) containing more than 2700 genes known to cause inherited disorders. We detected pathogenic variants in the TSEN54 gene in compound heterozygosity, NM_207346.2:c.[56 $+2 \mathrm{~T}>\mathrm{G} ; 160 \mathrm{C}>\mathrm{G}] ;[919 \mathrm{G}>\mathrm{T}]$. The parents are healthy heterozygous carriers of these variants, as was confirmed by Sanger sequencing. Pathogenic variants of the TSEN54 are causative in extremely rare pontocerebellar hypoplasia $(\mathrm{PCH})$ type $2 \mathrm{~A}, 4$ and 5 . The combination of splicing and missense variants has been described in only one case of PCH5 so far, resulting in recurrent fetal lethality. Therefore, we suggest the TSEN54 pathogenic variants could have been the cause of previous recurrent abortions in the presented family. This case showed the usefulness and effectivity of our molecular diagnostics algorithm enhanced by NGS approaches to elucidate the molecular genetic causes in heterogeneous neurodevelopmental disorders. Supported by the project of Faculty of Science of Masaryk University MUNI/A/1127/2019 and IP UH Brno 2019 PIG 5/19.

M. Wayhelova: None. V. Vallova: None. E. Hladilkova: None. H. Filkova: None. J. Oppelt: None. J. Soukalova: None. P. Kuglik: None. 


\section{E-P08.33}

\section{Intrafamilial variability of Refsum syndrome}

\section{S. Geuer, I. Schwaab, R. König}

\section{Bioscientia, Ingelheim, Germany}

A consanguine Pakistani couple with two daughters affected by intellectual disability and developmental delay has been investigated by genetic diagnostics. The younger girl has more severe development delay and shows in contrast to her sister microcephaly, craniosynostosis and autistic behaviour. Whole exome sequencing of the more severe affected sister revealed a homozygous pathogenic variant in PEX5 - a gene known to be causative for autosomal recessive Refsum syndrome. Segregation analysis confirmed homozygosity of the variant in the other sister and heterozygosity of the parents. Whole exome sequencing did not detect another potential pathogenic variant. Thus, it can be concluded that the detected PEX5 variant causes a Refsum syndrome with uncharacteristic features and a high intrafamilial variability.

S. Geuer: A. Employment (full or part-time); Significant; Bioscientia. I. Schwaab: A. Employment (full or parttime); Significant; Bioscientia. R. König: A. Employment (full or part-time); Significant; Bioscientia.

\section{E-P08.35}

A further patient with Skraban-Deardorff syndrome: Presentation of the clinical and molecular phenotype of a rare intellectual disability syndrome

\section{A. Busche, M. Vockel, A. Bohring}

Institut für Humangenetik, Westfälische Wilhelms-Universität, Münster, Germany

The Skraban-Deardorff syndrome (SKDEAS, OMIM\#617616) is a rare genetic condition characterized by intellectual disability, a history of febrile and/or non-febrile seizures, and a recognizable facial appearance. SKDEAS is caused by heterozygeous mutations in WDR26 (OMIM*617424) and is inherited in an autosomal dominant pattern. To our knowledge only 15 patients have been reported so far.

Here we present a 3 year and 3 months old boy with SKDEAS due to the heterozygous de novo mutation c.508G $>\mathrm{T}$; p.(Gly170*) in WDR26. He has moderate intellectual disability and shows the characteristic facial gestalt comprising prominent forehead, bitemporal narrowing, large appearing eyes with rounded palpebral fissures, depressed nasal root, rounded nasal tip, anteverted nares, short philtrum, tended upper lips, full checks, and low set ears. He has widely spaced teeth and elongated gums. Since the age of 8 months he has fever/infection associated seizures but needs no permanent medication.

In this report we present detailed clinical and molecular data of a new patient with SKDEAS to raise awareness of this clinically recognizable rare intellectual disability syndrome.

A. Busche: None. M. Vockel: None. A. Bohring: None.

\section{E-P08.36}

Two families with inherited protein truncating CHD3 variants suggest reduced penetrance in Snijders BlokCampeau syndrome

J. van der Spek ${ }^{l}$, J. den Hoed ${ }^{2}$, L. Snijders Blok ${ }^{1,2,3}$, C. W. Ockeloen ${ }^{l}$, M. E. H. Simon ${ }^{4}$, L. E. L. M. Vissers ${ }^{1,3}$, S. E. Fisher ${ }^{2,3}$, T. Kleefstra ${ }^{1,3}$

${ }^{I}$ Department of Human Genetics, Radboud University Medical Center, Nijmegen, Netherlands, ${ }^{2}$ Language and Genetics, Max Planck Institute for Psycholinguistics, Nijmegen, Netherlands, ${ }^{3}$ Donders Institute for Brain, Cognition and Behaviour, Nijmegen, Netherlands, ${ }^{4}$ Department of Medical Genetics, University Medical Center Utrecht, Utrecht, Netherlands

Chromodomain Helicase DNA-binding 3 (CHD3) is a chromatin remodeling ATPase which functions as an important subunit of the nucleosome remodeling and deacetylase (NuRD) complex. De novo mutations in CHD3 result in Snijders Blok-Campeau syndrome (\#MIM618205), characterized by global developmental delays and/or intellectual disability, speech delay, macrocephaly, hypotonia and facial dysmorphism. The vast majority ( 95\%) of pathogenic mutations reported so far are missense mutations. Interestingly, CHD3 is predicted to be extremely intolerant for loss of function variance. Non-penetrance and/ or variable expressivity has not been reported. Here we describe two cases with seemingly pathogenic, but inherited, protein truncating variants in $C H D 3$.

In a 4-year-old girl with global developmental delay, autism spectrum disorder, severe feeding difficulties, and facial dysmorphisms, fitting with the spectrum of Snijders Blok-Campeau syndrome, whole exome sequencing revealed a nonsense variant, p.(W1217*), in the CHD3 helicase domain. The variant was inherited from her healthy mother and grandmother.

In a 3-year-old boy with developmental delays, predominated by speech delay, another maternally inherited nonsense variant, p.(S1443*), was found. Facially, he resembled the gestalt previously described for Snijders Blok-Campeau syndrome. His mother, in whom the variant 
was de novo, had macrocephaly and was otherwise unaffected.

These two cases exemplify that protein truncating variants in CHD3 may result in reduced penetrance and/or variable expressivity. We are currently collecting more families with inherited $C H D 3$ variants to enhance our understanding of disease penetrance in Snijders Blok-Campeau syndrome. Additionally, we will perform functional studies of these CHD3 variants to increase our insight in the underlying pathogenic mechanisms.

J. van der Spek: None. J. den Hoed: None. L. Snijders Blok: None. C.W. Ockeloen: None. M.E.H. Simon: None. L.E.L.M. Vissers: None. S.E. Fisher: None. T. Kleefstra: None.

\section{E-P08.37}

\section{Snyder-Robinson syndrome with notable intrafamilial} clinical variability detected by WES and PEDIA

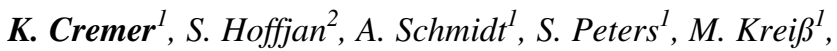
E. Mangold ${ }^{l}$, M. Mücke ${ }^{3}, H$. Hundertmark ${ }^{l}, F$. Brand ${ }^{4}, A$. Heimbach $^{1}$, A. Knaus ${ }^{4}$, A. Buness ${ }^{5}$, S. Sivalingam ${ }^{5}$, P. M. Krawitz $^{4}$, H. Engels ${ }^{1}$

${ }^{1}$ Institute of Human Genetics, University of Bonn, School of Medicine \& University Hospital Bonn, Bonn, Germany, ${ }^{2}$ Institute of Human Genetics, Ruhr-University Bochum, Bochum, Germany, ${ }^{3}$ Center for Rare Diseases Bonn (ZSEB), University Hospital Bonn, Bonn, Germany, ${ }^{4}$ Institute for Genomic Statistics and Bioinformatics, School of Medicine \& University Hospital Bonn, Bonn, Germany, ${ }^{5}$ Core Unit for Bioinformatics Data Analysis, University of Bonn, School of Medicine \& University Hospital Bonn, Bonn, Germany

Pathogenic variants in the spermine synthase (SMS) gene cause the X-linked recessive Snyder-Robinson syndrome (SRS, MIM \#309583), which is characterized by developmental delay / intellectual disability (DD / ID), speech abnormalities, muscle and bone abnormalities, growth retardation, epilepsy, and facial dysmorphism. We report on two brothers with non-specific DD and muscular hypotonia. The older, nine-year-old brother showed motor developmental delay, speech abnormalities, intelligence in the lower average range (total IQ 89), asthenic build with a borderline tall stature, long fingers and toes, hyperextensible joints, and mild facial dysmorphism. The younger, twoyear-old brother showed motor and speech developmental delay and a similar facial gestalt. Family history comprised a cousin (son of the mother's sister) with global developmental delay (total IQ 50), muscular hypotonia and EEG abnormalities. An X-linked ID syndrome was suspected. Whole exome sequencing (WES) of both brothers was performed within the TRANSLATE-NAMSE care project. The resulting variants were prioritized using phenotype and image data (PEDIA / Prioritization of Exome Data by Image Analysis). A probably pathogenic hemizygous variant c.410A>G;p.(Asp137Gly) in the SMS gene was detected in both brothers and subsequently also in the affected cousin. The mothers are heterozygous carriers of the SMS variant. We emphasize the marked intrafamilial variability of intelligence from low normal to mild / moderate ID of this rare and possibly recognizable X-linked DD / ID syndrome.

K. Cremer: None. S. Hoffjan: None. A. Schmidt: None. S. Peters: None. M. Kreiß: None. E. Mangold: None. M. Mücke: None. H. Hundertmark: None. F. Brand: None. A. Heimbach: None. A. Knaus: None. A. Buness: None. S. Sivalingam: None. P.M. Krawitz: None. H. Engels: None.

\section{E-P08.39}

X-linked TAF1 intellectual disability - decoding a new neurodevelopmental entity in the genomic era

\section{R. Gouveia, O. Moldovan, A. B. Sousa}

Serviço de Genética, Hospital de Santa Maria, Centro Hospitalar e Universitário Lisboa Norte, Centro Académico de Medicina de Lisboa, Lisboa, Portugal

Introduction: $T A F 1$ is a subunit of the transcription factor IID complex important in embryogenesis and neuronal development. Recently, X-linked mental retardation 33 syndrome (MRXS33; MIM\#300966) caused by pathogenic variants in $T A F 1$ was described, featuring intellectual disability (ID), hypotonia, neurological abnormalities, and dysmorphic features. To our knowledge, 36 missense and a splice site pathogenic variants have been reported so far.

Case Presentation: A 10-year-old boy with developmental delay was referred for genetic evaluation. He was the only child of non-consanguineous parents with no relevant family history. Atrial septal defect, restrictive muscle ventricle septal defect, mild Ebstein malformation, and axial hypotonia, were documented in the neonatal period. He evolved with moderate to severe ID, limited speech (barely 20 words), hypertonic lower limbs, ataxic gait, midline stereotypies, self-injurious behavior, and severe strabismus (surgically corrected). $\mathrm{He}$ had no autonomy for daily activities. Cerebral MRI was normal. On physical examination, he presented slight facial asymmetry, downslanted palpebral fissures, sunken eyes, bulbous nose, downturned corners of the mouth, micrognathia, and deep plantar creases. Karyotype, array-CGH, and metabolic investigation were normal.

Results: Whole exome sequencing was performed after extensive investigation, revealing a hemizygous TAF1 
variant: c. $412+4 \mathrm{~A}>\mathrm{G}$, affecting splicing. Maternal testing confirmed it was de novo.

Conclusions: This variant, which had not been previously reported, was classified as probably pathogenic. According to the literature, this is the second pathogenic splice site variant described in TAF1. Our case shares common features with previously described patients, but there is wide clinical variability making WES/WGS best suited to reach a diagnosis.

R. Gouveia: None. O. Moldovan: None. A.B. Sousa: None.

\section{E-P08.40}

Analysis of the effect of a novel splice site variant in TGFBR2 on MRNA level

G. Petraitytè $\dot{;}^{l}$, E. Siavrienè ${ }^{l}$, V. Mikštiene $\dot{e}^{1}$, R. Norvilas ${ }^{2}, B$. Burnytè $^{l}$, A. Utkus ${ }^{l}$, E. Preikšaitienè $\dot{e}^{l}$

${ }^{I}$ Department of Human and Medical Genetics, Institute of Biomedical Sciences, Faculty of Medicine, Vilnius University, Vilnius, Lithuania, ${ }^{2}$ Hematology, Oncology and Transfusion Medicine Center, Vilnius University Hospital Santaros Klinikos, Vilnius, Lithuania

Introduction: TGF- $\beta$ signaling pathway is a critical molecular pathway in a regulation of cell growth, development, and differentiation. One of the receptors of this pathway is encoded by TGFBR2 (MIM\#190182) gene. Heterozygous pathogenic variants in TGFBR2 are the cause of LoeysDietz syndrome 2 (LDS2; MIM\#610168), a disorder which mainly affects the connective tissue. Here we report a novel splice site variant in TGFBR2 gene and present its effect on mRNA level.

Materials and methods: DNA of the proband was analyzed by next generation sequencing gene panel technology. In order to elucidate the pathogenicity of the identified splice site variant in the TGFBR2 gene, total blood mRNA of the proband was isolated, template cDNA was synthesized and quantitative PCR using TaqMan gene expression assay designed for TGFBR2 gene was performed.

Results: $T G F B R 2$ gene acceptor splice site variant NC_000003.12(NM_001024847.2):c.1600-2A>G was detected in proband's DNA. Using different bioinformatics tools, this variant was predicted to be pathogenic and affecting mRNA splicing. Quantitative expression analysis of several TGFBR2 exons revealed that c. 1600-2A $>\mathrm{G}$ causes loss of 5' part of exon 8 - the last exon of analyzed transcript, presumably leading to a frameshift and a loss of part of Ser/Thr kinase domain and C-terminus of TGFBR2 protein.
Conclusions: According to the proposed analysis results, splice site variant NC_000003.12(NM_001024847.2): c. $1600-2 \mathrm{~A}>\mathrm{G}$ in $T G F B R 2$ gene disrupts mRNA splicing leading to a truncated protein and causing LDS2.

The work was funded by the Research Council of Lithuania (No. S-MIP-17-19/LSS-150000-1179).

Keywords: TGFBR2, Loeys-Dietz syndrome 2, qPCR

G. Petraityte;;: None. E. Siavrienė: None. V. Mikštienè: None. R. Norvilas: None. B. Burnytė: None. A. Utkus: None. E. Preikšaitienė: None.

\section{E-P08.41}

A novel de novo truncating TRIM8 mutation associated with intellectual disability and renal failure.

M. A. McClatchey ${ }^{\text {, }}$ Z. D. du Toit ${ }^{2}$, R. Vaughan ${ }^{1}$, S. D. Whatley ${ }^{3}$ S. Martins ${ }^{3}$, S. Hegde ${ }^{4}$, J. te Water Naude , D. H. Thomas ${ }^{6}$, D. F. Griffiths ${ }^{6}$, Genomics England Research Consortium, A. J. Clarke $^{1,3}$, A. E. Fry ${ }^{1,3}$

${ }^{1}$ Division of Cancer and Genetics, Cardiff University, Cardiff, United Kingdom, ${ }^{2}$ Department of General Medicine, Glangwili General Hospital, Carmarthen, United Kingdom, ${ }^{3}$ All Wales Medical Genomics Service, Cardiff, United Kingdom, ${ }^{4}$ Children's Kidney Centre, University Hospital of Wales, Cardiff, United Kingdom, ${ }^{5}$ Paediatric Neurology Service, University Hospital of Wales, Cardiff, United Kingdom, ${ }^{6}$ Department of Cellular Pathology, University Hospital of Wales, Cardiff, United Kingdom

Introduction: Truncating mutations in the C-terminus of the TRIM8 protein have been reported in patients with severe developmental delay, intellectual disability and epilepsy. Only six previous patients have been reported (see Assoum et al PMID: 30244534). Three of the previous patients were noted to have proteinuria and one (a patient with epileptic encephalopathy) was diagnosed with nephrotic syndrome.

Case Presentation: We describe an 8-year-old boy with mild intellectual disability who was found to have proteinuria at 2 years of age. Renal biopsy findings were suggestive of focal segmental glomerulosclerosis. The proteinuria persisted and the patient's kidney function declined despite treatment. He had a renal transplant at 7 years of age. Prior to the transplant he had three generalized seizures which were controlled with sodium valproate. Triobased whole genome sequencing as part of the 100,000 Genomes Project found a de novo heterozygous frameshift mutation in TRIM8 (NM_030912.2) c.1198_1220del, p. (Tyr400ArgfsTer2).

Conclusion: The experience of this patient is further evidence that TRIM8 truncating mutations cause both neurological and renal features. This case also suggests 
the spectrum of disease associated with TRIM8 mutations may be wider than previously thought - with the potential for more severe kidney problems but milder intellectual disability. We highlight the need for proteinuria screening in patients with TRIM8 mutations.

M.A. McClatchey: None. Z.D. du Toit: None. R. Vaughan: None. S.D. Whatley: None. S. Martins: None. S. Hegde: None. J. te Water Naude: None. D.H. Thomas: None. D.F. Griffiths: None. A.J. Clarke: None. A.E. Fry: None.

\section{E-P08.42}

A recurrent $Z C 4 H 2$ gene mutation associated with diverse neurological symptoms in a patient with Wieacker-Wolff syndrome

G. Büki ${ }^{1,2}, J . Z_{i m a}^{l}$, B. Galik ${ }^{2}$, A. Gyenesei ${ }^{2}$, K. Hadzsiev ${ }^{1,2}$, B. Melegh ${ }^{1,2}$, J. Bene ${ }^{1,2}$

${ }^{1}$ University of Pecs, Medical School, Clinical Center, Department of Medical Genetics, Pecs, Hungary, Pecs, Hungary, ${ }^{2}$ Szentagothai Research Center, University of Pecs, Pecs, Hungary, Pecs, Hungary

Introduction: Wieacker-Wolff syndrome is an X-linked recessive neurodevelopmental disorder presenting e.g. intellectual disability, developmental delay, characteristic facial features and contractures of the feet. Relatively few cases have been reported so far with high clinical heterogeneity, especially amongst heterozygous females. The disease is caused by mutations in the $\mathrm{ZC} 4 \mathrm{H} 2$ gene, altering a zinc finger domain-containing protein with four cysteine and two histidine residues.

Materials and methods: Whole-genome sequencing was performed in a 5-year-old male patient to identify the disease-causing mutation. Libraries were prepared using TruSeq ${ }^{\mathrm{TM}}$ DNA PCR-Free HT Library Prep Kit (Illumina) and sequenced on NovaSeq 6000 platform (Illumina) with $150 \mathrm{bp}$ paired-end chemistry. The detected mutation was validated by Sanger sequencing.

Results: A recurrent hemizygous missense mutation (c.593 G>A) in $Z C 4 H 2$ gene was discovered in our patient and not in his parents. This mutation leads to an arginineglutamine amino acid change and so far it was found only in one large family. However, our patient shares common hallmarks of the syndrome with the previously reported cases, the unique feature is that he does not present seizure and he has a severe speech delay.

Discussion: Intra- and interfamiliar phenotypic variability is a well-known phenomenon in several Mendelian disorders. Among patients with Wieacker-Wolff syndrome only one recurrent mutation was reported in the $\mathrm{ZC} 4 \mathrm{H} 2$ gene until now. Here we report another recurrent $\mathrm{ZC} 4 \mathrm{H} 2$ gene mutation with diverse neurologic features. Our case supports the interfamiliar variability of the same mutation in this gene.

G. Büki: None. J. Zima: None. B. Galik: None. A. Gyenesei: None. K. Hadzsiev: None. B. Melegh: None. J. Bene: None.

\section{E-P09 Neurogenetic and Psychiatric Disorders}

\section{E-P09.02}

Probably digenic Aicardi-Goutieres syndrome caused by heterozygous $A D A R$-and RNASEH $2 B$-mutations- an extended case report

\section{Hempel ${ }^{l}$, A. Bley ${ }^{l}$, T. Bierhals ${ }^{l}$, K. Radig ${ }^{l}$,} M. Lee-Kirsch ${ }^{2}$, C. Kubisch

${ }^{1}$ University Medical Center Hamburg-Eppendorf, Hamburg, Germany, ${ }^{2}$ Technical University Dresden, Dresden, Germany

Introduction: Aicardi-Goutières syndrome (AGS) is an inflammatory disease associated with a broad phenotypic spectrum although an infantile sub-acute encephalopathy represents the classical manifestation. Mutations in seven genes have been identified causing monogenic AGS: TREX1, ADAR, RNASEH2A, RNASEH2B, RNASEH2C, $S A M H D 1, I F 1 H 1$. The majority of patients carry biallelic mutations, however for specific variants in ADAR, TREXI and $I F 1 H I$ a dominant effect has been described.

Case report: We report on a boy presenting with muscular hypotonia and global developmental delay at the age of 6 months, who further developed slow regression, secondary microcephaly and movement abnormalities. A brain MRI revealed a leukencephalopathy with severe hypomyelination of cerebral white matter. Blood interferon signature showed the characteristic pattern of an interferonopathy type 1 .

Genetic results: Whole exome sequencing in the index and his healthy unrelated parents identified a maternally inherited recurrent mutation in RNASEH2B (p.Ala177Thr) and a paternally inherited very likely pathogenic variant in ADAR (p.Arg892Cys). Neither a second coding variant in these two genes nor other variants explaining the phenotype could be detected. Genome-wide CNV analysis did not show a relevant $\mathrm{CNV}$, in particular not in AGS-associated genes. RNA analysis from skin fibroblasts verified both variants in a heterozygous state but could not detect a second aberration affecting the RNASEH $2 B$ or $A D A R$ transcripts.

Discussion: The patient reported here is affected by classical AGS. Extended genetic analysis revealed combined heterozygous mutations in RNASEH $2 B$ and $A D A R$ as the most probably cause of disease strongly suggesting a digenic inheritance of AGS in this pedigree. 
M. Hempel: None. A. Bley: None. T. Bierhals: None. K. Radig: None. M. Lee-Kirsch: None. C. Kubisch: None.

\section{E-P09.03}

The connection of COMT and receptor DRD2 polymorphisms with alcohol craving and hunger in people treated due alcohol dependence

D. Czarnecki ${ }^{1}$, M. Ziótkowski ${ }^{1}$, A. Dtugosz ${ }^{2}$, J. Feldheim $^{2}$, N. Waszkiewicz ${ }^{3}$, A. Kułak-Bejda ${ }^{3}$, M. Gorzkiewicz ${ }^{4}$, J. Budzyński ${ }^{5}$,J. Chodkiewicz ${ }^{6}$, A. Junkiert-Czarnecka ${ }^{7}$

${ }^{1}$ Nicolaus Copernicus University, Department of Psychiatric Nursing, Torun, Poland, ${ }^{2}$ University of Technology and Life Sciences, Faculty of Chemical Technology and Engineering, Bydgoszcz, Poland, ${ }^{3}$ Medical University of Biatystok, Department of Psychiatry, Choroszcz, Poland, ${ }^{4}$ Nicolaus Copernicus University, Department of Molecular Genetics and Justice, Torun, Poland, ${ }^{5}$ Nicolaus Copernicus University, Department of Vascular and Internal Diseases, Torun, Poland, ${ }^{6}$ University of Lodz, Institute of Psychology, Department of Health Psychology, Eódz, Poland, ${ }^{7}$ Nicolaus Copernicus University, Department of Clinical Genetics, Torun, Poland

Introduction: In Poland about $25 \%$ patients maintains abstinence, after one year from finished of therapy. So it's important, to identify the predictors of relapse of alcohol drinking. To the predictors belong among others alcohol craving, which in some patients is the strongly connected with hunger before meal. It is consider that polymorphisms of COMT and DRD2 genes are associated with increase of alcohol drinking. Aim of study was evaluation of connection, feeling of alcohol craving and hunger in patients with alcohol dependence with polymorphism of DRD2 (c. $2137 \mathrm{G}>\mathrm{A}$ ) and COMT (c. 472G>A).

Material and Methods: Studied 184 patients during hospitalization due to alcohol dependence and 99 people with control group. In study, used the scales: PACS, scale of hunger before meals and also molecular testing of COMT and DRD2 polymorphism.

Results: Patients without COMT polymorphism, but with polymorphism of DRD2 (genotype G/A) felt high values of alcohol craving, which did not decrease during 4 weeks of therapy ( 7.18 vs. $7.00 ; \mathrm{p}>0.05)$. At the same time, patients felt strongly hunger, which did not decrease during 4 weeks of therapy (13 vs. 11; $\mathrm{p}>0.05$ ). The observation, suggest, that it can be extracted, based on the tested polymorphism, group of patients who feel chronic and co-existing alcohol craving and hunger, and this patients have got risk of relapse alcohol drinking or nutrition disorders.

Conclusions: The polymorphism of DRD2 can be connected with the strongly alcohol craving and hunger.
The investigation was financed by grant of Poland's National Health Program for 2016-2020 (no. 74/44/3.4.3/ 18/DEA).

D. Czarnecki: None. M. Ziółkowski: None. A. Długosz: None. J. Feldheim: None. N. Waszkiewicz: None. A. Kułak-Bejda: None. M. Gorzkiewicz: None. J. Budzyński: None. J. Chodkiewicz: None. A. Junkiert-Czarnecka: None.

\section{E-P09.04}

Case report: Alexander Disease in a 12-month-old male infant with epilepsy and global retardation

\section{N. Baba, K. Burghardt, I. Schreyer, C. A. Hübner}

Institute of Human Genetics, University Hospital Jena, Jena, Germany

We report on a 12-month-old male infant of healthy unrelated German parents who was presented in our genetic counseling center in September 2019 with global retardation. One month before, he had two focal clonic seizures predominantly on the left side of the body, which led to hospitalization. The family history revealed no epilepsy or other relevant neurological or genetic diseases. An MRI scan of the brain revealed a severe myelination defect with white matter abnormalities. Genetic diagnostic workup included: cytogenetic analysis, array-CGH and NGS-based panel diagnostics for familial focal epilepsies, extended regarding GFAP. NGS identified a likely pathogenic mutation in GFAP (c.235C $>$ G (p.Arg79Gly) heterozygous). To our knowledge this specific mutation has been reported in only one patient suffering from Alexander disease (AxD) (Gorospe et al., 2002). AxD is a very rare neurodegenerative disease which belongs to the leukodystrophies. Patients with AxD have unspecific symptoms like progressive motor and intellectual retardation, developmental regression and multiple other neurological symptoms including seizures. In most cases gain-of-function mutations in GFAP encoding the glial fibrillary acidic protein underlie this disorder, which result in the accumulation of the variant protein in astrocytes, then called Rosenthal fibers. The exact cellular pathogenesis remains unknown. Only about 500 cases of $\mathrm{AxD}$ have been reported worldwide. Survival time depends on the age at onset of $\mathrm{AxD}$, a median of 14 years was found in a larger cohort (Prust et al., 2011). AxD should be considered differential diagnosis of global developmental retardation, seizures and white matter abnormalities.

N. Baba: None. K. Burghardt: None. I. Schreyer: None. C.A. Hübner: None. 


\section{E-P09.07}

Copy number variants in autism spectrum disorders patients

S. M. Papuc ${ }^{l}$, A. Erbescu ${ }^{l}$, C. Iliescu ${ }^{2,3}$, M. Dobre ${ }^{l}$,

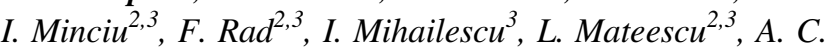
Tutulan-Cunita $^{1,4}$, R. Grozavesc ${ }^{2,3}$, E. Andrei $^{3}$, B. Budisteanu ${ }^{3}$, I. Focsa ${ }^{1,2}$, F. Linca ${ }^{3}$, D. Ioana ${ }^{3}$, G. Gaina ${ }^{1}$, L. Albulescu ${ }^{1}$, I. Dobrescu ${ }^{2,3}$, M. Budisteanu ${ }^{1,3,5}$, A. Arghir ${ }^{1}$

${ }^{1}$ Victor Babes National Institute of Pathology, Bucharest, Romania, ${ }^{2}$ Carol Davila University of Medicine and Pharmacy, Bucharest, Romania, ${ }^{3}$ Prof. Dr. Alex. Obregia Clinical Hospital of Psychiatry, Department of Pediatric Neurology, Bucharest, Romania, ${ }^{4}$ Cytogenomic Medical Laboratory, Bucharest, Romania, ${ }^{5}$ Titu Maiorescu University, Faculty of Medicine, Bucharest, Romania

Copy-number variants (CNVs) have been shown to be involved in etiology of neurodevelopmental disorders like autism spectrum disorders (ASDs), intellectual disability (ID), and other psychiatric disorders. Chromosomal microarray analyses (CMA) has offered a high detection rate of small and large CNVs associated with ASD, and led to discovery of new autism genes. We report on the results of chromosomal CMA investigation in a group of ASDs patients. Eighty patients were referred to our laboratory for genetic testing with ASDs as part of a broad phenotype including ID or other clinical features. A complete clinical evaluation was performed with focus on psychiatric examination and psychological evaluation with specific ASDs tests (ADOS, ADI-R). Array-CGH completed with karyotyping, FISH and qPCR tests were performed. A total of 21 pathological CNVs (14 deletions and 7 duplications), with a median length of $3.7 \mathrm{Mb}$ were detected in 17 patients. The involved genomic regions overlap 550 OMIM genes. Besides well-described syndromic regions associated with ASDs (e.g. deletions of 1q21.1, 22q13.3, 22q11.2, MBD5; Xq28 duplication), other genomic regions, rarely reported in patients with ASDs were identified (deletion of 8p11.2p21.2, 9q34.1, 9p13, duplication of 7p22.2p22.1, CHRNA7); thus having the potential to reveal new ASD genes. Our data illustrates the utility of array-CGH in the investigation of patients with ASDs, specifically in the context of complex phenotypes. Acknowledgment: The research leading to these results has received funding from the EEA Grant 2014-2021, under the project contract No 6/ 2019, and MRI Projects PN 92.033.02.03 and PN 16.22.05.01.

S.M. Papuc: None. A. Erbescu: None. C. Iliescu: None. M. Dobre: None. I. Minciu: None. F. Rad: None. I. Mihailescu: None. L. Mateescu: None. A.C. TutulanCunita: None. R. Grozavescu: None. E. Andrei: None.
B. Budisteanu: None. I. Focsa: None. F. Linca: None. D. Ioana: None. G. Gaina: None. L. Albulescu: None. I. Dobrescu: None. M. Budisteanu: None. A. Arghir: None.

\section{E-P09.09}

A new case of beta-propeller protein-associated neurodegeneration (BPAN) in a 7-year old male with a novel mosaic WDR45 mutation

\section{H. Wang, G. Fteeh}

Loma Linda University, School of Medicine, LOMA LINDA, CA, United States

Introduction: Beta-propeller protein-associated neurodegeneration (BPAN) is a neurodegeneration with brain iron accumulation disorder. BPAN is very rare, with few than 60 cases reported to date in which the vast majority of cases are female and sporadic, resulting from a de novo mutation in the WDR45 gene located at Xp11.23. Here we report a new case of BPAN in a male.

Case Description: This a 7 years old adoptive boy with history of autism, developmental delay, and seizures. He was not able to walk until age of 4 and nonverbal. He was diagnosed with Lennox Gastaut syndrome. EEG (during the sleep) showed frequent to abundant high to very high voltage sharp, spike and slow wave discharges present in right and left hemisphere. The brain MRI indicated prominent mineralization of the globus pallidi and substantia nigra considering iron accumulation or mitochondrial disorder.

Result: The initial workup of chromosome microarray was negative, the subsequent brain-iron accumulation gene panel reported as WDR45, X-linked, c.577C $>\mathrm{T}$ (p. Gln193*), zygosity (indeterminate, $46 \%$ allele fraction), pathogenic.

Discussion/Conclusion: BPAN is phenotypically similar in both male and female which is characterized by clustered spasm, hypsarrhythmia patterns on EEG and developmental delay or regress. The similarity in both genders was explained by somatic mosaicism in surviving males and germline or somatic mutations in females, as well as skewing of $\mathrm{X}$ chromosome inactivation. Only a few male cases were reported and this is a new male case with a novel mosaic mutation and presenting with characteristic clinical features.

H. Wang: None. G. Fteeh: None.

\section{E-P09.10}

New mendelian gene for bipolar disorder. 
M. F. Rossi ${ }^{1,2}$, D. Lopergolo ${ }^{1,2}$, M. A. Trusso ${ }^{3}$, E. Benetti, C. Fallerini ${ }^{1}$, S. Furini ${ }^{4}$, A. Goracci ${ }^{3,5}$, A. Fagiolini ${ }^{3,5}$, ${\text { A. } \text { Renieri }^{1,2}, \boldsymbol{F} \text {. } \text { Mari }^{1,2}}^{1,}$

${ }^{1}$ Medical Genetics, University of Siena, Siena, Italy, ${ }^{2}$ Genetica Medica, Azienda Ospedaliera Universitaria Senese, Siena, Italy, ${ }^{3}$ Department of Molecular Medicine and Development, University of Siena, Siena, Italy, ${ }^{4}$ Department of Medical Biotechnologies, University of Siena, Siena, Italy, ${ }^{5}$ Department of Mental Health; Psychiatry Unit, Azienda Ospedaliera Universitaria Senese, Siena, Italy

Introduction: Bipolar Disorder (BP) is a mental health condition characterized by extreme mood fluctuations that include emotional highs and lows. It affects about $1 \%$ of the world population and its estimated heritability is around $70 \%$, similar to Schizophrenia.

Materials and methods: A large autosomal dominant BP family with eleven affected subjects was included in the study. Mental health of seven family members was ascertained by clinical observation and by the use of specific tests. Exome sequencing was performed in all members. Variants prioritization was performed on the basis of the frequency $(<5 \%)$, evidence of pathogenicity and association with BP.

Results: Three members, the female proband, her mother and his maternal uncle, were diagnosed with BP and four members (two proband's sisters and two proband's male cousins) were categorized as healthy subjects. Exome analysis revealed one single variant that met the above given criteria present in all three affected members and in none of the healthy ones. It is a missense variant, absent in controls (Exome Sequencing Project or 1000 Genomes Project). Multiple lines of computational evidence support a deleterious effect of the variant (CADD 35). The mutated gene encodes for a protein involved in cell motility and extracellular matrix remodeling. A recent study revealed that this protein is downregulated in plasma samples of a large cohort of patients with mood disorder and its expression was reduced in a C. elegans model of Alzheimer's disease.

Conclusions: We were able to identify a gene that likely contributed to BP in our family.

M.F. Rossi: None. D. Lopergolo: None. M.A. Trusso: None. E. Benetti: None. C. Fallerini: None. S. Furini: None. A. Goracci: None. A. Fagiolini: None. A. Renieri: None. F. Mari: None.

\section{E-P09.11}

RASSF 10, a new gene associated to hereditary forms of Bipolar Disorder?
E. Dámaso ${ }^{1}$, A. Culiáñez-Casas ${ }^{2}$, I. Lara-Espejo ${ }^{2}$, M. Castillejo ${ }^{1}$, A. Castillejo $^{2}$, M. García-Escudero ${ }^{2}$, J. Soto $^{2}$

${ }^{1}$ Elche University Hospital - FISABIO, Elche, Spain, ${ }^{2}$ Elche University Hospital, Elche, Spain

Introduction: Bipolar disorder (BD) is a complex psychiatric condition with high heritability. Extended families with high prevalence of $\mathrm{BD}$ provide an opportunity to identify novel risk genes. We present findings from whole exome sequencing (WES) obtained from a Spanish family with high density of BD cases and dominant autosomal pattern of inheritance.

Materials and -Methods: Blood DNA was obtained from 8 family members: five patients diagnosed of BD, another patient with other mental disorder and two healthy individuals. WES was performed using SureSelect-HumanAllExonV6+UTR (AgilentTechnologies) libraries and sequencing on Illumina-NovaSeq6000. Alignment and variant calling was performed by SureCall and variant filtering by a custom pipeline for cohort analyses on Alissa Interpret softwares (AgilentTechnologies).

Results: The total size of captured region was $90 \mathrm{Mb}$. An average of $87 \%$ of the target regions had $30 \mathrm{X}$ coverage or better. The within-family association of rare coding and splice-site variants with predictable loss of function present in cases and absent in controls was investigated. We found an unreported stopgain candidate variant (NM_001080521.3:c.161C>A) at RASSF10 gene, co-segregating with $\mathrm{BD}(\mathrm{p}=0.036)$. RASSF10 plays an important role in regulating embryonic neurogenesis. It is expressed in normal brain tissue and epigenetically inactivated in brain tumors. These evidences might suggest an important role of RASSF10 in normal brain function.

Conclusions: RASSF 10 could be a new candidate gene associated to BD, although further studies are needed to be validated. New associated BD genes could shed light on understanding the molecular basis of mental disorders and defining possible targets for new treatments.

E. Dámaso: None. A. Culiáñez-Casas: None. I. LaraEspejo: None. M. Castillejo: None. A. Castillejo: None. M. García-Escudero: None. J. Soto: None.

\section{E-P09.12}

Brain malformations diagnosis and clinical management in pediatric patients

A. Erbescu ${ }^{1}$, S. M. Papuc ${ }^{1}$, C. Iliescu, ${ }^{2,3}$, C. Burloiu', O. Tarta-Arsene 2,3 D. Barca $^{2,3}$, C. Motoescu ${ }^{2,3}$, I. Minciu $^{2,3}$, N. Butoianu, ${ }^{2,3}$, B. Budisteanu ${ }^{2}$, A. C. Tutulan- 


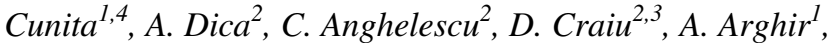
M. Budisteanu ${ }^{1,2,5}$

${ }^{1}$ Victor Babes National Institute of Pathology, Bucharest, Romania, ${ }^{2}$ Prof. Dr. Alex. Obregia Clinical Hospital of Psychiatry, Bucharest, Romania, ${ }^{3}$ Carol Davila University of Medicine and Pharmacy, Bucharest, Romania, ${ }^{4}$ Cytogenomic Medical Laboratory, Bucharest, Romania, ${ }^{5}$ Titu Maiorescu University, Faculty of Medicine, Bucharest, Romania

Brain malformations (BMs) encompass a large spectrum of disorders with various genetic etiologies, anatomic abnormalities, and clinical features. We report on the results of a study focused on diagnosis and clinical management of BMs in a Romanian pediatric population. One hundred and twenty patients with BMs and complex phenotype were selected from those referred for developmental delay and/or epilepsy, between 2010-2019, to our departments. General clinical examination, neurologic, dysmorphologic, psychiatric/psychologic evaluations and brain imaging studies were performed for all the children. Genetic investigations included array based comparative genomic hybridization (Agilent Technologies), and classical and molecular cytogenetics. Gyration anomalies, corpus callosum defects, cerebellar malformations, and heterotopia, isolated or in different combinations, associated with complex clinical presentations were detected in our patient group. Genomic imbalances, 4 deletions and 3 duplications, were detected in 7 patients. Syndromic regions (17p13.3, 1q21.1, 1q43-q44, 22q11.2) were prevalent in our study group; however, some of these regions are known to be clinically heterogeneous, thus our patients might contribute to further delineation of the phenotypic spectrum. In our study group, chromosomal defects were detected with a high incidence in patients with BMs as part of complex phenotypes. Epileptic seizures and/or developmental delay were the most common clinical features. Brain imaging and genetic studies were instrumental in the diagnostic algorithm, with impact on prognosis and genetic counselling of these cases. Acknowledgment: This work was supported by grants of the Romanian National Authority for Scientific Research Innovation, CCCDI-UEFISCDI, Projects number 87/2019 and 88/2019, COFUND-ERANET E-RARE 3HETER-OMICS-2, within PNCDI III

A. Erbescu: None. S.M. Papuc: None. C. Iliescu: None. C. Burloiu: None. O. Tarta- Arsene: None. D. Barca: None. C. Motoescu: None. I. Minciu: None. N. Butoianu: None. B. Budisteanu: None. A.C. Tutulan-Cunita: None. A. Dica: None. C. Anghelescu: None. D. Craiu: None. A. Arghir: None. M. Budisteanu: None.

\section{E-P09.13}

Whole exome sequencing greatly increases the chances for the differential diagnosis in complex clinical phenotypes involving cerebellar ataxia

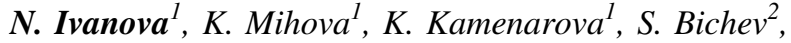 \\ T. Chamova ${ }^{3}$, S. Cherninkova ${ }^{3}$, J. Samuel ${ }^{3}$, \\ V. Guergueltcheva ${ }^{4}$, D. Avdjieva-Tzavella ${ }^{5}$, H. Kathom ${ }^{5}$, \\ A. Savov ${ }^{2}$, I. Kremensky ${ }^{l}$, V. Bojinova ${ }^{6}$, V. Mitev $^{l}$, \\ I. Tournev ${ }^{3,7}$, A. Jordanova ${ }^{1,8}, R$. Kaneva ${ }^{1}$
}

${ }^{1}$ Molecular Medicine Centre, Department of Medical Chemistry and Biochemistry, Medical University - Sofia, Sofia, Bulgaria, ${ }^{2}$ National Genetic Laboratory, University Hospital of Obstetrics and Gynaecology "Maichin dom", Sofia, Bulgaria, ${ }^{3}$ Neurology Clinic, University Hospital "Aleksandrovska”, Sofia, Bulgaria, ${ }^{4}$ Neurology Clinic, University Hospital "Sofiamed”, Sofia, Bulgaria, ${ }^{5}$ Specialized Hospital for Active Treatment Children's Hospital, Prof. Dr. Ivan Mitev", Sofia, Bulgaria, ${ }^{6}$ Neurological Clinic for Children, Multiprofile hospital for active treatment in neurology and psychiatry "St. Naum", Sofia, Bulgaria, ${ }^{7}$ Department of Cognitive Science and Psychology, New Bulgarian University, Sofia, Bulgaria, ${ }^{8}$ Molecular Neurogenomics Group, VIB-UAntwerp Center for Molecular Neurology, University of Antwerp, Antwerp, Belgium

Introduction: Cerebellar ataxias are genetically heterogeneous group of neurodegenerative disorders that could be present with complex and variable clinical phenotypes overlapping other neurological disorders. Most of them are extremely rare and therefore, poorly studied, difficult to diagnose and symptomatically treated. Introduction of nextgeneration sequencing in clinical practice provides a fast and effective decision for precise genetic diagnosis.

Materials and methods: We performed whole exome sequence (WES) to search for disease-causing variants in 13 patients with complex clinical phenotypes involving cerebellar ataxia and one or more of the following symptoms: spasticity, peripheral neuropathy, epilepsy, dysmorphic features, intellectual disability.

Results: WES-data analysis led to the identification of the potentially disease-causing variants in 10 families. They include five novel and four already known pathogenic variants in the SACS, PNPLA6, PRNP, SCN8A, SPG7, CoQ8A and KIF1A genes. After additional genetic analysis and thorough patients' phenotyping we were able to confirm genetic diagnosis in 9 families.

Conclusions: Our study enriches genetic and clinical databases with novel genetic variants and clinical cases of several rare genetic disorders that will help to delineate their molecular mechanisms. It added a new spot to the geographic distribution worldwide of the spastic ataxia of Charlevoix-Saguenay that has been previously limited to a specific geographic region. WES provides a fast and 
effective tool for precise genetic diagnosis that allows to destinguish among variaty of clinical phenotypes sharing common clinical feature of cerebellar ataxia.

Acknowledgements: This study was supported by Grants B02/3/2014 and D01-285/17.12.2019 National Science Fund/Ministry of Education and Science, Bulgaria.

N. Ivanova: None. K. Mihova: None. K. Kamenarova: None. S. Bichev: None. T. Chamova: None. S. Cherninkova: None. J. Samuel: None. V. Guergueltcheva: None. D. Avdjieva-Tzavella: None. H. Kathom: None. A. Savov: None. I. Kremensky: None. V. Bojinova: None. V. Mitev: None. I. Tournev: None. A. Jordanova: None. R. Kaneva: None.

\section{E-P09.14}

A novel mutation of $G D A P 1$ associated with severe form of Charcot-Marie-Tooth disease N. BENSLIMANE ${ }^{l}, F$. Miressi $^{1}$, C. Magdelaine
P.,
P. Faye
, F. Sturtz

${ }^{1}$ EA6309-Neuropathie périphérique et Maintenance Myélinique, Limoges, France, ${ }^{2}$ Laboratoire de Biochimie et Génétique Moléculaire CHU DUPUYTREN, Limoges, France

Introduction: Charcot-Marie-Tooth disease (CMT) is the most common hereditary neuropathy with a prevalence of $1 /$ 2500; also known as sensory-motor neuropathy. There are several forms of CMT which are differentiated by their mode of transmission (recessive, dominant, autosomal or Xlinked), and their electrophysiological aspect (demyelinating or axonal forms). Currently, more than 90 genes are involved in CMT.

Material and Methods: A 47-year-old patient presenting CMT disease was sampled with peripheral blood collected in EDTA tubes after giving her informed consent. Genomic DNA was extracted by standard methods. Next Generation Sequencing (NGS) strategy was performed using a 92 genes custom panel designed for CMT and associated neuropathies diagnosis.

Results: The patient was a 47-year-old woman, presenting a severe demyelinating form of CMT disease. The disease onset was in early childhood with a loss of walking around the age of 11 and since then a wheelchair dependence. Molecular biology analysis highlighted the presence of a new GDAPI mutation, never described to date. This mutation, c. $165 \mathrm{~T}>\mathrm{G}$, located on exon 2, was presented in the homozygous state and leads to the replacement of an aspartic acid in position 55 by a glutamic acid (p.Asp55Glu) and it is predicted pathogenic by two prediction softwares. Splicing involvement is under investigation. Asymptomatic parents presented the GDAPI mutation in the heterozygous state.

Conclusion: The homozygous c.165T $>\mathrm{G}$ detected in GDAPI in our patient is likely responsible for her CMT disease. This result is important in terms of diagnosis for molecular biologists and geneticists.

N. Benslimane: None. F. Miressi: None. C. Magdelaine: None. P. Faye: None. F. Sturtz: None. F. Favreau: None. A. Lia: None.

\section{E-P09.15}

Another case of Coffin-Siris-Syndrome 10 identified by trio exome sequencing.

L. Mulahasanovic ${ }^{l}$, H. Gabriel ${ }^{l}$, S. Biskup ${ }^{l}$, C. Schell-Apacik ${ }^{2}$, C. Bus ${ }^{3}$

${ }^{1}$ Praxis für Humangenetik, Tuebingen, Germany, ${ }^{2}$ Medicover Human Genetics Berlin-Westend MVZ, Berlin, Germany, ${ }^{3}$ Humangenetik Tübingen, Tuebingen, Germany

Coffin-Siris syndrome (CSS) is a rare genetic disorder characterized mainly by developmental delay, intellectual disability and characteristic facial features. Hypoplasia or absence of the fifth finger and toe nails are most common in affected individuals. Until now, ten responsible genes for CSS have been identified. Recently, de novo variants in the gene SOX4 were identified in four patients with typical features of CSS, particularly fifth finger clinodactyly (Zawerton et al., 2019). This new CSS type 10 is characterized by intellectual disability, microcephaly and facial abnormalities. SOX4 belongs to a family of SRYrelated transcription factors, which are playing a key role in multiple developmental pathways. Expression studies showed that $S O X 4$ is highly expressed during neurogenesis. All four pathogenic SOX4 variants identified before were located within the SOX family-specific HMG domain of the protein, which is crucial for DNA-binding. Here we report on a mildly affected female patient carrying the de novo variant c.278T $>\mathrm{A}$; p.Leu93Gln in the SOX4 gene, which is also located in the HMG domain. She could sit at the age of 4 months and started walking on his own with 15 months. She had delayed speech development and learning disability, was restless and agitated, and was diagnosed with ADHD at the age of 8 years. At the age of 21 , she presented with mild dysmorphic features (e.g. long face; deep-set, dysmorphic ears; low palpepral fissures; diffuse inserted eyebrows; wide mouth; long fingers; slight brachydactyly of the fifth fingers; broad big toes) and displayed anxiety and a lack of motivation.

L. Mulahasanovic: None. H. Gabriel: None. S. Biskup: None. C. Schell-Apacik: None. C. Bus: None. 


\section{E-P09.18}

Clinical features of three familial cases of Neuronal Intranuclear Inclusion Disease (NIID)

\author{
H. Furuya, Y. Miyamoto, T. Furushima, S. Ohtsuru, \\ Y. Morita, Y. Osaki
}

\section{Kochi Medical School, Kochi, Japan}

Background: Neuronal intranuclear inclusion disease (NIID)(MIM 603472) is a neurodegenerative disorder characterized by eosinophilic intranuclear inclusions in neuronal cells. Such inclusions are also found in non-neuronal cells. The clinical features and pathological findings in patients with NIID are highly varied. Here, we present three familial NIID cases accompanied with parkinsonism, which is not so common in this disorder.

Objective: Cases: All cases are adult onset NIID with Parkinsonism with dementia and severe psychosis.

Results: In MRI imaging, diffuse cerebral atrophy was observed in all cases. High intensity of the corticomedullary junction in diffusion-weighted imaging (DWI), which is characteristic findings in NIID, was seen in all NIID. In neurological examination, all NIID showed laterality or severely increased Pulmomental Reflex (PMR). Ante-mortem diagnosis was made by identification of intranuclear inclusions with skin biopsy in all NIID. Neuroimaging diagnosis for usual Parkinsonism shows normal with DAT scan and MIBG scintigraphy.

Conclusion: Intranuclear accumulation of abnormal proteins and/or dysfunction of protein degradation might underlie in the pathogenesis of NIID. However, NIID is considered as a heterogeneous disease entity and shows various clinical features. Although dementia is common in NIID, Parkinsonism is also often observed in this disorder. Achknowledgement: We also thank to Dr. Sone J and Prof. Sobue G (Department of Neurology, Nagoya University, Japan) for immune staining of biopsy specimen.

H. Furuya: None. Y. Miyamoto: None. T. Furushima: None. S. Ohtsuru: None. Y. Morita: None. Y. Osaki: None.

\section{E-P09.20}

The Complex Molecular Basis of Dystonias in a Turkish Cohort

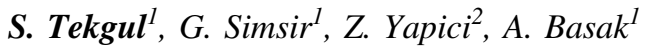 \\ ${ }^{1}$ Koç University, Istanbul, Turkey, ${ }^{2}$ Istanbul University, \\ Istanbul, Turkey
}

Introduction: Dystonias are neurological conditions in which abnormal movements or postures are observed, that are caused by sustained or intermittent muscle contractions. The major feature is over-activity of muscles required for movement. Dystonias can clinically vary in their manifestation and severity affecting all age groups and leading to considerable disability and impaired quality of life. With its high throughput capacity and proportionally low cost, next generation sequencing is now being extensively used in research/diagnostic settings to determine novel genes and mutations associated with dystonias.

Materials and methods: Genomic DNA was isolated from whole blood. Whole exome sequencing (WES), followed by family segregation analysis using Sanger sequencing, was performed on 21 probands with heterogenous dystonia phenotypes. The pathogenicity of the variant was scored by the prediction tools DANN, GERP, SIFT and MutationTaster.

Results: WES revealed 10 dystonia genes with varying frequencies and 12 variants, 4 of which are novel (Table 1). The diagnostic rate is $62 \%$. Table 1 Genes and variants identified in Turkish dystonia cohort

\begin{tabular}{|c|c|c|c|}
\hline $\begin{array}{l}\text { Genes (\# of } \\
\text { families) }\end{array}$ & Mutations & Zygosity & $\begin{array}{l}\text { Novel/ } \\
\text { Reported }\end{array}$ \\
\hline $\mathrm{GCH} 1(\mathrm{n}=3)$ & $\begin{array}{l}\text { c. } 284 \mathrm{C}>\mathrm{T} \text { p.Pro95Leu } \\
\text { c.631_632delAT p. } \\
\text { Met211ValfsTer38 } \\
\text { c.646C }>\text { T p.Arg216Ter }\end{array}$ & $\begin{array}{l}\text { het } \\
\text { het het }\end{array}$ & $\begin{array}{l}\text { Novel } \\
\text { Reported } \\
\text { Reported }\end{array}$ \\
\hline PTS $(n=2)$ & c. $84-3 \mathrm{C}>\mathrm{G}$ & hom & Reported \\
\hline TOR1A $(\mathrm{n}=1)$ & $\begin{array}{l}\text { c.907_909delGAG p. } \\
\text { Glu303del }\end{array}$ & het & Reported \\
\hline $\operatorname{SLC} 2 \mathrm{~A} 1(\mathrm{n}=1)$ & c. $972 \mathrm{G}>$ A p.Ser324Ser & het & Reported \\
\hline $\operatorname{DDC}(n=1)$ & c. $1040 \mathrm{G}>$ A p.Arg347Gln & hom & Reported \\
\hline KMT2B $(n=1)$ & $\begin{array}{l}\text { c.6863delC p. } \\
\text { Pro2289ArgfsTer36 }\end{array}$ & het & Novel \\
\hline $\mathrm{TH}(\mathrm{n}=1)$ & c. $698 \mathrm{G}>$ A p.Arg233His & hom & Reported \\
\hline ATP13A2 $(n=1)$ & $\begin{array}{l}\text { c.1551_1556delCCTCAC } \\
\text { p.Leu518_Thr519del }\end{array}$ & hom & Novel \\
\hline ATP1A3 $(n=1)$ & c. $1838 \mathrm{C}>\mathrm{T}$ p. Thr613Met & het & Reported \\
\hline $\operatorname{KIF} 1 \mathrm{C}(\mathrm{n}=1)$ & c. $2005 \mathrm{C}>\mathrm{T}$ p.Arg669Ter & hom & Novel \\
\hline
\end{tabular}

Conclusions: The 10 genes identified reveal a high locus heterogeneity. Further, ATP13A2 and KIF1C which also give rise to spasticity and ataxias, point to genetic and pathophysiological overlaps between neurological conditions.

S. Tekgul: None. G. Simsir: None. Z. Yapici: None. A. Basak: None.

\section{E-P09.21}

Genetic study of early-onset parkinsonism in Thailand: targeted next-generation sequencing 


\section{P. Phowthongkum}

\section{Faculty of Medicine, Bangkok, Thailand}

Background: Early-onset Parkinson disease (EOPD) is defined by PD with the onset age of less than 50 years. Patients may present predominantly with lower limb dystonia rather than typical resting tremor in late-onset patients which is the challenge in making the diagnosis in younger patients. Many patients apparently are responded dramatically with dopamine replacement but developed early motor complications, in contrast, to consistently respond to the treatment in DOPA Responsive Dystonia (DSD). Molecular diagnosis, therefore, helps in diagnosis EOPD, facilitates genetic counseling and treatment customization.

Methods: A total of 51 patients with early-onset parkinsonism from 49 families referred to Neurogenetics Center at King Chulalongkorn Memorial Hospital. Genetic test was performed with targeted next-generation sequencing panel containing 34 genes causing PD or dystonia.

Results: Eleven patients with EOPD (21.5\%) were found to carry likely pathogenic or pathogenic variants in $G B A$ (5/ 51),PARK2(4/51 from 2 families),PINK1 (2/51 from 2 families). Familial EOPD (2 families) harbored 3 different pathogenic variants in PARK2 (one family with homozygous deletion of exon7 and the other family with one heterozygous deletion of exon 5 and one heterozygous missense variant). They all have onset of less than 30 years.

Conclusions: Our findings add an understanding of the genetic architecture of EOPD in Southeast Asia. Targeted next-generation sequencing is an effective and affordable method for making genetic diagnosis for PD. Heterozygous $G B A$ definitely increases risk of EOPD in the Southeast Asian population. Individual seeking carrier testing includes $G B A$ should be informed about the risk of parents to develop neurological disorders.

P. Phowthongkum: None.

\section{E-P09.23 \\ NGS survey in neurological disorders - focus on variants in HLA class II genes}

\section{F. Shakola, I. Dimova, V. Peycheva, K. Mihova, K. Kamenarova, R. Kaneva}

\section{Molecular Medicine Center, MU Sofia, Sofia, Bulgaria}

Neuroinflammatory mechanisms may be involved in the genesis of epilepsies and autistic spectrum disorders. An NGS study with TrueSightOne gene panel was performed on 30 patients with neurological disorders (including seizures as a main component). In 10 patients pathogenic/ probably pathogenic mutations were discovered. We have performed comprehensive study of HLA gene variants discovered by NGS after lack of pathogenic mutations in patients affected by epilepsy with or without autism/mental retardation and compared with patients with other pathologies (referred as controls). We focused on variants annotated as Variants of Unknown Significance (VUS). We determined the frequency of such variants in DRB1 and DRB5, in cases and controls, as they were the most commonly found in both groups. The frequency varied between $11-15 \%$ in cases and $11-23 \%$ in controls. In addition, we reported variants found only in cases $(5$ variants in DRB5 and 1 in DRB1), only in controls (7 in DRB5 and 10 in DRB1) and both in cases and controls (5 in DRB5 and 6 in DRB1). We found 2 HLA-DMB and 2 HLA-G variants only in cases. This is a pilot study of the involvement of HLA class II gene variants in neurological disorders and it will be followed by the analysis of these individual variants' role.

F. Shakola: None. I. Dimova: None. V. Peycheva: None. K. Mihova: None. K. Kamenarova: None. R. Kaneva: None.

\section{E-P09.27}

Identification of the molecular etiology in early epileptic encephalopathy using whole exome sequencing

\author{
E. Isik ${ }^{1}$, T. Atik ${ }^{1}$, S. Keskin Yilmaz ${ }^{2}$, G. Aktan ${ }^{2}, H$. Onay $^{3}$, S. \\ Gokben $^{2}$, F. Ozkinay ${ }^{1,3}$
}

${ }^{1}$ Subdivision of Pediatric Genetics, Department of Pediatrics, Faculty of Medicine, Ege University, Izmir, Turkey, ${ }^{2}$ Subdivision of Pediatric Neurology, Department of Pediatrics, Faculty of Medicine, Ege University, Izmir, Turkey, ${ }^{3}$ Department of Medical Genetics, Faculty of Medicine, Ege University, Izmir, Turkey

Epileptic encephalopathies (EEs) are a group of devastating disorders caused by epileptic activity; resulting in deterioration in developmental, cognitive and motor functions. The number of genes identified as being responsible for EEs has been increasing rapidly. However, despite a comprehensive molecular analysis, a molecular diagnosis can only be established in $50 \%$ of cases. The aim of this project is to use whole exome sequencing to determine the molecular etiology of EEs in molecularly undiagnosed patients with a pedigree suggestive of an autosomal recessive single gene disease. Three EEs families, having either consanguineous parents of an affected individual and/or having more than one affected offspring were enrolled in the project. Prior to this project, the families had been evaluated using a next generation sequencing panel which included 16 EE genes; however, no molecular diagnosis could be established. In 5 cases from the 3 selected EEs families in our study genetic 
etiology was investigated using WES, with segregation analysis then being performed using Sanger sequencing. All patients in the study group had infantile onset epileptic seizures and severe developmental delay. WES revealed biallelic disease causing mutations in DENDD5A, GRN and $T B C D$ genes in Family 1, Family 2 and Family 3, respectively. In each family, the identified variants associated with the disease were segregated. Reverse phenotyping supported the molecular analysis. In conclusion, this study provides a valuable contribution to the genotype-phenotype relationship by determining rare epilepsy syndromes in a previously undiagnosable patient group. WES provides a useful diagnostic alternative; particularly in consanguineous families.

E. Isik: None. T. Atik: None. S. Keskin Yilmaz: None. G. Aktan: None. H. Onay: None. S. Gokben: None. F. Ozkinay: None.

\section{E-P09.28}

\section{Whole-exome sequencing in familial multiple sclerosis}

L. Torre Fuentes ${ }^{1}$, J. Matías-Guiu Antem ${ }^{2}$, V. Pytel ${ }^{1}$, P. Montero Escribano ${ }^{2}$, L. Hernández Lorenzo ${ }^{l}$, P. Maietta ${ }^{3}$, S. Álvarez ${ }^{3}$, U. Gómez Pinedo ${ }^{1}, J$. Matías-Guiu Guía $^{1,2}$

${ }^{1}$ Institute of Neuroscience, Health Research Institute of San Carlos Clinical Hospital, Madrid, Spain, ${ }^{2}$ Neurology department, San Carlos Clinical Hospital, Madrid, Spain, ${ }^{3}$ NIMGenetics, Madrid, Spain

Introduction: Multiple sclerosis (MS) is an inflammatory autoimmune disease of the central nervous system (CNS) which causes demyelination and axonal damage. MS is considered a polygenic disease where genetic risk factors can be more likely detected in familial MS. The aim of the study is to analyse genetic variations of signalling pathways and genes related to MS.

Materials and methods: Whole-exome sequencing (WES) with Ion Proton technology was performed in 138 individuals from 23 families including at least 2 MS patients. We compared patients with MS, patients with other autoimmune disease and healthy individuals. Signalling pathways and genes related to MS were analysed. Variant filtering and prioritization were performed with an own pipeline using criteria to include relevant and functional variants associated to MS.

Results: In our cohort, some variants matched criteria to be considered as a possibly pathogenic variable. The associated genes (NOD2, NLRP7, CYP24A1, TNFAIP3, TNFRSF 13B, LRP2, CIITA, ACE) were mainly involved in the immune system as well as in vitamin $D$ regulation.
These genes have been previously reported in literature associated to MS.

Conclusions: These findings support the idea that familial MS could present a genetic background. The variants found in this research require further study and could lead to a better understanding of MS.

L. Torre Fuentes: None. J. Matías-Guiu Antem: None. V. Pytel: None. P. Montero Escribano: None. L. Hernández Lorenzo: None. P. Maietta: None. S. Álvarez: None. U. Gómez Pinedo: None. J. Matías-Guiu Guía: None.

\section{E-P09.30 \\ Spontaneous rescue of a $F M R 1$ repeat expansion}

E. Erbs, M. Rasmussen, D. L. Lildballe

Department of Clinical Genetics, Lillebaelt Hospital, Vejle, Denmark

Introduction: Fragile X syndrome (FXS) is usually caused by CGG-repeat expansion in the 5' UTR region of the Fragile X Mental Retardation 1 (FMRI) gene of $>200$ repeats causing methylation of the region. Rare cases are caused by pathogenic variants within the coding region.

Materials and methods: Genetic testing for FXS was requested in a male infant born to a mother known to harbour a FXS full mutation ( $>200$ CGG-repeats). The maternal grandmother was a carrier of a FXS premutation. Analysis of CGG-repeats of the FMRI gene was performed using PCR, RP-PCR, and Sanger sequencing. STR markers were used in linkage analysis.

Results: An unexpected very short PCR fragment was detected in the male infant. Subsequent Sanger sequencing confirmed an 88 bp deletion (c.1-156_1-69del) in hemizygous form. The deletion included all CGGrepeats and flanking sequences. However, the deletion did not include FMR1 exons. Linkage analysis revealed that the allele, which has been expanded in the mother and the maternal grandmother, was passed on to the male infant.

Conclusion: Grønskov et al. previously reported a $\sim 150$ bp deletion of all CGG-repeats and flanking sequences in a normal female patient, who was hemizygous for the FMRI gene due to a large deletion of the other $\mathrm{X}$ chromosome. Western blot showed that the FMR1 protein level was unaffected. This reported deletion comprises the deletion detected in the male infant. Based on this report, we expect our patient to be unaffected.

In conclusion, a male infant was rescued from FXS by a spontaneous deletion.

E. Erbs: None. M. Rasmussen: None. D.L. Lildballe: None. 


\section{E-P09.32}

Genes with variants associated with frontotemporal dementia are enriched in extracellular matrix organization pathways

S. Karachanak-Yankova ${ }^{1,2}$, L. Balabanski ${ }^{1,3}$, D. Serbezov ${ }^{l}$, R. Vazharova ${ }^{3,4}$, O. Antonova ${ }^{l}$, D. Nikolova ${ }^{l}, M$. Mihaylova $^{l}$, R. Staneva ${ }^{l}, V$. Damyanova ${ }^{l}$, M. Ganev $^{l}, V$. Spasova $^{l}$, D. Nesheva ${ }^{l}$, Z. Hammoudeh ${ }^{l}$, S. Hadjidekoval, S. Mehrabian ${ }^{5}$, M. Petrova ${ }^{5}$, L. Traykov ${ }^{5}$, D. Toncheva ${ }^{1,6}$

${ }^{1}$ Department of Medical Genetics, Medical Faculty, Medical University-Sofia, Sofia, Bulgaria, ${ }^{2}$ Department of Genetics, Faculty of Biology, Sofia University "St. Kliment Ohridski", Sofia, Bulgaria, "Gynecology and assisted reproduction hospital "Malinov", Sofia, Bulgaria, ${ }^{4}$ Department of Biology, Medical genetics and Microbiology, Faculty of Medicine, Sofia University "St. Kliment Ohridski”, Sofia, Bulgaria, ${ }^{5}$ Depatment of Neurology, UH "Alexandrovska", Medical University-Sofia, Sofia, Bulgaria, ${ }^{6}$ Bulgarian Academy of Sciences, Sofia, Bulgaria

Introduction: Frontotemporal dementia (FTD) is a genetically and pathologically heterogeneous degenerative disorder, second to Alzheimer's disease as a cause of dementia in patients under 65 years of age. The present case-control study aims to broaden the understanding of the molecular pathogenesis of FTD by whole exome sequencing (WES) of pooled DNA samples.

Materials and methods: The analysis was undertaken on 140 FTD patients grouped in two DNA pools (original and replicate) and a control DNA pool of 100 age-matched healthy subjects. The WES was performed in BGI Genomics at a mean coverage of at least $250 \mathrm{x}$ per sample and the obtained variants were filtered by stringent criteria.

Results: The number of biallelic autosomal variants found in the control DNA pool is 180 692. The original and replicate patient DNA pool contain 18003 and 21555 variants not found in the control pool, respectively. Among these variants 2344 are found in both patient pools, as 175 have less than $1 \%$ frequency in non-Finish Europeans in GnomAD. These variants are located in 174 genes and can be considered as FTD associated. The pathway enrichment analysis of these genes using Reactome software platform showed over-represented pathways mainly in extracellular matrix organization.

Conclusion: The detection of over-represented extracellular matrix organization pathways in FTD contributes to the understanding of the pathogenetic mechanisms underlying neurodegeneration. Acknowledgment: KP-06-N33/5 from 13.12.2019 - National Science Fund of Bulgaria.

S. Karachanak-Yankova: None. L. Balabanski: None. D. Serbezov: None. R. Vazharova: None. O. Antonova: None.
D. Nikolova: None. M. Mihaylova: None. R. Staneva: None. V. Damyanova: None. M. Ganev: None. V. Spasova: None. D. Nesheva: None. Z. Hammoudeh: None. S. Hadjidekova: None. S. Mehrabian: None. M. Petrova: None. L. Traykov: None. D. Toncheva: None.

\section{E-P09.33}

Characterization of the PRNP gene mutations and the genotype-phenotype correlation in Hungarian patients with prion disease

H. Zeke ${ }^{1}$, Á. Palásti ${ }^{1}$, B. A. Fekete ${ }^{I}$, V. Molnár ${ }^{1}, A$. Gál $^{1}, Z$. Grosz $^{1}$, G. G. Kovács ${ }^{2}$, M. J. Molnár ${ }^{l}$

${ }^{I}$ Institute of Genomic Medicine and Rare Disorders, Semmelweis University, Budapest, Hungary, ${ }^{2}$ Department of Laboratory Medicine and Pathobiology, University of Toronto, Toronto, ON, Canada

Introduction: Abnormal accumulation of prion proteins are responsible for several fatal neurodegenerative diseases. $P R N P$ is the only gene in which pathogenic variants are known to cause genetic prion disease. However, several pathogenic mutations of $P R N P$ with heterogeneous type and position were published with disease-causing or modifying effect on other neurodegenerative disorders. In a significant portion, genetic cause can be explained with the most common pathogenic mutation E200K in the PRNP gene.

Materials and methods: Hundred-twenty-six patients were referred (age at referral: $57+13,7$ years) to our institute with clinically suggested prion disease during 2015-2019. Sanger sequencing was applied to analyze the entire coding region of PRNP gene.

Results: Altogether 25 unrelated prion-positive cases were detected during the 4-year observational period. Heterozygous c.598G $>$ A (E200K) variant was detected in $22 / 25$ cases. Besides this most common variant in one case the previously described c.350 $\mathrm{C}>\mathrm{T}(\mathrm{A} 117 \mathrm{~V})$ variant was found. In one family a rare, $168 \mathrm{bp}$ octapeptid-repeat insertion was observed, which is known to be associated with a very slow disease progression. In addition, a novel mutation c. 490 A $>\mathrm{G}$ (R164G) has been also identified which associated to a slow progression as well.

Conclusions: In our cohort $88 \%$ of the genetically confirmed prion disorders were associated to the most common E200K PRNP mutation. The type and location of the alteration of the $P R N P$ gene may have an important role in the determination of the phenotype, which makes the genetic counselling challenging.

Funding: This study was supported by the Hungarian Brain Research Program (KTIA_NAP_ 2017-1.2.1-NKP2017-00002). 
H. Zeke: None. Á. Palásti: None. B.A. Fekete: None. V. Molnár: None. A. Gál: None. Z. Grosz: None. G.G. Kovács: None. M.J. Molnár: None.

\section{E-P09.35}

Autosomal recessive axonal neuropathy with neuromyotonia; mutations in HINT1 are not uncommon in the Greek population

Z. Kontogeorgiou ${ }^{1}$, D. Pandis ${ }^{2}$, C. Kartanou ${ }^{1}$, M. Breza ${ }^{1}$, T. Zambelis ${ }^{2}$, M. Panas ${ }^{1}$, G. Karadima ${ }^{1}$, G. Koutsis ${ }^{l}$

${ }^{1}$ Neurogenetics Unit, 1st Department of Neurology, School of Medicine, Eginition Hospital, National and Kapodistrian University of Athens, Athens, Greece, ${ }^{2}$ Clinical Neurophysiology Unit, 1st Department of Neurology, School of Medicine, Eginition Hospital, National and Kapodistrian University of Athens, Athens, Greece

Autosomal recessive axonal neuropathy with neuromyotonia (ARAN-NM) has been recently shown to result from histidine triad nucleotide-binding protein 1 (HINT1) gene mutations. It accounts for around $10 \%$ of recessive axonal Charcot-Marie-Tooth disease (CMT) in European populations. A single case has been reported to date in the Greek population. We presently report a case-series of 3 Greek patients with ARAN-NM identified during screening of undiagnosed recessive axonal CMT cases with a combination of whole-exome and Sanger sequencing. Patient 1 was a 19 year-old female presenting with walking difficulty at the age of 8 years. She gradually developed lower limb atrophies and at 15 years old was also diagnosed with myoclonic epilepsy. On EMG she showed motor axonal neuropathy with neuromyotonia. She was compound heterozygous for mutations c.110G $>\mathrm{C}$ (p.Arg37Pro) and c.250T>C (p.Cys84Arg) in HINT1. Patient 2 was a 20 yearold woman presenting with walking difficulties at the age of 14 years. She gradually developed foot drop and upper limb distal weakness. On EMG she showed a motor axonal neuropathy with neuromyotonia. She was homozygous for the c.110G $>$ C (p.Arg37Pro) mutation in HINT1. Patient 3 was a 49 year-old male presenting with walking difficulties at the age of 15 years. He gradually developed foot drop and upper limb distal weakness. On EMG he showed a predominantly motor axonal neuropathy with neuromyotonia. He was homozygous for the c.110G $>C$ (p.Arg37Pro) mutation in HINT1. We conclude that HINT1-related CMT may be relatively common in Greece and should be further systematically screened for in appropriately selected populations.

Z. Kontogeorgiou: None. D. Pandis: None. C. Kartanou: None. M. Breza: None. T. Zambelis: None. M. Panas:
None. G. Karadima: B. Research Grant (principal investigator, collaborator or consultant and pending grants as well as grants already received); Modest; Pfizer. F. Consultant/ Advisory Board; Modest; Roche. G. Koutsis: B. Research Grant (principal investigator, collaborator or consultant and pending grants as well as grants already received); Significant; Teva Pharmaceuticals, Genesis Pharma. Other; Significant; Novartis, Genesis Pharma, Sanofi-Genzyme, Teva.

\section{E-P09.38}

Trio whole-exome sequencing confirms $K C N D 2$ de novo missense mutations as a cause of autosomal dominantly inherited severe seizures and developmental delay

\section{U. Zechner, D. Rosenkranz, H. Bolz.}

Senckenberg Centre of Human Genetics, Frankfurt, Germany

Trio whole-exome sequencing was carried out in a female proband with epileptic encephalopathy (delayed myelination, hypoplastic corpus callosum, abnormal EEG) and severe global retardation noticed from the age of 3 months (no psychomotor milestones acquired, muscle hypotonia, irregular eye movements and lack of fixation, stereotypic hyperkinetic arm and leg movements) and her healthy parents. The patient manifested intractable epileptic seizures from the age of 1 year; there was no significant psychomotor development. She died at the age of 21 years. A heterozygous de novo missense variant, c.1210G >C (p.(Val404Leu)), was detected in $K C N D 2$, the gene encoding $\mathrm{Kv} 4.2$, a major pore-forming subunit in somatodendritic subthreshold A-type potassium current (ISA) channels. The variant has not been described in the literature or in disease- and population-specific variant databases. It leads to the exchange of an evolutionarily highly conserved residue in the S6 transmembrane segment which is part of the channel's central pore for ion permeation. All 11 applied in silico prediction algorithms categorized the variant as deleterious. In a previous report on $K C D N 2$ as a disease gene, a monozygotic twin pair with epilepsy and developmental delay carried a heterozygous de novo missense variant affecting the same residue (Lee et al., PMID: 24501278). This p.(Val404Met) mutation (c.1210G >A) was shown to lead to enhanced inactivation of closed Kv4.2 channels and impaired inactivation of open channels (Lin et al., PMID: 29581270). Thus, our finding supports dominant de novo $K C N D 2$ missense variants as a cause of intractable, earlyonset epilepsy with severe global retardation.

U. Zechner: None. D. Rosenkranz: None. H. Bolz: None. 


\section{E-P09.39}

Correlation of genetic analysis and evolution of MRI changes leading to diagnosis of Labrune syndrome

\section{J. Pilch ${ }^{1}$, M. Machnikowska-Sokołowska ${ }^{2}$, M. Rydzanicz ${ }^{3}$, A. Pollak ${ }^{3}$, J. Kosinska ${ }^{3}$, P. Gasperowicz ${ }^{3}$, \\ K. Gruszczynska ${ }^{2}$, E. Emich-Widera ${ }^{1}$, R. Płoski ${ }^{3}$}

${ }^{1}$ Department of Pediatric Neurology, Medical University of Silesia, Katowice, Poland, ${ }^{2}$ Department of Diagnostic Imaging and Interventional Radiology, Department of Radiology and Nuclear Medicine, Medical University of Silesia, Katowice, Poland, ${ }^{3}$ Department of Medical Genetics, Medical University of Warsaw, Warsaw, Poland

Progressive leukoencephalopathy with calcification and cysts was first reported by Labrune in 1996. The pathology underlying the disease is a diffuse cerebral microangiopathy with development of micro-, macrocysts, tumour-like vascular hyperplasia, calcification, glial proliferation, demyelination, necrosis, iron deposition and haemorrhage. Mutations in the SNORD118 gene were associated with Labrune syndrome in 2012. Since then only individual cases have been presented.

We report a normally developing 10-years-old girl suffering from chronic headaches without neurological symptoms. Initially, Aicardi-Goutieres syndrome was suspected. We performed retrospective analysis of three annual brain MRI with MR angiography. In all MRI we found diffuse symmetrical white matter areas of increased signal with U-fibers sparing. Deep nuclei were affected. Cystic areas, enlarging in consecutive examinations were observed in thalami, with mass effect in last MRI. Multiple signals with blooming effect were spread both supra- and infratentorially. Partial peripheral contrast enhancement was seen in supratentorial changes. A suspicion of Labrune syndrome has arose after progression of cysts in degenerated white matter and appearance of calcifications. Complex heterozygous mutations 17:008173448-G $>\mathrm{C}$ and 17:008173570-G $>\mathrm{C}$ in the SNORD118 gene encoding small nuclear RNA were found in patient using whole exome sequencing. Both parents are carriers of identified mutations.

Conclusion: Follow up MRI examinations in normally developing child and correlation with in-depth genetic analysis led to diagnosis of a rare genetic metabolic syndrome. Collaboration between radiologists, geneticist and clinicians was crucial for establishing final diagnosis.

J. Pilch: None. M. Machnikowska-Sokołowska: None. M. Rydzanicz: None. A. Pollak: None. J. Kosińska: None. P. Gasperowicz: None. K. Gruszczynska: None. E. EmichWidera: None. R. Płoski: None.

\section{E-P09.42}

Early onset of complex seizures as a first sign of 16p11.2 deletion syndrome

E. Sukarova-Angelovska, V. Anastasovska, F. Duma, L. Muaremovska, D. Nestoroska, G. Ilieva, M. Pesevska, M. Velkov

Pediatric Clinic, Skopje, Macedonia, The Former Yugoslav Republic of

Background: Recent technologies enabled clarification of some previously undetected causes of intellectual disability and autism spectrum disorders. There are reports of $16 \mathrm{p} 11.2$ deletion in the literature, describing variable clinical presentation in patients. Most of them describe developmental delay, autism and seizures, however phenotypic pattern is undistinguishable and variable. There is still no sufficient clinical data of this $\mathrm{CNV}$ in babies.

Case report: We report on a patient with developmental delay and complex seizures. This is a second child in a family, the pregnancy and delivery was uneventful. Microcephaly, foramen ovale and generalized hypotonia were noticed shortly after birth. At the age of 3 months he developed infantile spasms, followed by profound developmental delay within the next several months. He has microcephaly, early closure of the fontanel, high forehead, bitemporal narrowing, wide nasal root, narrow palpebral features, upper lip notch, short neck, cryptorchidism. Karyotype was normal. aCGH analysis (using Affymetrix ${ }^{\circledR}$ CytoScan $^{\mathrm{TM}} 750 \mathrm{~K}$ Array) showed deletion of $16 \mathrm{p} 11.2$ $(1.430 \mathrm{~kb})$, also deletion on chromosome 14q11.2 (433 kb), both are considered as pathogenic according aDGV database.

Discussion and conclusion: Deletion of the 16p11.2 is mostly described in older children that developed autistic spectrum of disorder, ADHD, etc. Wide clinical variability is described between patients, mostly due to the variations on size and breakpoint of the deleted region. There are some inconsistencies in phenotype described in literature, however this could be a part of a changing phenotype with age. Since the baby had two pathogenic microdeletions, it is difficult to distinguish between two phenotypes.

E. Sukarova-Angelovska: None. V. Anastasovska: None. F. Duma: None. L. Muaremovska: None. D. Nestoroska: None. G. Ilieva: None. M. Pesevska: None. M. Velkov: None.

\section{E-P09.45}

Whole Exome Sequencing of consanguineous families of clinically diagnosed with Neurodevelopmental Disorders 
B. Turkgenc $^{l}$, K. Yararbas $^{2,3}$, H. Keskin Karakoyun ${ }^{1,4}$, A. Yesilyurt ${ }^{1}$, A. Gezdirici ${ }^{5}$, B. Tatll $^{6,7}$, N. H. Akcakaya ${ }^{8}, N$. E. Hacifazlioglu ${ }^{9}$, O. Coskun ${ }^{7}$, G. Yesil ${ }^{10}$, B. Ekicili, C. Ylldirım ${ }^{12}$, H. Onal ${ }^{13}$, G. Turanll ${ }^{14}$, Y. Alanay ${ }^{15,16}$

${ }^{1}$ Acibadem Genetic Diagnostic Center, Istanbul, Turkey, ${ }^{2}$ Acibadem Genetic Diagnosis Center, Istanbul, Turkey, ${ }^{3}$ Department of Medical Genetics, Faculty of Medicine, Acibadem University, Istanbul, Turkey, Istanbul, Turkey, ${ }^{4}$ Department of Biochemistry and Molecular Biology, Acibadem University, Istanbul, Turkey, ${ }^{5}$ Kanuni Sultan Süleyman Training and Research Hospital, University of Istanbul Health Sciences, Istanbul, Turkey, ${ }^{6}$ Pediatric Extreme NeuroTherapeutics Center, Istanbul, Turkey, ${ }^{7}$ Department of Pediatric Neurology, Faculty of Medicine, Istanbul University, Istanbul, Turkey, ${ }^{8}$ Department of Genetics, Aziz Sancar Institute of Experimental Medicine, Istanbul University, Istanbul, Turkey, ${ }^{9}$ Zeynep Kamil Women's and Children's Disease Training and Research Hospital, Istanbul, Turkey, ${ }^{10}$ Department of Medical Genetics, Faculty of Medicine, Bezmialem Foundation University, Istanbul, Turkey, ${ }^{11}$ Pediatric Neurology, Vital Fulya, Istanbul, Turkey, ${ }^{12}$ Department of Pediatric Neurology, Acibadem Altunizade Hospital, Istanbul, Turkey, ${ }^{13}$ Department of Pediatric Endocrine and Metabolism, Istanbul Kanuni Sultan Süleyman Training and Research Hospital, Istanbul, Turkey, ${ }^{14}$ Department of Pediatric Neurology, Faculty of Medicine, Medipol University, Istanbul, Turkey, ${ }^{15}$ Department of Pediatric Genetics, Acibadem Maslak Hospital, Istanbul, Turkey, ${ }^{16}$ Department of Medical Genetics, Faculty of Medicine, Acibadem University, Istanbul, Turkey

Introduction: Neurodevelopmental disorders (NDD) are heterogeneous conditions in which the development of the central nervous system is disturbed. Recent advancements in genomics have enabled scientists to identify numerous mutations underlying NDD.

Materials and methods: We used whole exome sequencing (WES) to identify the causative genes in 22 patients with NDD. Consanguinity was reported in all families. Genomic DNA was extracted using the QIAamp DNA Blood Mini QIAcube Kit (QIAGEN, Hilden, Germany). The DNA libraries were created by the Nextera DNA Exome kit (Illumina) and sequenced on NextSeq 500 sequencer using $2 \times 150 \mathrm{bp}$ sequencing kits (Illumina). Bioinformatic analyses were performed on Seqgenomize platform including an automated variant classification tool.

Results: In series of patients with NDD studied by WES, we found pathogenic/likely pathogenic variants in 17 different genes, which consist of 2 frameshift duplication, 4 frameshift deletion, 6 nonsense, 9 missense and a splice site variants in WDR62, CLP1, IGHMBP2, ADSL, GJC2,
EXOSC3, B4GALNT1, GBA2, WWOX, ERCC6, CHKB, TBCK, GLDC,TRAPPC9, CYP7B1, MID1 and TTN genes. CLP1 and GJC2 genes were found mutated twice. A total of 6 novel variants were identified. Interestingly, clinically relevant TTN and MID1 variants were detected in the same patient, explaining the combinatorial effects of the genes may contribute to phenotype complexity and variability.

Conclusions: Identification of pathogenic/ potentially pathogenic variants and novel variants may lead to the design of personalized therapeutic strategies and the implementation of genetic counseling of NDD as well as to monitoring and early intervention, even before the onset of the disorder.

B. Turkgenc: None. K. Yararbas: None. H. Keskin Karakoyun: None. A. Yesilyurt: None. A. Gezdirici: None. B. Tatlı: None. N.H. Akcakaya: None. N.E. Hacifazlioglu: None. O. Coskun: None. G. Yesil: None. B. Ekici: None. C. Yıldırım: None. H. Onal: None. G. Turanlı: None. Y. Alanay: None.

\section{E-P09.50}

Early-onset parkinsonian syndrome and PLA2G6 gene mutation

\section{Skrypnyk ${ }^{1}$, M. B. Falah ${ }^{2}$}

${ }^{1}$ Arabian Gulf University, College of Medicine and Medical Sciences, Al Jawhara Center for Molecular Medicine, Manama, Bahrain, ${ }^{2}$ Arabian Gulf University, University Medical Center, King Abdullah Medical City, Manama, Bahrain

Introduction: The parkinsonian syndromes encompass a number of entities grouped based on shared clinical features but separated by different pathologies. Non-motor features evolving coincident with progressive motor disability make the diagnosis very challenging.

Material and methods: Clinical assessment, brain MRI, EEG, biochemical profile and genetic testing were performed for a 25 years old male patient that presented with progressive neurological symptoms, generalized myoclonic jerks, unilateral left upper body tremor and rigidity, walking difficulties, swallowing difficulties, poor concentration and attention, behavioral changes and bouts of anger.

Results: The patient is the first child of healthy first degree cousins parents and has a positive family history of epilepsy. Behavioral changes were recorded since age 17 years old and neurological symptoms had onset at age 22 . CAG repeats testing was previously reported normal and ruled out Huntington disease. Brain MRI shown generalized cerebral and cerebellar atrophy without signs of iron 
accumulation or T2 lesions in basal ganglia. EEG revealed generalized epileptic complexes. Serum ceruroplasmin and copper values were normal. Rare forms of Parkinson, Multiple system cerebellar atrophy and Spinocerebellar ataxia were considered as differential diagnoses. Whole genome sequencing identified a PLA2G6 gene homozygous pathogenic missense mutation and clarified the diagnosis of Parkinson Disease type 14 (OMIM\#612953, ORPHA199351).

Conclusions: Whole genome sequencing is a very useful and fast tool in elucidating a complex and heterogeneous phenotype. PLA2G6 mutations should be considered in patients with atypical early-onset parkinsonian syndrome. Genetic testing is required when consanguinity associates with complex and early neurodegenerative onset.

C. Skrypnyk: None. M.B. Falah: None.

\section{E-P09.52}

Polymorphisms in genes for proinflammatory cytokines IL-6, IL-1 $\beta$, andTNF- $\alpha$ in relation with Parkinson's disease progression

M. Pesic ${ }^{l}$, N. Maksimovic ${ }^{1}$, A. Aleksic ${ }^{2}$, M. Gulic ${ }^{l}$, A. Djuranovic ${ }^{1}$, M. Grk ${ }^{l}$, M. Dusanovic Pjevic ${ }^{l}$, I. Stankovic ${ }^{3}$, V. Markovic ${ }^{3}$, A. Marjanovic ${ }^{3}$, I. Novakovic ${ }^{1}$, N. Dragasevic-Miskovic ${ }^{3}$, V. S. Kostic ${ }^{3}$

${ }^{1}$ Institute of Human Genetics, Belgrade, Serbia, ${ }^{2}$ Institute of Medical Statistics and Informatics, Belgrade, Serbia, ${ }^{3}$ Clinic for Neurology, Clinical Center of Serbia, Belgrade, Serbia

Introduction: Parkinson's disease (PD) is a progressive and complex neurological disorder with heterogeneous symptomatology. Chronic neuroinflammation contributes to the pathogenesis of PD. Activation of microglia and increased levels of pro-inflammatory mediators such as tumor necrosis factor alpha (TNF- $\alpha)$, interleukine- 1 beta (IL-1 $\beta$ ), and interleukine-6 (IL-6) have been reported after post-mortem analysis of the substantia nigra from PD patients. This study aimed to estimate the association of TNF- $\alpha$ rs1800629, IL-1 $\beta$ rs16944, and IL-6 rs1800795 with the disease progression in the initial motor stage, after fiveyear follow-up.

Material and methods: Our study included 64 consecutive PD outpatients at Hoehn and Yahr disease stage 1. The severity of PD, depression, anxiety, and cognitive impairment were evaluated using rating scales: Unified Parkinson Disease Rating Scale (UPDRS) Part (I-IV), MiniMental State Examination (MMSE) score, Hamilton Depression Rating Scale (HAM-D), and Hamilton Anxiety Rating Scale (HAM-A). Patients completed the same protocol at Years 1, 2, 3, and 5. Genotyping of TNF- $\alpha$ rs1800629, IL-1 $\beta$ rs16944, and IL-6 rs1800795 was performed using TaqMan real-time PCR assays.

Results: IL-1 $\beta$ rs16944 G allele in females has been associated with faster PD progression on UPDRS motor examination (Part III, $\mathrm{p}=0.014$ ), on motor complications (Part IV, $\mathrm{p}=0.026)$, and on UPDRS total $(\mathrm{p}=0.005)$. IL-6 rs1800795 GG genotype was statistically significant predictor of depression $(\mathrm{p}=0.047)$ and anxiety $(\mathrm{p}=0.004)$ progression on related scales.

Conclusion: Polymorphic variants in $I L-1 \beta$, and $I L-6$ genes influence Parkinson's disease course during five-year follow-up.

M. Pesic: None. N. Maksimovic: None. A. Aleksic: None. M. Gulic: None. A. Djuranovic: None. M. Grk: None. M. Dusanovic Pjevic: None. I. Stankovic: None. V. Markovic: None. A. Marjanovic: None. I. Novakovic: None. N. Dragasevic-Miskovic: None. V.S. Kostic: None.

\section{E-P09.53}

\section{Search for molecular genetic factors in Parkinson's} disease

\section{Shadrina ${ }^{1}$, S. Pchelina ${ }^{2}$, S. Illarioshkin ${ }^{3}$, P. Slominsky ${ }^{l}$}

${ }^{1}$ Institute of Molecular Genetics, Russian Academy of Sciences, Moscow, Russian Federation, ${ }^{2}$ The Petersburg Nuclear Physics Institute of the National Research Center, Kurchatov Institute, Russian Academy of Sciences, Gatchina, Russian Federation, ${ }^{3}$ Federal State Scientific Institution, Scientific Center of Neurology, Russian Academy of Sciences, Moscow, Russian Federation

Introduction: At present, there is no doubt about the complexity of the nature of the pathogenesis of Parkinson's disease (PD) due to disruption in the functioning of various metabolic systems, which leads to dopaminergic system deficiency. At the moment, there is no single picture of the etiopathogenesis of this disease.

Materials and methods: Toxic models of PD in rodents and various samples of patients with PD were studied using sequencing of full exome, transcriptome, and RT-PCR.

Results: An analysis of patients with a presumed autosomal dominant form of PD revealed eight new genes, mutations in which can lead to the development of the disease, was carried out. Three different variants were detected in the $S C N 3 A$ gene in three patients. Analysis of toxic models of PD revealed new ones that can play an important role at different stages of pathogenesis (vesicular transport, projection of neurons, RNA splicing and myelination processes). A detailed analysis of the mRNA level of individual genes in the peripheral blood of patients with PD revealed several genes, the level of which specifically varied in untreated patients with PD. 
Conclusion: Our data indicate a consistent involvement of the transcriptome in the pathogenesis of PD and highlight the independent role of various brain structures and individual parts of nerve cells in the formation of a response to the development of neurodegeneration. In addition, we showed the possible involvement of $S C N 3 A$ in the process of neurodegeneration. This work was supported by the Russian Science Foundation (grants 2015-00262).

M. Shadrina: None. S. Pchelina: None. S. Illarioshkin: None. P. Slominsky: None.

\section{E-P09.54}

Polymorphism of the functional promoter microsatellite locus of the arginine vasopressin receptor in Yakut men are linked to extraversion $\&$ neuroticism personality traits

S. S. Nakhodkin ${ }^{1}$, N. A. Barashkov ${ }^{1,2}$, V. G. Pshennikova ${ }^{1,2}$, S. A. Fedorova ${ }^{l}$

${ }^{1}$ M.K. Ammosov North-Eastern Federal University, Yakutsk, Russian Federation, ${ }^{2}$ Laboratory of Molecular Genetics, Yakut Scientific Centre of Complex Medical Problems, Yakutsk, Russian Federation

Polymorphism of the functional promoter microsatellite locus of the arginine vasopressin receptor (AVPR1A) can cause the formation of personality traits characterized by impulsivity, poor control of one's impulses, extravagance, anxiety, exaggeration of the expected danger and an acute reaction to social disapproval. In this paper, we evaluated the effect of the RS1 locus in the AVPR1A gene, in the formation of personal characteristics in 121 individuals of Yakut men, and also conducted a comparative analysis of the frequency distribution of alleles and genotypes. The study revealed the involvement of the polymorphic marker RS1 in the formation of such personal traits as "avoidance of damage" and "dependence on reward". The data obtained indicate a modulating effect of ethnicity on identifying associations of the AVPRA1 gene with personality traits. The frequencies of RS1 alleles differed in Yakut (Asian) volunteers compared to white (Finland, Ireland, Israel), and European white populations had a higher range of GATA track diversity. We identified seven different alleles of the RS1 marker. At the same time, short and long alleles (R8, R14, R15, R16) were absent in our sample in homozygous form. The Yakut population is characterized by a pronounced deficit of heterozygotes and a low level of actual heterozygosity, which can be explained by the founder effect in the Yakut population. The work was supported by the Ministry of science and higher education of the Russian Federation (the main part of NEFU) and with the support of RFBR grant (18-05-600035_arctic).

S.S. Nakhodkin: None. N.A. Barashkov: None. V.G. Pshennikova: None. S.A. Fedorova: None.

\section{E-P09.55}

Further evidence of the expanding clinical spectrum of PIGT-CDG: from epileptic encephalopathy to intellectual disability with epilepsy.

I. Ben Ayed ${ }^{1,2}$, F. Kamoun ${ }^{3}$, A. Souissi ${ }^{4}$, A. Bouzid ${ }^{4}$, M. Ben Said $^{4}$, S. Ben Nsir ${ }^{3}$, W. Bouchaala ${ }^{5}$, A. Tlili ${ }^{6,7}$, C. Triki ${ }^{3}, S$. Masmoudi $^{4}$

${ }^{1}$ Laboratory of Molecular and Cellular Screening Processes, Center of Biotechnology of Sfax, University of Sfax, Tunisia., SFAX, Tunisia, ${ }^{2}$ Medical Genetic Department, University Hedi Chaker hospital of Sfax, Sfax, Tunisia, ${ }^{3}$ Child Neurology department, Universitary Hedi Chaker hospitol of Sfax, SFAX, Tunisia, ${ }^{4}$ Laboratory of Molecular and Cellular Screening Processes, SFAX, Tunisia, ${ }^{5}$ Child Neurology department, Universitary Hedi Chaker hospitol of Sfax, SFAX, Tunisia, SFAX, Tunisia, ${ }^{6}$ Department of Applied Biology, College of Sciences, University of Sharjah, Sharjah, United Arab Emirates, ${ }^{7}$ Human Genetics and Stem Cell Laboratory, Research Institute of Sciences and Engineering, University of Sharjah, Sharjah, United Arab Emirates

Introduction: PIGT-CDG is a rare Glycosylphosphatidylinositol disorder. The patients show a broad clinical spectrum from epileptic encephalopathy to severe intellectual disability and treatable epilepsy with later age at onset.

Patient and Methods: Illumina ${ }^{\circledR}$ TruSight ${ }^{\mathrm{TM}}$ One clinical exome was performed in a 4-year-old girl with MiSeq nextgeneration sequencing platform in order to explore a profound developmental delay associated with severe hypotonia, seizure, and nystagmus.

Results: The analysis revealed a homozygous missense variant in PIGT (NM_015937.6): c.1519C $>$ T (p. Arg507Trp). Segregation analysis supports the recessive pattern of inheritance. The pathogenicity of p.Arg507Trp was estimated as deleterious by web-based prediction tools. So far 26 patients with a GPI anchor deficiency due to recessive PIGT variants have been described. Our patient shares the most common features associated with PIGTCDG: intellectual disability/development delay, seizures, nystagmus, and brain MRI anomalies. Most of the published PIGT deficient patients had evident cortical and cerebellar atrophy. Our patient demonstrated severe atrophy of the cerebral hemispheres with slight atrophy of the cerebellum at the age of 4 years. If the predominant presentation of patients is that of an epileptic encephalopathy including severe drug resistance, only some cases (4/26) with severe ID and treatable epilepsy with later age at onset were reported. Our patient had a global developmental 
delay with seizures; she became seizure-free with only one antiepileptic drug.

Conclusion: Here, a report a novel pathogenic missense variant in PIGT gene associated with treatable epilepsy strengthening the hypothesis that missense variant can lead to cause a milder phenotype with treatable seizures

I. Ben Ayed: None. F. Kamoun: None. A. Souissi: None. A. Bouzid: None. M. Ben Said: None. S. Ben Nsir: None. W. Bouchaala: None. A. Tlili: None. C. Triki: None. S. Masmoudi: None.

\section{E-P09.56}

Primary familial brain calcification manifesting as isolated paroxysmal dyskinesia: Case report of a patient with a homozygous MYORG change

\section{A. Westenberger ${ }^{l}$, G. Saranza $^{2}$, K. Grütz ${ }^{1}$, C. Klein $^{l}$,} A. E. Lang ${ }^{2,3}$

${ }^{1}$ Instituteof Neurogenetics, University of Lübeck, Lübeck, Germany, ${ }^{2}$ Edmond J. Safra Program in Parkinson's Disease and the Morton and Gloria Shulman Movement Disorders Clinic, Toronto Western Hospital, Toronto, ON, Canada, ${ }^{3}$ Division of Neurology, Department of Medicine, University of Toronto, Toronto, ON, Canada

Introduction: Primary familial brain calcification (PFBC) is a hereditary disorder characterized by calcium deposits in the brain and presenting clinically with neuropsychiatric symptoms, cognitive dysfunction, or a variety of movement disorders. Currently, changes in six genes are known to cause PFBC, with SLC20A2, PDGFB, PDGFRB, XPRI variants related to autosomal dominant forms and $M Y O R G$ and JAM2 variants to autosomal recessive ones.

Patients and Methods: We identified a 26-year-old female patient, presenting with periodic involuntary posturing of her limbs and symmetrical bilateral brain calcifications. Genetic testing was performed for PRRT2, SLC2OA2, $P D G F B, P D G F R B, X P R 1$, and MYORG. Her parents also underwent targeted $M Y O R G$ sequencing.

Results: In our patient, the paroxismal kinesigenic attacks first appeared at eight years of age, became more frequent in her teenage years (up to ten attacks per day) and were completely absent during her pregnancies. Neurologic examination was normal between episodes. Neuroimaging revealed symmetrical bilateral calcifications involving the cerebellum, basal ganglia, thalamus, midbrain, and the frontal and occipital cortical and subcortical areas. In addition, in her pregnancies, the placentae were noted to have widespread calcification. Our genetic analyses identified a homozygous previously reported and likely pathogenic missense variant in the MYORG gene (c.1831C $>\mathrm{T}$; $\mathrm{p}$. Arg611Trp) and no PRRT2 changes in our patients. Her father carried the same heterozygous MYORG variant and showed no brain calcification.

Conclusions: We report a homozygous $M Y O R G$ change as the most likely cause of PFBC presenting as paroxysmal dyskinesia in our patient and thus expand the known phenotypic spectrum of $M Y O R G$-associated PFBC.

A. Westenberger: F. Consultant/Advisory Board; Modest; Centogene. G. Saranza: None. K. Grütz: None. C. Klein: F. Consultant/Advisory Board; Modest; Centogene. A.E. Lang: None.

\section{E-P09.57}

A Portuguese family-based exome sequencing in primary headaches

A. Dias ${ }^{1,2}$, M. Alves-Ferreira ${ }^{1,2}$, J. Sequeiros ${ }^{1,2}$, J. Pereira Monteiro $^{1}$, A. Sousa ${ }^{l, 2}$, C. Lemos $^{1,2}$

${ }^{1} i 3 S$ - Instituto de Investigação e Inovação em Saúde, Porto, Portugal, ${ }^{2}$ ICBAS - Instituto Ciências Biomédicas Abel Salazar, Porto, Portugal

Introduction: Primary headaches comprises migraine with or without aura (MA/MO) and cluster headache $(\mathrm{CH})$. During the last 17 years, our group has been able to clinically characterize more than a thousand patients, relatives and controls and we performed several candidate-gene association studies in different pathways. Moreover, we found several variants involved in the vascular component, trigeminal nociceptive plasticity, neurogenic inflammation and in the release of neurotransmitters. However, these complex diseases are caused by several genetic factors. Whole-exome sequencing (WES) is a powerful approach to explore coding regions, particularly low-frequency variants. Our aim was to perform a WES in three families to unravel genetic factors involved in migraine and $\mathrm{CH}$ susceptibility.

Materials and methods: We gathered clinical information and DNA samples from 3 families with primary headaches and associated phenotypes. Afterwards, a WES was performed.

Results: We analysed 20 patients from the 3 families and we found common and rare variants in genes already associated with migraine subtypes as CACNAIA and $P R R T 2$ and in new genes that may open new pathways of study.

Conclusions: These preliminary results need to be further explored and interactions among these variants need to be understood to deepen the pathophysiological pathways of migraine. In the future, we will also correlate epigenetics and brain activity data to assess the risk of chronic and episodic migraine in women. Understanding the mechanisms underlying primary headache pathophysiology could lead to the development of more effective and better- 
tolerated therapeutic approaches to avoid the overlay of painful events.

A. Dias: None. M. Alves-Ferreira: None. J. Sequeiros: None. J. Pereira Monteiro: None. A. Sousa: None. C. Lemos: None.

\section{E-P09.58}

Prevalence of PTEN mutations in Turkish children with autism spectrum disorders and macrocephaly

H. Kaymakcalan ${ }^{1}$, I. Kaya $^{2}$, N. Cevher Binici ${ }^{3}$, B. Ozbaran ${ }^{4}$, G. Ozyurt ${ }^{5}$, S. Erbilgin ${ }^{6}$, E. Nikerel ${ }^{7}$, M. Aksoy ${ }^{4}$, D. Celik ${ }^{4}$, S. Kose ${ }^{4}$, N. Majroh ${ }^{4}$, S. Duruk ${ }^{3}$

${ }^{1}$ Demiroglu Bilim University, Istanbul, Turkey, ${ }^{2}$ Istanbul University, Istanbul, Turkey, ${ }^{3}$ Behcet Uz Hospital, Izmir, Turkey, ${ }^{4}$ Ege University, Izmir, Turkey, ${ }^{5}$ Katip Celebi University, Izmir, Turkey, ${ }^{6}$ Okmeydanı Hospital, Istanbul, Turkey, ${ }^{7}$ Yeditepe University, Istanbul, Turkey

Prevalence of PTEN mutations in Turkish children with autism spectrum disorders and macrocephaly Introduction: Recently, people with autism spectrum disorders (ASD) and macrocephaly were found to carry mutations in PTEN gene. Mutations in this gene cause PTEN Hamartoma Tumour Syndrome (PHTS). However, PHTS often goes undetected especially in children since the manifestations of the disease are variable and subtle and guidelines for testing are few. Studies show 1 to $17 \%$ prevalence rates of PTEN mutations in children with ASD and macrocephaly. The varying results on prevalence suggest the need for further studies. The aim of our study is to find PTEN prevalence in Turkish children with ASD and macrocephaly and increase awareness of this syndrome in Turkey. To the best of our knowledge this will be the first PTEN prevalence study done in Turkey and South Eastern Europe. This is also the largest pediatric cohort for PTEN prevalence study in patients with ASD and macrocephaly. Materials and methods: PTEN sequencing were done for 128 patients with ASD and head circumference size more than $97 \%$ on growth charts between the ages of 3 to 18 recruited from five different child and adolescent psychiatry clinics in Turkey. Result: We found 2 known pathogenic, 1 new pathogenic and 2 variants of insignificance (VUS) mutations out of 128 patients. The prevalence is $2,3 \%$ (VUS mutations are excluded). Conclusion: We recommend PTEN testing in patients with ASD and macrocephaly. Grant for this study is awarded by 'PTEN Research' in London, UK.

H. Kaymakcalan: None. I. Kaya: None. N. Cevher Binici: None. B. Ozbaran: None. G. Ozyurt: None. S. Erbilgin: None. E. Nikerel: None. M. Aksoy: None. D. Celik: None. S. Kose: None. N. Majroh: None. S. Duruk: None.

\section{E-P09.60}

Potential genetic modifiers of the inherited neurodegenerative disorder $\mathrm{SCA3}$

\author{
R. M. Burger ${ }^{1,2,3,4}$, M. L. Saraiva-Pereira ${ }^{5}$, M. Franca $^{6}$, \\ C. Gordon ${ }^{7}$, L. B. Jardim ${ }^{5}$, M. Cornejo-Olivas ${ }^{8}$, O. Rie $\beta^{1,2,3}$, \\ T. Schmidt ${ }^{1,2,3}$
}

${ }^{1}$ University of Tübingen, Medical Genetics and Applied Genomics, Tübingen, Germany, ${ }^{2}$ University of Tübingen, Center of Rare Diseases, Tübingen, Germany, ${ }^{3}$ University of Tübingen, NGS Competence Center of Tübingen (NCCT), Tübingen, Germany, ${ }^{4} I Z K F$, Graduate program of the University of Tübingen, Tübingen, Germany, ${ }^{5}$ Laboratório de Identificação Genética, Hospital de Clínicas de Porto Alegre, Porto Alegre, Brazil, ${ }^{6}$ Department of Neurology, University of Campinas-UNICAMP, Campinas, Brazil, ${ }^{7}$ Sackler Faculty of Medicine, Tel Aviv University,, Tel Aviv, Israel, ${ }^{8}$ Neurogenetics Research Center, Instituto Nacional de Ciencias Neurológicas, Lima, Peru

Background: Spinocerebellar ataxia type 3 (SCA3) is an autosomal-dominantly inherited neurodegenerative disease and the most common inherited ataxia worldwide. Its pathogenic mutation is an abnormal expansion of the CAG repeats over 55 triplet-repeats within the ATXN3 gene which encodes for an excessively long polyglutamine (polyQ) tract in the disease protein ataxin-3. Patients show a widely spread variation in age at onset and clinical variability of phenotype. Until today this phenomena is not fully explained. In the past, it was shown that the age at onset inversely correlates with the expanded CAG repeats. Within the wide range of age at onset between 5-75 years only 45$60 \%$ can be explained by the CAG repeat expansion.

Aim: The aim of our study is to identify genetic modifiers which can explain the wide range of age at onset as well as the clinical heterogeneity of SCA3.

Methods: Previously several genes (ATXN3, ATXN2, APOE, CACNAIA) were described as candidate genes in literature. We are genotyping two big cohorts EUROSCA (Europe) and EUSAge (Israel, Brazil, Peru) in terms of singlenucleotide polymorphism (SNP) within candidate genes.

Results and Conclusion: A variation in polymorphism can lead to different isoforms of the genes, functional changes of the encoding proteins and different interactions with molecules. A SNP in ATXN3 has already been found to influence the pathophysiology of SCA3. Therefore, SNPs of candidate genes seem to have an influence on the pathophysiology of SCA3 and may be promising genetic modifiers to observe further in order to learn more about the disease.

R.M. Burger: None. M.L. Saraiva-Pereira: None. M. Franca: None. C. Gordon: None. L.B. Jardim: None. M. Cornejo-Olivas: None. O. Rieß: None. T. Schmidt: None. 


\section{E-P09.61}

Co-ocurrence of two rare genetic diseases in a patient with intellectual disability, refractory epilepsy and skeletal abnormalities

\section{Rodrigues, M. P. Soares, P. Dias, A. B. Sousa}

Serviço de Genética Médica, Hospital de Santa Maria, Centro Hospitalar Universitário de Lisboa Norte EPE, Centro Académico de Medicina de Lisboa, Lisbon, Portugal, Lisbon, Portugal

Introduction: Whole-exome sequencing (WES) is a powerful tool to identify the genetic causes of rare mendelian disorders and can be particularly useful in patients with complex and genetically heterogeneous phenotypes. The authors describe a patient with phenotypic manifestations of two rare genetic diseases: autosomal recessive cerebellar ataxia type 23 (SCAR23, OMIM \#616949) and contractures, pterygia, and variable skeletal fusions syndrome1A (CPSKF1A, OMIM \#178110).

Methods and results: We report a 21-year-old male patient born to non-consanguineous parents, with irrelevant family history. He has language delay, mild to moderate intellectual disability (ID), refractory epilepsy, fine motor skill deficits, short neck, narrow shoulders, mild facial dysmorphisms, hypoplastic hypothenar eminence and hypoplastic flexion creases. His skeletal survey showed C4-C5 fusion. Brain MRI, karyotype, array-CGH and FMRI testing were normal. WES revealed two variants in TDP2 gene: c.345_348del, p.(IIe117Profs*7) (likely pathogenic) and c. $949 \mathrm{C}>\mathrm{T}$, p. $(\operatorname{Arg} 317 *)$ (variant of uncertain significance). These variants have not been previously reported and are in a compound heterozygous state. WES also showed a heterozygous deletion encompassing at least exons 4-7 of the MYH3 gene (likely pathogenic).

Discussion and conclusions: The presence of variants c.345_348del and c.949C $>\mathrm{T}$ in TDP2 gene may justify the neurological findings in our patient, namely ID and epilepsy, establishing the diagnosis of SCAR23. On the other hand, the heterozygous deletion of exons 4-7 of the MYH3 gene might explain the dysmorphic and skeletal findings of the patient, whose features are more suggestive of CPSKF1A. This case illustrates the challenge of diagnosing two genetic disorders co-occurring in the same patient.

M. Rodrigues: None. M.P. Soares: None. P. Dias: None. A.B. Sousa: None.

\section{E-P09.62}

DIO2Thr92Ala Reduces Deiodinase 2 Activity and Serum T3 Levels in Patients with Schizophrenia

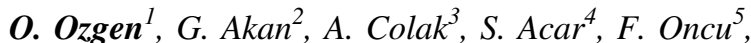

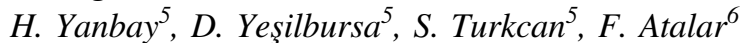

${ }^{I}$ Istanbul University Medical Faculty, Aziz Sancar Experimental Research Institute, Molecular Medicine Department, Istanbul, Turkey, ${ }^{2}$ MUHAS Genetics Laboratory, Biochemistry Department, School of Medicine, Muhimbili University of Health and Allied Sciences, Dar es Salaam, Tanzania, United Republic of, ${ }^{3}$ Department of Biomedical Engineering, Biomedical Engineering Institute, Bogazici University, Istanbul, Turkey, ${ }^{4}$ Department of Bioengineering, Yildiz Technical University, Istanbul, Turkey, ${ }^{5}$ Psychiatry Clinics, Turkish Ministry of Health Bakirkoy Research and Training Hospital for Psychiatry, Neurology and Neurosurgery, Istanbul, Turkey, ${ }^{6}$ Istanbul University Medical Faculty, Medical Genetics Department, Child Health Institute, Istanbul, Turkey

Background: Thyroid hormone modulation of crucial brain neurotransmitter systems and the misregulation of these pathways are of particular relevance in schizophrenia (SCH). Type II deiodinase enzyme (DIO2) has a critical potency on thyroid metabolism that converts prohormone thyroxine (T4) to the active hormone triiodothyronine (T3) also it seems that can particular relevance in schizophrenia. The aim of this study was to investigate the genetic contribution of the DIO2 gene to $\mathrm{SCH}$ by the genotype analysis of DIO2 Thr92Ala and ORFa-Gly3Asp polymorphisms and to compare the serum levels of free triiodothyronine (FT3), free thyroxine (FT4), and thyroid-stimulating hormone (TSH) concentrations in 290 unrelated Turkish $\mathrm{SCH}$ patients and 198 healthy controls.

Method and Results: We genotyped two single nucleotide polymorphisms (SNPs) Thr92Ala and ORFa-Gly3Asp located on DIO2 gene, and performed SNP association analysis along with the thyroid hormonal status in $\mathrm{SCH}$ patients and healthy controls. The distribution of Thr92Ala genotypes in $\mathrm{SCH}$ was significantly different compared to controls $(\mathrm{p}=0.045)$. In men cohort, both allele and genotype frequencies were found significantly different than controls $(p=0.03)$. However, neither allele and nor genotype frequencies of ORFa-Gly3Asp polymorphism were statistically significant in the study group. Decrease of mean FT3 $(\mathrm{p}<0.001)$ and TSH $(p=0.004)$ concentrations were observed in SCH patients compared to controls. Thr92Ala genotypes were found to be significantly associated with FT3 and TSH levels $(\mathrm{p}<0.05$, respectively).

Conclusion: Our results indicate that DIO2 Thr92Ala reduces Deiodinase-2 Activity and FT3 Levels in Turkish $\mathrm{SCH}$ patients. Our findings also show that the importance of thyroid hormone metabolism in $\mathrm{SCH}$. 
O. Ozgen: None. G. Akan: None. A. Colak: None. S. Acar: None. F. Oncu: None. H. Yanbay: None. D. Yeşilbursa: None. S. Turkcan: None. F. Atalar: None.

\section{E-P09.63}

Schizophrenia patients have significantly less f-SatIII and more rDNA repeats compared to healthy donors

V. Sergeeva ${ }^{l}$, A. Poletkina ${ }^{l}$, E. Ershova ${ }^{l}$, N. Veiko ${ }^{l}$, A. Martynov', J. Chudakoval, A. Artyushin', E. Malinovskaya ${ }^{1}$, A. Filev ${ }^{1}$, M. Konkova ${ }^{1}$, L. Bravve ${ }^{2}$, N. Zakharova ${ }^{2}$, G. Kostyuk ${ }^{2}$, O. Agafonova ${ }^{1}$, G. Shmarina ${ }^{1}$, S. Kostyuk ${ }^{l}$

${ }^{1}$ FSBI "Research Centre For Medical Genetics", Moscow, Russian Federation, ${ }^{2}$ N. A. Alexeev Clinical Psychiatric Hospital №1, Moscow Healthcare Department., Moscow, Russian Federation

Introduction: Dysregulation of rRNA biogenesis is implicated in some human diseases. rDNAcopy number $(\mathrm{CN})$ in the genome is a factor at play. F-SatIII is an AT-rich repeat ofpericentromeric heterochromatin in the first chromosome (1q12). rDNA and 1q12 interact ininterphase cells. rDNA $\mathrm{CN}$ might correlate with f-SatIII.

Materials and methods: We analyzed leukocytes of 469 healthy donors (HD) and 777 patientswith schizophrenia (F20.00, SP). CN was determined using non-radioactive hybridization withbiotin-labelled DNA probes.

Results: Genomes of SP contain increased levels of rDNA CN and decreased levels of f-SatIIIcompared to HD: $532 \pm 118$ copies with $18,0 \pm 5,9 \mathrm{pg} / \mathrm{ng}$ of f-SatIII and $413 \pm 105$ copies with21,6 $\pm 6,9 \mathrm{pg} / \mathrm{ng}$ of f-SatIII, respectively, $\mathrm{p}<10-80$. Amount of f-SatIII depends on rDNA CN:DNA of HD and SP containing low (200 - 350) or high $(>700) \mathrm{CN}$ of rDNA contained $10-30 \mathrm{pg} / \mathrm{ng}$ of $\mathrm{f}-$ SatIII. 55\% of SP have 500 - $600 \mathrm{CN}$ of rDNA and 15-20 $\mathrm{pg} / \mathrm{ng}$ of f-SatIII. Only $15 \%$ of HD have the same numbers. $16 \%$ of SP have $<20 \mathrm{pg} / \mathrm{ng}$ of f-SatIII and rDNA $\mathrm{CN}>$ 500 with their ratio $>40$, only $1.5 \%$ of $\mathrm{HD}$ have this ratio. $26 \%$ of $\mathrm{HD}$ have rDNA CN $<400$, f-SatIII $>20 \mathrm{pg} / \mathrm{ng}$ and their ratio $<20$ and only $1,9 \%$ of SP are in this area. Thus, there is acorrelation between rDNA $\mathrm{CN}$ and the size if heterochromatin regions.

Conclusions: SP have significantly less f-SatIII and more rDNA repeats compared to HD.

V. Sergeeva: None. A. Poletkina: None. E. Ershova: None. N. Veiko: None. A. Martynov: None. J. Chudakova: None. A. Artyushin: None. E. Malinovskaya: None. A. Filev: None. M. Konkova: None. L. Bravve: None. N. Zakharova: None. G. Kostyuk: None. O. Agafonova: None. G. Shmarina: None. S. Kostyuk: None.

\section{E-P09.65}

SORL1 variant associated with familial Parkinson's disease and dementia in a large Greek pedigree.

\section{T. Bourinaris ${ }^{1}$, G. Xiromerisiou ${ }^{2}$, H. Houlden ${ }^{1}$, A. Singleton ${ }^{3}$, SYNaPS study group, J. Hardy, J. Vandrovcoval}

${ }^{1}$ UCL Institute of Neurology, London, United Kingdom, ${ }^{2}$ University of Thessaly, Larissa, Greece, ${ }^{3}$ National Institute on Aging, Bethesda, MD, United States

Background: $10 \%$ of Parkinson's disease is caused by certain monogenic forms. Several causal genes have been identified today but the understanding of underlying genetic mechanisms has proved challenging. Many of the affected families remain genetically unsolved.

Methods: In this study we use molecular techniques to investigate a large pedigree with many affected individuals from an isolated village in central Greece affected by a high incidence of Parkinson's disease. We utilized whole exome sequencing and linkage analysis to identify coding variants that are of putative significance. We then apply association analysis across a wider population to highlight the significance of these variants in sporadic cases.

Results: Linkage to chromosome 11 was found with a LOD score of 2.2. The phenotypic spectrum of our patients was not uniform: 3 presented with Parkinson's disease without dementia, while another 2 presented with dementia that was later classified as Alzheimer's disease. All patients carried a novel heterozygous variant in SORL1 segregating with disease in the family.

Conclusions: This is the first report of the discovery of a likely pathogenic variant in SORL1 in a large family with some of the affected individuals presenting with Parkinson's disease without dementia at disease onset.

T. Bourinaris: None. G. Xiromerisiou: None. H. Houlden: None. A. Singleton: None. J. Hardy: None. J. Vandrovcova: None.

\section{E-P09.66}

Molecular Complexity of Spastic Ataxias and Hereditary Spastic Paraplegias in Turkey

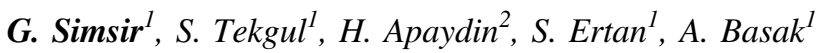 \\ ${ }^{1}$ Koç University, Istanbul, Turkey, ${ }^{2}$ Istanbul University- Cerrahpasa, Istanbul, Turkey}

Introduction: Hereditary spastic paraplegias (HSPs) and autosomal recessive cerebellar ataxias (ARCAs) are neurological diseases that may overlap genetically and clinically. Both can be associated with other neurologic or non- 
neurologic symptoms and complex phenotypes. Significantly, ARCAs frequently show pyramidal involvement, which results in spasticity. Molecular analysis is challenging due to complex genetics and rarity of these diseases. However, recent developments in next generation sequencing technologies help to understand complex genetic background of these diseases.

Materials and methods: DNA isolated from peripheral blood was used. 37 probands with spasticity and ataxia were subjected to whole exome sequencing (WES), followed by bioinformatic analysis. Most families were consanguineous.

Results: 26 mutations (15 novel) in 10 genes were identified (Table1). Table1 Genes and mutations identified by WES in the Turkish cohort under study

\begin{tabular}{|c|c|c|}
\hline $\begin{array}{l}\text { Genes (\# of } \\
\text { families) }\end{array}$ & Mutations & Novel/Reported \\
\hline SACS $(n=7)$ & $\begin{array}{l}\text { c. } 8867 \mathrm{~T}>\mathrm{C} \text { p.Leu2956Ser } \\
\text { c.12923_12927delAAGAA } \\
\text { p.Lys4308SerfsTer21 } \\
\text { c.4192T }>\text { C p.Cys1398Arg } \\
\text { c.11374C }>\text { T p.Arg3792Ter } \\
\text { c.7205_7206delTT p. } \\
\text { Leu2402ArgfsTer6 } \\
\text { c.3484G >T p.Glu1162Ter } \\
\text { c.7732_7734delGAT p. } \\
\text { Asp2578del and } \\
\text { c.3767A }>\text { G p.Tyr1256Cys }\end{array}$ & $\begin{array}{l}\text { Novel Novel Novel } \\
\text { Reported Reported } \\
\text { Reported Novel- } \\
\text { Novel }\end{array}$ \\
\hline SPG7 $(\mathrm{n}=5)$ & $\begin{array}{l}\text { c. } 1861 \mathrm{C}>\mathrm{T} \text { p.Gln621Ter } \\
\text { c. } 1715 \mathrm{C}>\mathrm{T} \text { p.Ala572Val } \\
\text { c. } 2096 \mathrm{~T}>\mathrm{C} \text { p.Met699Thr } \\
\text { c. } 1861 \mathrm{C}>\mathrm{T} \text { p.Gln621Ter / } \\
\text { c. } 1715 \mathrm{C}>\mathrm{T} \text { p.Ala572Val* }\end{array}$ & $\begin{array}{l}\text { Novel Reported } \\
\text { Novel Novel/ } \\
\text { Reported }\end{array}$ \\
\hline CAPN1 $(n=4)$ & $\begin{array}{l}\text { c. } 759+1 \mathrm{G}>\text { A c. } 397 \mathrm{C}>\mathrm{T} \text { p. } \\
\text { Arg133Ter c. } 907 \mathrm{~T}>\mathrm{G} \text { p. } \\
\text { Trp303Gly c. } 397 \mathrm{C}>\mathrm{T} \text { p. } \\
\text { Arg133Ter }\end{array}$ & $\begin{array}{l}\text { Reported Reported } \\
\text { Novel Reported }\end{array}$ \\
\hline SPG11 $(n=2)$ & $\begin{array}{l}\text { c.7158_7161dupACAA p. } \\
\text { His2388ThrfsTer6 } \\
\text { c.3809T>A p.Val1270Asp }\end{array}$ & Novel Reported \\
\hline HSD17B4 $(n=2)$ & c. $425 \mathrm{~A}>\mathrm{G}$ p.Asp142Gly & Novel \\
\hline MRE11A (n=1) & c. 1442 C $>$ A, p.Thr481Lys & Reported \\
\hline KIF1C $(n=1)$ & $\begin{array}{l}\text { c. } 866 \mathrm{~A}>\mathrm{C} \text { p. Gln289Pro and } \\
\text { c. } 940+1 \mathrm{G}>\mathrm{A}\end{array}$ & Novel-Novel \\
\hline $\operatorname{VPS} 13 \mathrm{D}(\mathrm{n}=1)$ & c. $12629 \mathrm{C}>\mathrm{T}$ p.Ala4210Val & Reported \\
\hline PNPLA6 $(n=1)$ & c. $940 \mathrm{C}>\mathrm{T}$ p.Arg314Trp & Novel \\
\hline SYNE1 (n=1) & c. $3371 \mathrm{~A}>$ G p.Asp1124Gly & Novel \\
\hline
\end{tabular}

All patients are true homozygotes except one compound heterozygote*

Conclusions: $25 / 37$ probands were solved, corresponding to $68 \%$ diagnostic yield. The genes identified point to a high heterogeneity and complexity of the cohort investigated. The results further reveal the well-known genetic overlap among HSPs and ARCAs.
G. Simsir: None. S. Tekgul: None. H. Apaydin: None. S. Ertan: None. A. Basak: None.

\section{E-P09.67}

A homozygous pathogenic variant in the STAMBPgene associated with microcephaly-capillary malformation syndrome - the first case report in a Roma patient

M. Giertlova ${ }^{1}$, M. Mistrik ${ }^{l}$, J. Saligova ${ }^{2}$, E. Zavadilikova ${ }^{2}$, A. Baranova ${ }^{2}$, M. Hyblova ${ }^{3}$, G. Minarik ${ }^{3}$

${ }^{1}$ Alpha medical Inc., Martin, Slovakia, ${ }^{2}$ Pediatric University Hospital and Medical faculty, University of P. J. Safarik, Kosice, Slovakia, ${ }^{3}$ Medirex Inc., Bratislava, Slovakia

Introduction: The gene STAMBP (STAM-binding protein) encoding a deubiquitinating enzyme is associated with microcephaly-capillary malformation syndrome (MICCAM) (OMIM 614261). We present a case study of the patient with the MIC-CAM phenotype with a homozygous pathogenic variant in the STAMBP gene.

Material and methods: The full-term newborn presented with microcephaly (-3.3SD) and small capillary malformations in different areas of the body. Focal myoclonic spasms developed from the $3^{\text {rd }}$ month of age. At the $6^{\text {th }}$ month, the focal EEG abnormalities, brain atrophy and profound developmental delay with hypotonia led to suspicion of the early infantile myoclonic encephalopathy. Similar clinical findings were documented in her older sister deceased at the $9^{\text {th }}$ month of age. Consanguinity was recorded in the family pedigree. The molecular diagnosis was found by whole-exome sequencing (Illumina NextSeq platform) using Human Core Exome Kit (Twist Bioscience) and confirmed by Sanger sequencing. The detected variants were annotated by Ingenuity Variant Analysis (Qiagen) and classified according to the ACMG criteria.

Results: Homozygous likely pathogenic missense variant in the STAMBP gene was identified (hg20: chr2:73831044A>G; ENST00000394070.7; c.188A>G; p. $\mathrm{Y} 63 \mathrm{C})$. This genetic finding is consistent with the MICCAM phenotype observed in our patient.

Conclusion: Less than 20 cases of MIC-CAM have been reported so far. We present the first case of MIC-CAM syndrome in a Roma origin patient with homozygous c. $188 \mathrm{~A}>\mathrm{G}$; p.Y63C variant in the STAMBP gene. The heterozygous variants occur in gnomAD Exomes exclusively in the European population with frequency 0.000014 . The hypothesized founder effect of the variant remains to be clarified.

M. Giertlova: None. M. Mistrik: None. J. Saligova: None. E. Zavadilikova: None. A. Baranova: None. M. Hyblova: None. G. Minarik: None. 


\section{E-P09.68}

Expressivity of mutations in $T U B B 2 B$ is highly variable

J. Dekker, K. E. M. Diderich, R. Schot, D. J. Smits,

M. Wilke, M. A. van Slegtenhorst, G. M. S. Mancini

\section{Erasmus MC, Rotterdam, Netherlands}

Microtubules play an important role during cortical development by regulating mitosis and neuronal migration. $T U B B 2 B$ codes for one the $\beta$-isoforms of tubulin and dominant negative mutations in this gene result in malformations of cortical development (MCD) like polymicrogyria, pachygyria, dysmorphic basal ganglia, epilepsy and intellectual disability. However, phenotypic heterogeneity has been reported in cases with isolated congenital fibrosis of extraocular muscles (CFEOM3A) and myoclonus-dystonia. Here we show three individuals in one family with a missense variant in $T U B B 2 B$, who exhibit an unusual phenotype. At 21 week of gestation advanced US revealed a foetus with relative small head, enlarged lateral ventricles, borderline hypoplastic cerebellum and a thin corpus callosum, which were less pronounced at MRI. Given the uncertain prognosis, the couple decided to terminate the pregnancy. Exome sequencing on foetal material identified a heterozygous maternally inherited variant in $T U B B 2 B$ (c.530A > T, p.Asp177Val), not present in GnomAD and predicted damaging. The healthy mother had only a language delay in childhood. The oldest son of the couple was also examined because of mild delay of both motor skills and speech. He follows regular education programme with extra support. The same variant in $T U B B 2 B$ was confirmed in the son. His MRI revealed decreased supratentorial brain volume, enlarged ventricles, mild cortical dysgyria, thin corpus callosum and dysmorphic vermis and basal ganglia, a pattern typical of tubulinopathies. Although we cannot exclude that the mother is a mosaic carrier, these results illustrate the wide phenotypic heterogeneity of tubulinopathies, including reduced penetrance and very mild expressivity.

J. Dekker :None. K.E.M. Diderich: None. R. Schot: None. D.J. Smits: None. M. Wilke: None. M.A. van Slegtenhorst: None. G.M.S. Mancini: None.

\section{E-P09.69}

An infant with two de novo variants causing recessive and dominant disorders: Diagnostic challenge

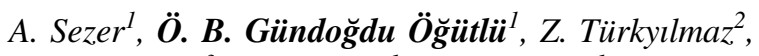

K. Gücüyener ${ }^{3}$, G. Kayhan ${ }^{1}$, F. E. Perçin ${ }^{l}$

${ }^{1}$ Gazi University Department of Medical Genetics, Ankara, Turkey, ${ }^{2}$ Gazi University Department of Pediatric Surgery,
Ankara, Turkey, ${ }^{3}$ Gazi University Department of Pediatric Neurology, Ankara, Turkey

Introduction: Inherited Glycosylphosphatidylinositol deficiencies (IGDs) are a subclass of Congenital Defects of glycosylation. Most common findings of IGDs are developmental delay, seizures, and persistent hyperphosphatasia. Moreover GRIN2B mutations cause another rare neurodevelopmental disorder which has overlapping findings with IGDs, e.g. developmental delay, intellectual disability and epilepsy. Herein, it is aimed to present a case diagnosed with two de novo variants in PIGO and GRIN2B genes: one is causing a dominant and the other is causing a recessive disorder.

Material Method: A seven-month-old girl was presented with hydronephrosis, seizure, microbrachycephaly, bilateral hypoplastic fifth fingernails, abnormal distal phalanx morphology of fingers on radiography and persistent hyperphosphatasia. There was no consanguinity of her parents. Whole-exome sequencing (WES) analysis was performed to evaluate genetic etiology.

Results: Two rare variants in PIGO (NM_032634.4) gene; c.1944C $>$ G (p.Cys648Trp) and c.2708_2710delTCT (p.Phe903del) were shown by WES analysis. Sanger sequencing performed to the parents, and unveiled that those two variants was maternal and de novo respectively. The variants were evaluated as in trans position, by using a heterozygous polymorphism (rs505297) and NGS readings of WES data. Further, a rare de novo variant was detected in GRIN2B (NM_000834.5) gene: c.1852G $>$ A (rs1428234478) (p.Val618Ile).

Conclusion: Some prospective and retrospective studies have been shown the underestimated number of multiple molecular diagnosis of which results in phenotypes overlapping or separated. In our case phenotypes of two diagnosis were overlapping. If one of multiple genetic diagnoses had been overlooked, this would change the present or future management options of disorders and genetic counseling to families.

A. Sezer: None. Ö.B. Gündoğdu Öğütlü: None. Z. Türky1lmaz: None. K. Gücüyener: None. G. Kayhan: None. F.E. Perçin: None.

\section{E-P10 Neuromuscular Disorders}

\section{E-P10.01}

Aicardi-Goutières Syndrome: unexpected another gene mutation which related epilepsy: a case report 
F. Duymus ${ }^{l}$, B. Goksel Tulgar ${ }^{1}$, D. Esin ${ }^{1}$, G. Turkoglu ${ }^{l}, M$. E. Sarl ${ }^{l}$, T. Cora ${ }^{l}$, A. Kartal ${ }^{2}$

${ }^{1}$ Selcuk University Faculty of Medicine, Medical Genetics Department, Konya, Turkey, ${ }^{2}$ Selcuk University Faculty of Medicine, Pediatric Neurology Department, Konya, Turkey

Aicardi-Goutières syndrome (AGS) is an inherited encephalopathy that mainly affects the brain, the immune system, and the skin. Several of the genes (TREX1, RNASEH2A, RNASEH2B, RNASEH2C, IFIH1, ADAR, SAMHD1) mutations can cause AGS. The syndrome can be seen as autosomal dominant/recessive. Generally, the onset of AGS occurs within the first few days or months of life. In our case, the most severe neonatal form of AGS1 is typically due to mutation in the TREX1 gene. In addition to our patient, SPTAN1 gene mutation was detected. This gene mutation can cause Early infantile epileptic encephalopathy type 5 which inherited autosomal dominant. We thought that the common point of the two mutations might be severe epilepsy and cerebellar atrophy for our patient. Her epileptic attacks started at about 4 months of age. On the physical examination microcephaly, joint stiffness, dystonia, abnormal eye movements, tetraplegic spasticity, and neuromotor developmental retardation were presented. In cranial MR, cerebral atrophy, intracerebral calcifications, and calcifications of the periventricular area were observed. Epilepsy attacks started at the age of 3 months. We performed RNASEH2B gene sequence analysis in the patient and could not detect a mutation. We detected two mutations with whole-exome sequencing. One of them was homozygous c.13_15dupATC mutation in the TREX gene and the other was heterozygous c.2882 C> T in the SPTAN1 gene. AGS is a very rare disease of unknown frequency. Our aim in presenting this case is to see two rare mutations together and think that the disease can change its severity.

F. Duymus: None. B. Goksel Tulgar: None. D. Esin: None. G. Turkoglu: None. M.E. Sarı: None. T. Cora: None. A. Kartal: None.

\section{E-P10.02}

Penetrance of Familial Amyloid Polyneuropathy in Huelva. One of the most important foci in worldwide

\section{Á. Gragera Martínez ${ }^{1}$, L. Herranz Arriero ${ }^{2}$, C. Borrachero Garro $^{l}$, F. Muñoz Beamud ${ }^{l}$}

${ }^{1}$ Juan Ramon Jimenez University Hospital, Huelva, Spain, ${ }^{2}$ Badajoz University Hospital, Badajoz, Spain

Introduction: Hereditary amyloidosis mediated by transthyretin (hATTR) is a hereditary, neurodegenerative, progressive, highly disabling and life-threatening disease in short term if an early treatment is not established. It is a rare disease, with prevalence less than $1 / 100,000$ habitants in worldwide. Huelva is one of the most important endemic foci of the disease worldwide and the second in Spain. Inherited with an autosomal dominant pattern characterized by a reduced penetrance, which varies according to age and geographic area. This poses big problems and big challenges to monitoring patients and their families.

Objectives: To know the penetrance in the endemic focus of Valverde del Camino, Huelva

Methodology: A retrospective, observational study was performed. For this work, medical records have been collected and statistically analyzed. Demographic details (sex, age at diagnosis of the disease, age at the onset of symptoms) were obtained

Results: Data from 112 patients have been analyzed

Conclusions: The prevalence obtained in our population is quite different between mens and womens. A higher proportion of men develop symptoms, most women with the pathogenic variant are asymptomatic. This could be explained by the presence of polymorphisms in the TTR gene that modulate disease expression as well as clinical symptoms. This is a very interesting field of study that will allow us to better understand the disease.

\begin{tabular}{llll}
\hline \multicolumn{2}{l}{ Population Penetrance } & \\
\hline $\begin{array}{l}\text { Age } \\
\text { (years) }\end{array}$ & $\begin{array}{l}\text { Overall } \\
\text { penetrance } \%\end{array}$ & $\begin{array}{l}\text { Mens } \\
\text { penetrances }\end{array}$ & $\begin{array}{l}\text { Womens } \\
\text { penetrance \% }\end{array}$ \\
\hline 30 & 3 & 4 & 0 \\
40 & 10 & 16 & 2 \\
50 & 16 & 20 & 9 \\
60 & 28 & 33 & 21 \\
70 & 34 & 40 & 25 \\
$>70$ & 51 & 60 & 37 \\
\hline
\end{tabular}

Á. Gragera Martínez: None. L. Herranz Arriero: None. C. Borrachero Garro: None. F. Muñoz Beamud: None.

\section{E-P10.04}

Amyotrophic lateral sclerosis (ALS) in the Faroe Islands, a genealogical approach

\author{
M. Johansen ${ }^{1,2}$, K. Svenstrup ${ }^{3}$, Ó. Mortensen $^{4}$, \\ G. Andorsdóttir ${ }^{4}$, M. S. Petersen ${ }^{1,2}$
}

${ }^{I}$ Department of Occupational Medicine and Public Health, The Faroese Hospital System, Tórshavn, Faroe Islands, ${ }^{2}$ Centre of Health Sciences, University of the Faroe Islands, Tórshavn, Faroe Islands, ${ }^{3}$ Neurological Department, University Hospital Copenhagen, Rigshospitalet and 
Bispebjerg Hospital, Copenhagen, Denmark, ${ }^{4}$ FarGen, The Genetic Biobank of the Faroe Islands, Tórshavn, Faroe Islands

ALS is a progressive neurodegenerative disease. In 5-10\% of cases, a family history is identified. Although the incidence of ALS in the Faroes is similar to other European countries, we have observed an overrepresentation of cases originating from the small geographically isolated island of Suðuroy, which lead to the study aim: estimation of familial ALS in the Faroese population. All ALS cases have been registered since 1987. In this study, 43 patients were included. First, familial ALS was recognized when occurring between first- or second-degree relatives. Secondly, the Faroese Multi Generation Register, which contains information about familial relationships since 1650, was used to construct pedigrees amongst patients to identify risk families ( $\geq 3$ cases within 3 generations). Fifteen cases originated from Suðuroy where the prevalence of ALS was more than 3 times higher than the nationwide prevalence. Six patients had familial ALS (13.9\%) of which four originated from Suðuroy. Through pedigrees, we identified six additional patients with possible familial ALS (28\%). This study is suggestive of familial clustering in excess of expected for ALS in Suðuroy and the proportion of familial ALS in the Faroe Islands was high. High levels of inbreeding and genetic substructure have been demonstrated in the Faroese population. Combined with these results, there is strong support of a genetic contribution to ALS in the Faroe Islands and further genetic studies are warranted. Funding: Aage og Johanne Louis-Hansens Fond, Minister Erna Hamiltons Legat for videnskab og kunst, Sjúkrakassagrunnurin, Betri Stuðul, Aase og Ejnar Danielsens Fond.

M. Johansen: None. K. Svenstrup: None. Ó. Mortensen: None. G. Andorsdóttir: None. M.S. Petersen: None.

\section{E-P10.05}

Efficiency of the MPS panel of 15 genes associated with genetically heterogeneous hereditary neuropathies

\section{D. Orlova, O. A. Schagina, O. P. Ryzhkova, \\ G. E. Rudenskaya, E. L. Dadali, A. V. Polyakov}

Research Centre for Medical Genetics, Moscow, Russian Federation

Introduction: Charcot-Marie-Tooth disease is a genetically heterogeneous pathology related to hereditary peripheral neuropathies. The purpose of the research is to evaluate the efficiency of panel sequencing for a highly genetically heterogeneous pathology.
Materials and methods: We have studied the DNA of 43 Russian CMT-patients (25 myelinopathy, 18 axonopathy) after excluding the most common cause of CMT - PMP22 duplications. MPS libraries were prepared with the use of Ion AmpliSeq ${ }^{\mathrm{TM}}$ reagents and sequencing by Ion S5 ${ }^{\mathrm{TM}}$ System. Panel includes 15 CMT genes: MFN2, MPZ, EGR2, FGD4, LITAF, PMP22, PRX, MORC2, SH3TC2, HINT1, HSPB1, NEFL, NDRG1, GDAP1, GJB1, mutations in which were previously identified as the cause of the disease for at least 2 unrelated Russian families.

Results: After the targeted MPS sequencing, a definite molecular diagnosis defined as a pathogenic or a likely pathogenic variant, was reached in $16 \%$ of cases $(n=7)$. Variants of unknown significance were found in $16 \%$ of cases $(n=7) .68 \%(n=29)$ of cases were molecularly undiagnosed. In most cases variants were found in the SH3TC2(5) and MFN2(3) genes. 6 patients had variants in the following genes: MPZ, GDAP1, HINT1, LITAF, PRX, MORC2.

Conclusion: Diagnoses by the MPS panel were confirmed for $16 \%$ of the patients, while the WES is efficient in $19-45 \%$ of cases. Thus, small MPS-panel is a useful tool for highly genetically heterogeneous pathology. It helps to avoid consequent tests of frequent genes associated with CMT with the Sanger sequencing while having the efficiency comparable to WES.

M.D. Orlova: None. O.A. Schagina: None. O.P. Ryzhkova: None. G.E. Rudenskaya: None. E.L. Dadali: None. A. V. Polyakov: None.

\section{E-P10.06}

Rapid exome sequencing with in silico variant prioritizing and the diagostic odyssey of a child with various neurological symptoms

\section{A. Illés ${ }^{l}, K$. Árvai $^{l}$, B. Kocsis-Deák ${ }^{1}$, Á. Herczegfalvi ${ }^{2}$, P. Lakatos ${ }^{3}$, J. Kósa ${ }^{1,3}$}

${ }^{1}$ PentaCore Laboratories, Budapest, Hungary, ${ }^{2} 2 n d$ Department of Pediatrics, Semmelweis University, Budapest, Hungary, ${ }^{3}$ Department of Internal Medicine and Oncology, Semmelweis University, Budapest, Hungary

Introduction: The hereditary motor and sensory neuropathies are a heterogenous disease group which could be caused by several gene mutations, such as deletions, duplications and single nucleotide substitutions. The complex genetic etiology could harden the differential diagnostics and lengthen the path to identify the genetic background.

Material and methods: In our case, a four-year-old boy was examined, who born with hypotonia and showed delayed motor development. At the age of three, EMG 
analysis showed axonal polyneuropathy and motor nerve conduction could not be detected in his legs. After the analysis of copy number variations of PMP22 and SMN genes, further neuropathies associated genes were sequenced without any results. Finally, whole exome sequencing was performed, and the results were analysed by focusing on primer neuropathy associated genes.

Results: A pathogenic heterozygous S72L mutation (rs 104894621) was identified in the PMP22 gene, which was previously associated with Charcot-Marie-Tooth disease. Sanger sequencing also confirmed the result.

Conclusions: Based on our result the clinical diagnosis was confirmed and the genetic counsellor could provide adequate information to the parents before the birth of the second child. It is important to highlight that in case of neuropathies, after screening copy number variations, performing whole exome sequencing could shorten and make easier the identification of potential pathogenic mutations in the background of the symptoms. The fast identification of causal variants could contribute to the selection of specific therapy and family planning.

A. Illés: None. K. Árvai: None. B. Kocsis-Deák: None. Á. Herczegfalvi: None. P. Lakatos: None. J. Kósa: None.

\section{E-P10.10}

A novel mutation of DYSF gene in a patient with Limb Girdle Muscular Dystrophy type 2b

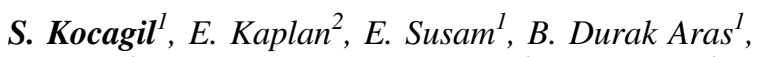 S. Artan ${ }^{l}$, E. Erzurumluoglu Gokalp ${ }^{l}$, O. Cilingir ${ }^{l}$}

${ }^{1}$ Eskisehir Osmangazi University Medical Genetics Department, Eskisehir, Turkey, ${ }^{2}$ Eskisehir Osmangazi University Pediatric Neurology Department, Eskisehir, Turkey

Dysferlinopathy muscular disease spectrum encompasses a group of phenotypes; including Limb Girdle Muscular Dystrophy type 2b, Myoshi myopathy, scapuloperoneal syndrome, distal myopathy with anterior tibial onset, elevated serum CK concentration only, and congenital muscular dystrophy. Biallelic pathogenic variants of DYSF gene is known to be responsible for dysferlinopathies. The prevalence of the disease is unknown but incidence is higher in the populations that consanguineous marriage is observed frequently. Here we report a 17 years-old female patient that was referred to our outpatient clinics for proximal muscle weakness. She had difficulty in walking and climbing stairs for the last 6 months. In her physical examination; her deep tendon reflexes of the lower extremities were diminished, her muscle strength was normal and she could walk on the tip of her toes and stand on her heels. Her serum creatine kinase levels were detected over 20.000 U/L repetitively therefore muscle biopsy was planned. Quadriceps femoris muscle histology revealed positive sarcoglycan proteins but reduced dysferline. Due to the muscle biopsy result we have planned DYSF gene sequence analysis and a novel c.754A $>\mathrm{G}$ (p.Thr252Ala) variant was detected in homozygous state. This variant was classified as likely pathogenic according to ACMG criteria and segregation analysis revealed the parents of the proband as carrier. According to her physical examination and the result of the molecular analysis we have concluded that this novel mutation generates a phenotype as Limb Girdle Muscular Dystrophy Type $2 b$ of the dysferlinopathy spectrum.

S. Kocagil: None. E. Kaplan: None. E. Susam: None. B. Durak Aras: None. S. Artan: None. E. Erzurumluoglu Gokalp: None. O. Cilingir: None.

\section{E-P10.12}

The retrieval of candidate genes of inverse comorbidity between cancer and neurodegeneration considering apoptosis as a key process

\author{
D. Gomboeva ${ }^{l}$, O. Saik ${ }^{2}$, E. Bragina ${ }^{l}$, V. Ivanisenko ${ }^{2}$, \\ V. Puzyrev ${ }^{l}$
}

${ }^{I}$ Tomsk National Research Medical Center, Tomsk, Russian Federation, ${ }^{2}$ Federal Research Center Institute of Cytology and Genetics, Novosibirsk, Russian Federation

Introduction: Epidemiological studies have revealed the reduced cancer incidence in patients with Huntington disease (HD), indicating a presence of inverse comorbidity or dystropy of HD and cancer. The one of promising biological process, which unites these two groups of diseases, is apoptosis. Cancer is characterized by inhibited apoptosis, while in HD, on contrary, apoptosis is up-regulated. Thus, identification of pro- and anti-apoptotic genes and study of their regulation is hopeful for investigation of inverse comorbidity mechanisms of HD and cancer.

Materials and methods: The genetic network was reconstructed using ANDSystem (Ivanisenko, 2015). Network included all genes and proteins associated with HD $(n=140)$. The list of genes of apoptosis was retrieved from KEGG (hsa04210 identifier, $n=136$ ). Prioritization was carried using ToppGene and functions of ANDSystem. The special criteria were calculated, including: betweenness centrality, stress centrality, closeness centrality, cross-talk centrality and cross-talk specificity (Saik et al., 2018). The search of miRNA was conducted using MirTarBase database.

Results: 10 genes were identified: $A P O E, B D N F$, HSPA4, HTT, IL6, INS, LEP, PSEN1, SPI, SQSTM1. Through the analysis at the miRNA level, we retrieved 5 common miRNA (let-7c-5p; miR-1-3p; miR-149-5p; miR$22-3 p$; miR-223-3p) regulate of expression of protein 
coding genes (SP1, HSPA4, BDNF, IL6). All genes are important for neurodegeneration and associated with cancer: play different roles in carcinogenesis, cancer progression dependent on type of cancer.

Conclusions: The approach uncovered miRNA- apoptotic genes regulation might give new insights into the mechanisms of inverse comorbidity between cancer and neurodegeneration. This work was supported by the RFBR grant No.19-015-00391.

D. Gomboeva: None. O. Saik: None. E. Bragina: None. V. Ivanisenko: None. V. Puzyrev: B. Research Grant (principal investigator, collaborator or consultant and pending grants as well as grants already received); Modest; Russian Foundation for Basic Research.

\section{E-P10.14}

A novel compound heterozygous MPV17 mutation causing axonal sensorimotor polyneuropathy

D. ESIN ${ }^{l}$, F. DUYMUS ${ }^{l}$, B. GOKSEL TULGAR ${ }^{l}$, G. TURKOGLU ${ }^{1}$, M. E. SARI ${ }^{1}$, T. CORA ${ }^{1}$, A. KARTAL ${ }^{2}$

${ }^{1}$ Selcuk University School Of Medicine, Medical Genetics Department, Konya, Turkey, ${ }^{2}$ Selcuk University School of Medicine, Pediatric Neurology Department, Konya, Turkey

Charcot-Marie-Tooth disease type 2EE is an autosomal recessive sensorimotor peripheral axonal neuropathy that is caused by mutations in the MPV17 gene. MPV17 encodes a channel-forming protein of the inner mitochondrial membrane. In this case, it is aimed to present the first clinical report of MPV17-related axonal sensorimotor neuropathy with compound heterozygous mutation. The patient was a 16-yearold male and these second child of healthy, consanguineous parents. He had severe distal muscle atrophy, pes cavus deformity, foot drop, hammertoes, atrophy of the intrinsic hand muscles and bilateral thenar and hypothenar muscles. Hyporeflexia of upper limbs was observed. The first symptom of the patient was the loss of balance and walking difficulty starting at the age of six. c.122G $>\mathrm{A}$ and c.376-9T> A mutations in the MPV17 gene was detected by whole-exome sequencing. The MPV17:c.122G $>$ A variant has already been described as a pathogenic variant. The MPV17:c.376-9T> A variant described as a variant of uncertain significance. To the best of our knowledge, the coexistence of these two heterozygous mutations was not found in the literature review. In this case report, we emphasize that a novel compound heterozygous MPV17 mutation can cause non-syndromic peripheral axonal neuropathy and should be considered in the differential diagnosis of juvenile-adult onset severe axonal sensorimotor neuropathy.
D. Esin: None. F. Duymus: None. B. Goksel tulgar: None. G. Turkoglu: None. M.E. Sari: None. T. Cora: None. A. Kartal: None.

\section{E-P10.15}

LGMD 2E: using sequence analysis to verify sarcoglycanopathy

L. Khlunovska ${ }^{\text {, I. Lastivka }}{ }^{l}$, V. Antsupova ${ }^{2}$, M. Ryznychuk ${ }^{l}$, L. Hodovaniuk ${ }^{3}$

${ }^{1}$ HSEE of Ukraine, "Bukovinian State Medical University", Chernivtsi, Ukraine, ${ }^{2}$ Bohomolets National medical university, Kyiv, Ukraine, ${ }^{3}$ Bukovynian Center for Rehabilitation and Integrated Rehabilitation of Children, Chernivtsi, Ukraine

Introduction: Muscular dystrophy of the lumbar girdle (LGMD) is a heterogeneous group of hereditary progressive neuromuscular diseases.

Material and Methods: A clinical case of LGMD 2E in a 9 year old child. Clinical, genealogical, laboratory and instrumental and molecular-genetic methods were used.

Results: Girl K., up to 8 years old, developed in accordance with age norms. At age 8 , the mother began to notice motor impairment in the child; at age 9, a duck walk appeared. It became difficult for the child to climb the stairs, squat and jump; there was pain in the calf muscles. The girl complained of fatigue during normal physical activity. Objective data: stimulation neuromyography - a decrease in the muscles of the lower extremities (47\% on the right and $61 \%$ on the left); a 35 -fold increase in creatinekinase levels. To clarify the diagnosis, a moleculargenetic examination was assigned: analysis and testing of the removal/duplication of 123 strength genes (Los Angeles, 2019). Two pathogenic variants $\quad$ c.2T $>C$ and c.341C $>\mathrm{T}$ (p.Ser114Phe) were identified in the SGCB gene. The SGCB gene is associated with an autosomal recessive LGMD type 2E. Final diagnosis: LGMD $2 \mathrm{E}$ (sarco-glycanopathy).

Conclusions: Significant difficulties arise in the diagnosis of genetic variants of neuromuscular diseases only on the basis of a clinical study. The use of sequence analysis makes it possible to clarify the type of muscular dystrophy. Given the hereditary nature of the disease, it is necessary to recommend genetic counseling and molecular genetic screening for the purpose of prophylaxis and/or prognosis of offspring.

L. Khlunovska: None. I. Lastivka: None. V. Antsupova: None. M. Ryznychuk: None. L. Hodovaniuk: None. 


\section{E-P10.18}

Screening of SMA carrier status in Republic of Moldova

\section{Coliban ${ }^{1}$, N. Ușurelu ${ }^{2}$, V. Sacar $\bigotimes^{l}$}

${ }^{1}$ Laboratory of Human Molecular Genetics, Institute of Mother and Child, Chisinau, Moldova, Republic of, ${ }^{2}$ Laboratory Prophylaxis of Hereditary Pathologies, Institute of Mother and Child,, Chisinau, Moldova, Republic of

Introduction: Spinal muscular atrophy (SMA) is an autosomal-recessive disease, with a frequency of carriers of 1:35 up to 1: 117 in the Caucasian population. The prevalence of SMA in the Republic of Moldova constitutes 8.43 $\pm 0,15$ : 100000 population which is significantly higher than the data from Orphanet $(2,93)$ and Russia $(0,31-3,91) .95 \%$ of SMA is caused by deletion of exon 7 of SMN1. If both persons in a couple are carriers, they have a 1-in-4 chance of having a child with SMA.

Materials and methods: 30 persons that formed 15 couples who addressed for family planning, without a family history of SMA were diagnosed for determining the status of exon 7 SMN1 by qPCR method, melting curve. The DNA concentration was measured by spectrophotometry. For PCR reaction each DNA sample was used as 2 replicates for $S M N 1$ exon 7 and 1 replicate for the external standard ( $A L B$, exon 12). EvaGreen was used as a DNAbinding dye.

Results: For all DNA samples, amplification occurred for both exon $12 A L B$ and exon 7 SMN1. According to the melting curves, for 22 persons the exon7 SMN1 was determined to be present and for 8 persons was determined heterozygous status (5 women and 3 men). Among those who are heterozygous, 2 people form the same couple.

Conclusions: Following the Hardy-Weinberg equilibrium $(0,23)$ for $26 \%$ of the patients, the status of carriers of the deletion was established. Screening of SMA carriers is an important step in assessing the risk of giving birth to an affected child.

I. Coliban: None. N. Ușurelu: None. V. Sacară: None.

\section{E-P10.19}

A case of Spinal Muscular Atrophy with Congenital Bone Fractures 2 expands ASCC1 prenatal presentation and mutational spectrum
G. Mastromoro ${ }^{1}, M$. Giuffrida ${ }^{2}$, V. Guida ${ }^{2}$,
D. Guadagnolo ${ }^{l}$, E. Marchionni, ${ }^{1}$ F. Di Palma ${ }^{l}$,

M. Truglio ${ }^{2}$, M. Fabbretti ${ }^{2}$, B. Torres ${ }^{2}$, T. Mazza ${ }^{2}$, A. De Luca ${ }^{2}$, M. Roggini ${ }^{1}$, L. Bernardini ${ }^{2}$, A. Pizzuti ${ }^{1}$

${ }^{1}$ Sapienza University of Rome, Rome, Italy, ${ }^{2}$ Casa Sollievo della Sofferenza IRCCS Foundation, San Giovanni Rotondo, Italy

Introduction: Spinal muscular atrophy with congenital bone fractures 2 (SMABF2) is a rare autosomal recessive neuromuscular disorder characterized by arthrogryposis multiplex congenita and prenatal fractures. Reported patients present biallelic loss-of-function single-nucleotide variants in ASCC1 gene, encoding a subunit of the transcriptional coactivator ASC-1 complex. We describe the first SMABF2 case with documented prenatal disease evolution, caused by an in-frame microdeletion and a nonsense nucleotide variant in a stillbirth.

Materials and methods: The stillbirth, born from nonconsanguineous parents, presented hydrops, lower limbs hyperextension with bending of the hips, bilateral club-foot and congenital fractures. Prenatal ultrasound had showed bilateral club-foot, closed fists and fetal biometric parameters at middle-lower limits. Microarray analysis was performed on DNA extracted from umbilical cord. Data were analyzed by CytoGenomics software. Next Generation Sequencing analysis was performed using the TruSightOne sequencing panel kit. NGS data were processed and analyzed using an in-house implemented pipeline.

Results: A $64 \mathrm{~Kb}$ microdeletion, involving four exons of ASCC1, was detected by microarray analysis. NGS disclosed a single-nucleotide variant of the same gene (c.1027C $>\mathrm{T}$; $\mathrm{p}$. Arg $343 *$, resulting in a premature termination codon.

Conclusions: This is the first case of ASCC1 due to an exonic microdeletion and a nucleotide variant in compound heterozygosity, expanding the mutational spectrum of this gene. This case provides further evidence that exonic copy number variants are an underestimated cause of diseasealleles and that the integrated use of the last generation genetic analysis tools, together with careful clinical evaluations, are fundamental for the characterization of rare diseases even in prenatal setting.

G. Mastromoro: None. M. Giuffrida: None. V. Guida: None. D. Guadagnolo: None. E. Marchionni: None. F. Di Palma: None. M. Truglio: None. M. Fabbretti: None. B. Torres: None. T. Mazza: None. A. De Luca: None. M. Roggini: None. L. Bernardini: None. A. Pizzuti: None.

\section{E-P10.20}

An interesting case: 30-year-old male patient with myotonic dystrophy typeI has got normal CTG repeats sequence in the DMPK gene, but it does not comply with its clinical features 
S. Tutgun Onrat ${ }^{1}$, M. Elmas ${ }^{1}, H$. Demirbas $^{2}$

${ }^{1}$ Afyon Health Science University, Faculty of Medicine, Department of Medical Genetics, Afyonkarahisar, Turkey, ${ }^{2}$ Afyon Health Science University, Faculty of Medicine, Department of Neurology, Afyonkarahisar, Turkey

Introduction: Myotonic dystrophy type 1 DM1 is inherited in an autosomal dominant pattern a multisystem disorder, caused by expansion of a CTG trinucleotide repeat in the $3^{\prime}$ untranslated region of the DMPK gene, with pathogenic alleles ranging from around 50 to over 1000 CTG triplets repeats (1). Some features include skeletal muscle weakness and myotonia. The age at onset is extremely variable in DM1, and inversely correlates with the expansion size and individual-specific differences in somatic instability (2-4).

Materials and methods: We want to present most interestingly our patient-male (30 years) with Myotonic Dystrophy Type I disease, the exact number of repeats of the CTG trinucleotide for both alleles of the DMPK1 gene was detect by PCR-Fragman analysis. Afterwards, the age of the disease, the clinical severity of the disease, radiological and laboratory findings were compared with these patients. In addition, detailed pedigree analysis of the patients was performed and thus anticipation and variable expressivity of the disease was determined.

Results: We find a result of the important in terms of myotonic dystrophy; he has got clinically myotonic discharges in EMG(electromyography) diagnosed as myotonic dystrophy, but when examined in terms of the number of repeats, the DMKP1 gene was a normal number of repeats in terms of $8 / 17$ for both alleles.

Conclusions: Myotonic dystrophies represent the most variable clinical phenotypes, so treatment stratification is key for any modern therapeutic approach. We still need much more understanding of the signs and symptoms of Myotonic dystrophies patients in correlation to their molecular origins.

S. Tutgun Onrat: None. M. Elmas: None. H. Demirbaş: None.

\section{E-P11 Multiple Malformation/Anomalies Syndromes}

\section{E-P11.001}

16p13.11 microduplication in a patient with seizures: A case report about an elusive syndrome

\section{S. Yildirim, A. B. Arslan, A. G. Zamani}

Necmettin Erbakan University, Konya, Turkey

Background: The short arm of chromosome 16, (16p) and especially the 16p13.11 region is involved in many structural variants. To date, deletions of this region is mostly considered as pathogenic; yet duplications are still controversial. Here a patient with $16 \mathrm{p} 13.11$ duplication that have findings concordant with previous reports and two additional findings is presented, aiming to further enhance our understanding of the nature of $16 p$ rearrangements.

Materials and methods: After the DNA was isolated from the peripheral blood sample of the patient, the data obtained by microarray using the "afyymetrix cytoscan optima array kit" were analyzed for gain or loss according to the GRCh37 (hg19) reference genome using ChAS 3.1.1.27 (Chromosome Analysis Suite) software.

Case Report: Patient is a 5 years and 2 months old female, admitted to pediatric neurology department with the complaints of seizures and speech delay. She was diagnosed with epilepsy and atypic autism. A peripheral blood karyotyping was normal $(46, \mathrm{XX})$. Microarray result of the patient revealed a $1833 \mathrm{kbp}$-long microduplication, involving the $16 \mathrm{p} 13.11$ region. (arr[hg19] 16p13.11 $(14,897,804-16,730,375) \times 3)$

Discussion: 16p13.11 duplications were previously described as rare benign variants whereas there are also some reports of pathogenicity; relating it to a phenotypic spectrum involving autism, intellectual disability, hypotonia, motor and speech delay, seizures, abnormal MRI and cardiac malformations and this spectrum applies for our patient as well. Additionally, upslanting palpebral fissures and preauricular skin tag are important phenotypic findings of our patient that were not reported before.

M.S. Yildirim: None. A.B. Arslan: None. A.G. Zamani: None.

\section{E-P11.004}

Phenotypic features in a patient with 1 p36 deletion syndrome

\section{F. Stipoljev ${ }^{1,2}$, A. Vicic $^{1,3}$, R. Gjergja-Juraski ${ }^{4,2}$}

${ }^{1}$ Clinical Hospital “Sveti Duh”, Zagreb, Croatia, ${ }^{2}$ Faculty of Medicine, Josip Juraj Strossmayer University of Osijek, Osijek, Croatia, ${ }^{3}$ Univesity of Applied Health Sciences, Zagreb, Croatia, ${ }^{4}$ Children's Hospital Srebrnjak, Zagreb, Croatia

Monosomy $1 \mathrm{p} 36$ is characterized by variable size deletions, while the phenotypic features include intellectual disability, developmental delay, distinctive facial dysmorphic features, hypotonia, seizures, cardiac defects, hearing impairment and ophthalmologic problems. Herein we present a patient with 1 p36 deletion syndrome. The girl was born with muscular VSD and the Pierre Robin sequence. She was operated due to the Pierre Robin at the early age and got tracheal canulla up to 3 years of age. MRI of the neuroaxis 
later revealed the syrinx of the spine. She has distinctive facial features including microbrachycephaly, deep-set eyes with straight eyebrows; midface hypoplasia with broad, flat nose and philtrum. Ears are low-set, rotated backwards and abnormally shaped. Compared to other described cases, her chin is rather small. There are no abnormalities of gastrointestinal system, kidneys, or genitalia. She is hypotonic, with severe intellectual disability, refractory epilepsy and temper tantrums; having vision, hearing and speech problems. Array CGH analysis using SurePrint G3 Human CGH $8 \times 60 \mathrm{~K}$ microarray revealed $4.3 \mathrm{Mb}$ loss in the region 1p36.33-p36.32. Deletion was confirmed with FISH analysis using subtelomeric $1 \mathrm{p}$ probe, and a karyotype was designated as 46,XX,del(1)(p36.32)dn. Cytogenetic analysis of parental peripheral blood revealed normal female karyotype in mother and a 46,XY,inv(10)(p11.2q21.2) karyotype in father, while FISH analysis showed normal distribution of signals characteristic for subtelomeres $1 p$ in both parents. In conclusion, our case emphasizes the significance of molecular cytogenetic analysis in order of establishing accurate diagnosis and patient management, as well as counseling parents regarding future pregnancies.

F. Stipoljev: None. A. Vicic: None. R. GjergjaJuraski: None.

\section{E-P11.005}

Identification of clinical features to predict 22q11.2 deletion syndrome, proven by multiplex ligation-dependent probe amplification

\section{K. Rojnueangnit ${ }^{1}$, T. Khetkham ${ }^{1}$, P. Onsod ${ }^{2}, T$. Chareonsirisuthigul ${ }^{2}$}

\section{${ }^{1}$ Thammasat University, Klong-Luang, Thailand, ${ }^{2}$ Mahidol} University, Bangkok, Thailand

Introduction: $22 \mathrm{q} 11.2$ deletion syndrome (22q11.2 DS) is the most common microdeletion syndrome with a wide variety of clinical features. However, there are no clinical criteria for diagnosis; confirmation is solely by genetic tests. Normally, these tests are only performed if clinicians recognize signs and symptoms; therefore, we aimed to identify any clinical features that may help clinicians be aware of 22q11.2 DS.

Materials and methods: Participants with at least two anomalies were enrolled from June 2015 - May 2018 at Thammasat University Hospital. Complete patient history and physical examinations were done; multiplex ligationdependent probe amplification analysis for $22 \mathrm{q} 11.2$ were performed with blood samples.

Results: Out of 48 participants, we identified 11 (23\%) with 22q11.2 DS. Palate anomalies, hypocalcemia, and $\geq 3$ affected body systems were highly significant presentations in the 22q11.2 DS group versus the group without deletion $(p<0.05)$. Conotruncal cardiac defects alone were insufficient for 22q11.2 DS prediction but sufficient when accompanied with other anomalies.

Conclusion: A comprehensive physical examination is crucial at identifying any subtle features which may lead to testing and a definite diagnosis. Grant from Thammasat University (Grant No.2/32/2558)

K. Rojnueangnit: None. T. Khetkham: None. P. Onsod: None. T. Chareonsirisuthigul: None.

\section{E-P11.006}

Diverse phenotypes in a family of six all with $22 q 11.2$ duplication or double duplication

A. Zagorac ${ }^{l}$, A. Golub ${ }^{l}$, B. Zagradišnik ${ }^{1}$, A. Erjavec $\check{S ̌ k e r g e t}^{l}$, D. Krgovic ${ }^{1}, \breve{S}$. Stangler Herodežl, A. Osterc Koprivšek ${ }^{1}$, N. Kokalj Vokač ${ }^{1,2}$

${ }^{1}$ University Medical Centre Maribor, Maribor, Slovenia, ${ }^{2}$ Medical Faculty Maribor, University Maribor, Maribor, Slovenia

Introduction: 22q11.2 duplications may be largely undetected, as a result of less-distinct, unpredictable, and/or milder phenotype. Double duplications 22q11.2 are extremely rare. We describe the family of six, all with 22q11.2 duplication or double duplication. The boy proband with duplication had a global developmental delay, seizures and dysmorphic features. The girl proband with double duplication, had sensorineural hearing loss, mild intellectual disabilities, mild speech delay and minor dismorphic features. Other relatives were unaffected, except for very mild possible manifestations in the mother.

Materials and methods: aCGH analysis using $8 \times 60 \mathrm{~K}$ array, microsatellite segregation analysis and QF-PCR for the informative marker on peripheral blood for all family, were performed. Clinical exsom analysis by NGS of the affected boy and the girl, were done.

Results: aCGH revealed 3 copies, approximately $2,8 \mathrm{Mb}$, of 22q11.2 in both parents, affected boy and one unaffected girl and 4 copies of the same region in one affected girl and another unaffected girl. Segregation microsatellite analysis and QF-PCR confirmed that both parents carry the duplication (2:1), two children inherited duplication from their mother, another two daughters inherited two copies of 22q11.2 (2:2) from each parent, it's a double duplication. NGS analysis didn't identify any additional mutations that would be clinically characterized as being pathogenic.

Conclusions: The family described illustrates the phenotypic variability associated with $22 \mathrm{q} 11.2$ duplication or double duplication. It provides support for the view that the 22q11.2 duplication/double duplication may not be 
pathogenic on its own and require additional genetic, epigenetic or nongenetic factors, to express the phenotype.

A. Zagorac: None. A. Golub: None. B. Zagradišnik: None. A. Erjavec Škerget: None. D. Krgović: None. Š. Stangler Herodež: None. A. Osterc Koprivšek: None. N. Kokalj Vokač: None.

\section{E-P11.007}

A homozygous WDR91 nonsense variant responsible of severe form of $3 \mathrm{C}$ syndrome

\section{N. BOURGON ${ }^{1,2}$, M. LEFEBVRE $E^{1,2}, A$. BRUEL $^{1,2}$, J. THEVENON ${ }^{1,2}$, J. RIVIERE ${ }^{1,2}$, C. POE ${ }^{1,2}$, M. CHEVARIN ${ }^{1,2}$, T. JOUAN ${ }^{1,2}$, Y. DUFFOUR ${ }^{1,2}$, L. FAIVRE ${ }^{1,2,3,4}$, C. THAUVIN ${ }^{1,2,3,4}$}

${ }^{1}$ UMR 1231, GAD team, DIJON, France, ${ }^{2} F H U$ TRANSLAD, Dijon, France, ${ }^{3}$ Centre de Génétique, CHU Dijon Bourgogne, Dijon, France, ${ }^{4}$ Centre de référence Anomalies du Développement, Dijon, France

Introduction: $3 \mathrm{C}$ syndrome is a rare genetic syndrome characterized by craniofacial anomalies, cardiac and cerebral related to WSHC5 (MIM \# 220210) and CCDC22 (MIM \# 300963) variants. Molecular bases of some cases suspected of 3C syndrome remained identified.

Patients and methods: We report 4 fetuses with $3 \mathrm{C}$ syndrome from a unique consanguineous family. Case 1 had a fetal hydrops with hygroma coli, cerebellar hypoplasia, severe tetraventricular hydrocephalus with macrocephaly, facial dysmorphia and interventricular septal defect. The 3 other cases have been suspected with fetal hydrops during the first trimester associated with brain abnormalities.

Results: ES performed in case 1 identified a homozygous WDR91 nonsense variant (NM_014149.3:c.240C $>$ G, p. Tyr80*) in a homozygous region. Family segregation confirmed the homozygous status of this variant in all affected fetuses and in the heterozygous status in both parents, as well as two healthy children and absence in the third healthy child. International datasharing and targeted sequencing of a cohort of $3 \mathrm{C}$ syndrome patients have so far failed to identify other patients with WDR91 variants. WDR91 interacts with WDR81 in the endosome-lysosome pathway for early-to-late endosome conversion. WDR91 appears to be essential, particularly for neuronal and brain development.

Conclusion: Identification of a homozygous WDR91 nonsense variant in several fetuses with $3 \mathrm{C}$ syndrome in a consanguineous family suggests that WDR91 is a new gene responsible for this syndrome. Identification of new similar cases thanks to international datasharing would allow us to conclude on the implication of this gene in a severe form of 3C syndrome.

N. Bourgon: None. M. Lefebvre: None. A. Bruel: None. J. Thevenon: None. J. Riviere: None. C. Poe: None. M. Chevarin: None. T. Jouan: None. Y. Duffour: None. L. Faivre: None. C. Thauvin: None.

\section{E-P11.008}

Allan Herndon Dudley syndrome due to a novel pathogenic variant in the $S L C 16 A 2$ gene

\section{T. N. Delchev, H. M. Kathom, D. M. Avdjieva-Tzavella}

\section{Medical University-Sofia, Sofia, Bulgaria}

Introduction: Allan-Herndon-Dudley syndrome (AHDS) is a rare, X-linked condition caused by various pathogenic variants in the SLC16A2 gene. In hemizygous males AHDS is characterized by dysthyroidism, hypotonia and feeding difficulties (in infancy), developmental delay and drugresistant seizures. Additional findings may include pyramidal and extrapyramidal signs as well as intellectual disability. Heterozygous females are rarely affected.

Materials and methods: We present a 9 month old boy with facial dysmorphism, psychomotor delay and myoclonus in addition to hypomyelination findings on brain MRI. Dysthyroidism (elevated T3, low T4, fT4 levels and normal TSH) alongside poor weight gain, irritability and axial hypotonia were also found. Based on those findings a targeted SLC16A2 gene sequencing was performed.

Results: A novel hemizygous variant, c.695del (p. Asn232Metfs*35), was identified in SLC16A2 confirming the diagnosis - Allan-Herndon-Dudley syndrome (MedGen UID: 208645). This variant is neither present in population databases (ExAC no frequency) nor reported in the literature in individuals with SLC16A2-related conditions. This sequence change creates a premature translational stop signal and has been classified as pathogenic.

Conclusions: Allan-Herndon-Dudley syndrome, however rare, should be considered in children manifesting the typical clinical, phenotypic, laboratory and imaging findings. In our case we found a specific constellation of developmental delay and dysthyroidism which lead to targeted sequencing analysis and the correct diagnosis. We hope that this novel pathogenic variant in SLC16A2 will provide additional information about AHDS and its genotype-phenotype correlations.

T.N. Delchev: None. H.M. Kathom: None. D.M. Avdjieva-Tzavella: None. 


\section{E-P11.009}

Clinical characterization of Aicardi-Goutières Syndrome 7 in an extreme preterm infant, due to a recurrent IFIHI variant

\section{E. Phillips, M. Kerr, A. Khan}

Departments of Medical Genetics and Pediatrics, University of Calgary Cumming School of Medicine, Alberta Children's Hospital Research Institute., Calgary, AB, Canada

Introduction: Aicardi-Goutières Syndrome (AGS) is a genetically heterogeneous disorder of interferon 1 upregulation. Approximately $10 \%$ of patients present at birth with a congenital infection-like syndrome with hepatosplenomegaly, thrombocytopenia, neurologic abnormalities and brain calcifications. Characterization of this disorder in premature infants is limited, and diagnosis in this group is challenging. We present a case of AGS type 7 in an extreme preterm infant, and highlight the role of whole exome sequencing (WES) in providing a timely diagnosis.

Materials and methods: A female infant was delivered at $27+2$ for pre-eclampsia, IUGR, and abnormal Dopplers. From birth, she had refractory thrombocytopenia necessitating almost-daily transfusions, as well as anemia, progressive hepatosplenomegaly, cholestasis, pulmonary hypertension, respiratory insufficiency, poor feeding, and punctate brain calcifications. Screening for TORCH infections and autoimmune thrombocytopenia was negative. She was treated twice for presumed culture-negative sepsis, with minimal clinical improvement. In the face of worsening multi-organ dysfunction, palliative measures were instituted and she died at 54 days.

Results: Singleton WES identified a likely pathogenic variant in IFIHI: c.2159G>A, p.(Arg720Gln). This missense variant has been reported twice in ClinVar, and published in 6 individuals with variable AGS. Pathogenic variants in IFIHI cause autosomal dominant AGS7, and account for $3-5 \%$ of cases.

Conclusion: AGS7 presents with a variable phenotype, including a neonatal-onset form. Phenotypic overlap with common disorders of prematurity complicates diagnosis in critically ill, extreme preterm infants. Rapid WES in such cases can provide a timely diagnosis, and inform counselling on prognosis, recurrence, and end-of-life decision making. Grant: MITO-FIND, funding from MITOCANADA

E. Phillips: None. M. Kerr: None. A. Khan: B. Research Grant (principal investigator, collaborator or consultant and pending grants as well as grants already received); Modest; MITO-FIND. F. Consultant/Advisory Board; Modest; MITOCANADA.

\section{E-P11.010}

The third family with TAF6-related phenotype: Alazami-Yuan syndrome

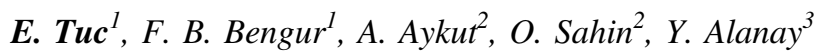

${ }^{1}$ Acibadem Mehmet Ali Aydinlar University School of Medicine, Istanbul, Turkey, ${ }^{2}$ Marmara University School of Medicine, Department of Ophthalmology, Istanbul, Turkey, ${ }^{3}$ Acibadem Mehmet Ali Aydinlar University School of Medicine, Department of Pediatrics, Pediatric Genetics Unit, Istanbul, Turkey

Introduction: Homozygous variants in TAF6 gene cause an autosomal recessive intellectual disability syndrome with Cornelia de Lange syndrome (CdLS)-like features, namely Alazami-Yuan Syndrome (ALYUS;MIM\#617126). We report the sixth patient from a third family with additional ocular and behavioral features.

Clinical Report: The proband was evaluated after family members with congenital nystagmus were referred. He is an 18-years-old male with moderate intellectual disability, mild dysmorphic features and stereotypic behaviors. His movements stop when verbally distracted and he responds with single words. Bilateral nystagmus was noticed in childhood. Flash electroretinogram recordings of cone and rod function were normal. Ophthalmological evaluation of other family members with congenital nystagmus demonstrated decreased visual acuity and impaired cone and rod function.

Results: Whole exome sequencing (WES) in family members revealed two different causative genetic conditions. Homozygous missense pathogenic variant, c.323T>C;p.(Ile108Thr) in TAF6 (NM_001190415.1) and heterozygous missense variant of uncertain significance, c.1708G>A;p.(Val570Met) in PDE6C (NM_006204.3) were identified in the proband. His sister and her son were both homozygous for the variant in PDE6C gene.

Conclusion: The proband shares the same homozygous missense variant, c.212T>C;p.(Ile71Thr) in TAF6, previously reported in a Saudi family by Alazami et al. Three siblings from the same Saudi family and a Turkish patient were simultaneously published by Yuan et al. Comparison of WES data from the proband and the published Saudi family revealed a shared haplotype of $3 \mathrm{Mb}$ in the region including TAF6 gene. This finding supports pathogenicity of this homozygous variant and suggests an ancient founder effect.

E. Tuc: None. F.B. Bengur: None. A. Aykut: None. O. Sahin: None. Y. Alanay: None. 


\section{E-P11.014}

The First Case Report Coexistence of Asymmetric Crying Face, Congenital Ptosis And Isotretinoin Embryopathy

\section{Seven ${ }^{1}$, A. Kalayci Yigin' ${ }^{l}$, M. T. Alay ${ }^{1}$, S. Sozer Tokdemir ${ }^{2}$}

${ }^{1}$ Department of Medical Genetics, Cerrahpasa Medical Faculty, Istanbul University-Cerrahpasa, Istanbul, Turkey, ${ }^{2}$ Department of Genetics, Aziz Sancar Research Institute of Experimental Medicine, Istanbul, Turkey

Introduction: Isotretinoin is categorized as $\mathrm{X}$ group of drug by Food and Drug Administration (FDA). Teratogenic effects are well defined and mainly includes: craniofacial abnormalities, cardiovascular, central nerve system, thymic/ parathyroid anomalies and ear abnormalities [1]. Asymmetric crying face $(\mathrm{ACF})$ is very rare finding of $0.2-0.6 \%$ and mimics the sign of peripheral facial paralyses. Generally, associated with left-sided predominance.

Clinical Findings and method: 28-day baby referred to our clinic for dysmorphological examination. Her mother was followed-up in our teratology clinic for isotretinoin usage during the pregnancy. On baby's physical examination; congenital ptosis in the right eye, ACF, nevus flammeus in the glabella, cleft in the right earlobe, retromicrognathia, bilateral sandal gap, radial deviation in wrist and metatarsus adductus were detected. The right lower lip of the patient was observed to be moving down, when crying. ACF was identified. In order to exclude facial nerve paralysis, electromyelography (EMG) was performed. His karyotype was normal.

Conclusion: In the literature, there is only one case report about isotretinoin embryopathy with ACF which was not confirmed by EMG. Congenital ptosis is a common sign and maybe associated with many abnormalities, however, to the best of our knowledge coexistence of congenital ptosis and ACF with isotretinoin embryopathy is the first reported case in here.

M. Seven: None. A. Kalayci Yigin: None. M.T. Alay: None. S. Sozer Tokdemir: None.

\section{E-P11.015}

Au-Kline syndrome due to a de novo missense variant in HNRNPK

\section{Fauth ${ }^{1}$, U. Albrecht ${ }^{2}$, E. Maurer ${ }^{1}$, J. Zschocke ${ }^{l}$}

Institute of Human Genetics, Medical University Innsbruck, Innsbruck, Austria, ${ }^{2}$ Department of Pediatrics I, Medical University Innsbruck, Innsbruck, Austria
Introduction: Haploinsufficiency of HNRNPK, which encodes the heterogeneous nuclear ribonucleoprotein $\mathrm{K}$, has recently been identified as cause of Au-Kline syndrome (MIM \#616580). Major findings include muscular hypotonia, developmental delay, intellectual disability, cardiac, urogenital and skeletal abnormalities, and a characteristic facial gestalt. With one exception, all patients with $\mathrm{Au}$ Kline syndrome published so far have loss-of-function mutations. There is only a single patient with a de novo missense variant (p.L155P) which is located in KH2, the second of three K-homology RNA-binding (KH) domains of HNRNPK which ensure sequence-specific RNA and DNA binding. Due to the limited number of patients, the phenotypic consequences of missense variants in $H N R N P K$ remain to be defined.

Methods and results: We report a second patient with a de novo missense variant in $H N R N P K$ and typical features of Au-Kline syndrome. The 10 year-old girl has severe global developmental delay, muscular hypotonia, cleft palate, a branchial cyst, hydronephrosis, vertebral segmentation anomalies and facial dysmorphism. Based on the clinical phenotype Au-Kline syndrome was suspected and confirmed by Sanger sequencing which revealed a de novo missense variant c.146C $>\mathrm{T}$, p. L49P in HNRNPK [NM_0021404]. This variant affects a moderately conserved amino acid, locates to $\mathrm{KH} 1$, the first K-homology RNA-binding domain of HNRNPK, is not listed in GnomAD and is predicted to be pathogenic by in silico tools. Together with the typical clinical phenotype this strongly suggests that the variant is disease-causing.

Conclusion: Our observation confirms that HNRNPK missense variants affecting $\mathrm{KH}$ domains can lead to the characteristic clinical phenotype of Au-Kline syndrome.

C. Fauth: None. U. Albrecht: None. E. Maurer: None. J. Zschocke: None.

\section{E-P11.016}

Clinical and molecular characterization of a rare case diagnosed with Bardet Biedl Syndrome

\section{O. Focsa ${ }^{1}$, M. Budisteanu ${ }^{2,3,4}$, C. Burloiu ${ }^{2}$, L. C. Bohiltea ${ }^{1}$, M. Balgradean ${ }^{5,6}$}

I"C.Davila" University of Medicine and Pharmacy, Bucharest, Bucharest, Romania, ${ }^{2}$ Prof. Dr. Alex. Obregia Clinical Hospital of Psychiatry, Bucharest, Romania, ${ }^{3}$ Victor Babes National Institute of Pathology, Bucharest, Romania, ${ }^{4}$ Titu Maiorescu University, Bucharest, Romania, ${ }^{5}$ Marie Curie Children's Clinical Hospital, Bucharest, Romania, ${ }^{6}$ Carol Davila University of Medicine and Pharmacy, Bucharest, Romania 
Bardet Biedl syndrome (BBS) is a rare, pleiotropic ciliopathy caused by mutations in 21 genes. BBS 12 gene mutations are responsible for $5-6 \%$ from molecular diagnosed cases. Clinical spectrum is highly variable in- and between families. The main features are: rod cone dystrophy, polydactyly, obesity, cognitive impairment, renal anomalies and urogenital malformations. We report on a new case of BBS caused by BBS12 mutation. The 7 years old girl is the first child, born at 42 weeks from healthy, unrelated, gypsy parents. Physical parameters at birth were normal. Post axial polydactyly in all four members, brachydactyly and 2-3 fingers syndactyly, were noted. Last physical exam reveals macrocephaly (OFC $>2 \mathrm{SD}$ ), obesity (weight $>6 \mathrm{SD}$ ), dysmorphyc features, rod cone dystrophy, genital malformations, severe intellectual disability, and behavior abnormalities (self aggressive behavior, hyperkinesia, food intake abnormalities). Over the last year, renal dysfunction and hypertension have occurred. Clinical diagnosis was established due to presence of more than four primary features included in consensus criteria. Molecular genetic tests confirmed the diagnosis, identifying an homozygous nonsense mutation in BBS12 gene (c. 1063C > T; p.Arg355*). The mutation was reported first in 2007 in two French gypsy families. Later, other two patients were reported. Our patient brings new evidence in the phenotype caused by this rare mutation. Further studies are needed for a better understanding of genotype-phenotype relation. Albeit there is a little experience in predicting the phenotype severity taking into account the mutational load, an early clinical and molecular diagnosis may be helpful for a good management of these patients.

I.O. Focsa: None. M. Budisteanu: None. C. Burloiu: None. L.C. Bohiltea: None. M. Balgradean: None.

\section{E-P11.017}

Improving the phenotype description of Basel-Vanagaite-Smirin-Yosef syndrome, $M E D 25$-related: the relevance of neuroimaging findings.

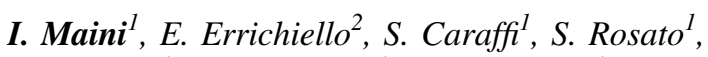

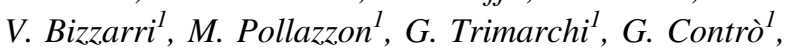

C. Gelmini ${ }^{1}$, M. Napoli ${ }^{3}$, R. Pascarella ${ }^{3}$, S. Rizzi $i^{4}$,

C. Fusco ${ }^{5}$, O. Zuffardi ${ }^{6}$, L. Garavelli ${ }^{1}$

${ }^{1}$ Medical Genetics Unit, Department of Maternal and Child Health Department, AUSL-IRCCS of Reggio Emilia, Reggio Emilia, Italy, ${ }^{2}$ Unit of Medical Genetics, Department of Molecular Medicine, University of Pavia, Pavia, Italy, ${ }^{3}$ Neuroradiology Unit, AUSL-IRCCS of Reggio Emilia, Reggio Emilia, Italy, ${ }^{4}$ 5. Child Neuropsychiatry Unit, AUSL-IRCCS of Reggio Emilia, Reggio Emilia, Italy, ${ }^{5}$ Child Neuropsychiatry Unit, AUSL-IRCCS of Reggio Emilia, Reggio Emilia, Italy, ${ }^{6}$ Unit of Medical Genetics,
Department of Molecular Medicine, University of Pavia, Pavia, Italy

Introduction: Basel-Vanagaite-Smirin-Yosef syndrome (BVSYS) is an extremely rare autosomal recessive genetic disorder characterized by severe developmental delay and variable congenital anomalies, caused by variants in the MED25 gene. Since 2015, 17 patients have been described with common clinical features and homozygous variants in the MED25 gene, leading to a better definition of the phenotype associated with BVSYS.

Materials and methods: Here we report two young sisters, of consanguineous parents, presenting typical dysmorphic features and neuroimaging findings of BVSYS, plus polymicrogyria.

Patient 1 showed features of BVSYS at physical examination (strabismus, large and overhanging nasal tip, short philtrum, down-turned corners of the mouth, micrognathia, bilateral 2nd-3rd toe syndactyly Y-shaped) and a delayed psychomotor development. Brain MRI showed bilateral perisilvian polymicrogyria, thin corpus callosum, temporal and parietal atrophy, cyst of the cavum septum pellucidum and cavum vergae, enlargement of the third and lateral ventricles.

Patient 2 (patient 1's sister) show similar clinical features at physical examination and brain MRI; she died at 1 year of age and authoptic investigations were not performed. Results (patient 1): Karyotype and array-CGH were normal; WES identified an homozygous frameshift variant c.1778_1779delAG in MED25. The variant leads to stop coding formation and results in a loss of function protein.

Conclusions: Our cases showed among neuroimaging findings also perisilvian polymicrogyria. which has never been described before in BVSYS. We suggest including it among the possible cerebral anomalies of BVSYS and emphasize the importance of a detailed clinical and neuroradiological evaluation of these patients.

I. Maini: None. E. Errichiello: None. S. Caraffi: None. S. Rosato: None. V. Bizzarri: None. M. Pollazzon: None. G. Trimarchi: None. G. Contrò: None. C. Gelmini: None. M. Napoli: None. R. Pascarella: None. S. Rizzi: None. C. Fusco: None. O. Zuffardi: None. L. Garavelli: None.

\section{E-P11.018}

Concurrent hypomethylation in both IC loci found in a Beckwith-Wiedemann syndrome case 
V. Anastasiadou ${ }^{1}$, I. Kyprianou ${ }^{2}$, A. Miltiadous ${ }^{2}$,

P. Gerasimou ${ }^{2}$, J. Chi ${ }^{3,2}$, I. Savvidou ${ }^{1}$, P. Costeas ${ }^{2,3}$

${ }^{1}$ Department of Clinical Genetics, Archbishop Makarios III Medical Centre, Nicosia, Cyprus, ${ }^{2}$ Molecular Hematologyoncology, Karaiskakio Foundation, Nicosia, Cyprus, ${ }^{3}$ The Center for the Study of Haematological Malignancies, Nicosia, Cyprus

Introduction: Beckwith-Wiedemann syndrome (BWS) is a clinically heterogeneous overgrowth syndrome associated with an increased risk of embryonal tumour development. BWS incidence ranges from 1 in $10,000-15,000$ newborns with $85 \%$ of the cases sporadic. Genetic or epigenetic alteration within two domains of imprinted growth regulatory genes on chromosome $11 \mathrm{p} 15$, IC1 (H19DMR) or IC2 (KvDMR), lead to deregulation and BWS phenotype. Other known causes of BWS are uniparental disomy (UPD), trisomy $11 \mathrm{p} 15$, mutations in the CDKN1C gene, as well as small deletions and translocations.

Materials and methods: We describe a 2-year-old girl, referred because of global developmental delay. On examination we noted macrosomia, midface and scalp hemangiomas and a protruding tongue. She suffers of choking episodes. Speech is limited. She is the first child and a product of sperm donation. Macrosomia was noted on prenatal ultrasound. Neonatal hypoglycemia was not reported. MS-MLPA analysis (MRC Holland) was performed to look for epimutations in the imprinted $11 \mathrm{p} 15$ region associated with BWS (IC2 loss of methylation or IC1 gain of methylation). The results were confirmed with a second sample from the proband.

Results: Concurrent hypomethylation on both imprinting loci, IC1 and IC2, was observed on chromosome 11p15. No copy number chromosomal abnormalities were detected.

Conclusions: We discuss the clinical and molecular findings of this case because of the rarity of simultaneous loss of methylation pattern in both IC1 and IC2 loci. Relevant literature is currently limited.

V. Anastasiadou: None. I. Kyprianou: None. A. Miltiadous: None. P. Gerasimou: None. J. Chi: None. I. Savvidou: None. P. Costeas: None.

\section{E-P11.020}

New heterozygous pathogenic variant in ABCC9 gene identified in two patients diagnosed with Cantu syndrome

K. Wojciechowska ${ }^{1}$, A. Tarkowska ${ }^{2}$, M. Kacprzak ${ }^{3}$, D. Loska ${ }^{3}$, A. Pietrzyk ${ }^{4}$, M. Babicz, M. Holweg ${ }^{5}$,
A. Mroczkowska ${ }^{5}$, P. Skowera ${ }^{5}$, B. Styka ${ }^{5}$, D. Winnicka ${ }^{5}$, M. Lejman $^{5}$, J. Kowalczyk ${ }^{1}$

${ }^{I}$ Department of Pediatric Hematology, Oncology and Transplantology, Children's University Hospital, Lublin, Poland, ${ }^{2}$ Department of Neonate and Infant Pathology, Children's University Hospital, Lublin, Poland, ${ }^{3}$ MedGen Medical Centre, Warsaw, Poland, ${ }^{4}$ Department of Clinical Genetics and Patomorphology, University of Zielona Gora, Zielona Gora, Poland, ${ }^{5}$ Laboratory of Genetic Diagnostic, Children's University Hospital, Lublin, Poland

Introduction: Cantu syndrome is characterized by congenital hypertrichosis, characteristic craniofacial dysmorphic features, cardiomegaly and osteochondrodysplasia. It is caused by mutations in $\mathrm{ABCC} 9$ or KCNJ8 genes. We would like to report two new cases of Cantu syndrome: 33year-old patient and her 3-month-old daughter. Mother during adolescence was diagnosed with hypogonadotropic hypogonadism. She had distinctive facial features, hypertrichosis on the face and polycystic ovaries. Neither signs of cardiomegaly nor osteochondrodysplasia were observed. Her daughter had similar coarse facial features, generalized hypertrichosis and enlarged tongue. She was born on time with normal length and weight parameters. After genetic consultation Whole Exome Sequencing was performed.

Materials and methods: The sequencing of isolated, enriched genomic DNA was performed on NovaSeq 6000. Raw sequences in fastq format were mapped to hs $37 \mathrm{~d} 5$ reference. The following in silico prediction software programs were used to assist with interpretation of pathogenicity of detected variant: SIFT, MutationTaster, PolyPhen-2 as embedded in Alamut visual v 2.9.0. The presence of the variant in control populations was checked in $1000 \mathrm{Genomes}$, the ExomeVariantServer, and the Exome Aggregation Consortium and gnomAD. Sanger sequencing was also performed.

Results: A new missense variant Arg1116Pro was found in one allele of ABCC9 gene. Considering the symptoms and the detected variant patients were diagnosed with Cantu syndrome.

Conclusion: We would like to present two patientsmother and her daughter with some distinctive clinical features for Cantu syndrome and new pathogenic variant in ABCC9 gene. Our report is a valuable contribution in expanding molecular and clinical spectrum of Cantu syndrome.

K. Wojciechowska: None. A. Tarkowska: None. M. Kacprzak: None. D. Loska: None. A. Pietrzyk: None. M. Babicz: None. M. Holweg: None. A. Mroczkowska: None. P. Skowera: None. B. Styka: None. D. Winnicka: None. M. Lejman: None. J. Kowalczyk: None. 


\section{E-P11.022}

Further delineation of putative CDK10 loss-of-function variants: a new form of ciliopathy phenotype

\section{G. Forzano ${ }^{1}$, R. Artuso ${ }^{2}$, E. Andreucci ${ }^{2}$, V. Palazzo ${ }^{2}$,}

A. Pagliazzil ${ }^{l}, F$. Vanderwert ${ }^{l}$, G. Gori ${ }^{1}, F$. Di Giovanni ${ }^{1}$, E. Fiorentini $^{1}$, S. Giglio $^{3}$

${ }^{1}$ Medical Genetics Unit, University of Florence, Florence, Italy, ${ }^{2}$ Medical Genetics Unit, Meyer Children's University Hospital, Florence, Italy, ${ }^{3}$ Medical Genetics Unit, University of Florence, Meyer Children's University Hospital, Florence, Italy

CDK10 encodes a protein kinase which interacts with cyclin to form a heterodimeric protein kinase that plays a central role in the regulation of cellular growth, ciliogenesis and primary cilium elongation. Bi-allelic loss-of-function CDK10 mutations have been recently reported associated with a distinct disorder, named Al-Kaissi syndrome, described so far in only 10 patients from 6 distinct families. We report the case of a 5-year-old boy with a complex phenotype: growth retardation, global developmental delay with absent speech, thin corpus callosum and paucity of white matter visible at the brain MRI, vertebral anomalies and a typical facial appearance (triangular face, bilateral epicanthic folds, hypertelorism, depressed nasal bridge, low-set and posteriorly rotated ears, small chin); besides these features, our patient was born with ostium secundum atrial septal defect and pulmonary valve stenosis. Array$\mathrm{CGH}$ and $\mathrm{CHD} 7$ molecular analysis were normal. Trio whole exome sequencing (WES) disclosed a homozygous splice site variant in the CDK10 gene. Our patient's phenotype resembles the clinical features described in patients affected by Al Kaissi syndrome, except for the congenital heart defect (CHD): none of the patients with the same mutation as our case were reported to have any heart anomalies; to date, only one patient with Al-Kaissi syndrome showed patent ductus arteriosus. The WES analysis excluded further variants of the genes described in association with heart malformations and therefore our case is likely to allow us to expand the clinical phenotype of a syndrome which is not yet fully characterised.

G. Forzano: None. R. Artuso: None. E. Andreucci: None. V. Palazzo: None. A. Pagliazzi: None. F. Vanderwert: None. G. Gori: None. F. Di Giovanni: None. E. Fiorentini: None. S. Giglio: None.

\section{E-P11.024}

A nonsense mutation in the first exon of CHD8 causes a multi-organ disease phenotype: Is it a novel syndrome or a detection of an additional gene resulting in CHARGE syndrome
T. Atik, S. Simsir, E. Isik, F. Ozkinay

Ege University Faculty of Medicine, Department of Pediatrics, Izmir, Turkey

Developments in the genomic era and large-scale exome/ genome sequencing studies have identified mutations in several members of the CHD (Chromodomain Helicase DNA-binding protein) gene family in neurodevelopmental disorders. Especially the group III CHD family consisting of CHD6, CHD7, CHD8 and CHD9 has well-documented roles in transcription regulation, impacting in organogenesis. These four enzymes are similar in their constituent domains. While mutations in the CHD2 and CHD8 have been linked to autism and intellectual disability. Additionally, CHD7 has been found to be associated with CHARGE syndrome which is a well-known congenital malformation syndrome. Here in this report, we present a case having intellectual dysability, autism and several congenital anomalies and a de-novo nonsense mutation in CHD8 gene as a possible cause of the clinical phenotype. A 5,5 year-old boy with macrocephaly, autism, intellectual disability and congenital anomalies including severe hypospadias, ventricular septal defect, inguinal hernia, cryptorchidism, anal atresia and hemivertebrae(T12) was referred to our department. He has also been treating for hypothyroidism. Trio whole exome sequencing(WES) was performed, and a de-novo heterozygous nonsense c.727C $>\mathrm{T}$ (p.Arg243*) mutation in the first exon of CHD8 was detected and it was found to be likely pathogenic according to ACMG 2015 criteria. We consider that the mutation found in the patient could be responsible for a congenital malformation syndrome similar to CHARGE syndrome, causing a disruption in CHD7-CHD8 complex. Further functional analysis is planned. As a conclusion, mutations in CHD8 gene, especially disrupting CHD7-CHD8 complex, could be responsible for a congenital malformation syndrome similar to CHARGE.

T. Atik: None. S. Simsir: None. E. Isik: None. F. Ozkinay: None.

\section{E-P11.025}

Chromoanasynthesis with involvement of chromosome 21: rare cause for dysmorphic features and developmental disorder

J. Lisfeld ${ }^{1}$, M. Pettersson ${ }^{2}$, S. Rudolph ${ }^{3}$, S. Fuchs ${ }^{1}$, J. Eisfeldt ${ }^{2}$, M. Hempel $^{1}$, A. Lindstrand ${ }^{2}$

${ }^{1}$ Institute of Human Genetics, University Medical Center Hamburg-Eppendorf, Hamburg, Germany, ${ }^{2}$ Department of Molecular Medicine and Surgery, Karolinska Institutet and Department of Clinical Genetics, Karolinska University 
Hospital, Stockholm, Sweden, ${ }^{3}$ Gemeinschaftspraxis für Humangenetik und Genetische Labore, Hamburg, Germany

Background: Although various complex chromosomal rearrangements have been described, the underlying pathomechanisms are still not fully understood. One of the models discussed is chromoanasynthesis, a replicationbased process that forms complex rearrangements during numerous cycles of fork stalling and template switching (FoSTeS) and microhomology-mediated break-induced replication (MMBIR). A commonly acknowledged mechanism for catalyzing these events is DNA polymerase slippage, which could lead to both inter- and intrachromosomal rearrangements.

Case report: We report on a 22-year-old man with moderate global developmental delay, short stature, microcephaly, severe hearing impairment and dysmorphic features resembling patients with Down syndrome. Brain MRI and echocardiography results were normal.

Methods and results: Chromosome analysis revealed a derivative chromosome 21. CGH array analysis could delineate one duplication on $21 \mathrm{p}$ and five duplications on 21q. Follow-up analysis using short-read paired-end wholegenome sequencing (WGS) revealed a complex rearrangement consisting of a total of seven duplications on chromosome 21. Four out of the total seven breakpoint junctions could be delineated on the nucleotide level and provided evidence of DNA polymerase slippage and errors during DNA replication as the underlying mechanism of formation. Chromosome analyses and WGS data for both parents were unremarkable, thereby indicating a de novo rearrangement.

Discussion: The patient described here is affected by a complex chromosomal rearrangement consisting of multiple partial duplications within chromosome 21. Duplications were mostly rearranged and one was inverted.

J. Lisfeld: None. M. Pettersson: None. S. Rudolph: None. S. Fuchs: None. J. Eisfeldt: None. M. Hempel: None. A. Lindstrand: None.

\section{E-P11.026}

Clinical results of chromosome 15 copy number variations

H. Gurkan ${ }^{l}$, E. Atll ${ }^{1}$, S. Yalcintepe ${ }^{1}$, E. \&. Atll ${ }^{l}$, S. Demir ${ }^{l}$, Y. Özen ${ }^{l}, H$. Tozkır ${ }^{2}$

${ }^{1}$ Trakya University, Medical Faculty, Department of Medical Genetics, Edirne, Turkey, ${ }^{2}$ Tekirdag, Namık Kemal University, Medical Faculty, Department of Medical Genetics, Tekirdag, Turkey
Chromosome 15 spans more than 102 million DNA base pairs and represents more than 3 percent of the total DNA in cells. Chromosome 15 likely contains 600 to 700 genes that provide instructions for making proteins. These proteins perform a variety of different roles in the body. Changes in the number or structure of chromosome 15 can cause intellectual disability, delayed growth and development, hypotonia, and characteristic facial features. These changes include an extra copy of part of chromosome 15 in each cell, a missing segment of the chromosome in each cell, and a circular structure called ring chromosome 15 . Sixteen patients who have a CNV on 15th chromosome as a result of the arrayCGH analysis made using 180K CGH + SNP oligoarray after they had been directed to Trakya University Hospital, Genetic Diseases Diagnosis Center between 20162019, were included in our study. The indications of the patients were intellectual disability, hypotonia, microcephaly, developmental delay, increased nuchal translucency, intrauterine growth retardation etc. Nine patients had 15q partial deletion and 7 patients had 15q partial duplication. As a result of the segregation analysis, 3 patients were diagnosed with Angelman Syndrome, one patient was diagnosed with Ring15. It was determined that 4 patients (del. in 2 patients, dup. in 2 patients) with $\mathrm{CNV}$ on chromosome $15 \mathrm{q}$ had inherited the CNV from a parent. As a result of segregation analysis, the diagnoses of the 8 patients, 4 having a deletion and the other 4 having a duplication, will be confirmed.

H. Gurkan: None. E. Atlı: None. S. Yalcintepe: None. E. \&. Atlı: None. S. Demir: None. Y. Özen: None. H. Tozkır: None.

\section{E-P11.027}

\section{Distal 18q deletion syndrome in girl}

O. Gumeniuk, Y. Chernenkov, S. Sanaeva, P. Golobokova, T. Ilysova

Saratov State Medical University, Saratov, Russian Federation

Distal $18 \mathrm{q}$ deletion syndrome is a rare hereditary with piece distal section of the long (q) arm of chromosome 18 is missing. Syndrome can lead to a wide variety of signs and symptoms. Purpose. To study the features distal $18 \mathrm{q}$ deletion syndrome in girl. Patient and methods. 14-year-old girl with physical and sexual delayed development, language delay, learning difficulties, muscle weakness, deformation of the foot, frequent non-diabetic ketoacidosis. Physical and instrumental examination, conventional chromosomal analysis was carried out. Results. Girl was born full term with normal birth weight, following a pathological pregnancy. 
Tetralogy of Fallot was diagnosed in neonatal period. Family History: congenital cataract in the mother and in the grandfather on the mother's side. She attends a special needs school and has poor school performance, with difficulty in attention, concentration, comprehension and obeying orders. She had delayed physical and sexual development, microcephaly, poor subcutaneous fat, asymmetric wide chest, long arms, short shoulders, gait disturbance, deformity of the foot on the right (Friedreich foot) and specific facial dysmorphies: wide nose bridge, hypertelorism, epicantum, thin upper lip, "carp mouth". Her IQ (intelligence quotient) was 53, and he showed mild mental retardation. Laboratory examination: increased level of $\mathrm{TSH}$, a decrease in T4 (primary congenital hypothyroidism, late form). Abdominal ultrasound: echo-signs gallbladder agenesis characteristic. Conventional chromosomal analysis on 30 metaphases from peripheral blood lymphocyte cultures showed two cell lines: 46, XX, del(18)(q21q21). Conclusions. The clinical features of the case were frequent non-diabetic ketoacidosis, gallbladder agenesis, severe heart disease and late genetic diagnosis.

O. Gumeniuk: None. Y. Chernenkov: None. S. Sanaeva: None. P. Golobokova: None. T. Ilysova: None.

\section{E-P11.028}

\section{A complex duplication/triplication of $20 \mathrm{q}$}

\section{Souto ${ }^{1}$, P. Botelho ${ }^{1}$, M. Martins ${ }^{2}$, O. Moutinho ${ }^{3}$,} R. Pinto Leite ${ }^{l}$

${ }^{1}$ Laboratório de Genética, Centro Hospitalar Trás-osMontes e Alto Douro, Vila Real, Portugal, ${ }^{2}$ Consulta de Genética, Centro Hospitalar Trás-os-Montes e Alto Douro, Vila Real, Portugal, ${ }^{3}$ Serviço de Ginecologia e Obstetrícia, Centro Hospitalar Trás-os-Montes e Alto Douro, Vila Real, Portugal

Introdution: Partial duplication of the long arm (q) of chromosome 20 occur very rarely. The phenotype usually consists of brachycephaly, epicanthus, anteverted nostrils, short neck, vertical chin dimple, and congenital heart defect or murmur. Some cases have development growth, speech, motor, and social interactions delay. The authors present a case of partial trisomy 20q.

Clinical Report: 8-year-old girl with short stature, minor dysmorphic features and global developmental delay. Blood culture and cytogenetic analysis were performed according standards protocols. Cytogenetics analysis revealed a chromosome marker in all metaphases analyzed. Parents karyotype were normal. Fluorescence in situ hybridization (FISH) technique identified the extra material as chromosome 20. Array Comparative Genomic Hybridization $(\mathrm{aCGH})$ technique revealed a gain in the 20q13.33 region: three copies of $400 \mathrm{Kbp}$ (without genes), four copies of $406 \mathrm{Kbp}$ (involving $\mathrm{CDH} 4$ gene), three copies of $543 \mathrm{Kbp}$ (containing three genes: $\mathrm{CDH} 4, \mathrm{MIR} 125, \mathrm{TAF} 4$ ) and four copies of $2.304 \mathrm{Mbp}$, comprising TAF4 and 86 more genes.

Discussion: In the present case conventional and molecular cytogenetics detected a marker chromosome derived from chromosome 20. The array clarified the amount and the segment involved. The extra chromosome comprised a complex duplication/triplication in the 20q13.3 region. The girl has some dysmorphic features and global developmental delay consistent with $20 \mathrm{q}$ duplication syndrome. Nevertheless, to our knowledge this is the first case involving only this region. Every new case of a rare chromosomal alteration should be reported in order to obtain a more precise genotype/ phenotype correlation, improving risk evaluation and genetic counselling.

M. Souto: None. P. Botelho: None. M. Martins: None. O. Moutinho: None. R. Pinto Leite: None.

\section{E-P11.030}

\section{A Rare Case Report: Cloves Syndrome}

\section{T. Alay $^{1}$, A. Kalayci Yigin ${ }^{1}$, B. Uyanik ${ }^{2}$, M. Seven ${ }^{1}$}

${ }^{1}$ Department of Medical Genetics, Cerrahpasa Medical Faculty, Istanbul University-Cerrahpasa, Istanbul, Turkey, ${ }^{2}$ Department of Medical Genetics, University of Health Sciences, Bakirkoy Dr. Sadi Konuk Training and Research Hospital, Istanbul, Turkey

Introduction: Clove(s) syndrome (CS) is one of the rare syndromes characterized with congenital adipose tissue excessive size, vascular malformation, epidermal nevus and scoliosis/spinal malformation. Although the etiology of the disease is not fully understood, it usually occurs as a result of post-zygotic mutations that function in the PIK3CA gene. CS syndrome can affect soft tissue, blood vessels, bone, and internal organs. Its symptoms vary widely between mild and severe anomalies.

Clinical Findings: A 3-year-old girl was referred to our outpatient clinic with a preliminary diagnosis of vascular malformation. In her physical examination, there was progressive growth in the abdomen, pigmented skin lesions in the left half of the body from birth, and capillary malformation (nevus flammeus) in the right lower limb and perivulvar region. Scoliosis, left hemihypertrophy, sandal gap and macrodactyly were detected in the toes.

Results: In the Clinical Exom DNA sequence analysis from the blood sample taken from the patient, a Heterozygous c.436G'A p.(Val146Ile) mutation was detected and confirmed by Sanger sequence. The PIK3CA gene is named 
as an oncogene encoding 23 exons, located on the PI3K/ $A K T$ signal pathway.

Conclusion: As a result of changes in the PIK3CA gene, congenital lipamatosis has been reported to cause anomalies and syndromes such as overgrowth, vascular malformations, epidermal nevus. Applying the diagnostic criteria of Keppler- Noreuil et al., our case was found to be compatible with the diagnosis of CS. CS is the first case reported in our country supported by molecular analysis.

Keywords: Cloves Syndrome, PIK3CA gene, Clinical Exome.

M.T. Alay: None. A. Kalayci Yigin: None. B. Uyanik: None. M. Seven: None.

\section{E-P11.031}

Cockayne Syndrome: a previous described heterozygous variant in homozygosity

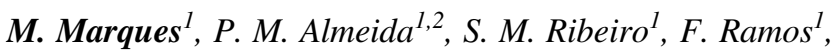
L. Ramos ${ }^{1,2}$

${ }^{1}$ Hospital Pediátrico, Centro Hospitalar e Universitário de Coimbra, Coimbra, Portugal, ${ }^{2}$ Faculty of Health Sciences, University of Beira Interior, Covilhã, Portugal

Cockayne syndrome (CS), is a rare autosomal recessive multisystem disorder, mainly characterised by postnatal growth failure, progressive neurological dysfunction, microcephaly, impaired vision and hearing, skeletal abnormalities and premature ageing. CS is caused by mutations in ERCC6 and in ERCC8 gene. Studies performed thus far have failed to delineate clear genotypephenotype relationships.

We present a one-year-old girl, from consanguineous parents, referred due to congenital cataracts, microcephaly, short stature and arthrogryposis. She had a history of reduced fetal movements, intrauterine growth restriction and hydramnios. At 6 months of life she had microcephaly $(-3,0 \mathrm{SD})$, growth failure and severe developmental delay. Brain MRI showed diffuse hypomyelination of the cerebral white matter, hypoplastic corpus callosum and small lens. We performed a broad gene panel analysis that identified a probably pathogenic missense variant [c.611C $>\mathrm{T}$ (p.Thr204Lys)] in homozygous form, on ERCC8 gene. Parental study confirmed that they are heterozygous for this variant.

CS belongs to the family of NER (nucleotide excision repair)-related disorders existing a large variation in severity. Our patient has CS type 2, which is a neonatal severe form, typically lethal in the first decade of life. Our patient has a homozygous probably pathogenic missense variant in the ERCC8 gene that was already described in a compound heterozygous state (c.611C $>\mathrm{A}$; c. $1122+1 \mathrm{delG})$ who has CS type I, without congenital cataracts, small lens, severe arthrogryposis and intrauterine growth restriction. The homozygous state in our patient can be the cause of the severe phenotype that was not described before.

M. Marques: None. P.M. Almeida: None. S.M. Ribeiro: None. F. Ramos: None. L. Ramos: None.

\section{E-P11.032}

Two Twin Sister With Cohen Syndrome And Hirsutism: A Case Report

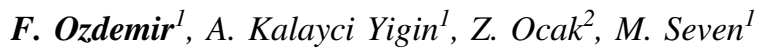

${ }^{I}$ Department of Medical Genetics, Cerrahpasa Medical Faculty, Istanbul University-Cerrahpasa, Istanbul, Turkey, ${ }^{2}$ Department of Medical Genetics, Medical Faculty, University of Istinye, Istanbul, Turkey

Introduction: Cohen syndrome is a genetic disease characterized by a rare neurodevelopmental delay, microcephaly and hypotonia. While phenotypic findings may differ among patients, it may appear normal in the neonatal period, as facial features are not evident. The first clinical signs include nutritional difficulties, hypotonia, microcephaly, neuromotor developmental retardation and joint hypermobility. Due to neutropenia, upper respiratory infections and oral aphthae could be observed. The disease has an autosomal recessive inheritance pattern and is caused by mutations in the VPS13B gene.

Clinical Findings and Method: In this study, we present two twin sister cases with hirsutism findings and neuromotor developmental retardation, microcephaly, mega sisterna magna in cranial MR and prominent antihelix, low hairline, camptodactyly and dysmorphic findings such as narrow forehead. DNA were isolated from peripheral blood samples taken from two siblings. The mutation was detected as a result of clinical exom analysis and confirmed by Sanger sequence. The results were evaluated with American College of Medical Genetics (ACMG) criterias and compared with genetic databases.

Result: As a result of the analysis, c. $412+1 \mathrm{G}>\mathrm{T}$ pathogenic variant was detected homozygous in the VPS13B gene in both sisters. Conclusion: This mutation supports Cohen's syndrome with clinical findings.

Keywords: Cohen Syndrome, Hirsutism, Clinical Exome Sequencing.

F. Ozdemir: None. A. Kalayci Yigin: None. Z. Ocak: None. M. Seven: None.

\section{E-P11.036}

A large homozygous deletion in two sibs suffering from multiple congenital disorders 
S. Seyedhassani ${ }^{1}$, M. Ebrahimi ${ }^{1}$, M. Tavakoli ${ }^{1}$, L. Najafi ${ }^{2}$, M. Neshan ${ }^{1,3}$

${ }^{l}$ Dr. Seyedhassani medical genetic center, Yazd, Iran, Islamic Republic of, ${ }^{2}$ Islamic azad university, Tehran, Iran, Islamic Republic of, ${ }^{3}$ National institute for genetic engineering and biotechnology, Tehran, Iran, Islamic Republic of

One family was referred to genetic counseling center because of multiple disabilities. They had three involved children resulting from consanguineous marriage with inbreeding coefficient about $6.25 \%$. A 10 months boy and a 8 years old girl suffering from delayed development, high crying, irritability, decreased head circumference, visual and hearing impairment and finally quadriplegic mental retardation. Another girl was dead in 11 years old with similar manifestations. This family had three normal children and was pregnant too.

Chromosomal study and CGH array were normal. Whole Exome Sequencing were used to enrich all exons of proteincoding genes as well as some important other genomic regions. Next generation sequencing was performed to sequence close to 100 million reads on Illumina Sequencer. In this test, point mutations and micro-insertion/deletions and duplication (<20bp) can be simultaneously detected. A large homozygous deletion around following region was predicted: chr11:653904710516, about 56600 bp, including genes: TMEM80, EPS8L2, DEAF1. DEAF1 gene acts as a regulator of transcription. Activity of this protein is important in the regulation of embryonic development. Mutations in this gene have been found in individuals with cognitive disability. EPS8L2 is a Protein Coding gene. Diseases associated with EPS8L2 include Deafness. TMEM80 is target gene of DEAF1 and decreased mRNA levels were observed for Tmem80 throughout the brain of DEAF1 knockout mice. Clinical manifestations were seen in involved sibs appeared as a result of dysfunction of deleted genes individually and interaction with other related genes. Prenatal diagnosis was done and fetus born in normal condition.

S. Seyedhassani: None. M. Ebrahimi: None. M. Tavakoli: None. L. Najafi: None. M. Neshan: None.

\section{E-P11.037}

Recessive inheritance of DISP1 variants in holoprosencephaly spectrum patients

A. Lavillaureix $^{1,2}$, C. Dubourg ${ }^{3,2}$, C. Quelin ${ }^{1}$, B. Keren ${ }^{4}$,

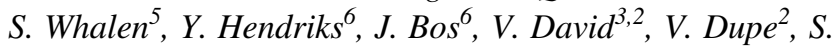
Odent $^{1,2}$

${ }^{1}$ Service de génétique clinique, Centre de Référence Maladies Rares, CLAD-Ouest, Centre Hospitalier
Universitaire de Rennes, ERN-ITHACA, Rennes, France, ${ }^{2}$ Rennes University, Institute of Genetics and Development of Rennes (IGDR) UMR6290 CNRS, Rennes, France, ${ }^{3}$ Service de Génétique Moléculaire et Génomique, Centre Hospitalier Universitaire de Rennes, Rennes, France, ${ }^{4} A P H P, G H$ Pitié Salpêtrière, Department of Genetics, Unit of Development Genomics, Paris, France, Paris, France, ${ }^{5}$ Unité Fonctionnelle de génétique clinique, Hôpital Armand Trousseau, Assistance publique-Hôpitaux de Paris, Centre de Référence Maladies Rares des anomalies du développement et syndromes malformatifs, Paris, France, ${ }^{6}$ Department of Clinical Genetics, Amsterdam UMC Locatie VUMC, Amsterdam, Netherlands

Holoprosencephaly (HPE; MIM\# 236100) is the most frequent congenital brain malformation ( 1 in 10,000 live births, 1 in 250 conceptuses). It results from incomplete midline division of the prosencephalon between $18^{\text {th }}$ and $28^{\text {th }}$ day of gestation, affecting both the forebrain and the face. The clinical spectrum is very wide, ranging from severe HPE with a single cerebral ventricle and cyclopia to clinically unaffected carriers in familial HPE. The full spectrum of HPE includes also microforms characterized by midline defects, with cleft lip/palate, hypotelorism, coloboma and/or single maxillary median incisor (SMMI). HPE is phenotypically and genetically heterogenous and was previously considered as a dominant disease with variable expression and incomplete penetrance. From the use of High Throughput Sequencing Technologies, it emerges that the penetrance and the phenotypic variability have digenic or oligogenic origin. To date, at least 18 genes are implicated in HPE and are all associated with key pathways of forebrain development including sonic hedgehog (SHH) pathway. Interestingly, among these genes, DISPI appears to have a specific mode of inheritance. DISP1 is a positive factor necessary for efficient secretion of the SHH morphogen and thus the establishment of its concentration gradient along the midline of the neural tube. We describe the clinical characteristics of three families with severe HPE or microform associated to compound heterozygous variants in DISP1. We will review the literature and discuss the genotype-phenotype correlations.

A. Lavillaureix: None. C. Dubourg: None. C. Quelin: None. B. Keren: None. S. Whalen: None. Y. Hendriks: None. J. Bos: None. V. David: None. V. Dupe: None. S. Odent: None.

\section{E-P11.038}

Detection of the pathogenic CNVs in the Macedonian patient with profound developmental delay 
V. Anastasovska ${ }^{l}$, E. Sukarova-Angelovska ${ }^{l}$, D. Nestoroska ${ }^{l}, N$. Zdraveska ${ }^{2}$, G. Ilieva ${ }^{l}$, M. Pesevska ${ }^{l}$

${ }^{1}$ Genetic Laboratory, Skopje, Macedonia, The Former Yugoslav Republic of, ${ }^{2}$ Department of Neonatology, Skopje, Macedonia, The Former Yugoslav Republic of

Introduction: The array Comparative Genomic Hybridization (aCGH) is now recognised as a first tier diagnostic test for patients with wideranging phenotypes and has led to greater sensitivity in the detection of sub-microscopic genomic changes and pathogenic copy number variants (CNVs).

Materials and methods: aCGH was performed in the Macedonian patient with clinical signs of dysmorphia and developmental delay using the Affymetrix ${ }^{\circledR}$ CytoScan $^{\text {TM }}$ $750 \mathrm{~K}$ Array (Applied Biosystems), that comprises $550 \mathrm{k}$ non-polymorphic and $200 \mathrm{k}$ SNP markers. The data was analysed using Chromosome Analysis Suite (ChAS) Software (v4.0).

Results: We describe a 2.5-year-old girl with dysmorphia and developmental delay. She has midfacial hypoplasia, facial asymmetry, prominent cheeks, deep set eyes, narrow rima oculi, short bulbous nose, simple and lop ears, round face and brachydactyly. Congenital hypothyroidism was detected at newborn screening, therefore she was on continuous 1-thyroxine therapy. At the age of 2 weeks the baby developed hemolytic crises, followed by apnoic episodes. Developmental delay was noticed at 6 months of age, both for global and fine motor skills. She started to walk at 2 years of age, and no speech development so far. Her height and weight are below 3 percentile. Karyotype was 46,XX, del18p- (11.1-pter). aCGH analysis was showed pathogenic 3 copies of the chromosomes 6q13q14.1 (4.081 kb) and 19p13.3 (650 kb) and one copy of chromosome 18p11.32p11.21 (14.919 kb), according to $\mathrm{aDGV}$ and ClinVar databases.

Conclusions: The array CGH analysis has become a widely accepted tool that supplements conventional karyotyping in patients with an unexplained phenotype.

V. Anastasovska: None. E. Sukarova-Angelovska: None. D. Nestoroska: None. N. Zdraveska: None. G. Ilieva: None. M. Pesevska: None.

\section{E-P11.039}

EBF3-related neurodevelopmental disorder - case report of female with phenotypic expansion and review of the literature

\author{
C. A. Chang $^{l}$, W. Al-Hertani ${ }^{2}$, M. Innes ${ }^{l}$ \\ ${ }^{1}$ Alberta Children's Hospital, Calgary, AB, Canada, ${ }^{2}$ Bos- \\ ton Children's Hospital, Boston, MA, United States
}

EBF3-related disorders were first described in 2017 (Sleven et al, Harms et al, Chao et al, Tanaka et al, Blackburn et al). Commonly reported features include global delays, mildsevere intellectual disability, ataxia, hypotonia, and mild facial dysmorphisms. Additional findings include high pain tolerance, febrile seizures, brain malformations, strabismus, feeding difficulties, scoliosis, genitourinary anomalies, short stature, perseverative social behaviours and motor stereotypies.

Herein, we report on a 19-year old female patient with a de novo likely pathogenic heterozygous variant in EBF3, c. $487 \mathrm{C}>\mathrm{T}$, p.(Arg163Trp). Our patient shows many symptoms of EBF3-related disorders, including cognitive delay, mental health concerns, hypotonia, strabismus, autonomic dysfunction, poor feeding, complex renal anomaly, neurogenic bladder, febrile seizures, unstable gait, and an unusual coronal pattern of sulcation in the cerebellar tonsils. Additionally, she had two unexplained episodes of significant elevation in AST and ALT (4000-6000 U/L), not previously reported.

Our report provides further expansion of the phenotypic spectrum of EBF3- related disorders. Long term outcomes are not well described; our patient represents the third oldest individual in the literature. The elevated liver transaminases remain unexplained and could be related to her EBF3related disorder, but the possible underlying mechanism is unknown. EBF3 encodes a member of the early B-cell factor transcription factor family. Haploinsufficiency has been proposed as a mechanism of EBF3-related disease (Lopes et al. 2017). This report contributes to the understanding of the long-term outcomes and health surveillance for affected individuals, and further supports the finding of multiple pleiotropic effects seen in EBF3-related disorders.

C.A. Chang: None. W. Al-Hertani: None. M. Innes: None.

\section{E-P11.040}

Expansion of phenotypic spectrum of EP300 mutations

\section{R. Kosaki ${ }^{l}$, K. Kosaki ${ }^{2}$}

${ }^{1}$ Division of Medical Genetics, National Center for Child Health and Development Hospital, Setagaya, Tokyo, Japan, ${ }^{2}$ Center for Medical Genetics, Keio University School of Medicine, Tokyo, Japan

Rubinstein-Taybi syndrome (RSTS) is a congenital malformation syndrome characterized by typical facial appearance, broad thumbs and halluces, and intellectual disability. Heterozygous mutations in CREBBP (50-60\%) or EP300 (8-10\%) have been detected. Recently, a heterogeneous group of patients with CREBBP or EP300 
mutations who have clinical features atypical for RSTS have been reported though exome analysis. We here report four unrelated patients with EP300 mutations to expand phenotypic spectrum. [Methods] Informed consent from the parents and approval from the local institutional review board were obtained. Medical exome analysis was performed. [Results] All the four patients had heterozygous mutation of EP300. Patient 1) 5-month-old male with some of RSTS including intellectual disability(ID), broad thumbs, and ventricular septal defect. Nonsense mutation p.Gln965* was detected. Patient 2) 2-year-old female with atypical features of RSTS including ID and normal thumbs. Missense mutation p.Val1656Ala was detected. Patient 3) 11month-old female with double outlet right ventricle, myelomenigocele, hydrocephalus, imperforate anus, coloboma, and hearing impairment. Dual mutations were detected: EP300 frameshift mutation p.Gln2048Thrfs*24 and SOS1 Thr778Pro mutation. Patient 4) 6-year-old female with PDA, PS, coloboma, and hearing impairment.: EP300 frameshift mutation p.Gln2048Thrfs*24 was detected.[Discussion] We demonstrated the clinical variability of patients with EP300 mutations. The combination of coloboma and imperforate anus has been reported in a patient with a frameshift mutation (p.H815Tfs*128) in EP300 (Masuda et al. 2015). Recurrence of such a specific combination of defects suggests causal relationship. Phenotypic spectrum of EP300 mutations may be much broader than has been previously appreciated.

R. Kosaki: None. K. Kosaki: None.

\section{E-P11.042}

\section{Deletion 2p24 and Feingold Syndrome Type 1}

C. Schell-Apacik ${ }^{1}$, S. Rauschke ${ }^{2}$, A. Weber ${ }^{2}$, M. Blankenburg ${ }^{2}$, L. Mulahasanovic ${ }^{3}$, H. Gabriel ${ }^{3}$, S. Biskup ${ }^{3}$, M. Stumm ${ }^{2}$

${ }^{1}$ Medicover Human Genetics Berlin-Westend MVZ, Berlin, Germany, ${ }^{2}$ Medicover Genetics GmbH, Berlin, Germany, ${ }^{3}$ Praxis für Humangenetik Tuebingen, Tuebingen, Germany

Introduction: Feingold Syndrome Type 1 (FS1 - OMIM \#164280) is a very rare syndromic microcephaly entity for which $M Y C N$ is the major disease-causing gene. The incidence of FS1 is currently unknown. About $10 \%$ of FS1 patients have deletions encompassing all or part of the $M Y C N$ gene. We report an additional FS1 case, carrying a 4.9 $\mathrm{Mb}$ de novo microdeletion of the chromosomal region $2 \mathrm{p} 24$.

Clinical data: The boy is the only child of unrelated parents. He was born in the 32th $(31+5)$ gestational week by Caesarean after preeclampsia. At the age of two days, a duodenal stenosis was treated surgically. Ultrasound revealed an agenesis of the left kidney, and a pancreas anulare. At the age of 9 months, he presented with microbrachycephaly, triangular facies, iris coloboma on both eyes, low-set ears, and partial cutaneous syndactyly of the 4th and 5the toes on the left foot, consistent with previous reports of patients with FS1.

Methods: Conventional chromosomal analysis (CA), array-CGH, quantitative PCR (qPCR) and Whole Trio Exome Sequencing (Trio-WES) were performed.

Results: GTG karyotyping showed a normal male result. Array CGH detected a $4.9 \mathrm{Mb}$ microdeletion of chromosome region 2 p24.3 to 2 p24.1, resulting in haploinsufficiency of MYCN. qPCR segregation analyses of the index patient and his parents demonstrated a de novo deletion. No pathogenic variants beside the 2 p24 microdeletion were revealed by Trio-WES of the patient and his parents.

Conclusion: Our results confirm that FS1 can be caused by microdeletions of the chromosomal region $2 \mathrm{q} 24$ as single abnormality.

C. Schell-Apacik: None. S. Rauschke: None. A. Weber: None. M. Blankenburg: None. L. Mulahasanovic: None. H. Gabriel: None. S. Biskup: None. M. Stumm: None.

\section{E-P11.044}

Ocular phenotype inpatients withGNB5variantsandimpact of variants onGbeta5 expressionand function

Z. Shao ${ }^{1,2}$, A. Tumber ${ }^{3}$, J. Maynes ${ }^{4}$, E. Tavares ${ }^{3}$, S. Hewson ${ }^{2}$ A. Schulze ${ }^{2}$, P. Kannu ${ }^{2}$, A. Vincent ${ }^{3}$

${ }^{1}$ Post-Graduate Medical Education, University of Toronto, Toronto, ON, Canada, ${ }^{2}$ Division of Clinical and Metabolic Genetics, Hospital for Sick Children, Toronto, ON, Canada, ${ }^{3}$ Department of Ophthalmology and Vision Sciences, Hospital for Sick Children, Toronto, ON, Canada, ${ }^{4}$ Division of Molecular Medicine, The Hospital for Sick Children Faculty of Medicine, University of Toronto, Toronto, ON, Canada

Biallelic mutations in $\mathrm{G}$ protein subunit beta 5 (GNB5) gene were first reported to cause a multi-system disorder with cardinal characteristics that include global developmental delay, seizures, generalized hypotonia, early-onset sinus node dysfunction and eye disease. We have previously reported the unique electroretinal signal of a 3-year-old female with a homozygous null variant in GNB5 (GNB5-L; NM_016194.3:c.1032C >A/p.Y344* and GNB5-S; NM_006578.3:c.906C $>$ A/ p.Y302*) representing dual retinal signalling defects that constitutes features of bradyopsia and rod ON-bipolar dysfunction. Subsequently, another female patient was identified with a homozygous missense variant in GNB5 (NM_016194.3:c.920T>G/p.L370R) 
through whole exome sequencing (WES). Furthermore, this patient also has a homozygous missense variant in MCCC1 (c.1394C > T p.T465I) that represents 3-methylcrotonyl-CoA carboxylase deficiency. The 3-MCC deficiency was confirmed enzymatically. Another heterozygous Hemoglobin E variant of codon 26 (GAG>AAG) [HBB c.79G $>A$ ] that represents $\mathrm{HbE}$ trait was also identified, consistent with patient's clinical presentation of splenomegaly. We therefore conducted a deep phenotypic analysis to segregate the contribution of each genetic variant with a focus on her ocular phenotype which involves high myopia. We also set out to study the impact of the GNB5 variants on gene expression, translation and protein-protein interaction using primary fibroblast culture from the patient. With increased use of WES in genetic diagnosis, more patients are found to have multiple molecular variants in syndromes with overlapping phenotypes. This study highlights the importance of detailed phenotyping and need of functional assays to aid variant classification. This work is supported by the Rare Disease Foundation and the BC Children's Hospital Foundation Microgrant.

Z. Shao: None. A. Tumber: None. J. Maynes: None. E. Tavares: None. S. Hewson: None. A. Schulze: None. P. Kannu: None. A. Vincent: None.

\section{E-P11.045}

Mutations in SALL1, TCL1 and TCL1B genes in patients with Goldenhar syndrome

\section{B. A. Marszalek-Kruk}

Department of Genetics, Wroclaw University of Environmental and Life Sciences, Wroclaw, Poland

Introduction: Goldenhar syndrome (GHS) is a rare inherited syndrome. The incidence of this disease is between 1:5600 to 1:25000. Syndrome is a multiple congenital disorder characterized by malformed ears, micrognathia, oral cavity malformations and dermal cysts. Due to malformation patients usually have problem with airway.

Materials and methods: DNA was isolated from peripheral blood leukocytes from the patients. Exons of genes SALL1 TCL1 and TCL1B were amplified by polymerase chain reaction (PCR) under optimal conditions, using specific primers. The PCR products were subjected to RFLP analysis and were purified on the DNA Gel Out columns followed by direct sequencing method.

Results: During examination were detected 3 substitutions. The c.3720G $>$ A mutation in exon 3 of the gene SALL1 which is a silent mutation (A1240A). Another of the described mutation is c. $166 \mathrm{G}>\mathrm{A}$ in exon 2 of TCL1 causing the conversion of $\mathrm{V} 56 \mathrm{I}$ and c. $277 \mathrm{G}>\mathrm{A}$ in exon 2 of TCL1B causing G93R. Additionally 4 polymorphisms were identified in introns: deletions c.76+36delAC in intron 1 SALL1 gene, c. $120+42 \mathrm{G}>\mathrm{T}$ in intron 1 of TCL1 and c.298_34C >T in intron 2 of TCL1 gene and substitution of c. $393+36 \mathrm{~T}>\mathrm{A}$ in intron 3 of TCL1B gene.

Conclusions: Molecular evaluation is of extreme importance for families with a history of GHS. Diagnosis, early detection of the syndrome and good management make possible of normal life of the patient.

B.A. Marszalek-Kruk: None.

\section{E-P11.046}

45, $\mathrm{X} / 46, \mathrm{XY}$ gonosomal mosaicism as an unexpected finding in a patient with lymphoproliferative disease

\section{J. Stellmachova ${ }^{l}$, V. Curtisova ${ }^{l}$, P. Rohon ${ }^{2}$, R. Vrtel ${ }^{l}$, P. Vrtel ${ }^{1}$, K. Adamoval ${ }^{l}$ D. Vrbicka $^{l}$, M. Prochazka ${ }^{l}$, M. Palova ${ }^{3}$}

${ }^{I}$ Department of Medical Genetics University Hospital and Palacky University, Olomouc, Czech Republic, ${ }^{2}$ Palacky University, Olomouc, Czech Republic, ${ }^{3}$ Department of Hematooncology University Hospital and Palacky University, Olomouc, Czech Republic

Introduction: We present a case of delayed diagnosis of a mixed gonadal dysgenesis in a 53 old woman.

Case presentation: The patient had short stature $(150 \mathrm{~cm})$, obesity, coarse facial features, mild male pattern hair loss, primary amenorrhea and borderline intellect. Her short stature and primary amenorrhea were never investigated. She was ascertained following a diagnosis of follicular lymphoma during a preventive mammography. Cytogenetic examination of the bone marrow detected complex changes including 46,XY clones prompting a request for genetic examination with following results: Karyotype from peripheral blood:45,X [12]/46,XY[38] (24\% monosomy X), FISH native peripheral blood: ish (DXZ1x1)[40]/(DXZ1x DYZ3x1)[160] (20\% monosomy $\mathrm{X})$, FISH buccal smear: ish (DXZ1x1)[68]/(DXZ1x DYZ3X1)[132] (34\% monosomy X). Her gonads were removed, and presence of both $45, \mathrm{X}$ and $46, \mathrm{XY}$ clones was detected by FISH. Patients with "45,X/46,XY mixed gonadal dysgenesis" syndrome can present with small stature have an increased risk of gonadoblastoma and congenital abnormalities of heart and kidneys (similar like Turner syndrome).

Conclusion: Early diagnosis of gonosomal mosaicism 45,X/46,XY enables multidisciplinary cooperation and treatment. Although the Czech Republic has a very good and longstanding tradition of primary care which enables the diagnosis in most cases to be reached by puberty there are still some adult patients who remain undiagnosed. This case highlights the usefulness of karyotype examination in 
women with unexplained primary amenorrhea and/or short stature.

Supported by MH CZ - DRO (FNOl, 00098892)

J. Stellmachova: None. V. Curtisova: None. P. Rohon: None. R. Vrtel: None. P. Vrtel: None. K. Adamova: None. D. Vrbicka: None. M. Prochazka: None. M. Palova: None.

\section{E-P11.048}

HDAC2 as a potential 'second hit' to KIAA0586. Novel 'two hit hypothesis' vs Digenic inheritance (DI) ?

\section{S. Kupchik ${ }^{l}$, S. Laul ${ }^{l}$, O. Beckett ${ }^{l}$, K. McWalter ${ }^{2}$, E. Normand ${ }^{3}$, Y. Kogelman ${ }^{1}$, P. Campeau ${ }^{4}$}

${ }^{1}$ Maimonides Medical Center, Brooklyn, NY, United States, ${ }^{2}$ Gene Dx, Gaithersburg, MD, United States, ${ }^{3}$ Gene Dx, Gaithesburg, MD, United States, ${ }^{4}$ University of Montreal, Montreal, QC, Canada

An 11 year old female with short stature, repaired open neural tube defect (NTD) and post axial polydactyly (PAP) was found on trio exome sequencing to have two frameshift variants, a paternally inherited pathogenic AR variant in KIAA0586: c.428delG:p.Arg143LysfsX4 and a de novo variant of unknown significance in HDAC2: c.1359_1362delAACA: p. Thr454ArgfsX51.KIAA0586 encodes a conserved centrosomal protein essential for ciliogenesis. Bi-allelic pathogenic variants in KIAA0586 result in a spectrum of autosomal recessive (AR) ciliopathy disorders ranging from short rib thoracic dysplasia with polydactyly-14 to Joubert syndrome. HDAC2, not currently known causative of disease, is a mammalian zinc finger transcription factor histone deacetylase 2 protein that acts both as positive and negative regulator of transcription. HDAC2 found expressed in mouse brain may play a role in normal proliferation and differentiation of neural precursor cells. There are reports of a de novo HDAC2 missense variant in an individual with a Cornelia de Langue syndrome like phenotype, NTD and PAP who also exhibited a 15q11.2-q11.3 duplication and in an individual with intellectual disability (ID). We hypothesize our patient's phenotypic manifestations could be explained by either digenic inheritance (DI) or by a novel 'two hit hypothesis' in which a single AR pathogenic allele reaches threshold of clinical expression due to presence of an additional de novo variant in a significant regulatory transcript namely HDAC2. If confirmed, it could have significant genetic counseling and genetic mechanisms of disease causation implications. A collaboration is under way to characterize phenotypic and biochemical consequences of HDAC2 mutations.

S. Kupchik: None. S. Lau: None. O. Beckett: None. K. McWalter: A. Employment (full or part-time); Significant; Gene Dx. E. Normand: A. Employment (full or part-time); Significant; GeneDx. Y. Kogelman: None. P. Campeau: None.

\section{E-P11.049}

New case of Bardet Bield Syndrome caused by novel biallelic mutations in IFT172 gene

M. Sánchez Soler ${ }^{l}$, M. Ballesta-Martínez $z^{2}$, A. SerranoAntón ${ }^{l}$, V. López-González ${ }^{2}$, L. Rodríguez-Peña ${ }^{l}$, E. Guillén-Navarro ${ }^{2}$

${ }^{I}$ Medical Genetic Sección, H. Clinic Universitary V. Arrixaca, IMIB-Arrixaca, El Palmar, Spain, ${ }^{2}$ Medical Genetic Sección, H. Clinic Universitary V. Arrixaca, IMIB-Arrixaca. CIBERER, El Palmar, Spain

Introduction: IFT172 is part of the intraflagellar transport (IFT), a bidirectional mechanism allowing the protein motility within the cilia and biallelic mutations in this gene have been described in patients with isolated retinosis pigmentosa, short-rib thoracic displasia 10 with or without polydactyly, joubert syndrome, recently with oro-fagiodigital syndrome and only in three cases of Bardet Bield syndrome (BBS).

Material and methods: Description of a new case of BBS caused by two novel mutations in IFT172 gene. Results5 years-old boy, first child of healthy non consanguineous parents. He had retinosis pigmentosa, myopia, language delay, obesity (weight $\mathrm{p}>99+4.8 \mathrm{SD}$ ), OFC $\mathrm{p}>99$ (2.7SD), generalized brachydactyly, abnormal palmar creases, round face, short palpebral fissures, small teeth and one cafe au lait spot. Cardiac and renal anomalies were ruled out and the hearing was normal. ArrayCGH and molecular study of Prader-Willi were normal. Firstly, commom mutations associated with BBS were studied without positive result. NGS of genes associated with retinosis pigmentosa detected two variants probably damaging in IFT172 gene: c. $2635 \mathrm{G}>\mathrm{A}$ and c. $4540-6 \mathrm{C}>\mathrm{G}$. The first was present in the mother and the second in the father, supporting their pathogenity.

Conclusions: We report on a new case of BBS due to biallelic mutations in IFT172 gene supporting its implication in this syndrome. We describe two new mutations in this gene. Achieving molecular diagnosis with NGS permits adequate genetic counseling in families.

M. Sánchez Soler: None. M. Ballesta-Martínez: None. A. Serrano-Antón: None. V. López-González: None. L. Rodríguez-Peña: None. E. Guillén-Navarro: None.

\section{E-P11.050}

Father-daughter incest: 30-year-old woman with Seckellike microcephaly and homozygous STIL pathogenic variant 
C. Ding ${ }^{l}$, J. Spranger ${ }^{2}$, S. Diederich ${ }^{l}$, D. Galetzka ${ }^{l}$, S. Schweiger ${ }^{l}$ O. Bartsch ${ }^{1}$

${ }^{1}$ Institut Institute of Human Genetics, University Medical Centre of the Johannes Gutenberg University, Mainz, Germany, ${ }^{2}$ Children's Hospital, University Medical Centre of the Johannes Gutenberg, Mainz, Germany

Among children resulting from incestuous matings, mental retardation and congenital malformations are known to be very common (PMID: 6779972, 5127404), but molecular studies to identify the underlying defects in these children are still scarce or lacking (PMID: 26320005, 20509080). We report here a woman resulting from a forced incestuous conception (father-daughter incest). For many years, her diagnosis had been Seckel syndrome due to extreme primary microcephaly (-10 SD), pre- and postnatal growth retardation $(-5 \mathrm{SD})$, prominent nose, and severe developmental retardation. Upon re-examination at age 30 years, she also displayed profound intellectual disability, absent speech, most severe behavioral abnormalities resulting in blindness (due to head banging and eye scratching) as well as tetraplegia. Microarray analysis revealed a highly increased loss of heterozygosity (LOH 25.3\%), consistent with incest. Of note, $\mathrm{LOH}>50 \%$ was detected for eight chromosomes. Whole exome sequencing revealed a homozygous variant c.3377A $>\mathrm{G}$ p.Tyr1126Cys in the STIL gene.

Pathogenic STIL variants result in non-syndromic autosomal recessive primary microvephaly type 7 (MCPH7, OMIM \#612703). The degree of intellectual disability among reported patients is very variable, ranging from mild to severe, and seems not to be genotype specific. Several features observed in the proband (including stereotypic behavior, tetraplegia, and profound global retardation) are not typical findings in MCPH7, suggesting that additional unrecognized recessive variants could possibly have contributed to the more complex phenotype in the patient, adding to the classic form of MCPH7.

C. Ding: None. J. Spranger: None. S. Diederich: None. D. Galetzka: None. S. Schweiger: None. O. Bartsch: None.

\section{E-P11.054}

The problem of identifying syndromes with a high degree of genetic heterogeneity and clinical polymorphism

\section{A. Galkina, A. V. Marakhonov, R. A. Zinchenko}

Research Center for Medical Genetics, Moscow, Russian Federation
Diagnosis and verification of multiple congenital dysmorphism (MCD) syndromes is often difficult due to the variety of clinical forms and inter- and intrafamilial clinical polymorphism. It is known that in sporadic cases, the diagnosis will be most reliable with the full range of phenotypic sings. Differential diagnosis of MCD syndromes can also be difficult due to the similarity of phenotypic features in different syndromic forms. In such cases, the problem of clarifying the diagnosis and resolving the issue of the possibility of conducting a prenatal diagnosis of the disease in the fetus in the family during subsequent pregnancy can be solved by the use of high-throughput DNA sequencing methods. Proband, a boy born in 2013, was first consulted with the aim of diagnosis at the age of 2 years old regarding complaints of short stature, congenital ptosis, nystagmus, severe hypotrichosis, renal hypoplasia, violation of pronation-supination (radio-lunar synostosis?), lobar holoprosencephaly, delayed development. Cartilage-hair hypoplasia and Noonan syndrome were excluded clinically. CMA was negative. The NGS revealed a small deletion in KIAA0556 gene (c.2373delC) in a homo- or hemizygous state. Thus, the diagnosis of Joubert syndrome (JS) type 26 was established. JS is a heterogeneous disease - 36 genetic forms are presented in the OMIM, all with AR type of inheritance which have far from classical phenotypic manifestations of this syndrome. A stricter approach to the description of phenotypes will probably solve the problem of such a high genetic heterogeneity. Supported by RFBR grant 18-015-00090.

V.A. Galkina: None. A.V. Marakhonov: None. R.A. Zinchenko: None.

\section{E-P11.055}

Co-occurrence of mutations in KIF7 and KIAA0556 in Joubert syndrome with ocular coloboma, pituitary malformation and growth hormone deficiency
M. Niceta ${ }^{1}$, M. L. Dentici, A. Ciolfi, R. Marini ${ }^{2}$, S. Barresi ${ }^{l}$, F. R. Lepri ${ }^{l}$, A. Novelli ${ }^{l}$, E. S. Bertini, ${ }^{1}$,

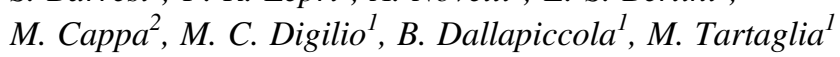

${ }^{1}$ Genetics and Rare Diseases Research Division, Ospedale Pediatrico Bambino Gesù, Rome, Italy, ${ }^{2}$ Unit of Endocrinology, Academic Department of Pediatrics, Ospedale Pediatrico Bambino Gesù, Rome, Italy

Introduction: Joubert syndrome is a recessive neurodevelopmental disorder characterized by clinical and genetic heterogeneity. Clinical hallmarks include hypotonia, ataxia, facial dysmorphism, abnormal eye movement, irregular breathing pattern cognitive impairment and, the molar tooth sign is the pathognomonic midbrain-hindbrain 
malformation on magnetic resonance imaging. The disorder is predominantly caused by biallelic mutations in more than 30 genes encoding proteins with a pivotal role in morphology and function of the primary cilium. Oligogenic inheritance or occurrence of genetic modifiers has been suggested to contribute to the variability of the clinical phenotype. We report on a family with peculiar clinical spectrum Joubert syndrome molecularly and clinically dissecting a complex phenotype, in which hypogonadism, pituitary malformation and growth hormone deficiency occur as major features.

Case Description and Methods: A 7 year-old male was enrolled in a dedicated "Undiagnosed Patients Program" for a peculiar form of Joubert syndrome complicated by iris and retinochoroidal coloboma, hypogonadism pituitary malformation, and growth hormone deficiency. The molecular basis of the complex phenotype was investigated by whole exome sequencing.

Results: The concomitant occurrence of homozygosity for mutations in KIF7 and KIAA0556 was identified, and the assessment of major clinical features associated with mutations in these two genes provided evidence that these two independent events represent the cause underlying the complexity of the present clinical phenotype.

Conclusion: Beside the clinical variability of Joubert syndrome, co-occurrence of mutations in ciliopathy-associated genes may contribute to increase the clinical complexity of the trait.

M. Niceta: None. M.L. Dentici: None. A. Ciolfi: None. R. Marini: None. S. Barresi: None. F.R. Lepri: None. A. Novelli: None. E.S. Bertini: None. M. Cappa: None. M.C. Digilio: None. B. Dallapiccola: None. M. Tartaglia: None.

\section{E-P11.056}

Neurodevelopmental disorders and chromatin remodeling dysregulation: the lessons from Kabuki syndrome spectrum and clinical presentation of Tunisian cases

N. B. ABDELMOULA, B. Abdelmoula, R. Louati, F. Abid, T. Sammouda, W. Smaoui, N. Abdellaoui, K. Trigui, O. Kaabi, W. Ltaif, S. Aloulou, S. Mallek, S. Kammoun, UR17ES36 members

\section{Medical University, Sfax, Tunisia}

Objective: Kabuki syndrome (KS) [MIM: 147920] is linked to dysregulated lysine degradation and chromatin organization pathways. Here, we report four Tunisian patients, who fulfilled clinical criteria of $\mathrm{KS}$, including neurodevelopmental deficits.

Clinical Presentation: During our genetic couselling at the medical university of Sfax (Tunisia), we recorded four patients with KS distinct face, neurodevelopmental deficit, congenital heart disease, and skeletal/dental malformations. Our patients have respectively partial atrio-ventricular septal defect, double outlet right ventricle, left ventricular hypertrophy and atrio-ventricular septal defect with atrial isomerism. Neurodevelopmental deficits were constant but variable with respectively developmental delay, behavioural troubles with autistic traits, perceptive sensory deafness, mental retardation and delayed speech with abnormal voice; learning disabilities and behavioural troubles; developmental delay, psycho-motor retardation, delayed speech, and mental retardation; and finally learning disabilities and hypernasal speech.

Conclusion: KS is a clinically recognizable syndrome and most patients have a pathogenic variant in the KMT2D or KDM6A genes. The five cardinal manifestations of KB defined in 1988 have been revisited recently (2018). The new consensus diagnostic criteria propose that a definitive diagnosis can be made in an individual of any age with a history of infantile hypotonia, developmental delay and/or intellectual disability, and one or both of other major criteria like pathogenic molecular variant. In fact, either with or without molecular genetic confirmation, it seems to be possible to go on toward a correct clinical diagnosis. The phenotypic variability is linked to the nature of the involved protein in the epigenetic machinery (readers, writers, erasers, or chromatin remodelers).

N.B. Abdelmoula: B. Research Grant (principal investigator, collaborator or consultant and pending grants as well as grants already received); Modest; Research Unit UR17ES36. B. Abdelmoula: B. Research Grant (principal investigator, collaborator or consultant and pending grants as well as grants already received); Modest; Research Unit UR17ES36. R. Louati: B. Research Grant (principal investigator, collaborator or consultant and pending grants as well as grants already received); Modest; Research Unit UR17ES36. F. Abid: B. Research Grant (principal investigator, collaborator or consultant and pending grants as well as grants already received); Modest; Research Unit UR17ES36. T. Sammouda: B. Research Grant (principal investigator, collaborator or consultant and pending grants as well as grants already received); Modest; Research Unit UR17ES36. W. Smaoui: B. Research Grant (principal investigator, collaborator or consultant and pending grants as well as grants already received); Modest; Research Unit UR17ES36. N. Abdellaoui: B. Research Grant (principal investigator, collaborator or consultant and pending grants as well as grants already received); Modest; Research Unit UR17ES36. K. Trigui: B. Research Grant (principal investigator, collaborator or consultant and pending grants as well as grants already received); Modest; Research Unit UR17ES36. O. Kaabi: B. Research Grant (principal 
investigator, collaborator or consultant and pending grants as well as grants already received); Modest; Research Unit UR17ES36. W. Ltaif: B. Research Grant (principal investigator, collaborator or consultant and pending grants as well as grants already received); Modest; Research Unit UR17ES36. S. Aloulou: B. Research Grant (principal investigator, collaborator or consultant and pending grants as well as grants already received); Modest; Research Unit UR17ES36. S. Mallek: B. Research Grant (principal investigator, collaborator or consultant and pending grants as well as grants already received); Modest; Research Unit UR17ES36. S. Kammoun: B. Research Grant (principal investigator, collaborator or consultant and pending grants as well as grants already received); Modest; Research Unit UR17ES36.

\section{E-P11.059}

\section{Lacrimo-auriculo-dento-digital LADD syndrome: first} case report of a Georgian patient

T. Tkemaladze ${ }^{1,2}$, E. Abzianidze ${ }^{l}$, E. Kvaratskhelia ${ }^{l}$, M. Ghughunishvili ${ }^{2,1}$, V. Skrahina ${ }^{3}$, A. Rolfs ${ }^{3}$

${ }^{1}$ Tbilisi State Medical University, Tbilisi, Georgia, ${ }^{2}$ Givi Zhvania Pediatric Academic Clinic, Tbilisi, Georgia, ${ }^{3}$ CENTOGENE AG, Rostock, Germany

Background: Lacrimo-auriculo-dento-digital (LADD) syndrome is extremely rare genetic disorder with only about 100 cases reported worldwide. LADD syndrome occurs due to pathogenic changes FGFR2, FGFR3 or FGF10 genes. Clinical signs and severity may vary, but almost uniformly there is involvement of the lacrimal systems, cup-shaped ears, dental and digital abnormalities.

Case Report: Here we report of a 9 years old girl with LADD syndrome from Georgia. At birth she presented with malformed left hand, ventricular-septal defect, congenital inguinal hernia and kidney aplasia. Dysmorphic features include lacrimal duct obstruction, low-set and cup-shaped ears, dental aplasia and salivary gland malformation. Her hearing loss started from 2,5 years and is slowly progressive. Interestingly she developed generalized seizures from 2,5 years which is not a common feature of LADD syndrome. Patient was on anti-epileptic treatment for 3 years and is now seizure-free and drug-free for already 4 years.

Methods: Whole exome sequencing (WES) was performed and c.1547C $>\mathrm{T}$ p.(Ala516Val) variant was identified in FGFR2 gene. In silico tools predict the change disrupts normal function of the FGFR2 protein. Additionally, parental testing confirmed de novo status of the variant.
Discussion: This is the first genetically confirmed case of LADD syndrome in Georgia, presenting with epilepsy as a new feature. Genetic testing lead to more accurate genetic and recurrence risk counseling. Our case expands the phenotypic and mutational spectrum of FGFR2 and confirms application of WES as a first-line diagnostic test in individuals with multiple congenital anomalies. More studies are needed to define whether genotype-phenotype correlations exist.

T. Tkemaladze: None. E. Abzianidze: None. E. Kvaratskhelia: None. M. Ghughunishvili: None. V. Skrahina: None. A. Rolfs: None.

\section{E-P11.060}

TGM1 mutation spectrum in patients with lamellar ichthyosis

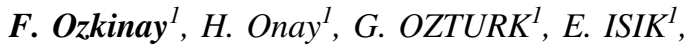
D. AYYILDIZ EMECEN ${ }^{l}$, E. ARSLAN ${ }^{l}, F . H_{A Z A N}^{2}$, T. Atik ${ }^{l}$

${ }^{1}$ Ege University Faculty of Medicine, Izmir, Turkey, ${ }^{2}$ S.B.U. DR.BEHCET UZ CHILDREN'S EDUCATION AND RESEARCH HOSPITAL, Izmir, Turkey

Aim: Autosomal recessive congenital ichthyosis (ARCI) is a heterogeneous group of keratinization disorders, caused by mutations in at least 13 different genes. Lamellar icthyosis (LI) which is one of the phenotypic features of ARCI presents with dark brown, plate-like scale with no erythroderma. TGM1 mutations is the most common cause of the classic LI phenotype. In this study, we investigated the clinical and molecular findings of eleven LI patients who had mutations in TGM1 gene.

Method: Ten patients clinically diagnosed as LI and two consangineous carrier parents (their child with LI had died) having mutations in TGM1 gene were included in the study. All patients (including death child) were examined by an experienced dermatologist and clinical geneticist. Patient histories; including demographic, clinical and laboratory findings, were obtained from hospital records. TGM1 gene analysis was performed using a next generation sequencing platform (Illumina MiSeq).

Results: Six patients were female, five male. Plate-like scale with no eritroderma, the typical features of LI, was defined in all patients. Eleven different mutations were identified; eight were missense, two frameshift and one nonsense. While ten patients carried the same mutations in both alleles, one was compound heterozygous (c.160C $>\mathrm{T}$; c.1469A $>$ G). Three of the eleven mutations (c.1148T $>\mathrm{G}$, c. $889 \mathrm{G}>\mathrm{A}$. C.2189delG) in the TGM1 gene had not previously been reported in the literature. 
Conclusion: By defining three novel mutations, this study enriched the molecular spectrum of ichthyosis, and also provided a further insight for genetic counselling.

F. Ozkinay: None. H. Onay: None. G. Ozturk: None. E. Isik: None. D. Ayyildiz emecen: None. E. Arslan: None. F. Hazan: None. T. Atik: None.

\section{E-P11.061}

\section{The Rare LIG4 Syndrome: A case report}

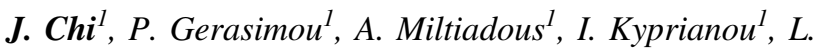
Koumas $^{1}$, L. Loizou ${ }^{2}$, E. Papachristodoulou ${ }^{2}$, E. Karaoli ${ }^{2}$, A. Loizou ${ }^{2}$, P. Costeas ${ }^{1}$

${ }^{1}$ Molecular Hematology-oncology, Karaiskakio Foundation, Nicosia, Cyprus, ${ }^{2}$ Pediatric Oncology-Hematology, Archbishop Makarios III Hospital, Nicosia, Cyprus

Introduction: The LIG4 syndrome is a rare hereditary disorder associated with impaired DNA double-strand break repair mechanisms. Patients show unusual facial features, microcephaly, growth and/or developmental delay, various skin abnormalities and pancytopenia, which is associated with combined immunodeficiency.

Case Description: 12-year old patient with obvious syndromic features with severe microcephaly, very short stature and a very low body weight. Bird-like facial features, resembling Seckel's syndrome or Fanconi anemia patients. He had no signs of acute illness nor malignancy. The patient is of Syrian descent and is the second child of a six-member family. Parents are first-degree relatives, both reported healthy.

Methodology: A custom HaloPlexHS (Agilent) NGS panel was used to investigate mutations in genes involved in Bone Marrow Failure/Severe Combined Neutropenia, Anemias and Chromosome Breakage Syndrome. Sanger sequencing performed parental carrier status.

Interpretation: Two variants in the LIG4 gene were identified, c.2440C $>\mathrm{T} \quad$ p.(Arg814Ter) and c.1273_1278delAGAGAA p.(Arg425_Glu426del). LIG4 c. $2440 \mathrm{C}>\mathrm{T}$ is a nonsense heterozygous variant which prematurely terminates the protein and is classified as pathogenic and LIG4 c.1273_1278del is an in-frame deletion classified as likely pathogenic according to ACMG recommendations. Compound heterozygous mutations in the LIG4 gene have been associated to LIG4 syndrome, an autosomal recessive disorder caused by homozygous or compound heterozygous mutations. Parental testing revealed that LIG4 c.2440C $>\mathrm{T}$ variant was maternally inherited and the LIG4 c.1273_1278delAGAGAA variant was paternally inherited.

Conclusion: The patient is compound heterozygous for the pathogenic (c.2440C $>\mathrm{T})$ and likely pathogenic (c.1273_1278del) variants in the LIG4 gene, consistent with LIG4 syndrome diagnosis. Parental testing confirmed compound heterozygosity.

J. Chi: None. P. Gerasimou: None. A. Miltiadous: None. I. Kyprianou: None. L. Koumas: None. L. Loizou: None. E. Papachristodoulou: None. E. Karaoli: None. A. Loizou: None. P. Costeas: None.

\section{E-P11.062}

A case ofantenatal Bartter's Syndrome with a novel $M A G E D 2$ variant manifesting with severe polyhydramnios and intrauterine fetal death

M. Nagasaka ${ }^{1,2}$, S. Nakago ${ }^{1}$, C. Nagano ${ }^{2}, N$. Morisada $^{2}, K$. $\mathrm{Nozu}^{2}$, K. Iijima ${ }^{2}$

${ }^{1}$ Takatsuki General Hospital, Takatsuki, Japan, ${ }^{2}$ Kobe University Graduate School of Medicine, Kobe, Japan

Introduction: Polyhydramnios is caused by various factors. Recently, the MAGED2 gene located on the X chromosome has been reported to be responsible for antenatal Bartter syndrome with most severe polyhydramnios.

Case: The patient was a girl died in utero at 31 weeks of gestation. The mother was a 29-year-old woman (gravida 3, para 2). The first child died in utero at 37 weeks of gestation without polyhydramnios. The second child was normally delivered at 36 weeks of gestation. She presented with severe polyhydramnios during the third pregnancy. Fetal development was good and no cardiac or morphological abnormalities were noted by fetal ultrasound. On fetal MRI at 27 weeks of gestation, no brain malformation or gastrointestinal obstruction was noted, but the bladder was large. She had been treated with amnioreduction six times in total, but the fetus died suddenly in utero at 31 weeks of gestation. The child was a girl weighing $1980 \mathrm{~g}$, and she had no apparent morphological abnormalities.

Genetic testing: Sequencing of DNA from the frozen umbilical cord of the third child revealed a de novo nonsense MAGED2 variant with heterozygosity. Skewed Xchromosome inactivation was observed via HUMARA assay.

Conclusion: We believe that our case showed a severe phenotype of antenatal Bartter's syndrome due to MAGED2 truncating variant and skewed $\mathrm{X}$-chromosome inactivation.

M. Nagasaka: None. S. Nakago: None. C. Nagano: None. N. Morisada: None. K. Nozu: None. K. Iijima: None.

\section{E-P11.066}

Intra-uterine fetal death in Meier-Gorlin Syndrome due to compound heterozygous variants of ORC6 gene with a novel mutation: a case report 
H. Thorn ${ }^{\text {I }}$, S. Drunat ${ }^{2}$, C. Fleury ${ }^{3}$, E. Alanio ${ }^{4}$, J. Bory ${ }^{5}$, S. Bonneau ${ }^{5}$, M. Doco-Fenzy ${ }^{6}$, M. Blocquaux ${ }^{7}$, C. Jacquin ${ }^{6}$, C. Poirsier ${ }^{8}$, M. Mozelle-Nivoix ${ }^{1}$

${ }^{I}$ Medical Genetic Service, CH Simone VEIL, Troyes, France, ${ }^{2}$ Laboratory of Molecular genetics, Hôpital Robert Debré, PARIS, France, ${ }^{3}$ Anatomical Pathology Laboratory, CHU de Reims, Reims, France, ${ }^{4}$ Anatomical Pathology laboratory, CHU de Reims, Reims, France, ${ }^{5}$ Obstetrics and Gynaecology Departments, CHU de Reims, Reims, France, ${ }^{6}$ Medical Genetic Service, CHU de Reims, Reims, France, ${ }^{7}$ Cytogenetics Laboratory and Laboratory of Molecular Genetics, CHU de Reims, Reims, France, ${ }^{8}$ Medical Genetic Service, CHU de Reims, Troyes, France

The Meier-Gorlin Syndrome (MGS) is a rare autosomal recessive disorder with characterized malformatives features. Among mutations of the pre-replication complex (ORC1, ORC4, ORC6, CDT1, CDC6), ORC1 and ORC4 mutations appear to be responsible of the most severe phenotypes. However, ORC6 mutations can lead to a severe abnormal embryological development.

We present the case of an intrauterine fetal death at 32 weeks during dichorionic diamniotic twin pregnancy. The fetus had a compound heterozygous ORC6 mutation, inherited from both parent.

At 16 weeks, a severe inadequate intra-uterine growth associated with a genu recurvatum and oligohydramnios were observed for one of the twins (ultrasound identification of the fetal sex: female). There was no family background aside the mother which presented neurofibromatosis type I. The parents were unrelated.

Fetal karyotype by amniocentesis on the pathological fetus was $46, X Y$. The genetic exploration was completed by MLPA. No quantitative chromosomal abnormalities were detected.

After caesarean delivery at 37 weeks, the fetal anatomopathological examination observed a male fetus with major inadequate intra-uterine growth, bilateral microtia, external genital hypoplasia and probable hypospadias, associated with abnormal joint morphology and skeletal malformations evoking a MGS. His twin brother was healthy.

A panel of genes responsible for primary microcephaly was performed and highlighted 2 ORC6 heterozygous VUS: c. $449+5 \mathrm{G}>\mathrm{A}$ variant, previously described in patients with Meier-Gorlin syndrome, and a new c.360-1G $>\mathrm{T}$ variant, never described before and that could also be responsible for Meier-Gorlin syndrome according to splicing prediction software. Study of alternative splicing confirmed the pathogenicity of the two variants.

H. Thorn: None. S. Drunat: None. C. Fleury: None. E. Alanio: None. J. Bory: None. S. Bonneau: None. M. Doco-
Fenzy: None. M. Blocquaux: None. C. Jacquin: None. C. Poirsier: None. M. Mozelle-Nivoix: None.

\section{E-P11.068}

A novel GTPBP2 splicing mutation in two siblings affected with microcephaly, generalized muscular atrophy, and hypotrichosis

\section{E. Akbariazar, I. Abdi Rad, A. Vahabi}

Urmia University of Medical Sciences, Urmia, Iran, Islamic Republic of

Background: One of the challenging pitfalls in the genetic counseling of patients with syndromic microcephaly is a definite clinical diagnosis to determine its etiology. Any unknown syndromic microcephaly necessitates piling up of clinical and molecular genetics data in the hope of getting a genotype-phenotype correlation.

Materials and methods: In this study, we identified a novel splice site mutation in the GTPBP2 gene by Whole Exome Sequencing in two siblings with microcephaly and progressive generalized muscular atrophy associated with hypotrichosis.

Results: This splice site mutation in the GTPBP2 gene is predicted to disrupt the highly conserved acceptor splice site of exon 4 in the GTPBP2 gene.

Conclusion: We describe syndromic microcephaly related to GTPBP2 gene in two siblings with an autosomal recessive pattern of inheritance, and to the best of our knowledge, is the first report of the GTPBP2 related hypotrichosis microcephaly with generalized muscular dystrophy.

Grant references: This research received no specific grant from any funding agency in the public, commercial, or not-for-profit sectors.

E. Akbariazar: None. I. Abdi Rad: None. A. Vahabi: None.

\section{E-P11.071}

Genotypes and phenotypes in $6 \mathrm{p} 25$ deletion syndrome

I. Bader ${ }^{\text {I, I. Reindl }}{ }^{2}$, G. Sander ${ }^{l}$, G. Kronberger ${ }^{1}$, A. Pleyers ${ }^{1}$, H. Mayr ${ }^{2}$, J. Koch ${ }^{2}$, J. Atzwanger ${ }^{2}$, C. Frenzel ${ }^{2}$, E. Haschke-Becher ${ }^{3}$, D. Kotzot ${ }^{1}$, W. Sperl ${ }^{2}$

${ }^{1}$ Unit for Clinical Genetics, Paracelsus Medical University Salzburg, Salzburg, Austria, ${ }^{2}$ Department of Pediatrics; Paracelsus Medical University, Salzburg, Austria, ${ }^{3}$ Department of Medical and Chemical Laboratory Diagnostics, Paracelsus Medical University Salzburg, Salzburg, Austria 
We report the phenotype and genotype of two children with a microdeletion in $6 \mathrm{p} 25$ :

1. A 4 years and 11 months old boy with a cMRI showing large bifronto-temporal arachnoidal cysts and multifocal white-matter-lesions involving the periventricular, deep, and subcortical cerebral white matter. The phenotype of the boy included mild developmental delay, strabism, slightly dysmorphic features and mild hearing impairment.

Molecular karyotyping detected a 3,9 $\mathrm{Mb}$ heterozygous microdeletion in $6 \mathrm{p} 25.3$ - $6 \mathrm{p} 25.2$ deleting 34 genes, 18 of which were OMIM annotated including the transcription factor FOXC1.

2. A 13 year old girl with Axenfeld-Rieger anomaly who developed glaucoma at the age of 12 years and had surgery for ASD II in the first year of life. The phenotype of the girl included typical dysmorphic features. She performs extremely well at high school (Gymnasium) and in sports. Molecular karyotyping detected $\mathrm{a} \sim 2,35 \mathrm{Mb}$ heterozygous Deletion in 6p25.3-6p25.2 deleting 17 genes, 8 of which are annotated in OMIM, including FOXC1.

From our cases and from review of the literature we conclude that a microdeletion in $6 \mathrm{p} 25$ should be considered as the cause in patients with unclear multifocal cerebral white matter abnormalities or Axenfeld-Rieger syndrome when additional dysmorphic features and congenital anomalies are present, even when intellectual abilities are within the normal range (Vernon et al. 2013).

I. Bader: None. I. Reindl: None. G. Sander: None. G. Kronberger: None. A. Pleyers: None. H. Mayr: None. J. Koch: None. J. Atzwanger: None. C. Frenzel: None. E. Haschke-Becher: None. D. Kotzot: None. W. Sperl: None.

\section{E-P11.074}

A novel deletion in $Z E B 2$ and biallelic frameshift variants in CNKSRI identified in a patient dysmorphologically diagnosed with Mowat-Wilson syndrome

\section{T. Kaname ${ }^{l}$, K. Yanagi ${ }^{l}$, M. Takeshita Th M. Omata $^{l}$,

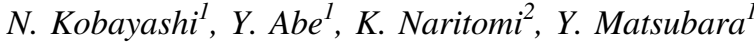 \\ ${ }^{1}$ National Center for Child Health and Development, Tokyo, Japan, ${ }^{2}$ Okinawa Nanbu Rehabilitation and Medical Center, Naha, Japan}

Mowat-Wilson syndrome (MOWS) is an autosomal dominant disorder characterized by distinctive facial appearance, intellectual disability, delayed motor development, microcephaly and short stature. It is known that most patients with MOWS have heterozygous mutations in the ZEB2 gene. We encountered a 3-year-old female patient with distinctive facial appearance (narrow bifrontal diameter, frontal bossing, telecanthus, epicanthal folds, prominent antihelices, prominent ears, uplifted earlobes, prominent nasal bridge), intellectual disability, delayed motor development, hypotonia and short stature. She was clinically diagnosed with MOWS. However, since direct sequencing for ZEB2 coding exons displayed no pathogenic variants in the patient, we performed whole exome analysis for trio in the family. No pathogenic SNVs in ZEB2 but biallelic frameshift variants (p.Thr282fs from mother and p.Ala503fs from father) in CNKSRl were identified in the patient. There are two reports that a recognizable autosomal recessive intellectual disability was caused by disruption of CNKSR1. The p.Thr282fs was recurrent and the p.Ala503fs was novel. However, clinical manifestations of the patient did not match the phenotypes of CNKSRl mutations, which were previously reported. In addition, a homozygous variant of p.Pro284HisfsTer74 in CNKSR1 was registered 224/ 273,674 in the gnomAD. Thus, copy number analysis was performed using whole exome data. We found an approximately $360 \mathrm{~kb}$ deletion around intron 7 of $Z E B 2$ in the patient, which was de novo. The deletion was confirmed by the brakepoint analysis using PCR and direct Sangersequencing. Based on these findings, we concluded that the patient was clinically and genetically diagnosed with MOWS caused by the novel deletion in ZEB2.<!--EndFragment-->

T. Kaname: None. K. Yanagi: None. M. Takeshita: None. M. Omata: None. N. Kobayashi: None. Y. Abe: None. K. Naritomi: None. Y. Matsubara: None.

\section{E-P11.075}

7q11.23 duplication in a Mayer-Rokitansky-KusterHauser syndrome patient

G. C. R. Cunha ${ }^{1}$, M. V. Zuben ${ }^{1}$, A. Lofrano-Porto', M. Soares ${ }^{2}$, P. Moretti ${ }^{1}$, R. S. Bonadio ${ }^{1}$, S. F. De Oliveira ${ }^{l}$, J. Mazzeu ${ }^{1}$, A. Pic-Taylor ${ }^{1}$

${ }^{1}$ University of Brasilia, Brasilia, Brazil, ${ }^{2}$ Hospital Universitario de Brasilia, Brasilia, Brazil

Mayer-Rokitansky-Küster-Hauser syndrome (MRKH) is a rare congenital disorderaffecting approximately 1:40005000 women. This condition is the second most common cause of primary amenorrhea and is characterized by absence or hypoplasic uterus, absence of the upper part of vagina and normal or hypoplasic fallopian tubes. A sexlimited (female) dominant autosomal inheritance with incomplete penetrance and variable expressiveness has been suggested. A range of gene and genomic alterations have been described and contributed to the identification of 
possible causative genes. For example, mutations in WNT4 and $L H X 1$ and CNV regions, especially 1q21.1, 16p11.2, 17q12, 22q11.21 and Xp22, were described in affected patients. Here we described a patient with MRKH presenting a single CNV known to cause the 7q11.23 microduplication syndrome. The 29 year old patient, born from unrelated parents, present primary amenorrhea, absence of uterus and a short vagina, characteristics of the MRKH syndrome, but also grade II hepatic steatosis, dorsal convex sinus scoliosis, anterolateral marginal osteophytes, ocular problems (myopia, astigmatism and strabismus), obesity and facial dysmorphisms, features that fit to $7 \mathrm{q} 11.23$ microduplication syndrome. The CMA (CytoScanTM 750k platform, Thermo Fisher Scientific, Affymetrix) revealed a $1,404 \mathrm{~Kb}$ duplication of $7 \mathrm{q} 11.23$ including 24 genes, the same recurrent duplication described in the 7q11.23 duplication syndrome. As the patient has MRKH and some of the features of 7q11.23 duplication syndrome, we propose that duplication of genes in this region might also be involved the etiology of MRKH in our patient

G.C.R. Cunha: None. M.V. Zuben: None. A. LofranoPorto: None. M. Soares: None. P. Moretti: None. R.S. Bonadio: None. S.F. De Oliveira: None. J. Mazzeu: None. A. Pic-Taylor: None.

\section{E-P11.079}

Variant of unknown clinical significance in the Verheij syndrome gene (PUF60) in a 5 months old boy with multiple neonatal anomalies

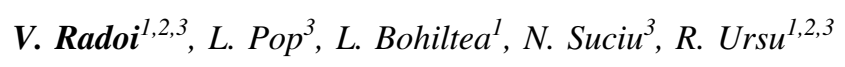

1 "Carol Davila" University of Medicine and Pharmacy, Department of Medical Genetics, Bucharest, Romania, ${ }^{2}$ Synevo Romania, Central Reference Laboratory, Department of Medical Genetics, Bucharest, Romania, ${ }^{3}$ National Institute for Mother and Child Health "AlessandrescuRusescu”, Bucharest, Romania

Reclassifying variants of unknown significance (VUS) coming from NGS sequencing is in many cases extremely challenging, needing a series of supporting criteria. We present the case of a 5 months old boy with a complex neonatal symptomatology including generalized hypotonia, global developmental delay, growth delay, hearing impairment, eye abnormalities, renal agenesis, a.o. The family history of the patient revealed a brother with polydactyly. The clinical suspicion at referral was CHARGE syndrome, with recommendations for genetic testing. Whole Exome Sequencing was performed on an Illumina platform. The results of the genetic analysis invalidated the CHARGE syndrome clinical suspicion, but revealed the presence of the heterozygous c.1604G $>C$ / p.Arg535Pro variant of unknown clinical significance (VUS, class 3 variant) in the PUF60 gene (Poly-U-Binding Splicing Factor, OMIM \#604819) on chromosome 8q24.3. The variant is a missense substitution and has never been described in literature before. Pathogenic variants in the PUF60 gene are documented to cause the autosomal dominant Verheij syndrome (OMIM \#615583), disorder with a complex but highly variable symptomatology with onset at birth which includes growth retardation, global developmental delay, eye abnormalities, renal agenesis. Considering the patient's phenotype and the clinical symptomatology of the syndrome produced by pathogenic mutations in the PUF60 gene, the identified c. $1640 \mathrm{~A}>\mathrm{G}$ variant may be reclassified as a class 4 (Likely Pathogenic) mutation. Parental testing for the identified mutation has been recommended, but also genetic testing of the brother with polydactyly might possibly be adviced.

V. Radoi: None. L. Pop: None. L. Bohiltea: None. N. Suciu: None. R. Ursu: None.

\section{E-P11.080}

A novel splice-variant in $N O N O$ in a boy with noncompaction cardiomyopathy and corpus callosum aplasia

V. Strehlow ${ }^{1}$, T. Bartolomaeus ${ }^{1}$, F. Cavallo ${ }^{2}$, M. Karnstedt ${ }^{1}$, S. Schubert ${ }^{l}$, M. Weidenbach ${ }^{2}$

${ }^{1}$ Institute of Human Genetics, University of Leipzig Medical Center, Leipzig, Germany, ${ }^{2}$ Department of Pediatric Cardiology, Heart Centre Leipzig, University of Leipzig, Leipzig, Germany

We describe a 6 month old boy with non-compaction cardiomyopathy (NCC), multiple VSDs, Ebstein's anomaly and aplasia of the corpus callosum. Prenatal examination revealed a VSD and corpus callosum aplasia. The boy was born at 38 weeks of gestation with low birth weight (-1.64 SD) and normal length and head circumference. The newborn needed respiratory support postnatally but could be discharged home at the age of 2 weeks. He was admitted to Heart Centre Leipzig at the age of 2.5 months due to failure to thrive and congestive heart failure. Echocardiography showed NCC of both ventricles, multiple ventricular septal defects and mild Ebstein's anomaly resulting in severe tricuspid regurgitation. Pulmonary artery banding was performed at the age of 4 months. Postsurgical course was prolonged but finally the child could be transferred to a specialized rehabilitation hospital at the age of 5 months. In the course of time the child showed a developmental delay, reduced spontaneous body movements and central hearing impairment. Exome sequencing revealed the hemizygous splice-variant c.651-1G>C, p.? in the NONO-gene. In the 
segregation analysis, a carriership for the $N O N O$-variant of the asymptomatic mother could be proven. RNA analysis of the NONO-gene revealed a splice effect on exon 7 and proved the pathogenic effect of the splice-variant. In summary, we report a case with a novel NONO variant (16 variants reported so far) and extend the phenotypic spectrum by NCC of both ventricles and corpus callosum aplasia.

V. Strehlow: None. T. Bartolomaeus: None. F. Cavallo: None. M. Karnstedt: None. S. Schubert: None. M. Weidenbach: None.

\section{E-P11.081}

Expanding the clinical and genetic spectrum of the Hermansky Pudlak syndrome type 9

M. M. Golas, S. von Hardenberg, T. Ripperger, J. Lentes, B. Schlegelberger, D. Steinemann, S. Morlot, B. Auber

Department of Human Genetics, Hannover Medical School, Hannover, Germany

Introduction: The Hermansky Pudlak syndrome (HPS) is a multi system autosomal-recessive disease that results from a dysfunction of lysosome-related organelles. HPS manifests as oculocutaneous albinism, bleeding diathesis and variable additional symptoms. Based on the causative gene, ten HPS subtypes have been described. HPS type 9 (HPS9) being caused by loss-of-function variants in BLOC1S6 is an exceedingly rare HPS subtype, and up to now, only three patients have been reported to be affected by HPS9.

Materials and methods: A DNA sample of the index patient was analyzed by whole exome sequencing and array comparative genomic hybridization (array-CGH).

Results: The 19-year-old female patient presented with oculocutaneous albinism, a bleeding disorder, and susceptibility to infections; however, the patient was neither affected by gastrointestinal nor pulmonary symptoms. On clinical examination, the patient presented with pale skin, blond hair as well as fair eyebrows and eyelashes. Nystagmus and photophobia were noted. Genetic analyses using whole exome sequencing identified a novel splice-site variant of BLOC1S6, c.83-2A>G p.? (ENST00000220531), in combination with a deletion of the second BLOC1S6 allele, which was confirmed as novel interstitial microdeletion using array-CGH (arr[GRCh37] 15q21.1 (45821145_46319127)x1). Based on the genetic analyses, the patient was diagnosed with HPS9 (MIM\#614171).

Conclusions: Our case suggests that immune deficiency may be associated with HPS9 and thus expands the clinical spectrum of HPS9. Rigorous genetic testing, including analyses for copy number alterations, is recommended for patients suspected for HPS to guide the clinical management and to define follow-up examinations based on the clinical spectrum of the HPS subtype.

M.M. Golas: None. S. von Hardenberg: None. T. Ripperger: None. J. Lentes: None. B. Schlegelberger: None. D. Steinemann: None. S. Morlot: None. B. Auber: None.

\section{E-P11.084}

Unusual clinical findings in a case with PACS2-related syndrome

D. Avdjieva-Tzavella ${ }^{1}$, S. Atemin ${ }^{2,3}$, T. Todorov ${ }^{4}$,

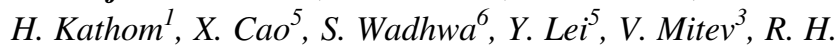
Finnell $^{6,7}$, A. Todorova ${ }^{4,3}$

${ }^{1}$ Department of Clinical Genetics, University Pediatric Hospital, Medical University, Sofia, Bulgaria, ${ }^{2}$ Genetic Medico-Diagnostic Laboratory "Genica", Sofia, Bulgaria, ${ }^{3}$ Department of Medical Chemistry and Biochemistry, Medical University, Sofia, Bulgaria, ${ }^{4}$ Genetic MedicoDiagnostic Laboratory "Genica”, Sofia, Bulgaria, ${ }^{5}$ Center for Precision Environmental Health, Department of Molecular and Cellular Biology, Baylor College of Medicine, Houston, TX, 77030,, TX, United States, ${ }^{6}$ Center for Precision Environmental Health, Department of Molecular and Cellular Biology, Baylor College of Medicine, Houston, TX, 77030, TX, United States, ${ }^{7}$ Departments of Molecular and Human Genetics and Medicine, Baylor College of Medicine, Houston, TX, United States

Introduction: Developmental and epileptic encephalopathies (DEE) are a heterogeneous clinical and genetic group of neurodevelopmental diseases. Recently, PACS2 gene mutations have been implicated in DEE with cerebellar dysgenesis and facial dysmorphism. Almost all described patients presented with a recurrent de novo missense mutation, c.625G>A (p.Glu209Lys). Their phenotype was characterized by early-onset epilepsy, cerebellar dysgenesis, dysmorphic facial features, moderate to severe developmental delay, and autism. We report the first Bulgarian case of this rare syndrome.

Case Report: The patient, a 17-month-old boy, was referred to genetic evaluation because of the developmental delay and neonatal convulsions started during the first week of life. He was born at term after complicated pregnancy. Birth weight was $3920 \mathrm{~g}$ and length was $51 \mathrm{~cm}$. Physical examination showed facial dysmorphism including hypertelorism, downslanted palpebral fissures, broad nasal root, broad flat nasal bridge, broad nasal tip, posteriorly rotated ears, downturned corners of the mouth and thin lips. Additional features include micropenis, inverted widely spaced nipples, one hypopigmented skin spot, one café au lait spot, nevus flammeus on the neck and coloboma of the iris. 
Results: The molecular genetic analysis was performed by whole exome sequencing (WES) of trio. The data interpretation revealed a pathogenic variant c.625G>A;p. Glu209Lys in the PACS2 gene. The variant was confirmed by Sanger sequencing.

Conclusions: Our study expands the clinical spectrum of PACS2 mutations. The coloboma of the iris is an unusual feature and it is difficult to determine whether it is etiologically related to this newly recognized syndrome or represents a coincidental finding.

D. Avdjieva-Tzavella: None. S. Atemin: None. T. Todorov: None. H. Kathom: None. X. Cao: None. S. Wadhwa: None. Y. Lei: None. V. Mitev: None. R.H. Finnell: None. A. Todorova: None.

\section{E-P11.086}

\section{Pendred syndrome in a 32-year-old woman- case report}

P. M. Pachniak, M. Malarska, H. Moczulska,

A. Lewandowska- Polak, J. Makowska, M. Borowiec

\section{Medical University of Lodz, Lodz, Poland}

Background: Pendred syndrome is an autosomal recessive inherited disease characterized by by congenital sensorineural hearing loss, hypothyroidism and Hashimoto's disease. There are significant problems with maintaining balance due to disturbances in the vestibular system of the internal ear.

Case: The patient is a32-year-old woman with rheumatoid arthritis, rheumatoid factor, ANA and anti CCP antibodies present in high titers, HLA-B27 negative. Patient has Hashimoto's disease, hyperinsulinemia with insulin resistance, problems with balance and permanent ear implants due to congenital hearing loss of unexplained cause. The $35 \mathrm{delG}$ heterozygote status was detected at the age of 18. Diagnostics was expanded adding exclusion of other deletions and insertions in the 12-72bp region of the 2nd exon of the GJB2 gene, with a particular indication of the exclusion of the $167 \mathrm{~T}$ deletion, the 314 deletion and the 333 deletion. The $1555 \mathrm{~A}>\mathrm{G}$ mitochondrial mutation was not found. The patient at the time was in good condition, with full verbal contact, actively working professionally. Due to presence of goiter, Hashimoto's disease, hyperinsulinemia and vestibular dysfunction, periodic dizziness Pendred's syndrome was clinically suspected. Genetic testing to confirm the syndrome is ongoing.

Conclusions: The pedrina protein mutations occurs in the thyroid follicles, internal ear, kidneys, brain, liver, as well as in semen. The disease picture can be very diverse. One should always remember to think about Pendred syndrome after excluding the most common genetic causes of hearing defects, because through adequate iodine supplementation, the development of goiter can be prevented or regress.

P.M. Pachniak: None. M. Malarska: None. H. Moczulska: None. A. Lewandowska- Polak: None. J. Makowska: None. M. Borowiec: None.

\section{E-P11.087}

Familial pericentric inversion of chromosome 3 predisposing to de novo interstitial microdeletion 3p14.3p21.31

R. V. Vazharova ${ }^{\text {I, I. Bradinova }}{ }^{2}$, S. Andonova ${ }^{2}$, S. Bichev ${ }^{2}$, A. Savov ${ }^{2}$

${ }^{1}$ Sofia University St. Kliment Ohridski, Faculty of Medicine, Department of Biology, Medical genetics and Microbiology, Sofia, Bulgaria, ${ }^{2}$ Medical University Sofia, UHOG "Maichin dom”, National genetic laboratory, Sofia, Bulgaria

Introduction: Pericentric chromosome inversions are rare (except the polymorphic inv(9)(p11;q12)) and are a known cause of reproductive failure or birth of individuals with chromosome imbalance. Here we report clinical and molecular findings of individuals from a family with a pericentric inversion of chromosome 3 .

Materials and methods: The family was revealed as part of a study of CNVs in a cohort of patients with intellectual disability and/or multiple congenital anomalies. According to the study protocol genetic tests included karyotyping, MLPA and arrayCGH. Array CGH analysis was performed using custom designed whole-genome oligonucleotide arrays (OGT, UK) with a median probe spacing of about $2.5 \mathrm{~kb}$ and median resolution of about $10 \mathrm{~kb}$. CytoSure Interpret (OGT, UK) software was used for CNV detection.

Results: In the reported family several relatives in four generations were found to carry an apparently balanced pericentric inversion of chromosome $3-\operatorname{inv}(3)(\mathrm{p} 21 ; \mathrm{q} 21)$. Four inversion carriers presented complex clinical features including mild/moderate intellectual disability, epilepsy, occult spina bifida and facial dismorphism. By arrayCGH the affected family members were found to carry a heterozygous $4.35 \mathrm{Mb}$ interstitial microdeletion (arr3p21.31p14.3(50,963,804-55,318,121)x1) encompassing a total of 79 genes and disrupting the DOCK3 gene.

Conclusions: Unusual findings in this family are that no carrier reported reproductive problems like infertility or miscarriages, all affected family members share the same interstitial microdeletion which occurred as de novo event in progenies of four different inversion carriers. This work was supported by funding from the Bulgarian Ministry of Education and Science / FNI, Grant: DTK02-51/2009.

R.V. Vazharova: None. I. Bradinova: None. S. Andonova: None. S. Bichev: None. A. Savov: None. 


\section{E-P11.088}

A Japanese girl with a novel variant of PIK3R1 showed deterioration of insulin resistance to biguanide treatment

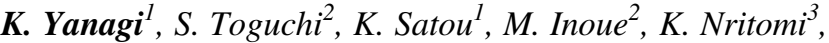 Y. Matsubara ${ }^{4}$, T. Kaname ${ }^{1}$}

${ }^{1}$ Genome Medicine, National Center for Child Health and Development, Tokyo, Japan, ${ }^{2}$ Department of Pediatrics, Naha City Hospital, Okinawa, Japan, ${ }^{3}$ Okinawa Nanbu Habilitation and Medical Center, Okinawa, Japan, ${ }^{4}$ National Center for Child Health and Development, Setagaya, Japan

SHORT syndrome (MIM\#269880) is defined by its acronym; short stature, hyperextensibility of joints or inguinal hernia or both, ocular depression, Rieger anomaly, and teething delay. Since the name was given by Gorlin (1975), fewer than 50 cases have been reported in the literature. About 10 heterozygous pathogenic variants of the PIK3RI gene in patients with SHORT syndrome have been reported to date. Here we report a Japanese girl with clinically and genetically diagnosed as SHORT syndrome. A patient is 17-year-old girl born to non-consanguineous parents at 40 weeks and 6 days with birth weight of $1,484 \mathrm{~g}$, height of $37.5 \mathrm{~cm}$ and head circumstance of $34 \mathrm{~cm}$. She had most of the clinically typical features of SHORT syndrome. Extensibility of joints were mild. Her face was triangular with telecanthus, deep-set eyes, wide nasal bridge, downturned corners of the mouth and large ears. Speech delay and hearing loss were also observed. Her bone age was equivalent to her age. Partial lipodystrophy of the face and upper limbs were recognizable. She had insulin-resistant diabetes whose blood glucose was difficult to control by biguanide treatment. A novel heterozygous missense variant in the PIK3RI gene (NM_181523) was identified in the patient by whole exome analysis. It was de novo. The variant was located in PI3K regulatory subunit P85 inter$\mathrm{SH} 2$ domain. A recent report shows that biguanide worsen insulin resistance in SHORT syndrome with a PIK3RI mutation. Her blood glucose became well controlled after changing medication of biguanide to mecarmin.

K. Yanagi: None. S. Toguchi: None. K. Satou: None. M. Inoue: None. K. Nritomi: None. Y. Matsubara: None. T. Kaname: None.

\section{E-P11.090}

New polydactyly phenotypes apparently associated with homozygous GLI3 variants
G. Van Winckel ${ }^{1}$, A. El Mouatani ${ }^{2}$, T. Attie-Bitach ${ }^{2}$, K. Zaafrane-Khachnaoui ${ }^{3}$, A. Superti-Furga ${ }^{l}$, H. Fodstad $^{l}$, F. Giuliano ${ }^{1}$

${ }^{1}$ Division of Genetic Medicine, Lausanne University Hospital (CHUV), Lausanne, Switzerland, ${ }^{2}$ Division of Genetics, Necker Hospital for Sick Children, Assistance PubliqueHôpitaux de Paris (AP-HP), Paris, France, ${ }^{3}$ Medical Genetics Unit 2, L'Archet Hospital, Nice, France

Heterozygous variants in GLI3 are involved in a clinical spectrum of polydactyly. Greig cephalopolysyndactyly and Pallister-Hall syndromes are the two main syndromic forms. Several isolated forms of postaxial or preaxial polydactyly are also recognized.

We report three unrelated individuals, all from consanguineous parents. An Afghan 10-year-old girl, presented bilateral postaxial polydactyly of the hands associated with developmental delay and multiple malformations. NGS disclosed a homozygous variant, c.668G >A (p.Ser223Asn), in GLI3. A newborn boy of Tunisian origin presented with an apparently isolated bilateral hand and feet postaxial polysyndactyly. He was found to have a homozygous variant c. $1673 \mathrm{C}>\mathrm{T}$ (p.Ser558Leu) in GLI3. Finally, an adult male was diagnosed with bilateral mesoaxial polydactyly of the hands associated with intellectual disability and multiple malformations. NGS disclosed an homozygous variant in GLI3, c.565_567delinsTCT (p.Pro189Ser).

To the best of our knowledge, only GLI3 monoallelic variants have been implicated in "GLI3 morphopathies" so far. Given (1) the similarity of the polydactyly phenotypes in our patients to those of classic GLI3 morphopathies, (2) the rarity of the variants observed, and (3) the absence of other pathogenic variants, we suggest that these homozygous variants in GLI3 could be causative. Of note is that the parents of these children, who are heterozygous for these GLI3 variants, are clinically unremarkable. This would indicate when monoallelic, these variants do not reach a pathogenic threshold necessary for clinical expression.

G. Van Winckel: None. A. El Mouatani: None. T. AttieBitach: None. K. Zaafrane-Khachnaoui: None. A. SupertiFurga: None. H. Fodstad: None. F. Giuliano: None.

\section{E-P11.091}

Prader-Willi syndrome caused by novel 3-7 exons deletion of SNRPN gene

R. Traberg, I. Nasvytiene, M. Sukys, V. Asmoniene

Lithuanian University of Health Sciences, Medical Academy, Department of Genetics and Molecular Medicine, Kaunas, Lithuania 
We report a 6 month old boy who was referred to clinical genetics consultation because of global development delay and failure to thrive. He was 4th child of non-consanguine Lithuanian couple. Older brother died due to sudden infant death syndrome at 12 months of age. The pregnancy was non complicated, full term and finished with caesarean section due to placental abruption. Muscular hypotonia and feeding difficulties noticed soon after birth. Basic biochemical blood tests, abdomen ultrasound scan, brain ultrasound scan were normal. Neurologic examination showed normal tendon reflexes but muscular hypotonia. On physical examination (6 months old): height $67 \mathrm{~cm}(25 \%)$, weight $6400 \mathrm{~g}(<3 \%$ ), head circumference $42 \mathrm{~cm}(10 \%)$. The patient was dysmorphic: almond-shaped eyes, narrow bifrontal diameter, narrow nasal bridge and thin vermilion of the upper lip, small hands and feet, unilateral cryptorchidism. We performed methylation specific multiplex ligation-dependent probe amplification (MS-MLPA) (SALSA MLPA Probemix ME028 Prader Willi/Angelman kit, MRC Holland) of 15q11-q13 region. The analysis showed 3-7 exons deletion of SNRPN gene with maternal methylation profile. Based on symptoms, clinical examination and molecular testing results the diagnosis of Prader-Willi syndrome was confirmed. The patient died at 8 months of age due to infection complications and there is no further data about clinical course of that case. There are only few reports about intragenic $S N R P N$ gene variants that cause Prader-Willi syndrome. We present 3-7 exons deletion of SNRPN gene as novel, not reported in scientific literature, possible cause of Prader-Willi syndrome.

R. Traberg: None. I. Nasvytiene: None. M. Sukys: None. V. Asmoniene: None.

\section{E-P11.092}

Report and investigation of regions of homozygosity in the autosomal genome of Brazilian individuals with intellectual disability and (or) multiple congenital anomalies previously investigated by chromosomal microarray analysis

G. R. Correia-Costa ${ }^{l}$, S. Spineli-Silva ${ }^{l}$, I. C. Sgardioli, A. P. dos-Santos ${ }^{l}$, J. R. Marques-Protal, R. Secolin ${ }^{l}$, N. Leijsten ${ }^{2}$, M. H. A. Ruiterkamp-Versteeg ${ }^{2}$, N. de Leeuw ${ }^{2}$, I. Lopes-Cendes ${ }^{1}$,

V. L. Gil-da-Silva-Lopes ${ }^{l}$, T. P. Vieira ${ }^{l}$

${ }^{1}$ Department of Medical Genetics and Genomic Medicine, School of Medical Sciences, State University of Campinas, Campinas, Brazil, ${ }^{2}$ Department of Human Genetics, Radboud University Medical Center, Nijmegen, Netherlands

Introduction: Regions of homozygosity ( $\mathrm{ROH})$ in the genome may be due to uniparental disomy or identity-bydescent and may be clinically relevant. The aim of this study was to report the frequency of $\mathrm{ROH} \geq 10 \mathrm{Mb}$ or $>1 \%$ in the autosomal genome of individuals with intellectual disability (ID) and (or) multiple congenital anomalies (MCA), and compare these data with a control group from the Brazilian population, including individuals from the BIPMed repository (www.bipmed.org).

Materials and methods: Calls of homozygosity from 265 patients and 289 controls tested with CytoScan ${ }^{\mathrm{TM}} 750 \mathrm{~K}$, CytoScan $^{\mathrm{TM}} H D$ or SNP $6.0^{\mathrm{TM}}$ arrays (Thermo Fisher Scientific Inc., Life Technologies, Carlsbad, CA, USA) were analyzed using the Chromosome Analysis Suite ChAS (version 4.0.0.385 (r28959) - Affymetrix ${ }^{\circledR}$ ) (hg19) with filters set as a minimum of 500 markers and size of $1,500 \mathrm{~kb}$. For complementary investigation, to date, whole exome sequencing (WES) was performed for 4 individuals with increased $\mathrm{ROH}$ in the genome.

Results: Single large $\mathrm{ROH} \geq 10 \mathrm{Mb}$ were detected in $1.9 \%$ of patients compared to $0.3 \%$ in the control group. $\mathrm{ROH}$ $>1 \%$ in the autosomal genome was detected in $7.2 \%$ of patients compared to $1.4 \%$ in the control group. WES revealed three pathogenic homozygous variants (ASPM, CTNS and DHCR7) in one patient with 5.56\% of ROH.

Conclusion: We found a higher proportion of ROHs in individuals with ID and (or) MCA. These results highlight the importance of investigating $\mathrm{ROHs}$ in this group of patients which with complementary tests, such as WES, can improve diagnostic yield. Financial support: CNPq, FAPESP(\#2018/08890-9), CAPES, FAEPEX-Unicamp.

G.R. Correia-Costa: None. S. Spineli-Silva: None. I.C. Sgardioli: None. A.P. dos-Santos: None. J.R. MarquesProta: None. R. Secolin: None. N. Leijsten: None. M.H.A. Ruiterkamp-Versteeg: None. N. de Leeuw: None. I. LopesCendes: None. V.L. Gil-da-Silva-Lopes: None. T.P. Vieira: None.

\section{E-P11.093}

A case with novel missense c.425T $>C$ mutation in RIPK4 Gene: Bartsocas Papas Syndrome

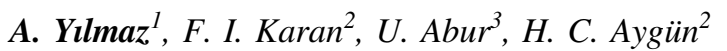 \\ ${ }^{I}$ Ondokuz Mayls University Medical Faculty, Department of \\ Pediatric Genetics, Samsun, Turkey, ${ }^{2}$ Ondokuz Mayıs \\ University Medical Faculty, Department of Neonatology, \\ Samsun, Turkey, ${ }^{3}$ Ondokuz Mayls University Medical \\ Faculty, Department of Medical Genetics, Samsun, Tur- \\ key
}

Backgound: Bartsocas Papas Syndrome (BPS) is a form of lethal popliteal pterygium syndrome, caused by homozygous mutation in the RIPK4 gene. Although BPS is 
described lethal, review of the literature reveals individuals who survive into childhood with this condition.

Case presentation: The patient was born after induction due to preeclampsia at 36 weeks gastation. The patient had cleft limb, small nose, cloudy cornea, popliteal and inguinal pterygia, complet syndactyly of all fingers and toes, absent of nails, pes equinovarus and ambigious genitale. Abdominal and transfontanelle ultrasonography was normal and no congenital heart malformation was detected on echocardiographic examination. Percutaneous gastrostomy tube was placed due to feding difficulty and tracheostomy was performed for apropriate follow up. BPS was considered with clinical findings. Next generation sequencing of RIBK4 revealed a novel homozygous missense mutation in exon 2 (c.425T $>$ C; p.Leu142Pro).

Conclusions: BPS is rare and lethal form of popliteal pterygium sendrome, characterized by cutaneous webbing across one or more major joints, cleft lip/palate, syndactyly, genital hypoplasia, ankyloblepharon, and ectodermal defects, absent eyelashes and eyebrows, and brittle nails. Our patient had a novel missense c.425T $>C$ mutation in RIBK4 gene. This mutation is predicted as pathological by in silico programs. Missense and nonsense mutations cause both the lethal and nonlethal recessive forms of multiple pterygium syndrome, characterized by severe organ anomalies of ectodermal origin. Although supportive treatments can be done life span is short so detection of mutation in lethal genetic diseases is very important for prenatal diagnosis and patient management in subsequent pregnancies.

A. Yllmaz: None. F.I. Karan: None. U. Abur: None. H.C. Aygün: None.

\section{E-P11.094}

Novel missense mutation c.1784A $>$ G, p.Tyr595Cys in RPS6KA3 gene responsible for Coffin Lowry Syndrome in a family with variable features and diabetes 2.

\section{T. YAMMINE ${ }^{1,2}$}

\section{${ }^{1}$ Medical Genetics Unit, Beirut, Lebanon, ${ }^{2}$ Saint Joseph University, Beirut, Lebanon}

Coffin Lowry syndrome (CLS) is an X-linked rare disorder, characterized by cognitive impairment, craniofacial dysmorphism, digit abnormalities, skeletal malformations, paroxysmal movement disorders and cardiac abnormalities. The gene altered in this disease is RPS6KA3 (ribosomal protein S6 kinase), located on Xp22.12. Previously identified mutations are distributed throughout the 22 exons of the gene. A large Lebanese family with several affected members, was referred to our Medical Genetics Unit for genetic evaluation. Analysis of RPS6KA3gene revealed the presence of a novel likely pathogenic variant, c.1784A $>\mathrm{G}$, p.Tyr595Cys in exon 19. This variant is located in the catalytic and ribosomal domain of the protein with a highly conserved amino acid. The physico-chemical difference between Tyrosine and cysteine are very significant. The variant was identified in all affected males and carrier females all displaying clinical features of variable severity, consistent with CLS. It is noteworthy that most of the affected or carrier individuals in the family developed diabetes type 2; further studies are required for a better delineation of the disorder and in order to investigate the correlation between type 2 diabetes and CLS.

T. Yammine: None.

\section{E-P11.095}

A novel PIK3R1 mutation in a Chinese female with SHORT syndrome and literature review

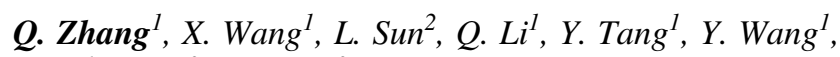
X. $\mathrm{Li}^{\mathrm{l}}, \mathrm{N} . \mathrm{Li}^{3}, \mathrm{~J}$. Wang ${ }^{3}$

${ }^{1}$ Department of Endocrinology and Metabolism, Shanghai Children's Medical Center, Shanghai Jiaotong University School of Medicine, Shanghai, China, ${ }^{2}$ Department of Pediatric and Adolescent Gynecology, The Children's Hospital of Zhejiang University School of Medicine, Hangzhou, China, Hangzhou, China, ${ }^{3}$ Department of Medical Genetics and Molecular Diagnostic Laboratory, Shanghai Children's Medical Center, Shanghai Jiao Tong University School of Medicine, Shanghai, China

Introduction: SHORT syndrome is a rare genetic disease named with the acronyms of short stature, hyper-extensibility of joints, ocular depression, Rieger anomaly and teething delay. It is inherited in an autosomal dominant manner confirmed by the identification of heterozygous mutations in PIK3R1. Only 43 cases have been confirmed with gene sequencing in the worldwide.

Materials and methods: Whole exome sequencing was performed on the patient and sanger sequencing confirmed the variant comparing to her parents. We made a literature review of all reported SHORT syndrome cases online before February 2020, trying to illustrate the genotypephenotype correlations.

Results: The patient was a 15-year-old female with intrauterine growth restriction, short stature, teething delay, characteristic facial gestalts, hirsutism and thyroid disease. Sequencing result indicated c.1960C $>$ T, a novel de novo nonsense mutation, leading to the termination of protein translation (p. Gln654*). We also thoroughly reviewed and presented the clinical manifestations of SHORT syndrome in 43 patients according to the mutations in different exons/ introns and in different domains of PIK3R1. 
Conclusions: We reported the first patient with SHORT syndrome in China, who was also the first patient with thyroid disease. Features described in the acronym of SHORT syndrome are not universally seen. Thyroid disease may be a new clinical symptom of patient with SHORT syndrome although it need more evidence to support.

Q. Zhang: None. X. Wang: None. L. Sun: None. Q. Li: None. Y. Tang: None. Y. Wang: None. X. Li: None. N. Li: None. J. Wang: None.

\section{E-P11.096}

The importance of molecular diagnosis in Silver Russell syndrome for the management of an adult patient

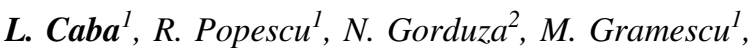
M. Panzaru', M. Vicol ${ }^{3}$, C. Rusul, E. Gorduza ${ }^{l}$

${ }^{1}$ University of Medicine and Pharmacy "Grigore T. Popa”, Medical Genetics Department, Iasi, Romania, " 2 "St. Spiridon" Hospital, Endocrinology Department, Iasi, Romania, ${ }^{3}$ University of Medicine and Pharmacy "Grigore T. Popa", Bioethics Department, Iasi, Romania

Introduction: Silver-Russell syndrome (SRS) is a rare disease associated with prenatal and postnatal growth retardation, characteristic craniofacial dysmorphic features (relative macrocephaly, prominent forehead), body asymmetry and feeding difficulties (Netchine-Harbison Clinical Scoring System). SRS is characterized by genetic heterogeneity because it could be determined by: hypomethylation of the imprinted control region 1 (ICR1) at 11p15.5, maternal uniparental disomy of chromosome 7 (mUPD7), duplications, deletions or translocations involving the imprinted region of chromosome 7 . We present a case with molecularly confirmed diagnosis of SRS at the age of 23 years.

Materials and methods: The patient was clinically diagnosed at 3 years of age with SRS based on delayed prenatal and postnatal growth, relative macrocephaly, triangular face, prominent forehead, dental malposition, facial and limb asymmetry, bilateral finger V clinodactyly. The karyotype was normal 46,XX. In time, bone changes of osteoarthritis of the elbow joint, osteopenia, and hypothyroidism were added. At age of 23 years, the patient was molecularly tested by MLPA technique. We used SALSA MLPA kit ME030 BWS/RSS (MRC Holland, Amsterdam, The Netherlands). The MLPA result confirmed the hypomethylation of H19/IGF2 IG-DMR at 11p15.5. In this situation, the recurrence risks in sibs of the proband and in offspring of the proband are low.

Conclusions: Although the Netchine-Harbison Clinical Scoring System is a sensitive diagnostic scoring system, molecular testing is especially important in adulthood for genetic counseling and the risk of recurrence, also for establishing the optimal prenatal diagnosis methods depending on the mechanism of disease.

L. Caba: None. R. Popescu: None. N. Gorduza: None. M. Gramescu: None. M. Panzaru: None. M. Vicol: None. C. Rusu: None. E. Gorduza: None.

\section{E-P11.100}

RBM10 (likely) pathogenic variants are associated with a broad phenotypic spectrum, beyond the classic TARP syndrome features.

C. Kumps ${ }^{l}$, E. D'Haenens ${ }^{l}$, S. Vergult ${ }^{l}$, B. Menten ${ }^{1}$, J. Leus ${ }^{2}$, R. Van Coster ${ }^{3}$, A. Jansen ${ }^{4}, K$. Devriendt ${ }^{5}$, A. Oostra ${ }^{6}$, O. Vanakker ${ }^{1}$

${ }^{1}$ Center for Medical Genetics, Ghent University Hospital, 9000 Ghent, Belgium, ${ }^{2}$ Medisch Centrum Maria Middelares, 9050 Gentbrugge, Belgium, ${ }^{3}$ Department of Pediatrics, Division of Pediatric Neurology and Metabolism, Ghent University, 9000 Ghent, Belgium, ${ }^{4}$ Pediatric Neurology Unit, Department of Pediatrics, UZ Brussel, 1090 Brussels, Belgium, ${ }^{5}$ Center for Medical Genetics, Katholieke Universiteit Leuven, 3000 Leuven, Belgium, ${ }^{6}$ Department of Pediatrics, Division of Pediatric Neurology and Metabolism, University Hospital Ghent, 9000 Ghent, Belgium

Introduction: TARP syndrome (Talipes equinovarus, Atrial septal defect, Robin sequence and Persistent left superior vena cava) is a rare $\mathrm{X}$-linked disorder caused by mutations in the RBM10 gene. So far, 22 patients have been reported. We present two novel patients with likely pathogenic RBM10 variants.

Case reports: The first male proband was born preterm with average low birth parameters. Postnatally, a VSD, aspecific brain abnormalities and optical nerve hypoplasia was noted. He displayed facial dysmorphism with telecanthus, upslanting palpebral fissures, a large mouth with downturned mouth corners and retrognathia. There was a growth and development delay. An RBM10 frameshift variant (c.413_416del, p.Ser138Trpfs*60) was identified, which was inherited from a mosaic $(13 \%)$ mother. The second male proband was born preterm with average birth measurements. After birth, limb contractures, a pectus carinatum, scoliosis and arachnodactyly were noted. Facial features included frontal bossing, crumpled ears and microretrognathia. A diagnosis of congenital contractural arachnodactyly (CCA) was made based on a FBN2 missense mutation. Upon follow-up, he displayed severe growth and developmental delay and brain abnormalities, atypical for CCA. Further investigations revealed a 
maternally inherited c. $1888+4 \mathrm{~A}>\mathrm{C}$ splice site variant in the RBM10 gene, that caused exon 15 skipping.

Conclusion: Initially RBM10 mutations were described in patients who featured the four cardinal features of TARP syndrome. However, recurrent clinical characteristics appear to include severe developmental delay, growth deficiency, heart defects, central nervous system, vision, skeletal and facial abnormalities. These 2 additional cases illustrate the highly pleiotropic nature of RBM10 mutations, beyond the classic TARP syndrome features.

C. Kumps: None. E. D’Haenens: None. S. Vergult: None. B. Menten: None. J. Leus: None. R. Van Coster: None. A. Jansen: None. K. Devriendt: None. A. Oostra: None. O. Vanakker: None.

\section{E-P11.101}

TBL1XR1 gene (\#608628) ¿Different phenotypes related to same variant?

\section{A. Serrano Antón ${ }^{1,2}$, M. Sánchez Soler ${ }^{1,2}$, M. Ballesta Martínez $^{1,2}$, V. López González ${ }^{1,2}$, L. Rodríguez Peña ${ }^{1,2}$, L. Rausell ${ }^{3}$, M. García Hoyos ${ }^{3}$, E. Guillén Navarro ${ }^{1,2}$}

${ }^{1}$ Sección Genética Médica. Hospital Clínico Universitario Virgen de la Arrixaca, IMIB-Arrixaca, Murcia, Spain, ${ }^{2}$ Grupo Clínico Vinculado al Centro de Investigación Biomédica en Red de Enfermedades Raras (CIBERER), Instituto de Salud Carlos III (ISCIII), Madrid, Spain, ${ }^{3}$ Departamento de Genética Médica. Instituto de Medicina Genómica (IMEGEN), Valencia, Spain

Introduction: Pierpont syndrome (PS) is a syndromic intellectual disability that comprises developmental delay, short stature, dysmorphic features and distal limb anomalies with deep palmar and plantar creases. Recurrent de novo missense variant (p.Tyr446Cys) in TBL1XR1 gene was identified as disease-causing. Recently, two patients with Pierpont-like syndrome and different de novo missense variants in TBL1XR1 have been reported.

Materials and methods: We describe the case of a 14year-old girl, born to healthy non-consanguineous parents. Pregnancy and neonatal period were uneventful. Postnatal growth with height at eighth centile and normal weight. She presented mild truncal hypotonia with psychomotor and expressive language delay. She attends to special school with major support and speech therapy. Clinical phenotype with narrow palpebral fissures, depressed nasal bridge, broad nose, long upper lip with flat philtrum, everted lower lip and prominent chin. Short and broad hands and feet with deep-set grooves in his palms and soles.

Results: Brain MRI, ophthalmological examination, renal ultrasound, metabolic analysis, Array-CGH, Fragile-X and targeted exome sequencing yielded normal results. Whole exome sequencing revealed a de novo heterozygous missense variant c.734A $>$ G; p.Tyr245Cys in TBL1XR1 gene.

Conclusions: We report on a new case characterized by clinical phenotype of PS and de novo missense variant c.734A $>\mathrm{G}$ in TBL1XR1 gene. This variant has been prior reported in patient given with developmental delay, short fingers and toes but who does not share distinctive facial features of PS. Hitherto, only ten patients with PS have been published. So, our report provided additional clinical and molecular data.

A. Serrano Antón: None. M. Sánchez Soler: None. M. Ballesta Martínez: None. V. López González: None. L. Rodríguez Peña: None. L. Rausell: None. M. García Hoyos: None. E. Guillén Navarro: None.

\section{E-P11.103}

Multi-country teamwork to investigate an infant with 45,X/46,X $\operatorname{der}(Y) t(Y ; 14)$, whirl-pooledtype pigmentary mosaicism, and ambiguous genitalia

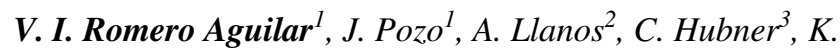
Hosomichi $^{4}$, A. Tajima ${ }^{4}$

${ }^{I}$ Universidad San Francisco de Quito, Cumbaya, Ecuador, ${ }^{2}$ Department of Genetics, Hospital General de las Fuerzas Armadas, Quito, Ecuador, ${ }^{3}$ Institut für humangenetik, Universitätsklinikum Jena, Jena, Germany, ${ }^{4}$ Department of Bioinformatics and Genomics, Kanazawa University, Kanazawa, Japan

Introduction: A newborn baby was born in Ecuador from a young non-consanguineous couple, with pigmentary mosaicism in whirl-pooled hyperpigmentation along the body, ambiguous genitalia with no visible testicles and a positive screening for suprarenal hyperplasia. A karyotype showed a trisomy 14 with an additional section on the extra chromosome. Trisomy 14 is one of the aneuploidies compatible with live in a mosaic state and many cases of rearrangements with chromosome 14 had been described in the literature. The predominant phenotype of trisomy 14 mosaicism includes prenatal and postnatal growth failure, ear abnormalities, congenital heart disease, developmental delay and genitourinary abnormalities. Our patient only shared some of phenotypic characteristics making additional genetic tests compulsory.

Materials and methods: We performed a multi-country teamwork investigation including karyotype in Ecuador, FISH in Germany and WES in Japan

Results: Karyotype and FISH results concluded 45,X/46, $\mathrm{X} \operatorname{der}(\mathrm{Y}) \mathrm{t}(\mathrm{Y} ; 14)$. WES identified no pathogenic nor likely pathogenic variants in the genes associated to suprarenal hyperplasia 
Conclusions: Our case is the first report of an infant with a $45, \mathrm{X} / 46, \mathrm{X} \operatorname{der}(\mathrm{Y}) \mathrm{t}(\mathrm{Y} ; 14)$. The pigmentary mosaicism is a common feature on trisomy 14 . The ambiguous genitalia were the result of the mosaicism which included Turner syndrome and an abnormal male cell-line. Finally, even though there was a positive test for suprarenal hyperplasia, WES analysis reported there were no damaging variants and steroids were suspended.

V.I. Romero Aguilar: None. J. Pozo: None. A. Llanos: None. C. Hubner: None. K. Hosomichi: None. A. Tajima: None.

\section{E-P11.104}

Partial trisomy 1q42.11-q44 clinical and molecular cytogenetic characterization. Case presentation

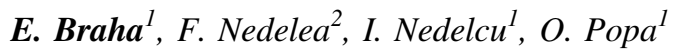

${ }^{I}$ National Institute of Endocrinology "CI Parhon", Bucuresti, Romania, ${ }^{2}$ Clinical Hospital Filantropia, Bucuresti, Romania

Partial 1q trisomy syndrome is an extremely rare chromosomal abnormality, with only a few cases reported. Frequent features were short stature, multiple minor anomalies, congenital heart defects especially for terminal duplication, learning difficulty, speech and developmental delay. Our goal is to contribute to clinical observation of 1q terminal duplication. We report a male infant (4 months old) with negative family history. Clinical features include intrauterine growth retardation with short stature at the time of clinical evaluation (-2.04 SD), mild facial dysmorphia (microretrognathia, thick eyebrows, hypertelorism, short nose, long smooth philtrum, thin upper lip vermillion, tented upper lip, dysplastic ears with small triangular earlobe), atrial septal defect ostium secundum, patent ductus arteriosus, partial 2-3 toe cutaneous syndactyly, little developmental progress (without head control at 4 months). Gbanded karyotype did not reveal any chromosomal abnormalities. The breakpoints were characterised by array CGH using the oligoarray platform CytoScan 750K Array, Gene Scan 3000 (Affymetrix). Our patient has a partial duplication of chromosome 1 between 1q42.11-q44 of $24.72 \mathrm{Mb}$ in lenght (nucleotides 224,499,474 $249,224,684$ ) according to NCBI 37/hg19. The parents karyotype was normal and FISH tests (for breakpoint) is to be performed. In conclusion we report a 4 months-old male with the distinctive clinical features and probably de novo 1q42.11q44 duplication without parental chromosomal abnormalities. The case requiers long-term medical followup for other clinical particulaties highlighted with aging.

E. Braha: None. F. Nedelea: None. I. Nedelcu: None. O. Popa: None.

\section{E-P11.107}

Possible effect of the imprinting on the Turner's phenotype

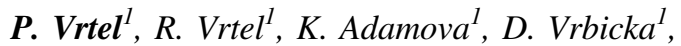
M. Prochazkal, J. Stellmachova ${ }^{l}$, E. Klaskova ${ }^{2}$, J. Zapletalova ${ }^{2}$, J. Pavlicek ${ }^{3}$, V. Hana ${ }^{4}$, V. Stara ${ }^{5}$, O. Soucek ${ }^{5}$ J. Lebl

${ }^{I}$ Department of Medical Genetics, University Hospital and Palacky University, Olomouc, Czech Republic, ${ }^{2}$ Clinic of Paediatrics, University Hospital and Palacky University, Olomouc, Czech Republic, ${ }^{3}$ Clinic of Paediatrics, University Hospital, Ostrava, Czech Republic, ${ }^{4} 3$ rd Department of Medicine - Department of Endocrinology and Metabolism, First Faculty of Medicine, Charles University and General University Hospital, Prague, Czech Republic, ${ }^{5}$ Department of Pediatrics 2nd Faculty of Medicine, Charles University and University Hospital Motol, Prague, Czech Republic

Monosomy of chromosome $\mathrm{X}$ - Turner syndrome (TS) is the only monosomy postnatally compatible with life. The incidence of this chromosomal abnormality is 1:2500 live female births. Their phenotype can be very variable. Common is low stature, congenital heart defects, renal abnormalities etc. Some of these defects correlate with karyotype - karyotype $45, \mathrm{X}$ is the most severe.

Other hypothesis is focused on parental origin of chromosome X. Specific defects can correlate with paternal or maternal origin of gonosome. We used molecular genetic analysis for detection microsatellite DNA markers on chromosome $\mathrm{X}$ to reveal the origin of these gonosome in TS patients.

We selected cohort of $50 \mathrm{TS}$ patients (plus their parents) with 45,X karyotype confirmed by karyotype and native FISH. We also tested patients with 46,XX karyotype (but Turner phenotype), for hypothesis of maternal $\mathrm{X}$ chromosome homology.

DNA was isolated from peripheral blood of the patients and the cells of the buccal mucosa of the parents. We used the Argus $\mathrm{X}-12 \mathrm{QS}$ forensic kit for the $\mathrm{X}$-chromosome analysis, which was optimized for our genetic testing. The method is based on detection of 12 STR markers from chromosome X.

Results suggest that $70 \%$ of $\mathrm{X}$ chromosomes are of maternal origin and $30 \%$ cases of paternal origin. The origin of X chromosome in 46,XX cohort was biparental. The next part of our project will be a correlation specific heart defects and renal abnormalities with the origin of gonosome. Supported by MH CZ - DRO (FNOI, 00098892) and grant nr. 17-29111A 
P. Vrtel: None. R. Vrtel: None. K. Adamova: None. D. Vrbicka: None. M. Prochazka: None. J. Stellmachova: None. E. Klaskova: None. J. Zapletalova: None. J. Pavlicek: None. V. Hana: None. V. Stara: None. O. Soucek: None. J. Lebl: None.

\section{E-P11.110}

Uniparental disomy of chromosome 16: a case report with a new cardiac malformation

F. Di Palma ${ }^{1}$, M. Goldoni ${ }^{2}$, E. Marchionni ${ }^{1}$, B. Caravale ${ }^{3}$, M. Mondovi ${ }^{3}$, G. Mastromoro ${ }^{1}$, D. Guadagnolo ${ }^{1}$,

P. Versacci ${ }^{4}$, V. D’Ambrosio ${ }^{5}$, A. Giancotti ${ }^{5}$, L. Bernardini $^{6}$, A. Pizzuti ${ }^{1}$

${ }^{1}$ Department of Experimental Medicine, Sapienza University, Rome, Italy, ${ }^{2}$ Medical Genetics Unit, Casa Sollievo della Sofferenza IRCCS Foundation, San Giovanni Rotondo, Italy, ${ }^{3}$ Department of Developmental and Social Psychology, Sapienza University, Rome, Italy, ${ }^{4}$ Department of Pediatrics, Sapienza University, Rome, Italy, ${ }^{5}$ Department of Maternal and Child Health and Urologic Science, Sapienza University, Rome, Italy, ${ }^{6}$ Medical Genetics Unit, Casa Sollievo della Sofferenza IRCCS Foundation, San Giovanni Rotondo, San Giovanni Rotondo, Italy

Introduction: Maternal uniparental disomy of chromosome 16 [UPD(16)mat] is the most often reported UPD other than $\mathrm{UPD}(15)$. In literature there is not a specific phenotype associated with upd(16)mat and few follow-up data are reported.

Materials and methods: We present the case of a 1-y.o. male of a healthy non-consanguineous parents. In prenatal diagnosis IUGR, polydramnios and single umbilical artery was reported. He born late preterm, small for gestational age, and showed facial dysmorphisms and congenital malformations (esophageal atresia, cardiac defects, mild bone alterations, hypospadias). Transesophageal echocardiography detected persistency of left superior vena cava draining into the left atrium through an unroofed coronary sinus. Brain ultrasound showed dilated and asymmetric lateral ventricles. At present, cognitive and language abilities are adequate for age but he has mild gross motor delay. Genomic DNA was extracted from peripheral blood and analyzed by SNP-array (Cytoscan HD; Thermo Fisher Scientific).

Results: SNP-array analysis showed a $147 \mathrm{~Kb}$ homozygous 16p13.3 microdeletion and a $114 \mathrm{~kb} 16 \mathrm{q} 24.3$ microtriplication, both segregated from the mother. Region of Homozigosity $(\mathrm{ROH})$ analysis showed two ROH of 7 and $13 \mathrm{Mb}$ on chromosome 16 compatible with the presence of UPD(16) of maternal origin. The possible presence of a residual mosaic trisomy 16 was excluded by karyotype analysis and FISH.

Conclusions: This is an additional case of the phenotypic characterization of $\operatorname{UPD}(16)$ mat. To date, it is the first time that an unroofed coronary sinus is described within cardiac malformations associated with UPD(16)mat. Moreover, according to literature data, it confirms the need to extend follow-up.

F. Di Palma: None. M. Goldoni: None. E. Marchionni: None. B. Caravale: None. M. Mondovì: None. G. Mastromoro: None. D. Guadagnolo: None. P. Versacci: None. V. D’Ambrosio: None. A. Giancotti: None. L. Bernardini: None. A. Pizzuti: None.

\section{E-P11.111}

Clinical analysis VACTERL/VACTER cases at children hospital in Bogotá, Colombia

\section{J. S. Rincon Redondo ${ }^{1}$, I. Rueda ${ }^{1}$, Y. Ladino ${ }^{2,3}$, M. C. Cuellar', L. A. Gonzalez', C. M. Restrepo ${ }^{4}$, O. I. Beltran ${ }^{1,2}$}

${ }^{1}$ Facultad de Medicina, Universidad Militar Nueva Granada, Bogotá DC, Colombia, ${ }^{2}$ Grupo GenHOMI, Hospital Pediátrico de la Misericordia, Bogotá DC, Colombia, ${ }^{3}$ Maestría en Genética Humana, Universidad Nacional de Colombia, Bogotá DC, Colombia, ${ }^{4}$ Unidad de Genética y Genómica, CIGGUR, Escuela de Medicina y Ciencias de la Salud, Universidad del Rosario, Bogotá DC, Colombia

Introduction: The VACTER-L/VATER association has been divided on three subtypes to improve clinical classification and epidemiologic analysis. It is an acronym that corresponds to a group of diverse and complex malformations that was initially described as the non-random occurrence of five anomalies together.

Methods: Descriptive study of case series of children with VACTER-L/VACTER diagnosis (using the criteria and classification of the EUROCAT) at Hospital Pediátrico de la Misericordia (April 2009-December 2016) approved by institutional ethical committee. Statistical analysis done SPSS program.

Results: Preliminary selection of 13 patients, one was excluded. The male: female ratio was 1,4 , age range (3 months - 9 years old), 1 case had family history of firstdegree consanguinity, 1 case had second degree consanguinity, and other had both. Four patients had VACTER-LIKE, three patients presented VACTER-STRICT, and five showed VACTER-PLUS diagnosis. Phenotypes were: VC, ATE, VR, VAT, VTL, ACR, VCR, VAC, VACR (X2), VCTRL, and VACRL. Additional anomalies in patients with VACTER-PLUS included: microtia, hemifacial microsomia, myelomeningocele, structural SNC malformation, 
these four had more than one anomaly. Eight patients had cytogenetic analysis (all normal) and only one case had normal sequencing exome.

Conclusions: VACTERL/VACTER association has a typical wide clinical variability, which was evident in our series. Consanguinity was a relevant factor, as forth part of the patients had it. VACTERL/VACTER has been extensively characterized by the 6 defined anomalies; nevertheless, diagnosis is not simple in VACTER-PLUS phenotype, as these cases might present with co-occurrence of multiple pathologies, atypical presentation of relatively common diseases or misdiagnosis.

J.S. Rincon Redondo: None. I. Rueda: None. Y. Ladino: None. M.C. Cuellar: None. L.A. Gonzalez: None. C.M. Restrepo: None. O.I. Beltran: None.

\section{E-P11.113}

\section{Whole Exome Sequencing in unsolved pediatric cases}

G. Moresco $^{l}$, O. Rondinone ${ }^{l}$, A. Mauri ${ }^{l}$, C. Santaniello ${ }^{l}, J$.

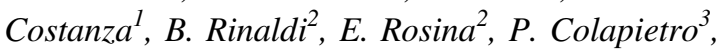
S. Tabano ${ }^{3,4}$, L. Fontana ${ }^{1}$, D. Milani ${ }^{2}$, M. Miozzo ${ }^{1,3}$

${ }^{1}$ Coordination Research Laboratories Unit, Fondazione IRCCS Ca' Granda, Ospedale Maggiore Policlinico, Milano, Italy, ${ }^{2}$ Highly Intensive Pediatric Care Unit, Fondazione IRCCS Ca' Granda, Ospedale Maggiore Policlinico, Milano, Italy, ${ }^{3}$ Department of Pathophysiology and Transplantation, Università degli Studi di Milano, Milano, Italy, ${ }^{4}$ Laboratory of Medical Genetics, Fondazione IRCCS Ca Granda Ospedale Maggiore Policlinico, Milano, Italy

Children suffering from undiagnosed diseases with a possible genetic origin underwent Whole-Exome Sequencing (WES) to disclose the causative variant. We evaluated twenty-two cases resulted negative to aCGH and karyotyping and found the causative lesion in four of them. Herein, we describe two paradigmatic solved cases: one without any definite clinical suspicion (F1), and the other in which the diagnosis was suggested (F2). Libraries were prepared using the SureSelect ${ }^{\mathrm{QXT}}$ Clinical Research Exome V2 kit (Agilent) on blood samples collected from patients and family members, and were run on NextSeq550 instrument (Illumina). F1: the female proband presents delayed psychomotor development, polymicrogyria, gastroesophageal reflux and facial dysmorphisms. The analysis identified the novel de novo missense variant c.C625G:p. H209D in the $D D X 3 X$ gene, an ATP-dependent RNA helicase involved in neurogenesis. Mutations in $D D X 3 X$ were described as causative of X-Linked Dominant Mental Retardation (OMIM \#300958) and variants mapping in the same domain are associated to the specific phenotypic traits of the proband (Lennox et al., bioRxiv, 2018). F2: two siblings were suspected to have Ochoa Syndrome (AR), characterized by congenital urinary bladder dysfunction. In both sibs, the analysis revealed the novel homozygous splicing variant c. $1478-2 \mathrm{~A}>\mathrm{G}$ in the LRIG2 gene (OMIM \#608869), associated with Ochoa Syndrome. This variant probably alters the proper splicing mechanism, leading to an aberrant mRNA maturation and unfunctional protein (ongoing experiments). Our report expands the genotypic spectrum of the pathogenetic variants of $D D X 3 X$ and LRIG2 disease-genes. In these cases, WES approach allowed us to get closer to the end of these diagnostic odysseys.

G. Moresco: None. O. Rondinone: None. A. Mauri: None. C. Santaniello: None. J. Costanza: None. B. Rinaldi: None. E. Rosina: None. P. Colapietro: None. S. Tabano: None. L. Fontana: None. D. Milani: None. M. Miozzo: None.

\section{E-P11.115}

A novel de novo frameshift mutation in SMC1A gene responsible for Cornelia de Lange syndrome 2

R. Szalai ${ }^{1,2}$, J. Bene ${ }^{1,2}$, L. Magyari ${ }^{1,2}$, A. Maasz ${ }^{1,2}$,

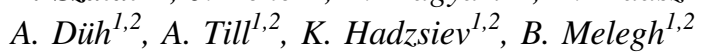

${ }^{1}$ University of Pecs, Clinical Center, Department of Medical Genetics, Pecs, Hungary, ${ }^{2}$ Szentagothai Research Center, University of Pecs, Pecs, Hungary

Introduction: Cornelia de Lange syndrome (CdLS) is a clinically heterogeneous developmental disorder characterized by malformations affecting multiple systems. CdLS have been reported in children with mutations of NIPBL, $S M C 1 A, S M C 3 A, R A D 21$, and HDAC8 genes. Although, the appearance of classic CdLS is well-characterised, the phenotypic traits of individuals with various SMC1A mutations have some similarities, but have other features that make them different from classic CdLS (e.g., small hands but no limb reductions or oligodactyly, prominent intellectual disability and seizures despite milder structural malformations, and a high rate of isolated cleft palate).

Materials and methods: We present a patient with CdLS2 features, including progressive microcephaly, severe psychomotor retardation, feeding difficulties, therapy resistant epilepsy, lack of expressive speech, nearsightedness, high arched palate and abnormal palmar creases. Whole Exome Sequencing (WES) analysis was performed using Agilent SureSelectXT library kit and Illumina sequencing technology. Sanger sequencing confirmed the presence of the pathogenic variant.

Results: WES analysis identified a c.3237_3238insT (p. Asn1080*) pathogenic variant in SMC1A gene in a heterozygous state. This alteration is a frameshift variant 
with a resulting stop codon leading to nonsense-mediated mRNA decay and loss of function, has never been reported.

Conclusions: The features of Cornelia de Lange syndrome vary widely, there are 5 distinct subgroups (CdLS1-5) according to the phenotype and affected genes. Here we report a pathogenic frameshift SMC1A mutation in a Hungarian patient with fitting but unique phenotype characteristics of Cornelia de Lange syndrome 2, still representing a novel phenotypic variant, as well.

R. Szalai: None. J. Bene: None. L. Magyari: None. A. Maasz: None. A. Düh: None. A. Till: None. K. Hadzsiev: None. B. Melegh: None.

\section{E-P12 Cancer Genetics}

\section{E-P12.07}

Genotyping of urinary bladder carcinomas for upregulated EGFR, PIK3CA, FGFR3 and $C D K N 2 A$ to determine the potential for targeted therapy

O. S. Antonova ${ }^{1}$, S. Rangelov ${ }^{2}$, B. Mladenov ${ }^{3}$, Z. Hammoudeh ${ }^{1}$, D. Nesheva ${ }^{1}$, O. Ivanovski ${ }^{4}$, S. Saidi $^{4}$, G. Petrusevska ${ }^{5}$, C. Slavov $^{6}$, A. Dimovski $^{7}, Z$. Yordanova ${ }^{8}$, D. Plaseska-Karanfilska ${ }^{7}$, Z. Popov ${ }^{4}$, A. Galabov ${ }^{9}$, D. Toncheval

${ }^{1}$ Department of Medical Genetics, Medical University of Sofia, Sofia, Bulgaria, ${ }^{2}$ Department of Urology, University Hospital ISUL-Tsaritsa Yoanna, Sofia, Bulgaria, ${ }^{3}$ Department of Urology, UMBALSM “N. I. Pirogov", Sofia, Bulgaria, ${ }^{4}$ University Clinic of Urology University of St. Cyrilius and Methodius, Skopje, Macedonia, The Former Yugoslav Republic of, ${ }^{5}$ Institute of pathology, Medical faculty, Skopje, Macedonia, The Former Yugoslav Republic of, ${ }^{6}$ Department of Urology, University Hospital ISULTsaritsa Yoannaf Sofia, Sofia, Bulgaria, ${ }^{7}$ Research Center for Genetic Engineering and Biotechnology "Georgi D. Efremov", Macedonian Academy of Sciences and Arts, Skopje, Macedonia, The Former Yugoslav Republic of, ${ }^{8}$ University of National and World Economy, Sofia, Bulgaria, ${ }^{9}$ The Stephan Angeloff Institute of Microbiology, Bulgarian Academy of Sciences, Sofia, Bulgaria

Background:The aim of our study was to identify mutational status of the uroepithelial bladder cancer samples for previously detected by us overexpression of EGFR, PIK3CA, FGFR3 and CDKN2A genes in order to determine the potential for targeted therapy.

Materials \& Methods: Tumour samples from 66 patients, staged pTa - T4 were collected. The epidemiological data related to age, gender, recurrence, smoking habits and occupational exposure were taken from patients' questionarie. RT-PCR for mutation detection in EGFR (exones 18, 19, 20, 21) and PIK3CA (A3140T, A3140G, C3139T) SensiScreen ${ }^{\circledR}$ as well as SNaPshot multiplex assay for activating FGFR3 mutations and MLPA for CDKN2A CNVs were performed.

Results and discussion: For the EGFR and PIK3CA only wild type of the tested genetic variants were detected. Mutations in FGFR3 were found in $50 \%$ from the tested tumors: $65 \%$ - S249C, 30\% Y375C and 5\% G372C. Mutations in CDKN2A were detected in 37,5\% from the tested tumors: 53,3\% - homozygous deletions, $40 \%$ heterozygous deletions and 6,7\% duplications.

Conclusion: The overexpression of the EGFR and PIK3CA was not found to be related to tested mutations and so far these two molecules are not convenient for bladder cancer targeted therapy. On the other hand the overexpression of FGFR3 and CDKN2A could be due to detected mutations.

Acknowledgements: BG NSF Grants No KP-06-OPR01/ 3-2018, MANU-BAN.

O.S. Antonova: None. S. Rangelov: None. B. Mladenov: None. Z. Hammoudeh: None. D. Nesheva: None. O. Ivanovski: None. S. Saidi: None. G. Petrusevska: None. C. Slavov: None. A. Dimovski: None. Z. Yordanova: None. D. Plaseska-Karanfilska: None. Z. Popov: None. A. Galabov: None. D. Toncheva: None.

\section{E-P12.08}

Germline variants in cancer-associated genes in pediatric and young adult patients with brain tumors
T. S. Lisitsa ${ }^{1,2}$, V. V. Semenova ${ }^{1}$, A. A. Mitrofanov $^{3}$, E. V. Zhukovskaya ${ }^{l}$, A. F. Karelin ${ }^{l}$, V. N. Kasatkin ${ }^{l}$, T. V. Nasedkina ${ }^{1,2}$

${ }^{1}$ Dmitry Rogachev National Medical Research Center Of Pediatric Hematology-Oncology and Immunology, Moscow, Russian Federation, ${ }^{2}$ Engelhardt Institute of Molecular Biology, Russian Academy of Sciences, Moscow, Russian Federation, ${ }^{3}$ N.N. Blokhin National Medical Research Center of Oncology, Moscow, Russian Federation

Introduction: Pediatric and young adult brain tumors are often associated with cancer predisposition syndromes. The knowledge of specific mutations in cancer-associated genes may alter the management of the cancer, offer novel targeted therapies and enable early detection of other malignancies.

Patients and Methods: Twenty-four patients with brain tumors aged 5-22 years were enrolled in the study, 15 boys and 9 girls. Astrocytoma was the most common histological type $(n=10)$, followed by medulloblastoma $(n=6)$, lowgrade glioma $(n=3), \quad$ ependymoma $\quad(n=2)$, 
craniopharyngioma $(n=1)$, ganglioglioma $(n=1)$, pineoblastoma $(n=1)$. Genomic DNA was isolated from peripheral blood leukocytes and next-generation sequencing on the NextSeq500 Illumina platform was performed. A multigene panel included coding sequences of 132 cancerassociated genes. The library was prepared using enrichment by hybridization with NimbleGen probes (Roche). For the analysis of NF1 large rearrangements MLPA assay was performed.

Results: In total, six patients carried NF1 mutations: frameshift deletions $(n=3)$, nonsynonymous missence $(n=2)$ or nonsense $(n=1)$ mutations. Patients with NF1 mutations had optic pathway glioma $(n=3)$, pylocytic astrocytoma $(n=2)$ and brain stem tumor $(n=1)$. Three patients also had clinical features of neurofibromatosis type 1, but no NF1 variants were revealed. Other findings included likely pathogenic variants in APC and CHEK2 genes. The results were validated by Sanger sequencing.

Conclusion: Germline variants associated with cancer were found in one-third of our patients, one-fourth had the mutations in NF1 gene. Thus, molecular analysis is important to reveal NF1-associated brain tumors for accurate diagnosis of the disease and personalized approach to treatment including targeted therapies.

T.S. Lisitsa: None. V.V. Semenova: None. A.A. Mitrofanov: None. E.V. Zhukovskaya: None. A.F. Karelin: None. V.N. Kasatkin: None. T.V. Nasedkina: None.

\section{E-P12.09}

Characterization of BRCA Genes' Variants in Turkish Hereditary Breast and Ovarian Cancer(HBOC) Patients

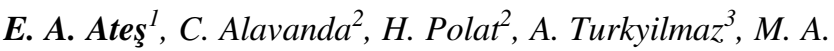
Soylemez ${ }^{2}$, B. B. Geckinli ${ }^{2}$, A. I. Guney ${ }^{2}$

${ }^{1}$ Marmara University Pendik and Research Hospital, Medical Genetics Department, Istanbul, Turkey, ${ }^{2}$ Marmara University School of Medicine, Medical Genetics Department, Istanbul, Turkey, ${ }^{3}$ Erzurum Region Training and Research Hospital, Medical Genetics Department, Erzurum, Turkey

Breast cancer is the most common cancer among women. BRCA genes are associated with \%10-15 of all breast cancers. Six hundred-four patients which 169 of them had family history and 435 had breast and/or ovarian cancer, applied to our clinic. After anamnesis, pedigree analyses and physical examinations, BRCA genes were sequenced via Next-Generation Sequencing(NGS) for all patients. Except from 67 patients with pathogenic mutations in BRCA genes, MLPA analysis was planned for all patients. Among patients that had pathogenic mutation, 36 had BRCA1 and 31 had BRCA2 gene mutations. The most common pathogenic mutations were c.5266dupC, c.3624dupA and c.5444G $>$ A in BRCA1 and c. $67+1 \mathrm{G}>\mathrm{A}$ in BRCA2. Sixty-one variants with unknown significance(VUS), mostly missense variants, were identified and the vast majority of them were in BRCA2 gene. In MLPA analyses seven gross deletions in BRCA1 were detected. The most common gross deletion in BRCA1 gene was exon 18-19 deletion. No gross deletion was detected in BRCA2 gene. Four novel mutations in BRCA1 and five novel mutations in BRCA2 gene were identified. In two of 5 patients with both breast and ovarian cancer, BRCA1 pathogenic mutation was detected and this was the highest mutation rate group. Among ten postmenopausal sporadic breast cancer patients, one had BRCA1 pathogenic mutation. This rate was higher than premenopausal breast cancer patients' rate with family history. This study determines the variants of BRCA genes, their frequencies and their clinical significance in Turkish population. Also, these results showed that screening indications for BRCA should be reviewed.

E.A. Ateş: None. C. Alavanda: None. H. Polat: None. A. Turkyilmaz: None. M.A. Soylemez: None. B.B. Geckinli: None. A.I. Guney: None.

\section{E-P12.11}

The potential of Dendrosomal curcumin (DNC) as a therapeutic agent for modulating the expression of p53 in both wild type and mutant type cancer cells

m. montazeri ${ }^{1}$, Y. Pilehvar Soltanahmadi ${ }^{2}$, N. Zarghami ${ }^{3}$, M. Sadeghizadeh ${ }^{4}$

${ }^{I}$ Department of Medical Biotechnology, Faculty of Advanced Science and Technology, Tehran Medical Sciences, Islamic Azad University, Tehran, Iran, Tehran, Iran, Islamic Republic of, ${ }^{2}$ Cellular and Molecular Research Center, Research Institute for Cellular and Molecular Medicine, Urmia University of Medical Sciences, Urmia, Iran, Urmia, Iran, Islamic Republic of, ${ }^{3}$ Department of Medical Biotechnology, Faculty of Advanced Medical Sciences, Tabriz University of Medical Sciences, Tabriz, Iran, Tabriz, Iran, Islamic Republic of, ${ }^{4}$ Department of Genetics, School of Biological Sciences, Tarbiat Modares University, Tehran, Iran, Tehran, Iran, Islamic Republic of

The effect of Dendrosomal curcumin (DNC) on the expression of p53 in both p53 mutant cell lines SKBR3 and p53 wild-type MCF7 in both RNA and protein levels, and also cell cycle distribution and apoptosis. Curcumin derived from rhizome of Curcumin longa modulates key signaling pathways in cellular processes, therefore, it is recently considered in cancer related researches for its cell 
growth inhibition properties against human tumors. It is evidenced that p53 is the most common tumor-suppressor gene involved in human cancers. Its mutation not only inhibits tumor suppressor activity but also promotes oncogenic activity. Toxicity of DNC was studied using MTT assay and cell cycle distribution, apoptosis study. Real-time PCR and Western blot analysis were used to analyze the expression of $\mathrm{p} 53$, and other apoptosis related proteins. DNC treatment during the time increased the cells in the SubG1 and gradually decreases them in the G1, S and G2/M phases, the percentages of early apoptosis also indicated the inhibition of cell growth in early phase. Real-Time PCR showed an increased mRNA expression of BAX, Noxa and p21 during the time with decreased expression of Bcl-2. The expression of p53 mutant decreased in SKBR3, and the expression of p53 wild-type increased in MCF7 cells. Consequently, DNC can drive the tumor cell lines toward the apoptosis by modulating the expression of genes involved in apoptosis and proliferation, furthermore, this study suggest p53 plays an important role in mediating the survival by DNC.

M. montazeri: None. Y. Pilehvar Soltanahmadi: None. N. Zarghami: None. M. Sadeghizadeh: None.

\section{E-P12.13}

The expression level of the EAF1 \& the EAF2 genes is significantly decreased in Iranian breast cancer patients

\section{S. Heydaran, M. Moghanibashi Mansurieh, S. Naeemi}

department of Genetics, Islamic Azad university, Kazerun, Iran, Islamic Republic of

Breast cancer death rate is increasing annually in Iran, and early diagnosis is important to reduce the mortality rate. Beside genetic predisposition, environmental factors are the most known risk factors. The EAF1 \& the EAF2 tumor suppressor genes belong to the ELL family, locating on chromosome 3 in the human genome, which antagonize the Wnt/ $\beta$-catenin signaling pathway in different cell lines by suppressing the tumorigenic activity. The change of the expression level of the EAF1 \& the EAF2 genes is reported in other cancer types such as intestinal and blood. Here, we investigated the fold change of these mentioned genes in breast cancer (invasive ductal carcinoma: IDC) in Iranian patients. Forty Tumor and marginal normal tissues from breast cancer patients (grade I 16\%, grade II 58\%, and grade III $25 \%$ ) were collected during surgery with immediately treatment of RNA later. All selected patients were HER2 positive, with an average age of 56 years old. Seventy percent of affected individuals were ER/PR[1] positive, and the other 30\% ER/PR negative. Quantitative analysis of the $E A F 1$ \& EAF2 gene expression in two sample groups (tumor \& normal) was performed by Real-time-PCR (cybergreen method). The expression of the EAF1 \& the EAF2 genes was in tumor tissue compared to normal samples significantly decreased (fold change $=-5.7$ for the EAF1 gene $[p=0.000] \&-4.4$ for the EAF2 gene [0.034]). Lower expression of the both mentioned genes is apparently associated with negative prognosis of breast cancer.

[1] ER: estrogen receptor \& PR: progesterone receptor

S. Heydaran: None. M. Moghanibashi Mansurieh: None. S. Naeemi: None.

\section{E-P12.15}

Age depending prevalence of $R U N X 3, B R C A 1$ genes comethylation among female breast cancer case

\author{
N. Medvedieva ${ }^{l}$, O. Lobanova ${ }^{2}$, Z. Rossokha ${ }^{l}$, \\ V. Vershyhora ${ }^{1}$, V. Cheshuk ${ }^{2}, R$. Vereshchako ${ }^{2}$, \\ N. Gorovenko ${ }^{3}$
}

${ }^{I}$ SI Reference Centere For Molecular Diagnostic of Public Health Ministry of Ukraine, Kyiv, Ukraine, ${ }^{2}$ Bogomolets National Medical University, Kyiv, Ukraine, ${ }^{3}$ P.L. Shupyk National Academy of Postgraduate Education, Kyiv, Ukraine

Introduction: Genes comethylation is important pathways of epigenetic mechanisms in the risk of breast cancer development. Hypermethylation of RUNX3 and BRCAI genes is involved in disrupted oncosuppression process among breast cancer cases. But no so much known about their co-influences on breast cancer (BC) risk in different age. Aim of the study was to evaluate RUNX3, BRCAl genes age depending comethylation status.

Materials and methods: Tumor tissue of $52 \mathrm{BC}$ female patients was obtained during surgery. Patients (mean age $53.05 \pm 1.86)$ subdivided depending of age: Group 1 (25-44 years), Group 2 (45-60 years), Group 3 (61-85 years). Obtained tumor was tested for BRCA1 and RUNX3 gene methylation status. Tumor samples saved using DNA/RNA Shield. DNA extracted with Quick-DNA Miniprep Plus Kit. Bisulfide conversion was done by dint of EZ DNA Methylation-Gold Kit. Converted DNA with methylspecific primers was used for PCR analysis.

Results: No significant differences were found for RUNX3 gene methylation. Patients of Group 1 had significantly higher methylation status $(41.2 \%)$ in BRCAl gene compared to patients of Group 2 (25\%) and Group 3 (26.6\%), respectively. Comethylation of hypermethylated 
BRCA1/RUNX3 was significantly prevalence also among Group 1 compared to others (Table).

Conclusions: Higher hypermethylated BRCA1/RUNX3 genes status was found among young patients (aged 25-44 years). Further careful studies are needed to understand cometylation effect of investigated genes on early breast cancer development.

Methylation status $R U N X 3$ hypermethylation $R U N X 3$ unmethylation

Group 1 (25-44 years) 17 patients

\begin{tabular}{|c|c|c|}
\hline $\begin{array}{l}B R C A 1 \\
\text { hypermethylation }\end{array}$ & $41.2 \%$ & $5.9 \%$ \\
\hline $\begin{array}{l}B R C A 1 \\
\text { unmethylation }\end{array}$ & $41.2 \%$ & $11.7 \%$ \\
\hline \multicolumn{3}{|c|}{ Group 2 (45-60 years) 20 patients } \\
\hline $\begin{array}{l}B R C A 1 \\
\text { hypermethylation }\end{array}$ & $25 \%$ & 0 \\
\hline $\begin{array}{l}\text { BRCAl } \\
\text { unmethylation }\end{array}$ & $60 \%$ & $15 \%$ \\
\hline \multicolumn{3}{|c|}{ Group 3 (61-85 years) 15 patients } \\
\hline $\begin{array}{l}B R C A 1 \\
\text { hypermethylation }\end{array}$ & $26.6 \%$ & $6.7 \%$ \\
\hline $\begin{array}{l}\text { BRCA1 } \\
\text { unmethylation }\end{array}$ & $60 \%$ & $6.7 \%$ \\
\hline
\end{tabular}

N. Medvedieva: None. O. Lobanova: None. Z. Rossokha: None. V. Vershyhora: None. V. Cheshuk: None. R. Vereshchako: None. N. Gorovenko: None.

\section{E-P12.19}

Impact of TP53 (rs1042522) and MDM2 (rs2279744) polymorphisms on cervical intraepithelial lesions and cervical cancer in North Macedonian women

\section{S. Duvlis ${ }^{1}$, D. Dabeski ${ }^{2}$, D. Plaseska-Karanfilska ${ }^{3}$}

${ }^{1}$ Institute of public health of Republic of North Macedonia, Skopje, Macedonia, The Former Yugoslav Republic of, ${ }^{2}$ Clinic for gynecology and obstetrics, Clinical center "Majka Tereza", Skopje, Macedonia, The Former Yugoslav Republic of, ${ }^{3}$ Research Center for Genetic Engineering and Biotechnology "Georgi D. Efremov", Skopje, Macedonia, The Former Yugoslav Republic of

Introduction: Variant in cell cycle regulation genes may influenced cervical intraepithelial lesions (CIN) and malignant progression to cervical cancer $(\mathrm{CCa})$. Single nucleotide polymorphisms (SNPs) rs1042522 within the codon 72 of TP53 and rs2279744 within MDM2 promoter genes are plausible factors that could have impact on cervical carcinogenesis conferring increased attenuation of p53 pathway. We investigated the association of these SNPs with the CIN and $\mathrm{CCa}$ among women from Republic of North Macedonia.

Methods: Using a multiplex PCR SNaPShot analysis we genotyped rs1042522 and rs2279744 in 131 women with CIN or CCa and 110 cytologicaly negative women.

Results: No significant difference in either genotype or allelic frequencies for rs1042522 and rs2279744 between cases and control was found. Stratification of the cases based on grade of the lesion, revealed lower frequency of $\mathrm{CC}$ genotype and $\mathrm{C}$ allele of rs1042522 in $\mathrm{CIN} 2+$ and $\mathrm{CCa}$ compared to $\mathrm{CIN} 1$ [GG vs $\mathrm{CC} ; \mathrm{p}=0.001, \mathrm{OR}=0.4 ; \mathrm{CG}$ vs $\mathrm{CC} ; \mathrm{p}=0.04, \mathrm{OR}=0.03$ and $\mathrm{CG}+\mathrm{GG}$ vs $\mathrm{CC} ; \mathrm{p}=0.004$, $\mathrm{OR}=0.2]$. Furthermore, $\mathrm{GG}$ genotype and $\mathrm{G}$ allele of rs2279744 showed significantly lower frequency in CIN2+ and $\mathrm{CCa}$ cases then in $\mathrm{CIN} 1$ [G vs $\mathrm{T} p=0.02, \mathrm{OR}=0.52$; $\mathrm{GG}$ vs TT; $\mathrm{p}=0.04, \mathrm{OR}=0.29$; TT vs $\mathrm{TG}+\mathrm{GG} ; \mathrm{p}=0.007$, $\mathrm{OR}=0.34]$.

Conclusion: The Arg variant of rs1042522 and T allele/ TT genotype of rs2279744 are associated with progression of CIN1 to CIN2+ or CCa and may be used as prediction markers in $\mathrm{CCa}$ management. Still, clinical importance of these variants warrants further validation in more comprehensive studies.

S. Duvlis: None. D. Dabeski: None. D. PlaseskaKaranfilska: None.

\section{E-P12.21}

Monitoring circulating tumor DNA in a breast cancer patient to provide real-time cancer progression information

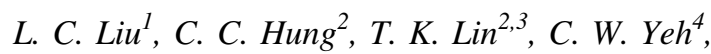
C. W. Huang ${ }^{4}$, Y. N. Su u $^{2,5}$

${ }^{1}$ Department of Medicine, College of Medicine, China Medical University, Taichung, Taiwan, ${ }^{2}$ Sofiva Genomics Co., Ltd., Taipei, Taiwan, ${ }^{3}$ Graduate Institute of Clinical Medicine, College of Medicine, National Taiwan University, Taipei, Taiwan, ${ }^{4}$ National Center for High Performance Computing, National Applied Research Laboratories, Hsinchu, Taiwan, ${ }^{5}$ Dianthus Maternal Fetal Medicine Clinic, Taipei, Taiwan

Background: Liquid biopsy is a mini-invasive test and easy to get specimen for cancer progression information based on circulating tumor DNA (ctDNA). In this study, we analyzed ctDNA dynamics and aim to push liquid biopsy toward clinical practice.

Method: We used next-generation sequencing (NGS)based panel assay and molecular barcoding technology to analyze variants in 197 genes. We examined a breast cancer patient after surgery and frequently monitoring of ctDNA.

Results: In April 2017, ERBB2 c.2264 T>C (2.00\%) was detected in ctDNA as well as ERBB2 c.2664 T>C (70.56\%) 
and PIK3CA c.1634 A>C (22.93\%) were detected in tissue of the patient. After seven months, ERBB2 c.2264 T>C (9.00\%) and PIK3CA c. $1634 \mathrm{~A}>\mathrm{C}(0.76 \%)$ were detected in ctDNA, and it seems the therapy did not work. The patient was suggested to change other chemotherapy and targeted therapy. Three months later, ERBB2 c.2264 T>C (0.59\%), PIK3CA c. 1625 A $>C(0.04 \%)$ and PIK3CA c.1634 A>C $(0.07 \%)$ were detected in February 2018. Then we follow the cancer genetic status every 1-2 months for the patient with given the same treatment. For ERBB2 c.2264T>C in particular, the mutation rates were shown $0.56 \%, 0.68 \%$, $1.96 \%$ and $3.48 \%$, respectively.

Conclusion: Using liquid biopsy to monitor ctDNA dynamics after surgery may be helpful for patient to estimate prognosis. The liquid biopsy can be used to monitor treatment effect in patient who receive cancer therapies.

L.C. Liu: None. C.C. Hung: A. Employment (full or parttime); Significant; Sofiva Genomics, Co., Ltd. T.K. Lin: A. Employment (full or part-time); Significant; Sofiva Genomics Co., Ltd.. C.W. Yeh: None. C.W. Huang: None. Y.N. Su: A. Employment (full or part-time); Significant; Sofiva Genomics Co., Ltd..

\section{E-P12.24}

Analysis of PPARG and UCP2 gene polymorphisms in colorectal cancer patients

\section{E. M. N. D. Ajaj}

\section{Institute Of Human Genetics, Belgrade, Serbia}

Introduction: Altered lipids metabolism is a hallmark characteristic of colorectal cancer (CRC). Peroxisome proliferator - activated receptor gamma (PPARG) and uncoupling protein 2 (UCP2) act as transcriptional regulators of lipid metabolism. Recent studies have shown that PPARG and UCP-2 could represent biomarkers for prognosis in $\mathrm{CRC}$ and possible target for cancer therapy. We aimed to investigate the association between PPARG Pro12Ala (rs1801282) and UCP2 45 bp +3474 ins/del polymorphisms with clinicopathological factors of tumor progression in CRC patients.

Material and methods: Our study included 160 CRC pateints, mean age $65,39 \pm 8.55$ years. Among them, there were 98 men $(61.3 \%)$ and $62(38.8 \%)$ women. PPARG Pro12Ala genotypes were detected by PCR-RFLPs while UCP2+3474 ins/del polymorphism was detected by PCR. all patients, data about tumor location, histologic differentiation, preoperative serum carcinoembryonic antigen level, the presence of lymph node metastases, distant metastases and stage of the disease were recorded.

Results: Analysis of PPARG Pro12Ala polymorphism has shown that carriers of Pro/Pro genotype had moderately and poorly differentiated tumor cells more frequently than patients with Pro/Ala genotype $(\mathrm{p}=0.01)$. Our analysis also revealed that colon cancer was more frequent than rectal in carriers of $\mathrm{UCP} 2+3474$ ins allele $(\mathrm{p}=0.02)$. Also, carriers of ins allele had distant metastases more frequently than patients with del/del genotype $(\mathrm{p}=0.024)$.

Conclusion: Our findings indicate that there is an association between PPARG gene Pro/Ala polymorphism and tumor cells differentiation and UCP2 gene +3474 ins/ del polymorphism with tumor location and the presence of distant metastases in CRC patients.

Key words: Colorectal cancer, PPARy, UCP2

E.M.N.D. Ajaj: None.

\section{E-P12.31}

\section{MGMT methylation status in glioblastomas multiforme}

\author{
A. V. Antonets, Y. G. Yakovlenko, S. V. Mordanov, \\ A. A. Tushev, V. A. Moldovanov, V. Y. Deribas, \\ S. S. Todorov
}

Rostov Medical State University, Rostov-on-Don, Russian Federation

Introduction: Epigenetic silencing of the methyl-guanine methyl transferase (MGMT) gene promoter by gene promoter methylation suggests a partial inability of the tumour to repair the chemotherapyinduced DNA damage. In retrospective analyses, MGMT methylation has been correlated with a response to or benefit predictive value of a methylated $M G M T$ status for response to or benefit from alkylating agent chemotherapy and thus improved outcome has been demonstrated repeatedly in glioblastomas. Moreover, MGMT methylation identifies a sub-population with a better prognosis irrespective of the applied treatment.

Materials and methods: We examined 10 patients with the diagnosis of glioblastoma multiforme treated in the neurosurgical department and have observed them within 12 months by present. DNA was extracted from freshly frozen tumor tissue. Detection of the MGMT gene hypermethylation was carried out with methyl-specific real time PCR.

Results: Hypermethylation of the MGMT gene was detected in 4 patients. We have not observed relapse in patients with hypermethylation by 12 months from surgery. One patient with unmethylated secondary glioblastoma multiforme after anaplastic astrocytoma had died.

Conclusion: In light of emerging human genetic and molecular knowledge, molecular diagnosis of oncological diseases might help in the establishment of prognosis and personalized treatment for patients and improve life quality. 
A.V. Antonets: None. Y.G. Yakovlenko: None. S.V. Mordanov: None. A.A. Tushev: None. V.A. Moldovanov: None. V.Y. Deribas: None. S.S. Todorov: None.

\section{E-P12.35}

$B R C A 1$ and $B R C A 2$ pathogenic variants spectrum in patients with hereditary breast and ovarian cancer syndrome

M. Šukys, V. Ašmonienè, D. Čereškevičius, I. Nasvytienè, I. Valančiūtè

Department Of Genetics And Molecular Medicine, Medical Academy, Lithuanian University of Health Sciences, Kaunas, Lithuania, Kaunas, Lithuania

Introduction: Hereditary breast and ovarian cancer syndrome is one of the most common cancer susceptibility syndromes, affecting 1 of 1600 women. Affected patients harbor pathogenic variant in BRCA1 or BRCA2 genes, which increases lifetime risk for women of developing breast or ovarian cancer to nearly $80 \%$, for men breast cancer - 8\%. Detection of such variant allows early screening and monitoring for better cancer management.

Methods: We report $B R C A 1$ and $B R C A 2$ analysis results of the Hospital of Lithuanian University of Health Sciences Kauno Klinikos from year 2015 till 2019. Analysis was performed for patients who were at increased risk of having BRCA1/2 genes pathogenic variants according to the local laws. Analysis was performed in two steps: firstly, it was analyzed with pyrosequencing kit for 6 common variants. Later, If needed, analysis on Sanger sequencing or Next generation sequencing for coding genes regions was continued.

Results: $B R C A 1$ and $B R C A 2$ analysis for 6 common pathogenic variants was performed for 1737 patients (including familial $B R C A 1$ and $B R C A 2$ variant cases). Pathogenic variant was found for $12,95 \%$ patients. Coding region sequencing was performed for 647 cases, as other patients testing is still ongoing, $10 \%$ pathogenic variants were found. From all pathogenic variants, most frequent were BRCA1: c.4035delA 43,4 \%; c.5266dupC - 23,1\%; c.181T > G - 10\%; BRCA2: c.657_658delTG 4,8\%.

Conclusion: The c.4035delA might be the most frequent variant in Lithuania, whereas in neighboring countries c.5266dupC is more common

M. Šukys: None. V. Ašmonienė: None. D. Čereškevičius: None. I. Nasvytienė: None. I. Valančiūtė: None.

\section{E-P12.37}

The case of Hutchinson-Gildford Syndrome associated with G608G mutation in LMNA gene and Methylenetetrahydrofolate reductase deficiency

\section{Y. Hrechanina, L. Molodan}

Interregional Specialized Medical Genetic Center - Center of Rare (Orphan) Diseases, Kharkiv National Medical University, Kharkiv, Ukraine

Introduction: Hutchinson-Gildford Syndrome (HGS) is an orphan disease from the group oncogenetic syndromes. The disease is based on a point mutation in Lamin A LMNA (1q21 / 2) gene, which leads to splicing disorder.

Materials and methods: HGS was diagnosed in 3 patients aged 4.5 to 8 years. Classic and modern technologies were used.

Results: Patient I., 4.5 years old with physical development delay, progeric skin and hair changes, prominent brain skull over facial, pronounced expanded venous network, microangiopathy. Progeric changes increase, the subcutaneous fat layer disappears, the skin becomes thin, dry patches of stretched skin alternate with patches sclerotic changes, alopecia develops, expansion of superficially located vessels. Molecular genetic analysis: a mutation in the LMNA gene (G608G). Pathology had a progressive course and manifested against of methylenetetrahydrofolate reductase deficiency. Biomarkers: glucose - $\downarrow$, lactate - $\uparrow$, cholesterol - $\uparrow$, LDL cholesterol - $\uparrow$, VLDL cholesterol $\uparrow \uparrow$, atherogenic coefficient - $\uparrow \uparrow$, homocysteine - $\uparrow \uparrow$ The comorbid pathology was diagnosed: a combination of HGS associated with mutation in the LMNA gene (G608G), methylenetetrahydrofolate reductase deficiency, hyperhomocysteinemia, lipid metabolism disorder. Therapy: positive dynamics was noted - skin condition improved, decreased dryness, skin became more elastic, hair growth appeared.

Conclusions: Search for metabolic disorders, clarification of their nature in the case of a combination of HGS with methylenetetrahydrofolate reductase deficiency have allowed to develop individual tactics of patient management taking into account revealed disorders, the correction has reduced clinical disease manifestations, improved the quality of life of the patient and family.

Y. Hrechanina: None. L. Molodan: None.

\section{E-P12.40}

KRAS, NRAS, BRAF mutation spectrum in Georgian patients with metastatic colorectal cancer

\section{Agladze 1,2,3 , S. Iordanishvili ${ }^{4}$, L. Margvelashvili, ${ }^{4,3}$, E. Kldiashvili ${ }^{4}$, O. Kvlividze $e^{1,5}$}

${ }^{1}$ School of Medicine, New Vision University, Tbilisi, Georgia, ${ }^{2}$ Research Institute of Clinical Medicine, Tbilisi, Georgia, ${ }^{3}$ Pediatric Surgery Center (KidCo), Tbilisi, Georgia, ${ }^{4}$ Petre Shotadze Tbilisi Medical Academy, Tbilisi, 
Georgia, ${ }^{5}$ Georgian Foundation for Genetic and Rare Diseases (GeRaD), Tbilisi, Georgia

Introduction: According to the NCDC data Colorectal Cancer (CRC) is the fifth among most common malignancy in Georgian population. In 2019326 new cases of CRC have been registered. Testing CRC for mutations in KRAS, NRAS, BRAF genes are common in most countries to determine resistance to anti-EGFR therapy.

Aim of the study: To detect most common mutations in KRAS, NRAS, BRAF genes of metastatic CRC cells in Georgian patients affected with mCRC and to compare the data with the results of similar studies in other countries.

Materials and methods: Study was made in Georgian population. Inclusion criteria was the morphologically diagnosed mCRC. DNA was extracted from 111 patients FFPE Tissue. KRAS (exon 2,3,4), NRAS (exon 2,3,4) and BRAF (exon15) were amplified. For mutations detection Sanger Sequencing method has been used.

Results: Analyzing data showed that in 111 tested patients mutation was detected in $21.6 \%$ cases. KRAS exon2 mutation was detected in $18.9 \%$ cases, NRAS ex4 mutation in $0.9 \%$, BRAF ex 15 mutation in $1.8 \%$. There was no mutation detected in KRAS ex 3 and 4 and NRAS ex2 and 3. From overall 24 detected mutations most common mutations are KRAS ex2 G12D 37.5\%, G12V 25\%, G12C 20.8\%, G13D 4.1\% and BRAF Ex15 V600E 8.3\%.

Conclusions: The study of 111 Georgian patients affected with mCRC showed that the most common mutations in KRAS gene is G12D, followed by the same codon mutations G12V and G12C. Similar studies already conducted in different countries in patients affected with mCRC showed the similar results.

D. Agladze: None. S. Iordanishvili: None. L. Margvelashvili: None. E. Kldiashvili: None. O. Kvlividze: None.

\section{E-P12.41}

Aberrant methylation of CDH13 and RASSF1 as classifiers of muscle-invasive, high grade bladder cancer.

\section{Pietrusinski ${ }^{l}$, E. Borkowska ${ }^{l}, M$. Constantinou ${ }^{l}$, Z. Jablonowski ${ }^{2}$, J. Salamunia ${ }^{l}$, M. Borowiec ${ }^{I}$}

${ }^{1}$ Medical University of Lodz, Department of Clinical Genetics, Lodz, Poland, ${ }^{2}$ Medical University of Lodz, 1 st Clinic of Urology, Lodz, Poland

Introduction: The mortality among men due to bladder cancer is approximately twice as high in Poland as in other European countries. Bladder cancer is a result of multistep accumulation of genetic and epigenetic alterations and exposure to environmental factors. Aberrant methylation of
CpG islands has been recognized as a potential early biomarker of carcinogenesis. Additionally, these changes affect tumor stage and grade, which may have an impact on the choice of the right treatment method.

Materials and methods: We aimed to investigate the methylation profiles of selected tumor suppressor genes to find out whether aberrant methylation can be significantly correlated with tumor stage and/or grade. Tumor DNA from carefully selected 44 patients diagnosed with NMIBC and MIBC was analyzed. Methylation profiles were examined by MS-MLPA technique. The capillary electrophoresis method provided data for calculating the percentage of methylation of particular genes. A value above $15 \%$ was considered as a positive result.

Results: Among the analyzed genes, the most frequently hypermethylated genes were $C D H 13, A P C$ and $R A R \beta$. RASSF1 methylation showed a significant correlation with the invasive stage. Hypermethylation of $\mathrm{CDH} 13$ (AUC = 0,75 ) and RASSF1 (AUC $=0,67$ ) has been statistically significantly associated with the histological high-grade bladder cancer and both genes are good classifiers for this condition.

Conclusions: This study confirms the association of $\mathrm{CpG}$ island methylation of $\mathrm{CDH} 13$ and RASSF1 with bladder cancer and suggests its potential role in the assessment of bladder cancer aggressiveness. Medical University of Lodz Grant No. 502-03/2-159-02/502-24-305.

M. Pietrusinski: None. E. Borkowska: None. M. Constantinou: None. Z. Jablonowski: None. J. Salamunia: None. M. Borowiec: None.

\section{E-P12.44}

A retrospective study of patients with Multiple Myeloma tested in Laboratory of Medical genetics, Varna, Bulgaria

V. Miteva, T. Ruseva, M. Hachmeriyan, M. Levkova, L. Angelova

Medical University Varna, Varna, Bulgaria

Introduction: Multiple myeloma (MM) is a clonal bone marrow disease of B-cells, at the last stage of differentiation and is the second most common hematologic neoplasia. $\mathrm{MM}$ is a heterogeneous disease characterized by very complex cytogenetic and molecular genetic aberrations.

Materials and methods: We performed a retrospective review of patients with MM referred to Laboratory of Medical Genetics, Varna, Bulgaria between 2017 and 2019. Bone marrow (BM) samples of 65 patients with MM at different stages of the disease were evaluated for cytogenetic analyses and 19 of them for interphase fluorescent 
in situ hybridization (iFISH). Chromosomes were classified according to the International System for Human Cytogenetic Nomenclature 2016 (ISCN 2016). FISH was performed on BM samples and 200 interphase nuclei were evaluated per sample. The iFISH was performed using commercially available probes (Kreatech ${ }^{\mathrm{TM}}$ FISH probes) for the detection of two oncohaematological markers: deletions of $17 \mathrm{p} 13$ (TP53) and rearrangements involving immunoglobulin heavy chain (IGH) region on $14 \mathrm{q} 32$ chromosome.

Results: Cytogenetic analysis was successful in 57 of the 65 patients $(87.7 \%)$. Six patients out of these $57(9.23 \%)$ had different abnormal karyotypes sustainable with the diagnosis MM.iFISH analysis was successful in all 19 patients. Deletion del17p13 was detected in seven patients $(36.84 \%)$ and rearrangements involving $14 \mathrm{q} 32$ in one $(5.26 \%)$.

Conclusion:The detection of chromosomal abnormalities in MM by conventional cytogenetics is limited due to the low mitotic index of malignant plasma cells. This disadvantage can be overcome with the use of FISH, which can detect specific target arrangements and chromosomal copy number changes.

V. Miteva: None. T. Ruseva: None. M. Hachmeriyan: None. M. Levkova: None. L. Angelova: None.

\section{E-P12.45}

Matrix metalloproteinases expression level in patients with oesophageal squamous cell carcinoma

S. Rakhimova ${ }^{1}$, U. Kozhamkulov ${ }^{1}$, A. Sharip ${ }^{l}$,

A. Molkenov', Y. Zhukov', M. Omarov', M. Tuleutaev ${ }^{2}$,

U. Kairov ${ }^{l}$, A. Akilzhanova ${ }^{l}$

${ }^{1}$ National Laboratory Astana, Nazarbayev University, NurSultan, Kazakhstan, ${ }^{2}$ Oncology center, Nur-Sultan, Kazakhstan

Introduction: More than $80 \%$ of oesophageal cancer cases occur in developing countries, Central and East Asia countries and the prevalent type is oesophageal squamous cell carcinoma (ESCC). The aim of our study was to analyse of major genes, anticancer drug targets based on whole transcriptome sequencing data for Kazakhstani patients.

Materials and methods: Tissue samples were collected from 25 ESCC-affected patients immediately after IvorLewis esophagectomy in Oncology Center. Whole transcriptome sequencing was performed regarding the TruSeq RNA Protocol. STAR software and DESeq2 package have been used for mapping and defining differentially expressed genes.

Results: 25 male and female (14 and 11 respectively) patients with ESCC were recruited for study. Average age of patient was $65.5 \pm 7.7$ years diagnosed with ESCC stages T1-T4. Expression level of 24 genes, targets of the main antitumor drugs was investigated in our research. Overexpression of metalloproteinase genes MMP1, MMP9, MMP10 during cancer progression (stages 2-4) was significantly found among patients with T2, T3 stages of ESCC (p-value<0.05). MMP1, MMP9 and MMP13 genes identified as up-regulated in patients with T4 stage (pvalue $<0.05$ ). According recent publications matrix metalloproteinases is frequently up-regulated and correlates with vascular invasion, metastasis and poor outcomes among patients with ESCC.

Conclusion: Our research study showed that metalloproteinase genes (MMP1, MMP9, MMP10 and MMP13) were overexpressed among patients with ESCC. Further research will be continued and additional validation of findings will be performed.

Grant reference: \#AP05134722, \#AP05135430 and \#AP05136106 MES

S. Rakhimova: None. U. Kozhamkulov: None. A. Sharip: None. A. Molkenov: None. Y. Zhukov: None. M. Omarov: None. M. Tuleutaev: None. U. Kairov: None. A. Akilzhanova: None.

\section{E-P12.56}

Resveratrol can miss laryngeal squamous cell carcinoma cells from immune response

B. Goksel Tulgar ${ }^{1}$, M. E. Sarl ${ }^{1}$, G. Turkoglu ${ }^{1}$, F. Duymus ${ }^{l}$, D. Esin' ${ }^{1}$, E. Marzioglu Ozdemir ${ }^{2}$, T. Cora ${ }^{1}$

${ }^{1}$ Selcuk University Faculty of Medicine, Medical Genetics Department, Konya, Turkey, ${ }^{2}$ Konya Education and Research Hospital, Genetic Diseases Diagnosis Unit, Konya, Turkey

The epidermal growth factor receptor (EGFR) is a receptor tyrosine kinase that mediates intracellular signals in response to various extracellular stimuli. Previous studies have been shown that EGFR is activated and overexpressed in many types of human epithelial cancer. In a limited study on epithelial cancer cells, resveratrol has been shown to have an inhibitory effect on EGFR tyrosine kinase activity in vitro. Programmed death-ligand 1(PD-L1) is normally expressed in healthy cells, is also expressed in cancer cells during cancer development and leads to the concealing of cancer cells from the immune system. In this study, we aimed to investigate the effect of resveratrol on EGFR and PDL-1 in Laryngeal squamous cell carcinoma cells(HEP2), which is epithelial cancer. We cultivated HEP2 and HEK293 (human embryonic kidney cell) cell lines as a control cell line. We determined the IC50 value as $100 \mathrm{uM}$. EGFR, MYC, and PD-L1 gene expression levels were 
evaluated by gene expression studies. GAPDH and ACTB genes were identified as housekeeping genes. As a result of the study, gene expression relative change levels for EGFR, MYC, and PD-L1 were determined as 1.145, 0.475, 2.695, respectively. According to these results, resveratrol did not cause a significant change in EGFR and MYC expression levels in the LSCC cell line, while it significantly increased PD-L1 levels. It has been found interesting because the resveratrol, which is normally included in the literature as an anticancer, increases levels of PD-L1 because it is likely to support escape from the immune response.

B. Goksel Tulgar: None. M.E. Sarı: None. G. Turkoglu: None. F. Duymus: None. D. Esin: None. E. Marzioglu Ozdemir: None. T. Cora: None.

\section{E-P12.57}

\section{Investigation of retroelement activity in childhood} leukemia

S. Z. Urazbakhtin ${ }^{l}$, M. V. Saliutina ${ }^{l}$, G. A. Nugmanov ${ }^{1}$, Y. B. Lebedev ${ }^{I}$, A. Y. Komkov ${ }^{l}$, Y. V. Olshanskaya ${ }^{2}$, E. A. Zerkalenkova ${ }^{2}$ I. Z. Mamedov ${ }^{I}$

${ }^{1}$ Shemyakin-Ovchinnikov Institute of Bioorganic Chemistry of Russian Academy of Science, Moscow, Russian Federation, ${ }^{2}$ Dmitry Rogachev National Medical Research Center Of Pediatric Hematology, Oncology and Immunology, Moscow, Russian Federation

Introduction: Retroelements (RE) are interspersed repeats that comprise a sufficient part of the human genome. The majority of them are currently inactive but functional copies can be a source of genetic instability associated with some types of cancer. Currently, there is very limited data about retrotranspositions in hematological malignancies. In this study we perform analysis of RE activity in childhood acute lymphoblastic and acute myeloblastic leukemia.

Materials and methods: Digested DNA obtained from paired tumor/normal samples was ligated to adapters with Unique Molecular Identifiers. Primers specific to young RE were used to selectively amplify sequences flanking RE insertions in the genome. These sequences were used to identify new insertions by custom pipeline. To test method sensitivity, we prepared a mixture of cells from 4 healthy donors, with different cell concentrations.

Results: Polymorphic RE insertions, distinguishing donors from each other, were used to determine method's sensitivity. We were able to detect $95 \%$ of insertions of a person, whose cells comprised $1 \%$ of the mixture at sequencing depth of 2,000,000 reads per Alu library and 300,000 per for L1 library. Using our method and pipeline we analyzed 14 T-ALL, 34 B-ALL and 18 AML paired tumor/normal samples.
Conclusion: We designed a sensitive method and sophisticated pipeline for tumor-specific RE insertion detection in as little as $1 \%$ of cells. The developed method was the first to evaluate RE activity in childhood leukemia.

Funding: This work was supported by RSF18-1400244 grant.

S.Z. Urazbakhtin: None. M.V. Saliutina: None. G.A. Nugmanov: None. Y.B. Lebedev: None. A.Y. Komkov: None. Y.V. Olshanskaya: None. E.A. Zerkalenkova: None. I.Z. Mamedov: None.

\section{E-P12.58}

Somatic Variant Interpretation in TET2 using two distinct next generation sequencing platforms

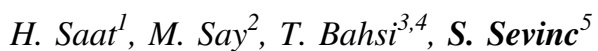

${ }^{1}$ Ankara Dıskapı Yıldırım Beyazıt Training and Research Hospital, Department of Medical Genetics, Ankara, Turkey, ${ }^{2}$ Gen Era Diagnostics, Bioinformatics Department, Istanbul, Turkey, ${ }^{3}$ Dr. Abdurrahman Yurtaslan Ankara Oncology Training and Research Hospital, Department of Medical Genetics, Ankara, Turkey, ${ }^{4}$ Ankara Provincial Health Directorate, Central Genetic Laboratory, Ankara, Turkey, ${ }^{5}$ Gen Era Diagnostics, Application Department, Istanbul, Turkey

Evans Syndrome is a rare autoimmune disorder in which the immune system produces antibodies that destroy erythrocytes, platelets and/or certain leukocytes. The patients may have different symptoms related to the type and extend of blood cells affected. The most common symptoms include hemolytic anemia and thrombocytopenia. Similarly, some database is included same phenotype knowledge for Myelodysplastic Syndrome. Herein, we have analyzed the DNA sample extracted from whole blood of a 19-year-old male with an initial diagnosis of Evans Syndrome starting from the age of 10. Next generation sequencing (NGS) was performed using Clinical Exome Solution (CES) of Sophia Genetics on the on the Illumina NextSeq $550^{\circledR}$ platform. has been analyzed for using. Through analysis of the CES data for single nucleotide and copy number variations has revealed a frameshift variant at TET2 gene with a variant fraction of only $6 \%$ in 120 reads. We have therefore decided to validate this result using the same biospecimen in an alternative platform, namely Archer $^{\oplus}$ Myeloid dedicated for somatic variant analysis. Accordingly, the identical variant was confirmed using the Archer platform with $13 \%$ variant fraction in 744 reads. Somatic mutations in TET2 encoding Tet methylcytosine dioxygenase 2 has been reported to be associated with myelodysplastic syndrome (MDS). MDS refers to a heterogeneous group of closely related clonal hematopoietic disorders characterized by ineffective 
hematopoiesis resulting in low blood counts and possibility of developing acute myeloid leukemia. The variant identified in TET2 can be considered as somatic and may aid correct diagnosis and treatment for the patient.

H. Saat: None. M. Say: None. T. Bahsi: None. S. Sevinc: None.

\section{E-P12.60}

A novel de novo splicing mutation in the TSC2 gene causes exon in a patient with tuberous sclerosis syndrome

\section{P. Gerasimou ${ }^{l}$, A. Miltiadou ${ }^{I}$, I. Kyprianou ${ }^{l}$, J. Chi ${ }^{l}$, V. Anastasiadou ${ }^{2}, P$. Costeas $^{1}$}

${ }^{1}$ Molecular Hematology-oncology, Karaiskakio Foundation, Nicosia, Cyprus, ${ }^{2}$ Department of Clinical Genetics, Archbishop Makarios III Medical Centre, Nicosia, Cyprus

Introduction: Tuberous sclerosis complex is an autosomal dominant disorder characterized by hamartomas in multiple organ including the brain, skin, heart and kidneys among other complications. Approximately 10 to $30 \%$ of cases are due to mutations in the TSC 1 gene. TSC 2 gene mutations frequency is consistently higher and are associated with more severe phenotype.

Case Description: Newborn with prenatal diagnosis of heart tumor. Suspected Tuberous Sclerosis.

Methodology: A custom HaloPlexHS (Agilent) NGS panel was used to investigate mutations in Syndromic associated genes as per manufacturer's instructions. Sanger sequencing performed for parental carrier status and RNA investigation for exon skipping.

Interpretation: A heterozygous novel splice-site mutation in the TSC2 gene was detected in the patient sample. The c.139-2A $>\mathrm{T}$ (NM_000548.5) mutation is located two nucleotides from the splice acceptor site between exons 2 and 3 of the TSC2 gene. It is classified as pathogenic according to ACMG recommendations and Bioinformatics tools predicted that this novel mutation have pathogenic effects on splicing machinery. RNA investigation revealed that this variation leads in exon 3 skipping. Parental testing showed that this is a de novo mutation in the patient as neither of the parents were carriers.

Conclusion: More than 1,100 mutations in the TSC2 gene have been identified in individuals with tuberous sclerosis complex. This study highlighted the presence of a novel de novo splicing mutation in the TSC2 gene that leads in exon 3 skipping and is responsible for the loss of 29 amino acids, which causes the production of an aberrant protein isoform.
P. Gerasimou: None. A. Miltiadou: None. I. Kyprianou: None. J. Chi: None. V. Anastasiadou: None. P. Costeas: None.

\section{E-P13 Genome Variation and Architecture}

\section{E-P13.3}

Copy number losses of 11p14.3-p13 and 11p13-p12 detected by CGH array revealed to be heterozygous deletion of 11p14.3-p12 on one chromosome 11 and heterozygous duplication within 11 p13 on the other chromosome 11 by metaphase FISH analysis

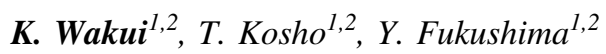

${ }^{1}$ Department of Medical Genetics, Shinshu University School of Medicine, Matsumoto, Japan, ${ }^{2}$ Center for Medical Genetics, Shinshu University Hospital, Matsumoto, Japan

Introduction: Wilms tumor-Aniridia-Genitourinary abnormalities-growth and mental Retardation (WAGR) syndrome $/ 11 \mathrm{p} 13$ deletion syndrome is a rare genetic condition associated with contiguous-gene deletions including PAX6 and WT1. An 8-month-old boy with aniridia and his parents were analyzed for possible genomic variations.

Methods: Cytogenomic microarray testing using a CGH array (135K [hg18], Roche NimbleGen) was performed to evaluate copy number variations (CNVs). We employed metaphase FISH analyses using selected BAC clones to identify the chromosomal rearrangements related to the CNVs.

Results: Copy number losses of 11p14.3-p13 (chr11:25,784,386-32,872,519; 7.1 Mb) including the PAX6 and WT1 genes and of 11p13-p12 (chr11:33,555,357$42,819,900 ; 9.3 \mathrm{Mb}$ ) were detected by the CGH array in the patient. And the FISH signals of the probes mapping the normal copy number region between the two copy number loss regions were absent in the abnormal chromosome 11, as were the probes mapping the distal and proximal copy number loss regions. Additional microarray analyses of the parents uncovered a copy number gain of $11 \mathrm{p} 13$ (chr11:32,910,676-33,522,409; 0.6 Mb) in the mother.

Conclusion: Comprehensive genomic evaluation of a case of WAGR syndrome ultimately revealed a heterozygous deletion of 11p14.3-p12 (chr11:25,784,386$42,819,900 ; 17.0 \mathrm{Mb})$ on one chromosome 11 and a heterozygous duplication of a part of $11 \mathrm{p} 13$ (chr11:32,910, $676-33,522,409 ; 0.6 \mathrm{Mb})$ on the other chromosome 11. Metaphase FISH analysis is indispensable in clarifying exact chromosomal rearrangements while distinguishing between homologous chromosomes.

K. Wakui: None. T. Kosho: None. Y. Fukushima: None. 


\section{E-P13.4}

Identification of unsolved rare genetic cases of North Cyprus

\author{
M. C. Ergoren ${ }^{1}$, E. Manara ${ }^{2}$, S. Paolacci ${ }^{2}$, G. Tuncel ${ }^{3}$, \\ S. G. Temel ${ }^{4}$, G. Mocan ${ }^{1}$, M. Dundar ${ }^{5}$, M. Bertelli $^{6}$ \\ ${ }^{1}$ Near East University, Faculty of Medicine, Nicosia, \\ Cyprus, ${ }^{2}$ MAGI Euregio, Bolzano, Italy, ${ }^{3}$ Near East \\ University, DESAM Institute, Nicosia, Cyprus, ${ }^{4}$ Bursa \\ Uludag University, Faculty of Medicine, Bursa, Turkey, \\ ${ }^{5}$ Erciyes University, Faculty of Medicine, Kayseri, Turkey, \\ ${ }^{6} 8 M A G I ' s$ LAB S.r.l., Roverato, Italy
}

Cyprus, which is the third largest island in the Mediterranean Sea, has been at the crossroads of multiple civilizations throughout human history. Today, the Turkish Cypriots and the Greek Cypriots constitute two major ethnic groups in Cyprus, along with some minor groups. Even though Turkish-Cypriot paternal lineages have an autochthonous quality, some Y-DNA haplotypes are shared between the Turkish Cypriots and the Greek Cypriots. It should be no surprise that the genetic characteristics of the current Turkish-Cypriot population have been determined by the interactive forces of migrations, admixture, genetic drift, etc. We aimed at elucidating the path from the interesting or unusual clinical phenotypes of rare diseases to potentially novel gene variants. In the present study, the most recent high-throughput genomic technologies were employed to study the genetic etiology of such diseases. Accordingly, WES was used to sequence trios. As a result, we identified a number of novel population-specific gene variants, each of which causes rhizomelic chondrodysplasia punctate type 5, WiedemanRautenstrauch Syndrome, tricho-rhino-phalangeal syndrome type 1, Joubert syndrome, etc. Lack of genetic databases in the region of middle eastern, the laboratory set up the capacity to produce DNA sequence data expands the number of contributors to DNA sequence databases, which is beneficial for science and may be helpful for any number of bioinformatic analyses. Overall, this study emphasizes the importance of revealing rare-disease-causing variants in a particular population for immediate use in accurate diagnosis, for improved understanding of the underlying molecular mechanisms and for developing effective preventive medicine strategies.

M.C. Ergoren: None. E. Manara: None. S. Paolacci: None. G. Tuncel: None. S.G. Temel: None. G. Mocan: None. M. Dundar: None. M. Bertelli: None.

\section{E-P13.5}

Parallel analysis of next generation sequencing data for small nucleotide and copy number variations and a follow-up approach using SNP array genotyping detect a biallelic variant in $T B X 1$ gene

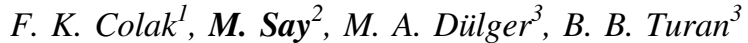

${ }^{I}$ Dr. Sami Ulus Obstetrics and Gynecology, Children Health and Disease Training and Research Hospital, Department of Medical Genetics, Ankara, Turkey, ${ }^{2}$ Gen Era Diagnostics, Bioinformatics Department, Istanbul, Turkey, ${ }^{3}$ Gen Era Diagnostics, Application Department, Istanbul, Turkey

Next-generation sequencing (NGS) platforms with dedicated software are increasingly being used to identify copy number variations $(\mathrm{CNVs})$ along with single nucleotide variants (SNVs) simultaneously. Array-based technologies including SNP genotyping has been be employed in parallel to NGS for further characterization of the CNV event. Such combined approaches may resolve challenging cases in medical genetics especially for recessive conditions when one copy of the gene is deleted while the other copy carries a pathogenic CNV. Herein, we have adopted Clinical Exome Solution (CES) of Sophia Genetics in a patient with an initial diagnosis of Pierre Robin syndrome. The capture-based target enrichment kit has enabled us to cover approximately 4490 genes. Sequencing was performed on the Illumina Next Seq $550^{\circledR}$ platform. Sophia DDM platform was used as a interface to evaluate SNVs and CNVs. HumanCytoSNP-12 v2.1 Bead Chip Kit at Illumina SNP array platform was used for follow-up detection of CNV events. Analysis of NGS data for CNV events has revealed one copy loss at chromosome 22q11 including the gene TBXI. The loss had exactly the same boundaries as deduced from SNP genotyping. Although, haploinsufficiency of TBXI is associated with DiGeorge syndrome, we have interestingly identified a novel missense pathogenic SNV in TBXI in the NGS data. Combined analysis of CNV events in NGS and SNP array platforms has enabled us to identify a deletion involving the $T B X 1$ gene in a patient. We propose that the SNV identified in the nondeleted copy may explain the phenotypic variability associated with $T B X I$ deletions.

F.K. Colak: None. M. Say: None. M.A. Dülger: None. B. B. Turan: None.

\section{E-P14 Cytogenetics}

\section{E-P14.01}

Both 17q12 duplication and deletion detected in the patient with normal phenotype and their segregation in family with variably affected members

H. Zunova, M. Stolfa, T. Rasplickova, D. Novotna, M. Vlckova

DBMG, 2nd Faculty of Medicine Charles University and University Hospital Motol, Prague, Czech Republic 
Chromosomal band $17 \mathrm{q} 12$ is a gene rich region flanked by segmental duplications which make the region prone to deletions and duplication by NAHR mechanism (non-allelic homologous recombination). While the deletions cause well described clinical unit with specific phenotype called RCAD (renal cyst and diabetes mellitus), the phenotype caused by reciprocal duplications of the same region still remains unclear especially due to variable expressivity and incomplete penetrance and fact, that they are often detected in healthy patients. Here we present unusual case of family where the mother is carrier of duplication (paternally inherited) and also deletion (inherited from mother with RCAD) of the identical 17q12 region. All of her children were diagnosed with $17 \mathrm{q} 12$ microduplication syndrome. Except of the mother and grandfather, all her children carrying duplication express variable degree of neurodevelopmental problems, such as epilepsy, mild intellectual disability, delayed speech development or attention deficit disorder, that correlate with the hypothesis of incomplete penetrance and variable phenotype published by many studies. As a potential causative genes are considered $L H X I$ for neurodevelopmental problems and gene $A C A C A$ for epilepsy. The simultaneous occurrence of deletion and duplication of the same chromosomal region in one family is very rare. This case supports hypothesis that $17 \mathrm{q} 12$ duplications are stable and may segregate in a family for several generations. Supported by: 00064203, NF-CZ11PDP-3-003-2014, AZV17-29423A

H. Zunova: None. M. Stolfa: None. T. Rasplickova: None. D. Novotna: None. M. Vlckova: None.

\section{E-P14.05}

Duplication of 10q22.2q23.1 as a cause for severe hypotonia in a child

\section{G. Ilieva, E. Sukarova-Angelovska, D. Nestoroska,}

S. Naunova-Timovska, M. Pesevska, V. Anastasovska

\section{University Pediatric Clinic, Skopje, Macedonia, The} Former Yugoslav Republic of

Introduction: Array comparative genomic hybridization (aCGH) technique enables high-resolution screening of the genome for segmental genomic copy number variations (CNVs). The technique today represents an unsurpassed tool for detecting minor chromosomal aberrations in children with expressed pathological clinical features, such as hypotonia, dysmorphia, motor and mental delay.

Materials and methods: aCGH analysis was performed in a child with clinical signs of dysmorphia and severe hypotonia using the Affymetrix ${ }^{\circledR}$ CytoScan $^{\mathrm{TM}} 750 \mathrm{~K}$ Array (Applied Biosystems). Each array was consisted of $200 \mathrm{k}$ SNP and $550 \mathrm{k}$ non-polymorphic markers. The data was analysed and interpreted using Chromosome Analysis Suite (ChAS) Software (v4.0).

Results: 7-month-old child had hypotonia, was unable to hold his head, to sit and did not develop fine motor skills. The clinical conditions included asthenia, frequent respiratory infections and atypical convulsion. Dysmorphological features followed wide forehead, protruded eyes, long eyelashes, triangular face, slender extremities and diminished fat tissue. Karyotype results were typical for a normal male (46, XY). The array CGH analysis revealed a pathological microduplication of a $10 \mathrm{q}$ segment - arr $10 \mathrm{q} 22.2 \mathrm{q} 23.1(75,709,593-80,912,470) \times 3$ that was $5230 \mathrm{~kb}$ long and includes 44 genes.

Conclusion: Application of array Comparative Genomic Hybridization (aCGH) in patient with severe hypotonia, dysmorphia and neurological disability represents a great diagnostic marker compared to conventional karyotyping. The results suggest that the main reason for the various clinical and dysmorphological features of the child has genetic background. Keywords: array Comparative Genomic Hybridization, karyotype, dysmorphia, hypotonia

G. Ilieva: None. E. Sukarova-Angelovska: None. D. Nestoroska: None. S. Naunova-Timovska: None. M. Pesevska: None. V. Anastasovska: None.

\section{E-P14.07}

Chromosome instability index: a potential human cancer biomarker?

\section{T. Sylaidy ${ }^{1}$, E. Lialiaris $^{2}$, T. S. Lialiaris $^{3}$}

${ }^{1}$ Demokritus University of Thrace, Dept of Mol. Biology and Genetics, Alexandroupolis, Greece, ${ }^{2}$ Aristotle University of Thessaloniki, Dept of Biology, Thessaloniki, Greece, ${ }^{3}$ Demokritus University of Thrace, Medical School, Dept of Genetics, Alexandroupolis, Greece

Introduction: Chromosome Instability Index (CII) is a biomarker that has been remarkably increased in cancer patients and it consists of three parameters: Sister Chromatid Exchange (SCE) frequencies, Proliferating Rate Index (PRI) and Mitotic Index (MI). The most important of the above is SCEs because they showed the most significant difference between patients and controls. The aim of the study was to investigate the CII as possible biomarker in different types of cancer.

Material and Method: We used our data of the SCE methodology as it is a simple, sensitive and rapid detection technique used to evaluate chromosomal fragility. Increased SCEs mean increased unspecific damage to the DNA molecule, which was caused by various mutants in lymphocyte cultures. 
Discussion: A significant alteration in both SCE frequency and PRI was observed in patients with different types of cancer, such as breast and stomach cancer or leukemia. Specifically, SCEs in cancer patients were almost twice than in controls. Additionally, as far as PRI is concert, there was a significant reduction in cancer patients compared to normals. We believe that, the co-calculation of SCEs, PRIs and MIs in an index, can predict the chromosomal instability in human cancer. So, CI Index was created as $\mathrm{CI}=20 * \mathrm{SCEs}+10 *(3-\mathrm{PRI})+2 / 10 *(60-\mathrm{MI})$. From the results it is revealed that CII (cancer) $>>\mathrm{CII}$ (control).

Conclusions: The results of the present study demonstrate that SCEs can offer reliable results for the studies of chromosome instability and so CII could be used as biomarker for cancer and this is of great importance in premature diagnosis.

T. Sylaidy: None. E. Lialiaris: None. T.S. Lialiaris: None.

\section{E-P14.08}

Genotoxicity of thimerosal and paracetamol in human lymphocyte culture Genotoxicity of thimerosal and paracetamol in human lymphocyte culture

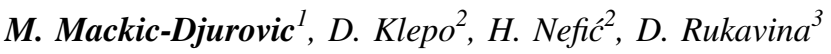 \\ ${ }^{1}$ Center for Genetics, Sarajevo, Bosnia and Herzegovina, ${ }^{2}$ Faculty of Natural Science and Mathematics, Sarajevo, Bosnia and Herzegovina, ${ }^{3}$ Veterinary Faculty, Sarajevo, Bosnia and Herzegovina}

Introdaction: Although its use has been reduced, thimerosal is still present as conservans in multidose containers of some vaccines or as a trace from pathogen inactivation process in vaccines production. Paracetamol, as widely used analgoantipyretic, is most often used for treatment of postimunisation pirexy. Profilactic use of paracetamol upon vaccination is still present, despite the fact that in this cases paracetamol decrease immune response on some vaccines.

Materials and methods: Blood samples of three healthy donors were included in the study. Tested substances were added 24 hours after culture were started and following concentration were examined: thimerosal $(1 \mu \mathrm{g} / \mathrm{ml}$ and $0.5 \mu \mathrm{g} / \mathrm{ml}$ ), paracetamol $20 \mu \mathrm{g} / \mathrm{ml}$, thimerosal with paracetamol $(0,5 \mu \mathrm{g} / \mathrm{ml} / 20 \mu \mathrm{g} / \mathrm{ml}$ and $0.5 \mu \mathrm{g} / \mathrm{ml} / 20 \mu \mathrm{g} / \mathrm{ml})$. Cultivations were lasted for 72 hours, and analysys of structural chromosomes aberations was performed.

Results: Results shown that frequency of chromosomal structural aberation was significantly incresed in all tretaed cultures in comparission with negative control. Number of chromosomal structural aberations was highest in culture treated with $1 \mu \mathrm{g} / \mathrm{ml}$ of tiomersal. In cells treated with thiomersal $1 \mu \mathrm{g} / \mathrm{ml}$ with presence of $20 \mu \mathrm{g} / \mathrm{ml}$ of paracetamol, the number of aberation was significatly decreased.
Conclusion: It is shown induction of chromosomal structural aberation as sign of genotoxicity for examined concentration of thimerosal and paracetamol and their common treatment of lymphocites cultures. Suppresing effect of paracetamol on thimerosal genotoxicity in lymphocytes culture treated with $1 \mu \mathrm{g} / \mathrm{ml}$ of thimerosal was shown as indicative for furter examination of paracetamol use in prevention of genotoxicity.

M. Mackic-Djurovic: None. D. Klepo: None. H. Nefić: None. D. Rukavina: None.

\section{E-P14.09}

Genetic diagnosis of true hermaphroditism; case report in an Angolan patient

N. Calumbuana ${ }^{l}$, C. Correia ${ }^{2}$, I. C. Capuepue ${ }^{l}$, J. C. V. D. Van-Dúnem ${ }^{l}$, M. M. P. Chimpolo ${ }^{1}$

${ }^{1}$ Faculdade de Medicina da UAN, Luanda, Angola, ${ }^{2}$ SynlabHealth Medical Genetics, Porto, Portugal

Introduction: The present work reports a case of marked gynecomastia, ambiguous genitalia and female body fat distribution in a young boy of 14 years of age with no family history of hermaphroditism. Scan tests have shown the presence of a uterus and a right ovary. Laboratory exams (FSH, LH and Testosterone levels) were also added to the clinical investigation.

Objectives: To report the case of a genetic diagnosis of an individual with sex differentiation disorder, seen by a urology consultant in Luanda.

Materials and methods: Initially, sexual markers were analysed by capillary electrophoresis followed by conventional cytogenetic analysis. 200 nuclei were analysed by FISH with a probe for the $S R Y$ gene and another for the $\mathrm{X}$ chromosome centromere for exact determination of the $\%$ of each line.

Results: The results of the molecular examination by capillary electrophoresis showed an atypical genotype, with tri-allelic markers, similar to those of mixtures. The cytogenetic analysis of 50 metaphases indicated 35 metaphases 46,XX and 15 metaphases 46,XY. The examination of FISH confirmed the presence of $70 \%$ of the nuclei showed two signals for the $\mathrm{X}$ chromosome centromere and none of the $S R Y$ and $30 \%$ showed one signal for the $\mathrm{X}$ chromosome and one $S R Y$ centromere.

Conclusion: We present a true chimera hermaphroditism with a 46XX/46XY karyotype and hypothesize that the patient is a tetra-gamete or chimera due to early postzygotic error and fusion of two distinct zygotes that gave rise to only one embryo. The results obtained will be used for clinical decision-making. 
N. Calumbuana: None. C. Correia: None. I.C. Capuepue: None. J.C.V.D. Van-Dúnem: None. M.M.P. Chimpolo: None.

\section{E-P14.10}

\section{A Rare Immune Deficiency Case With Dysmorphic Findings: ICF Syndrome}

\section{A. Kalayci Yigin, F. Ozdemir, A. Ozaydin, M. Seven}

Department of Medical Genetics, Cerrahpasa Medical Faculty, Istanbul University-Cerrahpasa, Istanbul, Turkey

Introduction: ICF (immune deficiency, centromeric region instability, facial anomalies) syndrome is a rare autosomal recessive inherited chromosomal breakage syndrome. In mitogen-induced lymphocytes, it is characterized by agamaglobulinemia or hypogammaglobulinemia, as well as rearrangements of chromosomes $1,9,16$, adjacent to the centromere, in the heterochromatin regions.

Clinical Findings: A 16-year-old male patient was referred to our outpatient clinic for genetic analysis with a preliminary diagnosis of ICF syndrome, due to recurrent lower and upper respiratory tract infections from a young age. The patient is the only surviving child of the family and no feature has been defined in his own and family history. In the physical examination; hypertelorism, nasal root flatness, deeply located eyes, flat and wide forehead, frequent hard and black hair were present. The ear was located downward and the bucket was facing forward.

Results: Immunoglobulin (Ig) levels: A, G and M were lower than normal. IgE levels were normal. In Ig $\mathrm{G}$ subtyping, IgG1, G2 and G4 levels are higher than normal. $\mathrm{CD}^{+}, \mathrm{CD}^{+}{ }^{+}, \mathrm{CD}^{+}{ }^{+}$were detected. Chromosomal analysis revealed $46 \mathrm{XY}$. Chromosomal instability in the perisentromeric heterochromatin region of chromosomes 1, 9 and 16, decondensation of chromosome 9 and specific multibranch chromosome structure was detected.

Conclusion: According to our karyotype analysis of the patient decondensation of chromosome 9, multi-branch chromosome structure, low levels of immunglobulins and accompaning dysmorphic face was considered as a ICF syndrome.

A. Kalayci Yigin: None. F. Ozdemir: None. A. Ozaydin: None. M. Seven: None.

\section{E-P14.11}

A case of mosaicism with two different sex cell lines detected by chromosome analysis in a second trimester spontaneous miscarriage

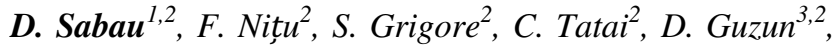
P. Apostol ${ }^{2}$

${ }^{1}$ Emergency Hospital "Sfantul Ioan" Bucharest/ Clinica Sante Bucharest, Bucharest, Romania, ${ }^{2}$ Clinica Sante, Bucharest, Romania, ${ }^{3}$ Fundeni Clinical Institute, Bucharest, Romania

Introduction: Miscarriage is a common event in a woman's life. Most occur before 12 weeks and it has been estimated only $2-3 \%$ of pregnancies end spontaneously in the second trimester. Second-trimester pregnancy loss has been defined as miscarriage diagnosed between the duration of gestation 12-24 weeks.

Material and methods: We report the case of a teenager who suffered a miscarriage in the second trimester (19 weeks). She was referred to our medical genetics department for further testing due to fetal malformation of the central nervous system, meningocele diagnosed by ultrasound. We performed karyotype analysis of the product of conception and analysed 70 cells.

Results: The final cytogenetic result according to ISCN 2016 was: mos 46,XYqh-,inv(7)(p12q21)[61]/46,XX[9]. The structural modification identified in the majority of cell lines (the male line), the inversion, has the break points most likely in the regions 7 p12 and $7 q 21$ and it could be the cause of the malformation discovered by ultrasound. The normal cell line (the female line), might be the result of vanishing twin syndrome, possible sexual ambiguity of the fetus or maternal contamination.

Conclusions: Considering even the possibility of maternal contamination, the diagnostic value for this case is of great importance, since it still offers the explanation for the pregnancy loss. To conclude, we consider karyotyping analysis of the product of conception to be of a great value when diagnosing the causes of miscarriages.

D. Sabau: None. F. Nițu: None. S. Grigore: None. C. Tatai: None. D. Guzun: None. P. Apostol: None.

\section{E-P14.12}

ADAMTS1, THBS1, RBFOX2 and WHSC1 gene expression as a biomarker of individual radiosensitivity in human lymphocytes
R. Savchenko ${ }^{1}$, S. Vasilyev ${ }^{1}$, V. Fishman ${ }^{2}$, E. Sukhikh ${ }^{3}$, O. Gribova ${ }^{4}$, Z. Startseva ${ }^{4}$, A. Murashkina ${ }^{5}$, A. Dorofeeva $^{6}$, D. Shunkova ${ }^{6}$, I. Lebedev ${ }^{1}$

${ }^{I}$ Research Institute of Medical Genetics, Tomsk National Research Medical Center of RAS, Tomsk, Russian Federation, ${ }^{2}$ Institute of Cytology and Genetics of SB RAS, Novosibirsk, Russian Federation, ${ }^{3}$ Tomsk Regional Oncology Center, Tomsk, Russian Federation, ${ }^{4}$ Cancer Research 
Institute, Tomsk National Research Medical Center of RAS, Tomsk, Russian Federation, ${ }^{5}$ National Research Tomsk State University, Tomsk, Russian Federation, ${ }^{6}$ Siberian State Medical University, Tomsk, Russian Federation

The search for genetic markers of human individual radiosensitivity is important. The microarray analysis of gene expression in human lymphocytes revealed the correlation of ADAMTS1, RBFOX2, THBS1 and WHSCl gene expression with the spontaneous $\gamma \mathrm{H} 2 \mathrm{AX}$ foci level and the radiation-induced micronuclei frequency. The aim of this study was to assess the possibility of predicting individual radiosensitivity using analysis of ADAMTS1, RBFOX2, THBS1 and WHSCl gene expression and methylation level of their promoters. We analyzed the blood lymphocytes obtained from 47 individuals, included 20 male and 27 female after exposure to 2 Gy of $\gamma$-rays in vitro. The micronucleus frequency was assessed using FISH-based micronucleus test with pancentromeric probes. The gene expression level was analyzed by real-time PCR. Targeted bisulfite analysis by NGS was used to estimate the methylation level of gene promoters. The micronucleus frequency was correlated with expression of ADAMTS1 $(\mathrm{R}=-0.52$, $\mathrm{p}=0.033)$, THBS1 $(\mathrm{R}=-0.52, \mathrm{p}=0.03)$ and WHSCl $(\mathrm{R}=-$ $0.37, \mathrm{p}=0.032)$ in non-irradiated cells and with expression of THBS1 $(\mathrm{R}=-0.53, \mathrm{p}=0.033)$ and RBFOX2 $(\mathrm{R}=-0.30$, $\mathrm{p}=0.027$ ) after irradiation. Simultaneous use of expression levels of all analyzed genes allowed to identify individuals with increased radiation-induced micronucleus frequency in groups of male $(\mathrm{p}=0.00026)$, female $(\mathrm{p}=0.048)$ and all individuals $(\mathrm{p}=0.0009)$. DNA methylation level was correlated with expression of $R B F O X 2$ only $(\mathrm{R}=-0.58$; $\mathrm{p}=0.009$ ). It is possible to identify individuals with increased radiation-induced micronucleus frequency using gene expression level of analyzed genes, but not the methylation level of gene promoters. This study was supported by Grant of the President of Russian Federation MK5944.2018.4 and RFBR grant №19-34-90143.

R. Savchenko: None. S. Vasilyev: None. V. Fishman: None. E. Sukhikh: None. O. Gribova: None. Z. Startseva: None. A. Murashkina: None. A. Dorofeeva: None. D. Shunkova: None. I. Lebedev: None.

\section{E-P14.14}

A retrospective study of indications and consent process for neonatal microarray analysis as a screen for genomic rearrangements

\section{K. A. Andrews ${ }^{* 1,2}$, M. Prapa ${ }^{1,2}$, E. J. Radford ${ }^{3,4}$,} I. Simonic ${ }^{\text {, S. Holden }}{ }^{1,2}$, G. Belteki ${ }^{3}$, * equally shared

${ }^{I}$ Department of Medical Genetics, University of Cambridge, Cambridge, United Kingdom, ${ }^{2}$ East Anglian Medical
Genetics Service, Cambridge, United Kingdom, ${ }^{3}$ Addenbrookes Hospital, Cambridge University NHS Foundation Trust, Cambridge, United Kingdom, ${ }^{4}$ Department of Paediatrics, University of Cambridge, Cambridge, United Kingdom

Introduction: Microarrays are increasingly requested as a first-line genetic investigation for chromosome anomalies in the neonatal population. Paediatricians, frequently trainees, usually choose the indication and take consent. This is often without specific training in how to consent for genetic tests.

Materials and methods: We conducted a retrospective study over one year on our tertiary neonatal unit to assess the indications, consent process, diagnostic yield and results delivery for microarray testing.

Results: Of 98 neonatal microarrays there was documented evidence of consent in $62 \%$ of cases and in only $2 \%$ was consent informed with documented discussion about prognostic information, variants of uncertain significance (VUS), incidental findings and possible implications for family members. Nearly a quarter of microarrays were requested for intra-uterine growth restriction without congenital anomalies, and in this group no likely pathogenic variants were identified. Four times more VUS were identified than likely pathogenic copy number variants. In $41 \%$ of cases, there was no documentation that the results had been communicated to the parents. However, all cases with clinically actionable findings were referred to clinical genetics.

Conclusions: There is a need to build greater consensus on the indications for microarray testing in neonates, and training for paediatricians regarding how to take adequately informed consent for genetic testing. Citation:Andrews K, Prapa M, Radford E, et al Taking consent for neonatal microarray analysis as a screen for genomic rearrangements: are paediatricians equipped for the genomic era? Archives of Disease in Childhood Published Online First: 28 September 2019. doi: 10.1136/archdischild-2019-317966

K.A. Andrews*: None. M. Prapa*: None. E.J. Radford*: None. I. Simonic: None. S. Holden: None. G. Belteki: None.

\section{E-P14.20}

Could be a supernumerary marker chromosome in mosaic associated with pregnancy loss? A case report

\section{P. APOSTOL, M. Cristea}

Clinica Sante - Genetic Expert Center, Bucharest, Romania

Fertility problems are became common disorder in the last time. Although cytogenetic abnormalities (e.g. the presence 
of a supernumerary marker chromosomes) represent an important cause of abortions, for some of couples the etiology remains unexplained.

A couple, both partners of 38 years old, was refereed to our center for genetic investigations. In their history a pregnancy loss in the first trimester due to foetal cardiac defects (ultrasonographically detected) is described. A peripheral blood sample was collected and karyotype was performed from two independent cultures. Chromosome analysis was performed using the classical G-banding method (650 bands resolution).

Cytogenetic analysis, done by two observers, shown a normal karyotype for the wife $(46, \mathrm{XX})$, while the husband was found to have a mosaic karyotype: mos 46,XY,22pstk $+[78] / 47, \mathrm{XY}, 22 \mathrm{pstk}+,+\operatorname{mar}[8]$ (according to ISCN 2016). For this case 86 metaphases were analysed - 78 metaphases with a normal polymorphism 22pstk + and 8 metaphases with a supernumerary marker chromosome with unknown chromosome origin. Further studies, including molecular analyses to highlight the breakpoint, are necessary in order to identify the origin of this marker chromosome. Few cases of supernumerary marker chromosome are described in the literature, most of them of small size and associated with different phenotypes, including infertility.

In conclusion, although only $9.3 \%$ of the investigated metaphases had the chromosome marker, proper genetic counseling is recommended for the couple. Although the potential risk of this supernumerary marker chromosomes for pregnancy loss cannot be defined, it should be considered for further pregnancies and prenatal genetic tests should be done.

P. Apostol: None. M. Cristea: None.

\section{E-P15 New Technologies and Approaches}

\section{E-P15.04}

\section{Towards Next-Generation Phenotyping (NPG) for cat- eye syndrome}

\section{T. Liehr, A. Al-Rikabi}

\section{Jena University Hospital, Institute of Human Genetics, Jena, Germany}

We presently work together with the company FACE2Gene, (https://www.face2gene.com/) to establish the possibility to recognize cat-eye syndrome just by analyzing facial pictures of an individual patient. Forms of next generation phenotyping (NPG) are needed to increase further the value of any kind of genetic approaches, including timely consideration of (molecular) cytogenetics during the diagnostic quest. As NGP we used in this study the facial dysmorphology novel analysis (FDNA) technology. To automatically identify facial phenotypes of patients with small supernumerary marker chromosomes we already established successfully Emanuel (ES) and Pallister-Killian Syndrome (PKS) identification from 2D facial photos. The comparison between ES or PKS and normal individuals expressed a full separation between the cohorts. These results show that NPG is able to help in the clinic, and could reduce the time patients spend in diagnostic odyssey. The same development is currently undertaken for cat-eye syndrome. It became possible thanks to a grant from NORD. The link for the present project to upload pictures of cat-eye syndrome will be provided on the poster. NPG also helps to differentiate ES or PKS from each other and other patients with small supernumerary marker chromosomes and most likely also from patients with cat-eye syndrome. Thus, especially in countries with no access to more sophisticated genetic approaches apart from banding cytogenetics NPG is a very timely approach. Inclusion of more facial pictures of patient with sSMC, like cat-eye-, isochromosome-18psyndrome or others may contribute to higher detection rates in future. Supported by NORD Research Program grant number 18002.

T. Liehr: None. A. Al-Rikabi: None.

\section{E-P15.05}

\section{Performance evaluation study of INNO-LiPA ${ }^{\oplus}$} CFTRiage

\author{
C. Dobbels ${ }^{1}$, L. Tremmerie ${ }^{1}$, M. Seia ${ }^{2}$, M. D'Apice ${ }^{3}$, \\ M. Lucarelli ${ }^{4}$, K. Stouffs ${ }^{5}$, S. Seneca ${ }^{5}$, A. Gheldof, \\ C. Orsini ${ }^{6}$, G. Mersch ${ }^{l}$, V. Kostanjevecki ${ }^{l}$
}

${ }^{1}$ Fujirebio, Gent, Belgium, ${ }^{2}$ Medical Genetics Lab, Milan, Italy, ${ }^{3}$ PTV Foundation, Rome, Italy, ${ }^{4}$ Dipartimento di Medicina Sperimentale, Rome, Italy, ${ }^{5}$ Center for Medical Genetics, Brussels, Belgium, ${ }^{6}$ Fujirebio Italia S.r.l., Rome, Italy

Objectives: The INNO-LiPA ${ }^{\circledR}$ CFTRiage assay based on reverse-hybridization is a simple method allowing simultaneous analysis of 88 CFTR mutations with automatic interpretation using LiRAS $^{\mathrm{TM}}$ for LiPA Cystic Fibrosis v.5 software. By performing just one amplification and hybridization with the INNO-LiPA CFTRiage $>95 \%$ of the samples can already be characterized. Worst case 2 additional confirmation strips, using the same amplicon, are required to obtain complete characterization. The aim of the study was to assess the success rate, agreement with a reference result and reproducibility of the INNO-LiPA $^{\circledR}$ CFTRiage assay.

Methods: To assess the success rate and agreement, 324 stored DNA samples from (suspected) CF patients, heterozygous carriers and healthy subjects were tested at 3 
external sites. The DNA was amplified with the OneAmp kit and consecutively tested with the INNO-LiPA ${ }^{\circledR}$ CFTRiage strips and, where applicable with the INNOLiPA $^{\circledast}$ CFTR confirmatory strips. For reproducibility testing, a panel of 6 DNA samples was tested at three sites (2 external, and 1 in-house), by different persons, on different days, on different instruments, and in-house on 3 different lots of the INNO-LiPA CFTRiage.

Results: The success rate was $100.0 \% \quad(95 \%$ CI $[99.2 \% ; 100.0 \%])$. An agreement of $99.7 \% \quad(95 \%$ CI [98.6\%;99.7\%]) between INNO-LiPA CFTRiage assay and the reference result was obtained. The reproducibility of the INNO-LiPA CFTRiage was $100.0 \%$ (95\% CI of $[98.6 \% ; 100.0 \%]$ ).

Conclusion: The study proved that the INNO-LiPA ${ }^{\circledast}$ CFTRiage assay has an excellent performance and is an easy to use assay to detect 88 CFTR mutations simultaneously.

C. Dobbels: None. L. Tremmerie: A. Employment (full or part-time); Modest; Fujirebio. M. Seia: None. M. D'Apice: None. M. Lucarelli: None. K. Stouffs: None. S. Seneca: None. A. Gheldof: None. C. Orsini: A. Employment (full or part-time); Modest; Fujirebio. G. Mersch: A. Employment (full or part-time); Modest; Fujirebio. V. Kostanjevecki: A. Employment (full or part-time); Modest; Fujirebio.

\section{E-P15.06}

Association of two rare inherited causes of neonatal intrahepatic cholestasis revealed by next generation sequencing

\section{H. Fredj ${ }^{l}$, S. Hizem ${ }^{l}$, Y. Elaribi ${ }^{l}$, I. Rejeb ${ }^{l}$, H. Jilani ${ }^{l}$, M. Sebai ${ }^{l}$, M. Chaabouni ${ }^{2}$, F. Broly ${ }^{3}$, L. Ben Jemaal}

\section{${ }^{I}$ Department of Congenital and Hereditary Diseases, Mongi Slim Hospital, La Marsa, Tunis, Tunisia, ${ }^{2}$ pediatric clinic, Sfax, Tunisia, ${ }^{3}$ Service Toxicologie et Génopathies. Institut de Biochimie et Biologie Moléculaire CHU de Lille, Lille, France}

Introduction: Neonatal cholestasis is a heterogeneous group of rare disorders of impaired bile flow in the newborn and young infant, some of which might be fatal if not identified and treated rapidly. More than 100 inherited etiologies were identified to cause cholestatic liver diseases with the initial presentation of jaundice. Clinical diagnosis of neonatal cholestasis is considered to be an extremely challenging process. With the advent of next generation sequencing (NGS), it becomes possible to rapidly identify the genetic cause of such disorders in a high-throughput manner. We report on a case of neonatal cholestasis investigated by an expanded panel-based NGS, revealing a rare association of citrin deficiency and progressive familial intrahepatic deficiency (PFIC 2).

Material and methods: The proband is a 3-month-old boy, born from consanguineous union. He presented with intermittent jaundice from 15 days after birth, with elevated transaminase and alpha-fetoprotein serum levels, very high serum bile acid concentration, and normal serum gammaglutamyltransferase (GGT) activity. An expanded panelbased NGS was performed on genomic DNA from the proband, including 29 genes implicated in PFIC, and additional 224 other genes for cholestasis.

Results: Two different homozygous variants were identified in the child: A c.1062T>A in $A B C B 11$ gene responsible for PFIC2, and a novel probably pathogenic variant in SLC25A13 gene responsible for citrin deficiency. Both parents were heterozygous for both variants.

Conclusions: Using large panel-based NGS allowed the identification of a combination of two rare intricate inherited causes of neonatal cholestasis, needing an appropriate treatment and dietary management to avoid complications.

H. Fredj: None. S. Hizem: None. Y. Elaribi: None. I. Rejeb: None. H. Jilani: None. M. Sebai: None. M. Chaabouni: None. F. Broly: None. L. Ben Jemaa: None.

\section{E-P15.08}

Sequencing Illumina DNA libraries on BGISEQ-500 sequencing platform using custom protocol

\section{J. A. Vnuchkova, P. A. Koshkin, D. V. Pyankov, S. A. Korostelev}

\section{Genomed Ltd, Moscow, Russian Federation}

Introduction: Convertion of Illumina DNA libraries to DNA nanoballs and subsequent sequencing on BGI/MGI sequencing platforms could resolve potential problem of obtaining optimal cluster density on Illumina platforms. In this study we describe a custom protocol for sequencing Illumina DNA libraries on BGISEQ-500 sequencer and present comparative analysis of data obtained from NextSeq 500 and BGISEQ-500 sequencers.

Materials and methods: Set of 48 DNA libraries was prepared using Swift 2S Turbo DNA Library Kit (Swift Bioscience) and NimbleGen SeqCap Hybridization reagents and probes (Roche). Sequencing was performed on NextSeq 500 and BGISEQ-500 platforms. Oligonucleotides for library convertion and BGISEQ-500 sequencing were ordered from Microsynth (Switzerland). Demultiplexing of BGISEQ-500 data was performed using custom tool. FastQC, aligning and coverage analysis were performed using FASTQC, BWA MEM and Mosdepth tools. Variant 
calling was performed using in-house pipeline for clinical variant interpretation based on ACMG criteria.

Results: Custom protocol for sequencing Illumina DNA libraries on BGISEQ-500 was developed. Comparative analysis of Illumina and BGI data was performed. We observed comparably good quality in both datasets according to various quality control metrics, even having a better quality for BGISEQ-500 data. Variant calling showed some diversity in general amount of obtained variants between two platforms, however all clinically significant variants were similar in both datasets.

Conclusions: Our study suggests that developed protocol is acceptable for sequencing hybridization-based DNA libraries. The limitation of the method is a necessity to use unique single indexes. The development of library convertion kit based on this study is potential.

J.A. Vnuchkova: None. P.A. Koshkin: None. D.V. Pyankov: None. S.A. Korostelev: None.

\section{E-P15.09}

\section{Investigation of the DNA methylation profile in children} presenting emergence delirium

V. Quintao ${ }^{1,2}$, Y. Gasparini ${ }^{2}$, V. Almeida ${ }^{2}$, A. Dias $^{2}$, M. Montenegro ${ }^{2}$, M. Carmona $^{l}$, L. Kulikowski ${ }^{2}$

${ }^{1}$ Hospital das Clinicas HCFMUSP, Faculdade de Medicina, Universidade de Sao Paulo, Sao Paulo, Brazil, ${ }^{2}$ Laboratorio de Citogenomica, Hospital das Clinicas HCFMUSP, Faculdade de Medicina, Universidade de Sao Paulo, Sao Paulo, Brazil

Introduction: Among cognitive changes related to anesthesia, emergence delirium is frequent in children and could be observed in up to $30 \%$ of cases. The occurrence of delirium may be related to postoperative cognitive and behavioral changes. Recent studies demonstrated the involvement of epigenetic processes in the behavioral changes related to anesthesia. Thus, performing the DNA methylation profiles is an important tool for studying the role of epigenetic factors in these events.

Materials and methods: After IRB approval, children between 1 and 12 years old, submitted to general anesthesia for endoscopic procedures, were recruited. Emergence delirium was considered as Pediatric Anesthesia Emergence Delirium Scale score $\geq 10$. DNA from blood lymphocytes were extracted by QIAamp DNA BloodMidi Kit (QIA$\left.\mathrm{GEN}^{\circledR}\right)$. The genomic array was performed using iScan (Illumina $^{\circledR}$ ) with HumanCytoSNP850K and Infinium MethylationEPIC BeadChips. Arrays data were treated and analyzed using Bluefuse $^{\circledR}$, GenomeStudio ${ }^{\circledR}$, and specific packages in $\mathrm{R}$ environment.
Results: We included 53 patients. Twenty-three children (43.4\%) presented emergence delirium. Eight children with emergence delirium and eight controls (after age and gender matching) were selected for methylation profile evaluation. Copy number variation analysis demonstrated no presence of pathogenic deletions or duplications. Analysis of methylation raw data showed a difference in the average beta value between cases and controls in selected chromosomes.

Conclusions: Our results suggest that DNA methylation profiles may present significant differences between children with emergence delirium and controls, including specific genomic regions. Therefore, we emphasize the importance of investigating and identifying epigenetic alterations to understand this relevant clinical adverse event.

V. Quintao: None. Y. Gasparini: None. V. Almeida: None. A. Dias: None. M. Montenegro: None. M. Carmona: None. L. Kulikowski: None.

\section{E-P15.10}

Gene expression profiling of RNA samples from frozen blood and optimization of sample storage conditions

\section{E. Koh, S. Yu, S. Hwang}

\section{Hanyang University, Ansan, Korea, Republic of}

Gene expression profiling is one of the most powerful technologies to understand bio-molecular alterations which are affected by external stimulus such as environmental toxicants. In order to obtain reliable interpretation of these data, integrity of RNA should be primarily qualified, which is determined from the very moment of sample collection and storage conditions. In the meantime, whole blood can be obtained with less-invasive way compared to others such as tissues. However, blood is vulnerable to freezing and often loses its availability for RNA downstream applications. In this study, we compared changes of RNA quality according to the several sample storage conditions including temperature and duration of incubation before freezing, long-term freezing and types of collection tubes. After simple QC steps, we performed mRNA-seq to compare gene expression patterns of RNAs from various storage conditions. We found that quality of RNA samples is obviously affected by storage conditions leading to changes in gene expression patterns compared to control (Fold changes $>|2|$ ). Differentially expressed genes in this study are grouped as snoRNA family, which is involved in structure of rRNA, tRNA and so on. As a results, we set the guideline for optimal blood sample storage. By proceeding to further study, it is expected to set the markers for RNA sample quality by inducing whether these genes take charge in RNA degradation in some ways. This study was 
supported by Korea Environment Industry \& Technology Institute(KEITI) through "the Environmental Health Action Program", funded by Korea Ministry of Environment (MOE) (2017001360005).

E. Koh: None. S. Yu: None. S. Hwang: None.

\section{E-P15.12}

Quick assessment of antioxidant effects in combined treatments measuring catalase gene activity in vitro

\section{N. Tomic, M. Hadzic, N. Lojo-Kadric, J. Ramic, L. Pojskic}

University of Sarajevo, Institute for Genetic Engineering and Biotechnology, Sarajevo, Bosnia and Herzegovina

Luteolin is the bioflavonoid with known protective roles. It inhibited genotoxicity experimentally induced by halogenated boroxine (HB). Statistically significant decrease in the frequency of micronuclei and nuclear buds was observed before, but mechanism of its anti-genotoxic activity is not clear. Since HB is recognized as inhibitor of catalase activity as a major antioxidant enzyme, we designed experiment to assess antioxidative effects of luteolin on level of relative expression of catalase (CAT) gene. Cell cultures from peripheral blood lymphocytes of five healthy donors were established and treated with luteolin, $\mathrm{HB}$, and luteolin and HB combined. After 72 hours incubation, total RNA was isolated from harvested cells and reverse-transcribed. SYBR based Real-Time PCR amplification method for analysis of relative gene expression in treated cultures was used. Analysis of results included normalization of ratio of target $(C A T)$ and housekeeping $(G A P D H)$ gene and statistical analysis $\left(\mathrm{REST}^{\circledR}{ }^{\circledR}\right.$. Significant up-regulation of $C A T$ expression on transcriptional level was found in simultaneously treated cultures when compared with cultures treated with each substance individually. In luteolintreated cultures when compared to HB-treated cultures $C A T$ expression was down regulated but not statistically significant. In comparison to control (DMSO), CAT expression was up regulated in simultaneously treated cultures but in individually treated cultures it was down regulated, with no statistical significance. Obtained results may indicate that new approaches to the treatment of skin changes with $\mathrm{HB}$ should include luteolin and monitoring corresponding antioxidant system. For more precise determination of luteolin effects and potential, more treatments and genes should be explored.

N. Tomic: None. M. Hadzic: None. N. Lojo-Kadric: None. J. Ramic: None. L. Pojskic: None.

\section{E-P15.13}

\section{Closing the diagnostic gap on muscular dystrophies}

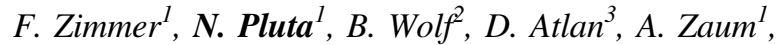
S. Rost ${ }^{l}$

${ }^{I}$ Institute of Human Genetics, Wuerzburg, Germany, ${ }^{2}$ iCoSys, Fribourg, Switzerland, ${ }^{3}$ Phenosystems SA, Braine le Chateau, Belgium

Introduction: Whole genome sequencing (WGS) is becoming more and more meaningful not only in human genetic research but also for future molecular genetic diagnostics. The main challenges in WGS lie in data analysis and variant interpretation rather than in the sequencing technology itself. The detection of disease-causing variants in those extensive genome data requires the application of appropriate software tools.

Materials and methods: Here we present the data analyses of four genomes from patients with muscular dystrophies (without mutations using gene panel or exome sequencing) by applying the software GensearchNGS (PhenoSystems). We used integrated variant calling, CNV tools and anomaly scans together with external tools (e.g. cnMops, Alamut, CADD) to analyze and validate the patient's data (see table).

\begin{tabular}{|c|c|c|c|c|}
\hline patient & 1 & 2 & 3 & 4 \\
\hline $\operatorname{sex}$ & $\mathrm{F}$ & M & $\mathrm{F}$ & M \\
\hline $\begin{array}{l}\text { gene of } \\
\text { interest } \\
\text { (goi) }\end{array}$ & $S G C G$ & $D M D$ & $D M D$ & $D M D$ \\
\hline $\begin{array}{l}\text { aligned } \\
\text { sequences }\end{array}$ & 1.262 .574 .555 & 1.072 .189 .661 & 666.864 .288 & 680.631 .017 \\
\hline fastq(Gb) & 90,9 & 77,9 & 48,1 & 48,9 \\
\hline $\begin{array}{l}\text { mean } \\
\text { coverage } \\
\text { (goi) }\end{array}$ & 64 & 29 & 34 & 18 \\
\hline $\begin{array}{l}\text { variants } \\
(\text { total })^{*}\end{array}$ & 5.706 .907 & 5.679 .385 & 5.635 .599 & 5.709 .193 \\
\hline $\begin{array}{l}\text { variants } \\
\text { (goi) }^{*}\end{array}$ & 453 & 2.321 & 3.151 & 2.003 \\
\hline $\begin{array}{l}\text { candidate } \\
\text { variant }\end{array}$ & $\begin{array}{l}\text { homozygous SNV } \\
\text { intron } 1\end{array}$ & pending & $\begin{array}{l}\text { heterozygous } \\
\text { SNV } \\
\text { intron } 63\end{array}$ & $\begin{array}{l}\text { hemizygous } \\
\text { deletion } \\
\text { intron } 2\end{array}$ \\
\hline $\begin{array}{l}\text { splice } \\
\text { effects (in } \\
\text { silico } \\
\text { prediction) }\end{array}$ & $\begin{array}{l}\text { cryptic acceptor } \\
\text { activated }\end{array}$ & pending & $\begin{array}{l}\text { cryptic } \\
\text { acceptor } \\
\text { activated }\end{array}$ & unknown \\
\hline comments & $\begin{array}{l}\text { muscle biopsy: } \\
\text { reduced gamma- } \\
\text { sarcoglycan } \\
\text { expression }\end{array}$ & $\begin{array}{l}\text { RNA-analysis of } \\
D M D \text { without } \\
\text { pathological } \\
\text { findings }\end{array}$ & $\begin{array}{l}\text { skewed X- } \\
\text { inactivation }\end{array}$ & $\begin{array}{l}\text { heterozygous } \\
\text { intronic } \\
\text { deletion } \\
(S G C G)\end{array}$ \\
\hline
\end{tabular}

*variant calling options: frequency $>20 \%$, coverage $>5$

Conclusion:In at least three out of four patients, definitely suffering from muscular dystrophies, we could detect promising structural variants and/or SNVs in the noncoding regions of known genes (DMD, SGCG). The most promising candidate variants are now under consideration 
for further functional studies in order to confirm/exclude the deleteriousness of those variants.

F. Zimmer: None. N. Pluta: None. B. Wolf: None. D. Atlan: A. Employment (full or part-time); Significant; Phenosystems SA. A. Zaum: None. S. Rost: None.

\section{E-P15.17}

Nanoscale automation of RNA sequencing library preparation on a microfluidic platform

A. Hamilton ${ }^{1}$, S. Chamnongpol ${ }^{2}$, J. Alipaz ${ }^{2}$, J. Brockman ${ }^{2}$,

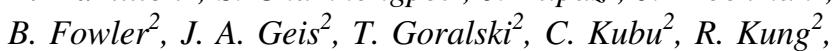

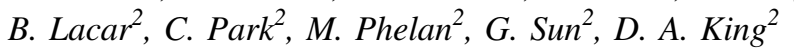

${ }^{1}$ Fluidigm GmBH, Munich, Germany, ${ }^{2}$ Fluidigm Corporation, South San Francisco, CA, United States

Insights from the analysis of expressed gene products by RNA sequencing enables human disease researchers to better understand the underlying structure and function of genes of interest. We developed a microfluidics-based solution that automates many tedious hands-on steps of the RNA-seq library prep workflow including poly(A) RNA selection. The method generates up to 48 random-primed stranded RNA-seq libraries from as little as $10 \mathrm{ng}$ of total RNA. Nanoliter reaction volumes significantly reduce reagent consumption to lower overall costs per sample. We are developing an option for use of unique dual index (UDI) tags to improve proper assignment of reads reported for sequencers with patterned flow cells, such as the Illumina ${ }^{\circledR}$ NovaSeq ${ }^{\mathrm{TM}}$. In this poster, results from an internal analytical study of the Advanta ${ }^{\mathrm{TM}}$ RNA-Seq NGS Library Prep Kit and a comparison to results obtained from the Illumina TruSeq ${ }^{\circledast}$ Stranded mRNA Kit are presented. Our study included $>900$ samples at 10 and $100 \mathrm{ng}$ of input that generated genome mapping rates $>80 \%$, with rRNA reads $<10 \%$. Technical replicate correlations were observed to be $>98 \%$ in all conditions. A comparable number of genes are detected in libraries prepared with either Advanta or TruSeq kits. The Advanta RNA-Seq Kit provides an RNA-seq library prep solution offering walkaway automation that substantially minimizes manual pipetting steps and handson time. The inclusion of UDIs further improves the workflow with high-data-output sequencers such as the NovaSeq. For Research Use Only. Not for use in diagnostic procedures.

A. Hamilton: A. Employment (full or part-time); Significant; Fluidigm. S. Chamnongpol: A. Employment (full or part-time); Significant; Fluidigm Corporation. J. Alipaz: A. Employment (full or part-time); Significant; Fluidigm. J. Brockman: A. Employment (full or parttime); Significant; Fluidigm. B. Fowler: A. Employment (full or part-time); Significant; Fluidigm. J.A. Geis: A.
Employment (full or part-time); Significant; Fluidigm. T. Goralski: A. Employment (full or part-time); Significant; Fluidigm. C. Kubu: A. Employment (full or part-time); Significant; Fluidigm. R. Kung: A. Employment (full or part-time); Significant; Fluidigm. B. Lacar: A. Employment (full or part-time); Significant; Fluidigm. C. Park: A. Employment (full or part-time); Significant; Fluidigm. M. Phelan: A. Employment (full or part-time); Significant; Fluidigm. G. Sun: A. Employment (full or part-time); Significant; Fluidigm. D.A. King: A. Employment (full or part-time); Significant; Fluidigm.

\section{E-P15.18}

Using RNA-Seq as complementary diagnostic tool in congenital heart defects: pilot study in a group of transplanted children

A. del Pozo ${ }^{l}$, C. Rodríguez-Antolín ${ }^{2}$, R. Martín-Arenas ${ }^{3}, A^{\text {. }}$ González-Rocafort ${ }^{4}$, M. Solís ${ }^{1}$, B. Ruz ${ }^{2}$, L. García Guereta ${ }^{4}$, L. Fernández García-Moya ${ }^{2}$, E. Vallespín ${ }^{1}$

${ }^{1}$ INGEMM - Hospital Universitario La Paz - CIBERER, Madrid, Spain, ${ }^{2}$ INGEMM - Hospital Universitario La Paz, Madrid, Spain, ${ }^{3}$ Genycell Biotech España, Madrid, Spain, ${ }^{4}$ Cirugía Cardiaca Infantil. Hospital Universitario La Paz, Madrid, Spain

The implantation of NGS in clinical routine has supposed a great advance in the diagnostic of mendelian diseases providing very valuable information for disease management. Still there are cases where NGS testing does not provide a conclusive diagnosis due to the limits that present studies based exclusively on DNA analysis specially in the interpretation of challenging variants as those within intronic, regulatory or splicing regions.

Recent works endorse RNA-Seq as a complementary technique that would provide more evidences to lead to a diagnostic. Although promising results, methodological and quality issues must be determined to ensure clinical validity/ utility of the study.

In this work, it had been selected a group of 9 pediatric patients who had undergone a heart transplant surgery during the period 2018-2019 due to a congenital heart defect with negative or inconclusive result for genepanel study.

It has been designed a pilot diagnostic scheme to achieve a robust diagnosis that includes the DNA information of the patients together with RNA-Seq data from their cardiac tissue from the discarded heart.

The RNA-Seq pipeline integrates several tools that compares a target sample against a control cohort selected from the GTEx project. It includes tools for: 1) aberrant splicing events identification, 2) aberrant expression 
quantification and 3) identification of allele-specific expression of variants determined both by DNA-Seq and RNASeq.

This study has enabled the characterization of a new candidate and the study of previous VUS variants what shows the potential of such approaches to be adopted in routine diagnosis.

A. del Pozo: None. C. Rodríguez-Antolín: None. R. Martín-Arenas: A. Employment (full or part-time); Significant; Genycell Biotech España. Á. González-Rocafort: None. M. Solís: None. B. Ruz: None. L. García Guereta: None. L. Fernández García-Moya: None. E. Vallespín: None.

\section{E-P15.20}

\section{Try to find the somatic mutation in the brain!}

\section{J. Kárteszi}

\section{Genetic Laboratory, Zalaegerszeg, Hungary}

It was demonstrated in recent years that somatic mutations happen in neuronal stem cells. However, the effect of somatic mutations during tumorigenesis had been proven long time ago. We also know that some clue oncogenes have important role in neurodevelopment and maintenance of brain integrity. Based on common regulatory pathways in both tumorigenesis and neurodegeneration I discuss the possible use of oncogenetic research evidences in the explanation of devastating neurogenic diseases. "Loss of heterozygosity" is widely used in tumour genetics. On the other hand, plenty of neurogenic disorders were described with "dominant negative effect" which meant loss of function of the effected genes. Data were collected that until the normal allele functioned well this effect didn't work. Somatic mutation in neuronal stem cells containing a germline mutation may destroy the function of the normal allele. I propose that neurodegeneration can be clonal disorder in some instances. The complicated pathogenesis of Parkinson disease is highly studied and GBA (beta-glucocerebrosidase) gene heterozygous mutations were found more frequently in the familial form of the disease. Gaucher disease is an autosomal recessive lysosomal storage disorder caused by glucocerebrosidase deficiency. It was detected that Parkinson disease is more frequent in Gaucher type I patients but also in the carrier parents. Liquid biopsy is well known in tumour research. Let's try to use it to find the somatic mutation in the brain! The first successful step in this direction may also lead to the understanding of a subset of autosomal dominant neuronal diseases with incomplete penetrance.

J. Kárteszi: None.

\section{E-P15.21}

The use of artificial intelligence to assess factors of genetic predisposition to cancer of the uterine body

\section{Ugarov ${ }^{I}$, V. Chernyh ${ }^{1,2}$, I. Sharkova ${ }^{1,2}$, N. Ivanov $^{l}$, V. Maslennikov ${ }^{l}$, D. Ostapenko ${ }^{l}, V$. Solovey ${ }^{l}$}

${ }^{1} x$ Gen Cybernetics, Moscow, Russian Federation, ${ }^{2}$ Federal state scientific budgetary Institution «Research Centre for Medical Genetics», Moscow, Russian Federation

Uterine Corpus Endometrial Carcinoma (UCEC) is a malignant multifactorial neoplasm which is characterized by high prevalence. Currently, there are no screening methods for assessing genetic predisposition to UCEC, so the development of an approach to analyzing the results of genetic testing, devoid of the previously described shortcomings and providing identification of variants characteristic of multifactorial pathology, is an actual problem. Objective: to develop an approach to identify patterns in the results of genetic testing that are typical for patients with multifactorial cancer and to develop a genetic panel for screening for predisposition to UCEC. Materials and methods: For a set of genes, the analysis of refereed scientific articles on the following keywords was performed:" Uterine Corpus Endometrial Carcinoma"," predisposition"," mutation","SNP". Data from TumorPortal was used to evaluate the model's performance (http://www.tumorportal.org/) and the Cancer Genome Atlas (TCGA). Based on the data, two groups were formed: patients with UCEC $(n=248)$ and a control cohort of patients with other nosologies $(\mathrm{n}=6498)$. Results: a method has been developed that allows us to identify screening patterns of multifactorial conditions. Patterns and a mathematical model based on the method takes into account genetic variants in 229 genes related to UCEC, showing $79 \%$ sensitivity and $85 \%$ specificity. Screening patterns cover all highly significant mutated genes according to Tumorportal data, excluding CCND1, ERBB3, ZNF471, and ING1. The use of artificial intelligence to assess factors of genetic predisposition to cancer of the uterine body and preclinical prevention.

I. Ugarov: None. V. Chernyh: None. I. Sharkova: None. N. Ivanov: None. V. Maslennikov: None. D. Ostapenko: None. V. Solovey: None.

\section{E-P15.22}

The first Albanian case of Waardenburg syndrome type 4A

\section{A. Laku - Babameto}

Service of Genetic Laboratory, University of Medicine, University Hospital Cenetr "Mother Teresa", Tirana, Albania 
Waardenburg syndrome type 4 (WS4), is a very rare congenital disorder with variable clinical expression, characterised by Hirschsprung disease, and abnormal melanocyte migration, resulting in pigmentary abnormalities and sensorineural deafness. Three disease-causing genes have been identified: mutations in the SOX10, END3, or EDNRB genes are known to cause WS type IV. Variants in the endothelin $\mathrm{B}$ receptor $(\mathrm{EDNRB})$ gene on $13 \mathrm{q} 22$ region have been found to cause the Waardenburg syndrome type IVA (WS 4A), that combines clinical features of pigmentary abnormalities of the skin, hair and irides, sensorineural hearing loss, and Hirschprung disease. We report a 15 months - old albanian boy who was diagnosed as WS IVA. He presented clinical manifestations as skin and hair hypopigmentation, hypertelorism, broad nasal bridge, epicanthal folds, bilateral blue homochromatic irides, mild developmental delay, muscular hypotonia, short stature and constipation. The entire exome has been evaluated for variants clinically relevant to the described phenotype by the whole exome sequencing, but it was not detected any variant indicative of the genetic diagnosis of the patient. The diagnosis was confirmed by chromosomal microarray analysis. It was detected a $23153 \mathrm{~kb}$ one copy loss on the long arm of chromosome 13. A pathogenic $\mathrm{CNV}$ has been detected in the 13q21.1q31.1 region, totally encompassing the EDNRB gene. The EDNRB gene has been found totally deleted in our patient. It is observed a variable phenotype in patients with heterozygous loss of function of EDNRB gene. This is the first case of WS 4A to be reported from Albania.

A. Laku - Babameto: None.

\section{E-P15.23}

Generation of whole-exome-libraries with a wide-range of fragment length with the Nextera Flex bead-linked transposome approach to improve genotypability

\section{B. Iadarola ${ }^{1}$, M. Rossato ${ }^{1}$, M. Paterno ${ }^{1}$, D. Lavezzari ${ }^{1}$, C. Degli Esposti ${ }^{1}$, C. De Quattro ${ }^{1}$, B. Giovannone ${ }^{1}$, G. Bhattacharjee ${ }^{2}$, R. Tim ${ }^{2}$, M. You ${ }^{2}$, M. Delledonne ${ }^{1}$ \\ ${ }^{1}$ University of Verona, Verona, Italy, ${ }^{2}$ Illumina, San Diego, $C A$, United States}

Introduction: Short-read sequencing in targeted resequencing prevents the identification of clinically-relevant genetic variants within or in close proximity to duplicate genomic regions. We recently found that the analysis of fragments longer than standard-size improves mapping quality and thus genotypability of many clinically relevant genomic regions. However, modulating fragment length in enzymatic-based library preparation is challenging, as optimal ratio between DNA/enzymes is not easily identifiable or because bead-linked transposome chemistry is setup for a standard DNA size. In this study we tested the possibility to modulate fragment length in exome-libraries using the standard or a modified version of the Nextera Flex for Enrichment bead-linked transposome (Illumina), extending fragment length up to 550bp.

M\&M: Genomic libraries were obtained with the standard Nextera Flex for Enrichment Library Prep kit or its new modified version (not commercially available yet), and subsequently enriched in exonic portions with the Illumina Exome Panel. Libraries have been sequenced in 150PE to 80X mapped coverage.

Results: Genomic libraries with different fragment lengths $(200,300,400,500 \mathrm{bp})$ have been successfully generated by adjusting simple experimental conditions such as bead-based size-selection and PCR extension time. Exome enrichment did not change the different fragment sizes, that were subsequently confirmed by sequencing. Target coverage and number of variants across the multiple fragment sizes are being evaluated.

Conclusions: The availability of two distinct bead-based transposome library prep kits will allow to generate libraries with a wide range of fragment size, thus permitting to modulate/improve genotypability in targeted resequencing.

B. Iadarola: None. M. Rossato: None. M. Paterno: None. D. Lavezzari: None. C. Degli Esposti: None. C. De Quattro: None. B. Giovannone: None. G. Bhattacharjee: A. Employment (full or part-time); Significant; Illumina. R. Tim: A. Employment (full or part-time); Significant; Illumina. M. You: A. Employment (full or part-time); Significant; Illumina. M. Delledonne: C. Other Research Support (supplies, equipment, receipt of drugs or other in-kind support); Modest; Illumina.

\section{E-P16 Diagnostic Improvements and Quality Control}

\section{E-P16.01}

Validation of a Next Generation Sequencing $B R C A$ assay using buccal DNA swabs to meet current and future demands in diagnostic field

E. De Paolis ${ }^{1}$, M. De Bonis ${ }^{1}$, E. Ferri ${ }^{2}$, C. Marchetti ${ }^{2,3}$, A. Pietragalla ${ }^{2}$, G. Scambia ${ }^{2,3}$, A. Urbani ${ }^{1,3}$, A. Fagotti $^{2,3}$, A. Minucci ${ }^{l}$

${ }^{1}$ Molecular Diagnostic and Genomics Laboratory, Fondazione Policlinico Universitario A. Gemelli IRCCS, Rome, 00168, Italy, ${ }^{2}$ Division of Oncological Gynecology, Department of Women's and Children's Health, Fondazione Policlinico Universitario A. Gemelli IRCCS, Rome, 00168, Italy, ${ }^{3}$ Catholic University of the Sacred Heart, Rome, Italy 
Introduction: Most of the commercial sequencing methods use blood as primary sample source of genomic DNA (gDNA). Buccal swab is an emerging sample collection device due to convenient and non-invasive characteristics. This study assesses the suitability of buccal swab as gDNA source and evaluates the performance of Next Generation Sequencing (NGS) Devyser kit for detecting BRCA pathogenic variants (PVs).

Materials and methods: Swabs and blood samples were collected from 5 healthy and 3 BRCA PVs carriers. Buccal swabs were obtained using FLOQ Swab (COPAN, Brescia, Italy) placed with NAO basket. Both sample types were extracted using the QIAmp DNA Mini kit (Qiagen, Hilden, Germany). NGS Devyser BRCA kit (Devyser, Hägersten, Sweden) was used for the $B R C A$ amplification. Sequencing was performed on MiSeq platform (Illumina, CA, US). Quality parameters were evaluated from sequencing data. NGS data were analyzed by Amplicon Suite (SmartSeq, Novara, Italy).

Results: Concentration and quality of DNA extracted from buccal swab samples were adequate for library preparation and sequencing. Comparable quality parameters result from the evaluation of NGS data across samples types. BRCA genetic alterations (SNPs, PVs, CNV) detected in buccal DNA swabs were concordant with those from matched blood samples.

Conclusions: Devyser BRCA NGS kit performs as well with buccal swabs collected using Copan FLOQ Swab as it does with samples collected from blood. Similar genotyping performances between the two DNA sources were observed, supporting the use of buccal swabs as alternative sample type in NGS. Larger study has been planned in order to perform the validation assay.

E. De Paolis: None. M. De Bonis: None. E. Ferri: None. C. Marchetti: None. A. Pietragalla: None. G. Scambia: None. A. Urbani: None. A. Fagotti: None. A. Minucci: None.

\section{E-P16.02}

Clinical utility of carrier status for autosomal recessive conditions in the Brazilian population

\section{W. A. R. Baratela, C. M. Moreira, P. R. Sacramento-}

Bobotis, G. M. N. Filho, M. G. Penna, R. R. F. Souza, A. C. C. Trindade, C. R. Furuzawa, D. P. Pereira, C. Olivati, G. M. Spolador, V. C. Pereira, R. A. da Silva, M. N. P. Santos, C. R. D. C. Quaio

\section{Fleury Medicina e Saúde, São Paulo, Brazil}

Introduction: Commercial tests for carrier status determination of common autosomal recessive conditions are currently offered in Brazil. Nevertheless, frequency of rare autosomal recessive alleles has not been established for the Brazilian population. Therefore, clinical utility of these tests is not determined for this specific population.

Methods: We actively searched for pathogenic variants in autosomal recessive conditions, not the primary objective of analysis, in 100 whole exomes performed for diagnostic purposes in a private laboratory in Brazil. Common variants (e.g., frequency $>1 \%$ ) in common recessive conditions (HFE, SERPINE1, BTD) were excluded from our analysis. We reviewed the methodology of three commercial tests for carrier status and assessed whether variants found in our cohort were covered by these commercial kits.

Results: Rare pathogenic variants in genes associated with autosomal recessive conditions were found in $37 \%$ of patients. Among these patients, 40,5\% presented more than one variant. Average number of genes tested by commercial kits was 433 . More than half $(54 \%)$ of variants found were not universally covered by all commercial kits assessed. As for individual laboratories, coverage of recessive variants varied from $45 \%$ to $66,5 \%$.

Conclusion: Information about limited sensitivity for carrier status panels for pathogenic variants in Brazilian patients should be addressed in genetic counselling to avoid false reassurance and adjusting expectations from couples. The usage of larger target gene panels, or even whole exome sequencing, might overcome this limitation.

W.A.R. Baratela: None. C.M. Moreira: None. P.R. Sacramento-Bobotis: None. G.M.N. Filho: None. M.G. Penna: None. R.R.F. Souza: None. A.C.C. Trindade: None. C.R. Furuzawa: None. D.P. Pereira: None. C. Olivati: None. G.M. Spolador: None. V.C. Pereira: None. R.A. da Silva: None. M.N.P. Santos: None. C.R.D.C. Quaio: None.

\section{E-P16.03}

Allele dropout caused by an AT-repeat in $M M E$

H. T. Hilmarsen ${ }^{1}$, P. F. Rosenegger ${ }^{2}$, Ø. L. Holla ${ }^{1}$, S. B. C. F. Reiter ${ }^{3}$, L. Strand ${ }^{1}$, G. J. Braathen ${ }^{1}$, J. Senderek ${ }^{4}, H$. Hфyer ${ }^{l}$, M. Auer-Grumbach ${ }^{4}$

${ }^{1}$ Telemark Hospital Trust, Skien, Norway, ${ }^{2}$ Medical University of Vienna, Vienna, Austria, ${ }^{3}$ Haukeland University Hospital, Bergen, Norway, ${ }^{4} L M U$ Munich University Hospital, Munich, Germany

Introduction: The $M M E$-gene encodes a widely expressed zinc-dependent metalloendopeptidase (Neprilysin). Biallelic sequence variants in $M M E$ cause axonal Charcot-MarieTooth disease and fetomaternal alloimmune membranous glomerulopathy, while heterozygous variants are susceptibility factors for axonal Charcot-Marie-Tooth disease. The frameshift deletion NM_007289.3(MME):c.467delC 
(p.Pro156Leufs*14) has been reported as a dominant susceptibility factor and as a cause of recessive neuropathy.

Materials and methods: We present three axonal neuropathy families and three simplex individuals carrying the $M M E$ c.467delC variant. Probands underwent NextGeneration-Sequencing (NGS); most relatives were Sanger sequenced.

Results: NGS identified heterozygous c.467delC in the probands with familial disease and in two simplex cases. The last simplex case was homozygous. Subsequent Sanger analysis confirmed these results in all cases. Six additional relatives appeared to be homozygotes by Sanger sequencing. However, NGS showed these six individuals to be heterozygotes. Further investigation revealed a highly polymorphic AT-repeat in close proximity to the variant. All heterozygous individuals who appeared homozygous for c.467delC were heterozygous for the AT-repeat (8AT/ 13AT). The heterozygous individuals were homozygous for the AT-repeat.

Conclusions: A heterozygous AT-repeat situated in close proximity to $M M E$ c. $467 \mathrm{delC}$ causes allele dropout on Sanger sequencing; heterozygotes appear to be homozygotes. An incorrect diagnosis and cascade testing may have a large clinical impact. This highlights that even a small difference in nucleotides outside the primer binding sites may cause allele dropout.

H.T. Hilmarsen: None. P.F. Rosenegger: None. Ø.L. Holla: None. S.B.C.F. Reiter: None. L. Strand: None. G.J. Braathen: None. J. Senderek: None. H. Høyer: None. M. Auer-Grumbach: None.

\section{E-P16.04}

Cancer care and a tale of three molecular "genomic" tests

N. Hook, J. Wallace, B. Zimmermann, N. Liang, R. Swenerton, U. Do, S. Sawyer, J. Saucier, S. Parmar, A. Aleshin

Natera, Inc, San Carlos, CA, United States

Introduction: Patient-specific, bespoke assays detect circulating tumor DNA (ctDNA) by analyzing cell free DNA to monitor molecular residual disease, recurrence, and treatment response in cancer patients. We present a case study of a patient diagnosed with cancer where three individual molecular tests were utilized in the care continuum.

Case Presentation: A 56 yo Caucasian female diagnosed with stage IIIc ovarian clear cell carcinoma was treated with total abdominal hysterectomy with bilateral salpingooophorectomy, omentectomy, appendectomy, lymph node sampling, peritonectomy, and optimal tumor debulking in June, 2018. Comprehensive genomic profiling for tumor molecules and diagnostic testing for hereditary cancer predisposition syndrome was performed to identify inherited risk and consider use of PARP inhibitor therapy. Treatment with first-line adjuvant chemotherapy concluded in October 2018. The patient relapsed (Jan 2019) and was enrolled in clinical trial GYO16, receiving pembrolizumab infusions with oral epacadostat. In Feb 2019, ctDNA was monitored using a bespoke assay designed from matched-normal whole exome sequencing. ctDNA was initially detected (4.61 $\mathrm{MTM} / \mathrm{mL}$ ) and continued to increase across multiple timepoints (15.33 MTM/mL), consistent with the patient's reported progressive disease and exit from GYO16 study in Nov. 2019. Positive ctDNA results allow opportunity to monitor treatment response and trending disease burden through longitudinal screening of ctDNA levels.

Conclusion: This case study utilized three different molecular tests to inform and monitor cancer treatment. There are an increasing number of molecular genomic tests incorporated into practice, each with a unique purpose in the patient continuum of care that genetic professionals need to be aware of.

N. Hook: A. Employment (full or part-time); Significant; Natera, Inc. J. Wallace: A. Employment (full or part-time); Significant; Natera, Inc. B. Zimmermann: A. Employment (full or part-time); Significant; Natera, Inc. N. Liang: A. Employment (full or part-time); Significant; Natera, Inc. R. Swenerton: A. Employment (full or part-time); Significant; Natera, Inc. U. Do: Other; Significant; Natera, Inc (past employee). S. Sawyer: A. Employment (full or part-time); Significant; Natera, Inc. J. Saucier: A. Employment (full or part-time); Significant; Natera, Inc. S. Parmar: A. Employment (full or part-time); Significant; Natera, Inc. A. Aleshin: A. Employment (full or part-time); Significant; Natera, Inc.

\section{E-P16.06}

Decade-long progress of molecular genetics diagnostics of cystic fibrosis in Khanty-Mansi region (Russia)

M. Donnikov ${ }^{1}$, V. Mescheryakov ${ }^{1}$, N. Satsuk ${ }^{2}$,

A. Vorobyov ${ }^{1}$, L. Kolbasin ${ }^{3}$, I. Urvantseva ${ }^{4}$, O. Glotov ${ }^{5}$,

A. Glotov ${ }^{6}$, L. Kovalenko ${ }^{1}$

${ }^{1}$ Medical Institute of Surgut State University, Surgut, Russian Federation, ${ }^{2}$ Regional Children Clinical Hospital, Nizhnevartovsk, Russian Federation, ${ }^{3}$ Medical Genetics Counseling Service of Diagnostics and Cardiovascular Surgery Center (Cardiology Clinic) of KHMAO-Ugra, Surgut, Russian Federation, ${ }^{4}$ Diagnostics and Cardiovascular Surgery Center (Cardiology Clinic) of KHMAOUgra, Surgut, Russian Federation, ${ }^{5}$ Department of Genomic Medicine, D. O. Ott Research Institute of Obstetrics, Gynecology and Reproduction, Saint Petersburg, Russian 
Federation, Saint Petersburg, Russian Federation, ${ }^{6}$ Department of Genomic Medicine, D. O. Ott Research Institute of Obstetrics, Gynecology and Reproduction, Saint Petersburg, Russian Federation

Introduction: Cystic fibrosis (CF) newborn screening since 2006 year provided patients surge requiring elucidation of genetic diagnosis but scarce molecular tools allowed detection of only major mutations specific for mixed Russian population. Introduction of high-resolution melting analysis (HRMA), MLPA, NGS techniques at regional genetics laboratory allowed to perform extensive genetic analysis of CFTR gene.

Materials and methods: gDNA was obtained by wholeblood column extraction; HRMA performed by "PrecisionMelt Software" (Bio-Rad). MLPA performed with SALSA Probemix P091-D2_CFTR (MRC-Holland). NGS performed on MiSeq (Illumina) using NimbleGen SeqCap EZ_CysFib kit, mean coverage 70x. Raw data processed with bwa, GenomeAnalysisToolKit, annotated by dbNSFP (SnpEff, SnpSift), variants filtered by SNVViewer.

Results: During 2009-2019 regional genetics laboratory studied 1,095 gDNA samples from newborns suspicious for CF. First three years only eight $C F T R$ mutations were tested by allele-specific PCR. Successive introduction of HRMA, MLPA, Sanger sequencing and NGS allowed to perform comprehensive genetic analysis since 2014 . We revealed 36 mutations ( 25 found once) in 110 alleles of 55 CF patients (with 2 CFTR mutations), including confirmed CF cases in 10 adult patients. Interestingly to note year-to-year increased proportion of North Caucasus mutations within regional mutation spectrum.

Conclusions: Introduction of state-of-the-art techniques in regional genetics laboratory allowed to perform timely comprehensive genetic analysis of CFTR gene and deduce changes in regional mutations structure related to high migration rate of North Caucasians to Siberia.

Mutant alleles list

\begin{tabular}{llll}
\hline № & CFTR alleles (HGVS; legacy) & $\begin{array}{l}\text { Number } \\
(\mathrm{n}=110)\end{array}$ & Frequency \\
\hline 1 & c.1521_1523delCTT(p.Phe508del) & 58 & 0.527 \\
2 & $\begin{array}{l}\text { c.1545_1546delTA(p.Tyr515X; } \\
\text { 1677delTA) }\end{array}$ & 6 & 0.055 \\
3 & c.274G>A(p.Glu92Lys; E92K) & 5 & 0.046 \\
4 & c.54-5940_273+10250del21kb (p. & 4 & 0.036 \\
& Ser18ArgfsX16; dele2,3) & & \\
5 & c.3196C>T (p.Arg1066Cys; R1066C) & 4 & 0.036 \\
6 & c.413_415dupTAC (p.Leu138dup) & 2 & 0.018 \\
7 & c.1397C>G(p.Ser466X; S466X & 2 & 0.018 \\
& (TAG)) & & \\
8 & c.1399C>T (p.Leu467Phe; L467F) & 2 & 0.018 \\
9 & c.3209G>A(p.Arg1070Gln; R1070Q) & 2 & 0.018
\end{tabular}

\begin{tabular}{llll}
\hline Mutant alleles list & & \\
\hline № $\quad$ CFTR alleles (HGVS; legacy) & $\begin{array}{l}\text { Number } \\
(\mathrm{n}=110)\end{array}$ & Frequency \\
\hline $\begin{array}{l}10- \\
36\end{array}$ & rare (published elsewhere) & 25 & 0.229 (sum) \\
\hline
\end{tabular}

M. Donnikov: None. V. Mescheryakov: None. N. Satsuk: None. A. Vorobyov: None. L. Kolbasin: None. I. Urvantseva: None. O. Glotov: None. A. Glotov: None. L. Kovalenko: None.

\section{E-P16.07}

Comparison of DNA extraction methods for samples from old blood collections

\section{S. Samut Tagliaferro, A. Zejnelagic, R. Farrugia,}

S. Bezzina Wettinger

\section{University of Malta, Msida, Malta}

Introduction: Limited information is available on which methods are suitable to extract DNA from 20-year-old blood samples and samples kept in non-ideal storage conditions. The aims of this study were to assess if DNA can be extracted from whole blood collected and stored at $-20{ }^{\circ} \mathrm{C}$ for 7-20 years, and to identify which commercial DNA extraction kit is most suitable for this purpose, yielding good quality DNA.

Materials and methods: Nine whole blood samples collected in 1999, 2001 and 2012, all having low (between $100 \mu \mathrm{L}$ to $200 \mu \mathrm{L}$ ) blood volumes, were used in this study. DNA extraction was performed using three different DNA extraction kits, according to their respective instructions.

Results: The Quick-DNA Miniprep Plus kit produced the highest DNA yield ranging from $0.51-20.2 \mu \mathrm{g}$, followed by the DNeasy Blood and Tissue kit which gave DNA yields ranging between $0.30 \mu \mathrm{g}$ and $2.92 \mu \mathrm{g}$. In contrast, the MagAttract HMW DNA kit was less efficient at extracting DNA from these samples, with DNA yields ranging between 0.16 and $0.80 \mu \mathrm{g}$. The average yield was not affected by the age of the sample.

Conclusion: From the results obtained, it can be concluded that good quality DNA can be extracted from whole blood samples biobanked for 18 to 20 years at $-20{ }^{\circ} \mathrm{C}$. This study was limited by the small number of samples. DNA concentrations were not affected by the number of years the blood sample was stored, but were affected by the type of kit used to extract the DNA.

S. Samut Tagliaferro: None. A. Zejnelagic: None. R. Farrugia: None. S. Bezzina Wettinger: None. 


\section{E-P16.08}

Low density oligonucleotide microarray for the detection of ethnospecific mutations causing five genetic disorders prevalent among the Yakut population

\section{Savvina, N. Maksimova, A. Sukhomyasova}

North-Eastern federal university, Yakutsk, Russian Federation

In yakut population of republic of Sakha (Yakutia) serious genetic disorders occur with relatively high frequency. There is a pressing need for comprehensive carrier screening and molecular diagnostic, but methods of genetic diagnostics that commonly used today are not able to afford it due to the high cost and time consumption for a single test. For such purposes, we developed an ethnicity-specific assay based on DNA microarray. Microarray is designed to address most prevalent mutations among yakut population: 4582_4583insT in CUL7 gene, c.5741G >A in NBAS gene, c.806C $>\mathrm{T}$ in DIA1 gene, c. $1090 \mathrm{G}>\mathrm{C}$ in $F A H$ gene, c. $-23+1 \mathrm{G}>\mathrm{A}$ in $G B J 2$ gene causing 3-M syndrome, SOPH-syndrome, Tyrosinemia type 1 , Methaemoglobinaemia type 1 , Nonsyndromic hearing loss and deafness (DFNB1) type 1A respectively. Oligonucleotide probes printed onto aldehyde group-coated glass slides using non-contact microarrayer. The assay based on reverse hybridization, which include two-step multiplex PCR reactions with production of one-stranded Cy5 labeled-PCR products following its hybridization with oligonucleotide probes. Using this microarray, we estimated heterozygose carrier frequency in 120 healthy people of yakut ethnicity and validated data by real-time PCR and PCR-RLFP methods. The evaluation of this microarray demonstrates that it is comprehensive, robust, sensitive, specific, and easily modifiable. The developed assay can be considered as an alternative cost-effective tool for genetic screening purposes and it is suitable for both carrier screening and disease diagnostics in yakut population in the republic of Sakha (Yakutia). The research is conducted under the state target program: project FSRG-2020-0014 "Genomic of Arctic: epidemiology, hereditary and pathology"

M. Savvina: None. N. Maksimova: None. A. Sukhomyasova: None.

\section{E-P16.09}

Lessons from 14 years of french cytogenetic EQA concerning clinical genetics

M. Doco-Fenzy ${ }^{1}$, D. Sanlaville ${ }^{2}$, C. Missirian ${ }^{3}$, I. Luquet ${ }^{4}$, c. Terre ${ }^{5}$, C. Schluth-Bolard ${ }^{2}$, M. Till ${ }^{2}$, C. Sarrauste de Menthières $^{6}$, J. Dupont ${ }^{7}$

${ }^{1}$ Service de Génétique, CHU-Reims, Reims, France, ${ }^{2}$ Service de Génétique, CHU-Lyon Bron, Lyon, France, ${ }^{3}$ Service de Génétique, CHU la Timone, Marseille, France, ${ }^{4}$ Service d'hématologie, CHU-Toulouse, Toulouse, France, ${ }^{5}$ Service de Génétique, CHU-Versailles, Versailles, France, ${ }^{6}$ Génétique, IGH, Montpellier, Montpellier, France, ${ }^{7}$ Service de Génétique, chu Cochin, Paris, France

The ACLF provides laboratories since 2005 with external quality assessments for the caryotype (including FISH) and array-CGH. More than 70 laboratories in constitutional cytogenetics, hematology and array-CGH participate each year. The evolution of our practices requires continuously adaptation but these EQAs and their European equivalents have made us aware of the state of knowledge and certain limitations reveal the loss of competence in classical cytogenetic analysis as well as of molecular pangenomic techniques. Indeed we have identified technical gaps or pitfalls that we will illustrate with 2 examples. Postnatal Cytogenetics: We proposed a pericentric chromosome 5 inversion inv $(5)(\mathrm{p} 13 \mathrm{q} 13)$ considered a variant in the literature. A large discrepancy was observed between laboratories reporting this chromosome a polymorphism and others reasoning as a classic pericentric inversion. The "youngest" cytogeneticists have lost experience regarding a variant not recently reported in the literature. Postnatal Array-CGH: The second proposed case involved a caryotype-validated pentasomy X. Surprisingy some laboratories missed the pentasomy. They concluded a trisomy or tetrasomy mosaicism. The explanation may lie in the limitations of the assessment of the copy number by algorithms used in the presence of unusual ratios especially at the level of gonosomes, a too fast analysis or a technique that did not allow to evaluate a large number of chromosome $\mathrm{X}$ copies. This exercise allowed us to observe the limitations of certain computational algorithms and remind us of the importance of maintaining a solid formation in classical cytogenetic analysis to best interpret the results from pangenomic molecular techniques (array-CGH, WGS/WES).

M. Doco-Fenzy: None. D. Sanlaville: None. C. Missirian: None. I. Luquet: None. C. Terre: None. C. Schluth-Bolard: None. M. Till: None. C. Sarrauste de Menthières: None. J. Dupont: None.

\section{E-P16.10}

Next-generation sequencing for the diagnosis of Spanish patients affected by congenital fibrinogen defects

I. Tirado, L. Romero, N. Vilalta, M. Carrasco, J. Souto, E. Martínez-Sánchez, V. Criado, M. Simón, L. Alías,

S. Quero, J. Mateo

Unitat d'Hemostàsia $i$ Trombosi, Servei d'Hematologia. Hospital de la Santa Creu i Sant Pau, Barcelona, Spain 
Introduction: The aim of our study was to characterize patients with fibrinogen (Fbg) deficiencies by analyzing $F G A, \quad F G B$ and $F G G$ genes using next generation sequencing (NGS).

Methods: Functional and immunologic Fbg levels were determined with Clauss $\mathrm{Fib}^{\mathrm{R}}$ (Stago) and NOR-Partigen Fibrinogen (Siemens), respectively. A custom Nonacus v1.2.1 Cell $3^{\mathrm{TM}}$ Target that includes the $F G A, F G B$ and $F G G$ genes was performed on a MiSeq instrument (Ilumina). All variants were confirmed by Sanger sequencing using an Applied Biosystems 3500.

Results: Genetic and phenotypic findings are summarized in Table 1.

\begin{tabular}{|c|c|c|c|}
\hline $\begin{array}{l}\text { Patient / Fbg } \\
\text { deficiency }\end{array}$ & Clinical & $\begin{array}{l}\text { Functional/ } \\
\text { antigen } \\
\text { Fbg g/L }\end{array}$ & NGS /Sanger \\
\hline 1Afibrinogenemia & $\begin{array}{l}\text { Intracerebral } \\
\text { haemorrhageMesenteric } \\
\text { thrombosis }\end{array}$ & $<0.0 / 0.0$ & $\begin{array}{l}\text { Homozygous } F G A \\
\text { c. } 532 \mathrm{C}>\mathrm{T} \text { p.R } 178 * \mathrm{New} \\
\text { stop codon }\end{array}$ \\
\hline $\begin{array}{l}\text { Son of the } \\
\text { patient } 1\end{array}$ & Asymptomatic & $2.4 / 2.6$ & $\begin{array}{l}\text { Heterozygous } F G A \\
\text { c. } 532 \mathrm{C}>\mathrm{T} \text { p.R } 178^{*} \\
\text { New stop codon }\end{array}$ \\
\hline $\begin{array}{l}2 \\
\text { Dysfibrinogenemia }\end{array}$ & Asymptomatic & $1.8 / 4.8$ & $\begin{array}{l}\text { Heterozygous } F G A \\
\text { c. } 112 \mathrm{~A}>\mathrm{G} \text { p.R38G } \\
\text { (Fibrinogen Aarhus. } \\
\text { Ref. 1) }\end{array}$ \\
\hline $\begin{array}{l}3 \\
\text { Dysfibrinogenemia }\end{array}$ & $\begin{array}{l}\text { Postpartum hemorrhagic } \\
\text { diathesis.Femoral } \\
\text { thrombosis }\end{array}$ & $<0.1 / 7.2$ & $\begin{array}{l}\text { Homozygous } F G A \\
\text { c. } 112 \mathrm{~A}>\mathrm{G} \text { p.R38G } \\
\text { (Fibrinogen Aarhus) }\end{array}$ \\
\hline
\end{tabular}

Table 1. Summary of patient data. FGA reference is NM_000508.3.

Conclusions: Two different variants have been found in the $F G A$ gene. NGS has been proven to be a useful tool for simultaneously sequencing all the genes involved in the fibrinogen deficiencies. Reference 1. Blomback, B et al. Biochemistry, Biological Functions, Gene Regulation and Expression. Amsterdam: Elsevier (pub.) 1988. Pp. 263-266. Funding: FIS PI18/01492.

I. Tirado: None. L. Romero: None. N. Vilalta: None. M. Carrasco: None. J. Souto: None. E. Martínez-Sánchez: None. V. Criado: None. M. Simón: None. L. Alías: None. S. Quero: None. J. Mateo: None.

\section{E-P16.11}

Extraction of genomic DNA from stabilized saliva using selected QIAGEN purification systems

\section{Lowe, L. Erkelenz, M. Schmitz}

\section{QIAGEN GmbH, Hilden, Germany}

Introduction: Saliva is increasingly used as a noninvasive alternative to blood for the examination of human DNA in ancestry testing or disease monitoring. Sample collection does not require trained professionals or dedicated facilities. The preanalytical workflow should minimize DNA degradation and fragmentation. This study demonstrates the compatibility of QIAGEN gDNA sample preparation kits with different saliva collection devices, as part of a workflow for extraction of high-quality, intact salivary gDNA.

Methods: Saliva was collected from 10 healthy individuals using six different collection devices, according to manufacturer's collection instructions. gDNA was extracted using either QIAamp technology (manual) or the QIAsymphony instrument (automated) and quality and quantity were spectrophotometrically assessed. A qPCR assay targeting human $\beta$-Actin and the QIAGEN Investigator Quantiplex Pro RGQ Kit, a multiplex gene assay, were used to evaluate compatibility with qPCR. The Agilent TapeStation 4200 was used for fragmentation analysis.

Results: High donor-to-donor variability was observed, but there was no significant difference in total yield between the saliva collection devices used. DNA purity was assessed by $A_{260} / A_{280}$ ratio, showing good sample quality with both extraction methods. Intact gDNA was recovered and showed low fragmentation of high-molecular-weight gDNA. Samples analyzed demonstrated consistent and stable performance in qPCR applications, showing no inhibition.

Conclusions: QIAGEN gDNA sample preparation kits are compatible with various saliva collection devices. The recovered gDNA is intact high-molecular-weight DNA and can be used in amplification-based qPCR assays.

C. Lowe: A. Employment (full or part-time); Modest; QIAGEN GmbH. L. Erkelenz: A. Employment (full or part-time); Modest; QIAGEN GmbH. M. Schmitz: A. Employment (full or part-time); Modest; QIAGEN GmbH.

\section{E-P16.12}

Approbation of the molecular genetic method for the diagnosis of Helicobacter pylori infection isolated from feces of patients with gastroduodenal diseases from individuals of Sakha Republic

\section{N. Gotovtsev ${ }^{1,2}$, N. Barashkov ${ }^{1,2}$, T. Borisova ${ }^{l}$, M. Pak ${ }^{1,3}$, M. Alekseeva ${ }^{3}, N$. Innokentieva ${ }^{l, 3}, K$. Loskutova ${ }^{1,3}$, V. Pshennikova ${ }^{1,2}$, S. Lekhanova ${ }^{l}$, S. Fedorova ${ }^{l}$ \\ ${ }^{1}$ M.K. Ammosov North-Eastern Federal University, Yakutsk, Russian Federation, ${ }^{2}$ Yakut Science Center of Complex Medical Problems, Yakutsk, Russian Federation, ${ }^{3}$ Republican hospital №1 - National Center of Medicine, Yakutsk, Russian Federation}

The International Agency for Research on Cancer in 1994 classified Helicobacter pylori $(\mathrm{Hp})$ infection to the I group of carcinogen, along with some of the radionuclides and 
radiation. The diagnosis of $H p$ infection includes molecular genetic research methods by PCR, based on amplification of $16 S$ rRNA because it's a quite specific and excludes the possibility of amplification of related species and strains closest to Hp (Campylobacter, Helicobacter cinaedi, Helicobacter mustelae and Wolinella succinogenes). Detection of $\mathrm{Hp}$ infection by PCR methods was not performed earlier in the Sakha Republic. Our aim was approbation of the molecular genetic method for the diagnosis of $H p$ infection isolated from feces of patients with gastroduodenal diseases from individuals of Sakha Republic. We studied 92 Yakut patients with gastroduodenal diseases confirmed by endoscopic and histological study. Two patients had $H p$ with high degree of dissemination. The urea breath test was positive because the results of determining the presence of $H p$ and showed high concentration of ammonia (high degree of dissemination). Subsequently, samples of evacuated feces were obtained from these two patients. Positive amplification of the $16 S$ rRNA marker gene was obtained from one of two examined patients. We were able to show that using the proposed method it is possible to determine the presence of $H p$ DNA in feces, which allows to pass endoscopic examination, in cases where there are contraindications to invasive research methods. This study was supported by Ministry of Science and Higher Education of Russian Federation (basic part to NEFU) and RFBR-grant \#18-05-600035_Arctika.

N. Gotovtsev: None. N. Barashkov: None. T. Borisova: None. M. Pak: None. M. Alekseeva: None. N. Innokentieva: None. K. Loskutova: None. V. Pshennikova: None. S. Lekhanova: None. S. Fedorova: None.

\section{E-P16.14}

Overcoming pitfalls in the genetic diagnosis of the Koolen-De Vries syndrome.

N. Ordonez-Herrera, J. Numrich, D. Diego Alvarez,

L. Ramirez, K. Kandaswamy, N. Ameziane, B. Peter, A. M. Bertoli-Avella

\section{Centogene AG, Rostock, Germany}

Normal 0 false false false DE X-NONE X-NONE /* Style Definitions */ table.MsoNormalTable \{mso-style-name:"Table Normal"; mso-tstyle-rowband-size:0; mso-tstyle-colband-size:0; mso-style-noshow:yes; mso-style-priority:99; mso-style-parent:""; mso-padding-alt:0in 5.4pt 0in 5.4pt; mso-para-margin-top:0in; mso-para-margin-right:0in; msopara-margin-bottom:10.0pt; mso-para-margin-left:0in; lineheight:115\%; mso-pagination:widow-orphan; fontsize:11.0pt; font-family:"Calibri", sans-serif; mso-asciifont-family:Calibri; mso-ascii-theme-font:minor-latin; mso-
hansi-font-family:Calibri; mso-hansi-theme-font:minorlatin; mso-bidi-font-family:"Times New Roman"; mso-biditheme-font:minor-bidi; mso-ansi-language:DE;\} Heterozygous single-nucleotide variants and large deletions disrupting the KANSL1 gene cause Koolen-De Vries syndrome (OMIM $^{\oplus}$ 610443). The KANSL1 gene is located on the $17 \mathrm{q} 21.31$ locus, which has been subjected to structural divergence in humans. The common structural $17 \mathrm{q} 21.31$ haplotypes diverge mainly in the direction of the inversion and the copy-number state of KANSL1, affecting exons 1 to 3 of the gene. The architecture of this genomic region signifies a challenge for the clinical interpretation of KANSL1 variants as reported by Koolen et al in 2016. We present several cases illustrating strategies to ascertain the putative clinical significance of single-nucleotide variants located within exons 1-3 of KANSL1 (NM_001193466.1). These cases corroborate that partial polymorphic duplications of the $17 \mathrm{q} 21.31$ region confound the clinical relevance of the KANSL1 variants. Consequently, the same pathogenic variant can be causative in some individuals, while clinically irrelevant in others. Hence, parental testing demonstrating the de novo state of the variant in index patients is critical evidence supporting its pathogenicity. Importantly, in cases where parental testing is not performed, the variant allele frequencies combined with copy number analysis can be used to disentangle the genomic architecture at the locus and consolidate (or exclude) the diagnosis of the syndrome.

N. Ordonez-Herrera: None. J. Numrich: None. D. Diego Alvarez: None. L. Ramirez: None. K. Kandaswamy: None. N. Ameziane: None. B. Peter: None. A.M. BertoliAvella: None.

\section{E-P16.15}

Incidental findings of Klinefelter's and Turner syndromes by cancer predisposition gene panel analysis

L. Golmard ${ }^{l}$, M. Schwartz ${ }^{l}$, M. Blanluet ${ }^{l}$, J. Le Gall ${ }^{1}$,

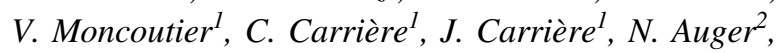
D. Stoppa-Lyonnet ${ }^{1,3}$, C. Colas $^{4}$, M. Bahuau ${ }^{5}$, E. Mouret-Fourme ${ }^{4}$

${ }^{1}$ Institut Curie, PSL Research University, Service de Génétique, Paris, France, ${ }^{2}$ Gustave Roussy, Service de Pathologie moléculaire, Villejuif, France, ${ }^{3}$ Université de Paris, Paris, France, ${ }^{4}$ Institut Curie, PSL Research University, Service de Génétique, St Cloud, France, ${ }^{5}$ Hôpital Henri Mondor, Service de Génétique médicale, Créteil, France

Klinefelter's syndrome (KS) and Turner syndrome (TS) are chromosome $\mathrm{X}$ aneuploidies, with an additional chromosome $\mathrm{X}$ in men affected with $\mathrm{KS}(47, \mathrm{XXY})$ and a missing chromosome $\mathrm{X}$ in women affected with TS $(45, \mathrm{X})$. KS and 
TS prevalence is estimated to be $1 / 600$ and $1 / 2500$, respectively, but their diagnosis often remains unknown. Here we report two incidental findings of KS and TS by cancer predisposition gene panel analysis on 3,460 patients. KS case was a man diagnosed with breast cancer at 67 years of age. TS case was a woman with personal (age 54) and family history of breast cancer. For both cases, breast cancer gene panel analysis did not detect any pathogenic variant but chromosome $\mathrm{X}$ control probes showed abnormal results: presence of two chromosomes $\mathrm{X}$ for the man and one chromosome $\mathrm{X}$ for the woman. Subsequent MLPA analysis suggested the presence of two chromosomes $\mathrm{X}$ and one chromosome $\mathrm{Y}$ for the man, and one chromosome $\mathrm{X}$ and no chromosome $\mathrm{Y}$ for the woman. Karyotype analysis could be performed only for the woman and confirmed the diagnosis of TS, with 45,X chromosomal content. Chromosome X control probes are used to detect errors of patient identity during sampling or experiment. Our report shows that they also allow the identification of sex chromosome aneuploidies for genetic testing performed for other disorders. It is important to inform biological and clinical geneticists about these incidental findings to perform additional analysis after detection of abnormal chromosome $\mathrm{X}$ number and to better anticipate patient specific follow-up.

L. Golmard: None. M. Schwartz: None. M. Blanluet: None. J. Le Gall: None. V. Moncoutier: None. C. Carrière: None. J. Carrière: None. N. Auger: None. D. StoppaLyonnet: None. C. Colas: None. M. Bahuau: None. E. Mouret-Fourme: None.

\section{E-P16.16}

Genetic analysis of mucopolysaccharidosis-plus syndrome (MPSPS) in the Republic of Sakha (Yakutia)

\section{S. Novgorodova}

\section{Genomic medicine, Yakutsk, Russian Federation}

Mucopolysaccharidosis is a group of hereditary metabolic diseases connected with the disturbance of glycosaminoglycan metabolism. The Yakut population according to the data given by a number of researchers is a homogenous population, and it has accumulation of about a dozen diseases of monogenous nature. A new hereditary disease with an autosomal recessive type of inheritance, belonging to the group of lysosomal diseases, with a mutation in the VPS33A gene called MPS plus (OMIM \# 617303), which occurs in children of Yakut nationality, leading to early infant mortality [Kondo et al., 2017]. We carried out a molecular genetic analysis for the carriage of mutations in the VPS33A gene among the Yakuts (94 cases). The method of express-diagnostics - mucopolysaccharidosis- plus syndrome in the Yakutia using real-time PCR has been developed. Molecular genetic analysis mutations in the VPS33A gene meets the following parameters: analysis time with the use of the developed method makes not more than 3 hours, including sample preparation and evaluation of the research results; The reliability of the analysis results by means of the developed method in the study of the sample $\mathrm{n}=100$ makes not less than 90\%. Molecular genetic analysis of the carriage of a mutation in the VPS33A gene among the Yakuts has proven its effectiveness and the quickness of execution among the targeted patients. 6 heterozygous carriers and 1 homozygous carriage in the VPS33A gene have been revealed. This diagnostic method on MPSPS allows to reduce the analysis time and is promising for patients.

S. Novgorodova: None.

\section{E-P16.18}

Exceptionally late diagnosis of patient with Prader-Willi Syndrome

\section{K. A. Zapart, M. Malarska}

\section{Medical University of Lodz, Łódź, Poland}

Prader-Willi Syndrome is an uncommon imprinting disorder, caused usually by the deletion of the paternal copy in $15 \mathrm{q} 11-\mathrm{q} 13$ region. Average age of patients diagnosed is not older than 3 years of age when syndromes like hipotonia, feeding difficulties and delayed development, including hypogonadism arise. Distinctive facial features in particular almond-shaped eyes, a narrowing of the head at the temples, a turned-down mouth and a thin upper lip are noticed. Nevertheless, the main complications come from sequelae of hyperfagia, thus obesity and intellectual impairment. Still, early diagnosis can prevent most of them.

This study presents a case of 27-year-old man, who was admitted to general practitioner because of memory problems. He had never been to genetics clinic before. Obese patient (BMI:47), with distinctive facial traits, reports learning difficulties and poor short term memory. One-toone tuition during his schooling age was necessary. During his childhood, a psychologist diagnosed him with personality disorders. Although he likes playing video games, he finds it difficult to focus on things. He also reports photophobia and constant hunger. Since all those symptoms were unusual for PWS he was finally referred to a specialist.

Because of nonspecific symptoms and low incidence, the diagnosis presents a challenge to pediatricians and requires skilled eye and experience. Such cases show us that increasing awareness among doctors about genetic conditions is still certainly needed. 
K.A. Zapart: None. M. Malarska: None.

\section{E-P16.20}

Rare no longer means unavailable - new concept of routine genetic diagnostics

K. Hirschfeldova, L. Obeidova, J. Stekrova, M. Urbanova, B. Janosikova, I. Zednikova, V. Elisakova, F. Liska, B. Chylikova, A. Horinek, A. Panczak, O. Seda

First Faculty of Medicine Charles University, Prague 2, Czech Republic

We would like to introduce the new concept of molecular diagnostics of genetic disorders intended for routine clinical laboratories in the Czech Republic. The concept is based on three levels of genetic analysis using the method of next generation sequencing. The first level is represented by a panel of genes called OIDIP (Open Interdisciplinary Diagnostic Panel) designed for routine differential diagnosis of both selected common and rare diseases. However, the provided genetic analysis is much more complex and, if relevant, the Polygenic Risk Score (PRS) calculation can be done on basis of co-analysed SNPs. Moreover, the detection of diverse clinically significant common variants (trombophilic mutations, lactose intolerance, warfarin drug response, etc.) is performed at the background. From the epidemiologic point of view, two genes are included to detect carriers of pathogenic alleles for cystic fibrosis and phenylketonuria to spread knowledge of associated risks within Czech population. The second level of genetic testing is represented by "clinical exome" suited for diagnosis of rare and genetically heterogenous diseases. The last level of genetic testing is featured by whole exome sequencing and is still intended for special cases. Appropriate clinical questionnaires are an integral part of the concept as well as website (www.oidip.cz), which should serve to connect clinical specialists and share the information. The aim is to provide rapid genetic diagnostics of a wide spectrum of even rare pathologies to general public. Supp. by the ESIF Operational programme Prague - Growth Pole of the Czech Rep. Reg. No. CZ.07.1.02/0.0/0.0/17_049/0000828

K. Hirschfeldova: None. L. Obeidova: None. J. Stekrova: None. M. Urbanova: None. B. Janosikova: None. I. Zednikova: None. V. Elisakova: None. F. Liska: A. Employment (full or part-time); Modest; GENvia, s.r.o.. B. Chylikova: None. A. Horinek: None. A. Panczak: None. O. Seda: None.

\section{E-P16.22}

Importance of Low and Moderate impact variants in whole exome sequencing (WES) analysis
R. Tripathy, Z. Yüksel, M. Drasdo

Bioscientia, Ingelheim, Germany

Introduction: Whole exome sequencing (WES) has become an indispensable tool in the field of genetic diagnostics. While we have observed an enormous rise in technical and medical standards of WES data analysis, there remain many limitations in the interpretation of the identified variants. Currently applied analytical strategies rely heavily on assumptions of variant filtering such as the commonly used parameter of variant type (e.g. missense, frameshift, canonical splice-site). The variants then undergo a subjective classification of the severity of consequence based on SNPEff: HIGH, MODERATE, LOW and MODIFIER. In this study, we concentrated on the pathogenicity of variants that are often declared to have low deleterious effects and hence overlooked during patient WES data analysis: synonymous (LOW), splice region (LOW) and intron (MODIFIER) variants according to VEP annotation in order to understand the pathogenicity of such variants.

Materials and methods: We determined only very rare synonymous, splice region and intron variants in patients in which WES was performed. We concentrated on those variants which showed significant overlap with the given patient phenotype. We studied the predicted effect of such variants on splicing using reverse transcriptase PCR (RTPCR).

Results: We identified several synonymous, splice region and intron variants that are usually underestimated by conventional filtering and/or annotation methods.

Conclusion: It is important to build WES variant filtering strategies based on the phenotypic overlap before concentrating on the uncovered regions or other genetic mechanisms. The diagnostic yield is increased with such filtering and conformational strategies such as RT-PCR.

R. Tripathy: A. Employment (full or part-time); Significant; Bioscientia GmbH. Z. Yüksel: A. Employment (full or part-time); Significant; Bioscientia GmbH. M. Drasdo: A. Employment (full or part-time); Significant; Bioscientia GmbH.

\section{E-P17 Bioinformatics and Statistical Methods}

\section{E-P17.04}

A GWAS of Listeria monocytogenes strains of food and clinical origins 
F. Laporte ${ }^{1}$, M. Maury ${ }^{2}$, E. Patin ${ }^{3}$, A. Leclercq ${ }^{2}$, L. Quintana-Murci ${ }^{3}$, R. Chikhi ${ }^{1}$, M. Lecuit ${ }^{2}$, H. Aschard ${ }^{1}$

${ }^{1}$ Department of Computational Biology-USR 3756 CNRS, Institut Pasteur, Paris, France, ${ }^{2}$ Biology of Infection Unit, National Reference Center and WHO Collaborating Center Listeria, Institut Pasteur, Paris, France, ${ }^{3}$ Human Evolutionary Genetics, Institut Pasteur, Paris, France

Introduction: Genome-wide Association Studies (GWAS) have been central to identify genetic variations associated with complex human phenotypes. There is now tremendous interest for implementing GWAS-like approaches to genomes of pathogenic bacteria in order to advance our understanding of infectious disease risk and underlying bacterial virulence factors. However, bacterial genomes harbour complex structure and long-range linkage disequilibrium (LD), making such analyses extremely challenging. We aim at identifying genetic variation in Listeria monocytogenes associated with food versus clinical sources using whole genome sequencing of 3718 strains of food and clinical origins collected in the context of Listeria surveillance in France.

Materials and methods: We used both real and simulated data to assess the performances of various GWAS approaches. First, we considered SNPs derived from the core genome, and unitigs obtained by compacting the de Bruijn graph. Second, we compared alternative ad hoc algorithms for deriving pairwise genetic relatedness between strains. Third, we assessed a range of logistic and linear mixed models (LMM) with various random effect. And fourth, we assessed solutions to account for the observed extensive LD.

Results and conclusion: We identified multiple critical issues in existing approaches, resulting in invalid GWAS results in our data. We developed solutions for each of those issues, implementing an adaptive inference of relatedness between strains, a stepwise LMM model selection, and a genome LD correction inspired from human GWAS. The developed and validated approach shows promising results in the actual set of Listeria collected prospectively, pointing multiple variations associated with food versus clinic origins.

F. Laporte: None. M. Maury: None. E. Patin: None. A. Leclercq: None. L. Quintana-Murci: None. R. Chikhi: None. M. Lecuit: None. H. Aschard: None.

\section{E-P17.05}

A benchmarking of germline small-variant calling from whole human genome and exome sequencing data

D. Jáspez ${ }^{l}$, A. Muñoz-Barrera ${ }^{l}$, L. A. Rubio-Rodríguez, ${ }^{l}$ A. Ínigo-Campos $^{l}$, V. Garcia-Olivares ${ }^{l}$,
A. Díaz-de Usera ${ }^{l}$, R. González-Montelongo ${ }^{l}$, J. M. Lorenzo-Salazar ${ }^{1}$, C. Flores ${ }^{1,2,3}$

${ }^{I}$ Genomics Division, Instituto Tecnológico y de Energías Renovables (ITER), Santa Cruz de Tenerife, Spain, ${ }^{2}$ Research Unit, Hospital Universitario N.S. de Candelaria, Universidad de La Laguna, Santa Cruz de Tenerife, Spain, ${ }^{3}$ CIBER de Enfermedades Respiratorias, Instituto de Salud Carlos III, Madrid, Spain

Introduction: Bioinformatics benchmarking is essential to provide guidance for improving the variant calling process and define standards and performance metrics. Following the GA4GH recommendations and the PrecisionFDA challenges, we aimed to assess the performance of our current whole-genome (WGS) and whole-exome (WES) sequencing and bioinformatic workflows for detecting single nucleotide variants (SNVs) and small insertion and deletion (indels) in human samples.

Materials and methods: We used the NIST-GIAB pilot sample NA12878/HG001 WGS provided by the PrecisionFDA, and WES sequences with PE75 at $>50 \mathrm{X}$ obtained on a HiSeq4000 (Illumina) in our laboratory, both used as internal references. Variants were called with the HaplotypeCaller following the Genome Analysis Toolkit Best Practices workflow recommendations (GATK v3.8 and v4.1.4). A high confidence set of variants accounting for read depth, and variant calling and mapping quality was compared against the GIAB gold standard.

Results: A relatively high accuracy was observed according to recall, precision and F-score metrics from the benchmark of WGS (>97.9\%) and WES $(>87.5 \%)$, with minor differences among SNVs and indels. The lower observed WES accuracy might be related to the nonuniform coverage found in WES enrichment when comparing to synthetic-WES reads from HG001.

Conclusions: We have developed and tested a custom pipeline to conduct WGS and WES-based benchmarking to ensure reproducibility. These results support that our current procedures and workflows are reliable albeit a fine-grained study of inaccuracies is needed.

Funding: Ministerio de Ciencia e Innovación (RTC2017-6471-1; MINECO/AEI/FEDER, UE); ITER agreement OA17/008. Ministerio de Educación y Formación Profesional (A.D.U.; FPU16/01435).

D. Jáspez: None. A. Muñoz-Barrera: None. L.A. RubioRodríguez: None. A. Ínigo-Campos: None. V. GarciaOlivares: None. A. Díaz-de Usera: None. R. GonzálezMontelongo: None. J.M. Lorenzo-Salazar: None. C. Flores: None. 


\section{E-P17.06}

Mining the MGI resource to unravel genes and molecular mechanisms driving ciliary dysfunction: lessons from ciliopathy mouse models

\section{A. V. Anagnostopoulos, M. Tomczuk, J. E. Richardson, C. L. Smith, C. J. Bult, The Mouse Genome Informatics Staff and Software Team}

\section{The Jackson Laboratory, Bar Harbor, ME, United States}

Ciliopathies comprise a broad spectrum of genetically and phenotypically heterogeneous disorders arising from mutations that disrupt ciliary structure or function, and ranging from mild single-organ pathologies to lethal multi-systemic syndromes. Phenotypic manifestations can include respiratory dysfunction, infertility, brain malformations, hydrocephalus, heterotaxia, polydactyly, renal anomalies, neurosensory deficits, and cognitive impairment. Moreover, the clinical features of syndromic ciliopathies are often variable and overlapping, depending on the causal gene, mutation type or site, and/or other genetic modifiers of penetrance or expressivity, rendering diagnosis challenging.

Mice present a premier model system to dissect the functional roles of motile and non-motile cilia in diverse tissue/cell types and developmental stages, discover new ciliopathy clinical entities and/or contributing loci, and evaluate therapeutic strategies. Mouse Genome Informatics (MGI, http://www.informatics.jax.org) integrates data from multiple sources to correlate mouse phenotypes and human disease symptoms and inform disease pathogenesis and treatment. Here, we employ MGI data mining tools to: explore an array of mouse mutations, alleles and phenotypes associated with ciliary dysfunction; assess phenotypic outcome in different genetic backgrounds; find experimental mouse models of known ciliopathies (e.g., Joubert syndrome); and delineate candidate genes or pathways associated with core ciliopathy features. We present enrichment analysis of 239 candidate ciliopathy genes and, as hydrocephalus (DOID:10908) emerges as the only enriched human condition exclusively associated with 5 genes (Dpcd, Kif27, Nme5, Stk36 and Ulk4), we prioritize Ulk4 for further characterization and corroborate its candidacy based on genomic, homology, expression, functional, pathway, phenotype and disease model data available at MGI. Supported by NIH grant HG000330.

A.V. Anagnostopoulos: None. M. Tomczuk: None. J.E. Richardson: None. C.L. Smith: None. C.J. Bult: None.

\section{E-P17.07}

Using AI for an automated CNV Classification based on the new ACMG/ClinGen recommendations

\section{Y. Einhorn}

Genoox, Tel-Aviv, Israel

Introduction: The ACMG and ClinGen recently published new technical standards for CNV classification in order to replace the old guidelines. These new standards are highly detailed and structured compared with the previous guidelines. Thus, while the detailed evaluation criteria will increase classification consistency between different labs, it can be time consuming and hold some computational challenges. Here, we present a novel AI-based variant classification engine (aiVCE) for CNV classification based on the new ACMG/ClinGen framework.

Methods: An aiVCE was developed for implementing the new recommendations and removing their computational and technical challenges. As no gold standard dataset with category level evidence exists for classified CNVs, to evaluate the aiVCE we benchmark against the dataset which was used for the recommendations creation where each CNV was evaluated by two different clinical labs.

Results: Comparing the results. shows high concordance without any strong conflict. Looking on the variants that both reviewer classified the same, $100 \%$ of the $\mathrm{P}$ variants were classified as P or VUS-Leaning-Pathogenic (VUS-P), $100 \%$ of the VUS variants were classified as VUS,VUS-P or VUS-Leaning-Benign (VUS-B)and $71 \%$ of the B variants were classified as B/VUS-B and the remaining (29\%) as VUS.

Conclusions: Herein we demonstrated how an AI-based algorithm can aid in following the new CNV classification recommendations, a task which is challenging, time consuming and error prone.

Table. Benchmarking an aiVCE for CNV classification against labs' classification

\begin{tabular}{llllll}
\hline labslaiVCE & B & VUS-B & VUS & VUS-P & P \\
\hline B & $\mathbf{1}$ & $\mathbf{4}$ & 2 & 0 & 0 \\
B/VUS & 0 & 0 & $\mathbf{5}$ & 0 & 0 \\
VUS & 0 & $\mathbf{2}$ & $\mathbf{3 9}$ & $\mathbf{6}$ & 0 \\
VUS/P & 0 & 0 & $\mathbf{8}$ & $\mathbf{9}$ & $\mathbf{4}$ \\
P & 0 & 0 & 0 & $\mathbf{6}$ & $\mathbf{1 7}$ \\
B/P & 0 & 0 & 1 & 1 & 0 \\
\hline
\end{tabular}

Y. Einhorn: A. Employment (full or part-time); Significant; Genoox. 


\section{E-P17.08}

CNVariome digitalized: a new approach towards CNV prioritization ( $\mathrm{CNV}$ laundering) for uncovering mechanisms of brain disorders

\author{
M. A. Zelenova ${ }^{1,2}$, Y. B. Yurov ${ }^{1,2}$, S. G. Vorsanova ${ }^{1,2}$, \\ I. Y. Iourov $^{1,2,3}$
}

${ }^{1}$ Mental Health Research Center, Moscow, Russian Federation, ${ }^{2}$ Academician Yu.E. Veltishchev Research Clinical Institute of Pediatrics, N.I. Pirogov Russian National Research Medical University, Ministry of Health of the Russian Federation, Moscow, Russian Federation, ${ }^{3}$ Russian Medical Academy of Continuous Professional Education of the Ministry of Health of the Russian Federation, Moscow, Russian Federation

Introduction: Brain disorders are constantly associated with copy number variations (CNVs). Increase in $\mathrm{CNV}$ burden is common in psychiatric disorders, leading to disruptions of shared biological processes. However, there is a lack of approaches used for uncovering pathogenic pathways/CNVs in brain diseases using genomic data.

Materials and methods: Here, we propose an algorithm - "CNV laundering" - for CNV/pathway prioritization in brain disorders. Algorithm's stages are (1) CNV data is obtained and checked for recurrence (recurrent CNVs excluded); (2) ontology and expression of CNV genes obtained (genes not expressed in the brain excluded); (3) gene-gene interactions are retrieved; (4) candidate pathways obtained by analyzing the set of interacting genes; (5) pathway prioritization and clusterization using a novel prioritization criterion (number of CNV genes in a pathway/ total number of pathway genes). Using the algorithm and Affymetrix CytoScan HD microarray, we analyzed 191 genomes of children with neurodevelopmental disorders and congenital abnormalities (patient inclusion criteria: absence of CNVs larger than $500 \mathrm{kbp}$ ).

Results: The most significant pathway clusters were determined as "proteasome", "neurodegenerative diseases", "regulation of TP53", "vesicles functioning", "signaling by NOTCH", "actin functioning".

Conclusions: "CNV data laundering" allows for pathway and $\mathrm{CNV}$ prioritization. The application of the algorithm provides for accumulation of knowledge about the mechanisms of brain disease both in individual cases and in certain disorders. Supported by RFFI and CITMA (project No. 18515-34005).

M.A. Zelenova: None. Y.B. Yurov: None. S.G. Vorsanova: None. I.Y. Iourov: None.

\section{E-P17.10}

New intronic variant at the Splicing site of the LMBR1 gene associated with Laurin-Sandrow syndrome in Colombia.

\section{J. Moreno-Giraldo ${ }^{1,2,3,4}$, M. Escobar-Muñoz ${ }^{1,4}$, D. Arturo-Terranova ${ }^{1,4}$, J. M. Satizábal Soto ${ }^{1,4}$}

${ }^{1}$ Universidad del Valle, Cali, Colombia, ${ }^{2}$ Universidad Santiago de Cali, Santiago de Cali, Colombia, ${ }^{3}$ Universidad Libre, Santiago de Cali, Colombia, ${ }^{4}$ Congenital diseases metabolism research group -Category A Colciencias 2019, Santiago de Cali, Colombia

Laurin-Sandrow Syndrome (LSS) is an autosomal dominant inherited disease of a malformities nature, its incidence does not exceed 16 cases worldwide. It is characterized by a complete polysyndactyly in hands, feet in the form of a mirror image and malformations at the level of the nose such as hypoplasia of the nasal alae and the short columella. To inform a new intronic variant associated with LSS in a Colombian female patient of 2 years and 11 months of age with multiple malformations associated with agenesis of bony parts, a molecular study was conducted using complete exome sequencing and afterward a bioinformatic prediction analysis of the splicing effect through the Human Splicing Finder prediction software. As a result, a variant was found in the LMBR1 gene c.1282-14G>A, Intron 16 Splicing, heterozygosis (69\%); associated with LSS. The bioinformatic study demonstrated the pathogenicity of the variant, which allows a phenotype-genotype association. Our findings inform the first case reported in the literature of a patient with LSS in Colombia. Besides, they suggest that this intronic variant plays a pathogenic role associated with the phenotype of the patient and allows us to conclude that the use of bioinformatics software for the prediction intronic variants are a useful tool to evaluate the clinical significance, promoting its use to provide directed clinical approaches.

L.J. Moreno-Giraldo: None. M. Escobar -Muñoz: None. D. Arturo-Terranova: None. J.M. Satizábal Soto: None.

\section{E-P17.11}

Cross platform bio-informatics data analysis using docker

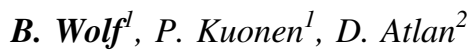

${ }^{I}$ University of applied sciences and arts western switzerland, Fribourg, Switzerland, ${ }^{2}$ Phenosystems SA, Wallonia, Belgium

Introduction: The field of bio-informatics has a big variety of tools to perform all sorts of data analysis. New and exciting tools are released constantly, relying on a variety of 
underlying technologies. While those different tools help to improve not only the quality of the data analysis but help to perform new types of analyses, they are often complicated to install and only run on Linux. Although bigger laboratories often run their analyses on clusters using pre-configured pipelines, this is often not the case in smaller laboratories which rely on windows machines with a limited informatics support.

Methods: We propose to use docker for Windows with WSL 2 to integrate bio-informatics tools into existing analysis tools like GensearchNGS with a minimal overhead. WSL 2 greatly reduces the I/O overhead previous versions of Docker or approaches likes emulation. We show

Results: We released a new version of GensearchNGS which allows users that have Docker installed to use a variety of bio-informatics tools (such as BWA or Bismark), previously only available to linux users.

Conclusions: We show that by using Docker combined with WSL 2, Linux only bio-informatics tools can be run with a minimal overhead on windows and integrated into existing data analysis tools. We demonstrate this by integrating NGS aligners into GensearchNGS, an existing cross-platform NGS data analysis tool. This new approach gives users a big flexibility and greatly reduces the complexity to run some of those tools.

B. Wolf: None. P. Kuonen: None. D. Atlan: A. Employment (full or part-time); Significant; Phenosystems SA.

\section{E-P17.13}

Insight into an artificial neural network, that learned to infer gender from funduscopies

M. A. Ibarra-Arellano ${ }^{l}, S$. Dieck $^{l}$, M. W. Yeung ${ }^{2}$, S. Thiele ${ }^{3}$, T. Pantel ${ }^{4}$, I. Moghul ${ }^{5}$, N. Pontikos ${ }^{5}$, P. Krawitz $^{1}$

${ }^{1}$ Universitätsklinikum Bonn, Bonn, Germany, ${ }^{2} U M C G$ University Groningen, Groningen, Netherlands, ${ }^{3}$ University Hospital Bonn, Bonn, Germany, ${ }^{4}$ Charité - Universitätsmedizin Berlin, Berlin, Germany, ${ }^{5}$ University College London, London, United Kingdom

Deep convolutional neural networks (DCNN) outperform human experts in many medical classification tasks. Yet, due to the "black-box" nature DCNNs remain dismissed in the clinic. Thus, extracting knowledge from artificial intelligence (AI) becomes an important bioinformatic's research topic. We analyzed a DCNN that learned to infer the gender from fundus images with high accuracy. This puzzled ophthalmologists since they haven't found any differential features.

We trained an inceptionv3 DCNN to classify gender in fundus images from UKBiobank. With an AUC of 0.82, we asked ophthalmologists for possible features that could explain this accuracy. We then tested the effect of hypothesis-driven data manipulation on the classification accuracy. 1) To highlight possible morphological features we created occlusion-sensitivity maps. 2) To assess the relevance of cone distribution we split the color channels of the fundus images. 3) We compared the distance between the fovea and the optic disc.

Color channel separation didn't improve the basal performance, discarding the cone distribution hypothesis. Distances between the fovea and the optic disc were similar across gender, based on a t-test ( $p$-value $>0.05$ ). Finally, we compared the performance of different occlusion-sensitivity maps. This allowed us to identify a distinctive vein structure close to the ocular disc in the nasal side of the eye, providing a differential feature to label images.

Image manipulation is a viable method to help interpret DCNN models and extract clinically relevant knowledge. Thus, our study shows that clinical expertise can narrow the feature space and help guide model training. http://www. eye2sex.com/ http://www.fundus2gender.org/

M.A. Ibarra-Arellano: None. S. Dieck: None. M.W. Yeung: None. S. Thiele: None. T. Pantel: None. I. Moghul: None. N. Pontikos: None. P. Krawitz: None.

\section{E-P17.15}

Deciphering the epidemiological, molecular and functional features of Variants of Uncertain Significance in Inherited Retinal dystrophies
I. Iancu, A. Arteche, A. Avila-Fernandez,
M. Del Pozo-Valero, R. Riveiro-Alvarez,
M. Trujillo-Tiebas, I. Perea, M. Corton, P. Minguez, C. Ayuso

Department of Genetics, Instituto de Investigación SanitariaFundación Jiménez Díaz University Hospital-Universidad Autónoma de Madrid (IIS-FJD, UAM), Madrid, Spain

Introduction: Inherited retinal dystrophies (IRD) are a highly heterogeneous group of rare diseases with a molecular diagnosis ratio $>50 \%$. Reclassification of Variants of Uncertain Significance (VUS) represents a challenge in their diagnosis. We aimed to study VUS epidemiological, molecular and functional features that can provide rules to improve VUS clinical management.

Methods: In a two years period, we collected 669 IRD cases studied by clinical exome sequencing and determined their diagnostic status. We identified 152 VUS in 129 unsolved cases, and studied their features in the context of the same cohort causal variants $(\mathrm{N}=379$ cases).

Results: While the type of commercial clinical exome used did not show any difference, the analyst in charge seems to be a source of discrepancy, as one of the most IRD experienced 
clinician showed greater VUS reporting $(\mathrm{p}=1.52 \mathrm{e}-04)$. We found significantly differential patterns for VUS frequency, according to inheritance classification and involved gene, being recessive cases significantly less frequent $(\mathrm{p}=0.01)$, and $A B C A 4$ and IMPG 2 showing the lowest and highest VUS frequency ratio, respectively $(\mathrm{p}=1.05-\mathrm{e} 08 ; \mathrm{p}<0.001)$. Moreover, frameshift and stop-gain variants are barely reported as VUS $(p=6.3 e-07)$. Last, we applied five pathogenicity predictors to VUS and causal variants respectively and extracted the best combination to annotate pathogenicity from the former to be applied to the VUS.

Conclusions: We identified patterns in IRD cases molecular analyses and interpretation related with increased probability to inform them with a VUS. Considering the biases found we have defined a rules-based algorithm to select VUS for further studies.

I. Iancu: None. A. Arteche: None. A. Avila-Fernandez: None. M. Del Pozo-Valero: None. R. Riveiro-Alvarez: None. M. Trujillo-Tiebas: None. I. Perea: None. M. Corton: None. P. Minguez: None. C. Ayuso: None.

\section{E-P17.16}

Validating DeepVariant: Assessment of a deep learning variant caller in Targeted Next Generation Sequencing data

\section{T. Loupis, D. Vrachnos, K. Zoi, D. Thanos, \\ P. Makrythanasis}

Greek Genome Center, Biomedical Research Foundation Academy of Athens (BRFAA), Athens, Greece

Introduction: In 2018, DeepVariant was introduced, a deep convolutional neural network tool which is reported to outperform other variant callers. The algorithm can be implemented in three different type of datasets: 1) Short read Whole Genome Sequencing 2) Short read Whole Exome Sequencing (WES) and 3) PacBio reads, excluding the commonly used Targeted Next Generation Sequencing (TNGS) panels or at least without validating the performance of DeepVariant in such data.

Materials and methods: We created artificial TNGS data, by downscaling WES data from the reference sample NA12878 to simulate an in-house TNGS custom designed gene panel (containing less than 100 genes), targeting clinical significant germline variants. Performed variant calling using DeepVariant's WES model and GATK's Best Practices germline variant calling pipeline (HaplotypeCaller, HC), comparing both results to the high confidence calls of the NA12878.

Results: DeepVariant achieved $100 \%$ accuracy with the artificial TNGS data calling all 51 of the high confidence variants of the NA12878 same as HC. Furthermore, 2 extra variants where called by DeepVariant, one common with $\mathrm{HC}$, both not in the high confidence set.

Conclusions: DeepVariant seems to adapt to targeted gene panel data, suggesting the possible implementation of the algorithm to accurate detect germline variants in human samples. We currently investigate the efficiency of DeepVariant, on a range of gene panels and genomic regions of different features, to accurately detect germline variants in any set of genomic intervals smaller than a WES.

T. Loupis: None. D. Vrachnos: None. K. Zoi: None. D. Thanos: None. P. Makrythanasis: None.

\section{E-P17.19 \\ INTERACTION ANALYSIS of SMAD4 COMPLEXES}

\section{N. Kawashita, A. Itagaki, K. Tamura}

\section{Kindai University, HigashiOsaka, Japan}

SMAD4 mediates this TGF- $\beta$ signaling, is frequently mutated in pancreatic cancer. In addition, SMAD4 mutations have been identified not only in pancreatic cancer but also in other cancers such as colorectal and gastric cancers. The analysis method used the fragment molecular orbital (FMO) method, which can calculate the molecular structure and the interaction between molecules. In this study, we investigated whether the effects of genetic mutations on pathogenicity could be estimated using the FMO method. The structure (1dd1.pdb) of the wild type SMAD4 trimer was obtained from the protein structure data bank (PDB), and the structure was corrected by MOE Structure Preparation. Pathogenic mutations were obtained from ClinVar, and the structure of mutants were constructed by MOE Homology modeling and wild type structure as a template. The computer used supercomputer K (AICS, RIKEN) and FX100 (Nagoya Univ). The calculation was performed at the calculation level of MP2/6-31G* using ABINIT-MP as the software. The sum of inter fragment interaction energies (IFIE-sum) was compared between the wild type and the mutant. Comparing the calculated results of the wild type and the mutant, the interaction energy was destabilized in the mutant such as R361C. R361C mutation destabilized the interaction between D537 and D351. R361, D537 and D351 form a hydrogen bond network during SMAD4 trimer formation. After R361C mutation, this hydrogen bond network could not be formed, and the results of computational analysis showed that this trimer formation was destabilized. It is thought that a similar analysis could predict a pathogenic mutation.

N. Kawashita: None. A. Itagaki: None. K. Tamura: None. 


\section{E-P17.20}

Evaluation of 4 splicing prediction algorithms in a NGS clinical diagnosis context.

\section{J. BURATTI ${ }^{1}$, E. LEJEUNE ${ }^{1}$, P. RICHARD ${ }^{2}$, C. METAY ${ }^{2}$, F. ADER ${ }^{2}$, C. SAINT-MARTIN ${ }^{l}$, C. LEDEUIL ${ }^{2}, M$.

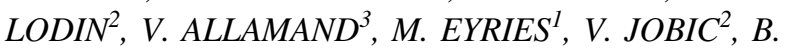 KEREN ${ }^{l}$, A. DE BECDELIEVRE ${ }^{2}$, C. BELLANNE- CHANTELOT ${ }^{l}$}

${ }^{1}$ Département de Génétique, Hôpital Pitié-Salpêtrière, APHP. Sorbonne Université, PARIS, France, ${ }^{2}$ Service de Biochimie Métabolique, Hôpital Pitié-Salpêtrière, AP-HP. Sorbonne Université, PARIS, France, ${ }^{3}$ Inserm UMRS 974, Institut de Myologie, PARIS, France

In molecular genetics, variant interpretation is a critical step in order to make a diagnosis. Presumed splice variants present specific analytical difficulties in clinical practice outside the natural splicing sites (GT and AG dinucleotides).

Laboratory tests to study splicing on RNA are expensive and time consuming, thus it is unrealistic to perform them on the large amount of variants identified by NGS. The use of in silico prediction tools is therefore necessary to quickly select the most convincing splicing variants before sending them to experimental validation. Many tools are available but often based on a web interface and limited to only one request at a time.

Consequently, we have selected 4 algorithms which allow automatic annotation of variants and simple integration into bioinformatics pipelines: MaxEntScan (2004), dbscSNV_ada (2014), SpliceAI (2019) and SPiP (2019). To assess the performance of these tools, we collected 47 variants suspected of altering splicing which were tested experimentally at the Pitié-Salpêtrière Hospital, Paris. 33 variants out of 47 have a demonstrated effect on RNA, 14 have not. We compared the 4 scores (percentage) with the real known effects, considering a predicted effect threshold at $15 \%$.

\begin{tabular}{lllll}
\hline 147 variants & MaxEntScan & dbscSNV & SpliceAI & SPiP \\
\hline No annotation & 13 & 19 & 2 & 1 \\
Consitstent prediction & 28 & 23 & 40 & 35 \\
Unconsistent prediction & 6 & 5 & 5 & 11 \\
\hline
\end{tabular}

In conclusion, there is no perfect prediction tool. However, we have shown that the 2 most recent algorithms offer better annotation and better performance on predicting the variant's impact on splicing.

J. Buratti: None. E. Lejeune: None. P. Richard: None. C. Metay: None. F. Ader: None. C. Saint-martin: None.
C. Ledeuil: None. M. Lodin: None. V. Allamand: None. M. Eyries: None. V. Jobic: None. B. Keren: None. A. De becdelievre: None. C. Bellanne-chantelot: None.

\section{E-P17.21 \\ Detection of non-reference sequences in population-scale WGS data}

\section{T. Krannich, B. Kehr}

\section{Berlin Institute of Health, Berlin, Germany}

Non-reference sequence insertions are a less frequently investigated class of genomic structural variation. Here, DNA sequences are found within an individual that are novel with respect to a given reference. These sequences occur primarily due to the fact that a reference genome lacks some ancestral sequence since it is mostly derived from a few or a single individual. Therefore, newly sequenced individuals can yield genomic sequences which are absent from a reference genome. To find such insertions in shortread sequencing data, it is necessary to perform a de novo assembly of the non-reference sequence, an algorithmically challenging and computationally expensive task. In recent years, only a few methods and tools have been developed to detect these non-reference sequence insertions and even fewer to perform the detection on large cohorts. One insertion caller that exploits the simultaneous analysis of multiple samples is PopIns. PopIns extracts unmapped reads from all individuals, assembles a non-redundant set of contig sequences across all individuals, anchors these sequences to the reference and finally genotypes each individual. We entirely redesigned the assembly module, a key step to combine samples. The novel assembly module uses a highly specialized data structure called compacted colored de Bruijn Graph. By applying this new approach to simulated data, the detected sequences are more precise while their computation requires less memory. Subsequently, this will considerably improve the detection of non-reference sequence insertions and, therefore, offer more comprehensive variant call sets for population and disease studies.

T. Krannich: None. B. Kehr: None.

\section{E-P17.23}

re-Searcher: a user-friendly GUI-based bioinformatics tool for simplified genomics data mining from big VCF files

D. Karabayev ${ }^{1}$, A. Molkenov ${ }^{1}, K$. Yerulanuly ${ }^{1,2}$,

A. Daniyarov ${ }^{l}$, A. Sharip ${ }^{l}$, A. Seisenova ${ }^{l}$, Z. Zhumadilov ${ }^{1,3}$, U. Kairov ${ }^{I}$

${ }^{1}$ Nazarbayev University, National Laboratory Astana, NurSultan, Kazakhstan, ${ }^{2}$ L.N. Gumilyov Eurasian National 
University, Nur-Sultan, Kazakhstan, ${ }^{3}$ Corporate fund "University Medical Center", Nazarbayev University, NurSultan, Kazakhstan

High-throughput sequencing platforms generate a massive amount of high-dimensional genomic datasets available for analysis. The availability of modern and user- friendly bioinformatics tools for analysis and interpretation of genomics data becomes an essential part during the analysis of sequencing data. The Variant Call Format (VCF) is a standard format that contains genomic information and variants of sequenced samples. Existent tools that process VCF files usually have command-line as their interface without an intuitive graphical interface that may be challenging for a broad biomedical community interested in genomics data analysis. We present re-Searcher, a new bioinformatics application tool with user-friendly GUI, developed to simplify genomic data mining from VCF files.

D. Karabayev: None. A. Molkenov: None. K. Yerulanuly: None. A. Daniyarov: None. A. Sharip: None. A. Seisenova: None. Z. Zhumadilov: None. U. Kairov: None.

\section{E-P18 Personalized Medicine and Pharmacogenomics}

\section{E-P18.01}

Comparison of genetic variation in ADME genes between the Croatian population and Europeans (gnomAD database)

Z. Celinscak, M. Zajc Petranovic, A. Stojanovic Markovic, M. Pericic Salihovic, N. Smolej Narancic, B. Janicijevic, T. Skaric-Juric

\section{Institute for Anthropological Reasearch, Zagreb, Croatia}

Introduction: The pharmacogenetic data have been studied extensively due to their clinical importance in the appropriate drug prescription.

Aim: to determine allele frequencies of 27 most important ADME polymorphisms (20 of them $1 \mathrm{~A}, 1 \mathrm{~B}$ or $2 \mathrm{~A}$ clinical annotation levels of evidence) in the Croatian population and to compare them with the European population.

Materials and methods: The 27 loci from 20 ADME genes were genotyped in the Croatian study sample consisted of 429 participants. Allele frequencies were combined with published data on the Croatian population (weighted frequencies according to sample sizes). The Croatian allele frequencies were compared with the European population average data taken from the gnomAD database (selection criteria: control cases only and nonFinnish).
Results: Results showed higher variant allele frequencies in the Croatian population at six loci (rs1045642, rs2279343, rs1799853, rs1057910, rs28371725, rs9923231) and lower at two loci (rs1695, rs1801272). The most significant differences were: 0.4608 vs 0.4880 in $A B C B 1,0.0932$ vs 0.2430 in $C Y P 2 B 6^{*} 4,0.1228$ vs 0.1470 in $C Y P 2 C 9 * 2,0.3325$ vs 0.2980 in GSTP1 and 0.3488 vs 0.4030 in VKORC1 genes, in Europeans and Croatians respectively.

Conclusions: Croatian population shows higher variant allele frequencies in several genes responsible for metabolism of anticoagulation drugs (warfarin, acenocumarol and phenprocoumon). This finding indicates higher bleeding and over-anticoagulation risk in Croatians, demanding lower drugs' prescription doses. Also, lower doses of phenytoin and celecoxib, while higher doses of digoxin, fentanyl, bupoprion and efavirenz are more frequently required in Croatians compared with European average. Acknowledgement: Croatian Science Foundation (HRZZ IP-01-2018-2497 and HRZZ-IP-2014-09-4454).

Z. Celinscak: None. M. Zajc Petranovic: None. A. Stojanovic Markovic: None. M. Pericic Salihovic: None. N. Smolej Narancic: None. B. Janicijevic: None. T. SkaricJuric: None.

\section{E-P18.04}

Construction of mental support system in cancer genomic medicine

\section{H. Fukushima ${ }^{1}$, M. Ishida ${ }^{2}$, T. Takahashi ${ }^{3}$, H. Onishi $^{2}$, T. Hamaguchi ${ }^{1}$}

${ }^{1}$ Department of Clinical Cancer genomics, Saitama Medical University International Medical Center, Hidaka, Japan, ${ }^{2}$ Department of Psycho-Oncology, Saitama Medical University International Medical Center, Hidaka, Japan, ${ }^{3}$ Department of Supportive Oncology and Palliative Medicine, Saitama Medical University International Medical Center, Hidaka, Japan

Introduction: The genomic medicine of cancer implemented in Japan, since two multigene-panel tests, Oncoguide NCC Oncopanel system and FoundationOne cancer genomic profile were covered by the national healthcare insurance system in September 2019. The reimbursement is restricted to solid tumor patients with no standard therapy, or completed or expected to complete it. According to the fact that only about 10 to 20 percent of the patients taking genomic profiling test will be able to find novel candidate drugs and therapies, most of the patients do not see them. Under the situation patients forced to remind the end of their anticancer therapy in time and may face the fear of their death caused by disease. The aim of this study is to 
identify the needs and find effective way for mental problem of the patients undergoing genomic profiling test.

Method: Current status and a series of procedures for genomic profiling test were reviewed by specialists of Psyco-oncology and supportive care division. And Investigate an interview with medical stuff involved in genomic profiling test to clarify individual problem.

Results: Consulting to supportive care specialist is recommended and description about availability of the team is added to the explanatory leaflet on genomic profiling. Psyco-oncologists and supportive care doctor join the expert-panel in order to advice in each case. Our goal is to construct support system of the patient receiving genomic medicine including sufficient mental care.

H. Fukushima: None. M. Ishida: None. T. Takahashi: None. H. Onishi: None. T. Hamaguchi: None.

\section{E-P19 Genetic Epidemiology, Population Genetics and Evolutionary Genetics}

\section{E-P19.01}

Divergent patterns of mitochondrial and nuclear ancestry are associated with the risk for preterm birth

N. Crawford ${ }^{1}$, D. B. Prendergast ${ }^{2}, J . W$. Oehlert $^{3}$, G. M. Shaw ${ }^{3}$, D. K. Stevenson ${ }^{3}$, N. Rappaport ${ }^{4}$, M. Sirota ${ }^{4}$, S. A. Tishkoff', N. Sondheimer ${ }^{5,1}$

${ }^{I}$ The University of Pennsylvania, Philadelphia, PA, United States, ${ }^{2}$ The Hospital for Sick Children, Toronto, ON, Canada, ${ }^{3}$ Stanford University, Palo Alto, CA, United States, ${ }^{4}$ The University of California San Francisco, San Francisco, CA, United States, ${ }^{5}$ The University of Toronto, Toronto, ON, Canada

Objective: To examine linkages between mitochondrial genetics and preterm birth by assessing the risk for preterm birth associated with the inheritance of nuclear haplotypes that are ancestrally distinct from mitochondrial haplogroup.

Study design: Genome-wide genotyping studies of cohorts of preterm and term individuals were evaluated. We determined the mitochondrial haplogroup and nuclear ancestry for individuals and developed a scoring for the degree to which mitochondrial ancestry is divergent from nuclear ancestry.

Results: Infants with higher degrees of divergent mitochondrial ancestry were at increased risk for preterm birth $(0.124$ for preterm vs 0.105 for term infants; $\mathrm{P}<.05)$. This finding was validated in 1 of 2 replication cohorts. We also observed that greater degrees of divergent ancestry correlated with earlier delivery within the primary study population, but this finding was not replicated in secondary cohorts born preterm.
Conclusions: Individuals with divergent patterns of mitochondrial and nuclear ancestry are at increased risk for preterm birth. These findings may in part explain the higher rates of preterm birth in African Americans and in individuals with a matrilineal family history of preterm birth.

Funded by The March of Dimes Prematurity Research Center at the University of Pennsylvania (22-FY17-890).

N. Crawford: None. D.B. Prendergast: None. J.W. Oehlert: None. G.M. Shaw: None. D.K. Stevenson: None. N. Rappaport: None. M. Sirota: None. S.A. Tishkoff: None. N. Sondheimer: None.

\section{E-P19.02}

The incidence of the commonest alpha-1-antitrypsin deficiency (AATD) alleles $(P I * Z$ and $P I * S)$ in the population of Polish newborns

\section{J. Chorostowska-Wynimko ${ }^{l}$, R. Struniawski ${ }^{1}$, M. Borszewska-Kornacka ${ }^{2}$, A. Jezela-Stanek ${ }^{l}$}

${ }^{1}$ National Institute of Tuberculosis and Lung Diseases, Department of Genetics and Clinical Immunology, Warsaw, Poland, ${ }^{2}$ Medical University of Warsaw, Neonatal and Intensive Care Department, Warsaw, Poland

Introduction: Alpha-1 antitrypsin deficiency (AATD, MIM613490) is listed among the most common genetic disorders in Caucasians. It considerably increases the risk of progressive obstructive lung diseases, as well as hepatic dysfunction, especially in early childhood. The protein is encoded by the SERPINAl gene, harbouring numerous polymorphisms. Among them, $\mathrm{Z}\left(\mathrm{PI}^{*} \mathrm{Z}\right)$ and $\mathrm{S}$ (PI*S), resulted from mutation at Glu342Lys and Glu264Val position, respectively, are most commonly occurring deficiency alleles. They lead to the synthesis of structurally abnormal AAT protein, enable to succeed a physiological conformation, and thus accumulating in hepatocytes ( $\mathrm{Z}$ protein) or degrading (S protein). Data on the prevalence of AATD and frequency of mentioned deficiency alleles in Poland are scarce, with only one published preliminary result and no studies with large enough group to be representative for the entire population. Our presentation aims to show the results of a study which investigates the prevalence of deficiency alleles $\mathrm{Z}$ and $\mathrm{S}$ in Poland.

Materials and methods: Real-time PCR genotyping and AAT blood concentration measurement by nephelometry were performed from the dry blood spots (DBS) samples of approx. 5000 newborns.

Results: Among 4948 newborns (preliminary data) both variants were identified in 221 children, including $\mathrm{PI}^{* \mathrm{Z}}$ allele in $115(2.3 \%)$ and $\mathrm{PI} * \mathrm{~S}$ allele in 106 neonates $(2.1 \%)$. 
Blood A1AT concentration was significantly lower in both groups.

Conclusions: The estimated prevalence of AAT deficiency alleles per 1.000 persons were $11,5(9,4-13,6)$ for $\mathrm{PI} * \mathrm{Z}$ and $10,6(8,6-12,6)$ for $\mathrm{PI}{ }^{*} \mathrm{~S}$. It indicates more frequent occurrence of the $\mathrm{Z}$ allele in Poland than in earlier reports.

J. Chorostowska-Wynimko: None. R. Struniawski: None. M. Borszewska-Kornacka: None. A. Jezela-Stanek: None.

\section{E-P19.03}

Incidence of anencephaly in the Czech Republic: Longterm population based study

A. Sipek Sr ${ }^{1,2,3}$, V. Gregor ${ }^{1,2}$, A. Sipek $\mathrm{Jr}^{1,4,5}$, J. Klaschka ${ }^{6,7}$, M. Maly ${ }^{6,8}$, J. Jirova 9

${ }^{1}$ Department of Medical Genetics, Thomayer Hospital, Prague, Czech Republic, ${ }^{2}$ Department of Medical Genetics, Pronatal Sanatorium, Prague, Czech Republic, ${ }^{3}$ GENNET, Prague, Czech Republic, ${ }^{4}$ Institute of Biology and Medical Genetics, First Faculty of Medicine, Charles University, Prague, Czech Republic, ${ }^{5}$ Institute of Medical Genetics, Third Faculty of Medicicne, Charles University, Prague, Czech Republic, ${ }^{6}$ Institute of Computer Science of the Czech Academy of Sciences, Prague, Czech Republic, ${ }^{7}$ Institute of Biophysics and Informatics, First Faculty of Medicine, Charles University, Prague, Czech Republic, ${ }^{8}$ National Institute of Public Health, Prague, Czech Republic, ${ }^{9}$ Institute of Health Information and Statistics of the Czech Republic, Prague, Czech Republic

Introduction: Anencephaly is a lethal anomaly of the central nervous system from the group of neural tube defects. The main goal of our study was to evaluate the incidence of anencephaly (and its possible trends) during the long-term period.

Methods: For this study, we have used data from the National Registry of Congenital Anomalies of the Czech Republic. We analyzed the incidence of anencephaly in births and in prenatally diagnosed cases in the 1964-2016 period. We also evaluated maternal age in these cases. The statistical analysis was performed by Poisson regression and Fisher's exact test.

Results: During the study period there were 6891062 children born in the Czech Republic. Among those, 1232 children were born with anencephaly. Another 966 cases of anencephaly were diagnosed during prenatal diagnosis and these pregnancies were electively terminated, the total number of anencephaly cases was 2198. The total incidence of anencephaly significantly decreased during the study period $(\mathrm{p}=0.0136)$. The incidence in births decreased significantly as well $(\mathrm{p}<0.001)$. We found statistically higher incidence of anencephaly in elder mothers (42 years and over).

Discussion: Gradual implementation of ultrasound prenatal diagnostics started in the 80s of the last century and lead to a very significant decrease in the incidence of anencephaly cases in births. Additionally, the total incidence of anencephaly decreased significantly as well. We found significantly higher incidence of anencephaly in elder mothers (42 years and over).

Supported by the Ministry of Health of the Czech Republic, grant $n r$. AZV 17-29622A

A. Sipek Sr: B. Research Grant (principal investigator, collaborator or consultant and pending grants as well as grants already received); Significant; Research grant provided by Ministry of Health of the Czech Republic, grant nr. AZV 17-29622A. V. Gregor: B. Research Grant (principal investigator, collaborator or consultant and pending grants as well as grants already received); Significant; Research grant provided by Ministry of Health of the Czech Republic, grant nr. AZV 17-29622A. A. Sipek Jr: B. Research Grant (principal investigator, collaborator or consultant and pending grants as well as grants already received); Significant; Research grant provided by Ministry of Health of the Czech Republic, grant nr. AZV 17-29622A. J. Klaschka: B. Research Grant (principal investigator, collaborator or consultant and pending grants as well as grants already received); Significant; Research grant provided by Ministry of Health of the Czech Republic, grant nr. AZV 17-29622A. M. Maly: B. Research Grant (principal investigator, collaborator or consultant and pending grants as well as grants already received); Significant; Research grant provided by Ministry of Health of the Czech Republic, grant nr. AZV 17-29622A. J. Jirova: B. Research Grant (principal investigator, collaborator or consultant and pending grants as well as grants already received); Significant; Research grant provided by Ministry of Health of the Czech Republic, grant nr. AZV 17-29622A.

\section{E-P19.04}

Assessment of knowledge and attitude towards biobanks among healthcare students and their willingness to donate biospecimens in Libya

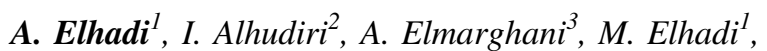
A. Msherghi ${ }^{1}$, A. Buhmeida ${ }^{4}$, N. Enattah ${ }^{2,5}$, A. Elzagheid $^{2}$

${ }^{1}$ Faculty of Medicine, University of Tripoli, Tripoli, Libyan Arab Jamahiriya, ${ }^{2}$ Genetic Engineering Department, Biotechnology Research Center, Tripoli, Libyan Arab Jamahiriya, ${ }^{3}$ Cell Biology Department, Biotechnology Research Center, Tripoli, Libyan Arab Jamahiriya, ${ }^{4}$ Center of Excellence in Genomic Medicine Research, King Abdulaziz, 
Jeddah, Saudi Arabia, ${ }^{5}$ University of Tripoli, Libya, Tripoli, Libyan Arab Jamahiriya

Introduction: Globally, many studies have assessed knowledge and attitude about biobanks and the willingness to donate specimens in health workers and medical students. The study aims to evaluate the knowledge and attitude of medical and biomedical students in Libya towards biobanks and their willingness to donate biological samples.

Materials and methods: A cross-sectional study was conducted among senior healthcare students at the main Libyan Universities. Students from faculties of Medical, Dental, Pharmacy and Medical Technology who were about to graduate at the end of the 2020/2021 academic year completed a structured and self-administered online questionnaire distributed in social media University pages and via emails. The questionnaire included information about personal and general health, attitudes towards biomedical research and donation and awareness of biobanks.

Results: A total of 324 students successfully completed and submitted the questionnaire. The majority of students approved medical research $(96 \%, 311 / 324)$ and $75 \%$ (244/ 324) were willing to participate in genetic research. Less than $5 \%$ of students were unwilling to participate in genetic research. Approximately 48\% (155/324) were aware of the term biobank and $74 \%$ (240/324) were willing to donate their specimens to a biobank.

Conclusions: This study provides novel information on the likelihood of donating biospecimens and although students were largely willing to donate biological samples, they were not aware of biobanking. It is therefore highly recommended to reinforce medical and biomedical curricula to educate students about biobanking, medical and genetic research.

A. Elhadi: None. I. Alhudiri: None. A. Elmarghani: None. M. Elhadi: None. A. Msherghi: None. A. Buhmeida: None. N. Enattah: None. A. Elzagheid: None.

\section{E-P19.06}

\section{Genetic variants of BRCA1 exon 11 in Libyan breast cancer patients}

\section{H. Elshwekh, I. Alhudiri, H. Abdul Jalil, A. Mohamed, A. Elkikli, N. Jornaz, N. Enattah}

\section{Biotechnology Research Centre, Tripoli, Libyan Arab Jamahiriya}

Introduction: The majority of hereditary breast and ovarian cancers are associated with mutations in two genes, breast cancer type 1 and 2 susceptibility genes (BRCA1 and BRCA2). Here we describe for the first time analysis of
BRCA1 exon 11 in 48 Libyan breast cancer patients with family history of cancer.

Materials and methods: All patients had family history of cancer and were included in the study only if they have family history of breast or ovarian cancer, male breast cancer or triple negative tumours. PCR was performed using specific primer pairs spanning BRCA1 exon 11 followed by Sanger sequencing. Genetic analysis was done using Sequencher ${ }^{\circledR} 5.1$.

Results: We identified 12 genetic variants in BRCA1 exon 11. Three variants were novel (c.1019T $>C$ c.2363T $>$ G, c. $3192 T>C$ ); c. $2363 T>G$ is predicted by SIFT as damaging. Six variants were of unknown significance (c. $918 \mathrm{~T}>\mathrm{C}, \quad$ c. $1853 \mathrm{G}>\mathrm{C}, \quad$ c. $1886 \mathrm{G}>\mathrm{A}, \quad$ c. $2215 \mathrm{~A}>\mathrm{G}$, c. $2612 \mathrm{C}>\mathrm{T}, \mathrm{c} .3113 \mathrm{~A}>\mathrm{C}, \mathrm{c} .3784 \mathrm{~T}>\mathrm{C}$ ), and 3 were classified as benign in ClinVar database (c.918T $>\mathrm{C}$, c. 2082C $>\mathrm{T}$, c. $2311 \mathrm{~T}>\mathrm{C}$ ).

Conclusions: Scanning of the entire BRCA1 is needed to identify any associated deleterious mutations. Although the clinical importance of unclassified variants is unknown, the association of certain variants with deleterious mutations and contralateral breast cancer warrants genetic testing and counseling in Libyan population.

Keywords: Genetic variants, Breast cancer, BRCA1, Libya

H. Elshwekh: None. I. Alhudiri: None. H. Abdul Jalil: None. A. Mohamed: None. A. Elkikli: None. N. Jornaz: None. N. Enattah: None.

\section{E-P19.07}

Penetrance of the CFTR 5T allele in congenital bilateral absence of the vas deferens

D. Seidl ${ }^{1,2}$, Á. Mikó ${ }^{1,2}$, A. Kaposi ${ }^{2}$, K. Tory ${ }^{1,2}$

${ }^{1}$ Semmelweis Univesity, 1st Department of Paediatrics, Budapest, Hungary, ${ }^{2}$ MTA-SE Lendület Nephrogenetic Laboratory, Budapest, Hungary

Introduction: Congenital bilateral absence of the vas deferens (CBAVD) is responsible for $2 \%$ of male infertility. One third (25-40\%) of patients with CBAVD are compound heterozygous for the CFTR 5T (c.1210-7_1210-6delTT) variant and another variant in trans. The $5 \mathrm{~T}$ allele, with a MAF of 3\% in the general European population, causes the loss of exon 10 in 95\% of mRNA. Using a formerly developed population-genetic algorithm we aimed to assign the penetrance of the 5T variant.

Materials and methods: We collected phenotype and genotype data from 3279 patients with biallelic CFTR mutations from PubMed. The penetrance $(\mathrm{P})$ of the 5T variant was calculated by comparing its allele count (AC) to the $\mathrm{AC}$ of the loss-of-function (LOF) variants in the 
European non-Finnish patient population and in the gnom $\mathrm{AD}$ as $\mathrm{P}=\left(\mathrm{AC}_{5 \mathrm{~T}} / \mathrm{AC}_{\mathrm{LOF}}\right)_{\text {patient }} /\left(\mathrm{AC}_{5 \mathrm{~T}} / \mathrm{AC} \mathrm{COF}_{\mathrm{LO}}\right)_{\text {gnomAD }}$, tested by Fisher's exact test.

Results: We found the 5T allele in 339/3279 (10.34\%) patients, trans-associated to LOF mutations in $187 / 635$ (29.3\%) of the patients with CBAVD. None of the compound heterozygous patients with the 5T allele (without other variant in cis) developed $\mathrm{CF}$. We found the penetrance of the $5 \mathrm{~T}$ variant to be $4.3 \%\left(\mathrm{p}=5 \times 10^{-389}\right)$.

Conclusions: According to our penetrance estimation, for a couple with heterozygous CFTR 5T allele in one parent and a LOF variant in another, the risk of a son being affected by CBAVD is $1 \%(25 \% \times 4.3 \%)$.

Supported by the ÚNKP-19-3-II New National Excellence Program of the Ministry for Innovation and Technology.

D. Seidl: None. Á. Mikó: None. A. Kaposi: None. K. Tory: None.

\section{E-P19.08}

analysis the interactions of IL1B, IL4, IL6 genes polymorphism in infected women with human papillomavirus

\section{a. Al bosale, e. v. Mashkina}

southern Federal University, Rostov-on-don, Russian Federation

Introduction: The presence of functional SNPs in the promoter regions can be considered as one reason for the imbalance in cytokines levels during HPV infection which leads to an imbalance of pro- and anti-inflammatory cytokines.

Materials and methods: In our work, we assessed the association of polymorphic variants of the IL1 $\beta-31 \mathrm{~T}>\mathrm{C}$, $-511 \mathrm{C}>\mathrm{T}$, IL4 $-589 \mathrm{C}>\mathrm{T}$ and IL6 $-174 \mathrm{G}>\mathrm{C}$ genes with highrisk HPV infection in women, ages 30 years and older (101 women infected with HPV with viral load more than $4 \mathrm{lg}$ and 93 women without HPV). Polymorphic variants detected by allele-specific PCR.

Results: The individual SNP genes IL1 $\beta-31 \mathrm{~T}>\mathrm{C}$, IL4 $-589 \mathrm{C}>\mathrm{T}$ and IL6 $-174 \mathrm{G}>\mathrm{C}$ did not reveal significant differences in all frequencies of genotypes and alleles. At the same time, the difference in frequency of the $-511 \mathrm{~T}$ allele and TT genotype of the IL1 $\beta$ gene between two groups was statistically significant $(\mathrm{OR}=1.71,2.02$ respectively). Analysis of intergenic interactions for all contribution allelic variants revealed the significant interaction for polymorphic variants IL1 $\beta$, IL4 and IL6 genes (OR for the combination of loci was 4,70 CI $95 \%(2,55-$ $8,67)(\mathrm{P}=0,0001)$. Haplotypes analysis revealed that the $-511 \mathrm{C} /-31 \mathrm{~T}$ haplotype is statistically significantly less common among women with a high concentration of HPV $(5.9 \%$ and $15.2 \% \mathrm{p}=0.002)$.

Conclusions: in Russian women, IL1B -511 T/T genotypes significantly associated with an increased risk of HPV infection and -511C/-31T haplotype, it considered as a preventive factor against the risk of HPV infection.

A. Al bosale: None. E.V. Mashkina: None.

\section{E-P19.09}

COMT Micro-haplotypes distribution in North African populations: Usefulness in population evolutionary studies

S. Boussetta ${ }^{\text {, L. Lhernil }}{ }^{1,2}$, A. J. Pakstis ${ }^{3}$, N. Ben Salem ${ }^{l}, S$. Elkamel $^{l}$, H. Khodjet-el-khil ${ }^{4}, K . K . K^{2}{ }^{3} d^{3}$, A. BenammarElgaaied $^{l}$

${ }^{1}$ Laboratory of Genetics, Immunology and Human Pathology, Faculty of Sciences of Tunis, University of Tunis El Manar, Tunis, Tunisia, ${ }^{2}$ High Institute of Biotechnology, University of Monastir, Monastir, Tunisia, ${ }^{3}$ Department of Genetics, Yale University School of Medicine, New Haven, $C T$, United States, ${ }^{4}$ College of Health Sciences. QU Health, Doha, Qatar

Introduction: The COMT gene encodes for catechol-Omethyl-transferase enzyme that has the major role in the regulation of synaptic catecholamine neurotransmitters. Many variants of the COMT gene have been described and some of them are in strong linkage disequilibrium defining haplotypes associated with the enzyme activity level. Our aim is to investigate the haplotypic distribution of the COMT gene markers in North African population in order to assess their usefulness in investigating North African population genetic structure.

Materials and methods: Four markers (rs2020917, rs4818, rs4680, rs9332377) have been typed in 275 North African populations using TaqMan technology. Haplotypes have been phased. Linkage disequilibrium (LD), Fst distance matrix and Principal Component Analysis (PCA), were performed. For comparative analyses, data from the 59 Kidd Lab populations and the 1000 Genome Project, were used.

Results: Our main findings show that COMT activity prediction, according to LD performance, could be limited to rs4818-rs4680 micro-haplotypes. North Africans and South Asians show intermediate frequency values $(20 \%$ and $25 \%$ respectively) of the low activity (CG) haplotype when compared to those of African populations and East Asians that show frequencies of $55 \%$ and $40 \%$ respectively, and to European populations frequency of $10 \%$. Fst genetic distance and PCA analysis showed genetic differentiation between Africans, North African, Europeans, East and 
South Asians according to their significant haplotype frequency differences.

Conclusion: The high level of genetic diversity of COMT haplotypes along with different LD levels can be useful tool for population genetic studies and included in ancestry informative panels. Grants: MHESR. LGIPH-E05. 15-20

S. Boussetta: None. L. Cherni: None. A.J. Pakstis: None. N. Ben Salem: None. S. Elkamel: None. H. Khodjet-el-khil: None. K.K. Kidd: None. A. Benammar-Elgaaied: None.

\section{E-P19.10}

The coverage of Russian CF patients with genetic testing in 2011-2018.

N. Kashirskaya ${ }^{l}$, N. Petrova ${ }^{l}$, E. Kondratyeva ${ }^{l}$, A. Voronkoval, Y. Melyanovskaya ${ }^{l}$, A. Zodbinova ${ }^{l}$, E. Zhekaite ${ }^{1}$, E. Amelina ${ }^{2}$, S. Krasovskiy ${ }^{2}$, M. Starinova ${ }^{1}$, A.

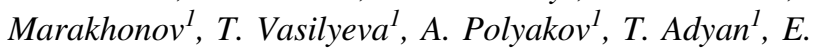
Ginter $^{l}$, S. Kutsev ${ }^{l}$, R. Zinchenko ${ }^{l}$

${ }^{1}$ Federal State Scientific Budgetary Institution «Research Centre for Medical Genetics», Moscow, Russian Federation, ${ }^{2}$ Pulmonology Research Institute under FMBA of Russia, Moscow, Russian Federation

Cystic Fibrosis Patient Registry of Russian Federation (CFPR-RF) 2011-2018 collects demographic and clinical data in accordance with the agreed European inclusion criteria and definitions.

Aim: To compare the coverage of $\mathrm{CF}$ patients with genetic testing in 2011 and 2018.

Materials and methods: CFPR-RF 2018 included data of 3142 CF patients while 2011 - 1015 patients.

Results: In 2018 molecular-genetic testing was performed in $94.3 \%$ (2011 -91.8\%) of CF patients (children$94.6 \%$, adults $-93.6 \%$ ). Two mutations were identified in $82.4 \%$, one - in $14.0 \%$, none - in $3.7 \%$ of patients. The total frequency of identified alleles was $89.3 \%$ (80.0\% in 2011). The worst situation is in the Volga region - only $87.9 \%$ of CF patients are genetically tested. The best is in the Central - 97.6\% and the Ural - 97.3\% RF regions (St. Petersburg $100.0 \%$, Moscow - 98.2\%). The allele frequency of the most common mutations in RF in 2018: F508del - 53.05\%, CFTRdele2, 3 - 6.09\%, E92K - 3.04\%, 3849+ 10kbC- >T $2.38 \%, 2143$ delT $-2.11 \%$. In total 223 pathogenic variants were identified and 100 of them repeatedly.

Conclusion: The number of CF centers and patients participating in CFPR-RF 2018 has tripled since 2011. CFTR genetic variants identification has been improved but there are still $\mathrm{CF}$ patients that are either not tested at all (5.7\%) or have unidentified one or two CFTR mutations.
The research was partially supported by grants RFFR №20-015-00061 and within the state task of the Ministry of education and science of Russia.

N. Kashirskaya: None. N. Petrova: None. E. Kondratyeva: None. A. Voronkova: None. Y. Melyanovskaya: None. A. Zodbinova: None. E. Zhekaite: None. E. Amelina: None. S. Krasovskiy: None. M. Starinova: None. A. Marakhonov: None. T. Vasilyeva: None. A. Polyakov: None. T. Adyan: None. E. Ginter: None. S. Kutsev: None. R. Zinchenko: None.

\section{E-P19.11}

Association of polymorphism of the DRD2 / ANKK1 Taq1A gene with depression in open male population of 45-64 years (epidemiological study)

V. Gafarov, E. Gromova, D. Panov, E. Krymov, I. Gagulin, A. Gafarova

Institute of Internal and Preventive Medicine - branch of Institute of Cytology and Genetics SB RAS, Novosibirsk, Russian Federation

Purpose: To study the association of the DRD2/ANKK1 Taq1A gene polymorphism with depression in open male population aged 45-64 years.

Methods: Under the IV screening of the international program "HAPIEE" and the WHO program "MONICApsychosocial" in 2003-2005 a representative sample of the population aged 45-64 years ( $\mathrm{n}=781$ of men) was surveyed. All study participants filled out the depression scale proposed by WHO MONICA-psychosocial program. The genotyping of the DRD2/ANKK1 Taq1A gene polymorphism was done in frame of the budgetary issue \# AAAAA17-117112850280-2.

Results: The prevalence of depression in the open population of men $45-64$ was $36.3 \%$ (13.5\% with a high level and $22.8 \%$ with middle level). A comparative intergroup analysis showed that odds ratio (OR) of a high level of depression was 3.86-fold higher in persons with $\mathrm{T} /$ $\mathrm{C}$ genotype than those with $\mathrm{C} / \mathrm{C}$ genotype without depression. OR of a high level of depression was in 3.28 times higher in persons with T/C genotype compared to persons with middle level of depression and with homozygous C/C genotype. Males with $\mathrm{T}$ allele of the DRD2 gene more likely to be at high level of depression in 2.63 times than persons with $\mathrm{C}$ allele who had more often no depression.

Conclusions: Reliable associations were found between the presence of Taq1A ( $\mathrm{T}$ allele) and depression in males aged 45-64 years.

V. Gafarov: None. E. Gromova: None. D. Panov: None. E. Krymov: None. I. Gagulin: None. A. Gafarova: None. 


\section{E-P19.12}

Pertinence of genetic testing ordered by different medical specialties. Experience from a reference laboratory in Colombia

\section{T. Bernal, S. J. Maradei, A. J. Muñoz, Y. M. Gómez}

Biotecgen S.A., Bogota, Colombia

Introduction: In the last decade, there has been a rapid increase in the technical capacity of multiple genetic tests to detect DNA variants that can be used to diagnose different pathologies and to guide specific diagnostic strategies. In Colombia, there has been a dramatic increase in the request for all types of genetic studies by the vast majority of medical specialties, without adequate guidance by clinical geneticists, regarding to the choice of molecular study and interpretation of results.

Materials and methods: We reviewed all the molecular studies received in our laboratory during the year 2019, grouping them by requesting specialty, type of study and result, determining the index of relevance and/or accuracy, and the diagnostic yield of each one.

Results: We received 834 orders for genetic studies, requested by 25 different medical specialties, with a total of 248 positive results $(29,7 \%), 119$ VOUS (14,2\%) and 467 negative results $(55,9 \%)$, mainly corresponding to NGS panels. The two specialties with the highest number of requests for molecular studies were Clinical Genetics and Oncohematology, the former having the highest diagnostic yield, the $40 \%$ of medical specialties had only negative results.

Conclusion: We strongly recommend that genetic tests prescribed by untrained medical staff should be reserved for cases with high clinical suspicion only, otherwise it leads to increased time for diagnosis and treatment, and economic detriment to the health system. Moreover, medical specialties should be instructed on the impact of VOUs and the importance of their reclassification.

I.T. Bernal: None. S.J. Maradei: None. A.J. Muñoz: None. Y.M. Gómez: None.

\section{E-P19.14}

Effect of dietary nutrients on modulation of heavy metal induced DNA damage in community dwelling Malaysian women

C. Y. $\mathbf{N g}^{1,2}$, A. B. Normina ${ }^{1}$, E. S. S. Tan ${ }^{l}$, M. S. Seghayat ${ }^{1}$, P. Y. Tan $^{3}$, S. R. Mitra ${ }^{3}$, E. S. C. $\mathrm{Ng}^{l}$, A. Muhamad ${ }^{4}$, R. Sharif ${ }^{5}$, A. Razak ${ }^{6}$, F. Amini

${ }^{1}$ School of Healthy Aging, Medical Aesthetics \& Regenerative Medicine, Faculty of Medicine and Health Sciences, UCSI University, Kuala Lumpur, Malaysia, ${ }^{2}$ Faculty of
Applied Science, UCSI University, Kuala lumpur, Malaysia, ${ }^{3}$ School of Biosciences, Faculty of Science and Engineering, University of Nottingham, Semenyih, Malaysia, ${ }^{4}$ Malaysia Genome Institute, National Institutes of Biotechnology, Bandar Baru Bangi, Malaysia, ${ }^{5}$ Centre of Healthy Ageing and Wellness, Faculty of Health Sciences, Universiti Kebangsaan Malaysia,, Kuala Lumpur, Malaysia, ${ }^{6}$ Faculty of Medicine and Health Sciences, UCSI University, Kuala Lumpur, Malaysia

Introduction: DNA damage due to toxic heavy metals (HMs) is a global public health concern. Unregulated DNA damage leads to aberrant gene expression and may initiate disease processes. This study aimed to investigate impact of dietary nutrients in modulating DNA damage due to HMs in non-occupationally exposed Malaysian women.

Methods: A cross-sectional study was conducted involving 134 Malay women aged between 20 to 50 years. Hair samples were collected from occipital region of the scalp. Concentrations of four HMs were quantified using Inductively Coupled Plasma Mass Spectrometry (ICP-MS). Degree of DNA damage was detected using AlkalineComet assay. Hundred cells were randomly selected and scored using online software for evaluating four comet assay parameters. Participant's daily energy, macro and micro nutrient intake were extracted from a food frequency questionnaire.

Results: The basal level of DNA strand breaks was $11.49 \pm 5.67$ (\%DNA in comet tail mean \pm SD). Mean concentrations of arsenic, mercury, chromium and cadmium were $0.1318 \pm 0.329,0.984 \pm 4.107,6.151 \pm 4.3487$, and $0.201 \pm 0.868 \mathrm{mg} / \mathrm{kg}$ respectively. After adjustment for covariates, significantly lower cadmium levels were found for high vitamin $\mathrm{D}$ intake $(\mathrm{p}=0.043)$ and significantly lower chromium levels were found for high calcium intake $(\mathrm{p}=0.043)$. High intake of manganese had positive impact $(\mathrm{p}=0.031)$ on comet tail DNA in individuals with high concentrations of hair arsenic.Conclusion: Specific dietary nutrients may modulate heavy metal induced DNA damage in non-occupationally exposed individuals.

The project was funded by UCSI Universities (Proj-InFMHS-029 and Proj-In-FMHS-019) and FRGS/1/2014/ SKK10/UCSI/03/1.

C.Y. Ng: None. A.B. Normina: None. E.S.S. Tan: None. M.S. Seghayat: None. P.Y. Tan: None. S.R. Mitra: None. E. S.C. Ng: None. A. Muhamad: None. R. Sharif: None. A. Razak: None. F. Amini: None.

\section{E-P19.16}

Origin and migration path of Val30Met in Familial Amyloid Polyneuropathy (TTR-FAP) in Portugal 
C. Leal ${ }^{1}$, T. Coelho ${ }^{2}$, M. Alves_Ferreira ${ }^{3}$, D. Santos ${ }^{3}$, I. Alonso ${ }^{3}$, J. Sequeiros ${ }^{3}$, C. Lemos ${ }^{1,3}$, A. Sousa ${ }^{1,3}$

${ }^{1}$ ICBAS, Porto, Portugal, ${ }^{2}$ CHUP, Porto, Portugal, ${ }^{3} i 3$, Porto, Portugal

Introduction: Variability in age-at-onset (AO) of Familial Amyloid Polyneuropathy (TTR-FAP Val30Met) is well known. Three independent Val30Met mutational events have been hypothesized by different authors, suggesting an association with AO differences among the main clusters. Whether there is a common origin for Val30Met mutation in the Portuguese cluster remains an open question.

Objectives: To distinguish between one or multiple mutational events among the Portuguese Val30Met TTRFAP kindreds, through characterization of the genetic background surrounding Val30Met locus and to estimate the age of ancestral mutational event(s).

Methods: From the largest Val30Met TTR-FAP database worldwide we carried out an extensive family-based haplotype study, in 45 families from two disease clusters (Northern Coast and Inland).

Results: Mean AO was significantly higher in patients from Inland than from Northern Coast $(\mathrm{p}<0.001)$. A common haplotype of different lengths was shared by almost all carriers, providing strong evidence of a major single-founder effect for Val30Met in these two disease clusters. We estimated that the mutational event occurred in the mid-VI century (AD) (1450-1475 years ago), spreading from Póvoa de Varzim/Vila do Conde to the Inland region. Although AO variability in Val30Met Portuguese kindreds does not seem associated to different mutational origins, striking differences in the extended haplotype downstream STR D18S1133 were found between Northern Coast vs. Inland.

Conclusions: 1) We were able to clarify Val30Met ancestral origins and its migration path between these two Portuguese cluster, 2) Exploring this genomic region may provide new insights on $\mathrm{AO}$ variability, with the identification of genetic modifiers.

C. Leal: None. T. Coelho: F. Consultant/Advisory Board; Modest; Pfizer. M. Alves_Ferreira: None. D. Santos: None. I. Alonso: None. J. Sequeiros: None. C. Lemos: None. A. Sousa: None.

\section{E-P19.18}

Incidence of $A P C$ and $M U T Y H$ mutation carriers of premalignant (tubular adenoma) versus malignant (adenocarcinoma) tumors of colon and rectum in Southern Turkey
M. K. Aktas $^{1}$, C. Mujde ${ }^{2}$, A. Rencuzogullari ${ }^{3}$, A. Bisgin ${ }^{2,4}$

${ }^{1}$ Cukurova University Faculty of Medicine, Adana, Turkey, ${ }^{2}$ Cukurova University AGENTEM (Adana Genetic Diseases Diagnosis and Treatment Center), Adana, Turkey, ${ }^{3}$ Cukurova University Faculty of Medicine, Surgical Oncology Department of Balcali Hospital and Clinics, Adana, Turkey, ${ }^{4}$ Cukurova University Faculty of Medicine, Medical Genetics Department of Balcali Hospital and Clinics, Adana, Turkey

Introduction: It has been reported that patients with adenomas or polyps in the colon or rectum may have an increased risk of developing colorectal cancer. However, it is still unclear in different populations that the $A P C$ and $M U T Y H$ genetic profiling might have effect. Thus, in study we determined the incidence of $A P C$ and MUTYH mutations in patients with tubular adenoma and adenocarcinoma. Material and Methods Peripheral blood samples of 18 patients were sequenced for APC and MUTYH genes via MiSeq NGS System (Illumina). Data was analyzed by QCIAnalyze and QCI-Interpret bioinformatics tools.

Results: 11 clinically significant variants in $A P C$ and MUTYH genes were detected in 55.5\% $(\mathrm{n}=10)$ of 18 patients whom $50 \%$ of tubular adenoma, $44 \%$ adenocarcinoma and only one of hyperplastic polyp. In tubular adenoma group, $77.7 \%$ of them had at least one mutation, 6 variants in $A P C$ gene and 2 variants in MUTYH gene retrospectively. Interestingly, while 1 of $A P C$ variants is a novel, two of them are variants classified as uncertain significant. On the other hand, only $25 \%$ of adenocarcinoma patients had mutations on $A P C$ and MUTYH genes ( 1 in $A P C$ and 1 in MUTYH).

Conclusions: Our data suggested that patients with both premalignant and malignant tumors of colorectum should undergo genetic screening for the benefit of patients and their families.

M.K. Aktas: None. C. Mujde: None. A. Rencuzogullari: None. A. Bisgin: None.

\section{E-P19.19}

Association between Interleukin 17A and Interleukin 17F Gene Polymorphisms and Periodontal Pathogens in Chronic Periodontitis among Libyans.

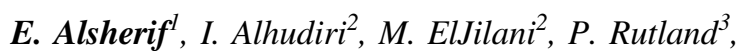 \\ N. Enattah ${ }^{1,2}$
}

${ }^{1}$ University of Tripoli, Tripoli, Libyan Arab Jamahiriya, ${ }^{2}$ Biotechnology Research Centre, Tripoli, Libyan Arab Jamahiriya, ${ }^{3}$ University College London, London, United Kingdom 
Background: Chronic periodontitis (CP) is triggered by periodontal pathogens and influenced by genetic and environmental factors. Recent studies suggest that antiinflammatory cytokines such as interleukin 17 (IL-17) plays a prominent role in the pathogenesis of $\mathrm{CP}$. This study aims to investigate the association between $\mathrm{CP}$ and interleukin IL- 17 (A and F) gene polymorphisms. We also aim to evaluate the association between eight subgingival pathogens (Porphyromonas gingivalis, Treponema denticola, Tannerella forsythia, Prevotella intermedia, Prevotella nigrescns, Campylobacter rectus, Aggregatibacter actinomycetemcomitans, Eikenella corrodens) and CP in Libyans.

Materials and methods: The study consisted of 100 Libyan individuals between the ages of 25 and 65 years including 50 cases and 50 controls. DNA was extracted from buccal swabs and paper points for subgingival pathogen samples. IL 17 (A\&F) genotyping was performed by PCR followed by Sanger sequencing. Specific $16 \mathrm{~S}$ rRNA primers for each pathogen were applied in a multiplex PCR reaction and visualized by agarose gel electrophoresis for detection of subgingival pathogens.

Results: IL17A AA, GG and AG (rs2275913) genotypes were detected in patients with CP. IL-17F CT (rs763780) genotype was seen in CP group only. A novel variant $\mathrm{c}$. $* 34 \mathrm{G}>\mathrm{A}$ in IL17F was found in $14 \%$ of patients. Significant association between the subgingival pathogens and CP (pvalue $=0.0001$ ) and high prevalence of subgingival pathogens in CP group.

Conclusion: IL-17 gene polymorphisms are associated with $\mathrm{CP}$, and strong association between subgingival pathogens and $\mathrm{CP}$ exist. Further large scale studies are needed to elucidate the association.

E. Alsherif: None. I. Alhudiri: None. M. ElJilani: None. P. Rutland: None. N. Enattah: None.

\section{E-P19.25}

Analysis of the association withinMMP-2gene polymorphisms and ischemic stroke outcome after thrombolytic therapy

M. G. Dusanovic Pjevic ${ }^{1}$, L. Vojvodic ${ }^{2}$, M. B. Grk ${ }^{l}$, N. Maksimovic ${ }^{l}$, T. Damnjanovic ${ }^{l}$, M. M. Pesic ${ }^{l}$, M. Gulic ${ }^{l}$, K. Kacar $^{3}$, B. Jekic ${ }^{1}$

${ }^{1}$ Institute of Human Genetics, Faculty of Medicine, Belgrade, Serbia, ${ }^{2}$ Euromedik Hospital, Belgrade, Serbia, ${ }^{3}$ St. Sava Hospital, Belgrade, Serbia

Introduction: Thrombolytic therapy has significantly improved recovery in ischemic stroke (IS) patients, reduced mortality, but is followed by the risk of hemorrhagic transformation (HT). Matrix metalloproteinases 2 (MMP-2) may have a significant role in the IS therapy outcome. Polymorphisms (rs243866, rs243865, rs243864, rs2285053) within the MMP-2 gene could potentially affect $M M P-2$ gene expression and consequently IS patients' recovery and the occurrence of HT.

Aim: To analyze the correlation between genotypes and haplotypes of the selected MMP-2 gene polymorphisms on IS patients' recovery, along with testing the occurrence of HT after thrombolytic therapy.

Methods: The study included 170 patients with IS treated with thrombolytic therapy. Patients' convalescence was determined using the Modified Rankin Scale (mRS) after 3 months from IS onset, where the good outcome has been defined with scores 0-1 and unfavorable with scores 2-6. Genotypisation was performed using Real-Time PCR (rs243866, rs243865, rs243864) and PCR-RFLP (rs2285053) methods. Haplotype analysis was performed by Haploview software.

Results: Obtained frequencies of genotypes and alleles were in accordance with Caucasian population results. We have observed no association between any genotypes or haplotypes with thrombolytic therapy outcome and HT occurrence. Haplotypes GCTC, ATGC, GCTT and GCGC (rs243866, rs243865, rs243864, rs2285053) were the most frequent among our patients $(73.2 \%, 17.0 \%, 5.6 \%, 1.5 \%$, respectively). Using Solid spine of LD method, haplotype block was defined among all investigated polymorphisms within the MMP-2 gene. Patients with GCTC haplotype had less frequently repeated IS $(60 \%$ Vs. $75.5 \%, \mathrm{p}=0.021)$.

Conclusion: $M M P-2$ promotor haplotype GCTC may be protective against recurrent IS.

M.G. Dusanovic Pjevic: None. L. Vojvodic: None. M.B. Grk: None. N. Maksimovic: None. T. Damnjanovic: None. M.M. Pesic: None. M. Gulic: None. K. Kacar: None. B. Jekic: None.

\section{E-P19.29}

Population genetic study from four polymorphism of the ADRB3, APOA5, ADIPOQ and PPARG genes associated with metabolic syndrome in Bucaramanga, Santander-Colombia

\section{Hernández Espinosa ${ }^{l}$, A. Castillo Pico ${ }^{l}$, G. Mantilla Mora $^{l}$, C. I. Vargas Castellanos ${ }^{1}$, F. Rondon Gonzalez ${ }^{2}, L$. Vera Cala ${ }^{3}$, V. L. Perez Romero ${ }^{4}$}

${ }^{I}$ Human Genetics Research Group. Universidad Industrial de Santander, Bucaramanga, Colombia, ${ }^{2}$ Microbiology and Genetics Research Group. Universidad Industrial de Santander, Bucaramanga, Colombia, ${ }^{3}$ GUINDESS Research Group. Universidad Industrial de Santander, Bucaramanga, Colombia, ${ }^{4}$ Basic Sciences Applied to Sustainability Research Group. Universidad de Santander, 


\section{Bucaramanga, Colombia}

Introduction: Metabolic Syndrome (MetS) is a cluster of alterations including increased fasting blood glucose levels, high triglycerides levels and blood pressure, abdominal obesity and lower HDL cholesterol levels. MetS is a multifactorial disorder and includes genetic-environmental interactions. ADRB3, APOA5, ADIPOQ and PPARG are among the most studied genes having roles in several metabolic pathways. A differential distribution of these polymorphic genes in different human populations has been reported by numerous studies worldwide. We aim to determine the genetic composition of Single Nucleotide Polymorphisms (SNPs) rs2075291 (G / C), rs2241766 (G / $\mathrm{T}), \operatorname{rs} 1800571(\mathrm{G} / \mathrm{T})$ and $\mathrm{rs} 4998(\mathrm{C} / \mathrm{A} / \mathrm{G})$ in a population sample of Bucaramanga, Colombia.

Materials and methods: A blood sample was collected from 1057 healthy subjects. DNA extraction was performed using the PrepFilerTM kit. Polymorphisms were amplified in a multiplex PCR, and a SNaPshot minisequencing reaction followed by analysis on an ABI PRISM 310. Allelic frequencies and Hardy-Weinberg test (EHW) were obtained by Arlequin v 3.5.

Results: The most frequent genotype and allele for each of the polymorphisms were: ADRB3 GG gene (0.738), G (0.859); APOA5 GG (0.766), G (0.875); ADIPOQ TT (0.561), T (0.749); and PPARG CC (0.861), C (0.928). These results agree with previous studies (Ziki 2016; Kim 2018). No deviations from EHW were found after performing Bonferroni correction.

Conclusions: The frequencies obtained do not differ from those previously reported. Finding SNPs under EHW contribute to determine the possible association of these polymorphisms with MetS

V. Hernández Espinosa: None. A. Castillo Pico: None. G. Mantilla Mora: None. C.I. Vargas Castellanos: None. F. Rondon Gonzalez: None. L. Vera Cala: None. V.L. Perez Romero: None.

\section{E-P19.30}

Analysis of association of MMP-2 gene promoter haplotype with efficacy and toxicity of methotrexate in patients with Rheumatoid arthritis

M. B. Grk ${ }^{1}$, V. Milic ${ }^{2}$, M. G. Dusanovic Pjevic ${ }^{1}$, N. Maksimovic ${ }^{l}$, T. Damnjanovic ${ }^{l}$, M. M. Pesic ${ }^{l}$, M. Gulic ${ }^{l}$, B. Jekic ${ }^{1}$

${ }^{1}$ Institute of Human Genetics, Belgrade, Serbia, ${ }^{2}$ Institute of Rheumatology, Belgrade, Serbia

Introduction: Rheumatoid arthritis (RA) is an autoimmune disease characterised by chronic synovitis. Invasive synovial pannus overproduces matrix metalloproteinase (MMP) and leads to synovial joints impairment. Expression of $M M P$ is controlled by cytokines at the transcriptional level. Methotrexate (MTX), as anchor treatment in RA, could indirectly influence MMP production through cytokine expression modulation. Hence, $M M P-2$ promoter haplotype could affect its expression, and as a result influence disease activity and MTX response. We investigated the association of $M M P$-2 promoter haplotype with MTX monotherapy efficacy/toxicity.

Material and methods: We included 109 RA patients. MTX efficacy was estimated using the changes in Disease activity score (DAS28) after 6 months of MTX monotherapy, according to EULAR response criteria. Adverse effects (AE) were collected during the follow-up period. Bone erosion appearance was estimated after 6 months of treatment. Genotyping for $M M P-2$ gene polymorphisms (rs243866, rs243865, rs2285053) was performed using the Real Time or PCR-RFLP methods. Haplotype analysis was performed using the Haploview software.

Results: Investigated $M M P-2$ haplotypes were not associated with MTX response or overall AE. Haplotypes GCC, ATC, GCT and GTC were most frequent among our patients $(64.3 \%, 19.2 \%, 11.8 \%, 3.6 \%$, respectively). Using Confidence intervals LD method, haplotype block was defined between rs243866 and rs243865 MMP-2 gene polymorphisms $\left(\mathrm{r} 2=0.74, \mathrm{D}^{\prime}=0.94\right)$. ATC haplotype was not observed among patients with nausea $(n=9)$ while it was present in $21 \%$ of patients without this $\mathrm{AE}(\mathrm{p}=0.03)$.

Conclusion: According to our study $M M P-2$ promoter haplotype does not influence MTX therapy outcome in RA patients.

M.B. Grk: None. V. Milic: None. M.G. Dusanovic Pjevic: None. N. Maksimovic: None. T. Damnjanovic: None. M.M. Pesic: None. M. Gulic: None. B. Jekic: None.

\section{E-P19.31}

MITF variation sites associated with intermediate pigmentation phenotypes in a Brazilian population sample

L. Marcorin $^{\text {, A. L. E. Pereira }}{ }^{1}$, G. Debortoli ${ }^{2}$, M. L. G. Oliveira ${ }^{1}$, T. M. T. Carratto ${ }^{3}$, N. C. A. Fracasso ${ }^{1}$, G. Valle-Silva ${ }^{3}$, A. B. C. Silva ${ }^{3}$, E. A. Donadi,

A. L. Simões ${ }^{1}$, E. C. Castelli ${ }^{5}$, H. L. Norton ${ }^{6}$, E. J. Parra ${ }^{2}$, C. T. Mendes-Junior ${ }^{3}$

${ }^{1}$ Departamento de Genética / FMRP-USP, Ribeirão Preto, Brazil, ${ }^{2}$ Department of Anthropology / University of Toronto, Mississauga, ON, Canada, ${ }^{3}$ Departamento de Química / FFCLRP-USP, Ribeirão Preto, Brazil, ${ }^{4}$ Departamento de Clínica Médica / FMRP-USP, Ribeirão Preto, Brazil, ${ }^{5}$ Department of Pathology / FMB-UNESP, Botucatu, 
Brazil, ${ }^{6}$ Department of Anthropology / University of Cincinnati, Cincinnati, OH, United States

Introduction: DNA Phenotyping is of large interest in the forensic field since it gives an alternative for identifying physical characteristics in cases that lack suspects. Unfortunately, the tools developed so far do not perform well when predicting phenotypes in admixed populations. MITF (Melanocyte Inducing Transcription Factor) is an essential transcription factor in melanin biosynthesis, regulating the transcription of TYR, TYRP1, DCT and SLC24A5 genes, and could be a good target for finding new variants for Forensic DNA Phenotyping. Here we report associations found between variation sites at the MITF gene and pigmentation phenotypes in a Brazilian population sample.

Materials and methods: Samples were collected from 498 volunteers in Ribeirão Preto, SP. Pigmentation phenotypes and melanin index measurements (spectrophotometry) were also collected. Genotype data was obtained using a) Infinium Multi-Ethnic Global-8 (MEGA) Kit Array (Illumina) and b) target sequencing (HaloPlex Target Enrichment System For Illumina Sequencing, Agilent Technologies), followed by alignment to hg19/ GRCh37 reference genome and genotyping by Genome Analysis Tool Kit (GATK) HaplotypeCaller. Association analysis were performed using logistic regression adjusting to ancestry composition and Fisher Exact test.

Results: Two variants in linkage disequilibrium in the

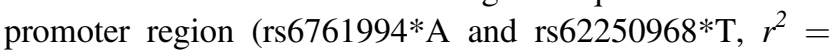
$0.698954)$ were associated with dark blond hair, green eyes and fair skin. Melanin index is significantly lower for individuals carrying these variants. Previous studies reported rs62250968 effects on regulatory motifs and on MITF expression.

Conclusions: Results showed that rs6761994 and rs62250968 are associated with intermediate phenotypes and could be good markers for DNA phenotyping in admixed populations.

L. Marcorin: None. A.L.E. Pereira: None. G. Debortoli: None. M.L.G. Oliveira: None. T.M.T. Carratto: None. N.C. A. Fracasso: None. G. Valle-Silva: None. A.B.C. Silva: None. E.A. Donadi: None. A.L. Simões: None. E.C. Castelli: None. H.L. Norton: None. E.J. Parra: None. C.T. Mendes-Junior: None.

\section{E-P19.33}

Scythian cultures genetic traces in the mitochondrial gene pool of modern Northern Eurasian populations
N. V. Ekomasova ${ }^{1,2}$, M. A. Dzhaubarmezov ${ }^{1,2}$, S. S. Litvinov ${ }^{1}$, L. R. Gabidullina ${ }^{2}$, K. Tambets ${ }^{3}$, E. K. Khusnutdinova ${ }^{1,2}$

${ }^{1}$ Institute of Biochemistry and Genetics - Subdivision of the Ufa Federal Research Centre of Russian Academy of Sciences (IBG UFRC RAS), UFA, Russian Federation, ${ }^{2}$ Bashkir State University, Ufa, Russian Federation, ${ }^{3}$ University of Tartu, Tartu, Estonia

Scythians of the Iron Age were previously described by historians and geneticists as a confederation of tribes of different origins. Nevertheless, to date it remains completely unclear whether there is a genetic continuity between the Scythian-Sarmatian archaeological culture of the Iron Age and modern population of Northern Eurasia. The aim of our study was to analyze mitochondrial DNA in 9 samples of the Scythian-Sarmatian historical and archaeological culture, followed by comparing the results with data from more than 1,000 individuals from modern Northern Eurasian populations. DNA samples from the Scythian and Sarmatian cultures were shown to belong to mtDNA haplogroups: W, W3a, T1a1, U5a2+16294, U5b2a1a2, U5b2a1a1, U5b2c, T1a1d, C1e. Screening using 1042 samples from modern populations of Northern Eurasia showed that haplogroup $\mathrm{W}$ was found in almost all analyzed samples and reached maximum in Balkar population (15.7\%) and was absent only in some ethno-territorial groups of Bashkirs. In turn, haplogroup W3 was detected only in populations of the Volga-Ural region, where it reaches its maximum among Udmurts (6.8\%) and Besermyans (5.1\%). The mtDNA haplogroup T1a1 was detected with a frequency of $1.7 \%$ in the Balkar population and a single variant in the group of Burzyansky Bashkirs (1.7\%). Haplogroup U5a2 +16294 was identified in single cases in Mordvins and Karachays, Haplogroup U5b2a1a was detected in Russians (3.8\%), and in single cases in Besermyans and Arkhangelsky Bashkirs. Haplogroups U5b2c, T1a1d, C1e were unobserved in modern populations studied. The reported study was funded by RFBR according to the research project №19-04-01195.

N.V. Ekomasova: None. M.A. Dzhaubarmezov: None. S. S. Litvinov: None. L.R. Gabidullina: None. K. Tambets: None. E.K. Khusnutdinova: None.

\section{E-P19.34}

Mitochondrial DNA in peripheral blood as a prognostic biomarker of acute respiratory distress syndrome

T. Hernandez-Beeftink ${ }^{1,2}$, B. Guillen-Guio ${ }^{l}$, H. RodríguezPérez ${ }^{1}$, I. Marcelino-Rodríguez ${ }^{1}$, J. Lorenzo-Salazar ${ }^{3}$, A. Corrales $^{1,4}$, N. Casanova ${ }^{5}$, M. González-Garay ${ }^{5}$, E. Espinosa $^{6}$, A. Muriel ${ }^{7}$, D. Domínguez ${ }^{6}$, A. García de 


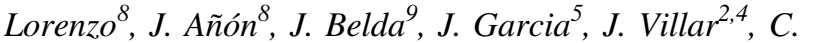
Flores $^{1,3,4}$

${ }^{1}$ Research Unit, Hospital Universitario N.S. de Candelaria, Universidad de La Laguna, Santa Cruz de Tenerife, Spain, ${ }^{2}$ Research Unit, Hospital Universitario Dr. Negrin, Las Palmas de Gran Canaria, Spain, ${ }^{3}$ Instituto Tecnológico de Energías Renovables, Granadilla, Santa Cruz de Tenerife, Spain, ${ }^{4}$ CIBER de Enfermedades Respiratorias, Instituto de Salud Carlos III, Madrid, Spain, ${ }^{5}$ Department of Medicine, The University of Arizona, Tucson, AZ, United States, ${ }^{6}$ Department of Anesthesiology, Hospital Universitario N.S. de Candelaria, Santa Cruz de Tenerife, Spain, ${ }^{7}$ Intensive Care Unit, Hospital Universitario Rio Hortega, Valladolid, Spain, ${ }^{8}$ Intensive Care Unit, Hospital Universitario La Paz, Madrid, Spain, ${ }^{9}$ Anesthesiology and Critical Care Department, Hospital Clinico Universitario of Valencia, Valencia, Spain

Introduction: Quantitative PCR measures of circulating cell-free mitochondrial DNA (mtDNA) have been associated with overall patient mortality in the Intensive Care Units (ICUs). Using an orthogonal approach to infer mtDNA levels in peripheral blood, we aimed to analyze the association with ICU mortality by acute respiratory distress syndrome (ARDS).

Material and Methods: We analyzed Axiom GenomeWide CEU1 and Axiom Genome-Wide PanAFR array data from patients with sepsis-associated ARDS: 269 Spanish from GEN-SEP study and 137 African Americans. Estimates of the mtDNA levels were obtained from the intensities of selected probes, with $100 \%$ identity with mtDNA and with the largest number of mismatches with the nuclear sequences. Values were normalized across the individual-probe intensities. Logistic regression was used for association with 28-day mortality and ICU mortality in Spanish and African Americans, respectively.

Results and conclusions: A significant association was obtained in the Spanish study $(\mathrm{OR}=1.47 ; 95 \% \mathrm{CI}=1.11$ $1.93 ; \mathrm{p}=6.98 \mathrm{e}-3)$. The result was not significant in African Americans $(\mathrm{OR}=0.98 ; 95 \% \mathrm{CI}=0.72-1.32 ; \mathrm{p}=0.88)$. Our results support that circulating mtDNA levels could be a prognostic biomarker among sepsis-associated ARDS.

Funding: Instituto de Salud Carlos III (PI14/00844; PI16/ 00049; PI17/00610; FI17/00177; FI18/00230), co-financed by the European Regional Development Funds, "A way of making Europe" from the EU; ITER agreement OA17/008 to strengthen scientific and technological education, training, research, development and innovation in Genomics, Personalized Medicine and Biotechnology.

T. Hernandez-Beeftink: None. B. Guillen-Guio: None. H. Rodríguez-Pérez: None. I. Marcelino-Rodríguez: None. J. Lorenzo-Salazar: None. A. Corrales: None. N. Casanova:
None. M. González-Garay: None. E. Espinosa: None. A. Muriel: None. D. Domínguez: None. A. García de Lorenzo: None. J. Añón: None. J. Belda: None. J. Garcia: None. J. Villar: None. C. Flores: None.

\section{E-P19.36}

Age structure of neurodegeneration onset is consistent with multistep aetiology

\section{R. L. McLaughlin}

\section{Smurfit Institute of Genetics, Dublin, Ireland}

Introduction: Most neurodegenerative diseases are highly heritable, implying genetically determined disease risk and onset. Despite this, neurodegeneration does not typically begin until later in life, with peak incidence between the sixth and eighth decades. Using an epidemiological method classically applied to cancer, a linear relationship between log-scaled incidence and log-scaled age was recently demonstrated for amyotrophic lateral sclerosis, supporting a multistep model of disease onset due to six discreet timeassociated steps (or exposures) on average. In this study, we sought to determine whether other ICD-10 classified diseases of the nervous system appear to be multistep in nature.

Materials and methods: We used least squares regression to fit the model $\log$ (incidence) $\sim(\mathrm{n}-1) \log ($ age $)$ in published estimates of age-specific incidence of neurological diseases to estimate $n$, the average number of steps required for disease onset.

Results: Onset of age-related neurodegeneration is consistent with multistep aetiology. For Parkinson's disease, the average number of steps required across both sexes in a catchment population of 2 million white Americans was $8.4\left(r^{2}=1 ; p=6.1 \times 10^{-5} ; 474\right.$ cases $)$. For Alzheimer's disease, 13.0 steps were required on average $\left(r^{2}=0.98 ; p=8.6 \times 10^{-4} ; 114\right.$ cases $)$. This contrasts with epilepsy, which showed no evidence of a multistep aetiology $\left(r^{2}=0.01 ; p=0.76 ; 501\right.$ cases from 0.9 million Icelanders).

Conclusions: This model supports the conclusion that neurodegenerative diseases begin later in life because of the accumulation of multiple time-dependent steps or exposures. These could be environmental risk factors or ageassociated biological changes in (eg) gene expression or brain cell type composition, potentially interacting with genetic risk.

R.L. McLaughlin: None.

\section{E-P19.37}

Epigenetic variability in Neuromedin $U$ gene and metabolic parameters in a cohort of Italian adults 
A. Marotta ${ }^{l}$, F. Noro ${ }^{l}$, A. Gialluisi ${ }^{l}$, R. Parisi ${ }^{l}$,

A. De Curtis ${ }^{I}$, S. Costanzo ${ }^{l}$, A. Di Castelnuovo ${ }^{1,2}$, C. Cerletti ${ }^{l}$, M. B. Donati ${ }^{l}$, G. de Gaetano ${ }^{l}$, B. Izzi $^{l}$, L. Iacoviello ${ }^{1,3}, F$. Gianfagna ${ }^{3,2}$, on behalf of Moli-sani Study Investigators

${ }^{1}$ IRCCS Neuromed, Pozzilli, Italy, ${ }^{2}$ Mediterranea Cardiocentro, Napoli, Italy, ${ }^{3}$ University of Insubria, Varese, Italy

Background: Neuromedin $\mathrm{U}(\mathrm{NmU})$ is a multifunctional neuropeptide involved in adiposity regulation. We aimed to analyse the association between epigenetic variants in gene encoding for $\mathrm{NmU}$ and metabolic phenotypes, and the potential mediating role of low-grade inflammation.

Methods: A subcohort of 1,160 subjects were randomly selected from the Italian Moli-sani cohort (2005-2010, 235years). Methylation levels at $17 \mathrm{CpG}$ sites of NMU regions 76 (promoter) and 32 (intronic) were analysed using pyrosequencing. Principal factor analysis (PFA) was performed to identify the main methylation patterns. Multivariable regressions (age, sex, smoking, alcohol and vegetables consumption as covariates) were performed to estimate the associations between resulting factors and metabolic phenotypes (BMI, waist, blood pressure, glucose, lipids and apolipoproteins $\mathrm{A}$ and $\mathrm{B}$ ). False discovery rate (FDR) was used to correct for multiple comparisons. Mediation analyses were performed using a composite index of circulating inflammation based on hsCRP, granuocyte-to-lymphocyte ratio, platelet and white blood cell count (INFLA-score).

Results: PFA on CpG sites identified two factors for each region. F1 of region 76 was significantly associated with lipid-related parameters (total cholesterol, $4.5 \pm 1.4 \mathrm{mg} / \mathrm{dL}$, $10.8 \%$ of variance explained, $\mathrm{p}=0.001 ; \mathrm{ApoB}, 0.03 \pm 0.01 \mathrm{~g} /$ $\mathrm{L}, 12.2 \%$ of variance explained, $\mathrm{p}=0.0004)$. $\mathrm{F} 1$ was also significantly associated with granulocyte-to-lymphcyte ratio, in turn associated with total cholesterol and $A p o B$ (all $\mathrm{p}<0.0001$ ) and mediated part of the total effect of $\mathrm{F} 1$ on the lipid-related phenotypes $(24.1 \%, \mathrm{p}=0.012$, and $25.4 \%$, $\mathrm{p}=0.003$ ).

Conclusion: Methylation patterns in the promoter region of the NMU gene could jointly modulate lipid-related parameters and low-grade-inflammation. Funded by Italian Ministry of Health GR2011-02351736

A. Marotta: None. F. Noro: None. A. Gialluisi: None. R. Parisi: None. A. De Curtis: None. S. Costanzo: None. A. Di Castelnuovo: None. C. Cerletti: None. M.B. Donati: None. G. de Gaetano: None. B. Izzi: None. L. Iacoviello: None. F. Gianfagna: None.

\section{E-P19.41}

Paraoxonase -1 polymorphisms L55M and Q192R in patients with recurrent spontaneous abortion
A. Kotsifaki ${ }^{l}$, S. Nasis ${ }^{l}$, K. Agiannitopoulos ${ }^{l}$, D. Mavrogianni ${ }^{2}$, P. Drakakis $^{2}$, K. Lamnissou ${ }^{l}$

${ }^{I}$ Department of Biology, National \& Kapodistrian University of Athens, Athens, Greece, ${ }^{2} 1$ st Department of Obstetrics and Gynecology, Alexandra Hospital, School of Medicine, National \& Kapodistrian University of Athens, Athens, Greece

Background: Paraoxonase-1 (PON-1) is a calcium depended antioxidant glycoprotein and one of the HDL structures. The PON-1 coding region has the two polymorphisms L55M $(163 T>C)$ and Q192R $(575 A>G)$. The purpose of the present study was to investigate the possible association between these PON-1 polymorphisms with the risk of recurrent spontaneous abortion (RSA) in a Caucasian Greek population.

Materials and methods: A total of 280 women were enrolled in this case-control study. The patient group consisted of 150 women identified as RSA patients, and the controls were 130 women with at least two successful pregnancies and without history of abortions. All individuals were genotyped for the PON-1 polymorphisms by PCR-RFLP method.

Results: Statistical analysis of the results indicated that for PON-1 L55M variant the differences of genotype or allele frequencies between RSA patient and control groups were statistically significant. In contrary, no significant differences have been observed in genotype or allele frequencies among the patient and the control groups for the PON-1 Q192R variant.

Conclusion: Our results indicated that the PON-1 polymorphism L55M (163T>C) may represent a useful biomarker of RSA susceptibility in the Greek Caucasian population and with combined effects of environmental factors might contribute to RSA prevalence.

\section{References:}

Machness M and Machness B (2015) Human paraoxonase-1 (PON1): gene structure and expression, promiscuous activities and multiple physiological roles. Gene, 2015, 567:12-21. Shunmoogam N, Naidoo P, Chilton R (2018) Paraoxonase (PON)-1: a brief overview on genetics, structure, polymorphisms and clinical relevance. Vasc Health Risk Manag 18;14:137-143.

A. Kotsifaki: None. S. Nasis: None. K. Agiannitopoulos: None. D. Mavrogianni: None. P. Drakakis: None. K. Lamnissou: None.

\section{E-P19.42}

Influence of $e N O S$ gene haplotypes and iNOS rs2297518 gene variant on severe complications and mortality in surgical patients with secondary peritonitis 
M. Gulic ${ }^{1}$, N. Maksimovic ${ }^{1}$, K. Doklestic ${ }^{2,3}$, M. Grk ${ }^{l}$, M. Svircev ${ }^{3}$, M. Dusanovic Pjevic ${ }^{1}$, M. Kulic ${ }^{3}$, I. Novakovic ${ }^{1}$

${ }^{1}$ Institute of Human Genetics, Belgrade, Serbia, ${ }^{2}$ Clinic for Emergency Surgery, Clinical Center of Serbia, Belgrade, Serbia, ${ }^{3}$ Faculty of Medicine, Belgrade, Serbia

Introduction: Secondary peritonitis is a peritoneal inflammation caused by perforation, necrosis or penetrant injuries of gastrointestinal tract. Sepsis susceptibility and poor outcome have been observed, independently from patients age, gender and comorbidities. Nitric oxide (NO) synthesized by endothelial NO (eNOS) or inducible NO (iNOS) synthase, plays significant role in pathogenesis of sepsis. INOS has been associated with NO overload and hypotension in sepsis. ENOS-derived NO is linked to the upregulation of proinflammatory cytokines and production of superoxides. This study aimed to assess the influence of eNOS haplotypes (T786C, 4b/4a, G894T polymorphisms) and iNOS rs2297518 gene variant on severe complications and mortality in surgical patients with secondary peritonitis.

Material and methods: Study included 149 surgical patients with secondary peritonitis who underwent emergency laparotomy, surgical source control, antibiotic therapy and intensive care support. We evaluated the following outcomes: septic shock, multiple organ dysfunction syndrome (MODS), mechanical ventilation and mortality. Genotyping was performed using Real-time PCR ( $r$ 22297518) or PCR-RFLP method (T786C, 4b/4a, G894T). ENOS gene haplotypes were evaluated using the Haploview 4.2 software.

Results: Patients with TbG eNOS haplotype were more frequently in ICU $(p=0.045)$ and required mechanical ventilation support $(\mathrm{p}=0.003)$ more often than patients with other haplotypes. Analysis of $r s 2297518$ using multiple logistic regression adjusted for confounding factors (age, APACHE II score and comorbidities) showed that MODS was more frequent in patients with GG genotype ( $\mathrm{p}=0.017)$.

Conclusion: Our findings suggest that $e N O S$ and $i N O S$ polymorphisms may play a role in development of severe complications in surgical patients with secondary peritonitis.

M. Gulic: None. N. Maksimovic: None. K. Doklestic: None. M. Grk: None. M. Svircev: None. M. Dusanovic Pjevic: None. M. Kulic: None. I. Novakovic: None.

\section{E-P19.45}

The rs13107325 polymorphism of the SLC39A8 gene and body mass index in two teenage, European populations.
M. Kafyra ${ }^{1,2}$, M. Stathopoulou ${ }^{l}$, S. Siest ${ }^{l}$, G. Dedoussis ${ }^{1,2}$

${ }^{1}$ University of Lorraine, Nancy, France, ${ }^{2}$ Harokopio University of Athens, Athens, Greece

Introduction: Research on the effect of genetic background on Body Mass Index (BMI) in teenagers and its potential influence on adult BMI values, has unveiled the effect of various single nucleotide polymorphisms (SNPs), including the rs13107325, in the SLC39A8 gene. The present analysis, focuses on the role of the rs13107325 SNP on the BMI of two teenage, European populations.

Materials and methods: We used data from two European cohorts; the Greek TEENAGE study (data used for $n=654$ ) and the French STANISLAS Family Study-SFS (data used for $n=509$ ). We examined the potential effect of the rs13107325 polymorphism on the z-scores (zBMI) and $\log B M I$ of the teenagers. Analyses adjusted for age and gender, were conducted via linear regression modelling in SPSS, for the TEENAGE cohort. Given that some of the teenagers of the STANISLAS cohort could belong to the same family, we tested for potential association using the $\mathrm{R}$ statistical software, the GWAF and kinship2 packages, to correct for familial resemblance.

Results: The rs13107325 polymorphism was related both to zBMI $(\beta=0.23, p=0.006)$ and $\log B M I \quad(\beta=0.01$, $\mathrm{p}=0.005$ ) of the Greek teenagers. The polymorphism did not display any significant associations, neither with zBMI $(\mathrm{p}=0,63)$, nor with $\log \mathrm{BMI}(\mathrm{p}=0.48)$, when referring to the French teenagers.

Conclusion: Genetic background plays a major part in obesity development, even from adolescence. Variations in the effect of loci display great interest and lay the foundation for the creation of genetic scores, for the prediction of the genetically-associated obesity development risk.

Funding: Région Grand Est, France - GUTENBERG chair

M. Kafyra: None. M. Stathopoulou: None. S. Siest: None. G. Dedoussis: None.

\section{E-P19.46}

Genetic Variations Associated with Smoking Relapse in a Chinese population of Taiwan: a 2-year Followup Study

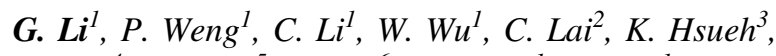

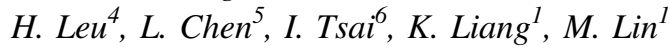

${ }^{1}$ Institute of Public Health, National Yang-Ming University, Taipei, Taiwan, ${ }^{2}$ Department of Family Medicine, Taipei Veterans General Hospital, Taipei, Taiwan, ${ }^{3}$ Department of Family Medicine, Kaohsiung Veterans General Hospital, 
Kaohsiung, Taiwan, ${ }^{4}$ Healthcare Center, Taipei Veterans General Hospital, Taipei, Taiwan, ${ }^{5}$ Department of Psychiatry, Washington University, St. Louis, MO, United States, ${ }^{6}$ Institute of Health Policy and Welfare, National Yang-Ming University, Taipei, Taiwan

Introduction: Many smokers have successful quit attempts, but it is easy to end up with smoking relapse. So far, few studies identified the susceptibility genes of smoking relapse around the world. The study was aimed to investigate the genetic variants associated with smoking relapse in Taiwan.

Materials and methods: A total of 600 current smokers having at least one quitting attempt during the 2-year follow-up were recruited. Fifteen SNPs in seven candidate genes (CHRNA3, CHRNA5, CYP2A6, CYP2B6, 5HT $2 \mathrm{~A}$, $5 \mathrm{HT}_{3 \mathrm{~A}}, 5 \mathrm{HT}_{3 \mathrm{~B}}$ ) were genotyped. Logistic regression and Cox proportional hazard models were used to explore the association between the genetic variants and smoking relapse as well as their association over time.

Results: After adjusted for gender, age, smoking durations and cigarettes per day, we found that the TC genotype of rs578776 in CHRNA3 gene compared with TT genotype $(\mathrm{OR}=0.68,95 \%$ C.I. $=0.47-0.97)$ and the CT genotype of rs518425 in CHRNA5 gene compared with $\mathrm{CC}$ genotype $(\mathrm{OR}=0.68, \quad 95 \%$ C.I. $=0.48-0.98)$ would decrease the risk of smoking relapse. Moreover, the TT genotype of rs33940208 in $5 \mathrm{HT}_{3 \mathrm{~A}}$ gene compared with CC genotype would increase the risk of smoking relapse $(\mathrm{OR}=2.80$, 95\%C.I. $=1.19-6.60)$. Smokers carrying an additional $\mathrm{T}$ allele of $\mathrm{rs} 33940208$ would relapse more easily $(\mathrm{OR}=1.40,95 \%$ C.I. $=1.05-1.86)$. However, we did not find any associations between other four genes and smoking relapse.

Conclusions: Our findings suggested that the genetic variations in CHRNA3, CHRNA5 and $5 \mathrm{HT}_{3 \mathrm{~A}}$ genes were associated with the risk of smoking relapse in a Chinese population of Taiwan.

G. Li: None. P. Weng: None. C. Li: None. W. Wu: None. C. Lai: None. K. Hsueh: None. H. Leu: None. L. Chen: None. I. Tsai: None. K. Liang: None. M. Lin: None.

\section{E-P19.48}

Can genetic determinants of brain structure be detected soon after birth?

H. Cullen ${ }^{l}$, K. Dimitrakopoulou ${ }^{2}$, D. Batalle ${ }^{l}$, O. Gale-Grant ${ }^{1}$, H. Patel ${ }^{3}$, C. Curtis ${ }^{4}$, R. Chung ${ }^{4}$, A. Schuh ${ }^{5}$, L. Cordero-Grande ${ }^{l}$, E. Hughes ${ }^{l}$, A. Price ${ }^{l}$, D. Rueckert ${ }^{6}$, J. Hajnal ${ }^{1}$, S. Smith ${ }^{7}$, A. Edwards ${ }^{l}$

${ }^{1}$ Centre for the Developing Brain, King's College London, London, United Kingdom, ${ }^{2}$ Translational Bioinformatics
Platform, NIHR Biomedical Research Centre, Guy's and St Thomas' NHS Foundation Trust and King's College London, London, United Kingdom, ${ }^{3}$ NIHR Maudsley Biomedical Research Centre, King's College London, London, United Kingdom, ${ }^{4}$ NIHR BioResource Centre Maudsley, NIHR Maudsley Biomedical Research Centre, King's College London, London, United Kingdom, ${ }^{5}$ Imperial College, London, United Kingdom, ${ }^{6}$ Biomedical ImageAnalysis Group, Department of Computing, Imperial College London, London, United Kingdom, ${ }^{7}$ Oxford University Centre for Functional MRI of the Brain, Oxford, United Kingdom

Introduction: We look at whether associations between single nucleotide polymorphisms (SNPs) and subcortical brain volumes, established in adult cohorts, are present at birth. We examine four SNP-volume associations for the putamen, pallidum, caudate and hippocampus in a neonatal cohort.

Methods: Data was obtained as part of the Developing Human Connectome Project. Our full, mixed-ancestral cohort comprised 411 term-born infants with accompanying MRI and genetic data (European subsample 202). Infant DNA was genotyped for SNPs genome-wide and standard quality control and imputation were performed. MRI images were acquired at term equivalent age. Volume estimates for the caudate, putamen, pallidum and hippocampus were extracted using a neonatal atlas. We used a linear regression model with an additive model of association for each genetic variant, including gestational age at birth, postmenstrual age at scan, intracranial volume, gender and ancestry principal components as covariates. The main analysis was with a European cohort (four imaging phenotypes, corrected p-value, $\mathrm{p}<0.0125)$. Significant results were replicated in the full mixedancestral cohort.

Results: In the European cohort there was a significant association between SNP rs945270 and putamen volume $(\mathrm{p}=0.0027, \beta=0.14, \mathrm{SE}=0.04)$ and a borderline association between SNP rs61921502 and hippocampal volume $(p=0.012, \quad \beta=-0.12, \quad S E=0.046)$. The putamen result replicated in the full mixed-ancestral cohort $\left(\mathrm{p}=3.81 \times 10^{-}\right.$ ${ }^{4}, \beta=0.12, \mathrm{SE}=0.03$ ) suggesting this relationship is robust across different ancestries at birth.

Conclusions: SNP rs945270 was significantly associated with putamen volume in a European and mixed-ancestral neonatal cohort showing the relationship previously described in adults, is present at birth.

H. Cullen: None. K. Dimitrakopoulou: None. D. Batalle: None. O. Gale-Grant: None. H. Patel: None. C. Curtis: None. R. Chung: None. A. Schuh: None. L. CorderoGrande: None. E. Hughes: None. A. Price: None. D. 
Rueckert: None. J. Hajnal: None. S. Smith: None. A. Edwards: None.

\section{E-P19.49}

Evaluation of tagged single nucleotide polymorphisms (SNPs) in identifying type 1 diabetes associated high-risk HLA-DRB1 and -DQB1 alleles in North India

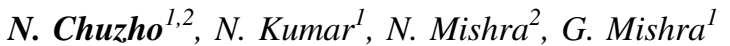 \\ ${ }^{1}$ Indian Council of Medical Research - National Institute of Pathology, New Delhi, India, ${ }^{2}$ Symbiosis School of Biological Sciences, Symbiosis International (Deemed University), Pune, India}

The human leukocyte antigen (HLA) region with its multiple genes and extreme polymorphism is known to be the greatest contributor to the genetic susceptibility to type 1 diabetes (T1D). To evaluate the risk of developing T1D, it is important to define the individual's HLA type, particularly HLA-DRB1 and -DQB1 allele. Individuals who are positive for HLA-DQA1*05:01-DQB1*02:01 (DQ2) on HLA-DRB1*03 (DR3) and DQA1*03:01-DQB1*03:02 (DQ8) on HLA-DRB1*04 (DR4) have the highest risk of developing T1D. For determining HLA-DR/ DQ types relevant to T1D, there are various HLA typing methods but most of these are time consuming and expensive Hence, we used four SNPs, namely, rs3104413, rs285427, rs9273363 and rs2187668 to identify the HLA-DR/DQ types relevant to T1D, viz., DR3/4, DR3/3, DR3/X, DR4/4, DR4/X, and $\mathrm{DRX} / \mathrm{X}$ (where $\mathrm{X} \neq \mathrm{DR} 3$ nor DR4). We genotyped 135 samples of ( $80 \mathrm{~T} 1 \mathrm{D}$ and 55 healthy controls) for the SNPs using ARMS-PCR. The PCR parameters were standardized and performed using in-house standardised protocol. Further, we validated our SNP-based HLA-DR/DQ screening using HLA-DRB1/DQB1 combi-genotyping SSP kits (BAG Healthcare, Germany). Out of the 80 T1D patients, 63 patients $(78.6 \%)$ and 10 out of 55 healthy individuals (18.18\%) were found to be positive for high-risk HLA-DRB $1 * 03-D Q B 1 * 02$ haplotype $(\mathrm{p}<0.01)$. For HLADRB1*04-DQB1*03 haplotype, 17 out of 80 T1D (23.75\%) and 8 out of 55 healthy individuals $(14.55 \%)$ were positive $(\mathrm{p}<0.01)$. Our SNP-based screening method showed $100 \%$ concordance with the kit-based method. Using our SNP-based HLA typing, we were able to predict HLA-DR/ DQ types relevant to T1D accurately in a rapid and costeffective manner.

N. Chuzho: None. N. Kumar: None. N. Mishra: None. G. Mishra: None.

\section{E-P19.50}

Genetic variation of VDR, CYP24A1 genes and relation to susceptibility to tuberculosis in Kazakhstan
U. Kozhamkulov ${ }^{l}$, M. Sadykov ${ }^{2}$, A. Azizan ${ }^{2}$,

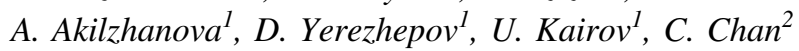

${ }^{I}$ National Laboratory Astana, Nazarbayev University, NurSultan, Kazakhstan, ${ }^{2}$ Department of Biomedical Sciences, School of Medicine, Nazarbayev University, Nur-Sultan, Kazakhstan

Introduction: Variation of a number of genes in humans has been found to play a role in TB susceptibility. A number of genes related to vitamin D metabolism were also implicated to contribute to the susceptibility to persistent TB infection among which vitamin D receptor (VDR) has been well studied. Aim of the study is to evaluate the possible role of the VDR polymorphisms and polymorphisms of the other genes involved in vitamin D pathway on TB susceptibility in Kazakhstani population.

Materials and methods: DNA samples from 84 participants were included to the current study. SNPs were genotyped on a SNP array using qualitative real-time PCR on a Life Technologies Quantstudio 12K Real Time PCR system using the cycle relative threshold (Crt) method. 11 SNPs from 8 genes of the vitamin D metabolic pathway were subsequently analyzed.

Results: We specifically analyzed 11 SNPs belonging to the following genes: DHCR7, CYP2R1, GC-1, CYP24A1, CYP27A1, CYP27B1, VDR and TNF $\alpha$. These genes play a number of different roles including synthesis, activation, delivery and binding of the activated vitamin D. Our preliminary results indicate significant association of VDR (Vitamin D receptor) SNPs (rs1544410, BsmI, with $\mathrm{OR}=0.425, \mathrm{CI}=0.221-0.816, \mathrm{p}=0.009$ and $\mathrm{rs} 731236$, Taq I with $\mathrm{OR}=0.443, \mathrm{CI}=0.228-0.859, \mathrm{p}=0.015)$ and CYP24A1 (rs6013897 with $\mathrm{OR}=0.436, \mathrm{CI}=0.191-0.996$, $\mathrm{P}=0.045$ ) with $\mathrm{TB}$.

Conclusion: Interaction of genetic variation of VDR and CYP24A1 may impact susceptibility to TB. The findings provided initial clues to understand individual genetic differences in relation to susceptibility and protection to TB. Grant references: AP05134737; MES RK.

U. Kozhamkulov: None. M. Sadykov: None. A. Azizan: None. A. Akilzhanova: None. D. Yerezhepov: None. U. Kairov: None. C. Chan: None.

\section{E-P20 Functional Genomics and Epigenomics}

\section{E-P20.1}

Borderline Personality Disorder - BION Study: Epigenetic changes observed after clinical intervention 
S. Herms ${ }^{1,2,3}$, I. Bölükbas ${ }^{4}$, M. Busmann ${ }^{5}$, U. Lang $^{5}$, M. Walter ${ }^{5}$, J. Wrege ${ }^{5}$, P. Hoffmann ${ }^{1,2,3}$, S. Cichon ${ }^{2,6}$, S. Euler

${ }^{1}$ Human Genomics Research Group, Department of Biomedicine, Basel, Switzerland, ${ }^{2}$ Institute of Medical Genetics and Pathology, University Hospital Basel, Basel, Switzerland, ${ }^{3}$ Institute of Human Genetics, University of Bonn, School of Medicine \& University Hospital Bonn, Bonn, Germany, ${ }^{4}$ Department of Psychosomatics and Psychotherapy, Psychiatric University Hospital Basel, University Basel, Basel, Switzerland, ${ }^{5}$ Department of Psychosomatics and Psychotherapy, Psychiatric University Hospital Basel, Basel, Switzerland, ${ }^{6}$ Human Genomics Research Group, Department of Biomedicine, University of Basel, Basel, Switzerland

Introduction: Borderline personality disorder (BPD) is a common psychiatric condition which is characterized by impaired social behavior and dysregulated affect and impulse control. To elucidate the genetic predictors of treatment outcome as well as to investigate epigenetic alterations in the context of treatment, we performed a study with about 300 patients. During 80 inpatient days, the treatment consisted of two weekly $45 \mathrm{~min}$ sessions of individual short- term psychodynamic psychotherapy (Leichsenring et al., 2004) and three weekly 75 min sessions of Mentalization-Based Group Therapy (Karterud, 2015).

Material / Methods: 79 samples had a complete data set of all questionnaires, two blood samplings at entry and exit of the study, and informed consent. DNA was extracted and processed on Illumina's GlobalScreeningArray 24v2-0-MD and HumanMethylationEPIC array.

Analysis: Genotype data: All samples achieved high call rates and did not show larger structural aberrations. Samples were checked for ethnicity, gender and hidden relatedness. Methylation data: Samples were checked for detection pvalue and identity using fingerprinting markers. First line methylation analysis focused on methylation beta values at entry versus exit. A more detailed analysis focused on the differences in methylation changes within a given sample.

Results: We present preliminary data on the group comparison and the more detailed per individual analysis. The most significant result in the group differential analysis is $\operatorname{cg} 23577102$ which is located in the gene LAYN. The individual based analysis revealed a region on chromosome 15 having several consecutive markers with identical changes around the gene DYX1C1.

S. Herms: None. I. Bölükbas: None. M. Busmann: None. U. Lang: None. M. Walter: None. J. Wrege: None. P. Hoffmann: None. S. Cichon: None. S. Euler: None.

\section{E-P20.3}

Liquid biopsy for human cutaneous melanoma staging. New circulating miRNAs as potential prognostic biomarkers in melanoma

B. Sánchez-Sendra ${ }^{1,2}$, J. F. González-Muñoz ${ }^{1}$, J. L. GarcíaGiménez $^{1,3,4}$, L. Navarro ${ }^{5}$, A. Murgui ${ }^{6}$, L. Terrádez ${ }^{7}, I$. Pinazo $^{8}$, J. M. Martín ${ }^{8}$, C. Monteagudo ${ }^{1,2,7}$

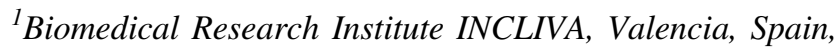
${ }^{2}$ Pathology Department, University of Valencia, Valencia, Spain, ${ }^{3}$ Centro de Investigación Biomédica en Red de Enfermedades Raras (CIBERER), Valencia, Spain, ${ }^{4}$ Physiology Department, University of Valencia, Valencia, Spain, ${ }^{5}$ Consortium Hospital General Universitario de Valencia, Valencia, Spain, ${ }^{6}$ Biochemistry and Molecular Biology Department, University of Valencia, Valencia, Spain, ${ }^{7}$ Pathology Department, Hospital Clínico Universitario de Valencia, Valencia, Spain, ${ }^{8}$ Dermatology Department, Hospital Clínico Universitario de Valencia, Valencia, Spain

Introduction: Circulating miRNAs are emerging as a new class of non-invasive biomarkers of cancer. This study aimed to identify circulating prognostic biomarkers in order to select melanoma patients at high risk of tumor progression at the time of diagnosis. We hypothesized that circulating miRNAs could provide information about melanoma progression at the time of primary tumor excision.

Materials and methods: We performed RNA-Seq on 25 plasma samples from patients with primary cutaneous melanoma. We investigated the differential expression of miRNAs in stages I-III at the time of diagnosis. Functional analysis of the involved molecular pathways was also performed.

Results: miR-182-5p was downregulated, and miR-199a5p, miR-877-3p, miR-1228-3p and miR-3613-5p upregulated in stage III in comparison with stage IA melanoma patients. Functional analysis of this set of circulating miRNAs revealed their involvement in molecular pathways related to cancer.

Conclusions: Low circulating levels of miR-182-5p, and high miR-199a-5p, miR-877-3p, miR-1228-3p and miR3613-5p levels, are associated with advanced melanoma stage at the time of primary tumor excision, and may therefore serve to recommend sentinel lymph node biopsy and to guide surveillance follow-up and imaging studies schedule for tumor staging. Our findings may serve to anticipate the stage at the moment of the initial diagnosis and, therefore, to avoid a delay in the initiation of the adjuvant therapy.

Work supported by PROMETEO II/2015/009 from Generalitat Valenciana, Spain; PI17/02019 from ISCIII, 
Spain; FEDER European funds, and Reto Everest from AECC. We want to acknowledge the INCLIVA BioBank (PT17/0015/0049 B.0000768) for its collaboration.

B. Sánchez-Sendra: None. J.F. González-Muñoz: None. J. L. García-Giménez: None. L. Navarro: None. A. Murgui: None. L. Terrádez: None. I. Pinazo: None. J.M. Martín: None. C. Monteagudo: None.

\section{E-P20.4}

Publication-Based Analysis of miR-210-Dependent biomarkers of Pre-Eclampsia

\author{
A. S. Glotov ${ }^{1,2}$, A. Tkachenko ${ }^{3,1}$, R. A. Illarionov ${ }^{4,1}$, \\ E. Vashukoval
}

${ }^{1}$ D.O. Ott Research Institute of Obstetrics, Gynecology and Reproductology, Saint-Petersburg, Russian Federation, ${ }^{2}$ St. Petersburg State University, Saint-Petersburg, Russian Federation, ${ }^{3}$ ITMO University, Saint-Petersburg, Russian Federation, ${ }^{4}$ Saint Petersburg State Technological Institute (Technical University), Saint-Petersburg, Russian Federation

Introduction: MicroRNAs are potential biomarkers of most pregnancy complications. In recent years, miR-210 has been shown as one of the main biomarkers, associated with various diseases, including pre-eclampsia (PE).

Materials and methods: We obtained miRNAs linked with preeclampsia from the manually curated pregnancy pathology database PregMiR was developed us early (https://pregmir.ott.ru/). miRNA were included in the analysis only if their differential expression between samples with PE and normal samples was significant (adjusted $\mathrm{p}<0.05$ ).

Results: We observed that miR-152, miR-1 and miR$193 \mathrm{~b}$ were only detected in studies with a changed miR-210 level, whereas miR-27a, miR-29a, miR-130a and miR-519b were detected in studies without miRNA-210 differential expression. It can be assumed that the most likely mechanisms of PE development in the early pregnancy stage are either upregulation of miR-210, miR-152, miR$518 \mathrm{~b}$ and downregulation of miR-126; or upregulation of miR-126 and downregulation of miR-182 and miR-518b. Late stages of PE are determined by miR-210, miR-152, miR-518b, miR-21, miR-155, miR-181a, miR-182, miR193b-3p, miR-517c, miR-518e (upregulation) and miR-126, miR-18a, miR-195, miR-223, let-7f (downregulation); or miR-27a, miR-29a, miR-130a and miR-519d, miR-517c, miR-518e miR-155, miR-126, miR-181a, miR-195 (upregulation) and miR-223, miR-18a, miR-182 (downregulation).

Conclusions: The presented results allow speculation about the influence of certain miRNAs on PE development in the context of the presence or absence of miR-210 differential expression, but additional experimental studies are required to evaluate the findings. This study was financially supported by Russian Scientific Foundation, grant № 19-75-20033.

A.S. Glotov: None. A. Tkachenko: None. R.A. Illarionov: None. E. Vashukova: None.

\section{E-P20.7}

C-leptin type receptor 5 and IFN-gamma gene expression and polymorphisms as markers for 17DD Yellow Fever vaccine immune response

T. Azamor ${ }^{l}$, A. M. V. Silva ${ }^{l}$, C. X. Carvalho ${ }^{l}$, L. Alvarado-Arnez ${ }^{2}$, J. G. Melgaço ${ }^{1}$, C. Bayma ${ }^{1}$, A. D. Ano

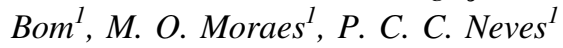

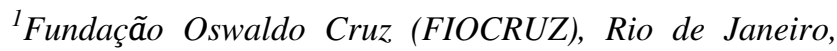
Brazil, ${ }^{2}$ Universidad Privada Franz Tamayo - UNIFRANZ, Bolivia, Cochabamba, Bolivia, Plurinational State of

Introduction: The Yellow Fever (YF) vaccine is one of the most effective ever made, although some rare serious adverse events may occur after vaccination due to genetic innate deficiency. YF primary immunization resulted in early synthesis of IFN- $\gamma$ and C-leptin type receptor 5 (CLEC-5A) is associated with severity in other flaviviral infections. Herein, we have investigated association of single nucleotide polimorphisms (SNP) in $I F N G$ and CLEC5A and vaccine efficacy, looking for elucidate the genotypic and phenotypic mechanisms that may also be correlated to post-vaccination adverse events.

Material and Methods: Blood samples from thirty volunteers vaccinated with YF17DD were collected 0,4 , 7 and 10 after immunization (DAI). RT-qPCR assay was carried out to quantify expression of CLEC5A, IFNG, IL6, RNASEL, IRF7, IRF9, STAT2 and OAS1. SNP Allelic discriminations was carried using TaqMan assays. Blood cell were stimulated with the envelope viral protein and phenotyped by flow cytometry.

Results: We observed an association between CLEC5A rs13237944 (AC/CC, $\mathrm{p}=0.04$ ), and $I F N G$ rs2069718 (AA/ TT, $\mathrm{p}=0.01)$ and $\mathrm{rs} 2430561$ (AG/AA, $\mathrm{p}=0.04)$ and high antibody levels, also presenting high expression levels of CLEC5A and IFNG mRNA respectively. Additionally we observed a phenotype polarization of CLEC5A at 4DAI both in gene expression and flow cytometry.

Conclusion: These results indicated that genetically regulated early expression of CLEC5A and IFNG after YF immunization is related to 17DD immunogenicity. The polarized profile of CLEC5A gene expression and abundance in cell surface after 17DD immunization could be 
dependent of rs13237944 genotype, indicating CLEC5A as a novel role in the response against $\mathrm{YF}$ vaccine.

T. Azamor: None. A.M.V. Silva: None. C.X. Carvalho: None. L. Alvarado-Arnez: None. J.G. Melgaço: None. C. Bayma: None. A.D. Ano Bom: None. M.O. Moraes: None. P.C.C. Neves: None.

\section{E-P21 New Treatments for Genetic Disorders}

\section{E-P21.2}

Amazing matching sequences of olive genome with human genome; results of multiple alignments

M. Senkal ${ }^{1}$, I. Akalin ${ }^{2}$

${ }^{1}$ Istanbul Medeniyet University, Faculty of Medicine, Phase IV medical student, istanbul, Turkey, ${ }^{2}$ Istanbul Medeniyet University, Department of Medical Genetics, Istanbul, Turkey

Introduction: Both cardiovascular and carcinogenic benefits of olive and its products (olive oil) have been demonstrated especially as dietary intake. Here, we were curious about whether or not there is possible forthcoming genomic benefits on the molecular basis.

Materials and methods: The olive (Olea europae, Genome ID:10724) genome sequence in the Pubmed genome database was aligned with the human genome using website NucleotidBlast. The variations of the matching sequences with nearest restriction sites were investigated in online databases such as Pubmed, PolyPhen2, Exac.browser.

Results: Multiple matched oligo-sequences (at least 28 to more than $500 \mathrm{bp}$ in length) were found in 67 genes with a similarity rate of $73-100 \%$. Among these genes, remarkable sequence matches were found in cancer related genes (BRAF, BRMSIL, SDHA) and calmodulin (CALMI, CALM3) genes. There are many pathological (Egs; rs267607276, rs1060602608, rs1064796271, rs155814427 on CALM1 gene leading to Long QT syndrome) and nonpathological variations defined on the human genome at the matched sequences, but interestingly the wild-types of them were present in the olive genome. Another notable finding was the presence of diverse restriction enzyme sites within the matching sequences.

Conclusions: Presence of wild type DNA sequences at the plant genome while the disease causing mutations reported in the similar human genome sequences give the clues of possible genomic based therapies could emerge as the phytotherapy protocols. Moreover, we wondered if oral consumption of genome carrying seeds might give rise to therapy regimens as the DNA pills. Those questions would need more detailed investigations to elucidate them.
M. Senkal: None. I. Akalin: None.

\section{E-P21.4}

The usefulness of high throughput sequencing for the diagnosis of hereditary diseases of neuromuscular system

\section{S. A. Garifullina, A. Marakhonov, S. Kutsev, R. Zinchenko}

Research Center for Medical Genetics, Moscow, Russian Federation

Hereditary diseases of the neuromuscular system represent a large group of genetically heterogeneous hereditary diseases, which are characterized by marked clinical polymorphism. High-throughput sequencing (HTS) methods allow an advanced search of genetic causes to make the most accurate diagnosis.

A family with two affected brothers was referred to the Research Center for Medical Genetics with a primary diagnosis of spinal muscular atrophy. Kids from a normal pregnancy. One of the affected children had moved with support, at the moment of examination moves with the wheelchair, mental development without abnormalities. The chest is flattened, stiffened spine with curvature, hypermobility of upper limbs, diffuse muscle atrophy, areflexia. Parents are healthy, the family has a third healthy child. Whole exome sequencing was performed for one of the probands and revealed two novel small frame-shifting deletions of 28 and 2 nucleotides affecting COL6A2 gene encoding the $\alpha 2$-chain of type VI collagen. The pathogenic variants in the COL6A2 gene are associated with Bethlem myopathy 1 and Ullrich congenital muscular dystrophy 1 . Segregation analysis in the family confirmed the compound heterozygous state in probands. Thus, Ullrich congenital muscular dystrophy was diagnosed. The marked clinical polymorphism and the presence of a significant number of genes involved in neuromuscular pathology make accurate diagnose difficult and lead to the need for a comprehensive search for the causes using HTS methods.

S.A. Garifullina: None. A. Marakhonov: None. S. Kutsev: None. R. Zinchenko: None.

\section{E-P22 Genetic Counselling / Services / Education}

\section{E-P22.01 \\ Role of Genetic Nurse in the Gastrointestinal Cancers Clinic at Sultan Qaboos University Hospital in Oman}




\section{Alhaddabi, A. AlSayegh, Z. Bruwer, K. Al-Kharusi}

SQUH, Muscat, Oman

Introduction: The cancer genetics service was introduced at Sultan Qaboos University Hospital in 2011 run by a cancer geneticist and genetic nurse. The principle goal of the clinic is to provide genetic counselling and testing to individuals affected with or at increased risk of an inherited cancer.

Aim: This study aimed to look into the number of families that were seen for gastrointestinal cancer GI during the last five years and to describe the role of the genetic nurse within the clinic.

Method: The database was reviewed to identify all GI cancer cases that were tested from 2015 till 2019 seen. The role of the genetic nurse was explored over the five year period and the involvement in clinic related procedures was documented.

Results: Two hundred and twenty patients were identified with a pathogenic variant. Predictive testing was offered to 136 family members (greatest number seen in 2018 -2019). Initially the role of the genetic nurse was limited to admin related tasks like arranging follow-up appointments and referrals to gastroenterologist. This developed to include educating affected individuals about the importance of family screening and surveillance, facilitating group sessions for family support and promoting a multidisciplinary approach with greater involvement of the GI team.

Conclusion: A large number of cases are being seen in Oman in our tertiary hospital. Predictive testing has increased exponentially over the last two years. This is likely related to the improvements that have been put in place by the cancer genetics team and genetic nurse.

I. Alhaddabi: None. A. AlSayegh: None. Z. Bruwer: None. K. Al-Kharusi: None.

\section{E-P22.03}

\section{Communicating complex diagnoses with the help of} caricatures

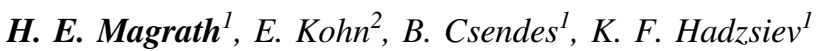 \\ ${ }^{1}$ Department of Medical Genetics, University of Pecs, Pecs, Hungary, ${ }^{2}$ Faculty of Cultural Sciences, Education and Regional Development, University of Pecs, Pecs, Hun- gary}

Effective patient-doctor communication is not only a fundamental part of patient satisfaction, but also contributes to better medicinal outcomes by building a caring relationship with patients. Communicating complex diagnoses to patients, explaining the effects of rare mutations (or even the very concept of a DNA mutation) can be challenging in medical genetics. What is obvious to us medical professionals in genetics, may not be obvious to anyone else. Having realised that words might not be the ultimate form of communication, we have started an experimental method of creating individualised, caricature-esk diagrams sometimes assembled into comic strips to explain difficult concepts to patients and their primary health care providers. Mental pictures rendered into actual images with the complete avoidance of jargon and confusing expressions are not only an effective method of communicating complex issues, but it might be a successful way of reaching out to difficult populations, such as parents with anti-vaccine beliefs.

H.E. Magrath: None. E. Kohn: None. B. Csendes: None. K.F. Hadzsiev: None.

\section{E-P22.04}

Universal, centralized, equal and fully comprehensive genetic services within an insular region of Europe: The Balearic experience

D. Heine Suñer ${ }^{1}$, N. Voloj Soffer ${ }^{2}$, V. J. Asensio Landa ${ }^{l}$, A. Obrador-Hevia ${ }^{1}$, C. Vidal Lampurdanes ${ }^{2}, J_{\text {. Rosell }}^{2}$, N. Govea ${ }^{2}$, A. Perez-Granero ${ }^{2}$, M. Bernues ${ }^{2}$, R. MartorellRiera $^{2}$, A. de Juan Socias ${ }^{2}$, M. Rosado-Muñoz ${ }^{2}$, M. PradoFarnos $^{2}$, M. Santos-Pons ${ }^{2}$, M. Montaño Mera ${ }^{2}$, M. Coll Ferrer $^{2}$, O. Exposito Perez ${ }^{2}$, C. Salleras Gelabert ${ }^{2}, B$. Ortega Vila ${ }^{2}$, R. Rodriguez Zafra ${ }^{2}$, I. Martinez-Lopez ${ }^{l}$

${ }^{1}$ Hospital Universitari Son Espases/Institut d'Investigació Sanitaria de Palma (IDISBA), Palma, Spain, ${ }^{2}$ Hospital Universitari Son Espases, Palma, Spain

Healthcare in the Balearic Islands, with a population of 1.150 .000 , is provided by a combination of public and private hospitals and medical centers. Furthermore, last year, the islands received more than 16 million tourists and many of them used the public health system during their stay, genetic tests included. The Balearic Islands Department of Health oversees and regulates the public health facilities through the Health Service (IB-Salut). In 2017, IB-Salut created the Genetics and Genomics Unit (GENIB). Its objective is to elaborate a plan of dynamic and transversal Community action and guarantee that any person affected by a genetic-based disease, at risk of suffering it in the future, at risk of transmitting it or whose health problem requires genetic or genomic analysis techniques, have equal access to clinical evaluation, genetic counseling and relevant tests. This is implemented through a centralized core Clinical Genetics and Diagnostic Department. Diagnostic tests are designed, interpreted and implemented through Molecular Committees organized by specialty where clinical cases are presented 
and the protocol to be followed is decided. The battery of tests to request follows an economy of scale and includes from traditional cytogenetics to NGS techniques such as clinical exome and complete exome. The number of applications made in 2019 for the entire Balearic community has been 7,750 . Their distribution has been $61 \%$ molecular studies; $31 \%$ cytogenetic studies and $7 \%$ family studies. The origin of the tests has been mostly Clinical Genetics (1,046) and Pediatrics (1,044), Obstetrics and Reproduction (912) and Cardiology (149).

D. Heine Suñer: None. N. Voloj Soffer: None. V.J. Asensio Landa: None. A. Obrador-Hevia: None. C. Vidal Lampurdanes: None. J. Rosell: None. N. Govea: None. A. Perez-Granero: None. M. Bernues: None. R. MartorellRiera: None. A. de Juan Socias: None. M. Rosado-Muñoz: None. M. Prado-Farnos: None. M. Santos-Pons: None. M. Montaño Mera: None. M. Coll Ferrer: None. O. Exposito Perez: None. C. Salleras Gelabert: None. B. Ortega Vila: None. R. Rodriguez Zafra: None. I. MartinezLopez: None.

\section{E-P22.07}

The thin line between the need for early diagnostic and dealing with unexpected findings

\section{M. Serban ${ }^{1}$, C. L. Serban ${ }^{1}$, A. Dobrescu ${ }^{2}$, N. Andreescu ${ }^{1}$,} A. Chiriță Emandi ${ }^{1}$, M. Puiu ${ }^{1}$

${ }^{1}$ Victor Babes University of Medicine and Pharmacy, Timisoara, Romania, ${ }^{2}$ University of Medicine and Pharmacy Craiova, Craiova, Romania

Background: NGS testing has become widely available. Clinicians are recommending testing, hoping for an early diagnosis. This may lead to early treatment; however, in some situations testing results are unexpected. We report a newborn with long QT syndrome.

Methods: Targeted NGS, with a panel of 174 gene related to cardiologic phenotype (TruSightCardio Illumina) was used. The panel includes the KCNQ1, KCNH2, SCN5A, $A N K 2, K C N E 1, K C N E 2, K C N J 2, C A C N A 1 C, C A V 3$, SCN4B, AKAP9, SNTA1, KCNJ5, CALM1 genes. Results. An eutrophic newborn male, with a 41-42 weeks gestational period, with normal evolution of neonatal jaundice, with a normal level of creatine kinase, without hypotonia was examined. During labor, a fracture of clavicula and lesion of the eye have been reported. While being monitored, a unique episode of bradycardia was observed and ECG was suggestive for long-QT syndrome. The NGS result did not indicate any genetic cause to explain patient's phenotype, but did report, as incidental finding in $R Y R 1$, with a heterozygote, probably pathogenic, NM_000540.2: c.2390G $>$ A, and VUS NM_000540.2:c.10648C $>$ T.
Pathogenic variants in RYRl gene were associated with high clinical variability and multiple phenotypes: Susceptibility to Malignant Hyperthermia (OMIM 145600), AD; Central core disease, OMIM 117000, AD, AR; KingDenborough Syndrome (OMIM 145600), AD; Minicore myopathy with external ophthalmoplegia, (OMIM 255320), AR; Congenital neuromuscular disease with uniform fiber type 1 (OMIM 117000) AD, AR. Attributing clinical significance for the variants was very difficult due to lack of phenotype details and age of patient. Thus, susceptibility for Malign Hyperthermia, was reported as main possible association.

Conclusions: Without a clear indication and a specific phenotype, genetic testing can create distress for the family.

D.M. Serban: None. C.L. Șerban: None. A. Dobrescu: None. N. Andreescu: None. A. Chiriță Emandi: None. M. Puiu: None.

\section{E-P22.08}

Noonan syndrome-molecular spectrum and genotypephenotype correlation

L. C. Popa, N. Andreescu, S. Farcas, P. Tutac,

A. Chirita-Emandi, M. Puiu

Genetics Discipline, Center of Genomic Medicine, "Victor Babes," University of Medicine and Pharmacy, Timisoara, Romania

Background: Noonan syndrome is an autosomal dominant genetic disorder, characterized by a wide spectrum of symptoms and clinical features, being characterized by short stature, skeletal malformations, hypertelorism, low-set ears, pulmonary stenosis, congenital heart defect and thorax deformations. The most frequent involved genes are PTPN11 (50\%), SOS1 (10-13\%), RAF1 (5\%), RIT1 (5\%), KRAS (less than 5\%).

Aim: This study aims to correlate the genotype with the phenotype in NS patients in order to identify the characteristic features for each gene involved.

Materials and method: 12 patients, aged 0 to 17 years old, with Noonan syndrome were diagnosed at the Timisoara Centre for Genomic Medicine. The molecular testing was performed on MiSeq platform (Illumina) using TruSight Cardio Sequencing Panel. As expected, the most commonly involved genes we found were: PTPN11 (c.1504T>G, c. $923 \mathrm{~A}>\mathrm{G}$, с. $922 \mathrm{~A}>\mathrm{G}$, c. $236 \mathrm{~A}>\mathrm{G}$, c. $188 \mathrm{~A}>\mathrm{G}$, c. $767 \mathrm{~A}>\mathrm{G})$ and SOS1 (c.512T>C, c. $1322 \mathrm{G}>\mathrm{A}$, c. $1277 \mathrm{~A}>\mathrm{G}, \mathrm{c.} 842 \mathrm{G}>\mathrm{T}$ ).

Results: The main facial features characteristic for NS, mentioned above, were present in all cases, but we also found significant clinical differences between the patient's phenotype. The patients with PTPN11 pathogenic variants showed higher prevalence in developing aortic 
regurgitation, atrial septal defect, "café au lait" spots, exophthalmia, umbilical hernia and thrombocytopenia while the patients with SOS1 were presenting hypertrophic cardiomyopathy, spastic tetra paresis, coarctation of the aorta, lymphedemas and joint laxity.

Conclusion: The variety of phenotypes in NS represent different genes with diverse mutations. The genetic variability of NS makes the establishment of a reliable genotype-phenotype correlation essential for the management of these patients.

L.C. Popa: None. N. Andreescu: None. S. Farcas: None. P. Tutac: None. A. Chirita-Emandi: None. M. Puiu: None.

\section{E-P22.09}

Opinion on genetic testing in relation to reproductive choice among Russian geneticists.

\section{G. Zobkova ${ }^{1}$, E. Baranova ${ }^{2}$, V. Izhevskaya ${ }^{3}$}

${ }^{1}$ City Clinical Hospital № 67 of Moscow Health Department, Moscow, Russian Federation, ${ }^{2}$ Federal State Budgetary Educational Institution of Further Professional Education Russian Medical Academy of Continuous Professional Education of the Ministry of Healthcare of the Russian Federation, Moscow, Russian Federation, ${ }^{3}$ Research Centre for Medical Genetics, Moscow, Russian Federation

Introduction: Respect for the autonomy of the individual and independent decision-making based on patient's informed choice is the basis of genetic counseling, especially when counseling concerns the patient's reproductive choice. The aim of this study was to elicit the opinion of Russian geneticists on genetic testing in relation to reproductive choice.

Materials and methods: For the pilot study, a questionnaire was developed containing questions for evaluating the main approach to patient counseling. The survey was attended by 30 geneticists.

Results: All 30 geneticists answered that the prevention of the birth of children with a genetic disease is extremely important for them. 27/30 specialists agreed with the statement "Before marriage, people should know if they or their partners are carriers of genetic diseases". The majority of respondents (23/30) agreed that "The government should make it mandatory to test for carriage of mutations in genes for common hereditary diseases. Less than half of the doctors (11/30) expressed their disagreement with the patient's right not to know the results of their study.

Conclusions: Results indicate some ethical issues associated with genetic counseling for patient's reproductive choices. These issues will be discussed in more detail in the following broad studies. The study was carried out with a grant from the Russian Science Foundation (project No. 19-18-00422)

G. Zobkova: None. E. Baranova: None. V. Izhevskaya: None.

\section{E-P22.10}

Students' achievements in medical genetics are linked to their state of happiness

\section{R. M. Dragotoiu, T. S. Grozescu}

Medical and Pharmacy University Carol Davila, Bucuresti, Romania

Viewing the world as having positive or negative effects on life depends on previously constructed neuronal connections, that are constantly stimulated by the memory of specific events, that generated feelings either of joy, happiness, satisfaction, love or anger, hurt, grief, guilt, etc. Medical students will have to face many exams during their training. Teaching them to leave behind all educated negative feelings about an exam, and showing them how to participate with open minds and hearts instead of fear and frustration in an examination, can make a difference in the way they perceive this examination and future ones. This paper shows a possible connection between feeling peaceful and fearless during an examination in medical genetics and respectively the better marks obtained by the participating students. At the end of the first term, in January 2019, 105 first year medical students were asked to try to relax before an open book examination. The 2 - 4 minutes long relaxation technique began with deep breathing, followed by directing the mind to a place of peace, safety, support and love, and it ended with suggesting feelings of happiness and joy, when seeing the high grade each one of them obtained. Not all students were compliant. Students' papers were evaluated and ranked to see if the usual normal distribution of the marks was still present. The results showed marks extremely skewed to the right, letting us conclude that by attentively and gradually learning to manage stress levels students could improve their academic performance.

R.M. Dragotoiu: None. T.S. Grozescu: None.

\section{E-P22.11}

Diagnosis and management of Turner syndrome at one tertiary medical center in Japan

A. Kondo ${ }^{l}$, A. Hayashi ${ }^{l}$, M. Yamasaki ${ }^{l}$, M. Morine ${ }^{l}$,

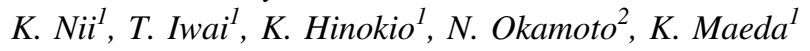

${ }^{1}$ Shikoku Medical Center for Children and Adults, Zentsuji, Japan, ${ }^{2}$ Osaka Women's and Children's Hospital, Izumi, Japan 
Background: Turner syndrome occurs in one 1,000 live female births. Generally, this condition is characterized by the partial or complete absence of one $\mathrm{X}$ chromosome. Majority of girls with Turner syndrome can be diagnosed within childhood, but about $20 \%$ might be diagnosed at adolescence period. Short stature is the most common trigger to have evaluation for those girls.

Material and Method: We looked at the patient visited to outpatient with short statue as main complaint from 2013 to 2019. Retrospective data on age at diagnosis, reason for karyotype analysis and presenting clinical features was collected from the medical records.

Result: We have seen 53 girls with short statue. All the patients had been through karyotyping as primary screening of short statue. Of those, 5 were diagnosed as Turner syndrome. 2 had other genetic condition and 10 was diagnosed as persistent short statue related small for gestational age. Most of them were diagnosed as idiopathic. All the girls with Turner syndrome have been on regular check-up with pediatrician and/or gynecologist.

Conclusion: Girls with Turner syndrome were diagnosed at early childhood. Once they are referred to large medical center, it is not difficult to find a diagnosis and also continuous treatment and check-up are feasible. Next step might be promoting height-screening for early diagnosis and education for girls with Turner syndrome for continuous medical support.

A. Kondo: None. A. Hayashi: None. M. Yamasaki: None. M. Morine: None. K. Nii: None. T. Iwai: None. K. Hinokio: None. N. Okamoto: None. K. Maeda: None.

\section{E-P23 Ethical, Legal and Psychosocial Aspects in Genetics}

\section{E-P23.1}

A "Sisyphus burden": Recurrent acute porphyrias attacks in a cohort of Brazilian patients

\section{M. Lourenco ${ }^{l}$, M. Carinhani de Cico ${ }^{l}$, A. Sanches Mateus $^{l}$, B. Forte Giacheto ${ }^{l}$, Z. Carneirol ${ }^{\text {, L. L. Vagnini }}{ }^{2}$, I. Bussman ${ }^{3}$}

${ }^{1}$ Faculdade de Medicina - Centro Universitario Estacio de Ribeirao Preto, Ribeirao Preto, Brazil, ${ }^{2}$ Centro Paulista de Diagnostico e Pesquisa, Ribeirao Preto, Brazil, ${ }^{3} A B R A P O$ Associacao Brasileira de Porfiria, Ribeirao Preto, Brazil

Introduction: Acute porphyrias are mostly autosomal dominant inborn metabolic errors of the heme biosynthetic pathway. Clinical manifestations involve central and peripheral nervous system. Patients can experience recurrent acute attacks that can be not only life-threating, but also chronically debilitating.
Material and Methods: Retrospective data from medical records and clinical questionnaires with patients followed in a reference center in Brazil Results 24 patients (20 females and four males) were enrolled. Media age of symptoms onset was 22 years (range: 12-47 years). All patients reported recurrent porphyria related symptoms, such as pain, neurological and/or psychiatric disorders, nevertheless other systemic complications as hypertension and chronic kidney disease were seen in 10 patients. The median frequency of attacks in the last year was 3 times, and the duration of every attack was 8 days. Analgesic dependency to opioid was seen in 12 patients. Heme therapy was initiated in all patients with remission of the symptoms in most of the patients for more than 6 months in its first use.

Conclusion: Our cohort of patients showed frequent recurrent attacks of acute porphyria requiring in most of them intravenous heme therapy. Although for patients with recurrent attacks prophylactic heme infusions may be benefic in remitting the symptoms, a subset of patients showed less response to this therapy overtime. Acute porphyria patients who suffer from recurrent attacks also report a low quality of life (QoL) and a negative impact on several aspects of everyday life, such as unemployment, personal relationships and long-term disability.

C.M. Lourenco: None. M. Carinhani de Cico: None. A. Sanches Mateus: None. B. Forte Giacheto: None. Z. Carneiro: None. L. Vagnini: None. I. Bussman: None.

\section{E-P23.2}

Formal approach to biosociality: autonomy and biobank informed consent

\section{R. Belyaletdinov}

Institute of Philosophy RAS; Institute of Scientific Information for Social Sciences, Moscow, Russian Federation

Introduction: The expansion of the role of genes in the treatment and diagnosis develops biosociality and requires personal reflection in case of taking decision on informed consent as far as the more it becomes known about how genes affect human health and life trajectories, the more responsible are those, who share genetic information with biobanks.

Materials and methods: Methodologically different approaches to structure of beneficence and autonomy are compared to describe model of current approaches to biobank science among professionals in Russia. Due to different research situations there are at least several types of informed consent: broad informed consent, dynamic informed consent and project specified informed consent as models for patient reflective approaches to biobank biomedical researches. The results of study of informed consent for biobanks among Russian researches is analyzed 
in the perspective of connection between science value and solidarity as social beneficence (understood as biological information), which must dominates over autonomy.

Results: The article propose view how local development of biobanks are related to process of development of technoscientific biosociality and what impact it may have on ideas about interaction of benefits and autonomy in local context of fast growing biobank system.

Conclusion: The key conclusion of the paper paradoxically informed consent (and autonomy) is formal though still important working procedure for biobanks, possibly because of biosocial value of biobanks. The study was supported by the Russian Science Foundation (project №19-18-00422).

R. Belyaletdinov: None.

\section{E-P23.3}

Development of ethical clinical conduct for expanded use of genetic diagnostic data: semi-structured expert interviews

\section{B. Vijlbrief, D. Houtman, S. Riedijk, R. Hofstra}

\section{Erasmus MC, Rotterdam, Netherlands}

Introduction: The amount of data per patient that clinical genetics departments produce is increasing, in large part due to innovations in sequencing and array technologies. In light of trends such as big data analysis and value-based healthcare, which respectively emphasize efficient data utilization and positive patient outcomes, extended use of genetic data to provide care is being discussed. This care may or may not be relevant to an initial indication. Examples include providing polygenic risk scores, certain incidental findings or pharmacogenetic passports. This study aims to explicate the values and principles of involved experts that underlie expanded use of genetic data to establish a starting point for ethical clinical conduct, and to clarify how experts think patients might benefit.

Methods: Semi-structured interviews, based on prevalent principles in medical ethics, have been conducted among 8 experts involved in expanding the scope of clinical genetics at the Erasmus Medical Center, including clinical geneticists, lab technicians, and bio-informaticians.

Results: Common principles in medical ethics (most notably autonomy, beneficence, non-maleficence and justice) prove useful in describing ethical conditions for clinical conduct, but were specified and supplemented in ways that depart from current clinical practice, and differed among experts. Motivation for expanded use of genetic data, expected patient benefit, and expectations concerning departmental responsibility showed only moderate overlap between experts.
Conclusions: Identified values and principles can provide the fundaments for developing and evaluating ethical clinical conduct in expanded genetic diagnostics, but further explication is required for convergence between experts.

B. Vijlbrief: None. D. Houtman: None. S. Riedijk: None. R. Hofstra: None.

\section{E-P23.4}

Legal and ethical issues of genetic passportization

V. D. Ruzanova, A. A. Inyushkin, E. S. Kryukova, I. S. Povarov, V. I. Belyakov

Samara National Reaserch University, Samara, Russian Federation

In the legislation of Russia, the implementation of genetic passportization and the formation of a genetic profile of the population is considered as one of the main tasks of chemical and biological safety. It is determined that such work should be carried out considering the need to protect data on the genome. There are legal provisions related to both human rights and fundamental freedoms, and the regime of genetic information as a type of personal data. This means that the legal component of the genetic passportization goes far beyond addressing the issue of creating genetic passports. According to the International Declarations on Human Rights research in this area should not prevail over respect for fundamental freedoms and dignity of people. Scientists warn against possible abuses in this area talking about the creation of two types of passports: a forensic passport and a passport that reflects risks to human health and other features of his body. We proceed from the need to develop specialized genetic passports. The main use of genetic information today is limited to medicine and forensics. Meanwhile, the possibilities in this area are immeasurably wider as soon as the use of human genetic data for non-medical purposes is also allowed by the International Declaration on Human Genetic Data. The study was funded by RFBR according to the research project № 18-29-14073.

V.D. Ruzanova: None. A.A. Inyushkin: None. E.S. Kryukova: None. I.S. Povarov: None. V.I. Belyakov: None.

\section{E-P23.5}

The principle of confidentiality in genomic research

I. S. Povarov, E. S. Kryukova, V. D. Ruzanova, A. A. Inyushkin

Samara National Research University, SAMARA, Russian Federation 
The principle of confidentiality is one of the key legal and ethical principles for building relationships between parties in many areas of public life. Its essence is that the information obtained in the course of work is not subject to deliberate or accidental disclosure, and in a situation where it is necessary to transfer it to third parties, it must be presented in a form that excludes its use against the interests of its owner. The principle of confidentiality accompanies the entire set of actions carried out with genetic information. It is inextricably linked to such legal institutions as "medical secrecy", "personal data", principle of informed consent, "autonomy of the patient's will" etc. Within the genetic research, the content and forms of manifestation of this principle have a certain specificity, which requires special attention in the legal regulation of the relevant provisions. Thus, the information obtained during genetic research is not strictly individual, since it may relate to family members of the owner of genetic information. In this regard, in many cases there is a need in making a compromise decision. This aspect leads to cases of deviation from the strict rules of confidentiality. Russian legislation requires the development of a clear and consistent algorithm for performing appropriate actions by a specialist. The study was funded by RFBR according to the research project № 18-29-14073.

I.S. Povarov: None. E.S. Kryukova: None. V.D. Ruzanova: None. A.A. Inyushkin: None.

\section{E-P23.6}

Human embryo genome editing in Russia: bioethical analysis of one case

\section{E. Grebenshchikova}

\section{INION RAS, Moscow, Russian Federation}

Introduction: The statement of the Russian researcher Denis Rebrikov about his readiness to edit the embryo genome aroused wide interest of the international scientific community. Initially, Rebrikov's research team worked with the CCR5 gene related to HIV resistance. But their methodology was different from that used by He. They tested it on abnormal embryos that could not be transferred to the uterus due to genetic defects. Then he changed his goal and decided to edit the GJB2 gene, causing hereditary deafness. He said in the media that already has several families that could take part in an experiment on editing embryos.

Materials and methods: the content analysis method was used to analyze publications in the Russian media.

Results: this problem should be considered from three perspectives: clinical ethics (normative regulation of research, ethical aspects of safety and reliability of technologies); ethical and philosophical issues ("designed baby", the emergence of new forms of social inequality, interference with the evolution of humankind, etc.); and research ethics (reputation of a scientist in the scientific world, biotechnological race, IRB).

Conclusion: a consensus of the scientific community has been reached in Russia, there are positive steps to interact with society, a law is being developed. The research was supported by the grant of the Russian Scientific Foundation, project № 19-18-00422.

E. Grebenshchikova: None.

\section{E-P23.7}

Human germline gene editing: A comparison of reports and recommendations by two leading European national ethics advisory bodies (Nuffield Council and German Ethics Council)

\section{R. Denkhaus}

\section{Zentrum für Gesundheitsethik, Hannover, Germany}

Introduction: Human germline gene editing (HGGE) has been the subject of numerous position papers by professional organisations and ethics advisory bodies. On a national level, both the British Nuffield Council and the German Ethics Council have published comprehensive reports on ethical aspects of HGGE. Analysis of the two documents may provide valuable insight into the current state of opinion-formation in Europe and help to clarify the prospect for developing a common European policy on HGGE.

Materials and methods: Analysis and comparison of the Nuffield Council's report, Genome Editing and Human Reproduction" (2018) and the German Ethics Council's opinion, Intervening in the Human Germline“ (2019).

Results: Even though public attitudes towards genetic and reproductive technologies have traditionally been much more sceptical in Germany than in the UK, conclusions on HGGE reached by the Nuffield Council and (the majority of) the German Ethics Council are rather similar. Both committees are open towards further exploration of the potential of HGGE. Differences appear when it comes to the way ethical questions on HGGE are framed, but also with regard to a number of substantial issues (e.g. embryo research or the relevance of the therapy/enhancement distinction).

Conclusion: Despite remaining differences, the two councils' reports reflect tendencies towards convergence of opinion between the UK and Germany (and maybe within Europe more generally) on genetic and reproductive technologies. Agreement on basic standards governing the use of HGGE does not seem out of reach. A moratorium on 
clinical uses of HGGE could provide time for further public debate.

R. Denkhaus: None.

\section{E-P23.8}

Evaluating the quality, readability, and scientific accuracy of sickle cell disease gene therapy online patient education materials

\section{A. Stewart ${ }^{1,2}$, K. M. Lippold, Ph.D., M.P.H $H^{3,4}$, V. L. Bonham ${ }^{l}$}

${ }^{1}$ National Human Genome Research Institute, National Institutes of Health, Bethesda, MD, United States, ${ }^{2}$ National Institutes of Health, Bethesda, MD, United States, ${ }^{3}$ Office of the Chief Medical Officer, Office of the Assistant Secretary for Health (OASH), U.S. Department of Health and Human Services (DHHS), Washington DC, DC, United States, ${ }^{4}$ Oak Ridge Institute for Science and Education (ORISE) Fellow, Oak Ridge Associated Universities, Rockville, MD, United States

Introduction: A growing number of gene therapy clinical trials for sickle cell disease (SCD) are open in Europe and North America. The SCD communities require high quality, accurate, and accessible information about novel curative genetic therapies.

Methods: Searched the world wide web (WWW) for patient education materials (PEM) on gene therapy, gene editing and curative genetic therapies for SCD. News media articles, magazines, personal stories and scientific publications were excluded. A secondary search was conducted by a medical librarian to identify additional educational materials. PEM quality was evaluated using the United States Agency for Healthcare Research and Quality (AHRQ) Patient Education Materials Assessment Tool for Print or Audiovisual materials (PEMAT-P; PEMAT-AV). The readability of PEM was assessed utilizing the FleschKincaid Grade Level (FKGL) and the Simple Measure of Gobbledygook (SMOG). Scientific Accuracy Score (SAS) was assessed by two reviewers and a gene therapy clinical expert. The assessment of the scientific accuracy included an evaluation of the risks and benefits.

Results: Authors identified on the WWW twelve patient education materials: 8 webpages, 1 infographic and 3 educational videos. Findings include FKGL, SMOG and SAS scores. PEMAT understandability scores ranged from $60-93 \%$ ( \pm 9.78$)$, average $77 \%$, and the actionability scores ranged from $0-70 \%( \pm 33.8)$, average $40 \%$.

Conclusion: Access to quality PEM about novel curative genetic therapies for SCD is a requirement for disease advocacy community engagement and study participant recruitment for ongoing clinical trials. Further development of high quality, international, and open-source PEM for gene therapy is needed.

C.A. Stewart: None. K.M. Lippold, Ph.D., M.P.H: None. V.L. Bonham: None.

\section{E-P24 Late (breaking) e-Posters}

\section{E-P24.01}

RLIM is a candidate dosage sensitive gene for individuals with varying duplications of $\mathrm{Xq13}$, intellectual disability and recognizable facial features.

E. E. Palmer ${ }^{1,2}$, R. Caroll $^{3}$, M. Shaw ${ }^{4}, R$. Kumar $^{3}$, U. Nawaz ${ }^{3}$, A. Minoche ${ }^{5}$, M. Leffler ${ }^{1}$, L. Murray ${ }^{1}$, R. Macintosh ${ }^{6}$, D. Wright ${ }^{7}$, C. Troedson ${ }^{7}$, F. McKenzie ${ }^{8}, S$.

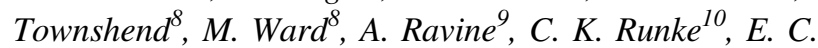
Thorland $^{10}$, M. Hummel ${ }^{11}$, N. Foulds ${ }^{12}$, O. Pichon ${ }^{13}$, B. Isidor $^{13}$, C. Le Caignec ${ }^{14}$, A. Bye ${ }^{15}$, R. Sachdev ${ }^{6}$, E. P. Kirk ${ }^{6}$, M. J. Cowley ${ }^{16}$, M. Field ${ }^{17}$, J. Gecz ${ }^{3}$

${ }^{1}$ Genetics of Learning Disability (GoLD) Service, Waratah, Australia, ${ }^{2}$ University of New South Wales, Sydney, Australia, ${ }^{3}$ Adelaide Medical School and the Robinson Research Institute, Adelaide, Australia, ${ }^{4}$ Adelaide Medical School and the Robinson Research Institute, Waratah, Australia, ${ }^{5}$ Kinghorn Centre for Clinical Genomics, Sydney, Australia, ${ }^{6}$ Sydney Children's Hospital, Randwick, Australia, ${ }^{7}$ Children's Hospital at Westmead, Sydney, Australia, ${ }^{8}$ School of Paediatrics and Child Health, University of Western Australia, Perth, Australia, ${ }^{9}$ Pathwest Laboratory Medicine WA, Perth, Australia, ${ }^{10}$ Genomics Laboratory, Department of Laboratory Medicine and Pathology, Mayo Clinic, Scottsdale, AZ, United States, ${ }^{11}$ West Virginia University School of Medicine, Morganville, WV, United States, ${ }^{12}$ Wessex Clinical Genetics Services, Southampton, United Kingdom, ${ }^{13} \mathrm{CHU}$ de Nantes, Nantes, France, ${ }^{14}$ Hopital Purpan, Toulouse, France, ${ }^{15}$ Sydney Children's Hospital, Sydney, Australia, ${ }^{16}$ Children's Cancer Institute, Sydney, Australia, ${ }^{17}$ Genetics of Learning Disability Service, WARATAH, Australia

Interpretation of the significance of maternally inherited Xchromosome variants in males with neurocognitive phenotypes continues to present a challenge to clinical geneticists and diagnostic laboratories. Here we report eleven males from eight families with copy-number gains at the Xq13.2q13.3 locus with a common facial phenotype, intellectual disability (ID), distinctive behavioral features, and a seizure disorder in two cases. All tested carrier mothers had normal intelligence. The duplication arose de novo in three mothers where grandparental testing was possible. In one family the duplication segregated with ID across three generations. $R L I M$ is the only gene common to all duplications 
investigated. It encodes a RING (Really Interesting New Gene) domain-containing zinc-finger protein with diverse cellular roles including in transcriptional regulation and ubiquitination-mediated protein degradation. Missense variants in RLIM have recently been identified to cause $\mathrm{X}$ linked ID in males, with heterozygous females typically having normal intelligence and highly skewed X-chromosome inactivation. We detected consistent and significant increase of RLIM mRNA and RLIM protein levels in cells derived from eight affected males from five families with the duplication. Subsequent analysis of MDM2, as one of the targets of the RLIM E3 ligase activity, showed its consistent downregulation in cells from the affected males. However, all the carrier mothers displayed normal RLIM mRNA levels and had highly skewed $\mathrm{X}$ inactivation. We propose duplications at Xq13.2-13.3 including RLIM cause a recognizable, but mild neurocognitive phenotype in hemizygous males.

E.E. Palmer: None. R. Caroll: None. M. Shaw: None. R. Kumar: None. U. Nawaz: None. A. Minoche: None. M. Leffler: None. L. Murray: None. R. Macintosh: None. D. Wright: None. C. Troedson: None. F. McKenzie: None. S. Townshend: None. M. Ward: None. A. Ravine: None. C.K. Runke: None. E.C. Thorland: None. M. Hummel: None. N. Foulds: None. O. Pichon: None. B. Isidor: None. C. Le Caignec: None. A. Bye: None. R. Sachdev: None. E.P. Kirk: None. M.J. Cowley: None. M. Field: None. J. Gecz: None.

\section{E-P24.02}

Cis-regulatory architecture of human ESC-derived hypothalamic neuron differentiation aids in variant-togene mapping of relevant common complex traits

S. H. Littleton ${ }^{l}$, M. C. Pahl ${ }^{l}$, C. A. Doege $e^{2}$, K. M. Hodge , M. E. Leonard ${ }^{1}$, S. Lu ${ }^{l}$, J. A. Pippin ${ }^{l}$, J. P. Bradfield ${ }^{1}$, R. K. Hammond $^{l}$, K. Boehm ${ }^{l}$, R. I. Berkowitz ${ }^{3}$, C. Lasconi ${ }^{1}$, C. $\mathrm{Su}^{l}$, A. Chesi ${ }^{1}$, M. E. Johnson ${ }^{1}$, A. D. Wells ${ }^{1,3}$, B. F. Voight ${ }^{3}$, R. L. Leibel ${ }^{2}$, D. L. Cousminer ${ }^{1,3}$, S. F. A. Grant ${ }^{1,3}$

${ }^{1}$ Children's Hospital of Philadelphia, Philadelphia, PA, United States, ${ }^{2}$ Columbia University, New York, NY, United States, ${ }^{3}$ University of Pennsylvania, Philadelphia, PA, United States

Dysregulation of hypothalamus-mediated metabolic homeostasis influences BMI, pubertal timing and sleep. However, the gene regulatory landscape of the hypothalamus remains unclear. We characterized the physical relationships among gene promoters and their corresponding cis-regulatory elements in human embryonic stem cell (ESC)-derived hypothalamic cells by integrating RNA-seq, ATAC-seq and promoter-focused Capture $\mathrm{C}$ profiles. We conducted this effort in an in vitro model of hypothalamic neurons using human ESCs at 3 stages of differentiation: ESCs, hypothalamic progenitors (HPs) and hypothalamic-like neurons (HNs). We identified 87,170 open chromatin regions (OCRs) contacting promoters in at least 1 stage of $\mathrm{HN}$ differentiation. Interestingly, we observed that regardless of gene expression pattern during differentiation, overall accessibility of interacting OCRs first increased during the transition to HP, then decreased following differentiation to HN. In order to relate these findings to GWAS data, we tested for enrichment of SNPs within promoter-interacting OCRs. We found significant associations with BMI, sleep traits, age at menarche (AAM) and major depressive disorder (MDD). We mapped these trait-associated variants to their physically interacting genes. We identified 2 putative effector genes for AAM (RPS26 and SUOX) and 1 for BMI (DHRS11), which were also supported by colocalized hypothalamus eQTLs in GTEx. Additionally, HyPrColoc analyses revealed several putative effector genes associated with $>1$ trait, including ZRANB2 for AAM, BMI and MDD, and FEZF1 for AAM and BMI. Taken together, our analyses provide insight into cis-regulatory architecture during hypothalamic development and how it may contribute to hypothalamus-associated phenotypes.

Funding: R01 HG010067, R01 HD056465, NIH/NICHD 1K99HD099330-01

S.H. Littleton: None. M.C. Pahl: None. C.A. Doege: None. K.M. Hodge: None. M.E. Leonard: None. S. Lu: None. J.A. Pippin: None. J.P. Bradfield: None. R.K. Hammond: None. K. Boehm: None. R.I. Berkowitz: None. C. Lasconi: None. C. Su: None. A. Chesi: None. M.E. Johnson: None. A.D. Wells: None. B.F. Voight: None. R.L. Leibel: None. D.L. Cousminer: None. S.F.A. Grant: None.

\section{E-P24.03}

Developmental consequences of defective ATG7mediated autophagy in humans

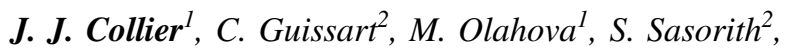 F. Suomi ${ }^{3}$, F. Piron-Prunier ${ }^{4}$, D. Zhang ${ }^{5}$, N. Martinez- Lopez $^{6}$, N. Leboucq ${ }^{7}$, A. Bahr ${ }^{8}$, S. Azzarello-Burri, ${ }^{8}$, S. Reich ${ }^{9}$, L. Schols ${ }^{9}$, T. M. Polvikoski ${ }^{10}$, P. Meyer ${ }^{11}$, L. Larrieu', A. M. Schaefer ${ }^{1}$, S. Zuchner ${ }^{12}$, I. A. Barbosa ${ }^{13}$, C. Deshpande ${ }^{14}$, A. Pyle ${ }^{1}$, A. Rauch ${ }^{8}$, M. Synofzik ${ }^{9}, M$. Ryten $^{5}$, A. Delahodde ${ }^{4}$, F. Rivier ${ }^{11}$, R. McFarland ${ }^{1}$, T. G. McWilliams $^{3}$, M. Koenig ${ }^{2}$, R. W. Taylor ${ }^{l}$}

${ }^{I}$ Wellcome Centre for Mitochondrial Research, Newcastle University, Newcastle, United Kingdom, ${ }^{2}$ EA7402 Institut Universitaire de Recherche Clinique, and Laboratoire de Génétique Moléculaire, University of Montpellier and University Hospital, Montpellier, France, ${ }^{3}$ Translational Stem Cell Biology \& Metabolism Program, Research 
Programs Unit, Faculty of Medicine, University of Helsinki, Helsinki, Finland, ${ }^{4}$ Institut de Biologie Intégrative de la Cellule, Gif-sur-Yvette, France, ${ }^{5}$ Reta Lila Weston Research Laboratories, Department of Molecular Neuroscience, University College London (UCL) Institute of Neurology, London, United Kingdom, ${ }^{6}$ Radiation oncology, Albert Einstein College of Medicine, Bronx, NY, United States, ${ }^{7}$ Neuroradiologie, CHU de Montpellier, Montpellier, France, ${ }^{8}$ Institute of Medical Genetics, University of Zurich, Zurich, Switzerland, ${ }^{9}$ Hertie-Institute for Clinical Brain Research (HIH) and Center of Neurology, University of Tubingen, Tubingen, Germany, ${ }^{10}$ Translational and Clinical Research Institute, Faculty of Medical Sciences, Newcastle University, Newcastle, United Kingdom, ${ }^{11}$ Department of Neuropédiatrie and CR Maladies Neuromusculaires, CHU de Montpellier, Montpellier, France, ${ }^{12}$ Dr. John T. Macdonald Foundation, Department of Human Genetics, Miami, FL, United States, ${ }^{13}$ Division of Genetics and Molecular Medicine, King's College London School of Medicine, Guy's Hospital, London, United Kingdom, ${ }^{14}$ Clinical Genetics Unit, Guys and St. Thomas' NHS Foundation Trust, London, United Kingdom

Background: Autophagy is the major intracellular degradation route in mammalian cells, driving the endolysosomal removal of toxic protein aggregates, damaged organelles and pathogens. Impaired autophagy has been associated with a range of complex human diseases, yet congenital core autophagy disorders are exceedingly rare.

Patients: Whole exome sequencing identified pathogenic bi-allelic variants in the novel disease gene $A T G 7$, encoding a core autophagy protein, in seven individuals from four families who display complex neurodevelopmental disorders distinguished by selective neurological, neuromuscular and endocrine dysfunction. Contrasting Atg7-null mice that demonstrate perinatal lethality, all patients investigated are now adults.

Results: $A T G 7$ variants lead to diminished levels or loss of ATG7 protein, causing severe attenuation or complete absence of autophagic reactions including LC3 lipidation in patient-derived fibroblasts and a marked reduction in starvation-induced long-lived protein degradation. Expression of mutant atg7 failed to rescue the autophagic defect displayed by $\Delta a \operatorname{tg} 7$ yeast. Skeletal muscle biopsy with no detectable ATG7 protein demonstrated myopathic changes including accumulation of p62 and focal mitochondrial dysfunction. Despite the loss of ATG7, we detected autophagosomes and autolysosomes in patient fibroblasts and muscle, in support of an alternative autophagy pathway driving their biogenesis. Moreover, RNA-sequencing of patient fibroblasts highlighted dysfunctional spinocerebellar ataxia-associated ITPRI-signalling.
Conclusion: Our study provides the first clinical, genetic and mechanistic demonstration that mutated $A T G 7$ leads to profound neurological disease in humans who are able to survive for decades in the absence of canonical autophagy, defining the importance of this pivotal pathway in human health.

J.J. Collier: None. C. Guissart: None. M. Olahova: None. S. Sasorith: None. F. Suomi: None. F. Piron-Prunier: None. D. Zhang: None. N. Martinez-Lopez: None. N. Leboucq: None. A. Bahr: None. S. Azzarello-Burri: None. S. Reich: None. L. Schols: None. T.M. Polvikoski: None. P. Meyer: None. L. Larrieu: None. A.M. Schaefer: None. S. Zuchner: None. I.A. Barbosa: None. C. Deshpande: None. A. Pyle: None. A. Rauch: None. M. Synofzik: None. M. Ryten: None. A. Delahodde: None. F. Rivier: None. R. McFarland: None. T.G. McWilliams: None. M. Koenig: None. R.W. Taylor: None.

\section{E-P24.04}

A transcriptome-wide Mendelian randomization study to dissect the molecular aetiology of autoimmune disease and cancer outcomes

\section{Prince, T. G. Richardson}

MRC Integrative Epidemiology Unit, Population Health Sciences, University of Bristol, Bristol, United Kingdom

Developing functional understanding into the causal molecular drivers of immunological disease is a critical challenge in genomic medicine. Here we use Mendelian randomization (MR) methods to investigate the effect of genetically predicted gene expression on 12 autoimmune and 4 cancer outcomes. Gene expression data from the eQTLGen consortium $(n=31,684)$ was leveraged to construct weakly correlated genetic instruments $(\mathrm{r} 2<0.1)$ for 15,392 genes. Applying the inverse-variance weighted MR method transcriptome-wide whilst accounting for linkage disequilibrium structure identified 773 unique genes with evidence of a genetically predicted effect on at least one disease outcome. We next applied genetic colocalization to investigate whether these effects may be confined to specific cell-types using data derived from 18 types of immune cells. This highlighted various cell-type specific relationships, including $P R K C Q$ and asthma which appeared to be T-cell specific, as well as $P T K 2 B$ and inflammatory bowel disease, which was restricted to monocytes and neutrophils. Furthermore, as the use of human genetics can help improve the efficacy and safety rates of drug targets, we undertook a phenome-wide MR analysis for each of these 773 genes on 335 health-related outcomes. This atlas of results can be used to prioritise which of these potential therapeutic targets is least likely to cause unanticipated adverse effects. Our 
findings provide insight into the mechanisms and biological pathways which predispose individuals to autoimmune disease and cancer. As future work we intend to apply this framework to COVID19 data to improve our capability to prevent and treat the cause of the current global pandemic.

C. Prince: None. T.G. Richardson: None.

\section{E-P24.08}

The T1D-associated IncRNA Lnc13 modulates human pancreatic $\beta$-cell inflammation by allele-specific stabilization of STAT1 mRNA.

I. Gonzalez-Moro ${ }^{1,2}$, A. Olazagoitia-Garmendia ${ }^{1,2}$, M. L. Colli ${ }^{3}$, N. Cobo-Vuilleumier ${ }^{4}$, T. S. Postler ${ }^{5}$, L. Marselli, ${ }^{6}$ P. Marchetti ${ }^{6}$, S. Ghosh ${ }^{5}$, B. R. Gauthier, ${ }^{4,7}$, D. L. Eizirik ${ }^{3,8}$, A. Castellanos-Rubio ${ }^{1,2,9}$, I. Santin ${ }^{1,2,7}$

${ }^{1}$ University of the Basque Country, Leioa, Spain, ${ }^{2}$ Biocruces Bizkaia Research Institute, Barakaldo, Spain, ${ }^{3} U L B$ Center for Diabetes Research, Brussels, Belgium, ${ }^{4}$ Andalusian Center for Molecular Biology and Regenerative Medicine, Sevilla, Spain, ${ }^{5}$ Columbia university, New York, NY, United States, ${ }^{6}$ Cisanello University Hospital, Pisa, Italy, ${ }^{7}$ CIBER de Diabetes y Enfermedades Metabólicas Asociadas (CIBERDEM, Madrid, Spain, ${ }^{8}$ Indiana Biosciences Research Institute, Indianapolis, IN, United States, ${ }^{9}$ Ikerbasque, Basque Foundation for Science, Bilbao, Spain

Introduction: Type 1 diabetes (T1D) is an autoimmune disease in which insulin-producing $\beta$-cells are specifically destroyed by the immune system. The majority of T1Dassociated polymorphisms lie in noncoding regions of the genome and many are predicted to alter the secondary structure and expression of long noncoding RNAs (lncRNAs). We performed a complete functional characterization of a lncRNA that harbors a T1D-associated SNP, namely Lnc13.

Materials and methods: Overexpression and disruption of Lncl3 were performed in the human pancreatic $\beta$-cell line EndoC- $\beta \mathrm{H} 1$ using allele-specific overexpression vectors and CRISPR-Cas9 mediated deletion. Polyinosinic: polycytidylic acid (PIC) was used to mimic a virus-related inflammatory response. RNA antisense purification (RAP) and RNA immunoprecipitation (RIP) assays were implemented to characterize the binding partners of Lncl3. In vitro experiments were further corroborated with expression analysis of human pancreatic islets.

Results: Human pancreatic islets harboring the T1Dassociated Lnc13 risk allele (rs917997*CC) showed higher STAT1 expression than islets harboring the heterozygous genotype (rs917997*CT). Upregulation of Lncl3 in pancreatic $\beta$-cells allele-specifically increased activation of the pro-inflammatory STATI pathway, correlating with increased production of chemokines. In contrast, Lnc13 disruption partially counteracted PIC-induced STAT1 and pro-inflammatory chemokine expression. In the presence of PIC, Lncl3 translocated from the nucleus to the cytoplasm acting as a linker between the 3'-UTR of STATI and the protein PCBP2, allowing the formation of a complex that stabilizes STAT1 mRNA and sustains $\beta$-cell inflammation.

Conclusions: Our results demonstrate that Lncl3 regulates virus-induced inflammation in pancreatic $\beta$-cells through a molecular mechanism that involves allele-specific activation of the STAT1 signaling pathway.

I. Gonzalez-Moro: None. A. Olazagoitia-Garmendia: None. M.L. Colli: None. N. Cobo-Vuilleumier: None. T. S. Postler: None. L. Marselli: None. P. Marchetti: None. S. Ghosh: None. B.R. Gauthier: None. D.L. Eizirik: None. A. Castellanos-Rubio: None. I. Santin: None.

\section{E-P24.09}

A transcriptome-wide Mendelian randomisation study of cardiovascular disease to prioritise genetic targets for therapeutic intervention

\section{G. M. Leyden, T. R. Gaunt, T. G. Richardson \\ University of Bristol, Bristol, United Kingdom}

Cardiovascular disease (CVD) is the leading cause of death worldwide, however its complex etiology presents a challenge for clinical intervention. Here we have applied a Mendelian randomization (MR) framework using genetically predicted variation in gene expression to prioritize candidate drug targets for the treatment of CVD.

We systematically evaluated the association between 8,851 genes each had $\geq 2$ cis-eQTLs based on results from the eQTLGen Consortium) and 5 CVD risk-factors from the UK Biobank (UKB) (body-mass index, systolic and diastolic blood pressure, low-density lipoprotein cholesterol and triglycerides). Associated genes were then analyzed using factorial MR to evaluate evidence of an additive CVD lowering effect compared to an $H M G C R$ score acting as a proxy for statin inhibition. Phenome-wide analyses were conducted to evaluate the associations with between 569 traits to elucidate potential adverse effects.

We identified 377 genetic associations with CVD risk factors $\left(\mathrm{P}<1.13 \times 10^{-6}\right)$ with factorial MR analyses highlighting 20 genetically predicted targets with evidence of a CVD lowering effect in combination with statin treatment $\left(\mathrm{P}<7.36 \times 10^{-4}\right)$. Genes such as FDFT1 were predicted to have added benefit with statin therapy $(\mathrm{OR}=0.93 ; 95 \%$ $\mathrm{CI}=0.91-0.95 ; \mathrm{P}=2.21 \times 10^{-10}$ ) but are unlikely to make a safe target based on associations with potential adverse side-effects detected in our phenome-wide analysis. In 
contrast, targets such as PRKCE provided evidence of an additive CVD lowering effect $(\mathrm{OR}=0.94 ; \mathrm{CI}=0.91-0.96$; $\mathrm{P}=1.72 \times 10^{-9}$ ) without predicted adverse effects.

Through the application of a systematic factorial MR analysis, we have prioritized (and deprioritized) potential drug targets predicted to reduce CVD risk in addition to statin therapy.

G.M. Leyden: None. T.R. Gaunt: None. T.G. Richardson: None.

\section{E-P24.10}

TBX3 acts as tissue-specific component of the Wnt/betacatenin enhanceosome

D. Zimmerli ${ }^{1,2}$, C. Borrelli ${ }^{3}$, A. Jauregi-Miguel ${ }^{* 4,5}$, S. Söderholm ${ }^{4,5}$, S. Brütsch ${ }^{1}$, N. Doumpas ${ }^{1}$, J. Reichmuth ${ }^{1}$, F. Murphy-Seiler ${ }^{6}$, M. Aguet ${ }^{6}$, K. Basler $^{1}$, A. Moor $^{3}$, C. Cantù $^{4,5}$

${ }^{1}$ Department of Molecular Life Sciences, University of Zurich, Zurich, Switzerland, ${ }^{2}$ Division of Molecular Pathology, The Netherlands Cancer Institute, Amsterdam, Netherlands, ${ }^{3}$ Institute of Molecular Cancer Research, University of Zurich, Zurich, Switzerland, ${ }^{4}$ Wallenberg Centre for Molecular Medicine, Linköping University, Linköping, Sweden, ${ }^{5}$ Department of Biomedical and Clinical Sciences, Faculty of Health Science, Linköping, Sweden, ${ }^{6}$ Swiss Institute for Experimental Cancer Research (ISREC), Ecole Polytechnique Fédérale de Lausanne (EPFL), School of Life Sciences, Lausanne, Switzerland

Introduction: Aberrant regulation of the Wnt/ $\beta$-catenin signaling pathway is a prevalent theme in cancer biology. BCL9 and PYGO, the well-known $\beta$-catenin transcriptional cofactors, have been proposed as therapeutic targets to diminish Wnt signaling output in patients with colorectal cancer (CRC).

Material and methods: We combined mouse genetics with molecular high-throughput genomic technologies on ex vivo and in vitro human cells to discover the molecular nature of CRC-causing Wnt signaling machinery.

Results: Here we find that, in CRC cells and in developing mouse forelimbs, BCL9 proteins sustain the action of $\beta$-catenin in a largely PYGO-independent manner. Our genetic analyses implied that BCL9 necessitates other interaction partners in mediating its transcriptional output. We identified the transcription factor TBX3 as a candidate tissue-specific member of the $\beta$-catenin transcriptional complex. In developing forelimbs, TBX3 and BCL9 cooccupy a large number of Wnt-responsive regulatory elements, genome-wide. Moreover, mutations in $\mathrm{Bcl} 9$ affect the expression of TBX3 targets in vivo, and modulation of TBX3 abundance impacts on Wnt target genes transcription in a $\beta$-catenin- and TCF/LEF-dependent manner. Finally, TBX3 overexpression exacerbates the metastatic potential of Wnt-dependent human CRC cells.

Conclusion: Our work implicates TBX3 as a new, context-dependent component of the $\mathrm{Wnt} / \beta$-catenin-dependent enhanceosome. Our study suggests a new paradigm in which tissue-specific co-factors might be the key to understand the spectrum of possible transcriptional outputs observed downstream of Wnt/ $\beta$-catenin signaling. Grants: Knut and Alice Wallenberg Foundation, Cancerfonden (CAN 2018/542), the Swiss National Science Foundation and the Canton of Zurich, and the Swiss National Science Foundation (grant PCEPP3_181249).

D. Zimmerli*: None. C. Borrelli*: None. A. JauregiMiguel*: None. S. Söderholm: None. S. Brütsch: None. N. Doumpas: None. J. Reichmuth: None. F. Murphy-Seiler: None. M. Aguet: None. K. Basler: None. A. Moor: None. C. Cantù: None.

\section{E-P24.11}

\section{Complex genomic rearrangements in $M E C P 2$ duplica- tion syndrome}

M. Gandhi ${ }^{1}$, D. Pehlivan ${ }^{1}$, C. M. Grochowski ${ }^{1}$, B. Suter ${ }^{2}$, J. M. Fatih ${ }^{l}$, H. Y. Zoghbi ${ }^{1}$, J. R. Lupski ${ }^{1}$,

C. M. B. Carvalhol

${ }^{1}$ Baylor College of Medicine, Houston, TX, United States, ${ }^{2}$ Texas Children's Hospital, Houston, TX, United States

MECP2 Duplication Syndrome (MDS) is a neurodevelopmental disorder caused by copy-number gains of the $M E C P 2$ gene at the $\mathrm{Xq} 28$ locus. The clinical presentation is variable, but there are several predominant features, including infantile hypotonia, severe developmental delay/ intellectual disability, recurrent respiratory infections, and epilepsy. One possible explanation for this variability is the distinct structural variants (SVs) among MDS patients at this locus, $26 \%$ of which were previously reported as complex genomic rearrangements (CGRs). To pursue a robust genotype-phenotype correlation, a combination of custom high-resolution array comparative genomic hybridization (aCGH), droplet digital PCR (ddPCR), wholegenome sequencing (WGS), and genomic optical mapping were used to resolve $M E C P 2 \mathrm{SVs}$ in $89 \mathrm{MDS}$ probands. This analysis revealed CGRs constitute $46.1 \%$ (41/89) of our cohort, including $15.7 \%$ (14/89) triplications (5/14 involving triplicated MECP2), 5.6\% (5/89) DUP-NMLDUP (two duplications separated by a copy-number neutral segment), and $10.1 \%$ (9/89) involving a translocation. De novo SVs were observed in $18.1 \%(12 / 66)$ of probands: 4 translocations, 4 terminal duplications, 3 single duplications, and 1 DUP-NML-DUP rearrangement. Interestingly, 
our data demonstrate that CGRs are observed in almost half of MDS patients, with triplications being amongst the most common copy-number finding. In 9/14 cases with triplications, MECP2 did not map within the triplicated interval. Probands who carry a de novo $M E C P 2$ duplication have a higher likelihood of carrying a translocation (4) or large terminal duplication (4), which may contribute to a more severe phenotype. Further studies are in progress to investigate genotype-phenotype correlations with molecular characterization of CGRs at this locus.

\section{NHGRI:UM1HG006542}

M. Gandhi: None. D. Pehlivan: None. C.M. Grochowski: None. B. Suter: None. J.M. Fatih: None. H.Y. Zoghbi: None. J.R. Lupski: E. Ownership Interest (stock, stock options, patent or other intellectual property); Modest; 23andme. F. Consultant/Advisory Board; Modest; Regeneron Pharmaceuticals, Novartis. C.M.B. Carvalho: None.

\section{E-P24.12}

Genetic dissection of spermatogenic arrest through exome analysis. Clinical implications for the management of azoospermic men

A. Riera-Escamilla ${ }^{1}$, D. Moreno-Mendoza ${ }^{1}, K$ Holleman $^{2}$, F. Cioppi ${ }^{3}$, F. Algaba ${ }^{4}$, M. Pybus ${ }^{5}$, C. Friedrich ${ }^{6}$, M. J. Wyrwoll ${ }^{6}$, E. Casamonti ${ }^{3}$, S. Pietroforte ${ }^{3}$, L. Nagirnaja ${ }^{7}$, A. M. Lopes ${ }^{8}$, S. Kliesch ${ }^{9}$, A. Pilatz ${ }^{10}$, D. T. Carrell ${ }^{11}$, D. F. Conrad ${ }^{7}$, E. Ars ${ }^{5}$, E. Ruiz-Castañé ${ }^{\text {, }}$, K. I. Aston ${ }^{11}$, W. M. Baarends ${ }^{2}$, F. Tüttelmann ${ }^{6}$, C. Krausz ${ }^{3}$

${ }^{1}$ Andrology Department, Fundació Puigvert, Universitat Autònoma de Barcelona, IIB-Sant Pau, Barcelona, Spain, ${ }^{2}$ Department of Developmental Biology, Erasmus MC University Medical Centre, Rotterdam, Netherlands, ${ }^{3}$ Department of Biomedical, Experimental and Clinical Sciences "Mario Serio", University of Florence, Florence, Italy, ${ }^{4}$ Pathology Section, Fundació Puigvert, Universitat Autonoma de Barcelona, Barcelona, Spain, ${ }^{5}$ Molecular Biology Laboratory, Fundació Puigvert, Instituto de Investigaciones Biomédicas Sant Pau (IIB Sant Pau), Universitat Autònoma de Barcelona, Barcelona, Spain, ${ }^{6}$ Institute of Human Genetics, University of Münster, Münster, Germany, ${ }^{7}$ Division of Genetics, Oregon National Primate Research Center, Oregon Health \& Science University, Portland, OR, United States, ${ }^{8}$ Instituto de Patologia e Imunologia Molecular da Universidade do Porto, Porto, Portugal, ${ }^{9}$ Centre of Reproductive Medicine and Andrology, Department of Clinical and Surgical Andrology, University Hospital Münster, Münster, Germany, ${ }^{10}$ Clinic for Urology, Pediatric Urology and Andrology, Justus Liebig University, Giessen, Germany, ${ }^{11}$ Andrology and IVF Laboratories, Division of Urology, Department of Surgery, University of Utah School of
Medicine, Salt Lake City, UT, United States

Introduction: Azoospermia affects $1 \%$ of men and it can be the consequence of spermatogenic maturation arrest (MA). Although the etiology of MA is likely to be of genetic origin, only 13 genes have been reported as recurrent potential causes of MA.

Material and Methods: Exome sequencing in 147 selected MA patients (discovery cohort and two validation cohorts).

Results: We identified 5 novel genes responsible for MA (ADAD2, TERB1, SHOC1, MSH4, and RAD21L1), for which mouse knockout (KO) models are concordant with the human phenotype. Four of them were validated in the two independent MA cohorts. In addition, 9 patients carried pathogenic variants in 7 previously reported genes -TEX14, DMRT1, TEX11, SYCE1, MEIOB, MEI1 and STAG3 allowing to upgrade the clinical significance of these genes for diagnostic purposes. Our meiotic studies provide novel insight into the functional consequences of the variants, supporting their pathogenic role.

Conclusions: Diagnosing complete MA based on a genetic test is clinically relevant because affected patients should not undergo invasive testis surgery (TESE). Our findings contribute substantially to the development of a pre-TESE prognostic gene panel. Wider implications include the understanding of potential genetic links between NOA and cancer predisposition, and between NOA and premature ovarian failure.

Grant References: Instituto Carlos III-FIS (FIS/FEDERPI14/01250; PI17/01822), European Commission (REPROTRAIN, project number: 289880), German Research Foundation Clinical Research Unit 'Male Germ Cells: from Genes to Function' (DFG CRU326), the National Institutes of Health (R01HD078641)

A. Riera-Escamilla: None. D. Moreno-Mendoza: None. K. Holleman: None. F. Cioppi: None. F. Algaba: None. M. Pybus: None. C. Friedrich: None. M.J. Wyrwoll: None. E. Casamonti: None. S. Pietroforte: None. L. Nagirnaja: None. A.M. Lopes: None. S. Kliesch: None. A. Pilatz: None. D.T. Carrell: None. D.F. Conrad: None. E. Ars: None. E. RuizCastañé: None. K.I. Aston: None. W.M. Baarends: None. F. Tüttelmann: None. C. Krausz: None.

\section{E-P24.13}

Diagnostic experience of whole-genome sequencing in a series of ophthalmic cases from The 100,000 Genomes Project

S. Nutan ${ }^{1}$, G. Arno ${ }^{1,2,3}$, E. Clement ${ }^{4}$, A. Buckton ${ }^{1}$, A. Lemaigre ${ }^{1}$, E. Schiff , H. Knight ${ }^{2}$, A. Gimenez ${ }^{2}$, S. Malka ${ }^{2}$, G. Wright ${ }^{2}$, N. Jurkute ${ }^{2,3}$, R. Ba-Abbad ${ }^{2,3}$, 


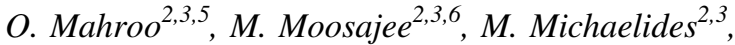
P. Yu-Wai-Man ${ }^{2,3,7}$, A. Webster ${ }^{2,3}$

${ }^{I}$ North Thames Genomic Laboratory Hub, Great Ormond Street NHS Foundation Trust, London, United Kingdom, ${ }^{2}$ Moorfields Eye Hospital NHS Foundation Trust, London, United Kingdom, ${ }^{3}$ UCL Institute of Ophthalmology, London, United Kingdom, ${ }^{4}$ Dept of Clinical Genetics, Great Ormond Street Hospital, London, United Kingdom, ${ }^{5} \mathrm{Sec}-$ tion of Ophthalmology, King's College London, London, United Kingdom, ${ }^{6}$ Ophthalmology Dept, Great Ormond Street Hospital, London, United Kingdom, ${ }^{7}$ John van Geest Centre for Brain Repair and MRC Mitochondrial Biology Unit, Department of Clinical Neurosciences, University of Cambridge, Cambridge, United Kingdom

Introduction: Current molecular diagnostic testing for inherited eye diseases (IED) is focused on targeted gene panel analysis with variable diagnostic rates. The recent completion of return of results from The 100,000 Genomes Project for Moorfields Eye hospital NHS Foundation Trust highlighted the vast potential of Whole-Genome Sequencing (WGS) to maximise diagnosis in IED.

Methods and Results: As part of the 100,000 Genomes project (pilot and main study), 1673 recruited cases were analysed. WGS analysis consisted of evaluation of potentially disease-causing variation, based on the ACMG evidence framework and application of suitable curated virtual panel(s) from PanelApp (https://panelapp. genomicsengland.co.uk/), resolving 54\% (903/1673) of cases. In an additional 17\% (291/1673) of cases, variants identified outside of this filtering strategy included noncoding, structural and those that were otherwise filtered out or outside the gene panel.

Examples included: 1) Strength of genetic evidence: e.g. curation and identification of known disease alleles in $A B C A 4$ (high frequency in control populations and those escaping standard in silico assessment) encompassed an additional 5\% of total diagnoses. 2) Strength of phenotypic evidence: e.g. NR2E3 biallelic variants and pathognomonic clinical presentation of Enhanced S-cone syndrome enabled identification of poorly annotated pathogenic variants in this gene.

Conclusion: Crucial to improving diagnostic services will be the widespread implementation of WGS and incorporating multidisciplinary team meetings. In order to maximise WGS effectiveness, expanding the analysis outside of the coding regions of the targeted gene panel and evaluating the pathogenicity of different classes of variants will be an important step forward in the postgenome age.
S. Nutan: None. G. Arno: None. E. Clement: None. A. Buckton: None. A. Lemaigre: None. E. Schiff: None. H. Knight: None. A. Gimenez: None. S. Malka: None. G. Wright: None. N. Jurkute: None. R. Ba-Abbad: None. O. Mahroo: None. M. Moosajee: None. M. Michaelides: None. P. Yu-Wai-Man: None. A. Webster: None.

\section{E-P24.15}

HDAC8: a newly identified role on mitotic fidelity and euploidy safeguard

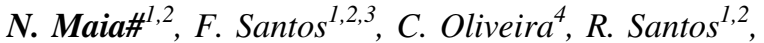 \\ B. Porto ${ }^{4}$, A. P. M. de Brouwer ${ }^{5}$, P. Jorge\# $\#^{1,2}$, \\ A. Jacome\#
}

${ }^{1}$ Unidade de Genética Molecular, Centro de Genética Médica Doutor Jacinto de Magalhães (CGMJM), Centro Hospitalar Universitário do Porto, Porto, Portugal, ${ }^{2}$ Unidade Multidisciplinar de Investigação Biomédica (UMIB), Instituto de Ciências Biomédicas Abel Salazar (ICBAS), Universidade do Porto, Porto, Portugal, ${ }^{3}$ Chromosome Instability and Dynamics Lab. (CID), Instituto de Inovação e Investigação (i3S), Universidade do Porto, Porto, Portugal, ${ }^{4}$ Laboratório Citogenética, Instituto de Ciências Biomédicas Abel Salazar (ICBAS), Universidade do Porto, Porto, Portugal, ${ }^{5}$ Department of Human Genetics, Donders Institute for Brain, Cognition and Behaviour, Radboud University Nijmegen, Nijmegen, Netherlands

HDAC8, a class I histone deacetylase is also responsible for the deacetylation of non-histone substrates, such as SMC3 located at the cohesin complex. The mechanism by which post-transductional modifications influence the mitotic apparatus and the mitotic fidelity remains poorly understood. Recent studies in mouse oocytes showed that Hdac8 controls meiotic spindle assembly. Using time-lapse imaging, we have found that HDAC8 co-localizes on spindle microtubules and the midbody, and therefore appears to be involved in chromosome alignment and accurate spindle assembly. We used HeLA cells and human-derived lymphoblastoid cell lines expressing a mutant HDAC8 allele as in vitro culture systems, both capable of recapitulating the in vivo events. These models proved to be valuable tools to understand how HDAC8 depletion affects genomic stability during mitosis. We observed that mutant HDAC8 led to the formation of anaphase bridges and lagging chromosomes, tripolar cells and inefficient chromosome congression. We found strong evidence that HDAC8 acts as genome euploidy guardian, and understanding the underlying mechanism in mitosis raised new questions. Overall, our findings indicate that HDAC8 deficiency promotes 
aneuploidy in human cells, providing some insight into the mechanisms behind HDAC8-related disorders, such as Cornelia de Lange Syndrome 5 (OMIM \#300882). Furthermore, the existence of a therapeutic HDAC8-targeted molecule paves the way for new tailored treatments. Funding: Unit for Multidisciplinary Research in Biomedicine is supported by National Funds through the Fundação para a Ciência e Tecnologia in the frameworks of the UID/ Multi/0215/2016 project -UMIB/ICBAS/UP. Nuno Maia received a research grant 2015 DEFI-CHUP, E.P.E. \#Equal contributors

N. Maia\#: None. F. Santos: None. C. Oliveira: None. R. Santos: None. B. Porto: None. A.P.M. de Brouwer: None. P. Jorge\#: None. A. Jacome\#: None.

\section{E-P24.16}

Biallelic mutations in M1AP are associated with meiotic arrest, severely impaired spermatogenesis and male infertility

C. Friedrich ${ }^{* 1}$, S. G. Temel ${ }^{2}$, L. Nagirnaja ${ }^{3}$, M. S. Oud ${ }^{4}$, A. M. Lopes $^{5,6}$, G. W. van der Heijden ${ }^{4,7}$, J. Heald ${ }^{8}$, N. Rotte ${ }^{1,9}$, J. Wistuba ${ }^{9}$, M. Wöste ${ }^{10}$, S. Ledig ${ }^{l}$, H. Krenz ${ }^{10}$, R. M. Smits ${ }^{7}$, F. Carvalho ${ }^{11,6}$, J. Gonçalves ${ }^{12,13}$, D. Fietz $^{14}$, B. Türkgenç ${ }^{15}$, M. C. Ergören ${ }^{16}$, M. Çetinkaya ${ }^{17}$, M. Başar ${ }^{18}$, S. Kahraman ${ }^{19}$, K. McEleny ${ }^{20}$, M. J. Xavier ${ }^{8}$, H. Turner $^{21}$, A. Pilatz ${ }^{22}$, A. Röpke ${ }^{1}$, M. Dugas ${ }^{10}$, S. Kliesch ${ }^{9}$, N. Neuhaus $^{9}$, GEMINI Consortium, K. I. Aston ${ }^{23}$, D. F. Conrad $^{3}$, J. A. Veltman ${ }^{4,8}$, M. J. Wyrwoll ${ }^{1}, F$. Tüttelmann ${ }^{*}$

${ }^{1}$ Institute of Human Genetics, University of Münster, Münster, Germany, ${ }^{2}$ Bursa Uludag University, Faculty of Medicine, Department of Medical Genetics \& Department of Histology \& Embryology \& Health Sciences Institute, Department of Translational Medicine, Bursa, Turkey, ${ }^{3}$ Division of Genetics, Oregon National Primate Research Center, Oregon Health \& Science University, Portland, OR, United States, ${ }^{4}$ Department of Human Genetics, Donders Institute for Brain, Cognition and Behavior, Radboud University Medical Center, Nijmegen, Netherlands, ${ }^{5}$ Instituto de Patologia e Imunologia Molecular da Universidade do Porto, Porto, Portugal, ${ }^{6}$ Instituto de Investigação e Inovação em Saúde (i3s), Universidade do Porto, Porto, Portugal, ${ }^{7}$ Department of Obstetrics and Gynecology, Radboud University Medical Center, Nijmegen, Netherlands, ${ }^{8}$ Biosciences Institute, Faculty of Medical Sciences, Newcastle University, Newcastle upon Tyne, United Kingdom, ${ }^{9}$ Centre of Reproductive Medicine and Andrology (CeRA), University Hospital Münster, Münster, Germany, ${ }^{10}$ Institute of Medical Informatics, University of Münster, Münster, Germany, ${ }^{11}$ Serviço de Genética, Departamento de Patologia, Faculdade de
Medicina da Universidade do Porto, Porto, Portugal, ${ }^{12}$ Departamento de Genética Humana, Instituto Nacional de Saúde Dr. Ricardo Jorge, Lisboa, Portugal, ${ }^{13}$ ToxOmics Centro de Toxicogenómica e Saúde Humana, Nova Medical School, Lisboa, Portugal, ${ }^{14}$ Institute of Veterinary Anatomy, Histology and Embryology, Justus Liebig University, Gießen, Germany, ${ }^{15}$ University of Acibadem, Acibadem Genetic Diagnostic Centre, Istanbul, Turkey, ${ }^{16}$ Near East University, Faculty of Medicine, Department of Medical Biology, Nicosia, Cyprus, ${ }^{17}$ Istanbul Memorial Hospital, Assisted Reproductive Technologies and Reproductive Genetics Centre, Istanbul, Turkey, ${ }^{18}$ Istanbul Memorial Hospital, Department of Urology \& Andrology, Istanbul, Turkey, ${ }^{19}$ Istanbul Memorial Hospital, Assisted Reproductive Technologies and Reproductive Genetics, Istanbul, Turkey, ${ }^{20}$ Newcastle Fertility Centre, The Newcastle upon Tyne Hospitals NHS Trust, Newcastle upon Tyne, United Kingdom, ${ }^{21}$ Newcastle upon Tyne Hospitals NHS Foundation Trust, Newcastle upon Tyne, United Kingdom, ${ }^{22}$ Clinic for Urology, Pediatric Urology and Andrology, Justus Liebig University, Gießen, Germany, ${ }^{23}$ Andrology and IVF Laboratories, Department of Surgery, University of Utah School of Medicine, Salt Lake City, UT, United States

Male infertility affects $\sim 7 \%$ of men, but its causes remain poorly understood. The most severe form is non-obstructive azoospermia (NOA), which is, in part, caused by an arrest at meiosis, but so far only few validated causal genes have been reported. To address this gap, we performed whole exome sequencing in 58 men with unexplained meiotic arrest and identified in three unrelated men the same homozygous frameshift variant c.676dup (p.Trp226LeufsTer4) in MIAP, encoding meiosis 1 arresting protein. This variant results in a truncated protein lacking $57 \%$ of its full-length as shown in vitro by heterologous expression of mutated M1AP. Next, we screened four large cohorts of 1904 infertile men from the International Male Infertility Genomics Consortium (IMIGC) and identified three additional cases carrying homozygous c.676dup and three carrying combinations of this and other likely causal variants in MIAP. Moreover, a homozygous missense variant p.(Pro389Leu) segregated with infertility in five men from a consanguineous Turkish family (LOD score $=3.28$ ). The common phenotype between all affected men was NOA, but occasionally spermatids and rarely a few spermatozoa in the semen were observed. A similar phenotype was described for mice with disruption of Mlap. Collectively, these findings demonstrate that mutations in M1AP cause autosomal recessive severe spermatogenic failure and male infertility. In view of the evidences from several independent groups and populations, MIAP should be included in the growing list of validated male infertility genes. 
This work was supported by DFG Clinical Research Unit "Male Germ Cells: from Genes to Function" (CRU326).

C. Friedrich*: None. S.G. Temel*: None. L. Nagirnaja: None. M.S. Oud: None. A.M. Lopes: None. G.W. van der Heijden: None. J. Heald: None. N. Rotte: None. J. Wistuba: None. M. Wöste: None. S. Ledig: None. H. Krenz: None. R. M. Smits: None. F. Carvalho: None. J. Gonçalves: None. D. Fietz: None. B. Türkgenç: None. M.C. Ergören: None. M. Çetinkaya: None. M. Başar: None. S. Kahraman: None. K. McEleny: None. M.J. Xavier: None. H. Turner: None. A. Pilatz: None. A. Röpke: None. M. Dugas: None. S. Kliesch: None. N. Neuhaus: None. K.I. Aston: None. D.F. Conrad: None. J.A. Veltman: None. M.J. Wyrwoll*: None. F. Tüttelmann*: None.

\section{E-P24.18}

A set of random forest models for each amino acid substitution to predict pathogenicity of missense variants in the human genome

\section{J. Takeda, K. Nanatsue, R. Yamagishi, M. Ito, K. Ohno}

Division of Neurogenetics, Center for Neurological Diseases and Cancer, Nagoya University Graduate School of Medicine, Nagoya, Japan

Introduction: When we predict the pathogenicity of a nonsynonymous single nucleotide variant (nsSNV), a radical change in amino acid (AA) properties is prone to be classified as being pathogenic. However, not all such nsSNVs are associated with human diseases.

Materials and methods: We generated random forest (RF) models individually for each AA substitution to differentiate pathogenic nsSNVs in the Human Gene Mutation Database (HGMD) and common nsSNVs in dbSNP. We named a set of our models "Individual Meta RF (InMeRF)".

Results: Ten-fold cross-validation of InMeRF showed that the area under the curves (AUCs) of receiver operating characteristic (ROC) and precision-recall (PR) curves were on average 0.941 and 0.957 , respectively. Variants of InMeRF and seven recently reported tools were generated by the same training dataset, and were compared using the same trifecta of benchmark datasets. Among the eight tools, ROC-AUC and PR-AUC of InMeRF were ranked first on average. Application of InMeRF to nsSNVs in the MUSK gene causing congenital myasthenic syndromes (CMS) showed the sensitivity and specificity of $1.000(7 / 7)$ and $0.857(12 / 14)$, respectively. With regard to the VANGL1 gene causing spina bifida, the sensitivity and specificity showed $0.933(14 / 15)$ and $1.000(8 / 8)$, respectively.

Conclusions: We showed that individual modeling each AA substitution made the performance of InMeRF superior to the previously reported tools. We made the InMeRF web service program, and also made genome-wide InMeRF scores available online at https://www.med.nagoya-u.ac.jp/ neurogenetics/InMeRF/.

Grant references

JSPS, MHLW, AMED, and NCNP.

J. Takeda: None. K. Nanatsue: None. R. Yamagishi: None. M. Ito: None. K. Ohno: None.

\section{E-P24.21}

Genetic basis of inherited retinal disease in a molecularly characterised cohort of over $\mathbf{3 0 0 0}$ families from the United Kingdom

D. A. Jaramillo-Calle ${ }^{l}$, N. Pontikos ${ }^{1,2}$, G. Arno ${ }^{l}$, N. Jurkute ${ }^{l}$, E. Schiff ${ }^{1}$, R. Ba-Abbad ${ }^{1}$, S. Malkal, A. Gimenez ${ }^{1}$, M. Georgiou ${ }^{1}$, A. T. Moore ${ }^{1}$, M. Michaelides ${ }^{1}$, A. R. Webster ${ }^{1}$, O. Mahroo ${ }^{1}$

${ }^{1}$ UCL Institute of Ophthalmology, London, United Kingdom, ${ }^{2}$ Moorfields Eye Hospital, London, United Kingdom

Introduction: In our cohort of $>3000$ molecularly characterised inherited retinal disease (IRD) families managed at the Genetics Service of Moorfields Eye Hospital (UK), we investigated proportions with disease attributable to causative variants in each gene.

Methods: We calculated proportions of families with IRD attributable to variants in each gene in the whole cohort, a cohort < 18 years, and a "current" cohort (patients seen in last two years). Additionally, we explored correlation between numbers of families and gene transcript length.

Results: We identified 3195 families with a molecular diagnosis (variants in 135 genes), including 4236 affected individuals. The paediatric cohort comprised 452 individuals from 411 families (66 genes). The current cohort comprised 2614 families (131 genes; 3130 affected individuals). The paediatric cohort showed a higher proportion of families with $\mathrm{X}$-linked disease. The 20 most frequently implicated genes accounted for $71.8 \%$ of all families (Table 1). Spearman coefficients for correlation between numbers of families and transcript length were $0.20 \quad(\mathrm{p}=0.025)$ overall, and 0.27 $(p=0.017),-0.17(p=0.46)$ and $0.71(p=0.047)$ for genes in which variants exclusively cause recessive, dominant or $\mathrm{X}$ linked disease respectively.

Conclusions: Our findings quantify the burden of inherited retinal disease attributable to each gene. Over $70 \%$ of families had pathogenic variants in one of 20 genes. Transcript length (relevant to gene delivery strategies) correlated significantly with numbers of affected families (but not for dominant disease).

Table 1: Gene prevalence in IRD cohort. 


\begin{tabular}{|c|c|}
\hline Gene & Pct of families \\
\hline$A B C A 4$ & 20.8 \\
\hline USH $2 A$ & 9.1 \\
\hline$R P G R$ & 5.1 \\
\hline PRPH2 & 4.6 \\
\hline BEST1 & 3.9 \\
\hline$R S 1$ & 3.5 \\
\hline$R P 1$ & 3.3 \\
\hline RHO & 3.3 \\
\hline CHM & 2.7 \\
\hline$C R B 1$ & 2.1 \\
\hline PRPF31 & 1.8 \\
\hline MYO7A & 1.7 \\
\hline OPA1 & 1.6 \\
\hline$C N G B 3$ & 1.4 \\
\hline RPE65 & 1.2 \\
\hline$E Y S$ & 1.2 \\
\hline$G U C Y 2 D$ & 1.2 \\
\hline PROM1 & 1.2 \\
\hline$C N G A 3$ & 1.1 \\
\hline RDH12 & 1.1 \\
\hline
\end{tabular}

D.A. Jaramillo-Calle: None. N. Pontikos: B. Research Grant (principal investigator, collaborator or consultant and pending grants as well as grants already received); Significant; Moorfields Eye Charity. G. Arno: None. N. Jurkute: None. E. Schiff: None. R. Ba-Abbad: None. S. Malka: None. A. Gimenez: None. M. Georgiou: None. A.T. Moore: None. M. Michaelides: None. A.R. Webster: None. O. Mahroo: None.

\section{E-P24.22}

The genetic architecture of left ventricular non-compaction reveals both substantial overlap with other cardiomyopathies and a distinct aetiology in a subset of cases

F. Mazzarotto ${ }^{1}$, M. H. Hawley ${ }^{2}$, M. Beltrami ${ }^{3}$, L. Beekman $^{4}$, A. de Marvao ${ }^{5}$, K. A. McGurk ${ }^{5}$, B. Statton ${ }^{5}$, B. Boschi ${ }^{3}$, F. Girolami $^{6}$, A. M. Roberts ${ }^{5}$, E. M. Lodder ${ }^{4}$, M. Allouba ${ }^{7}$, S. Romeih $^{7}$, Y. Aguib ${ }^{7}$, A. Baksi ${ }^{5}$, A. Pantazis ${ }^{8}$, S. K. Prasad ${ }^{5}$, E. Cerbai , M. H. Yacoub , D. P. O'Regan ${ }^{5}$, S. A. Cook ${ }^{10}$, J. S. Ware ${ }^{5}$, B. Funke ${ }^{11}$, I. Olivotto ${ }^{9}$, C. R. Bezzina ${ }^{4}$, P. J. R. Barton $^{5}$, R. Walsh

${ }^{1}$ University of Florence - Department of Experimental and Clinical Medicine, Florence, Italy, ${ }^{2}$ Laboratory for Molecular Medicine, Cambridge, MA, United States, ${ }^{3}$ Careggi University Hospital, Florence, Italy, ${ }^{4}$ Amsterdam UMC, Amsterdam, Netherlands, ${ }^{5}$ Imperial College London, London, United Kingdom, ${ }^{6}$ Meyer University Hospital, Florence, Italy, ${ }^{7}$ Aswan Heart Centre, Aswan, Egypt, ${ }^{8}$ Royal
Brompton and Harefield NHS Foundation Trust, London, United Kingdom, ${ }^{9}$ University of Florence, Florence, Italy, ${ }^{10}$ National Heart Centre Singapore, Singapore, Singapore, ${ }^{11}$ Harvard Medical School/Massachusetts General Hospital, Boston, MA, United States

Background: Left ventricular non-compaction (LVNC) is characterised by myocardial hypertrabeculation and is subject to considerable conjecture in the extent to which it represents a distinct pathology, a secondary phenotype associated with other cardiac diseases (particularly cardiomyopathies), or physiological adaptation.

Methods: We performed rare variant association analysis of 840 LVNC cases and 125,748 gnomAD population controls, and compared results to similar analyses on dilated cardiomyopathy (DCM) and hypertrophic cardiomyopathy (HCM).

Results: We observed substantial overlap in genes and variant classes associated with LVNC and with DCM/ HCM, indicating that LVNC often represent a secondary phenotype occurring in certain patients with DCM or HCM. In contrast, truncating variants (TV) in $M Y H 7, A C T N 2$ and PRDM16 were uniquely associated with LVNC and may reflect a distinct LVNC aetiology. MYH7 TV, generally considered non-pathogenic for cardiomyopathies, were 20fold enriched in LVNC cases over controls. Carriers of MYH7 TV in the UK Biobank and healthy volunteers also displayed significantly greater non-compaction compared to matched controls, with $50 \%$ meeting the diagnostic criteria for LVNC. Large deletions in RYR2 and missense variants in the transmembrane region of $\mathrm{HCN} 4$ were also enriched in LVNC, supporting prior reports of association with arrhythmogenic LVNC phenotypes.

Conclusions: While LVNC is characterised by substantial genetic overlap with DCM and HCM, it can also present with a distinct genetic profile involving TV in $M Y H 7$, ACTN2 and PRDM16 and with variants associated with joint LVNC/arrhythmia phenotypes. These results will enhance the application of LVNC genetic testing and help to distinguish pathological from physiological noncompaction.

F. Mazzarotto: None. M.H. Hawley: None. M. Beltrami: None. L. Beekman: None. A. de Marvao: None. K.A. McGurk: None. B. Statton: None. B. Boschi: None. F. Girolami: None. A.M. Roberts: None. E.M. Lodder: None. M. Allouba: None. S. Romeih: None. Y. Aguib: None. A. Baksi: None. A. Pantazis: None. S.K. Prasad: None. E. Cerbai: None. M.H. Yacoub: None. D.P. O'Regan: None. S.A. Cook: Other; Significant; Enleofen Bio PTE Ltd. J.S. Ware: D. Speakers Bureau/Honoraria (speakers bureau, symposia, and expert witness); Modest; MyoKardia. B. Funke: None. I. Olivotto: B. Research Grant (principal 
investigator, collaborator or consultant and pending grants as well as grants already received); Significant; MyoKardia. D. Speakers Bureau/Honoraria (speakers bureau, symposia, and expert witness); Modest; MyoKardia. C.R. Bezzina: None. P.J.R. Barton: None. R. Walsh: None.

\section{E-P24.23}

\section{Reverse regression increases power for detecting trans- eQTLs}

\author{
S. Banerjee ${ }^{l}$, F. L. Simonetti ${ }^{l}$, K. E. Detrois ${ }^{2}$, A. Kaphle ${ }^{l}$, \\ R. Mitra $^{3}$, R. Nagial ${ }^{3}$, J. Söding ${ }^{1}$
}

${ }^{1}$ Max Planck Institute for Biophysical Chemistry, Gottingen, Germany, ${ }^{2}$ George August University, Gottingen, Germany, ${ }^{3}$ Indian Institute of Technology, Kanpur, India

Trans-acting expression quantitative trait loci (trans-eQTLs) are SNPs regulating the expression of distant target genes $(>1 \mathrm{Mb})$. Trans-eQTLs account for $\geq 70 \%$ heritability of gene expression levels and have great potential to uncover the underlying biological mechanisms of complex diseases. However, trans-eQTLs are more challenging to identify than locally-acting cis-eQTLs because of their small effect sizes and the severe multiple-testing burden. Furthermore, strong gene expression correlations entails strong correlations among SNP-gene association P-values. Our method Tejaas can discover trans-eQTLs by performing L2-regularized 'reverse' multiple regression of each SNP on all gene expression levels, aggregating evidence from many small trans-effects while being unaffected by the strong expression correlations. Tejaas, coupled with a novel knearest neighbors confounder correction, discovers 18851 unique trans-eQTLs across 49 tissues from the GTEx(v8) data. They are enriched to occur in various functional regions of the genome, including DHS sites (1.32x enrichment), open chromatin (1.19x), enhancer (1.31x) and promoter $(1.10 \mathrm{x})$ regions. They overlap with known ciseQTLs (1.24x enrichment) from the GTEx analysis and are $1.09 \mathrm{x}$ enriched to be located within $100 \mathrm{~kb}$ of known transcription factors. They are also enriched in reporter assay QTL regions of K562 (1.79x) and HepG2 (3.29x) cells indicating regulatory activity. Many trans-eQTLs overlap with disease-associated SNPs from GWAS, revealing tissue-specific transcriptional regulation mechanisms that drive disease etiology. For example, trans-eQTLs discovered in whole blood show enrichment in GWAS for blood traits, while heart and aorta have enrichment in cardiometabolic traits. Thyroid and pancreas trans-eQTLs show enrichments for endocrine system diseases. Tejaas is open-source and available online (https://github.com/ soedinglab/tejaas).
S. Banerjee: None. F.L. Simonetti: None. K.E. Detrois: None. A. Kaphle: None. R. Mitra: None. R. Nagial: None. J. Söding: None.

\section{E-P24.24}

TERT Hypermethylated Oncogenic Region, A new player in the activation of the $h T E R T$ gene in cutaneous T-cell lymphomas.

A. Chebly ${ }^{1,2}$, J. Ropio ${ }^{1,3}$, J. Peloponese ${ }^{4}$, S. Poglio ${ }^{1}$, M. Prochazkova-Carlotti ${ }^{l}, F$. Cherrier ${ }^{l}$, J. Ferrer ${ }^{1}$, Y. Idrissi ${ }^{1}$,E. Segal-Bendirdjian ${ }^{5}$,E. Chouery ${ }^{2}$, C. Farra ${ }^{2,6}$, A. Pham-Ledard ${ }^{1,7}$, M. Beylot-Barry ${ }^{1,7}$, J. Merlio ${ }^{1,8}, R$. Tomb $^{2,9}$, E. Chevret ${ }^{1}$

${ }^{1}$ Bordeaux University, INSERM U1053 Bordeaux Research in Translational Oncology (BaRITOn), Cutaneous Lymphoma Oncogenesis Team, Bordeaux, France, ${ }^{2}$ Saint Joseph University, Faculty of Medicine, Medical Genetics Unit (UGM), Beirut, Lebanon, ${ }^{3}$ Porto University, Institute of Biomedical Sciences of Abel Salazar, Instituto de Investigação e Inovação em Saúde, Institute of Molecular Pathology and Immunology (Ipatimup), Cancer Biology group, Porto, Portugal, ${ }^{4}$ University of Montpellier, CNRS IRIM-UMR 9004, Research Institute in Infectiology of Montpellier, Montpellier, France, ${ }^{5}$ INSERM UMR-S 1124, Team: Cellular Homeostasis Cancer and Therapies, Université de Paris, Paris, France, ${ }^{6}$ Saint Joseph University, Faculty of Medicine, Genetics Department, Beirut, Lebanon, ${ }^{7}$ Bordeaux University Hospital Center, Dermatology Department, Bordeaux, France, ${ }^{8}$ Bordeaux University Hospital Center, Tumor Bank and Tumor Biology Laboratory, Pessac, France, ${ }^{9}$ Saint Joseph University, Faculty of Medicine, Dermatology Department, Beirut, Lebanon

Cutaneous T-cell lymphomas (CTCL) encompass a heterogeneous group of $\mathrm{T}$ lymphoproliferative disorders. We have previously shown that CTCL are telomerase positive tumors expressing $h T E R T$ in which neither amplification nor promoter hotspot mutations could explain the hTERT re-expression. Since the $h T E R T$ promoter region is rich in CpG islands, we investigated the contribution of DNA methylation in hTERT expression. We compared hTERT promoter methylation status in CTCL cell lines, Sézary syndrome (SS) patient-derived cells, fresh SS patient cells, and in normal $\mathrm{CD} 4+$ and $\mathrm{CD} 34+$ cells from healthy donors. Bisulfite Sanger sequencing revealed in tumor cells a common methylation pattern encompassing a hypermethylated distal region from $-650 \mathrm{bp}$ to $-150 \mathrm{bp}$ and a hypomethylated proximal region from $-150 \mathrm{bp}$ to $+150 \mathrm{bp}$ (relatively to the transcription start site). Interestingly, the identified hypermethylated region in CTCL cells matches 
with the recently described TERT Hypermethylated Oncogenic Region (THOR) reported to be associated with telomerase reactivation in many cancers, but so far not studied in lymphomas. Moreover, we assessed the effect on THOR methylation of two histone deacetylase inhibitors (HDACi), romidepsin and vorinostat, both approved for the treatment of CTCL patients. Overall, our results show the contribution of THOR hypermethylation in hTERT re-expression in neoplastic CTCL cells and provide new insights into the effects of epigenetic drugs (HDACi) on THOR methylation for the first time in lymphomas.

A. Chebly: A. Employment (full or part-time); Significant; Saint Joseph University of Beirut, Faculty of Medecine, Medical Genetics Laboratory. B. Research Grant (principal investigator, collaborator or consultant and pending grants as well as grants already received); Modest; Hubert Curien Partnership (PHC-CEDRE), ERASMUS plus scholarships. J. Ropio: B. Research Grant (principal investigator, collaborator or consultant and pending grants as well as grants already received); Modest; Hubert Curien Partnership (PHC-PESSOA, PAUILF), ERASMUS plus scholarships. J. Peloponese: None. S. Poglio: None. M. Prochazkova-Carlotti: None. F. Cherrier: None. J. Ferrer: None. Y. Idrissi: None. E. Segal-Bendirdjian: B. Research Grant (principal investigator, collaborator or consultant and pending grants as well as grants already received); Significant; Ligue Nationale contre le Cancer, National Center for Scientific Research (CNRS). E. Chouery: None. C. Farra: None. A. Pham-Ledard: None. M. Beylot-Barry: None. J. Merlio: None. R. Tomb: None. E. Chevret: B. Research Grant (principal investigator, collaborator or consultant and pending grants as well as grants already received); Significant; National Institute of Health and Medical Research (INSERM), French Society of Dermatology (SFD).

\section{E-P24.26}

Cross-population genetic variation of loci identified by Genome-Wide Association Studies conducted in British participants of European-descent from the UK Biobank

\section{A. De Lillo ${ }^{l}$, S. D'Antona ${ }^{l}$, M. Fuciarelli ${ }^{1}$, R. Polimanti ${ }^{2,3}$}

${ }^{1}$ Department of Biology, University of Rome Tor Vergata, Rome, Italy, ${ }^{2}$ Department of Psychiatry, Yale University School of Medicine, West Haven, CT, United States, ${ }^{3}$ VA CT Healthcare Center, West Haven, CT, United States

Introduction: There is a well-known European-ancestry bias in genome-wide association studies, where only a minority of them are conducted in non-European populations. To provide novel insight regarding the inter-population diversity of loci associated with complex traits, we integrated genome-wide data from UK Biobank (UKB) and 1,000 Genomes Project $(1 \mathrm{KG})$ data representative of the genetic diversity among worldwide populations.

Materials and methods: We investigated genome-wide data of 4,359 traits of 361,194 UKB participants. Using $1 \mathrm{KG}$ data, we explored the allele frequency differences and linkage disequilibrium (LD) structure across worldwide populations. ENCODE data were used to identify regulatory elements and evaluate the tagging properties of significant variants.

Results: No significant difference was observed in allele frequency between UKB and 1KG GBR. Considering other ancestries, we identified genome-wide significant alleles with frequencies different from what expected by chance: UKB vs. $1 \mathrm{KG}$ EUR without GBR (rs74945666; allele=T [0.908 vs. 0.03], standing height $p=1.48 \times 10^{-17}$ ), UKB vs. $1 \mathrm{KG}$ AFR (rs556562; allele $=\mathrm{A}[0.942$ vs. 0.083], platelet count $p=4.84 \times 10^{-15}$ ), UKB vs. 1 KG AMR (rs1812378; allele $=\mathrm{T}\left[0.931\right.$ vs. 0.089], standing height $p=4.23 \times 10^{-12}$ ), UKB vs. $1 \mathrm{KG}$ EAS (rs55881864; allele $=\mathrm{T}[0.911$ vs. $0.001]$, monocyte count $p=7.29 \times 10^{-13}$ ), and UKB vs. SAS (rs74945666; allele $=\mathrm{T}[0.908$ vs. 0.061], standing height $p=1.48 \times 10^{-17}$ ). LD-structure analysis and computational prediction showed differences in how some of these alleles tag functional elements across human populations.

Conclusions: We provide novel evidence regarding how the human genetic diversity affects GWAS results where certain loci appear to be affected by local adaptation while others may be biased by population stratification.

A. De Lillo: None. S. D'Antona: None. M. Fuciarelli: None. R. Polimanti: None.

\section{E-P24.30}

22q11 microdeletions and -duplications: How are they detected prenatally?

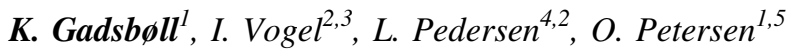

${ }^{1}$ Center for Fetal medicine, Pregnancy and Ultrasound, Copenhagen University Hospital, Rigshospitalet, Copenhagen, Denmark, ${ }^{2}$ Department of Clinical Genetics, Aarhus University/Aarhus University Hospital, Aarhus, Denmark, ${ }^{3}$ Center for Fetal Diagnostics, Aarhus University/Aarhus University Hospital,, Aarhus, Denmark, ${ }^{4}$ Department of Obstetrics and Gynecology, Aarhus University Hospital, Aarhus, Denmark, ${ }^{5}$ Department of Clinical Medicine, University of Copenhagen, Copenhagen, Denmark

Objectives: With chromosomal microarray prevailing in many countries, smaller but disease-causing, chromosomal aberrations are detected. 22q11 aberrations are the most commonly occurring microdeletions and -duplications causing a spectrum of features. We aim to assess whether 
fetuses with $22 \mathrm{q} 11$ aberrations have common features enabling us to detect them from first trimester combined screening (cFTS) parameters.

Methods: From regional databases, all pregnancies in Central Denmark Region were identified between January $1^{\text {st }}, 2008$, and December 31, 2018. Pregnancy data was linked to genetic outcome data and fetuses with $22 \mathrm{q} 11.2$ aberrations (hg19 chr22:18.8mio-23.7mio) were identified and compared to a reference group.

Results: We included 200,991 pregnancies and 12,616 prenatal genetic tests. 42 fetuses were diagnosed with $22 \mathrm{q} 11$ aberrations (23 with microdeletions and 19 with -duplications). One fetus was also diagnosed with Trisomy 21 and excluded from the analysis. Prenatal detection was based on: An elevated risk estimate $(n=20)$, malformations $(\mathrm{n}=14)$, familial predisposition $(\mathrm{n}=5)$, and test after stillbirth $(n=2)$. Compared to the reference group, fetuses with 22q11 aberrations had a significantly larger nuchal translucency (NT), and lower PAPP-A and $\beta$-hCG MoM $(p<0.05)$ but with substantial overlap in the distributions (Figure 1). Risk estimates for Trisomy 13, 18, and 21 were significantly higher for $22 \mathrm{q} 11$.

Conclusion: Of 41 prenatally identified cases with isolated 22q11 microdeletions and -duplications, 20 (49\%) were identified by cFTS in the first trimester. They differed significantly from the reference group on all cFTS parameters except age but with substantial overlap in the distributions, supporting the need for more complex prediction models.

K. Gadsbøll: None. I. Vogel: None. L. Pedersen: None. O. Petersen: None.

\section{E-P24.31}

The new normal for human genetics - scenario prototypes from inductive evidence

\section{T. Vrijenhoek ${ }^{1}$, T. Rigter ${ }^{2,3}$}

${ }^{1}$ Department of Genetics, University Medical Center Utrecht, Utrecht, Netherlands, ${ }^{2}$ Department of Clinical Genetics, Section Community Genetics and Amsterdam Public Health Research Institute, Amsterdam University Medical Center, Vrije Universiteit, Amsterdam, Netherlands, ${ }^{3}$ Centre for Health Protection, National Institute for Public Health and the Environment, Bilthoven, Netherlands

Introduction: As many from our genetics community put great effort in combatting COVID-19, society as a whole prepares for the 'new normal'. We present qualitative preand peri-lockdown data indicating that now is also the time to redefine the contribution of human genetics to a reformed society.
Methods: We interviewed 77 traditional and unconventional actors in human genetics - of which 28 during lockdown - to unravel, characterise and structure the emerging transition. We applied iterative coding to the interview transcripts. Inductive reasoning (grounded theory) revealed transition points, elements of culture, and emerging (adjacent) regime changes as major drivers for the (future) human genetics regime.

Results: Actors unanimously consider clinical genetics as dominant for the structure, culture and practice in today's human genetics. They point out three historic transition points that have shaped this evolution; WWII and its postwar anti-eugenic sentiments, legitimisation of clinical genetics as a medical specialty, and the introduction of next-generation sequencing. The interviews initiate a reflection on how we do research, who we should care for, and where our money comes from. The latest data generated during lockdown - particularly indicate a strong urge to anticipate an emerging transition in human genetics, moving beyond The Diseases Formerly Known As Monogenic (TDFKAM).

Conclusions: Based on the results we present scenario prototypes for the role of human genetics in the context of glocal societies, data-driven governance, hybrid healthcare, and participatory research

T. Vrijenhoek: None. T. Rigter: None.

\section{E-P24.32}

Duplex sequencing uncovers age-related increase in the frequency of de novo indels in mouse mitochondrial DNA

E. Torres-Gonzalez ${ }^{l}$, B. Arbeithuber ${ }^{l}$, J. Hester ${ }^{1}$, M. A. Cremona ${ }^{2}$, N. Stoler ${ }^{l}$, B. Higgins ${ }^{l}$, K. Anthony ${ }^{l}$, F. Chiaromonte ${ }^{1,3}$, F. J. Diaz ${ }^{1}$, K. D. Makova ${ }^{1}$

${ }^{1}$ Pennsylvania State University, University Park, PA, United States, ${ }^{2}$ Laval University, Quebec City, QC, Canada, ${ }^{3}$ Sant'Anna School of Advanced Studies, Pisa, Italy

To study the evolution of mitochondrial DNA (mtDNA), an understanding of the frequency and pattern of mutations, and the potential effect of age on these mutations, are essential. This is especially true for de novo mutations which can be difficult to detect with conventional sequencing techniques due to their high error rates. Duplex sequencing permits the detection of mutations at very low frequencies. Our study examined the frequency of mtDNA de novo insertions and deletions (indels) in mouse germ cells (single and pooled oocytes) and somatic tissues (brain and skeletal muscle). Two groups of mice were included: eight 10-month-old mothers and 28 1-month-old pups. The 
de novo indel frequency in mouse mtDNA was measured to be $1.16 \mathrm{e}-7$ indels per site, 4-fold lower than for de novo substitutions. Most de novo indels were small (1- to 3-bp long), but we also detected events as long as 58 bp. Deletions were more common than insertions. We observed a higher indel frequency at homopolymeric tracts $(\geq 5 \mathrm{bp}$ in length) than in the rest of the mitochondrial genome. We also found a significantly higher frequency of de novo deletions in skeletal muscle of mothers vs. pups, who were separated by only nine months in age from each other. Our results demonstrate the power of duplex sequencing in studying de novo mtDNA mutations, which has allowed us to study their frequency and pattern, as dependent on age, despite such a small difference in age between the studied animals.

E. Torres-Gonzalez: None. B. Arbeithuber: None. J. Hester: None. M.A. Cremona: None. N. Stoler: None. B. Higgins: None. K. Anthony: None. F. Chiaromonte: None. F.J. Diaz: None. K.D. Makova: None.

\section{E-P24.34}

The neuroendocrine $C R H R 1$ gene contributes to depression and type $\mathbf{2}$ diabetes comorbidity in Italian families

L. Del Bosque-Plata ${ }^{1}$, R. Gonzalez-Ramirez ${ }^{2}$, D. Gordon ${ }^{3}$,

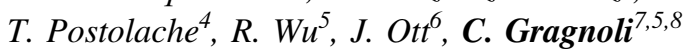

${ }^{1}$ National Institute of Genomic Medicine, Mexico City, Mexico, 2"Dr. Manuel Gea González" General Hospital, Mexico City, Mexico, ${ }^{3}$ Rutgers University, Piscataway, NJ, United States, ${ }^{4}$ University of Maryland, Baltimore, MD, United States, ${ }^{5}$ Penn State College of Medicine, Hershey, PA, United States, ${ }^{6}$ Rockefeller University, New York City, NY, United States, ${ }^{7}$ Thomas Jefferson, Philadelphia, PA, United States, ${ }^{8}$ Molecular Biology Laboratory, Bios Biotech Multi-Diagnostic Health Center, Rome, Italy

MDD increases T2D risk by $60 \%$, independently from antidepressants. The genetics of the MDD-T2D comorbidity is still understudied. Hypercortisolism occurs in MDD and a T2D-patients' subgroup. Given their hypercortisolism, MDD-patients have inappropriately normal cerebrospinalfluid-CRH and plasma-ACTH levels, indicating impaired negative-feedback. The $\mathrm{CRH}$ receptor-1 (CRHR1) may explain the MDD- and T2D-hypercortisolism. CRHRlvariants predisposing to impaired CRHR1-dosage/function may lead to hypercortisolism (substrate for serotonin dysfunction and MDD), and hyperglycemia, insulin resistance, and increased visceral fat, all T2D-traits. CRHRl-variants contribute to MDD. CRHR1 mediates glucose-stimulated insulin-secretion and, if impaired, may lead to T2D. We hypothesize that $C R H R l$-variants may impair the stress and cortisol response, conferring MDD-T2D comorbidity-risk in
MDD-drug naïve subjects. Using 212 Italian families with T2D, enriched T2D-familial history, and MDD, we amplified and tested 163 CRHRI SNPs by two-point parametriclinkage and linkage-disequilibrium (LD) with the models: dominant (D) complete-penetrance (1), D incompletepenetrance (2), recessive (R) 1 and R2 (Pseudomarker). We detected linkage and/or LD to MDD for 98 SNPs/D1, 59 SNPs/D2, 95 SNPs/R1, and 14SNPs/R2, and to T2D for 15 SNPs/D1, 19 SNPs/D2, 12 SNPs/R1 and 10 SNPs/R2. Three independent SNPs are comorbid. We found LDblocks' implications in the comorbidity. In-silico analysis detected functional gene and tridimensional protein changes in a risk-SNP. Ours is the first report of $C R H R l$-linkage/LD in MDD and T2D in T2D-families. CRHRl contribution to MDD appears stronger than and antecedes T2D. Genetically-impaired stress/cortisol response may lead to comorbid MDD-T2D, likely a molecular-clinical entity in a families' subset. Findings should be replicated in other ethnicities.

L. Del Bosque-Plata: None. R. Gonzalez-Ramirez: None. D. Gordon: None. T. Postolache: None. R. Wu: None. J. Ott: None. C. Gragnoli: None.

\section{E-P24.36}

Role of Specific micro-RNAs, Cellular Retinol Binding Protein-I (CRBPI) and Retinoic Acid Receptor (RAR $\alpha)$ in Lung Adenocarcinoma

\section{F. Centofanti, E. Doldo, S. Agostinelli, G. Costanza, M. Pocci, A. Ferlosio, A. Orlandi}

Dep. of Biomedicine and Prevention, Anatomic Pathology Section, Rome, Italy

Lung adenocarcinoma (AC) is one of the most common histological subtypes in the word. Epidermal growth factor receptor (EGFR) is the most common oncogene mutated in lung AC. Cellular Retinol Binding Protein-1 (CRBP1) is a small cytoplasmatic protein regulating retinol bioavailability and transcriptional activities and it is linked to EGFR signalling. Micro-RNAs (miRNAs) are major epigenetic factor that play a key role in the pathogenesis of lung cancer, in the regulation of retinoic acid and are also implicated in Copy Number Variation (CNV). In order to find a possible regulatory mechanism in the pathology of lung $\mathrm{AC}$, we investigated the relationship between CRBP1 and EGFR through the analysis of miRNAs expression. miRNAs, CNV analysis, gene and protein expression were performed in lung $\mathrm{AC}$ patients with high $\left(\mathrm{CRBP} 1^{\mathrm{HIGH}}\right)$ and low (CRBP1 ${ }^{\text {LOW }}$ ) CRBP1 expression, and in adenocarcinoma cells transfected with CRBP1 (CRBP1+ A549 cells). Twenty-three miRNAs were found deregulated in CRBP1+ A549 cells and eight in patients with 
$\mathrm{CRBPI}^{\mathrm{HIGH}}$. Among them, has-miR-148a-3p and has-miR$30 e-5 p$ bind Tumour Suppressor Gene p53 (TP53) gene; has-miR-4286 is located in chromosome 8 regions and it is implicated in microdeletion; has-miR-1915 regulates different cellular pathways and interacts with Retinoic Acid Receptor $(\operatorname{RAR} \alpha)$. Gene expression analysis discloses 21 genes deregulated in CRBP1+A549 cells, with TP53 gene downregulated; while CNV analysis identified single copy deletions and amplifications in 15 chromosomes in lung AC $\mathrm{CRBPI}^{\mathrm{HIGH}}$ patients, with TP53 genes showing 3 copies. Our findings provide new insights concerning a new regulatory mechanism of gene expression in Lung AC.

F. Centofanti: None. E. Doldo: None. S. Agostinelli: None. G. Costanza: None. M. Pocci: None. A. Ferlosio: None. A. Orlandi: None.

\section{E-P24.38}

A Homozygous Splicing Mutation in PDE2A in a Family with Atypical Rett Syndrome.

Z. Haidar ${ }^{1}$, N. Jalkh', S. Corbani ${ }^{1}$, J. Abou-Ghoch ${ }^{1}$, A. Fawaz ${ }^{2}$, C. Mehawej ${ }^{1}$, E. CHOUERY ${ }^{1}$

${ }^{1}$ Saint-Joseph University, Beirut, Lebanon, ${ }^{2}$ Lebanese University, Beirut, Lebanon

Rett syndrome (RTT) is an X-linked dominant neurodevelopmental disease characterized by a normal early postnatal development followed by an arrest of growth. There are two main types of RTT: typical and atypical delineated on the bases of clinical and genetic criteria. In the atypical form, patients present with many of the clinical criteria of RTT but do not essentially have all of the features of the disorder. Dysmorphic features were observed in few RTT cases. Rettlike syndrome is a recently introduced term, used when a patient presents with an overlap of features between typical and atypical RTT. While $M E C P 2$ mutations are linked to the majority of typical RTT cases and some atypical forms, the molecular etiology of many entities remains unelucidated. Two affected individuals from a consanguineous family presenting with atypical Rett phenotype were studied. MECP2 sequencing followed by a strategy combining whole-exome sequencing and chromosomal microarray analysis were performed. MECP 2 gene sequencing ruled out the implication of this gene in the disease of this family. A strategy combining WES data and CMA allowed the identification of a homozygous splice-site mutation (c.323 $+1 \mathrm{G}>\mathrm{A})$ in $P D E 2 A$ in both patients. The mutation segregates with the disease in the family and is predicted to disrupt the 5'-splice site of exon 4 of $P D E 2 A$, which was confirmed by RNA studies. Here we report the second case of $P D E 2 A$ mutations in humans and the first in patients with atypical RTT syndrome. Our findings further support the involvement of PDE2A in the regulation of physiological neurological mechanisms.

Z. Haidar: None. N. Jalkh: None. S. Corbani: None. J. Abou-Ghoch: None. A. Fawaz: None. C. Mehawej: None. E. Chouery: None.

\section{E-P24.39}

Views of adults with $22 q 11$ deletion syndrome on reproductive medicine options

\section{A. Mcneill, M. Freeth}

\section{University of Sheffield, Sheffield, United Kingdom}

The $22 q 11$ deletion syndrome (22q11DS) is one of the most common genomic disorders. $10 \%$ of cases 22q11DS follows an autosomal dominant pattern of inheritance. Several reproductive medicine options are available for adults with 22q11DS who wish to have an unaffected child, chiefly prenatal testing with termination of affected pregnancies or preimplantation genetic diagnosis (PGD). The views of people with 22 q11DS on these options have not previously been studied. An inductive qualitative design using semistructured interviews was used, to explore the views of adults with 22 q11DS on reproductive medicine options. Three overarching themes emerged: 1 . Personal and family impact of $22 q 11 \mathrm{DS}, 2$. Attitudes towards reproductive medicine, and 3. Lack of accessible information. The interviews revealed that 22q11DS has an impact on family life in ways which could influence reproductive decision making. Despite the concerns about the implications of having a child with $22 \mathrm{q} 11 \mathrm{DS}$, there was reluctance to terminate a 22q11DS pregnancy. PGD was viewed more positively, because it did not involve termination. Currently available information resources on reproductive medicine options are not designed for people with intellectual disability, participants identified that current information resources were not accessible to them. Our study highlights the need for health services to have strategies to offer patients with genetic conditions such as 22q11DS information on reproductive medicine options. For example, by designing accessible co-produced information resources, offering access to genetic counselling and developing an informatics infrastructure for identification of individuals with 22q11DS who may benefit from discussion of reproductive options

A. mcneill: None. M. Freeth: None.

\section{E-P24.40}

Outcome of cochlear implantation in NLRP3-related autoinflammatory inner ear disorders 


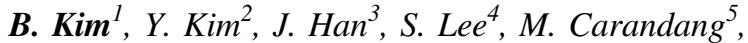

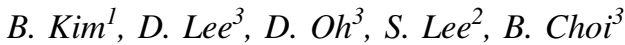

${ }^{1}$ Chungnam National University, Daejeon, Korea, Republic of, ${ }^{2}$ Seoul National University Hospital, Seoul, Korea, Republic of, ${ }^{3}$ Seoul National University Bundang Hospital, Seongnam, Korea, Republic of, ${ }^{4}$ Seoul National University Bundang Hospital, Seoul, Korea, Republic of, ${ }^{5}$ East Avenue Medical Center, Manila, Philippines

Introduction: Hearing loss is a highly penetrant phenotype in NLRP3-related autoinflammatory disorders. A recent study revealed potential medical treatment of hearing loss with anakinra (anti-interleukin 1 receptor antagonist) in selected cases. However, for severely aggravated hearing loss not responsive to anakinra, cochlear implantation (CI) would be the last resort for hearing rehabilitation. Outcome of CI is not clearly elucidated yet in this emerging type of hearing loss related to NLRP3-related autoinflammatory disorders, which has a systemic nature and ubiquitous distribution of resident macrophages involving the spiral ganglion neuron and the cochlear nerve. Here, we report the successful outcome of CI in NLRP3-related autoinflammatory disorder.

Materials and methods: Three subjects representing each type of NLRP3-related autoinflammatory disorder: CINCA (chronic infantile, neurological, cutaneous, and articular) syndrome; MWS (Muckle-Wells syndrome); and DFNA34, who underwent CI for auditory rehabilitation, were recruited. Phenotypes as well as genotypes were reviewed in the subjects and audiologic performances before/after CI over time were evaluated.

Results: All three subjects showed excellent audiological outcomes with rapid improvement of speech perception test result reaching plateau at 3 months after CI, although distribution of the disease predicted poor outcome based on theoretical analysis.

Conclusions: This is the first report to present outcome of CI among subjects with a confirmed NLRP3 genetic etiology and resultant systemic inflammation, and suggests that $\mathrm{CI}$ is a viable treatment option in this disease entity.

Grant references: Ministry of Education (2018R1A2B2001054BYC) Ministry of Health \& Welfare (HI17C0952BYC) SNUBH Research Fund (13-2020013BYC) CNUH Research Fund, 2019 (BJK)

B. Kim: None. Y. Kim: None. J. Han: None. S. Lee: None. M. Carandang: None. B. Kim: None. D. Lee: None. D. Oh: None. S. Lee: None. B. Choi: None.

\section{E-P24.41}

Artificial intelligence for genomic medicine - a policy analysis

\section{S. Raza}

\section{PHG Foundation, Cambridge, United Kingdom}

Introduction: The AI techniques, machine learning and deep learning, offer great potential for advancing genomic medicine. However, their application has yet to generate clearly improved outcomes in clinical genomics, and the discovery potential within genomic datasets remains largely untapped.

Objectives and methods: Our analysis sets out to determine the early stage practical policy actions for capitalising on the opportunities AI presents for genomic medicine, minimising harms and expediting its effective delivery into healthcare. Informed by a combination of literature review and expert interviews, we examine the state of AI applications for genomic medicine and determine the major challenges to realising its full potential.

Results: We identify a number of interconnected issues and barriers to progress, including: the quality and accessibility of AI training data; ethnic bias in genomic datasets; challenges in replicating AI methods and results; fragmentation of skills and infrastructure; data security and privacy concerns; and regulatory uncertainty around requirements and the status of AI algorithms within clinical genomics. The imperative to address these issues is heightened given the fast pace of AI activity in genomics.

We set out seven urgent policy priorities to meeting these challenges. They include taking action to establish the right skills, digital infrastructure, and cross-discipline collaborations; mitigate against algorithmic bias; prioritise AI development for the most pressing and clinically relevant problems; support community driven efforts to benchmark and review new algorithms; and establish the clinical governance arrangements for AI applications in clinical genomics. Action to achieve these priorities is key to harnessing AI.

S. Raza: None.

\section{E-P24.42}

Supporting reproductive decision making for adolescents and young adults with Li-Fraumeni Syndrome: Interprofessional perspectives from a U.S. National Cancer Institute study

A. Werner-Lin ${ }^{1}$, C. Rising ${ }^{2}$, P. Boyd ${ }^{2}$, C. Wilsnack ${ }^{2}$, A. Sleight ${ }^{2}$, S. P. Hutson ${ }^{3}$, S. A. Savage ${ }^{2}$, P. P. Khincha ${ }^{2}$

${ }^{1}$ University of Pennsylvania, Philadelphia, PA, United States, ${ }^{2}$ National Cancer Institute, Rockville, MD, United 
States, ${ }^{3}$ University of Tennessee, Knoxville, TN, United States

Introduction: Li-Fraumeni Syndrome (LFS) is a rare genetic condition characterized by lifetime cancer risk approaching $100 \%$, common pediatric onset, and limited data to support a clear management strategy beyond intensive cancer screening. For adolescents and young adults (15-39 years) with LFS, the inability to predict when cancer may develop or determine prognoses contribute to substantial uncertainty regarding how or whether to pursue family formation.

Materials and methods: An interdisciplinary team, including medicine, social work, health communication, psychology, occupational science, and nursing developed and conducted IRB-approved, in-depth interviews with 38 AYA individuals with LFS. Most participants were female $(n=26)$ and white $(n=31)$. Interviews were recorded, transcribed, and analyzed using modified grounded theory analysis.

Results: Reproductive concerns evolved based on age, cancer diagnostic and treatment history, relationship status, and caregiving responsibilities, and contributed prominently to LFS-related distress. Participants balanced developmental readiness with pressured timelines to achieve family formation goals and survivorship concerns. Decisions regarding fertility preservation, pre-implantation genetic diagnosis, or adoption were intertwined with identity, divergent spiritual or family beliefs, financial resources, and ongoing cancer worry. Limited provider guidance or referrals for reproductive or mental health consultation caused frustration and dissatisfaction with care.

Conclusions: Optimal LFS care for AYAs must include providers trained to support developmental transitions over time, specifically regarding family formation contextualized in lifelong cancer risk and survivorship. Established interprofessional partnerships can streamline referrals and enable holistic, collaborative care. Mental health providers with expertise in rare disease can treat distress and support decision-making that pairs medical recommendations with developmental readiness.

A. Werner-Lin: None. C. Rising: None. P. Boyd: None. C. Wilsnack: None. A. Sleight: None. S.P. Hutson: None. S. A. Savage: None. P.P. Khincha: None.

\section{E-P24.43}

Hypoxia-induced câncer stem cell accumulation in primary cell culture of head and neck squamous cell carcinoma

\author{
M. A. S. Bezerra, L. A. M. Ferreira,
}

R. S. Kawasaki-Oyama, C. I. Cuzziol,
M. C. A. Nascimento, M. M. U. Castanhole-Nunes, É. C. Pavarino, J. V. Maniglia, E. M. Goloni-Bertollo

São José do Rio Preto Medical School, São José do Rio Preto, Brazil

Introduction: Hypoxia is one of the essential biological phenomena inherent in aggressiveness of a diversity solid tumors. Reduction of $\mathrm{O}_{2}$ can modify tumor microenvironment influencing the tumor development and progression and maintenance of cancer stem cells (CSC). Thus, our aimed was induce CSC accumulation in head and neck squamous cell carcinoma (HNSCC) through hypoxia.

Materials and methods: Three samples of HNSCC were obtained after surgical resection. They were cultured in DMEM with $10 \% \mathrm{FBS}$ and $1 \% \mathrm{AB} / \mathrm{AM}$, at $37^{\circ} \mathrm{C}$ and $5 \%$ $\mathrm{CO}_{2}$. Approximately $2 \times 10^{6}$ cells were subjected to hypoxia $\left(2 \% \mathrm{O}_{2}\right)$ for 12 hours at $37^{\circ} \mathrm{C}$ using hypoxia chamber. Cells were immunophenotyped by flow cytometry using CD44/ CD133/ALDH1 markers immediately after hypoxia and after 45 days of cultivation. The presence of CSC was confirmed by invasion, migration and tumor sphere formation assays. ALDH1, CD44 and CD133 gene expressions were determined by qRT-PCR. Normoxic cells were used as control.

Results: Immunophenotyping showed that 45 days after hypoxia was 1.5 times more effective in CSC accumulation. The presence of CSCs was confirmed by migration, invasion $(\mathrm{p}<0.0001 ; \mathrm{p}<0.0001$, respectively) and tumor sphere formation assays (hypoxic cells formed more spheroids). Gene expression data are summarize in Table 1.

Conclusions: We demonstrate hypoxia-induced CSC accumulation. This procedure will help the study of these rare cells of great importance in oncogenesis process

Table $1-A L D H 1, C D 44$ and $C D 133$ gene expressions

\begin{tabular}{lccr}
\hline Sample & & RQ & \\
& ALDH1 & CD44 & CD133 \\
\hline 1 & 36.6 & 22.8 & 5.3 \\
2 & 13.9 & 24.3 & 167.2 \\
3 & 1.6 & 2.0 & 0.7 \\
\hline
\end{tabular}

Grants: FAPESP (2016/20087-1 and 2018/26166-6).

M.A.S. Bezerra: None. L.A.M. Ferreira: None. R.S. Kawasaki-Oyama: None. C.I. Cuzziol: None. M.C.A. Nascimento: None. M.M.U. Castanhole-Nunes: None. É. C. Pavarino: None. J.V. Maniglia: B. Research Grant (principal investigator, collaborator or consultant and pending grants as well as grants already received); Significant; Fundação de Amparo à Pesquisa do Estado de 
São Paulo (FAPESP). E.M. Goloni-Bertollo: B. Research Grant (principal investigator, collaborator or consultant and pending grants as well as grants already received); Significant; Fundação de Amparo à Pesquisa do Estado de São Paulo (FAPESP).

\section{E-P24.44}

Breast cancer subtypes and predictive factors from a NorthCyprus Cohort Analysis

\section{A. Ulgen}

Department of Epidemiology, Mailman School of Public Health, Columbia University, New York, NY, United States

We present a first epidemiological survey from North Cyprus to determine predictive factors for breast cancer subtypes. More than 300 breast cancer patients with subtype information are surveyed from the State Hospital in Nicosia between 2006-2015 for their demographic, reproductive, genetic, epidemiological factors. The breast cancer subtypes, Estrogen receptor (ER) +/-, Progesterone receptor (PR) +/-, and human epidermal growth factor 2 (HER2) $+/$ - status, are determined. Single and multiple variable, regularized regressions, with predictive factors as independent variables, breast cancer subtypes as dependent variables are conducted. Our cohort differs significantly from larger cohorts (eg Breast Cancer Family Registry), in age, menopause status, age of menarche, parity, education, oral contraceptive use, breast feeding but the distribution of breast subtypes is not significantly different. Subtype distribution in our cohort is also not different from another Turkish cohort. We show that the $\mathrm{ER}+$ subtype is positively related to post-menopause and negatively associated with hormone therapy; $\mathrm{ER}+/ \mathrm{PR}+$ is positively associated with age and breast feeding, and negatively associated with cancer grade and having first-degreerelative with cancer. HER $2+$, which is negatively correlated with $\mathrm{ER}+$ and $\mathrm{ER}+/ \mathrm{PR}+$, is positively related to having firstdegree-relative with cancer, and negatively associated with post-menopause. Regressions identify older age to be positively correlated to $\mathrm{ER}+$ and $\mathrm{ER}+/ \mathrm{PR}+$, negatively correlated to HER2+. Assuming ER+ and $\mathrm{ER}+/ \mathrm{PR}+$ to have better prognostic, post-menopause and breast-feeding are beneficial, hormone therapy treatment is detrimental. Next steps include biomarkers and cure models to determine longterm survivors

Funding:Fulbright Visiting Research Scholarship Grant by the US Department of State

A. Ulgen: None.

\section{E-P24.45}

Revealing novel splicing mutations in RAB3GAP1 gene causing Warburg Micro syndrome and a case including microduplication of $3 q 29$
B. B. Geckinli ${ }^{1}$, A. Turkyilmaz ${ }^{2}$, C. Alavanda ${ }^{1}$, H. Taslidere ${ }^{3}$, G. Sager ${ }^{4}$, E. Arslan Ates ${ }^{5}$, M. A. Soylemez ${ }^{1}$, A. Arman ${ }^{1}$

${ }^{1}$ Marmara University School of Medicine, Department of Medical Genetics, Istanbul, Turkey, ${ }^{2}$ S.B.U Erzurum Regional Training and Research Hospital, Department of Medical Genetics, Erzurum, Turkey, ${ }^{3}$ S.B.U Istanbul Haseki Training and Research Hospital, Genetic Diseases Diagnostic Center, Istanbul, Turkey, ${ }^{4}$ Marmara University School of Medicine, Department of Pediatrics, Istanbul, Turkey, ${ }^{5}$ Marmara University Pendik Training and Research Hospital, Department of Medical Genetics, Istanbul, Turkey

Introduction: Warburg Micro syndrome type1 (WARBM1) is a rare autosomal recessive syndrome caused by homozygous mutation in the RAB3GAP1 gene. It is characterized by microcephaly, microphthalmia, congenital cataracts, optic atrophy, cortical dysplasia, mental retardation and hypogonadism. We are reporting three cases with two novel splicing mutations and one including microduplication of 3q29.

Materials: Case 1 is a 8-year-old female with neuromotor retardation and spasticity. Parents were consanguineous having 3 affected children. She had microphthalmia, nystagmus, bilateral optic atrophy, operated congenital cataracts and corpus callosum hypoplasia. Case 2 is a sixmonth old male with microcephaly, microphthalmia, congenital cataracts, diffuse cerebral atrophy and cryptorchidism. Case 3 is a 21-month-old female having neuromotor retardation, hypotonia and epilepsy. She had microphthalmia, congenital cataracts, cerebral atrophy and corpus callosum hypoplasia.

Methods: RAB3GAP1 gene was analyzed by DNA sequencing. In Case 2 mRNA was isolated to generate to cDNA by reverse transcription, and exon 7-9 were analyzed.

Results: In case 1 homozygous c.974-2A $>\mathrm{G}$ novel splicing mutation was detected in two sisters tested. In case 2 homozygous c. $748+1 \mathrm{G}>\mathrm{A}$ mutation was identified. cDNA analysis of exon 7-9 showed exon 8 skipping. In case 3 homozygous c. $2606+1 \mathrm{G}>\mathrm{A}$ novel splicing mutation was detected. A-CGH analysis revealed $1.7 \mathrm{Mb}$ microduplication of 3q29.

Conclusions: Novel splicing mutations in case 1 at 11 . intronic splice site and in case 3 at 22. intronic splice site probably affects splicing where functional studies are planned. Functional study in case 2 showed exon 8 skipping. Case 3 is the first reported case with WARBM1 and microduplication of $3 \mathrm{q} 29$. 
B.B. Geckinli: None. A. Turkyilmaz: None. C. Alavanda: None. H. Taslidere: None. G. Sager: None. E. Arslan Ates: None. M.A. Soylemez: None. A. Arman: None.

\section{E-P24.46}

\section{Haplotype landscapes of genes associated with gallstone} formation

\section{O. K. Clay, S. A. Dingsdag, G. A. Quintero}

Translational Microbiology and Emerging Diseases (MICROS), School of Medicine and Health Sciences, Universidad del Rosario, Bogotá, Colombia

Introduction: Gallstone disease or cholelithiasis, resulting from gallstone formation/growth, is a costly and common health problem. Large prevalence differences exist among age groups, geographic locations or ethnic backgrounds including between men and women. Thus, in Native Americans from North America, $64.1 \%$ of women and $29.5 \%$ of men were reported to have gallstones. A genetic component is established, although individual common variants (including a variant of the $A B C G 8$ gene responsible for hepatic cholesterol secretion) appear to have attributable risks less than 10\%. Environment, including diet, and microbiota are also relevant factors. In parallel to phenotypic studies, corresponding genes and possible effects of their common variation have been identified (e.g., Weber et al., 2019, Curr. Opin. Gastroenterol. 35:82-87; Bustos et al., 2019, Sci. Rep. 9:772).

Materials and methods: Using data from the 1000 Genomes Project spanning populations of all continents, we performed a global analysis of haplotypes and haplotype blocks containing genes that are associated with increased cholelithiasis risk. Restriction of attention to biallelic SNPs with MAFs above 3-5\% facilitates the recognition, analysis and interpretation of haplotype blocks and haplotype classes.

Results: Full analysis, across world populations, of the haplotype landscapes in which gallstone loci are embedded allows association findings to be integrated with results for the genes/proteins, and facilitates interpretation at both SNP and haplotype-block resolutions.

Conclusions: The analyses presented represent a step toward assembling a concerted, haplotype-based view of how common variation in human genes may promote or prevent gallstone formation and disease.

Funding: Universidad del Rosario, intramural grant (2019-2022).

O.K. Clay: None. S.A. Dingsdag: None. G.A. Quintero: None.

\section{E-P24.47}

Differences in the frequency of CRC-associated pathogen variants in colorectal adenomas and cancers from the Hungarian population

\section{A. Kalmar ${ }^{l}$, O. Galamb ${ }^{1}$, G. Szabo ${ }^{2}$, B. Bartak ${ }^{3}$, Z. Nagy, S. Zsigrai ${ }^{3}$, K. Szigeti ${ }^{3}$, O. Pipek ${ }^{4}$, A. Medgyes-Horváth ${ }^{4}$, I. Csabai $^{4}$, Z. Tulassay ${ }^{1}$, P. Igaz ${ }^{1}$, B. Molnar ${ }^{1}$}

${ }^{1}$ Semmelweis University, 2nd Department of Internal Medicine; Hungarian Academy of Sciences, Molecular Medicine Research Unit, Budapest, Hungary, ${ }^{2}$ Hungarian Academy of Sciences, Molecular Medicine Research Unit, Budapest, Hungary, ${ }^{3}$ Semmelweis University, 2nd Department of Internal Medicine, Budapest, Hungary, ${ }^{4}$ Department of Physics of Complex Systems, Eötvös Loránd University, Budapest, Hungary, Budapest, Hungary

Colorectal cancer (CRC) has high incidence in EasternEurope, especially in Hungary. In addition to environmental factors, the genetic and epigenetic background of this phenomenon still remains unknown. We aimed to perform whole exome sequencing along the colorectal adenomacarcinoma sequence on tissue samples in order to identify characteristic germline and somatic variants. Genomic DNA was isolated from 27 adenomas (AD), 52 CRC, 18 healthy patients $(\mathrm{N})$ and from 2 colorectal cancer cell lines. Exome enrichment was done with the Nextera DNA Exome Kit and sequencing was performed using the NextSeq 500 Instrument (Illumina). Germline and somatic variants were determined by Genome Analysis Toolkit (GATK) and MuTect2 algorithms and were confirmed by ddPCR and in situ hybridization. Certain CRC-associated pathogenic variants could be detected in a relatively lower [e.g. APC $63.46 \%)$ ], or with relatively higher frequency compared to the European statistics [e.g. KRAS 40.38\%]. Among the germline variants of the one-carbon metabolism, MTHFR A1298C, MTR A2756G and MTRR A66G variants were detected with a relatively higher frequency in the Hungarian population compared to the European average. Alterations in the frequencies of CRC-associated somatic variants compared to the European averages might contribute to the high CRC incidence observed in the Hungarian population. The germline variants of one-carbon metabolism were observed with a relatively higher frequency in our cohort having an effect on the DNA methylation regulation system that might be a link between the genetic and epigenetic alterations of the CRC development.

A. Kalmar: None. O. Galamb: None. G. Szabo: None. B. Bartak: None. Z. Nagy: None. S. Zsigrai: None. K. Szigeti: None. O. Pipek: None. A. Medgyes-Horváth: None. I. Csabai: None. Z. Tulassay: None. P. Igaz: None. B. Molnar: None. 


\section{E-P24.48}

Longitudinal follow up on individuals with SNORD118 related Leukoencephalopathy with Calcification and Cysts illustrates a complex structure function interaction.

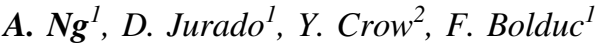 \\ ${ }^{1}$ University of Alberta, Edmonton, AB, Canada, ${ }^{2} M R C$ Institute of Genetics and Molecular Medicine, Edinburgh, United Kingdom}

Mutations in SNORD118 have recently been linked to leukoencephalopathy with calcifications and cysts. Our longitudinal follow up of 2 individuals with SNORD118 pathogenic variants illustrates the complexity of the disorder, suggesting seizures may be observed at different time point in relation to calcification and that clinical presentation may be not be directly correlated with lesion burden on MRI. Indeed, we observed that before MRI lesions became visible, the 2 sibliings had transient clinical seizure which resolved until recently and now became co-localized with MRI changes. In addition, we observed significant intrafamilial variability in clinical and MRI changes. Despite having the same mutation, the younger sibling had a more severe phenotype. Nonetheless, both siblings had similar EEG findings of left focal epileptiform abnormalities, with focal clinical seizures. MRI brain showed coarse calcification in basal ganglia, thalami, white matter and dentate nuclei. Our findings raise the importance of considering SNORD118 in patients with calcification but also with early onset seizure.

A. Ng: None. D. Jurado: None. Y. Crow: None. F. Bolduc: None.

\section{E-P24.49}

diagnosis of phosphoglycerate dehydrogenase deficiency by untargeted metabolomic analysis in plasma

\section{A. Lizcano-Gil ${ }^{1}$, M. A. Luna-Luna ${ }^{2}$}

${ }^{1}$ Genetica Humana, Bogotá, Colombia, ${ }^{2}$ Virrey Solis, Bogotá, Colombia

Introduction: Phosphoglycerate dehydrogenase deficiency is a rare inborn error of serine metabolism that causes severe neurological abnormalities.

Objective: We describe the usefulness of metabolomic analysis patients with the severe form of this condition, whom usually present symptoms during the first weeks of life.

Methods: We present the case of a five months Colombian patient, who presented focal symptomatic refractory epilepsy, hypotonia and bilateral cataracts. Metabolic studies showed normal or non-conclusive results. By the time when the metabolomic test was considered, the clinical condition was critical, with severe hypotonia, refractory seizures, and sepsis, and finally, the patient dies. Analysis by non-targeted metabolomics in plasma was performed (Metabolon Inc.) This test identifies molecules between 50-1,500 Daltons (Da). The identification is performed using four different types of high-performance Ultra Performance Liquid Chromatography (UPLC) instruments paired with Mass Spectrometry (UPLC/MS). The identification of each molecule is confirmed against a proprietary chemical library consisting of accurate molecular weight/mass plus information on any adductation, in source fragmentation, and/or polymerization, retention time/ index on the chromatography columns, and mass spectral fragmentation patterns.

Results: Metabolomic analysis demonstrated low levels of glycine, lactate, and serine. Phosphoglycerate dehydrogenase deficiency was considered, because all the biomarkers associated with the metabolomic biochemical signature of this disorder were outside the expected range.

Conclusion: Metabolomic analysis is useful for the diagnosis of Phosphoglycerate Dehydrogenase Deficiency, and it is also a valuable tool for the study of patients with suspected metabolic disease.

L.A. Lizcano-Gil: None. M.A. Luna-Luna: None.

\section{E-P24.50}

Genetic study on the patients with refractory rickets

B. Marik, A. Bagga, A. Sinha, P. Khandelwal, P. Hari, A. Sharma

All India Institute of Medical Sciences (AIIMS), New Delhi, New Delhi, India

Introduction: Refractory rickets (RR) is a heterogenous genetic disorder caused by mutations in genes encoding proteins involved in renal phosphate reabsorption process and vitamin $\mathrm{D}$ metabolism.

Methods: Clinical, radiological and pedigree information was noted from 84 patients with RR. Whole exome sequencing was performed with application of Ingenuity Variant Analysis tool and ACMG 2015 criteria for the identification of causal variants.

Results: Eighty-four patients (45 females; 39 males) belonged to 66 families with disease onset at $2.8+1.3$ years. Sixty-six different genetic variations in 84 patients have been identified, of which, 24 were PHEX, two ENPP1, three $C L C N 5$, three $C T N S$, six $S L C 2 A 2$, seven $S L C 4 A 1$, four $A T P 6 V 1 B 1$, five $C Y P 27 B 1$, four $V D R$ and one $F G F 23$, DMPI, SLC34AI, EHHADH, GATM, HRAS and FGFRI 
each. Among them, 30 were novel and 36 were previously reported. On the basis of the genetic variations identified, the patients of RR were classified as- Hypophosphatemic rickets (PHEX, FGF23, DMP1 and ENPP1; $\mathrm{n}=40 ; 48 \%$ ); Dent disease $(C L C N 5 ; \mathrm{n}=3,4 \%)$; Cystinosis $(C T N S ; \mathrm{n}=4$; $5 \%)$; Fanconi-Bickel syndrome (SLC2A2; $\mathrm{n}=5,6 \%)$; Fanconi renotubular syndrome (SLC34Al $\mathrm{n}=1,1 \%$; EHHADH; $\mathrm{n}=2,2 \%$;); Renal Fanconi syndrome GATMrelated $(G A T M ; \mathrm{n}=1,1 \%)$; Distal renal tubular acidosis (SLC4A1, ATP6VIB1 and ATP6VOA4; $\mathrm{n}=15 ; 18 \%$ ); Vitamin D dependent rickets type 1A (VDDR1A) (CYP27B1; $\mathrm{n}=3,4 \%) ;$ VDDR2A (VDR; $\mathrm{n}=7,8 \%)$; Parathyroid adenoma $(H R A S ; \mathrm{n}=1 ; 1 \%)$ and Osteoglophonic dysplasia (FGFRI; $\mathrm{n}=2,2 \%)$.

Conclusion: This is the first study in India on the genetic basis of RR and it suggests that hypophosphatemic rickets is the chief form of RR in Indian children.

B. Marik: None. A. Bagga: None. A. Sinha: None. P. Khandelwal: None. P. Hari: None. A. Sharma: None.

\section{E-P24.52}

SLC29A3 founder mutation and founder haplotype shared by $\mathrm{H}$ syndrome Tunisian patients

H. Chouk $^{1,2}$, M. Ben rejeb ${ }^{3}$, Y. Halleb ${ }^{2}$, H. El mabrouk ${ }^{1,2}$, M. Ben jomaa 2 , S. Nadjar ${ }^{2}$, M. Denguezli $i^{3}$ A. SAAD ${ }^{2}$, L. Boussofara ${ }^{3}$, D. Hmida ${ }^{2}$

${ }^{1}$ Higher Institut of Biotechnology of Monastir, Sousse, Tunisia, ${ }^{2}$ Laboratory of Cytogenetics, Molecular Genetics and Biology of Human Reproduction, Sousse, Tunisia, Sousse, Tunisia, ${ }^{3}$ Service of Dermatology, Farhat Hached Hospital, Sousse, Tunisia, Sousse, Tunisia

Introduction: $\mathrm{H}$ syndrome and Rosai-Dorfman disease (RDD) are rare autosomal recessive genodermatoses with multisystemic manifestations mainly caused by mutations in the SLC29A3 gene which encodes the equilibrative nucleoside transporter hENT3.

Materials and methods: Mutations analysis of SLC29A3 gene, was performed, using direct Sanger sequencing, for three unrelated Tunisian family with phenotypic overlap, associating features of RDD and $\mathrm{H}$ syndrome.

Results: In this study, molecular analysis revealed the presence of a known homozygous missense mutation c.1088G > A: p.Arg363Gln in 6 patients, 5 of whom were related. A heterozygous compound mutation c. $300+1 \mathrm{G}>$ A / 1088G $>$ A was also noted in a patient with $\mathrm{H}$ syndrome. Five of our patients carrying the same mutation c.1088G $>A$ and belonging to the same family have presented with distinct clinical manifestations, indeed 4 were carriers of the $\mathrm{H}$ syndrome, whereas the 5th patient was diagnosed with
RDD. The analysis of the haplotype of patients originated from the center and south of Tunisia is considered to determine if it is a founding haplotype. We carried out a haplotype-based analysis by way of genotyping 5 microsatellite markers flanking the SLC29A3 gene spanning a region of $2 \mathrm{Mb}$. Microsatellite markers analysis in all affected sibling indicates that all c.1088G $>$ A mutation carriers harbored the same haplotype, indicating common ancestor.

Conclusions: This finding could be explained by the high rate of consanguinity in our country. Other factors, genetic, epigenetic and/or environmental may have a role in determining intra and interfamilial variation in tissue involvement and severity of the disease.

H. Chouk: None. M. Ben rejeb: None. Y. Halleb: None. H. El mabrouk: None. M. Ben jomaa: None. S. Nadjar: None. M. Denguezli: None. A. Saad: None. L. Boussofara: None. D. Hmida: None.

\section{E-P24.53}

Feasibility of Identifying Familial Hypercholesterolaemia in Malaysian Primary Care

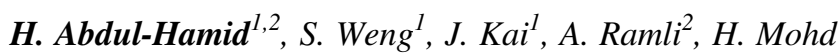
Nawawi $^{2}$, N. Qureshi ${ }^{l}$

${ }^{1}$ University of Nottingham, Nottingham, United Kingdom, ${ }^{2}$ Universiti Teknologi MARA, Selangor, Malaysia

Introduction: Familial Hypercholesterolaemia (FH) identification has been low worldwide, including in Malaysia. Primary care as initial contact for patients can play a vital role in the identification of individuals with $\mathrm{FH}$

Methods: Patients attending two Primary Care Clinics in Malaysia between July to December 2019 were recruited to the study. Consented patients were required to fill in sociodemographic and family history questionnaires, and patients' lipid profile were obtained from their clinical notes. Patients' risk of FH were calculated using Simon Broome(SB), Dutch Lipid Clinic Criteria(DLCC) and FAMCAT criteria. Patients with high risk of $\mathrm{FH}(\mathrm{SB}$ possible and definite, DLCC $\geq 6$, and FAMCAT $>1$ in 200) were then referred to the Specialist Lipid Clinic for confirmation through genetic testing.

Results: 1,191 patients were approached during the study period, out of which $637(53.48 \%)$ patients agreed to participate. Only 619 (51.97\%) patients met the eligibility criteria. $65(5.46 \%)$ patients had high risk of $\mathrm{FH}$ and required referral to Lipid Clinic, however, only 52 (4.37\%) patients agreed to be referred. Among the 619 patients recruited, the mean age was 58.09 (SD 12.44), male 310 (50.08\%), majority is of Malay ethnicity $(542,87.56 \%$ ), married $(548,88.53 \%)$, and had tertiary education (297, 
$47.98 \%$ ). The mean lipid parameters for these patients (in mmol/L) were: TC: 5.34 (SD 1.29); LDL-C: 3.27 (SD 1.16); triglycerides: 1.66 (SD 1.10); and HDL-C: 1.34 (0.34).

Conclusions / Implications:Recruitment of patients for FH assessment in Malaysian primary care setting is feasible. Increased training and awareness among the physicians and public are vital to increase identification.

H. Abdul-Hamid: None. S. Weng: None. J. Kai: None. A. Ramli: None. H. Mohd Nawawi: None. N. Qureshi: None.

\section{E-P24.54}

\section{Porokeratosis of Mebelli in the Tunisian population}

H. El Mabrouk ${ }^{1,2}$, H. Chouk $k^{1,2}$, M. Denguezli ${ }^{3,4}$, A. Saad $^{3,2}$, L. Boussofara ${ }^{3,4}$, D. H'mida ${ }^{3,2}$

${ }^{1}$ Higher Institute of Biotechnology, Monastir, Tunisia, ${ }^{2}$ Laboratory of Human Cytogenetics, Molecular Genetics and Reproductive Biology, Farhat Hached University Hospital, Sousse, Tunisia, ${ }^{3}$ Faculty of Medicine Ibn El Jazzar, Sousse, Tunisia, ${ }^{4}$ Department of Dermatology and Venerology, Farhat Hached University Hospital, Sousse, Tunisia

Introduction: Porokeratosis (PK) is a rare group of acquired or hereditary genodermatoses characterized by an epidermal differentiation disorder and sharing common histology. Their etiopathogenesis still not fully understood. To date, several genes such as PMVK, MVK, MVD and FDPS, members of the mevalonate pathway, involved in PK are identified. To date, several phenotypic variants of $\mathrm{PK}$ are described and rare are the genetic studies carried out in Tunisia.

Materials and methods: $5 \mathrm{PK}$ patients belonging to a cohort of 3 families from central Tunisia were sequenced for $P M V K$ and $M V K$ genes by the Sanger technique.

Results: Direct sequencing provides genetic evidence of PK. We identified a known nonsense variant, c.412C $>\mathrm{T}$ at a heterozygous state in exon 4 of the $P M V K$ gene in 4 patients. A second synonym variant, newly described, c.147A $>\mathrm{G}$ was identified at a heterozygous state in exon 2 of the same gene in 3 patients with porokeratosis of Mibelli (PM) and 3 of their relatives.

Discussion: The same mutation noticed among our patients, members of 2 unrelated families from 2 different Tunisian regions, very likely a founder mutation in our population. The newly described synonym variant found exclusively in our PM patients, could be a modifier gene.

Conclusion: We report the first genetic study of PK in the Tunisian population. Given the phenotypic heterogeneity of our patients, further molecular investigations are required.

H. El Mabrouk: None. H. Chouk: None. M. Denguezli: None. A. Saad: None. L. Boussofara: None. D. H'mida: None.

\section{E-P24.56}

Smith-Lemli-Opitz: About 1 case

\section{Ben Jemaa}

\section{Farhat Hached Hospital, Sousse, Tunisia}

Introduction: Smith-Lemli-Opitz syndrome (SLO) is a rare autosomal recessive disease caused by mutations in DHCR7 gene, resulting in deficiency of 7-dehydrocholesterol reductase (7-DHC) enzyme. Clinical phenotype is heterogeneous ranging from dysmorphia with behavioral disorders, to severe malformations.

Patients and methods:Our patient is dysmorphic with hands polydactyly, syndactyly of the 2-3e toes bilaterally, feet deformation, bilateral cataract, pyloric stenosis, horseshoe kidneys, micropenis, hypospadias, microcephaly, growth and psychomotor delay. Cholesterolemia is lowered and 7DHC is raised.

Results: The karyotype was normal. Molecular analysis of DHCR7 gene revealed the presence of compound heterozygous mutation c. $278 \mathrm{C}>\mathrm{T}$ : p.Thr93Met and c.724 C> T: p.Arg242Cys respectively in exon 4 and 7 . The diagnosis of SLO has been confirmed, the parents have been reconvened for genetic counseling and a proposal for a DPN, and cholesterol and simvastatin supplementation has been suggested to the pediatrician.

Discussion: Over 130 different DHCR7 mutations have been identified, such as c.964-1G $>\mathrm{C}$ responsible for $28 \%$ of SLOS, p.T93M (10\%), p.W151X (6\%), p.R404C (5\%) and p.V326L (5\%). Sequencing of exons 6-9 identifies approximately $85 \%$ of DHCR7 mutations. Sterol analysis demonstrating elevated blood or tissue 7DHC levels confirms SLO diagnosis. Mutation analysis is used to resolve borderline biochemical results. In rare cases, sterol analysis of fibroblasts or lymphoblasts are used. These techniques can be performed on amniotic fluid for prenatal diagnosis.

Conclusion: SLO is a metabolic disease with various phenotypes and weak genotype-phenotype correlation. Biochemical and molecular tests are important for the diagnosis, and eventually for the decision of cholesterol supplementation.

M. Ben Jemaa: None. 